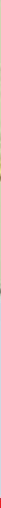

\title{
IntechOpen
}

\section{Advances in Bioengineering}

Edited by Pier Andrea Serra
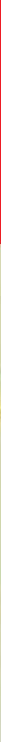



\section{ADVANCES IN BIOENGINEERING}

Edited by Pier Andrea Serra 


\section{Advances in Bioengineering}

http://dx.doi.org/10.5772/58642

Edited by Pier Andrea Serra

\section{Contributors}

Rodica-Mariana Ion, Rino Morent, Gaelle Aziz, Nathalie De Geyter, Toru Yazawa, Michelle Yongmei Wang, Chris Zwilling, Shlomo Margel, Michal Kolitz-Domb, Pieter Cools, Farzad Asgarian, Mohsen Judy, Alireza Akhavian, Gaofeng Zhou, Ifana Mahbub, Syed Kamrul Islam, Farhan Quaiyum, Md Sakib Hasan, Mauro C. Moreira, Eduardo Bauer, Yasmim Guterres, Alexandre Campos, Yu Nagase, Yosuke Okamura, Mario Letelier, Jaroslav Turánek, Zuzana Kauerova, Robert Lukáč, Josef Mašek, Štěpán Koudelka, Andrew Miller, Milan Raška

\section{(c) The Editor(s) and the Author(s) 2015}

The moral rights of the and the author(s) have been asserted.

All rights to the book as a whole are reserved by INTECH. The book as a whole (compilation) cannot be reproduced, distributed or used for commercial or non-commercial purposes without INTECH's written permission.

Enquiries concerning the use of the book should be directed to INTECH rights and permissions department (permissions@intechopen.com).

Violations are liable to prosecution under the governing Copyright Law.

\section{(c) BY}

Individual chapters of this publication are distributed under the terms of the Creative Commons Attribution 3.0 Unported License which permits commercial use, distribution and reproduction of the individual chapters, provided the original author(s) and source publication are appropriately acknowledged. If so indicated, certain images may not be included under the Creative Commons license. In such cases users will need to obtain permission from the license holder to reproduce the material. More details and guidelines concerning content reuse and adaptation can be foundat http://www.intechopen.com/copyright-policy.html.

\section{Notice}

Statements and opinions expressed in the chapters are these of the individual contributors and not necessarily those of the editors or publisher. No responsibility is accepted for the accuracy of information contained in the published chapters. The publisher assumes no responsibility for any damage or injury to persons or property arising out of the use of any materials, instructions, methods or ideas contained in the book.

First published in Croatia, 2015 by INTECH d.o.o.

eBook (PDF) Published by IN TECH d.o.o.

Place and year of publication of eBook (PDF): Rijeka, 2019.

IntechOpen is the global imprint of IN TECH d.o.o.

Printed in Croatia

Legal deposit, Croatia: National and University Library in Zagreb

Additional hard and PDF copies can be obtained from orders@intechopen.com

Advances in Bioengineering

Edited by Pier Andrea Serra

p. cm.

ISBN 978-953-51-2141-1

eBook (PDF) ISBN 978-953-51-6378-7 


\section{We are IntechOpen, \\ the world's leading publisher of Open Access books}

Built by scientists, for scientists

\section{$3,800+$}

Open access books available

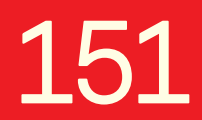

Countries delivered to

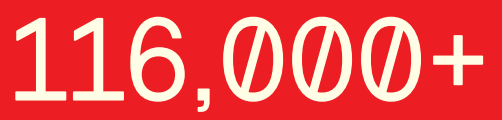

International authors and editors
$120 \mathrm{M}+$

Downloads

Our authors are among the

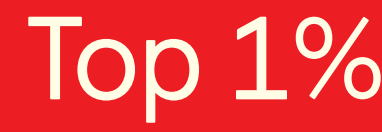

most cited scientists

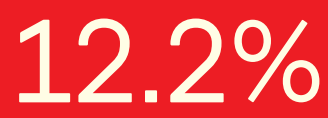

Contributors from top 500 universities

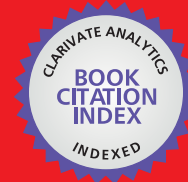

WEB OF SCIENCE ${ }^{\mathrm{TM}}$

Selection of our books indexed in the Book Citation Index in Web of Science ${ }^{\mathrm{TM}}$ Core Collection (BKCI)

Interested in publishing with us?

Contact book.department@intechopen.com

Numbers displayed above are based on latest data collected.

For more information visit www.intechopen.com

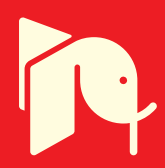





\section{Meet the editor}

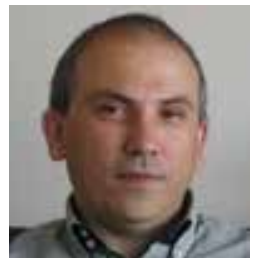

Pier Andrea Serra is Associate Professor of Pharmacology at Medical School at the University of Sassari. He received his degree as Medical Doctor at Sassari University and studied the Neurochemistry of Parkinson's Disease using microdialysis and voltammetry. Prof. Serra received his $\mathrm{PhD}$ in Pharmacology and obtained a Postdoctoral Fellowship at the University College of

Dublin and at National University of Maynooth (Ireland). During last year, Professor Serra worked as Visiting Professor in the Laboratory of Sleep and Consciousness directed by Prof. Giulio Tononi and Prof. Chiara Cirelli at the University of Wisconsin, Madison (USA). Professor Serra's research program focuses on design and application of microsensors, biosensors, EEG, EMG and neurotelemetry for electrochemical monitoring of brain signalling systems during sleep and in neurodegenerative diseases. 



\section{Contents}

Preface XI

Section 1 Materials Science 1

Chapter 1 Synthesis of New Biocompatible Polymers and Fabrication of Nanosheets 3

Yu Nagase and Yosuke Okamura

Chapter 2 Incorporation of Primary Amines via Plasma Technology on Biomaterials 21

Gaelle Aziz, Nathalie De Geyter and Rino Morent

Chapter 3 Engineering of Novel Proteinoids and PLLA-Proteinoid Polymers of Narrow Size Distribution and Uniform Nano/ Micro-Hollow Particles for Biomedical Applications 49 Michal Kolitz-Domb and Shlomo Margel

Chapter 4 Lipid-Based Nanoparticles and Microbubbles - Multifunctional Lipid-Based Biocompatible Particles for in vivo Imaging and Theranostics 79

Jaroslav Turánek, Andrew D. Miller, Zuzana Kauerová, Róbert Lukáč, Josef Mašek, Štěpán Koudelka and Milan Raška

Chapter 5 Plasma Modified Textiles for Biomedical Applications 117 Pieter Cools, Rino Morent and Nathalie De Geyter

Section 2 Biosensors, Electronics and Telemetry 149

Chapter 6 Electronic Sensor Interfaces With Wireless Telemetry 151 Ifana Mahbub, Farhan Quaiyum, Md Sakib Hasan and SyedKamrul Islam 
Chapter 7 Biomedical Sensor, Device and Measurement Systems 177

Gaofeng Zhou, Yannian Wang and Lujun Cui

Section 3 Light Therapy 229

Chapter 8 High Brightness LEDs Supplied by Electronics Converters Used in Tissue Healing and Cell Rejuvenation 231

Alexandre Campos, Yasmim Guterres, Eduardo B. Bauer and Mauro

C. Moreira

Chapter 9 Photodynamic Nanomedicine Strategies in Cancer Therapy and Drug Delivery 253

Rodica-Mariana Ion

Section 4 Computing and Analysis Techniques 289

Chapter 10 Data Reduction Techniques in Neural Recording

Microsystems 291

Mohsen Judy, Alireza Akhavian and Farzad Asgarian

Chapter 11 Multivariate Computing and Robust Estimating for Outlier and Novelty in Data and Imaging Sciences 317

Michelle Yongmei Wang and Chris E. Zwilling

Chapter 12 Energy Transfer and Dissipation in non-Newtonian Flows in non-Circular Tubes 337

Mario F. Letelier

Chapter 13 Quantifying Stress in Crabs and Humans using Modified DFA 359

Toru Yazawa 


\section{Preface}

The Biological engineering is a scientific discipline that is experiencing a period of expansion with important impacts in all areas of medicine. The technological approach and the high level of innovation make bioengineering extremely dynamic and this forces researchers to continuous updating. It involves the publication of the results of the latest scientific research.

This book covers a wide range of aspects and issues related to advances in bioengineering research, with a particular focus on innovative technologies and applications. The book consists of 13 scientific contributions divided in four sections: Materials Science; Biosensors, Electronics and Telemetry; Light Therapy; Computing and Analysis Techniques.

In the first section, consisting of five chapters, issues concerning the chemical synthesis of new (nano)materials and physical modifications of biomaterials are addressed. In the section entitled "Biosensors", two chapters treat the issues concerning the electronics of analytical devices and the wireless transmission of acquired data. The third section addresses themes linked to light therapy. The last four chapters, grouped in the section entitled "Computing and Analysis Techniques", show new computational approaches suitable for solving bioengineering problems.

Pier Andrea Serra

Dept. Of Clinical and Experimental Medicine

University of Sassari

Italy 

Section 1

Materials Science 



\title{
Chapter 1
}

\section{Synthesis of New Biocompatible Polymers and Fabrication of Nanosheets}

\author{
Yu Nagase and Yosuke Okamura \\ Additional information is available at the end of the chapter \\ http://dx.doi.org/10.5772/59633
}

\section{Introduction}

Synthetic polymers have been investigated for the applications in the medical field as biomaterials, and used for processing biomedical devices and artificial organs which could be used in living organs. However, most of the polymers are not suitable for a long-term implantation when the materials contact with flowing blood or internal organs, because the material surface could not avoid the initiation of the process leading to thrombosis. Therefore, the development of the materials, which are continuously showing a stable biocompatibility during the longterm use, is desired for the advanced medical devices. For example, segmented polyurethanes have been widely used in practical applications for medical devices due to their high mechanical strength and biocompatibility [1,2].

On the other hand, the phosphorylcholine (PC) group is a polar component of phospholipid molecules, which cover the surface of cell membranes. It has been well known that synthetic polymer materials containing PC group exhibit biocompatibility including nonthrombogenicity. Firstly, Ishihara et al. has been developed 2-methacryloyloxyethyl phosphorylcholine (MPC) polymer as an excellent biocompatible material, which efficiently reduces the adhesion of cells and proteins to the polymer surface [3-7]. The design of the MPC polymer was inspired by the chemical structure of the phospholipid polar group in biomembranes. Then, in recent years, the MPC polymer has been widely applied in biological and medical fields. Furthermore, the applications of MPC polymer to medical devices and other uses have been greatly advanced in these years [8 - 15]. However, most of MPC polymers do not possess the thermal stability and the mechanical strength, which were derived from the polymethacrylate type main chain. Then, if these physical properties of MPC polymers improved satisfactorily while maintaining the excellent biocompatibility, novel biocompatible polymer materials could be developed. 
In these years, we have succeeded in the syntheses of novel diamine and diol monomers containing PC moiety for the preparations of polyamides, polyimides, polyesters and polyurethanes from these monomers [16 - 23]. It was found that the obtained polymers exhibited the excellent biocompatibility derived from PC unit in addition to the processability, the durability to solvents, the thermal stability and the mechanical strength, which were derived from the main chain components.

By the way, the development of practical biomaterials will desire the collaborations among chemists, biologists, material scientists. We focused in the field of nanotechnology, especially the processing for free-standing ultrathin films consisting of polymers with a thickness less than $100 \mathrm{~nm}$ (often called nanosheets), which exhibited the unique properties such as high adhesive strength, flexibility, transparency and smoothness [24-26]. If the nanosheets could be fabricated from such PC-containing polymers, the applications as new biomaterials would be significantly advanced. Then, we attempted to prepare the nanosheets from the obtained copoly(ester-urethane)s and to investigate the physical properties and the biocompatibility of the nanosheet surface.

This chapter covered the subject of our recent study to develop new biomaterials containing a phospholipid moiety. We describe the preparations of aromatic polyimides and segmented polyurethanes containing PC group, which are obtained by polycondensation or polyaddition using PC-containing diamine and diol monomers. In addition, the fabrication of ultra-thin films, so called nanosheets, composed of these PC-containing polymers is described in detail. The obtained nanosheets exhibited the high adhesive strength, indicating that the nanosheets could conform closely to the desired surfaces due to their exquisite flexibility and low roughness. In this chapter, the physical properties such as thermal stability, biological function as blood compatibility, and surface property of the obtained polymers and nanosheets are discussed to reveal the possibility of a new biocompatible polymer material.

\section{Preparations of polymer materials}

\subsection{Syntheses of monomers containing PC group}

In order to prepare polyamides, polyimides and polyurethanes, we have investigated the syntheses of diamine and diol monomers containing PC moiety. At first, the synthesis of 2(3,5-diaminophenylcarbonyloxy)ethyl phosphorylcholine (DAPC) was carried out to prepare PC-containing polyamide [16]. Then, copolyamides were prepared by the polycondensation of DAPC with isophthaloyl chloride and another diamine comonomer. It was revealed that the obtained copolyamide films exhibited the excellent blood compatibility. These results would be due to the PC unit located at the surface of the polymer film, where the surface is covered with PC unit, and the interaction between the polymer surface and blood ingredients such as cells and platelets is very weak. However, the molecular weight and the PC content of copolyamides from DAPC were not enough to produce a self-standing film and to exhibit the higher biocompatibility, respectively, which would be due to the low reactivity and also the highly hygroscopic property of DAPC. Thus, we have developed a new structure of high 
molecular weight polymer in order to create the practical biomaterials for several applications, which exhibit the excellent biocompatibility in addition to the processability, the durability to solvents, the thermal stability and the mechanical strength [19].

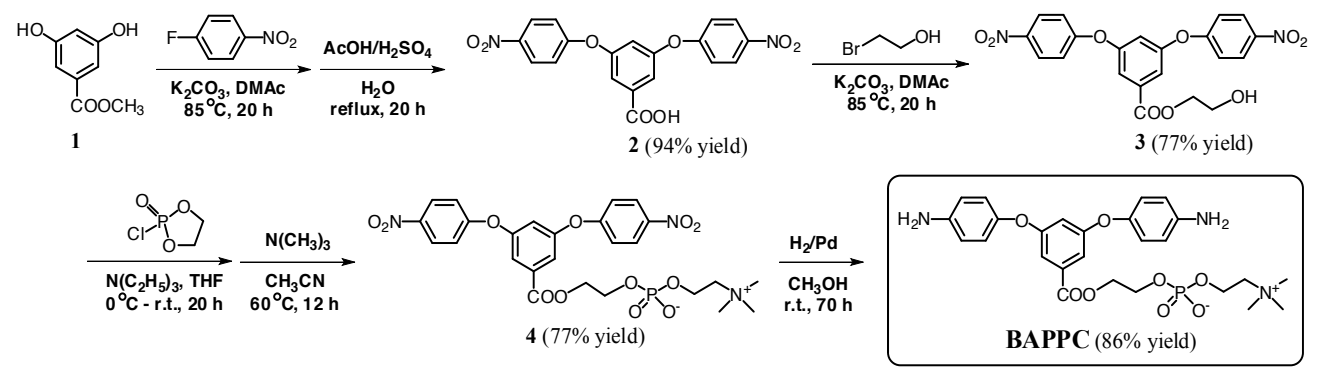

Sheme 1. Synthesis of diamine monomer containing PC moiety (BAPPC).

For this purpose, we designed a new diamine monomer containing PC group, 2-[3,5-bis(4aminophenoxy)phenylcarbonyloxy]ethyl phosphorylcholine (BAPPC). BAPPC is expected to show the higher reactivity in the polymerization than DAPC, which would be due to the relatively higher reactive amino groups on $p$-position of phenoxy groups. The synthetic route of BAPPC is outlined in Scheme 1. The compound 3 was prepared as an intermediate, which were obtained by the esterification of $\mathbf{2}$ with 2-bromoethanol. Then, the reaction of $\mathbf{3}$ with 2chloro-2-oxo-1,3,2-dioxaphospholane (COP) yielded a phospholane compound. The purification of the product by a silica-gel column chromatography was difficult because it was easily hydrolyzed. Therefore, the extraction of the crude products with chloroform followed by washing with distilled water gave the pure phospholane compound. Finally, BAPPC (melting point $=109^{\circ} \mathrm{C}$ ) was obtained by opening the cyclic phosphoric ester moiety with trimethylamine, followed by the reduction of the nitro groups of 4 with $\mathrm{H}_{2}$ catalyzed by $\mathrm{Pd}$. Although the several reaction steps are necessary to prepare this monomer, all of the reaction steps proceeded smoothly in high yields [18].

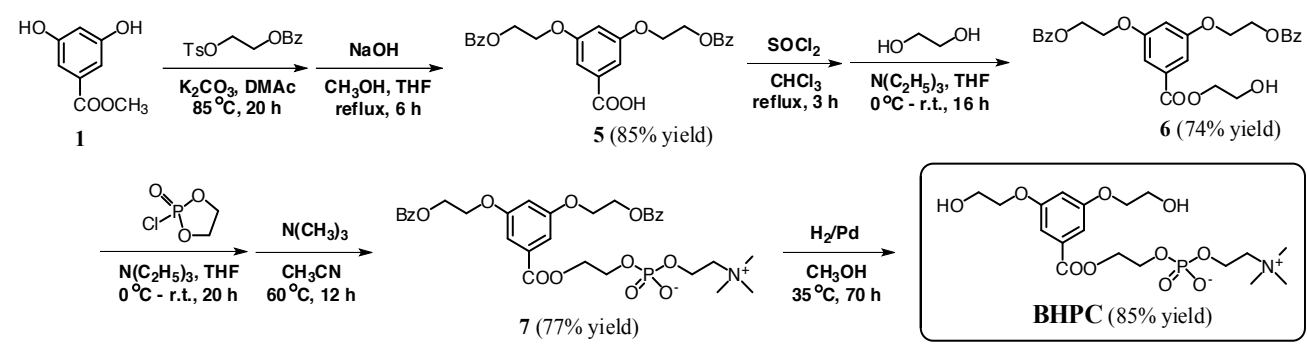

Sheme 2. Synthesis of diol monomer containing PC moiety (BHPC). 
On the other hand, the synthesis of diol monomer containing PC unit was investigated to prepare polyurethanes or polyesters [21]. The synthetic route of the desired diol monomer, 2(3,5-bis(2-hydroxyethoxy)benzoyloxy)ethyl phosphorylcholine (BHPC), is outlined in Scheme 2. The terminal benzyl groups of compounds 5, 6 and 7 were introduced as a protection group of the diol moiety. The key intermediate, $\mathbf{6}$, was synthesized by esterification of 5 with ethylene glycol, and the incorporation of the PC group was achieved by the reaction of 6 with COP, followed by the ring-opening reaction of the cyclic phosphoric ester moiety with trimethylamine. Finally, deprotection of the benzyl groups of 7 by Pd-catalyzed hydrogen reduction with $\mathrm{H}_{2}$ gas to afford the desired diol monomer, BHPC. This reaction proceeded quantitatively to give the pure product of $\mathrm{BHPC}$ as a white solid (melting point $=34^{\circ} \mathrm{C}$ ), although it was so hygroscopic that the obtained solid softened when exposed to moisture.

\subsection{Syntheses and properties of polyimides}

In these years, we have achieved the syntheses of polyamides, poly(urethane-urea)s and poly(amide-ester)s containing PC moiety by polycondensation or polyaddition using the novel diamine monomer, BAPPC, and investigated the physical and biological properties of the obtained polymers, as described in our literatures [18 - 20]. These aromatic polymers containing PC group showed the thermal stability up to $c a .250^{\circ} \mathrm{C}$, where the thermal degradation of PC component would started over $200^{\circ} \mathrm{C}$ that was confirmed by the thermogravimetric analysis of polymers. The heat resistance of these PC-containing polymers over $200^{\circ} \mathrm{C}$ is enough to use for biomaterial devices, for example, for the thermal sterilization process over $150^{\circ} \mathrm{C}$. In addition, the tough films could be prepared by solvent casting from poly(urethaneurea)s and poly(amide-ester)s, which were copolymerized with polycarbonate diol as the soft segment, and the elastomeric property was observed in these films [20]. Furthermore, it has been found that these PC-containing aromatic polymers efficiently reduced the adhesion of proteins and platelets, where the number of adhered platelets of PC-containing polymers was reduced in nearly one-tenth amount as compared with that of polymers without PC group. These results indicate that the PC unit plays an important role for the blood compatibility of the polymers. The amount of adhered proteins and platelets decreased as the increase of the content of PC unit in the copolymers, therefore, the composition of the PC unit was a dominant factor in the reduction of the adhesion of proteins and platelets.

In this chapter, we will describe our recent study for the synthesis of polyimide containing PC group as a biocompatible hard material. The desired copolyimides were carried out by the polycondensation of BAPPC and bis(p-diaminophenyloxy)benzene (BAPB) with 4,4'-hexafluoroisopropylidene diphthalic anhydride (6FDA), followed by the chemical imidization with triethylamine and acetic anhydride, as shown in Scheme 3. As the acid anhydride, 6FDA was used to make the polyimide soluble in some solvents. Table 1 summarizes the compositions and molecular weights of the obtained copolyimides. Four kinds of copolyimides with PC content were prepared by changing the ratio of BAPPC and BAPB in the feed of polymerization. The obtained copolyimides showed the number-average molecular weights $(\mathrm{Mn})$ at around $1 \times 10^{4}$. 
<smiles>CN(C)CCOP(=O)(O)OCCOc1cccc(Oc2ccc(N)cc2)c1</smiles>

BAPPC (diamine monomer)

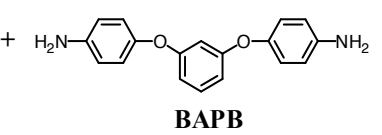

BAPB

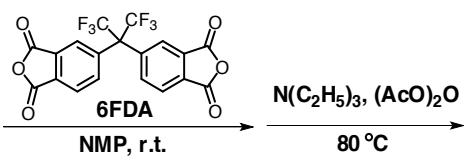

NMP, r.t.

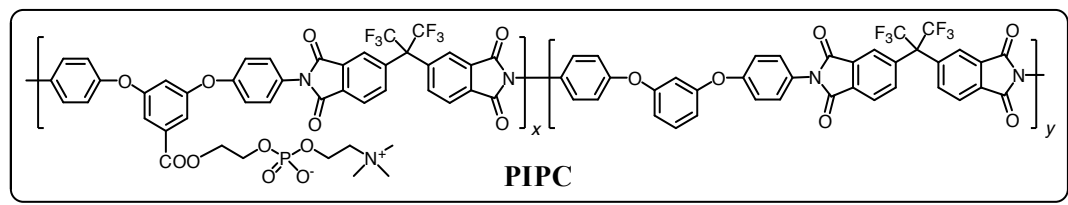

Sheme 3. Preparation of copolyimides containing PC moiety (PIPC).

\begin{tabular}{|c|c|c|c|c|}
\hline & BAPPC/BAPB ${ }^{\text {a }}$ & PC content $\left.{ }^{b}\right)$ & $M \mathrm{n}^{\mathrm{c}}$ & \\
\hline \hline Code & $(\mathrm{mol} \%)$ & $(\mathrm{mol} \%)$ & $(\mathrm{x} \mathrm{10})$ & $M \mathrm{w} / M \mathrm{n}$ \\
\hline PIPC-1 & $20 / 80$ & 14 & 17.4 & 1.44 \\
\hline PIPC-2 & $25 / 75$ & 24 & 10.4 & 1.37 \\
\hline PIPC-3 & $30 / 70$ & 40 & 10.1 & 1.52 \\
\hline PIPC-4 & $50 / 50$ & 48 & 7.29 & 1.30 \\
\hline
\end{tabular}

a) The molar ratio of monomers, BAPPC and BAPB, in the polymerazition.

b) $\mathrm{PC}$ contents in the copolymers were estimated by ${ }^{1} \mathrm{H}-\mathrm{NMR}$ spectra.

c) Number-avarage and weight-avarage molecular weights, $M n$ and $M w$, were determinated by GPC using $20 \mathrm{mM} \mathrm{LiBr}$ solution in DMF as an eluent.

Table 1. Composition and molecular weight of polyimides (PIPC).

These copolyimides were soluble in aprotic polar solvents, such as dimethylformamide (DMF), dimethylsulfoxide (DMSO) and N-methyl-2-pyrrolidinone (NMP), at room temperature, whereas they were insoluble in water, methanol, ethanol and acetone. This solubility in specific solvents is advantageous in the processing for medical devices, and the insolubility in other solvents enables the material durable to these solvents. For the solubility in some solvents, the solubility of these copolyimides depended on the PC content of polymers. For example, only PIPC-1 in Table 1 was soluble in chloroform and tetrahydrofuran (THF), but PIPC-2, 3 and 4 were insoluble in these low boiling point solvents. By the way, polyimide without PC unit, which was prepared from 6FDA and BAPD, was soluble in chloroform and THF. Therefore, the solubility of these copolyimides decreased with increasing PC content, where the maximum PC content that allowed the solubility in chloroform and THF was 15-20 wt. \%. It was speculated that polar PC groups in the side chains would have a strong interaction, which would make the polymer insoluble in these solvent.

On the other hand, it was found by the thermogravimetric analysis that the weight loss of the PIPC series started at $c a .250^{\circ} \mathrm{C}$, similar to polyamide and poly(urethane-urea) containing PC 
group, whereas the decomposition temperature of the polyimide without PC group was over $400^{\circ} \mathrm{C}$. In addition, the hard but brittle films were prepared from these polyimides by solvent casting method.

\subsection{Syntheses and properties of segmented polyurethane}

Segmented polyurethanes generally consist of short alternating blocks of soft and hard segments, and exhibit an elastomeric property. The biocompatibility of segmented polyurethane is thought to arise from the microphase separation of the soft and hard segments. However, the biostability of segmented polyurethane is not suitable for long-term implantation. It has been suggested that the biodegradation and cracking of polyurethane that occurred in vivo was due to the adsorption of proteins, adhesion of macrophages and peroxide formation [27 - 29], which resulted in the reduction of the mechanical strength of segmented polyurethane. Moreover, the soft segment of segmented polyurethane was reportedly degraded by oxygen radicals produced by adherent macrophages [30]. Therefore, several studies of surface or chemically modified segmented polyurethanes have been conducted to improve biostability by reducing the adhesion of cells and proteins [31 - 35]. Ishihara et al. have also investigated a polymer composite consisting of segmented polyurethane and MPC polymer to reduce protein adsorption to the polymer surface and to improve the biocompatibility of segmented polyurethane [36-41].

In our previous studies, we have prepared segmented poly(urethane-urea)s containing PC group by using the PC-containing diamine monomer, BAPPC, as a coupling reagent in the polyaddition of diols with diisocyanate [19]. The obtained polymers exhibited excellent biocompatibility, the film surface of which efficiently reduced the adhesion of human platelets. In addition, stress-strain measurements revealed that the poly(urethane-urea) films exhibited high elastic mechanical properties, where the Young's modulus increased with increasing PC content. The aim of the next study was to prepare another type of PC-containing polyurethane from diol monomer (BHPC). Cooper and his co-worker have reported that a PC-containing polyurethane could be prepared using glycerophosphorylcholine as a diol monomer [42]. We have designed the BHPC molecule based on the concepts that BHPC would be more hydrophobic than glycerophosphorylcholine and easier to handle as a monomer for polycondensation or polyaddition. We expected that both of the primary hydroxyl groups of BHPC would make the polymer have a high molecular weight due to its higher reactivity than glycerophosphorylcholine with its secondary hydroxyl group. Recently, Khan et al. have reported a potential application of poly(carbonate-urethane) as a long-term biomedical implant material due to its resistance to biodegradation and its biocompatibility [43,44]. Therefore, we selected polycarbonate diol (PCD) to construct the soft segment of PC-containing segmented polyurethane.

As shown in Scheme 4, the syntheses of segmented polyurethanes containing PC group and polycarbonate segment with different contents were carried out by polyaddition of BHPC and PCD with 4,4'-diphenylmethane diisocyanate (MDI). The compositions and the molecular weights of the obtained polymers are summarized in Table 2. The observed PC contents in mol 
$\%$ were determined by ${ }^{1} \mathrm{H}-\mathrm{NMR}$ and were in good agreement with the molar ratio of $\mathrm{BHPC}$ and PCD in the feed of polymerizations.

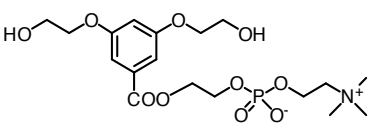

BHPC (diol monomer)
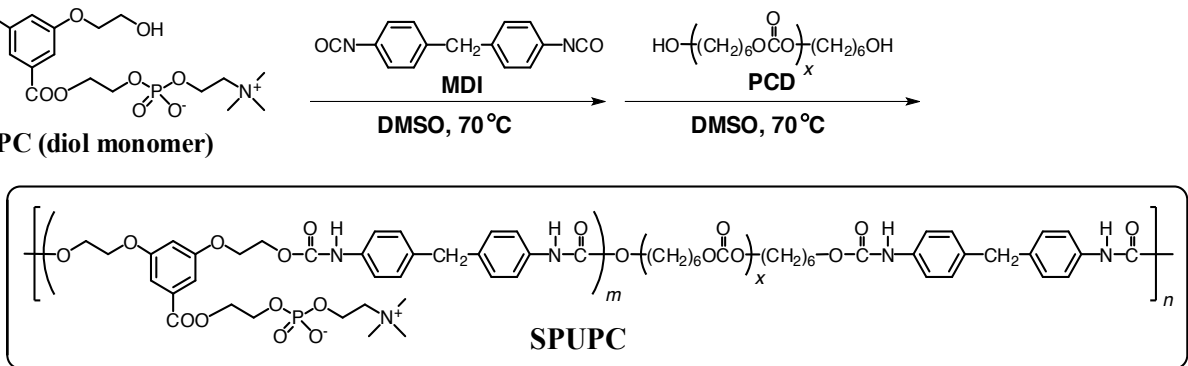

Sheme 4. Preparation of segmented polyurethane containing PC moiety (SPUPC).

\begin{tabular}{|c|c|c||c|c|}
\hline & BHPC/PCD $^{\mathrm{a})}$ & PC content $^{\mathrm{b})}$ & $M \mathrm{n}^{\mathrm{c}}$ & \\
\hline \hline Code & $(\mathrm{mol} \%)$ & $(\mathrm{mol} \%)$ & $(\mathrm{x} \mathrm{10})$ & $M \mathrm{w} / M \mathrm{n}$ \\
\hline SPUPC-1 & $20 / 80$ & 20 & 189 & 1.38 \\
\hline SPUPC-2 & $30 / 70$ & 34 & 266 & 1.25 \\
\hline SPUPC-3 & $40 / 60$ & 41 & 44.3 & 4.25 \\
\hline SPUPC-4 & $50 / 50$ & 50 & 92.5 & 1.91 \\
\hline
\end{tabular}

a) The molar ratio of monomers, BHPC and PCD, in the polymerization.

b) $\mathrm{PC}$ contents in the copolymers were estimated by ${ }^{1} \mathrm{H}-\mathrm{NMR}$ spectra.

c) Number-avarage and weight-avarage molecular weights, $M n$ and $M w$, were determinated by GPC using DMF as an eluent.

Table 2. Compositions and molecular weights of segmented polyurethanes (SPUPC).

The obtained polyurethanes, SPUPC-1, 2 and 3, exhibited a good solubility in aprotic polar solvents such as DMF, DMSO and NMP at room temperature, whereas SPUPC- 4 was insoluble in these solvents. In addition, SPUPC-1 and 2 were soluble in the low boiling solvents, chloroform and THF, but SPUPC-3 and 4 were insoluble in chloroform and THF. Therefore, a trade-off relation was observed between the solubility and the PC content of polyurethanes, which was similar to PIPC series. It would be due to the strong interaction of polar PC group in the side chain and the highly polar urethane bond in the main chain. To obtain a soluble polymer with high PC content, a different concept for the molecular design or the polymerization process should be developed the solubility of these PC-containing polymers. Recently, we have developed PC-containing poly(ester-urethane)s, the solubility of which was improved to some extent by the introduction of ester bond in addition to the highly polar urethane component [22, 23]. 
The flexible and self-standing films could be prepared from these segmented polyurethanes by a solvent casting method using DMSO as a solvent. Then, the elastic mechanical properties were observed for these segmented polyurethane films, where the Young's modulus increased with increasing PC content. Furthermore, the introduction of such a polar phospholipid group was effective in improving the resistance to protein and platelet adhesions on the polymer film, which was the result of surface properties derived from the PC moiety [21].

\section{Fabrication of ultra-thin films (nanosheets)}

\subsection{Preparation and characterization of nanosheets composed of PIPC and SPUPC}

Self-standing nanosheets are easily fabricated by a "sacrificial layer method" as depicted in Fig. 1a [26]. The sacrificial layer can be dissolved with appropriate solvents which do not dissolve nanosheets themselves. In parallel, solvents used for dissolving polymers of nanosheets must not dissolve the sacrificial layer. To this end, we selected poly(vinyl alcohol) (PVA) as a water-soluble sacrificial layer to obtain self-standing nanosheets composed of PIPC- 1 and SPUPC-2, because these polymers were soluble in chloroform which did not dissolve PVA. A fabrication procedure of PIPC-1 nanosheet is described as follows. First, an aqueous solution of $10 \mathrm{mg} / \mathrm{mL}$ PVA was dropped onto a silicon oxide $\left(\mathrm{SiO}_{2}\right)$ substrate, which has an extremely flat surface. The substrate was spin-coated at 4,000 rpm for $20 \mathrm{~s}$ and then dried. Next, a chloroform solution of $10 \mathrm{mg} / \mathrm{mL}$ PIPC- 1 was spin-coated on the PVA-coated substrate under the same conditions. When the substrate was immersed into distilled water, the PIPC-1 nanosheet was detached from the substrate due to dissolution of only the PVA layer with water. The obtained PIPC-1 nanosheet was transparent, amazingly flexible, and maintained the size and shape of the $\mathrm{SiO}_{2}$ substrate (Fig. 1b, left). In fact, the thickness was $42 \pm 2 \mathrm{~nm}$ and the roughness was nanometer scale. Furthermore, the thickness was easily controlled by adjusting the concentration of PIPC-1 just before spin-coating as shown in Fig. 1c. As described in section 2.2, the solubility of PIPC series is dependent on the content of the PC unit. For instance, PIPC-4 with high PC content was insoluble in chloroform but soluble in the aprotic polar solvents as DMSO. However, the PVA sacrificial layer is dissolved with DMSO. To this end, we can select other component of the water-soluble sacrificial layer, e.g. sodium alginate (Na-Alg), which is insoluble in DMSO. According to this technique, we could prepare the selfstanding nanosheets composed of SPUPC series [45]. In the case of a chloroform solution of 10 $\mathrm{mg} / \mathrm{mL}$ SPUPC-2, the thickness of the nanosheet was $66 \pm 4 \mathrm{~nm}$. Intriguingly, these nanosheets composed of SPUPC series, which were elastic polymers, tended to shrink after detaching from the substrate as seen in Fig. 1b, right. This tendency suggested that the nanosheet extended on the substrate by the centrifugal force during the spin-coating, and resulted in shrinking due to its elasticity when they were released from the substrate. This tendency was not observed in the nanosheets composed of non-elastic polymers such as PIPC series. In these years, we have prepared the self-standing nanosheets composed of versatile polymers such as polystyrene and poly (methyl methacrylate), etc., and typical biodegradable polymers such as poly(lactic acid), their copolymers and polycaprolactone, etc. [24, 25]. 
(a)

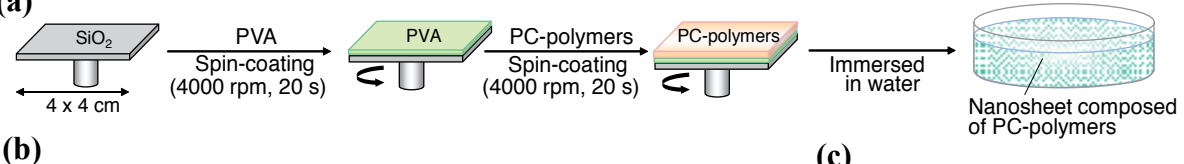

(b)

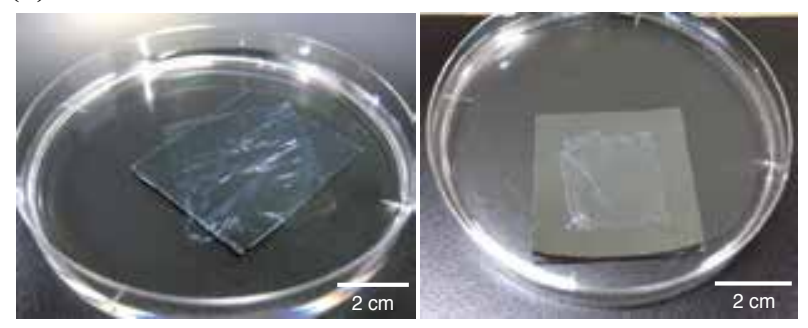

(c)

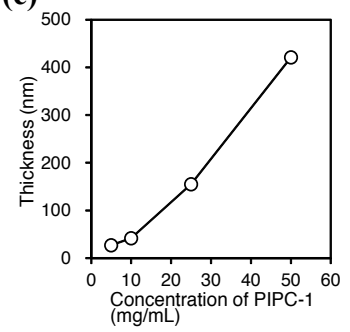

Figure 1. Fabrication of self-standing nanosheets composed of PIPC-1 and SPUPC-2. (a) Fabrication procedure of the nanosheets by spin-coating. (b) Macroscopic images of PIPC-1 (left) and SPUPC-2 (right) nanosheets suspended in water. (c) Relationship between thickness of the PIPC-1 nanosheets and concentration of PIPC-1 solution before spin coating.

We analyzed the mechanical properties of the nanosheets by using a bulging test developed for nanosheets [46]. In fact, the PIPC-1 nanosheet with a thickness of approximately $40 \mathrm{~nm}$ was physically adhered to a steel plate with a hole as shown in Fig. 2a. The plate was fixed to a custom-made chamber and air was supplied with a syringe pump until bursting the nanosheets. During the analysis, pressure applied to the nanosheets and its deflection was monitored in real time by a differential pressure gauge and a stereomicroscope, respectively. Based on the equations as shown in Fig. 2a, we obtained a strain-stress curve as shown in Fig. $2 b$. From the slope of the elastic region of the curve, the Young's modulus of the PIPC-1 nanosheet was calculated to be $196 \pm 9 \mathrm{MPa}$. This value was 10-folds lower compared to that of the bulk polyimide films (3-7 GPa), indicating that the PIPC-1 nanosheet was softer than the bulk polyimide film. We have demonstrated that the poly(lactic acid) nanosheets with a thicnkess less than $100 \mathrm{~nm}$ represent the same tenency [24]. Mattsson et al. have explored the relationship between the glass transition temperature $\left(T_{\mathrm{g}}\right)$ and thickness of the ultra-thin films of polystyrene using a Brillouin light scattering method. In fact, $T_{\mathrm{g}}$ of polystyrene films with a thickness of approximately $20 \mathrm{~nm}$ was decreased to $37^{\circ} \mathrm{C}$ compared to that of bulk polystyrene $\left(T_{\mathrm{g}}\right.$ : $109^{\circ} \mathrm{C}$ ), explaining that the interactions between polymer chains decreased in the ultra-thin films [47]. This may be one of the reasons why the $T_{g}$ of the PIPC-1 nanosheet would be lower than that of bulk polyimide.

Next, we analyzed the relationship between adhesive strength of the PIPC-1 nanosheets and their thickness with a scratch tester for thin films [48]. As depicted in Fig. 3a, the nanosheets were physically adhered on the $\mathrm{SiO}_{2}$ substrate, and the surface of the nanosheets were horizontally scratched with a diamond tip under the following conditions; radius of curvature of a diamond tip: $25 \mu \mathrm{m}$, scratch length: $100 \mu \mathrm{m}$, and scratch rate: $10 \mu \mathrm{m} / \mathrm{s}$. Critical loads just after detaching the nanosheet from the substrate were monitored. Then, the adhesive strength of the nanosheets was difined as the critical loads divided by the thickness of the nanosheets. 

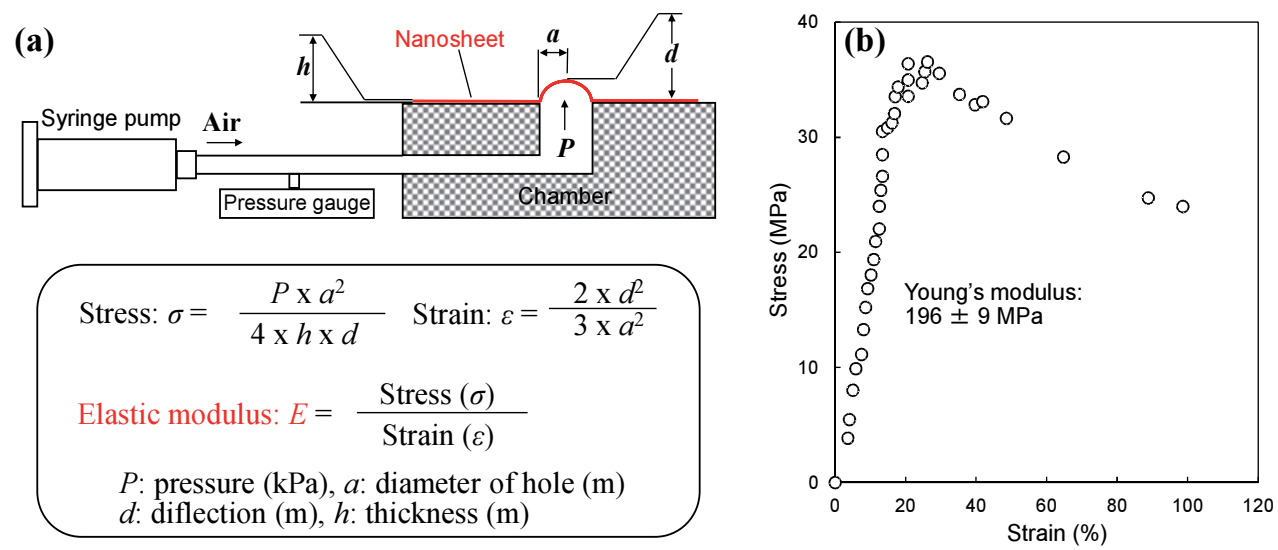

Figure 2. Mechanical properties of the PIPC-1 nanosheet analyzed by a bulging test. (a) Schematic image of the bulging test. (b) Represetative stress-strain curve of the PIPC-1 nanosheet with a thickness of $42 \pm 2 \mathrm{~nm}$.

The critical loads of the PIPC-1 nanosheet with thicknesses of 27 and $42 \mathrm{~nm}$ were calculated to be $(1.6 \pm 0.3) \times 10^{5}$ and $(1.4 \pm 0.4) \times 10^{5} \mathrm{~N} / \mathrm{m}$, respectively as shown in Fig. 3b. However, in the region of the thickness over $100 \mathrm{~nm}$, the critical roads were obviously decreased to $(0.8 \pm$ $0.2) \times 10^{5} \mathrm{~N} / \mathrm{m}$ (thickness: $155 \mathrm{~nm}$ ) and $(0.4 \pm 0.2) \times 10^{5} \mathrm{~N} / \mathrm{m}$ (thickness: $421 \mathrm{~nm}$ ). This would be the reason that the nanosheets could conform to the roughness of the substrate due to its flat surface and amazingly flexibility. Actually, these nanosheets can be adhered to various surfaces such as plastics, glasses, steels, and tissues without the utilization of adhesive agents. Once the nanosheets were dried on these surfaces, it was often hard to detach with even washing with water. Consequently, we have demonstrated that the greatest benefit of the nano-thickness is high potential to adhere. This phenomenon has been also observed with the poly(lactic acid) nanosheets with the thicnkesses less than $100 \mathrm{~nm}[24]$.

(a)

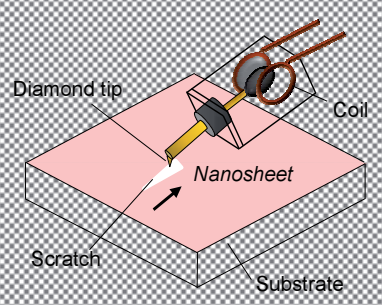

(b)

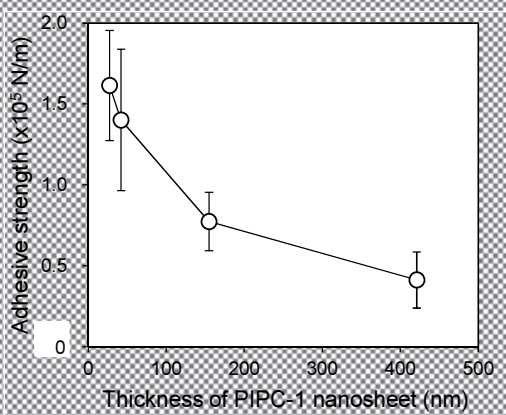

Figure 3. Adhesive strength of the PIPC-1 nanosheet. (a) Schematic image of scratch tester for thin films. (b) Correlation of adhesive strength of the PIPC-1 nanosheet with its thickness. 


\subsection{Biocompatibility of nanosheet surface}

Platelets are one of blood cells and involved in both normal hemostasis and pathological thrombosis [49]. In development of biocompatible materials with the possibility to contact with blood, what the most critical point is to inhibit non-specific interactions between platelets and the surface of the materials. To this end, we evaluated the blood compatibility of the surface of the nanosheets composed of PC polymers. Poly(ethylene terephtalate) (PET) plates were used as model surfaces, to which the nanosheets were adhered. The nanosheet-coated PET plates were immersed into $0.5 \mathrm{~mL}$ of platelet-rich plasma (PRP) obtained from healthy volunteers and incubated at physiological temperature for $2 \mathrm{~h}$. Finally, PRP was removed and the substrates were washed out with phosphate buffered saline. The surface of the plates was observed with a scanning electron microscope. As shown in Fig. 4, platelets with filopodial extensions were non-specifically adhered to the bared PET plate and the nanosheet-coated PET plate without PC units (PI and SPU). PI is a polyimide obtained by the polycondensation of BAPB with 6FDA followed by the chemical imidization, and SPU is a segmented polyurethane obtained by the polyaddition of 3,5-bis(2-hydroxyethoxy)benzene and PCD (molar ratio: 70/30) with MDI. In the case of the PET plates coated with PIPC-1 and SPUPC-2 nanoheets, reduction of platelet adhesion was clearly observed as compared with PET plate and PI/SPU coated plates. Therefore, it was confirmed that the surface of PC-polymer nanosheets exhibited the good blood compatibility. In other words, these results indicate that sealing of the nanosheets could act as a surface modifier to convert the surface property of the PET plates.
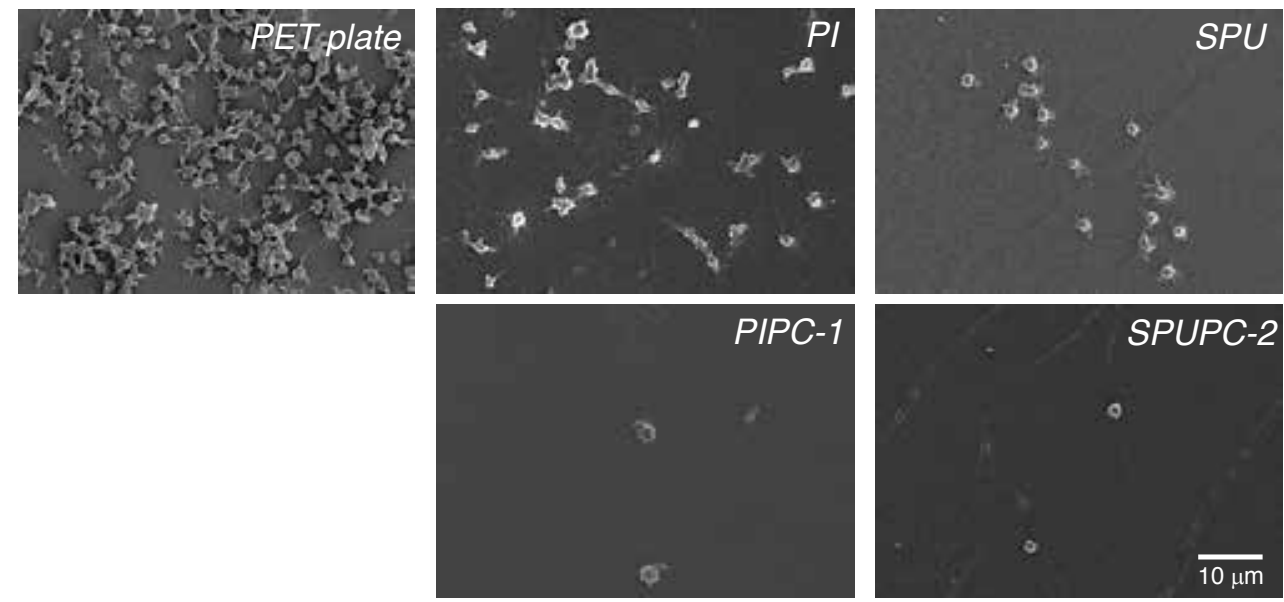

Figure 4. SEM images of nanosheet surfaces with or without PC unit after contact with plateled-rich plasma for $2 \mathrm{~h}$ at $37^{\circ} \mathrm{C}$

\subsection{Fragmentation of the nanosheets to coat irregular and uneven surfaces}

As described above, we have succeeded in the preparation of the self-standing nanosheets, which represent unique properties such as good adhesiveness, amazingly flexibility and high 
transparency. However, such nanosheets possess centimeter size and are only suitable for adhesion to relatively broad surfaces. They are often difficult to adhere to irregular and uneven surfaces because of centimeter size. In our recent study, we have discovered that the fragmented submillimeter-sized nanosheets composed of poly(lactic acid) were adhered to the various surfaces in a spread out configuration that looks like "patchwork" [25, 26]. Once the nanosheets dried on the surface, they were difficult to detach from the surface by even washing with water. Moreover, we have demonstrated that the irregular and uneven surfaces such as a needles and rubbers etc. are effectively coated with the patchwork-like coating of the fragmented nanosheets by just casting or dipping [25, 26]. In this section, we introduce the fragmented nanosheets composed of PIPC and SPUPC series to coat irregular and uneven surfaces and the evaluation of blood compatibility.

(a)

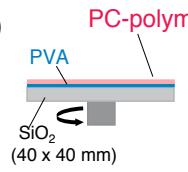

(b)

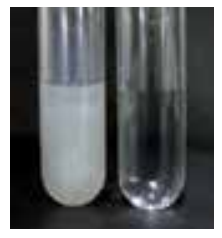

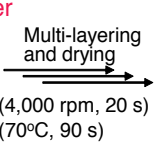

(c)

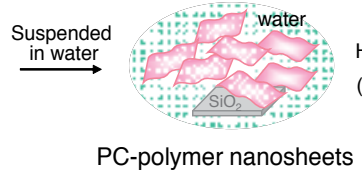

PC-polymer nanosheets

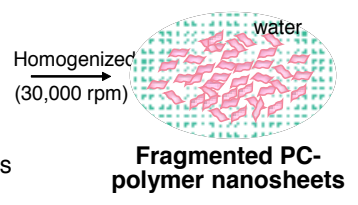

polymer nanosheets

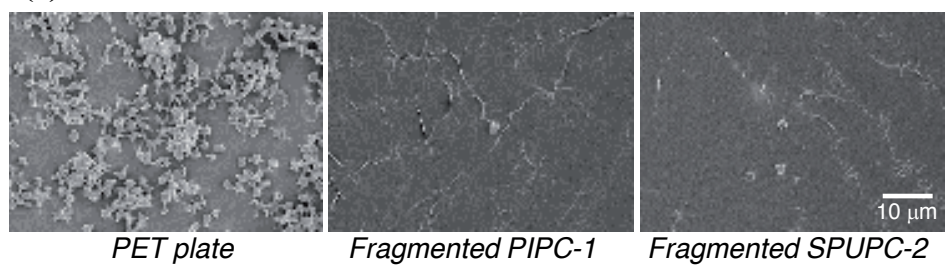

Figure 5. (a) Fabrication of fragmented nanosheets composed od PIPC-1 and SPUPC-2. (b) Macroscopic image of fragmented PIPC-1 nanosheets (left tube)suspended in distilled water. Right tube shows only distilled water. (c) SEM images of fragmented nanosheet surfaces after contact with platelet-rich plasma for $2 \mathrm{~h}$ at $37^{\circ} \mathrm{C}$.

We herein focus on the fragmented PIPC-1 nanosheets as follows. First, we fabricated abundant self-standing nanosheets with centimeter size by a simple multi-layering process of watersoluble PVA and PIPC nanosheets combined with a peeling technique, according to our reports $[25,26]$. Concretely, a $100 \mathrm{mg} / \mathrm{mL}$ solution of PVA as a water-soluble sacrificial layer was first spin-coated on a $\mathrm{SiO}_{2}$ substrate at $4000 \mathrm{rpm}$ for $20 \mathrm{~s}$, followed by a drying process as depicted in Fig. 5a. Next, a chloroform solution of $10 \mathrm{mg} / \mathrm{mL}$ PIPC- 1 was spin-coated on the PVA-coated substrate under the same conditions. Moreover, the multi-layering of PVA and PIPC-1 was repeated twenty times on the substrate. By dissolution of PVA layers in water, tewenty sheets of PIPC-1 nanosheets were obtained. Next, the obtained PIPC-1 nanosheets were fragmented with a homogenzer. When the PIPC-1 nanosheets (size: $40 \times 40 \mathrm{~mm}$, thickness: $42 \mathrm{~nm}$ ) in distilled water were homogenized at 30,000 rpm for $10 \mathrm{~min}$, they were instantly fragmented. The obtained nanosheets were homogeneously suspended in water and the turbidity of the suspension was quite increased as shown in Fig. 5b. In fact, the surface area of one fragmented nanosheet $10 \mathrm{~min}$ after homogenizaion was significantly decreased to $6800 \pm 208 \mu \mathrm{m}^{2}$, estimating that the average size of the nanosheet was approximately $80 \mu \mathrm{m}$. Using the same 
prosedure, we also prepared the fragmented nanosheets composed of SPUPC-2 (surface area: $3900 \pm 1300 \mu \mathrm{m}^{2}$, thickness: $66 \mathrm{~nm}$ ).

In order to evaluate the blood compatibility, the fragmented PIPC-1 or SPUPC-2 nanosheets were adhered to a bared PET plate as a model surface. They consisted of a patchwork-like coating in the same manner as the fragmented PLLA nanosheets [25, 26]. The nanosheet-coated PET plates were immersed into PRP and incubated at $37^{\circ} \mathrm{C}$ for $2 \mathrm{~h}$. As shown in Fig. $5 \mathrm{c}$, very few platelets were adhered to the PIPC- 1 and SPUPC-2 coated PET plate. Moreover, some lines were observed on the plates, that correspond to wrinkles (not cracks) formed during drying of patchwork-like coating. In the case of the bared PET plates, abundant platelets were activated and non-specifically adhered. Therefore, we demonstrated that patchwork-like coating with the fragmented nanosheets with PC units acts as an aqueous surface modifier to provide blood compatibility.

\section{Conclusions}

We have synthesized novel aromatic diamine and diol monomers containing PC group to develop the new biocompatible polycondensation-or polyaddition-type polymers. The obtained polymers exhibited good solubility with aprotic polar solvents and thermostability unlike MPC polymers. Using these polymers, we have succeeded in the fabrication of selfstanding nanosheets with a thickness less than $100 \mathrm{~nm}$. The PC-polymer nanosheets exhibited high adhesiveness to the various surfaces, and the surface of adhered nanosheets represented the good blood compatibility based on the platelet adhesion test. Furthermore, we have developed the fragmented nanosheets with submillimeter-size to coat irregular and uneven surfaces by controlling the size of the nanosheets. In fact, fragmented nanosheets were effectively coated with the patchwork-like adhesion behavior by just casting or dipping and provided blood compatibility to the various surfaces. Hence, these nanosheets composed of PC-containing polymers may be great promise as novel coating materials and surface modifiers to provide the biocompatibility to the surface of various medical devices such as catheters, artificial organs, microfluidic devices, etc.

\section{Author details}

Yu Nagase ${ }^{1^{*}}$ and Yosuke Okamura ${ }^{2}$

*Address all correspondence to: yunagase@tokai-u.jp

1 Department of Applied Chemistry, School of Engineering, Tokai University, Japan

2 Institute of Innovative Science and Technology, Tokai University, Japan 


\section{References}

[1] M.D. Lelah, S.L. Cooper, Polyurethanes in Medicine (1986), CRC Press, Boca Raton.

[2] C.D. Eisenbach, K. Fischer, H. Hayen, H. Nefzger, A. Ribbe, E. Stadler, Polyurethane elastomers, segmented (non-hydrogen bonding systems), Polymeric Materials Encyclopedia, Vol. 9 (1996) 6957-6968, CRC Press, Boca Raton.

[3] K. Ishihara, T. Ueda, N. Nakabayashi, Preparation of phospholipid polymers and their properties as polymer hydrogel membranes, Polymer Journal 22 (5) (1990), 355-360.

[4] K. Ishihara, R. Aragaki, T. Ueda, A. Watanabe, N. Nakabayashi, Reduced thrombogenicity of polymers having phospholipid polar groups, Journal of Biomedical Materials Research 24 (8) (1990), 1069-1077.

[5] K. Ishihara, N.P. Ziats, B.P Tierney, N. Nakabayashi, J.M. Anderson, Protein adsorption from human plasma is reduced on phospholipid polymers, Journal of Biomedical Materials Research 25 (11) (1991), 1397-1407.

[6] T. Ueda, H. Oshida, K. Kurita, K. Ishihara, N. Nakabayashi, Preparation of 2-methacryloyloxyethyl phosphorilcholine copolymers with alkyl methacrylates and their blood compatibility, Polymer Journal 24 (11) (1992), 1259-1269.

[7] Y. Iwasaki, A. Mikami, K. Kurita, N. Yui, K. Ishihara, N. Nakabayashi, Reduction of surface-induced platelet activation on phospholipid polymer, Journal of Biomedical Materials Research 36 (4) (1997), 508-515.

[8] S. Sawada, Y. Iwasaki, N. Nakabayashi, K. Ishihara, Stress response of adherent cells on a polymer blend surface composed of a segmented polyurethane and MPC copolymers, Journal of Biomedical Materials Research 79A (3) (2006), 476-484.

[9] J. Patel, Y. Iwasaki, K. Ishihara, J. Anderson, Phospholipid polymer surfaces reduce bacteria and leukocyte adhesion under dynamic flow conditions, Journal of Biomedical Materials Research 73A (3) (2005), 359-366.

[10] T. Uchiyama, J. Watanabe, K. Ishihara, Pressure-induced change in permeation of insulin through a polymer alloy membrane for an implantable insulin pump, Journal of Membrane Science 210 (2) (2002), 423-431.

[11] S.H. Ye, J. Watanabe, M. Takai, Y. Iwasaki, K. Ishihara, High functional hollow fiber membrane modified with phospholipid polymers for a liver assist bioreactor, Biomaterials 27 (9) (2006), 1955-1962.

[12] T. Goda, K. Ishihara, Novel Soft Contact Lens Biomaterials by Bioinspired Phospholipid Polymers, Expert Review of Medical Devices 3 (2) (2006), 167-174.

[13] T.A. Snyder, H. Tsukui, S. Kihara, T. Akimoto, K.N. Litwak, M.V. Kameneva, K. Yamazaki, W.R. Wagner, Preclinical biocompatibility assessment of the EVAHEART 
ventricular assist device: Coating comparison and platelet activation, Journal of Biomedical Materials Research, 81A (1) (2007), 85-92.

[14] K. Ishihara, M. Takai, Bioinspired interface for nanobiodevices based on phospholipid polymer chemistry, Journal of the Royal Society Interface 6 (3) (2009), S279-S291.

[15] Y. Inoue, T. Nakanishi, K. Ishihara, Elastic Repulsion from Polymer Brush Layers Exhibiting High Protein Repellency, Langmuir 29 (39) (2013), 10752-10758.

[16] Y. Nagase, M. Oku, Y. Iwasaki, K. Ishihara, Preparations of aromatic diamine monomers and copolyamides containing phosphorylcholine moiety and the biocompatibility of copolyamides, Polymer Journal 39 (7) (2007), 712-721.

[17] Y. Nagase, S. Nakajima, M. Oku, Y. Iwasaki, K. Ishihara, Synthesis and properties of segmented poly(urethane-urea)s containing phosphorylcholine moiety in the sidechain, Polymer Journal 40 (12) (2008), 1149-1156.

[18] K. Horiguchi, N. Shimoyamada, D. Nagawa Y. Nagase, Y. Iwasaki, K. Ishihara, Syntheses of a novel diamine monomer and aromatic polyamides containing phosphorylcholine group. Transactions of the Material Research Society of Japan 33 (4) (2008), 1261-1264.

[19] Y. Nagase, K. Horiguchi, Biocompatible Polyamides and Polyurethanes Containing Phospholipid Moiety, Biomedical Engineering-Frontiers and Challenges, Chapter 11 (2011), 217-232, InTech, Croatia.

[20] Y. Narita, W. Sirithep, Y. Okamura, Y. Nagase, Syntheses and Biocompatibility of Elastomers Containing Phospholipid Polar Groups, Kobunshi Ronbunshu 70 (5) (2013), 199-208.

[21] Y. Sakagami, K. Horiguchi, Y. Narita, W. Sirithep, K. Morita, Y. Nagase, Syntheses of a novel diol monomer and polyurethane elastomers containing phospholipid moieties, Polymer Journal 45 (11) (2013), 1159-116.

[22] W. Sirithep, Y. Narita, Y. Nagase, Syntheses and Physical Properties of Polyester and Poly(ester-urethane) Containing Phosphorylcholine Moiety, Transactions of the Material Research Society of Japan 38 (3) (2013), 473-476.

[23] W. Sirithep, K. Morita, A. Iwano, T. Komachi, Y. Okamura, Y. Nagase, Syntheses and properties of elastic copoly(ester-urethane)s containing a phospholipid moiety and the fabrication of nanosheets, Journal of Biomaterials Science, Polymer Edition (2014) in press. (http://dx.doi.org/10.1080/09205063.2014.929430)

[24] Y. Okamura, K. Kabata, M. Kinoshita, D. Saito, S. Takeoka, Free-standing biodegradable poly(lactic acid) nanosheet for sealing operations in surgery, Advanced Materials 21 (43) (2009), 4388-4392. 
[25] Y. Okamura, K. Kabata, M. Kinoshita, H. Miyazaki, A. Saito, T. Fujie, S. Ohtsubo, D. Saito, S. Takeoka, Fragmentation of poly(lactic acid) nanosheets and patchwork treatment for burn wounds, Advanced Materials 25 (4) (2013), 545-551.

[26] Y. Okamura, Fabrication of ultra-thin nanosheets with unique properties for biomedical applications, Kobunshi Ronbunshu 70 (8) (2013), 351-359.

[27] Q.H. Zhao, N. Topham, J.M. Anderson, A. Hiltner, G.M. London, C.R. Payet, Foreign-body giant cells and polyurethane biostability: In vivo correlation of cell adhesion and surface cracking, Journal of Biomedical Materials Research 25 (2) (1991), 177-183.

[28] Q.H. Zhao, A.K. McNally, K.R. Rubin, M. Renier, Y.V. Wu, Human plasma $\alpha 2$-macroglobulin promotes in vitro oxidative stress cracking of pellethane 2363-80A: In vivo and in vitro correlations, Journal of Biomedical Materials Research 27 (3) (1993), 379-388.

[29] Y. Wu, Q.H. Zhao, J.M.Anderson, A. Hiltner, G.M. London, C.R. Payet, Effect of some additives on the biostability of a poly(ether urethane) elastomer, Journal of Biomedical Materials Research 25 (6) (1991), 725-798.

[30] K. Stokes, R. McVenes, J.M. Anderson, Polyurethane Elastomer Biostability, Journal of Biomaterial Applications 9 (4) (1995), 321-354.

[31] I.K. Kang, O.H. Kwon, M.K. Kim, Y.M. Lee, Y.K. Sung, In vitro blood compatibility of functional group-grafted and heparin-immobilized polyurethanes prepared by plasma glow discharge, Biomaterials 18 (16) (1997), 1099-1107.

[32] R.G. Flemming, R.A. Proctor, S.L. Cooper, Bacterial adhesion to functionalized polyurethanes, Journal of Biomaterials Science, Polymer Edition 10 (6) (1999), 679-697.

[33] A.B. Mathur, T.O. Collier, W.J. Kao, M. Wiggins, M.A. Schubert, A. Hiltner, J.M. Anderson, In vivo biocompatibility and biostability of modified polyurethanes, Journal of Biomedical Materials Research 36 (2) (1997), 246-257.

[34] H.W. Roh, M.J. Song, D.K. Han, D.S. Lee, J.H. Ahn, S.C. Kim, Effect of cross-link density and hydrophilicity of PU on blood compatibility of hydrophobic PS/hydrophilic PU IPNs, Journal of Biomaterials Science, Polymer Edition 10 (1) (1999), 123-143.

[35] J.H. Lee, Y.M. Ju, D.M. Kim, Platelet adhesion onto segmented polyurethane film surfaces modified by addition and crosslinking of PEO-containing block copolymers, Biomaterials 21 (7) (2000), 683-691.

[36] K. Ishihara, H. Hanyuda, N. Nakabayashi, Synthesis of phospholipid polymers having a urethane bond in the side chain as coating material on segmented polyurethane and their platelet adhesion-resistant properties, Biomaterials 16 (11) (1995), 873-879.

[37] K. Ishihara, S. Tanaka, N. Furukawa, K. Kurita, N. Nakabayashi, Improved blood compatibility of segmented polyurethanes by polymeric additives having phospholi- 
pid polar groups. I. Molecular design of polymeric additives and their functions, Journal of Biomedical Materials Research 32 (3) (1996), 391-399.

[38] K. Ishihara, N. Shibata, S. Tanaka, Y. Iwasaki, T. Kurosaki, N. Nakabayashi, Improved blood compatibility of segmented polyurethane by polymeric additives having phospholipid polar group. II. Dispersion state of the polymeric additive and protein adsorption on the surface, Journal of Biomedical Materials Research 32 (3) (1996), 401-408.

[39] K. Ishihara, Y. Iwasaki, Biocompatible elastomers composed of segmented polyurethane and 2-methacryloyloxyethyl phosphorylcholine polymer, Polymers for Advanced Technologies 11 (8-12) (2000), 626-634.

[40] Y. Iwasaki, Y. Aiba, N. Morimoto, N. Nakabayashi, K. Ishihara, Semi-interpenetrating polymer networks composed of biocompatible phospholipid polymer and segmented polyurethane, Journal of Biomedical Materials Research 52 (4) (2000), 701-708.

[41] N. Morimoto, Y. Iwasaki, N. Nakabayashi, K. Ishihara, Physical properties and blood compatibility of surface-modified segmented polyurethane by semi-interpenetrating polymer networks with a phospholipid polymer, Biomaterials 23 (24) (2002), 4881-4887.

[42] L.L. Yung, S.L. Cooper, Neutrophil adhesion on phosphorylcholine-containing polyurethane, Biomaterials 19 (1-3) (1998), 31-40.

[43] I. Khan, N. Smith, E. Jones, D.S. Finch, R.E. Cameron, Analysis and evaluation of a biomedical polycarbonate urethane tested in an in vitro study and an ovine arthroplasty model. Part I: materials selection and evaluation, Biomaterials 26 (6) (2005), 621-631.

[44] I. Khan, N. Smith, E. Jones, D.S. Finch, R.E. Cameron, Analysis and evaluation of a biomedical polycarbonate urethane tested in an in vitro study and an ovine arthroplasty model. Part II: in vivo investigation, Biomaterials 26 (6) (2005), 633-643.

[45] A. Iwano, K. Morita, W. Sirithep, Y. Okamura, Y. Nagase, Synthesis of biocompatible elastic polyurethane containing phospholipid moiety, Transactions of the Material Research Society of Japan (2014) in press.

[46] S. Markutsya, C. Jiang, Y. Pikus, V.V. Tsukruk, Freely suspended layer-by-layer nanomembranes: Testing micromechanical properties, Advanced Functional Materials 15 (5) (2005), 771-780.

[47] J. Mattsson, J.A. Forrest, L. Börjesson, Quantifying glass transition behavior in ultrathin free-standing polymer films, Physical Review E 62 (2000), 5187-2000.

[48] S. Baba, T. Midorikawa, T. Nakano, Unambiguous detection of the adhesive failure of metal films in the microscratch test by waveform analysis of the friction signal, Applied Surface Science 144-145 (1999), 344-349. 
[49] S.J. Shattil, P.J. Newman, Integrins: dynamic scaffolds for adhesion and signaling in platelets, Blood 104 (15) (2004), 1606-1615. 
Chapter 2

\title{
Incorporation of Primary Amines via Plasma Technology on Biomaterials
}

\author{
Gaelle Aziz, Nathalie De Geyter and Rino Morent \\ Additional information is available at the end of the chapter
}

http://dx.doi.org/10.5772/59691

\section{Introduction}

\subsection{Tissue engineering}

A shortage of organs and tissues for transplantation has been present throughout most of the history of transplantation. Over the past few decades, the increasing incidence of vital organ failure and the severe shortage of donors have created a wide gap between organ supply and organ demand, which resulted in very long waiting times to receive an organ as well as an increasing number of deaths while waiting. Moreover, all manners of projections indicate that this gap will continue to widen making this a main challenge to modern medicine $[1,2]$.

Over the years and in order to overcome many challenges in the area of healthcare, technological advancements rapidly evolved and became a crucial part of modern medicine by helping ensure a better lifestyle and an increased life expectancy. Hence, the field of tissue engineering (TE) emerged in response to that growing need for tissues and organs for transplantation and has rapidly become one of the most exciting advances in regenerative medicine. TE is a multidisciplinary field combining principles of biology, medicine and engineering that aim at generating completely biocompatible fully functional organs or tissues that could be used to replace damaged or missing tissues in reconstructive surgery [3,4]. The numerous and complex problems arising when replacing tissues set very high and diverse requirements on the used materials: biodegradability, enabling cell attachment and proliferation and mechanical strength are some of the possible demands.

It is very difficult to find an adequate material that meets all requirements to function properly in a bio-environment. A way is to select a material having the required bulk properties such as mechanical strength and sometimes biodegradability and modify its surface properties via a surface treatment. 
The success of an implant is determined by the response of its surrounding biological environment. This is governed to a large extent by the surface properties of the biomaterial where the interaction happens. Correspondingly, considerable efforts have been focused on surface engineering of biomaterials in order to give them the ability to promote cell adhesion, proliferation and to maintain cell functions. Therefore, a clear characterization of the physical and chemical properties of the biomaterial surface has a major scientific importance on cellbiomaterial interactions allowing the evaluation of the bioactivity of the surface engineered biomaterial.

Up to present, a large number of surface engineering techniques for improving biocompatibility have been well established. The work generally contains three main steps: after the surface modification of the biomaterial is done, chemical and physical characterizations are conducted followed by a biocompatibility assessment through in vitro cell culture $[5,6]$.

In the past decades, surface treatment of biomaterials with plasma has been extensively studied [7-9]. Plasma modification of biomaterials gives the opportunity to change the surface characteristics to achieve better biocompatibility without altering the bulk properties. At the same time, plasma surface modification is a very versatile technology: the results of plasma modification can be easily controlled by choosing suitable gases or monomers and the appropriate conditions in the plasma [10].

\subsection{Plasma}

In order to introduce plasma it is often stated that plasma is the fourth state of matter in the sequence: solid, liquid, gas, and plasma. The state of matter changes from solid to liquid to gas to plasma by increasing the temperature of the material under consideration (see figure 1).

When gas atoms are subjected to energy (thermal, electrical or light) they become ions by releasing some of their electrons. Collisions between electrons and molecules and bond breaks in molecules create radicals. Energy will also create excited species that will generate photons. This is how plasma is created with a unique mixture of electrons, ions, radicals, photons and neutral atoms and molecules [11, 12].

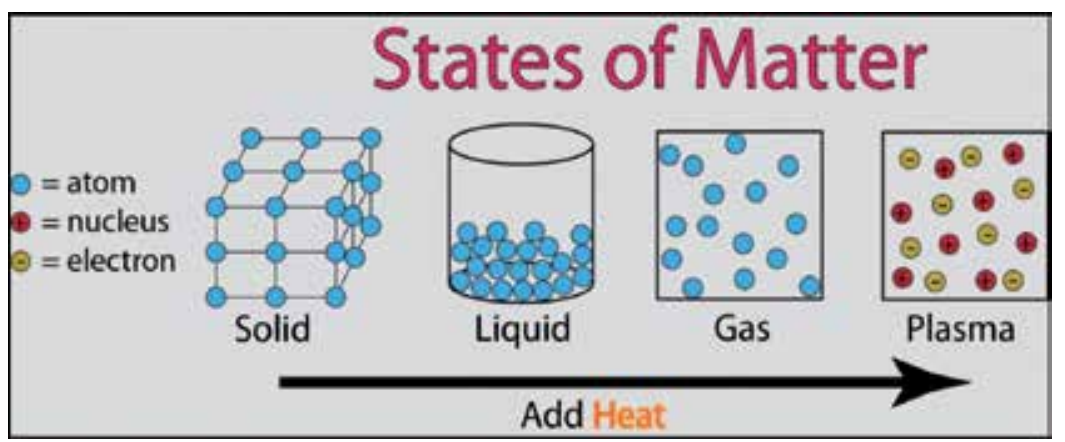

Figure 1. States of matter [90] 
Based on the relative temperatures of electrons, ions and neutrals, plasmas are classified as thermal "equilibrium" and non-thermal "non-equilibrium". Due to the light mass of the electrons present in the plasma, these electrons are instantly accelerated by the electric field to higher velocities than the heavier ions in the time available between collisions. When the collision occurs only a small fraction of the electron energy is lost. This is why the electron temperature in the plasma is initially higher than that of heavy particles. The resulting plasma is a non-thermal or cold plasma in which the electron temperature $\left(\approx 10000^{\circ} \mathrm{C}\right)$ is much higher than the gas temperature $\left(<200{ }^{\circ} \mathrm{C}\right)$. However, if the pressure is so high that the charged particles do not move very far before the next collision or the electrical field is very low, the energy of the electrons may tend towards that of the heavy particles. In this case, the resulting plasma is a thermal or hot plasma.

Plasmas used in "plasma technology" are usually cold plasmas in the sense that only a small fraction of the gas molecules are ionized which can provide electrons and ions at the right energy without excessive heat enabling the use of plasma on heat-sensitive materials such as polymers [13]. The most commonly used method for generating and sustaining a lowtemperature plasma for technological and technical applications is applying an electric field to a pure or mixed gas.

Different plasma sources are available and their dimensions are determined largely by the particular application for which the plasma is intended. There are distinct differences not only in the physical shape of various plasma sources, but also in the temporal behavior of the plasmas that are generated in different sources.

\section{Plasma generation}

\subsection{Plasma sources}

As already mentioned, for surface modification treatments, various plasma sources are available. Each of them has its own characteristics such as density, temperature, chemical composition, etc., and leads to different results. The choice of the proper source for the specific task requires the study of the characteristics of the various plasmas. In this section, a brief summary of the most common plasma sources used for the incorporation of amine functional groups in tissue engineering will be given.

\subsubsection{Microwave (MW) plasma}

MW discharges are electrical discharges generated by electromagnetic waves with frequencies between $300 \mathrm{MHz}$ and $10 \mathrm{GHz}$. MW discharges represent a simple way of plasma generation both with high $\left(>100 \mathrm{~W} / \mathrm{cm}^{3}\right)$ and low $\left(<1 \mathrm{~W} / \mathrm{cm}^{3}\right)$ power levels and can be used over a wide region of operating pressures (from $10^{-3} \mathrm{~Pa}$ up to atmospheric pressure). Nowadays, these discharges are widely used for generation of quasi-equilibrium and non-equilibrium plasmas for different applications because of the simplicity of control of the plasma internal structure by means of changes of the plasma characteristics and the possibility of plasma generation 
both in small and large chambers. The plasma absorbed power can be high enough and runs up to $90 \%$ of the incident power [14, 15].

\subsubsection{Radiofrequency $(R F)$ plasma}

RF discharges usually operate in the frequency range $\mathrm{f}=1-100 \mathrm{MHz}$. The power coupling in RF discharges can be accomplished in different ways: capacitively coupled discharges and inductively coupled discharges.

- Capacitively coupled plasma (CCP)

$\mathrm{CCP}$ is generated with high-frequency RF electric fields, typically $13.56 \mathrm{MHz}$. In its simplest form, the RF voltage is applied across two parallel metal plates, generating an oscillating electric field between them. This field accelerates electrons leading to an ionization avalanche. The parallel electrodes which are separated by a distance of a few centimeters may be in contact with the discharge or insulated from it by a dielectric. Gas pressures are typically in the range $1-10^{3} \mathrm{~Pa}$. In a capacitively coupled RF discharge, the electron density is in the range $\mathrm{n}_{\mathrm{e}}=10^{9}-10^{10} \mathrm{~cm}^{-3}$ and densities up to $10^{11} \mathrm{~cm}^{-3}$ are possible at higher frequencies.

- Inductively coupled plasma (ICP)

ICP is similar to CCP but the electrode consists of a coil wrapped around the discharge volume that inductively excites the plasma. ICP is excited by an electric field generated by a transformer from an RF current in a conductor. The changing magnetic field of this conductor induces an electric field in which the plasma electrons are accelerated. ICPs can achieve high electron densities $\left(\mathrm{n}_{\mathrm{e}}=10^{12} \mathrm{~cm}^{-3}\right)$ at low ion energies $[15,16]$.

\subsubsection{Dielectric Barrier Discharge (DBD)}

Dielectric barrier discharges (silent discharges) are non-equilibrium discharges that can be conveniently operated over a wide temperature and pressure range. DBDs are characterized by the presence of one or more insulating layers in the current path between metal electrodes in addition to the discharge space. At a sufficient $\mathrm{AC}$ voltage, electrical breakdown occurs in many independent thin current filaments. These short-lived microdischarges have properties of transient high pressure glow discharges with electron energies ideally suited for exciting or dissociating background gas atoms and molecules.

Due to charge build up on the dielectric, the field at the location of a microdischarge is reduced within a few nanoseconds after breakdown thus terminating the current flow at this location. The current density in a microdischarge channel can reach 100 to $1000 \mathrm{Acm}^{-2}$. Due to the short duration, this normally results in very little transient gas heating in the remaining channel. The dielectric barrier limits the amount of charge and energy deposited in a microdischarge and distributes the microdischarges over the entire electrode surface. As long as the external voltage is rising, additional microdischarges will occur at new positions because the presence of residual charges on the dielectric has reduced the electric fields at positions where microdischarges have already occurred. When the voltage is reversed, however, the next microdischarges will form in the old microdischarge locations. 


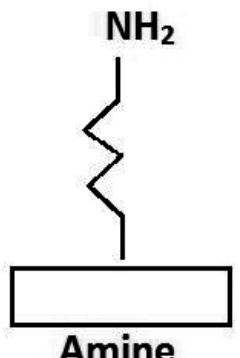

Amine

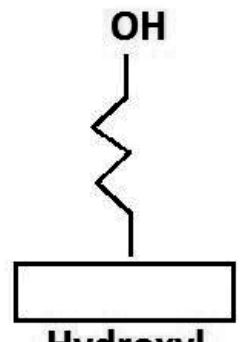

Hydroxyl

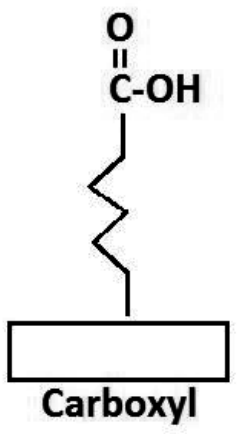

Figure 2. Primary amine, hydroxyl and carboxyl functionalities

Its flexibility with respect to geometrical configuration, operating medium and operating parameters is unprecedented. Conditions optimized in laboratory experiments can easily be scaled up to large industrial installations.

Although DBD configurations can be operated between line frequency and microwave frequencies the typical operating range for most technical DBD applications lies between 500 $\mathrm{Hz}$ and $500 \mathrm{kHz}$ [17].

Plasma can be used in the continuous wave $(\mathrm{CW})$ or pulsed mode. In the continuous wave mode, plasma with a specific power is turned on for a specific amount of time. In the pulsed mode, plasma is intermittently generated with a fixed duty cycle $(\Delta)$.

$$
\Delta=\mathrm{t}_{\text {on }} /\left(\mathrm{t}_{\text {on }}+\mathrm{t}_{\text {off }}\right)
$$

where $t_{\text {on }}$ is the time during which the plasma is turned on and $t_{\text {off }}$ is the time during which the plasma is turned off. The mean power $\left(\mathrm{P}_{\text {mean }}\right)$ is then defined by equation $(2)$ and represents the average energy dissipated in the plasma period, with $\mathrm{P}_{\text {peak }}$ the power injected during $\mathrm{t}_{\text {on }}[18]$.

$$
\mathrm{P}_{\text {mean }}=\Delta * \mathrm{P}_{\text {peak }}
$$

\subsection{Plasma media}

Among other physical and chemical techniques to improve the surface biocompatibility [19-22], plasma surface modification is used to adapt the surface properties by functionalization of the material surface to control the biological response. Different functionalities have been investigated, such as carboxyl [23], hydroxyl [24] and primary amine [25] (see figure 2). In this chapter, we will focus on plasma surface modification of biomaterials by means of primary amine group incorporation. 
Amino groups are usually incorporated on the surface using either non-polymer-forming gases (amine plasma activation) such as ammonia $\left(\mathrm{NH}_{3}\right)$ or nitrogen $\left(\mathrm{N}_{2}\right)$, or amine monomers (amine plasma polymerization) in the plasma medium. The former etches the biomaterial surface and introduces nitrogen functionalities; the latter deposits a plasma polymer layer containing nitrogen groups on the surface [26].

Plasma polymerization of amine-based monomers is an efficient way to prepare bioactive amino functionalized polymer surfaces. Amine-functionalized surfaces have previously been obtained through plasma polymerization using different monomers such as allylamine [27, 28], ethylenediamine [29-31], n-heptylamine [32, 33], propylamine [34, 35], cyclopropylamine [18], diaminocyclohexane [36, 37], and butylamine [26].

In the biomedical field, the most common used plasma media are ammonia as non-polymer forming precursor and allylamine as polymer forming precursor.

Primary amine $\left(-\mathrm{NH}_{2}\right)$ functional groups can promote covalent immobilization with biomolecules such as protein like antibodies, collagen and DNA [28, 38]. Moreover, protonated amines can introduce a localized positive charge in aqueous solution at physiological $\mathrm{pH}$ value, which can potentially be used for electrostatic interactions with negatively charged cells and proteins and is propitious to promote cell adhesion and proliferation [39, 40].

In this chapter we will focus more on amine plasma polymerization since this is the most commonly used plasma technique for the incorporation of primary amines. Plasma polymerization has unique practical advantages which include (i) ultra-thin film deposition, (ii) good adhesion to the substrate material, and (iii) chemically stable and physically durable nature of the polymers [38].

\section{Characterization techniques}

Since the surface of an implant will be readily in contact with the biological environment, the surface characteristics of a biomaterial will have a major influence on the cell-material interactions. These characteristics should be analyzed and studied in order to correlate them with the material biocompatibility and thus try to improve it by changing them.

In order to evaluate the effect of plasma treatments on the surfaces, physical and chemical characterizations are performed using different techniques, such as: water contact angle measurement (WCA), Fourier-transform infrared (FTIR) spectroscopy, X-ray photoelectron spectroscopy (XPS), atomic force microscopy (AFM) and scanning electron microscopy (SEM). In this section, a brief definition of each of these techniques and their correlation with amine plasma treatments will be given.

\subsection{Water Contact Angle measurements (WCA)}

WCA analysis is a simple and widely used test to evaluate the wettability of a surface by measuring the static contact angle of small droplets of distilled water or other liquids on the 
surface. The contact angle of a material is where a liquid/vapour interface meets a solid surface. It quantifies the wettability of a solid surface and thus the relative amounts of adhesive (liquidto-solid) and cohesive (liquid-to-liquid) forces acting on a liquid [41].

If the WCA is smaller than $90^{\circ}$, the solid surface is considered hydrophilic and if it is larger than $90^{\circ}$, the solid surface is considered hydrophobic (see figure 3) [42].
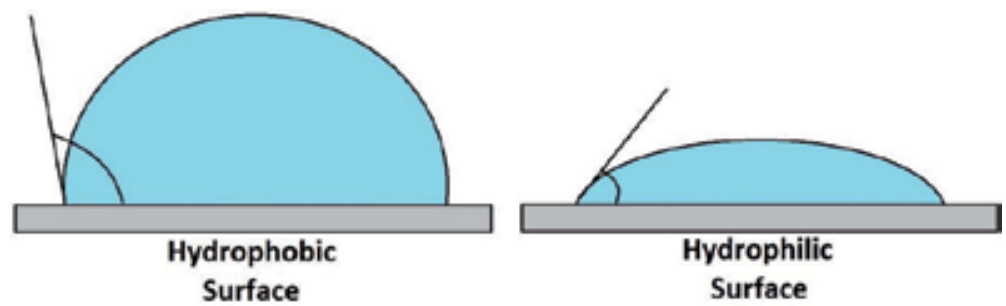

Figure 3. Principle of contact angle measurement

Amine plasma treatment causes a decrease in WCA due to the incorporation of hydrophilic nitrogen and oxygen moieties [43-45] onto the sample surface. Oxygen functionalities can be incorporated via two different processes. First, the vacuum level does not guarantee total absence of oxygen impurities in the plasma chamber [46, 47]. Second, after plasma treatment, samples are exposed to air which can induce a post plasma functionalization by reaction with oxygen and water vapor $[47,48]$.

Additionally, depending on the gas and general conditions of the plasma treatment, it is possible to promote some surface etching/abrasion which can induce changes in surface topography, thus having a certain effect on the wettability. Material removal occurring during plasma treatment increases roughness and contributes to improve the wettability properties of the plasma treated film $[44,49]$.

\subsection{X-ray Photoelectron Spectroscopy (XPS)}

X-ray photoelectron spectroscopy is a very powerful surface analysis technique also known as electron spectroscopy for chemical analysis (ESCA). It works by irradiating a sample with mono-energetic soft X-rays causing surface electrons to be ejected. The identification of the elements in the sample can be made directly from the kinetic energies of these ejected photoelectrons, and the relative concentrations of elements can be determined from the photoelectron intensities.

An important advantage of XPS is its ability to obtain information on chemical states from the variations in binding energies, or chemical shifts, of the photoelectrons [50].

Since a wide range of chemical functionalities is introduced on the surface of a plasma treated material [43], each of these groups might cause the observed binding energy of a particular peak (e.g. C 1s or N 1s) to shift to varying degrees. The resulting overall peak shape is a superposition of many components which are not clearly resolved. 
Curve-fitting can be a powerful method of extracting additional information from XPS data in which contributions from different chemical species can be quantified.

A high amount in nitrogen atoms does not necessary impart a high amount of amine groups on the polymer surface. Peak fitting of the high-resolution spectrum of the $\mathrm{N} 1$ s peak confirms the addition of different nitrogen functionalities to the surface during plasma treatment. The assignments of the different nitrogen groups under the $\mathrm{N}$ 1s peak found in literature are: amines (398.9-399.3 eV) [32, 51, 52], nitriles (399.6 eV) [53], amides (399.8 eV) [32, 51-53], imides $(400.5 \mathrm{eV})[32,51-53]$ and quaternary amines (401.3-401.5 eV) [52-54]. Moreover, in the peak fitting of $C 1 \mathrm{~s}$ in amine plasma deposited films two additional peaks might be observed: 286.4 $\mathrm{eV}$ corresponding to $\mathrm{C}-\mathrm{N}$ (amine) and $288.0 \mathrm{eV}$ corresponding to $\mathrm{N}-\mathrm{C}=\mathrm{O}$ (amide) groups [45, 55]. The areas under the photoelectron peaks in the spectrum are used to calculate the atomic concentrations.

Another more meaningful identification and quantification method of the present chemical groups is a combination of XPS with chemical derivatization. Chemical derivatization consists in inducing a chemical reaction between a targeted chemical group of the amine plasma polymer film and a chemical reactant containing at least one atom different from the ones composing the sample. For a successful derivatization process, selectivity of the reagent towards a particular functional group, its detectivity, kinetics of the reaction, and stability of the derivatized species are some of the factors necessary to identify [55].

In the case of amine plasma treatments, to selectively probe $-\mathrm{NH}_{2}$ groups, several reactants such as 4-trifluoromethyl-benzaldehyde (TFBA) [18, 36, 39, 55-60], pentafluoro-benzaldehyde (PFBA) [61, 62], and para-chlorobenzaldehyde [63] have been used.

The derivatization reaction performed by exposing the treated sample to TFBA vapor is the most commonly used and it consists in a nucleophilic addition on the carbonyl group $(\mathrm{C}=\mathrm{O})$ that converts an $\mathrm{NH}_{2}$ group into an imine (see figure 4). Hence, $\mathrm{NH}_{2}$ groups are selectively probed by the reagent $\mathrm{CF}_{3}$ terminal group with a ratio of one $\mathrm{CF}_{3}$ for one $\mathrm{NH}_{2}$. After the derivatization step, the $\% \mathrm{NH}_{2}$ is calculated from XPS according to [56]:

$$
\% \mathrm{NH} 2=\frac{\left[\mathrm{NH}_{2}\right]}{[\mathrm{N}]}=\frac{([\mathrm{F}] / 3)}{[\mathrm{N}]} \cdot 100 \%
$$

where $\left[\mathrm{NH}_{2}\right],[\mathrm{N}]$ and $[\mathrm{F}]$ represent respectively the relative concentration of primary amines, nitrogen and fluorine at the sample surface.

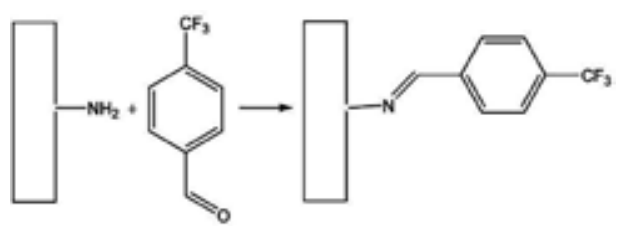

Figure 4. Reaction scheme for TFBA derivatization of primary amine functionalities [91] 


\subsection{Fourier-Transform Infrared (FTIR) spectroscopy}

FTIR is based on the interaction of an oscillating electromagnetic field with a molecule. In a specific compound, a particular structural group reveals IR absorption bands within characteristic spectral regions. In this way, FTIR can be used for assignation of functional groups and identification of pure compounds.

During plasma polymerization, the monomer undergoes reorganization due to the breakage and the recombination of bonds. Amine groups are partially transformed into amide, imine or nitrile functional groups [36, 64]. In various allylamine plasma polymerization studies, one can see after comparison with the pristine monomer that some bands are significantly broadened, some disappeared while new bands also appeared. An example of these spectra can be seen in figure 5. Double peaks of primary amine N-H stretching vibrations at 3380-3290 $\mathrm{cm}^{-1}$ are well resolved on the spectra of the monomer but a wide absorption band is found on the polymer at $3390 \mathrm{~cm}^{-1}$ which can originate from a primary amine, a secondary amine or an imine as well. The deformation vibration of primary amines $\left(1510-1650 \mathrm{~cm}^{-1}\right)$ is observed in both spectra, but is considerably broadened in the spectrum of plasma polymerized allylamine (PPAa), which indicates the presence of alkene groups or imines. A new band appears for the polymer at $2200 \mathrm{~cm}^{-1}$ which is associated with the stretching vibration of nitrile $(\mathrm{C} \equiv \mathrm{N})$ groups $[43,64,65]$.

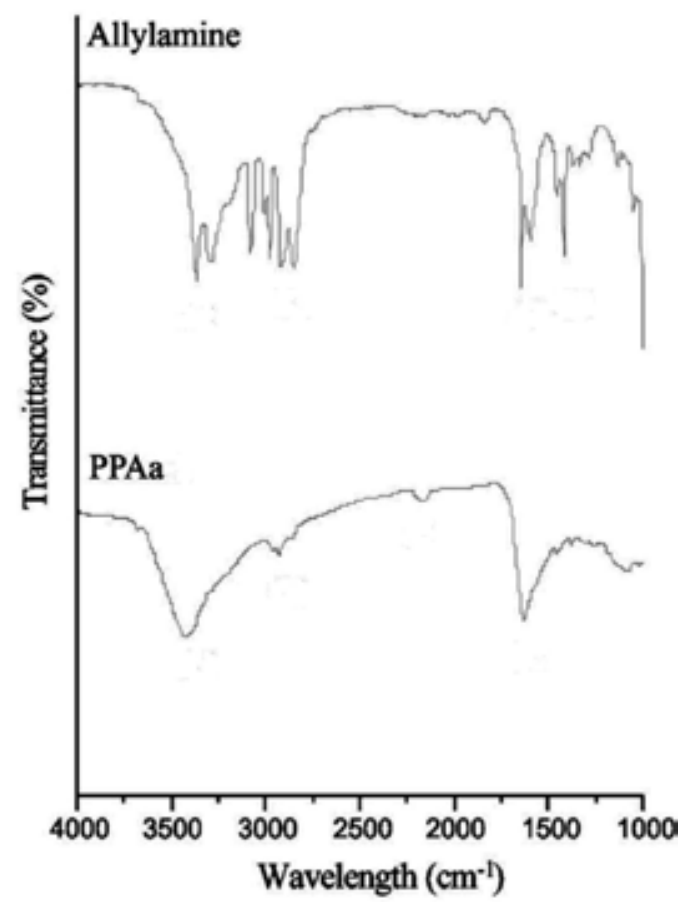

Figure 5. FTIR spectra of allylamine and PPAa [92] 


\subsection{Scanning Electron Microscopy (SEM)}

SEM images the sample surface by scanning it with a high-energy beam of electrons. Depending on the instrument, the resolution can fall somewhere between less than $1 \mathrm{~nm}$ and $20 \mathrm{~nm}$.

SEM has been used in different studies involving plasma treatments. For instance, in a study by Hamerli et al. [43], SEM images showed that allylamine plasma polymerization yields homogenous pinhole-free layers. In another study by Sanchis et al. [44], SEM images showed that nitrogen plasma treatment formed micro-cracks on the sample surface. In this way, SEM can be used on plasma treated samples to give information regarding the deposited film morphology and the treatment effect on the sample surface.

\subsection{Atomic Force Microscopy (AFM)}

AFM is a mechanical imaging instrument that measures the three-dimensional topography as well as physical properties of a surface with a sharpened probe. Typical AFM resolutions are well below $1 \mathrm{~nm}$.

AFM can be used to study the way plasma polymers grow: AFM images have been used to show the surface morphology of the deposited films while varying the treatment time [66]. AFM analysis is useful, not only in a qualitative way but also for quantitative determinations, since it allows a 3D representation of the treated surface and quantifies the effects of the plasma-etching mechanism by calculation of the surface roughness (root-mean-squared roughness, $R_{\text {rms }}$ ) [44].

\section{Effect of plasma process parameters on final layer properties}

Many parameters, such as the nature of the precursor, the precursor flow rate, the reactor chamber's pressure, the reaction time, the power and frequency of the discharge, the geometry and physical dimensions of the reactor, etc. are believed to influence the surface properties of the resulting thin film [67].

In this section, a summary of the effect of plasma process parameters on various aminedeposited film properties as elucidated by researchers in this field will be given. Process parameters will be discussed based on different studies, but one should always keep in mind that various results emerging from different studies are hinted by the possible role of the distinctive processes (different reactor, plasma media and plasma source) used in each case.

\subsection{Discharge power and deposition time effect}

Electrical power and treatment time have a large impact on the nature and concentration of the molecular species formed in the reactor, which in turn significantly influences the deposition rate and the final atomic composition of the deposited layers.

Effect on deposition rate / film thickness: Lucas et al. [61] studied the power variation (between 3 and $100 \mathrm{~W}$ ) effect on the deposition rate of allylamine plasma polymers. They found that 
deposition rate increases with increasing power. The same effect was observed by Myung et al. [64] with power variation between 30 and $90 \mathrm{~W}$. The thickness of the film increases with increasing plasma power since high power generates high plasma density which increases the deposition efficiency. However, in another study by Lejeune et al. [57], the increase of deposition rate with power was found to be valid only up to a certain limit. In fact, the deposition of a film relies on a dynamic equilibrium between a process of deposition and a process of sputtering by incoming particles from the plasma. At low power of deposition $(\mathrm{P}<$ $\left.\mathrm{P}_{\text {limit }}\right)$, the first process dominates the equilibrium. The amount of low energetic particles arriving on the surface of the film increases and the rearrangement of these deposited particles is low. This growth mode favors the formation of a low density structure (low cross-linking degree) with a high growth rate. With the increase of the power, the incoming particles have a higher energy and can penetrate more deeply in the growing film. Structural reorganization processes such as cross-linking of the polymeric chains and re-sputtering phenomena can occur. When the power is high enough $\left(\mathrm{P}>\mathrm{P}_{\text {limit }}\right)$, the second process of the dynamic equilibrium acts effectively on the deposition: due to the sputtering effect, the growth rate becomes constant and due to the bombardment effect, the density of the film increases (high crosslinking degree) (see figure 6). For Lejeune et al. the limiting power was $30 \mathrm{~W}$, but as already mentioned, results from different sources can only be compared to a certain extent and this limiting power varies from one treatment to another by taking into account the plasma media, plasma source, plasma parameters and reactor geometry.

Myung et al. [64] also investigated the effect of treatment time on the deposition rate. They found that the thickness of the deposited layers increases with the increase of plasma polymerization time. Martin et al. [68] investigated the synergistic effect of plasma power and deposition time on n-heptylamine plasma polymerized (HApp) film thicknesses using AFM step height measurements combined with a surface masking technique. The results showed a dramatic difference between conditions involving high power and long deposition (thickness average of $47 \mathrm{~nm}$ ) to those involving low power and short deposition time (thickness average of $3 \mathrm{~nm}$ ) (see figure 7).

Effect on film's atomic composition: Martin et al. [68] also studied the effect of power on the atomic composition of the layers using XPS analysis. High power yielded relatively lower surface concentration of nitrogen atoms than the use of low power: by varying the power from $80 \mathrm{~W}$ to $10 \mathrm{~W}$ nitrogen content increased by ca. $15 \%$. This is due to the more successful breakdown of the monomer molecule achieved at higher power, yielding layers which present less nitrogen atoms. This behavior has also been observed by Shard et al. [35]. However, deposition time and time-power interaction do not have a significant effect on the atomic composition of the layers [68].

In the study conducted by Lucas et al. [61], the use of XPS coupled to derivatization reactions for allylamine plasma polymerization showed that $\% \mathrm{NH}_{2}$ decreases with the increase of power. This was also observed by Lejeune et al. and Müller et al. [57, 69]. The effect of plasma on the retention of the precursor functional group also depends on the plasma mode. Basarir et al. [70] worked on plasma polymerization of allylamine using both CW and pulsed modes. Results showed that pulsed plasma polymerization further increased amine density. In fact, 


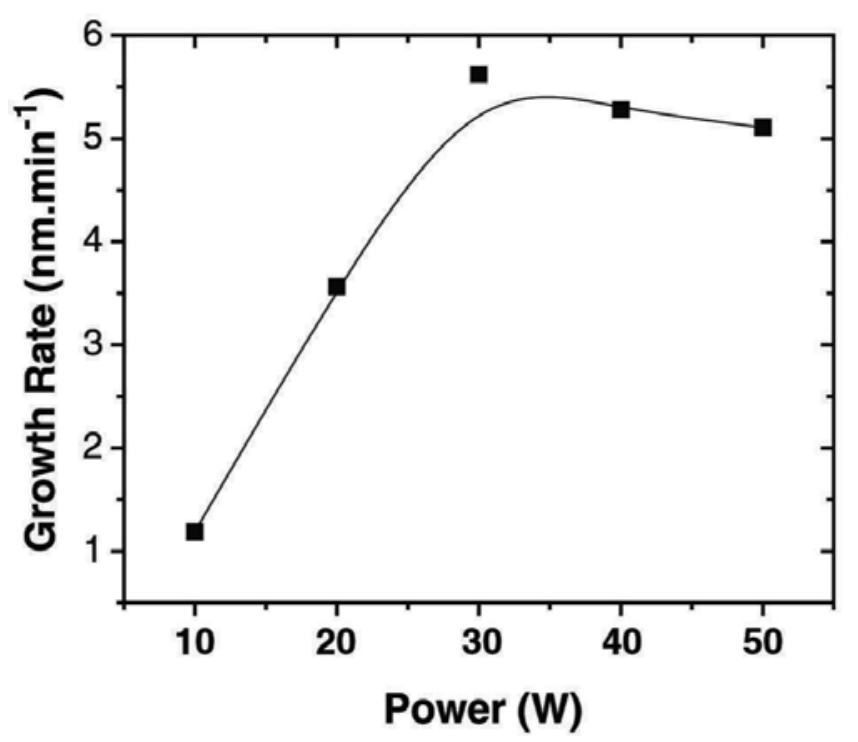

Figure 6. Growth rate of plasma polymerized allylamine films as a function of power [93]

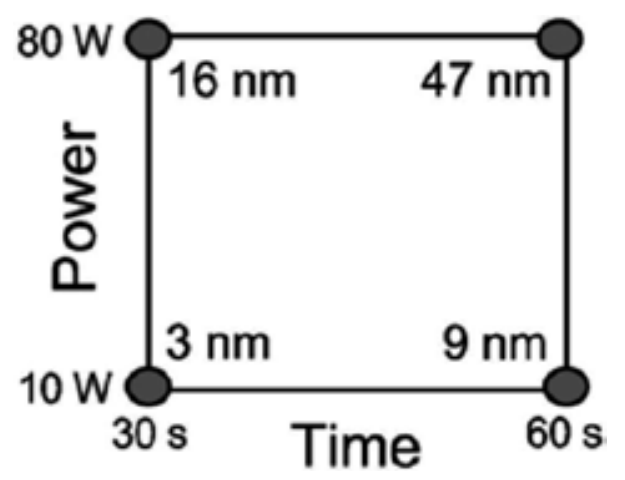

Figure 7. Square plot summarizing the influence of power and deposition time on the thickness of HApp layers [94]

in the pulsed mode, the mean power per precursor molecule is lower than that in the CW mode. In the off-time, dissociated monomers react with each other instead of continuing to dissociate. Moreover, the use of lower $P_{\text {mean }}$ in pulsed plasma polymerization results in a higher retention of the primary amine functional group $[18,56]$.

By using IR spectroscopy on allylamine plasma deposited films, Myung et al. [64] noticed changes in film composition by varying input power between 30 and $90 \mathrm{~W}$. High-power plasma led to a higher ratio of $\mathrm{C} \equiv \mathrm{N}$ to $\mathrm{CH}$ than the ratio of $\mathrm{NH}$ to $\mathrm{CH}$, thus to a recombination of amine functionalities into nitrile $(\mathrm{C} \equiv \mathrm{N})$ groups. This was also observed in two studies by Hamerli et al. [43, 47]. At high power, monomer fragmentation is accelerated and results in the formation of imine groups and nitrile groups. A high retention of amine groups is mainly favored by low input power $(<30 \mathrm{~W})$. This has been confirmed by Wen-Juan et al. [71] where 
power was varied between 5 and $30 \mathrm{~W}$. The increase of plasma power caused more formation of $\mathrm{C}=\mathrm{N}$ groups which can be explained by the sufficient fragmentation of monomer at higher applied powers.

Furthermore, Myung et al. [64] correlated the contact angle to the plasma input power. The contact angle increases with increasing input power thus causing a decrease of the surface free energy i.e. a decrease of the surface hydrophilicity. The main factors which resulted in this effect were the loss of amine functional groups and the formation of cross-linked structures due to more fragmentation of allylamine by increasing input power. In another study by Sanchis et al. [44], treatment time effect on surface morphology was investigated via AFM imaging. Different surface morphologies with a slight increase in surface roughness were observed as exposure time increased.

As we can see, power has a great influence on the chemical and physical properties of the deposited films. The synthesis conditions for deposition of plasma polymer films with high functional group concentration are characterized by low powers thus leading to low precursor fragmentation. This will result in the reduction of the plasma polymer film cross-linking degree [56]. However, the control of the cross-linking degree is an important factor for the optimization of the plasma polymer film stability and mechanical and thermal properties [72]. Therefore, it is important to evaluate the cross-linking degree in addition to the plasma and polymer film chemistry in order to choose the appropriate power [70, 73].

\subsection{Monomer flow rate effect}

Monomer flow rate is an important plasma process parameter that has been investigated in order to correlate it to the deposited film properties.

Hamerli et al. [43] and Basarir et al. [70] investigated monomer flow effect on amine functionality retention. At constant power and treatment time, higher monomer flow rates yielded higher amine retention. The increased amine density with increased flow rates can be explained by less dissociation of monomers, owing to the decreased plasma power for each molecule as the monomer flow rate increased.

Another study by Martin et al. [68] showed that the monomer flow rate does not influence the thickness of the deposited layers.

\subsection{Precursor type effect}

The precursor type is one of the most important factors in plasma treatments. For instance, in plasma polymerization, the use of a non-saturated monomer like allylamine is advantageous compared to saturated ones because in the former case less energy is needed for the polymerization process. In fact, the double bond in allylamine encourages a deposition by a combination of plasma and conventional free-radical polymerization. Because of that, allylamine typically polymerizes at low energies. Due to less fragmentation, a higher amount of primary amines can be retained in the plasma polymer [34,59]. Furthermore, each precursor is characterized by its own chemical structure and thus its particular bond breaking energies 
which influence to a great extent the selectivity of the fragmentation processes in the plasma. Therefore, depending on the kind of monomer used, various chemical compositions are obtained after each plasma treatment [18, 25, 55]. For example, Hamerli et al. [47] used ammonia and allylamine as plasma precursors. A higher amine concentration was found on the allylamine modified samples. Mangindaan et al. [60] used allylamine, propylamine and propargylamine (another unsaturated monomer) as precursors. XPS coupled to derivatization showed that allylamine incorporates the highest amount of amine functionalities into the corresponding thin films compared to those synthesized from the two other precursors.

The precursor type also influences the growth mode and thickness of the deposited layers. Michelmore et al. [66] noticed that films grown from n-heptylamine initially show "islandlike" growth before a continuous smooth film is formed. In contrast, films from allylamine grow smoothly from the very earliest stages. Moreover, it has been found that monomers containing double bonds polymerize faster in plasma than their saturated counterparts. Gancarz et al. [25] have investigated the plasma polymerization of n-butylamine and allylamine and observed that the deposited layers are much thicker for allylamine plasmas. This observation has also been confirmed in a study performed by Mangindaan et al. [60].

\section{Interaction between plasma induced amino groups and cells}

In this section, an overview of literature on plasma treatments used for the incorporation of amine functionalities and their influence on cell-material interactions will be given. It is important to note that each precursor compound and each plasma treatment is unique and will yield different surface chemical and physical properties. Hence, for the same plasma media, a different plasma treatment will yield specific surface characteristics. And for the same plasma treatment, a different plasma media will also yield specific surface characteristics. Moreover, a specific type of cell reacts differently with specific surface properties.

Cell adhesion and proliferation on amine plasma polymer films deposited onto different substrates have been widely investigated. Different plasma methods have been used and different cells and biomolecules interactions with the treated surfaces have been investigated for various biomedical applications. In the following, only some of the main studied bio applications will be explored. An overview of the various cited works on cell-material interactions for different amine plasma treatments is given in table 1.

One important studied bio application is hemocompatibility of biomaterials used in blood contacting devices [74]. Hemocompatibility is considered to be one of the most critical aspects of biocompatibility. In order to achieve better hemocompatibility, a common approach is to immobilize heparin on the surface of the implant. Heparin is a strongly acidic, negatively charged polysaccharide (see figure 8) used in preventing thromboembolic complications due to its high affinity binding to antithrombin III (ATIII) resulting in its conformational change thus accelerating its ability to inactivate the coagulation enzymes [75, 76]. Metallic biomaterials used for vascular devices have excellent mechanical properties and corrosion resistance but have insufficient long-term hemocompatibility. Yang et al. [77] modified stainless steel using 
radio frequency plasma and a mixed gas of allylamine vapor and $\mathrm{NH}_{3}$. After plasma treatment, an FTIR and XPS study of the deposited film showed a good retention of the allylamine structure and thus the presence of primary amine functionalities on the surface. Nevertheless, the appearance of some new peaks in the spectra of the deposited film suggests that the primary amine groups are partially transformed into nitrile, amide or imine functional groups. As a consequence, the hydrophilicity was greatly improved. The water contact angle decreased from 70.8 to $62.7^{\circ}$. Hemocompatibility improvement was successfully achieved by heparin immobilization on the plasma polymerized allylamine. Cell culture tests were also conducted using endothelial cells which were found to adhere and proliferate in a better way on the plasma polymerized allylamine coating. Both improved hemocompatibility, adhesion and proliferation of endothelial cells were attributed to a combined effect of increased wettability and amine based surface chemistry. In another study [78], the improvement of hemocompatibility of polymeric vascular prosthesis such as polytetrafluoroethylene (PTFE), polystyrene and silicon was investigated. After depositing diamond-like carbon (DLC) films on the polymer substrates via acetylene plasma, functionalization was done using ammonia plasma. The $\mathrm{NH}_{3}$ plasma exposure time was varied from 0 to $300 \mathrm{~s}$ resulting in a different heparin coverage. For the different treatment times, heparin was successfully immobilized on the functionalized DLC leading to an extended blood coagulation time. The maximum of bound heparin was obtained at a $30 \mathrm{~s}$ treatment. Moreover, different heparin coverages were obtained for the different substrates. Based on these observations, the authors suggested that the initial surface roughness and the plasma treatment time i.e. the surface chemical structure influence the final heparin coverage.

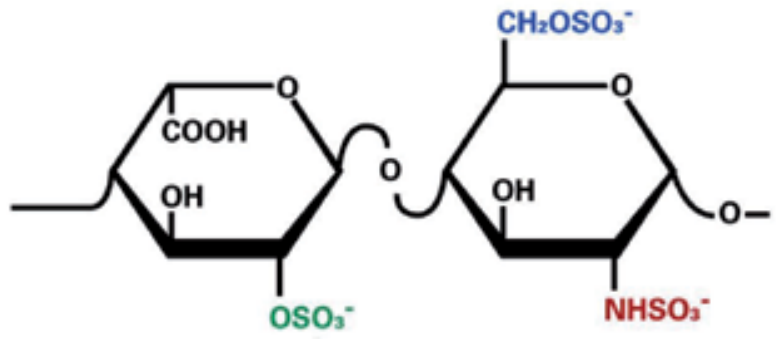

Figure 8. Chemical structure of heparin molecule [95]

In another important studied bio application related to biomedical implant devices, cell adhesion is very important because it is considered to be the determinant of the success or failure of implantation. Anchorage-dependent cells such as fibroblasts and osteoblasts need the adhesion for cell growth, division and spreading [79]. It was found that attachment, proliferation and function of these anchorage-dependent cells are highly dependent on the surface properties of biomaterials [80]. The variation of biocompatibility after amine plasma treatment of many biomaterials has been studied by the means of fibroblast and osteoblast cell cultures. Wen-Juan et al. [71] and Ren et al. [81] modified silicon surfaces with allylamine dielectric barrier discharge and microwave plasma respectively. After plasma exposure, the 
contact angle decreased considerably due to the formation of various nitrogen functionalities as determined by FTIR. Cell culture tests with fibroblasts showed that both cell adhesion as well as cell proliferation could be improved by allylamine plasma treatment. Comparable results were obtained by Zelzer et al. [82] using glass substrates.

In a study by Hamerli et al. [43], the surface of polyethylene terephtalate (PET) was modified via allylamine microwave plasma polymerization. Plasma process parameters such as power (MW power), monomer flow rate $\left(\varnothing_{\text {Allylamine }}\right)$ and duty cycle were varied which allowed the formation of different film chemical compositions. FTIR and XPS indicated that nitrogen as well as oxygen functionalities were incorporated which resulted in an increased hydrophilicity. Pictures from scanning electron microscopy showed that homogeneous pinhole-free allylamine plasma polymer (PPAa) films were obtained. Cell tests revealed improved cell attachment and spreading on PPAa coated PET compared to plain PET (see figure 9) with a greater improvement of biocompatibility on plasma polymerized allylamine coated surfaces containing amine functionalities in relatively high concentration. This is in agreement with other researches indicating that amino groups rather than others are favorable for protein adhesion [83, 84].
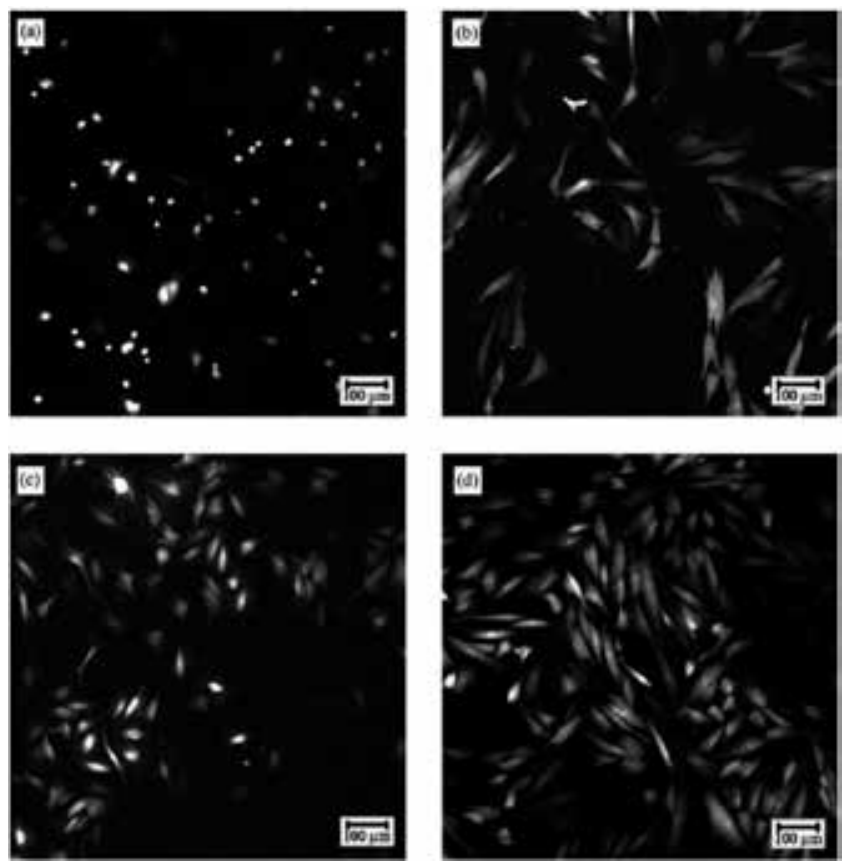

Figure 9. Photomicrographes of fibroblasts adhering on PET and PPAa membrane surfaces; $(\mathrm{a}, \mathrm{c}) 4 \mathrm{~h}$ of cultivation, (b,d) $24 \mathrm{~h}$ of cultivation, $(\mathrm{a}, \mathrm{b})$ PET membrane, and $(\mathrm{c}, \mathrm{d})$ PPAa coated PET membrane "MW power of $1200 \mathrm{~W}$ and $\varnothing_{\text {Allylamine }}$ of $30 \mathrm{sccm}^{\prime \prime}$ [96]

In another study by Hamerli et al. [47], PET membranes were modified by ammonia and allylamine microwave plasma treatments. Both plasma treatments yielded an approximately 
similar decrease in contact angle. Cell tests showed that fibroblast adhesion and spreading was improved for both plasma treatments compared to plain PET with higher proliferation on allylamine-modified samples in comparison to ammonia-plasma modified samples. This is mainly attributed to the higher amine concentration on allylamine modified PET.

Other studies involving osteoblast cell tests were also conducted on amine plasma polymerized films resulting in an improved cell adhesion and proliferation [39, 59, 65].

\begin{tabular}{|c|c|c|c|c|}
\hline Substrate & Plasma media & $\begin{array}{l}\text { Cell / biomolecule } \\
\text { type }\end{array}$ & Observations & Ref \\
\hline 316L stainless steel & $\begin{array}{c}\text { Allylamine } \\
+ \\
\text { Ammonia } \\
+ \\
\text { Ar }\end{array}$ & $\begin{array}{l}\text { Heparin, endothelial } \\
\text { cells }\end{array}$ & $\begin{array}{l}\text { Good immobilization of heparin/ } \\
\text { improved hemocompatibility/ } \\
\text { promotion of adhesion and } \\
\text { proliferation of endothelial cells }\end{array}$ & [77] \\
\hline Silicon & $\begin{array}{c}\text { Allylamine } \\
+ \\
\text { Ar }\end{array}$ & fibroblast & Improved cell adhesion and growth & {$[81]$} \\
\hline Silicon & $\begin{array}{l}\text { 1,2-diaminocyclo- } \\
\text { hexane }\end{array}$ & Plasma proteins & Improved protein adsorption & [83] \\
\hline Polysulfone & $\begin{array}{l}\text { Ammonia/n- } \\
\text { butylamine/ } \\
\text { allylamine }\end{array}$ & Glucose isomerase & $\begin{array}{l}\text { Successful immobilization of } \\
\text { enzyme }\end{array}$ & [26] \\
\hline BK7 glass & Allylamine & DNA & Successful immobilization of DNA & [85] \\
\hline Polysiloxane & Allylamine & Spinal cord cells & $\begin{array}{c}\text { Cell attachment and growth after } 14 \\
\text { days culture }\end{array}$ & [86] \\
\hline Silicon & $\begin{array}{c}\text { Allylamine } \\
+ \\
\mathrm{Ar}\end{array}$ & Fibroblast & $\begin{array}{l}\text { Promotion of cell growth and } \\
\text { maintenance of their activity }\end{array}$ & {$[71]$} \\
\hline Glass & Allylamine & Fibroblast & $\begin{array}{l}\text { Improved cell adherence and } \\
\text { proliferation }\end{array}$ & {$[82]$} \\
\hline Polyethylene terephtalate & $\begin{array}{c}\text { Allylamine } \\
+ \\
\text { Ar }\end{array}$ & Fibroblast & $\begin{array}{l}\text { Improved cell attachment and } \\
\text { viability }\end{array}$ & [43] \\
\hline Titanium alloy & Allylamine & $\begin{array}{l}\text { Lysozyme and } \\
\text { protein-4 (BMP-4) }\end{array}$ & $\begin{array}{l}\text { Immobilization of biomolecules and } \\
\text { retained activity on high amino } \\
\text { groups density surfaces }\end{array}$ & [87] \\
\hline Titanium & Allylamine & $\begin{array}{c}\text { Monocyte, } \\
\text { macrophage, T- }\end{array}$ & $\begin{array}{c}\text { Minor reduction of inflammatory } \\
\text { reactions in low water solubility } \\
\text { films }\end{array}$ & {$[58]$} \\
\hline
\end{tabular}




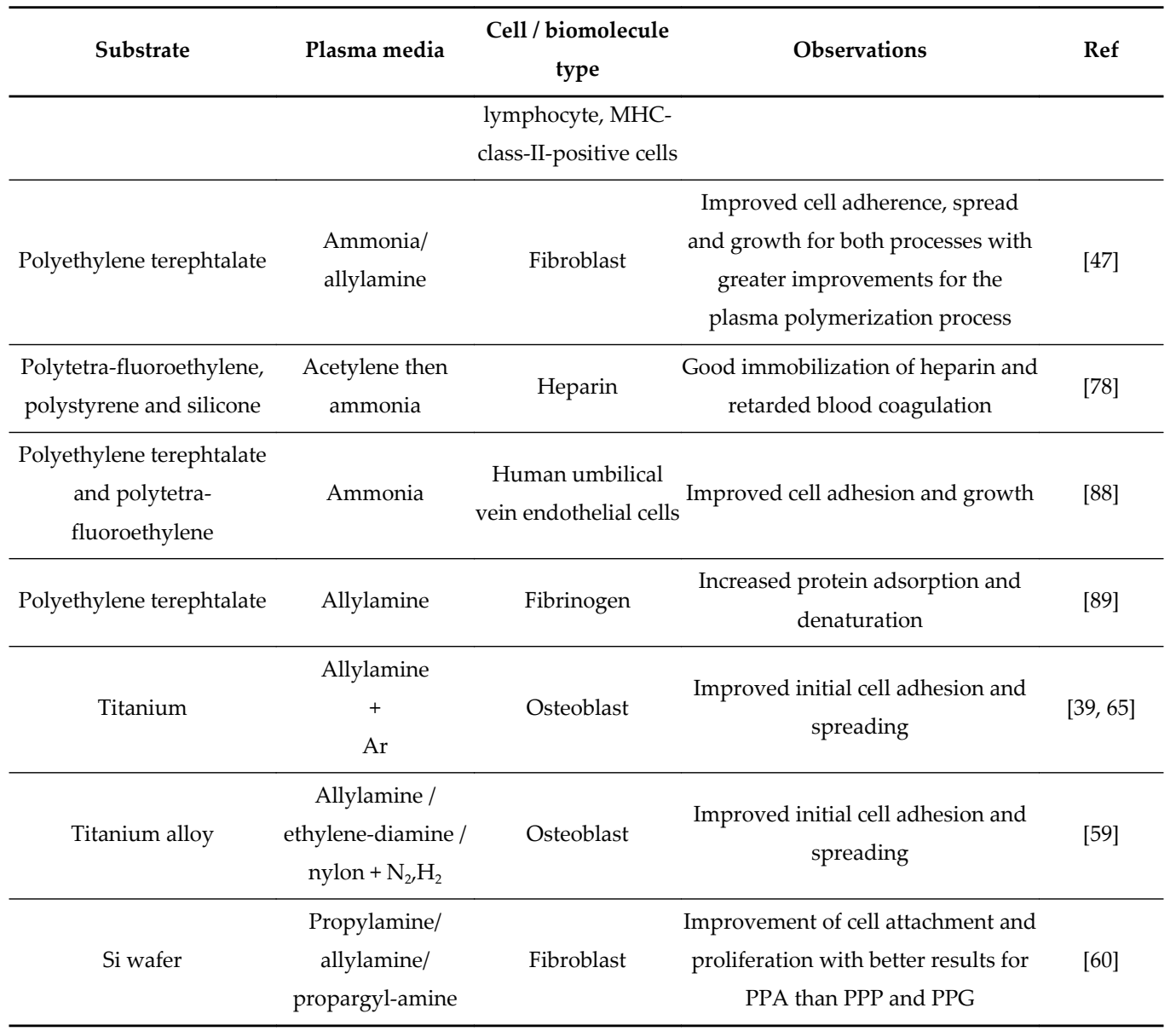

Table 1. Representative overview of plasma processes for the incorporation of amine groups and their influence on cell-material interactions

\section{Conclusions and future trends}

From the work presented in this chapter, it is clear that amine plasma based techniques are powerful versatile tools to modify a material surface in order to make it more biocompatible. However, despite the vast array of choices offered by plasma processing techniques, there remain a significant number of hurdles that must be overcome to allow this methodology to realize its full potential in the area of biocompatible materials. Challenges include issues associated with analytical characterization, material structure, plasma processing, and uniform composition and thickness following treatment. A better understanding of the cellbiomaterial interactions is of crucial importance providing us with essential clues about which strategies should be pursued. As our fundamental understanding of these complex phenomena further develops, better control over cell adhesion, proliferation and differentiation will 
be achievable. Up till now, plasma surface modification is mostly studied on two-dimensional substrates but it can be expected that the study of 3D porous scaffolds will become more important. It is believed that, in the future, a broader exploration of various plasma strategies, monomers and biomaterials will enable us to achieve homogeneous scaffolds with desired mechanical, chemical and biodegradation properties in an industrialized setting.

\section{Author details}

Gaelle Aziz , Nathalie De Geyter and Rino Morent

*Address all correspondence to: Gaelle.Aziz@ugent.be

Ghent University, Faculty of Engineering \& Architecture, Department of Applied Physics, Research Unit Plasma Technology (RUPT), Ghent, Belgium

\section{References}

[1] Siminoff L A, Arnold R M, Caplan A L, Virnig B A, Seltzer D L. Public Policy Governing Organ and Tissue Procurement in the United States: Results from the National Organ and Tissue Procurement Study. Annals of internal medicine 1995;123(1) 10-17.

[2] Opar A. As demand for organs expands, so does transplant technology. Nature Medicine 2008;14(3) 225-225.

[3] Katti D, Vasita R, Shanmugam K. Improved biomaterials for tissue engineering applications: surface modification of polymers. Current topics in medicinal chemistry 2008;8(4) 341-353.

[4] Nerem R M, Sambanis A. Tissue Engineering: From Biology to Biological Substitutes. Tissue engineering 1995;1(1) 3-13.

[5] Ma Z, Mao Z, Gao C. Surface modification and property analysis of biomedical polymers used for tissue engineering. Colloids and Surfaces B: Biointerfaces 2007;60(2) 137-157.

[6] Griffith L G, Naughton G. Tissue Engineering-Current Challenges and Expanding Opportunities. Science 2002;295(5557) 1009-1014.

[7] Morent R, De Geyter N, Desmet T, Dubruel P, Leys C. Plasma surface modification of biodegradable polymers: a review. Plasma processes and polymers 2011;8(3) 171-190. 
[8] Desmet T, Morent R, De Geyter N, Leys C, Schacht E, Dubruel P. Nonthermal plasma technology as a versatile strategy for polymeric biomaterials surface modification: a review. Biomacromolecules 2009;10(9) 2351-2378.

[9] Chu P K, Chen J Y, Wang L P, Huang N. Plasma-surface modification of biomaterials. Materials science and engineering R 2002;36(5-6) 143-206.

[10] Felten A, Bittencourt C, Pireaux J J, Van Lier G, Charlier J C. Radio-frequency plasma functionalization of carbon nanotubes surface O2, NH3, and CF4 treatments. Journal of applied physics 2005; 98(7) 074308-9.

[11] Christophorou L G, Olthoff J K. Fundamental Electron Interactions with Plasma Processing Gases. Springer; 2004.

[12] Plasmas international. Perspectives on plasmas. http://www.plasmas.org/what-areplasmas.htm (accessed 2 July 2014).

[13] De Geyter N. Plasma modification of polymer surfaces in the subatmospheric pressure range. PhD thesis. Gent University Faculty of engineering and architecture department of applied physics; 2007-2008.

[14] Lebedev Yu A. Microwave discharges: generation and diagnostics. Journal of Physics: Conference Series 2010;257(1) 1-12.

[15] Conrads H, Schmidt M. Plasma generation and plasma sources. Plasma sources Science and Technology 2000; 9(4) 441-454.

[16] Chen F F. Radiofrequency plasma sources for semiconductor processing. Advanced plasma technology. Wiley-VCH Verlag GmbH \& Co. KGaA; 2008. p99-115.

[17] Kogelschatz U, Eliasson B, Egli W. Dielectric-barrier discharges. Principle and applications. Journal de Physique IV 1997; 7(C4) C4-47-C4-66.

[18] Denis L, Marsal P, Olivier Y, Godfroid T, Lazzaroni R, Hecq M, Cornil J, Snyders R. Deposition of Functional Organic Thin Films by Pulsed Plasma Polymerization: A Joint Theoretical and Experimental Study. Plasma Processes and Polymers 2010;7(2) 172-181.

[19] Altankov G, Thom V, Groth Th. Modulating the biocompatibility of polymer surfaces with poly(ethylene glycol): Effect of fibronectin. Journal of biomedical materials research 2000;52(1) 219-230.

[20] Sbarbati R, Gianessi G, Cenni MC. Pyrolitic carbon coating enhances Teflon and Dacron fabric compatibility with endothelial cell growth. The international journal of artificial organs 1991;14(8) 491-498.

[21] Singh S K, Singh M K, Kulkarni P P, Sonkar V K, Grácio J J A, Dash D. Amine-modified graphene: thrombo-protective safer alternative to graphene oxide for biomedical applications. American chemical society 2012;6(3) 2731-2740. 
[22] Nanci A, Wuest J D, Brunet P. Chemical modification of titanium surfaces for covalent attachment of biological molecules. Journal of biomedical materials research 1998;40(2) 324-335.

[23] Rossini P, Colpo P, Cecone G. Surface engineering of polymeric films for biomedical applications. Materials science and engineering: C 2003; 23(3) 353-358.

[24] Lee J H, Park J W, Lee H B. Cell adhesion and growth on polymer surfaces with hydroxyl groups prepared by water vapour plasma treatment. Biomaterials 1991;12(5) 443-448.

[25] Gancarz I, Poźniak G, Bryjak M, Tylus W. Modification of polysulfone membranes 5. Effect of n-butylamine and allylamine plasma. European Polymer Journal 2002;38(10) 1937-1946.

[26] Gancarz I, Bryjak J, Poźniak G, Tylus W. Plasma modified polymers as a support for enzyme immobilization II. Amines plasma. European polymer journal 2003;39(11) 2217-2224.

[27] Yameen B, khan H U, Knoll W, Förch R, Jonas U. Surface initiated polymerization on pulsed plasma deposited polyallylamine: a polymer substrate-independent strategy to soft surfaces with polymer brushes. Macromolecular rapid communications 2011;32(21) 1735-1740.

[28] Hook A L, Thissen H, Quinton J, Voelcker N H. Comparison of the binding mode of plasmid DNA to allylamine plasma polymer and poly(ethylene glycol) surfaces. Surface science 2008;602(10) 1883-1891.

[29] Nakamura R, Muguruma H, Ikebukuro K, Sasaki S, Nagata R, Karube I, Pedersen H. A Plasma-Polymerized Film for Surface Plasmon Resonance Immunosensing. Analytical chemistry 1997;69(22) 4649-4652.

[30] Choukourov A, Biederman H, Kholodkov I, Slavinska D, Trchova M, Hollander A. Properties of Amine-Containing Coatings Prepared by plasma polymerization. Journal of Applied Polymer Science 2004;92(2) 979 -990.

[31] Kim J, Shon H K, Jung D, Moon D W, Han S Y, Lee T G. Quantitative chemical derivatization technique in time-of-flight secondary ion mass spectrometry for surface amine groups on plasma-polymerized ethylenediamine film. Analytical chemistry 2005;77(13) 4137-4141.

[32] Gengenbach T R, Chatelier R C, Griesser H J. Characterization of the Ageing of Plasma-deposited Polymer Films: Global Analysis of X-ray Photoelectron Spectroscopy Data. Surface and interface analysis 1996;24(4) 271-281.

[33] Vermette P, Meagher L. Immobilization and Characterization of Poly(acrylic acid) Graft Layers. Langmuir 2002;18(26) 10137-10145. 
[34] Fally F, Doneux C, Riga J, Verbist J J. Quantification of the functional groups present at the surface of plasma polymers deposited from propylamine, allylamine, and propargylamine. Journal of Applied Polymer Science 1995;56(5) 597-614.

[35] Shard A G, Whittle J D, Beck A J, Brookes P N, Bullett N A, Talib R A, Mistry A, Barton D, McArthur S L. A NEXAFS Examination of Unsaturation in Plasma Polymers of Allylamine and Propylamine. The journal of physical chemistry B 2004;108(33) 12472-12480.

[36] Choukourov A, Biederman H, Slavinska D, Trchova M, Hollander A. The influence of pulse parameters on film composition during pulsed plasma polymerization of diaminocyclohexane. Surface and Coatings Technology 2003;174-175 863-866.

[37] Gölander C-G, Rutland M W, Cho D L, Johansson A, Ringblom H, Jönsson S, Yasuda $\mathrm{H}$ K. Structure and surface properties of diaminocyclohexane plasma polymer films Journal of Applied Polymer Science 1993;49(1) 39-51.

[38] Puleo DA, Kissling RA, Sheu MS. A technique to immobilize bioactive proteins, including bone morphogenetic protein-4 (BMP-4), on titanium alloy. Biomaterials 2002;23(9) 2079-2087.

[39] Finke B, Luethen F, Schroeder K, Mueller P D, Bergemann C, Frant M, Ohl A, Nebe B J. The effect of positively charged plasma polymerization on initial osteoblastic focal adhesion on titanium surfaces. Biomaterials 2007;28(30) 4521-4534.

[40] Meyer-Plath A A, Schröder K, Finke B, Ohl A. Current trends in biomaterial surface functionalization-nitrogen-containing plasma assisted processes with enhanced selectivity. Vacuum 2003;71(3) 391-406.

[41] Rame-hart, Surface Science Instruments. Wetting, adhesion and cohesion. http:// ramehart.com/newsletters/2009-02_news.htm (accessed 5 July 2014).

[42] Rame-hart, Surface Science Instruments. Information on contact angle. http://ramehart.com/contactangle.htm (accessed 5 July 2014)

[43] Hamerli P, Weigel Th, Groth Th, Paul D. Surface properties of and cell adhesion onto allylamine-plasma-coated polyethylenterephtalat membranes. Biomaterials 2003;24(22) 3989-3999.

[44] Sanchis M R, Calvo O, Fenollar O, Garcia D, Balart R. Characterization of the surface changes and the aging effects of low-pressure nitrogen plasma treatment in a polyurethane film. Polymer testing 2008;27(1) 75-83.

[45] Nitschke M, Schmack G, Janke A, Simon F, Pleul D, Werner C. Low pressure plasma treatment of poly(3-hydroxybutyrate): toward tailored polymer surfaces for tissue engineering scaffolds. Journal of Biomedical Materials Research 2002;59(4) 632-638. 
[46] Dhayal M, Alexander M R, Bradley J W. The surface chemistry resulting from lowpressure plasma treatment of polystyrene: the effect of residual vessel bound oxygen. Applied surface science 2006;252(22) 7957-7963.

[47] Hamerli P, Weigel Th, Groth Th, Paul D. Enhanced tissue-compatibility of polyethylenterephtalat membranes by plasma aminofunctionalisation. Surface and coatings technology 2003;174-175 574-578.

[48] Ortiz-Magán A B, Pastor-Blas M M, Ferrándiz-Gómez T P, Morant-Zacarés C, Martín-Martínez J M. Surface modifications produced by N2 and O2 RF plasma treatment on a synthetic vulcanized styrene-butadiene rubber. Plasmas and polymers 2001;6(1-2) 81-105.

[49] Mokbul H M. Plasma technology for deposition and surface modification. Vol. 4. Logos Verlag Berlin GmbH; 2008.

[50] Engelhard M. X-ray photoelectron spectroscopy XPS. http://emsl.pnl.gov/capabilities/spectroscopy/engelhard_xps.pdf (accessed 23 July 2014).

[51] Gengenbach T R, Chatelier R C, Griesser H J. Correlation of the nitrogen 1s and oxygen 1s XPS binding energies with compositional changes during oxidation of ethylene diamine plasma polymers. Surface and interface analysis 1996;24(9) 611-619.

[52] Lawrie G, Grøndahl L, Battersby B, Keen I, Lorentzen M, Surawski P, Trau M. Tailoring Surface Properties To Build Colloidal Diagnostic Devices: Controlling Interparticle Associations. Langmuir 2006;22(1) 497-505.

[53] Tyler B J, Castner D G, Ratner B D. Regularization: A stable and accurate method for generating depth profiles from angle-dependent XPS data. Surface and Interface Analysis 1989;14(8) 443-450.

[54] Boonaert C J P, Rouxhet P G. Surface of Lactic Acid Bacteria: Relationships between Chemical Composition and Physicochemical Properties. Applied and environmental microbiology 2000;66(6) 2548-2554.

[55] Keen I, Broota P, Rintoul L, Fredericks P, Trau M, Grøndahl L. Introducing amine functionalities on a poly(3-hydroxybutyrate-co-3-hydroxyvalerate) surface: comparing the use of ammonia plasma treatment and ethylenediamine aminolysis. Biomacromolecules 2006;7(2) 427-434.

[56] Denis L, Cossement D, Godfroid T, Renaux F, Bittencourt C, Snyders R, Hecq M. Synthesis of allylamine plasma polymer films: correlation between plasma diagnostic and film characteristics. Plasma processes and polymers 2009;6(3) 199-208.

[57] Lejeune M, Brétagnol F, Ceccone G, Colpo P, Rossi F. Microstructural evolution of allylamine polymerized plasma films. Surface and coatings technology 2006;200(20-21) 5902-5907.

[58] Hoene A, Walschus U, Patrzyk M, Finke B, Lucke S, Nebe B, Schroeder K, Ohl A, Schlosser M. In vivo investigation of the inflammatory response against allylamine 
plasma polymer coated titanium implants in a rat model. Acta Biomaterialia 2010;6(2) 676-683.

[59] Finke B, Hempel F, Testrich H, Artemenko A, Rebl H, Kylián O, Meichsner J, Biederman H, Nebe B, Weltmann K-D, Schröder K. Plasma process for cell-adhesive titanium surfaces based on nitrogen-containing coatings. Surface and coatings technology 2011;205(2) S520-S524

[60] Mangindaan D, Kuo W H, Chang C C, Wang S L, Liu H C, Wang M J. Plasma polymerization of amine-containing thin films and the studies on the deposition kinetics. Surface and coatings technology 2011;206(6) 1299-1306.

[61] Lucas S, Genard G, Michiels C, Masereel B, Feron O, Gallez B, Vander Borght T, Moreau N. Production and preliminary characterization of DC plasma polymerized allylamine film (PPAA) by NRA, ERD and XPS. Nuclear instruments and methods in physics research B 2008;266(10) 2494-2497.

[62] Kim J, Jung D, Park Y, Kim Y, Moon D W, Lee T G. Quantitative analysis of surface amine groups on plasma-polymerized ethylenediamine films using UV-visible spectroscopy compared to chemical derivatization with FT-IR spectroscopy, XPS and TOF-SIMS. Applied surface science 2007;253(9) 4112-4118.

[63] Snyders R, Zabeida O, Roberges C, Shingel K I, Faure M P, Martinu L, Klemberg-Sapieha J E. Mechanism of adhesion between protein-based hydrogels and plasma treated polypropylene backing. Surface science 2007;601(1) 112-122.

[64] Myung S W, Choi H S. Chemical structure and surface morphology of plasma polymerized-allylamine film. Korean Journal of Chemical Engineering 2006;23(3) 505-511.

[65] Nebe B, Finke B, Lüthen F, Bergemann C, Schröder K, Rychly J, Liefeith K, Ohl A. Improved initial osteoblast functions on amino-functionalized titanium surfaces. Biomolecular engineering 2007;24(5) 447-454.

[66] Michelmore A, Martinek P, Sah V, Short R D, Vasilev K. Surface morphology in the early stages of plasma polymer film growth from amine-containing monomers. Plasma processes and polymers 2011;8(5) 367-372.

[67] Truica-Marasescu F, Wertheimer M R. Nitrogen-rich plasma-polymer films for biomedical applications. Plasma processes and polymers 2008;5(1) 44-57.

[68] Martin Y, Boutin D, Vermette P. Study of the effect of process parameters for n-heptylamine plasma polymerization on final layer properties. Thin solid films 2007;515(17) 6844-6852.

[69] Müller M, Oehr C. Plasma aminofunctionalisation of PVDF microfiltration membranes: comparison of the in plasma modifications with a grafting method using ES$\mathrm{CA}$ and an amino-selective fluorescent probe. Surface and coatings technology 1999;116-119 802-807. 
[70] Basarir F, Cuong N, Song W-K, Yoon T-H. Surface modification via plasma polymerization of allylamine for antibody immobilization. Macromolecular symposia 2007;249-250(1) 61-66.

[71] Wen-Juan H, Fen-Yan X, Qiang C, Jing W. Amine-containing film deposited in pulsed dielectric barrier discharge at a high pressure and its cell adsorption behaviors. Chinese physics B 2009;18(3) 1276-1282.

[72] Denis L, Thiry D, Cossement D, Gerbaux P, Brusciotti F, Van De Keere I, Goossens V, Terryn H, Hecq M, Snyders R. Towards the understanding of plasma polymer film behaviour in ethanol: A multi-technique investigation. Progress in organic coatings 2011;70(2-3) 134-141.

[73] Prikryl R, Cech V, Zajickova L, Vanek J, Behzadi S, Jones F R. Mechanical and optical properties of plasma-polymerized vinyltriethoxysilane. Surface and coatings technology 2005;200(1-4) 468-471.

[74] Waterhouse A, Yin Y, Wise S G, Bax D V, McKenzie D R, Bilek M M M, Weiss A S, $\mathrm{Ng}$ M K C. The immobilization of recombinant human tropoelastin on metals using a plasma-activated coating to improve the biocompatibility of coronary stents. Biomaterials 2010;31(32) 8332-8340.

[75] Hirsh J, Raschke R, Warkentin T E, Dalen J E, Deykin D, Poller L. Heparin: Mechanism of Action, Pharmacokinetics, Dosing Considerations, Monitoring, Efficacy, and Safety. American college of chest physicians 1995;108(4) 258S-275S.

[76] Riesenfeld J, Olsson P, Sanchez J, Mollnes T E. Surface modification with functionally active heparin. Medical Device Technology 1995;6(2) 24-31.

[77] Yang Z, Wang J, Luo R, Maitz M F, Jing F, Sun H, Huang N. The covalent immobilization of heparin to pulsed-plasma polymeric allylamine films on $316 \mathrm{~L}$ stainless steel and the resulting effects on hemocompatibility. Biomaterials 2010;31(8) 2072-2083.

[78] Steffen H J, Schmidt J, Gonzalez-Elipe A. Biocompatible surfaces by immobilization of heparin on diamond-like carbon films deposited on various substrates. Surface and interface analysis 2000;29(6) 386-391.

[79] Hamdan M, Blanco L, Khraisat A, Tresguerres I F. Influence of Titanium Surface Charge on Fibroblast Adhesion. Clinical implant dentistry and related research 2006;8(1) 32-38.

[80] Groth T, Seifert B, Malsch G, Albrecht W, Paul D, Kostadinova A, Krasteva N, Altankov G. Interaction of human skin fibroblasts with moderate wettable polyacrylonitrile-copolymer membranes. Journal of biomedical materials research 2002;61(2) 290-300.

[81] Ren T B, Weigel T, Groth T, Lendlein A. Microwave plasma surface modification of silicone elastomer with allylamine for improvement of biocompatibility. Journal of biomedical materials research part A 2008;86A(1) 209-219. 
[82] Zelzer M, Majani R, Bradley J W, Rose F R, Davies M C, Alexander M R. Investigation of cell-surface interactions using chemical gradients formed from plasma polymers. Biomaterials 2008;29(2) 172-184.

[83] Lassen B, Gölander C-G, Johansson A, Elwing H. Some model surfaces made by RF plasma aimed for the study of biocompatibility. Clinical materials 1992;11(1-4) 99-103.

[84] Webb K, Hlady W, Tresco P A. Relative importance of surface wettability and charged functional groups on NIH 3T3 fibroblast attachment, spreading, and cytoskeletal organization. Journal of biomedical materials research 1998;41(3) 422-430.

[85] Zhang Z, Knoll W, Förch R. Amino-functionalized plasma polymer films for DNA immobilization and hybridization. Surface and coatings technology 2005;200(1-4) 993-995.

[86] Harsch A, Calderon J, Timmons R B, Gross G W. Pulsed plasma deposition of allylamine on polysiloxane: a stable surface for neuronal cell adhesion. Journal of neuroscience methods 2000;98(2) 135-144.

[87] Puleo D A, Kissling R A, Sheu M S. A technique to immobilize bioactive proteins, including bone morphogenetic protein-4 (BMP-4), on titanium alloy. Biomaterials 2002;23(9) 2079-2087.

[88] Pu F R, Williams R L, Markkula T K, Hunt J A. Effects of plasma treated PET and PTFE on expression of adhesion molecules by human endothelial cells in vitro. Biomaterials 2002;23(11) 2411-2428.

[89] Tang L, Wu Y, Timmons R B. Fibrinogen adsorption and host tissue responses to plasma functionalized surfaces. Plasma functionalized surfaces 1998;42(1) 156-163.

[90] Readily available on: http://connorssciencelab.com/tag/ionization/ (accessed $18 \mathrm{Au}$ gust 2014).

[91] Reprinted with permission from (Keen I, Broota P, Rintoul L, Fredericks P, Trau M, Grøndahl L. Introducing amine functionalities on a poly(3-hydroxybutyrate-co-3-hydroxyvalerate) surface: comparing the use of ammonia plasma treatment and ethylenediamine aminolysis. Biomacromolecules 2006;7(2) 427-434). Copyright (2006) American Chemical Society.

[92] Adapted with permission from (Hamerli P, Weigel Th, Groth Th, Paul D. Surface properties of and cell adhesion onto allylamine-plasma-coated polyethylenterephtalat membranes. Biomaterials 2003;24(22) 3989-3999). Copyright (2003) Elsevier Science Ltd.

[93] Reprinted with permission from (Lejeune M, Brétagnol F, Ceccone G, Colpo P, Rossi F. Microstructural evolution of allylamine polymerized plasma films. Surface and coatings technology 2006;200(20-21) 5902-5907). Copyright (2005) Elsevier B.V. 
[94] Reprinted with permission from (Martin Y, Boutin D, Vermette P. Study of the effect of process parameters for $n$-heptylamine plasma polymerization on final layer properties. Thin solid films 2007;515(17) 6844-6852). Copyright (2007) Elsevier B.V.

[95] Readily available on: http://www.glycoforum.gr.jp/science/word/proteoglycan/ PGC01E.html (accessed 19 August 2014).

[96] Reprinted with permission from (Hamerli P, Weigel Th, Groth Th, Paul D. Surface properties of and cell adhesion onto allylamine-plasma-coated polyethylenterephtalat membranes. Biomaterials 2003;24(22) 3989-3999). Copyright (2003) Elsevier Science Ltd. 

Chapter 3

\title{
Engineering of Novel Proteinoids and PLLA-Proteinoid Polymers of Narrow Size Distribution and Uniform Nano/Micro-Hollow Particles for Biomedical Applications
}

\author{
Michal Kolitz-Domb and Shlomo Margel \\ Additional information is available at the end of the chapter \\ http://dx.doi.org/10.5772/59626
}

\section{Introduction}

Proteinoids, polymers made of amino acids by thermal condensation polymerization, are unique synthetic polymers studied by Fox and coworkers [1-7]. When present in certain concentrations in aqueous solutions, the proteinoids form small microscopic structures called "proteinoid microspheres" or protocells [4]. The proteinoid, made of most common amino acids, goes through a self-assembly process in specific conditions, as some of the amino acids incorporated into proteinoid chains are more hydrophobic than others, thus proteinoids cluster together like droplets of oil in water [5-7].

The amino acids, either natural or synthetic, are polymerized by step-growth polymerization in a special procedure discovered by Fox and Harada [8-12]. The procedure involves heating amino acids until they melt at first, and then the polymerization is initiated to give the proteinoid. The polymerization takes place at a relatively high temperature (e.g., $180{ }^{\circ} \mathrm{C}$ ), in absence of a solvent or a catalyst, and in an inert atmosphere. The accepted explanation of this polymerization process is that glutamic acid is used as a solvent for the other monomers, as it is condensed into pyroglutamic acid. The pyroglutamic acid initiates the polymerization with the rest of the present amino acids [11-14]. A brief description of the polymerization process is shown in Figure 1.

An important advantage in using biodegradable polymers bearing one or more stereogenic centers- optically active polymers- is the high variety of features they offer, when used as carriers for drug delivery systems [15]. This study is intended to broaden the scope of 


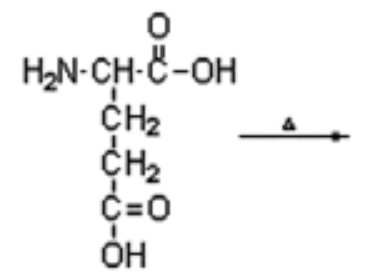<smiles>O=C1CCC(C(=O)O)N1</smiles>

Pyroglutamic acid

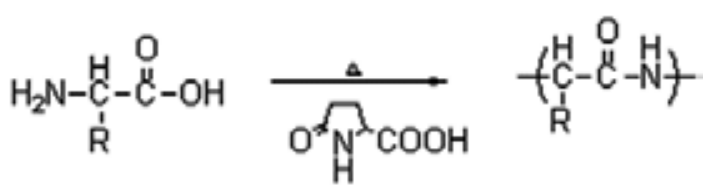

$\mathrm{R}=$ any amino- acid side chain

Figure 1. Thermal polymerization of amino acids through pyroglutamic acid catalysis.

biodegradable polymers by synthesizing new proteinoids carrying various stereogenic centers. The main goal is to provide a large choice of biodegradable proteinoids with predetermined characteristics, while using amino acids as building blocks. Hence, the new selection is based on chiral building blocks carrying positive/negative charge, hydrophilic/ hydrophobic nature or any desired combination of the above [16]. Homopolymers, random copolymers and block-copolymers of two amino acids or more can be designed and synthesized, presenting thereby new proteinoid materials with specific, desired nature. The large variety of amino acids, either natural or synthetic, makes it possible to obtain a large library of different proteinoids, by simply changing the amino acids ratios. One proteinoid is completely different from another, by the fact that they are made of different amino acid monomers. This fact provides each proteinoid special features, and possibly influences the character of particles made from it [17-20].

Up until now, most, if not all, of the reported proteinoids in the literature were synthesized from at least four amino acids and possessed relatively low molecular weights [21,22]. Thus, high molecular weight proteinoids of narrow size distribution made of two to three natural amino acids, along with proteinoids containing poly(L-lactic acid) (PLLA) segments are presented in this study. Furthermore, the study presents NIR fluorescent proteinoid-based particles, which can be used for colon cancer detection. Some of the methods used have been published in a previous work [23].

\section{Incorporation of poly(L-lactic acid) into the proteinoid backbone}

Poly(lactic acid) or PLA is the thermoplastic aliphatic polyester of lactic acid, derived from renewable sources. It is the one of the most important bioplastics in the world in terms of 
consumption volume. PLA can be processed by extrusion, injection molding, film and sheet casting, and spinning, providing access to a wide range of materials and applications. Two racemic structures of PLA, the $\mathrm{L}$ form and the D form, are known as PLLA and PDLA, respectively [24].

PLA is already used in many biomedical applications, as it is able to degrade into innocuous lactic acid and is very safe [25-29]. Medical implants made of PLA and approved by the FDA are anchors, screws, plates, pins, rods, and as a mesh. Depending on the specific type used, the implants degrade inside the body within 6 to 24 months. The strength characteristics of PLA and PLLA implants is well documented [30,31].

PLA can also be used as a compostable packaging material, either cast, injection molded, or spun. Also, cups, bags, hygene products and diapers have been made from PLA [32].

\section{Proteinoid microspheres}

After preparation, the crude proteinoids can self-assemble to form micro- and nano-sized particles [33, 34]. As opposed to polystyrene microparticles, for example, which are formed during the polymerization process of styrene in the presence of a surfactant [35], the proteinoid particles are formed through a self-assembly process. This process is completed only after the polymerization itself. The procedure involves either dissolving the dried crude proteinoid in water by heating and then cooling slowly,[36] or by $\mathrm{pH}$ changes of the proteinoid solution in water [37]. This way, particles of the size range of several nanometers to $10 \mu \mathrm{m}$ may be formed [38].

The idea standing behind the unique self-assembly process lies in the existence of many functional groups as a part of the random polymer backbone. The preparation of proteinoid microspheres does not require the addition of any toxic cross-linking agents or surfactants. The conversion of the polypeptidic proteinoid solution into particles with a slight change of the environment involves a complex process of functional group changes occurring in the polymer. The great amount of carboxylates in the backbone, or aminium ions when dealing with a lysine-rich proteinoid, cause the solubility of the proteinoid in certain conditions and insolubility in the reverse conditions [39]. The hydrophobic portions of the proteinoid are assembled within the particle matrix, and the carboxylic acid moieties are exposed, as illustrated in Figure 2 [40].

When the self-assembly procedure is done in the presence of a suitable molecule such as drug or dye, a proteinoid particle containing the molecule is formed [41]. The proteinoids may be used to encapsulate materials such as drugs for drug delivery purposes, e.g. for the oral delivery of methotrexate [40], Hydroxyapetite [42], Cholesterol [37] and for diagnostics [21,43-48]. 


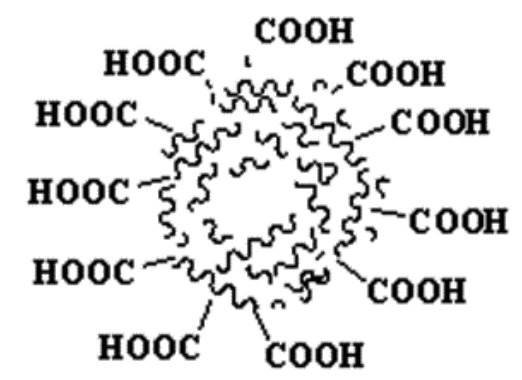

Figure 2. Schematic representation of the self-assembled proteinoid particles. Hydrophobic moieties are represented by scribbled lines. When lysine is also a part of the proteinoid, as in Prot5-7, some carboxyl groups are exchanged with amine groups.

\section{Preparation of proteinoids by thermal condensation polymerization}

L-glutamic acid was heated to the molten state $\left(180^{\circ} \mathrm{C}\right)$ in an oil bath, under nitrogen atmosphere. The molten mass was stirred at $180^{\circ} \mathrm{C}$ for $30 \mathrm{~min}$. To this, different contents of additional L-amino acids were added to give a total monomer weight of 5-5.01 g, as specified in Table 1, and kept at $180^{\circ} \mathrm{C}$ under nitrogen. The mixture was mechanically stirred at $150 \mathrm{rpm}$ for $3 \mathrm{~h}$. The product is a highly viscous orange-brown paste, which hardens to give a glassy mass when cooled to room temperature. Then, water $(10 \mathrm{~mL})$ was added to the crude product, and the mixture was stirred for $20 \mathrm{~min}$. The solution was then intensively dialyzed through a cellulose membrane (3500 Da MWCO) against distilled water. The content of the dialysis tube was then lyophilized to obtain a yellow-white proteinoid powder.

\begin{tabular}{llllll}
\hline Polymer & \multicolumn{5}{c}{ Amino acid content (g)a } \\
\cline { 2 - 6 } & L-Glu & L-Asp & L-Lys & L-Phe & PLLA \\
\hline Prot1 & 5 & - & - & - & - \\
\hline Prot2 & 2.5 & 2.5 & - & - & - \\
\hline Prot3 & 2.5 & - & - & 2.5 & - \\
\hline Prot4 & 1.25 & 2.5 & - & 1.25 & - \\
\hline Prot5 & 1.67 & 1.67 & 1.67 & - & - \\
\hline Prot6 & 1.67 & - & 1.67 & 1.67 & - \\
\hline Prot7 & 1.25 & - & 2.5 & 1.25 & - \\
\hline Prot8 & 2.25 & - & - & 2.25 & 0.5 \\
\hline
\end{tabular}

Table 1. Amino acid content of the different proteinoids. ${ }^{a}$ In all proteinoids made by thermal condensation polymerization the total monomer content was 5-5.01 g; ${ }^{\mathrm{b}}$ made by microwave-assisted polymerization.

\subsection{Polymerization kinetics study at different temperatures}

Polymerization kinetics was studied by collecting proteinoid samples from the reaction vessel at different time periods of the polymerization at 180,190 and $200^{\circ} \mathrm{C}$. The samples 
were then analyzed by both ninhydrin test for the determination of the primary amine groups content and Biuret test for the determination of the amide groups content of the various proteinoids [49]. The results shown in Figure 3 refer to the synthesis of Prot3, consisting of L-glutamic acid and L-phenylalanine. Similar results were observed for the other systems, Prot1-8, as well. It can be seen that at all temperatures, the polymerization takes place mainly over the first $100 \mathrm{~min}$. After that, both ninhydrin (A) and Biuret (B) tests show no significant drop of the Ninhydrin signal indicating the amount of free amines or rise of the peptide bonds shown by the Biuret signal. This figure also shows that as the temperature of the reaction was raised, the rate of the reaction increased. However, the preferred reaction temperature is $180^{\circ} \mathrm{C}$, as the yield of the reaction is higher since the amino acids decompose faster at the higher temperatures.

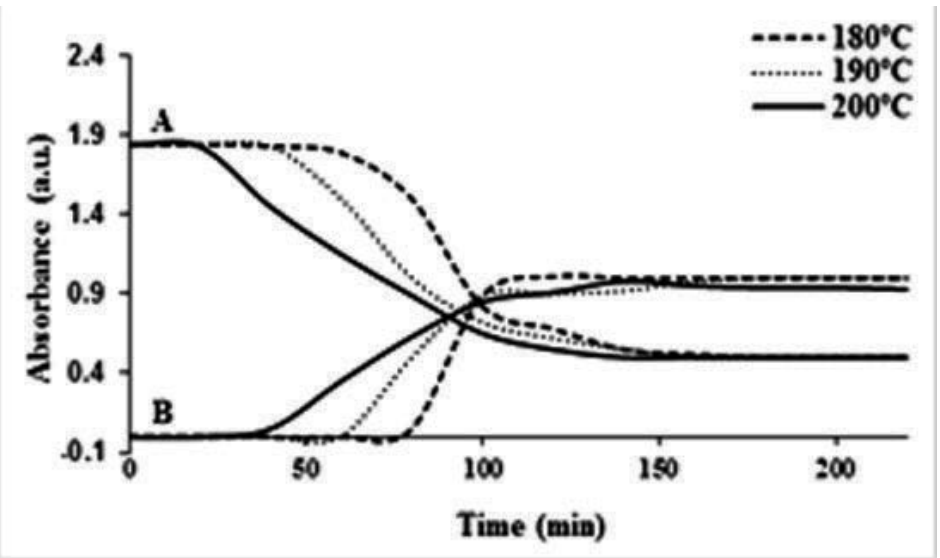

Figure 3. Thermal polycondensation kinetics of (L)glutamic acid and (L)phenylalanine to yield Prot3 at different temperatures by two tests: ninhydrin test (A) and Biuret test (B).

\section{Proteinoid analysis and characterization}

The molecular weights and polydispersity index of the dried crude proteinoids were determined using Gel Permeation Chromatography (GPC) consisting of a Waters Spectra Series P100 isocratic HPLC pump with an ERMA ERC-7510 refractive index detector and a Rheodyne (Coatati, CA) injection valve with a $20 \mu \mathrm{L}$ loop (Waters, MA). The samples were eluted with super-pure HPLC water through a linear BioSep SEC-s3000 column (Phenomenex) at a flow rate of $1 \mathrm{~mL} / \mathrm{min}$. The molecular weights were determined relative to poly(ethylene glycol) standards (Polymer Standards Service-USA, Silver Spring, MD) with a molecular weight range of 100-450000 Da, using Clarity chromatography software. The optical activities of the proteinoids were determined using a PE 343 polarimeter (PerkinElmer). All of the measurements were done in water, at $589 \mathrm{~nm}$ at $25^{\circ} \mathrm{C}$. Table 2 shows the characteristic molecular weights, polydispersity and optical activity of the prepared proteinoids. 


\begin{tabular}{|c|c|c|c|c|c|}
\hline Proteinoid $^{\mathrm{a}}$ & $\begin{array}{l}\mathrm{Mw} \\
(\mathrm{Da}) \mathrm{b}\end{array}$ & $\begin{array}{c}\mathrm{Mn} \\
(\mathrm{Da}) \mathrm{b}\end{array}$ & $\begin{array}{c}\mathrm{Mp} \\
(\mathrm{Da}) \mathrm{b}\end{array}$ & PDIc & $\begin{array}{l}\text { Optical Activity } \\
{[\alpha] \mathrm{D} 25^{\circ} \mathrm{C}\left({ }^{\circ}\right) \mathrm{d}}\end{array}$ \\
\hline Prot1 & 26250 & 11300 & 11320 & 2.32 & +6.5 \\
\hline Prot2 & 181540 & 144940 & 195300 & 1.25 & -4.4 \\
\hline Prot2 ${ }^{e}$ & 500240 & 497280 & 503070 & 1.01 & +8.1 \\
\hline Prot3 & 164930 & 138250 & 158740 & 1.19 & -9.0 \\
\hline Prot4 & 87660 & 84410 & 85250 & 1.04 & -3.3 \\
\hline Prot5 & 195080 & 165870 & 191440 & 1.17 & -7.4 \\
\hline Prot6 & 190390 & 163290 & 204050 & 1.16 & -15.1 \\
\hline Prot7 & 72260 & 56880 & 42870 & 1.27 & +2.8 \\
\hline Prot8 & 168300 & 156600 & 136800 & 1.07 & -4.6 \\
\hline
\end{tabular}

Table 2. Mw, Mn, Mp, PDI and optical activity of the various proteinoids. ${ }^{\text {aThe }}$ proteinoids were prepared at $180^{\circ} \mathrm{C}$ according to section 3.2.1; ${ }^{\mathrm{b}}$ molecular masses were measured by GPC, Mp is the molecular mass at the peak; ${ }^{\mathrm{CPDI}}$ is the polydispersity index, given by $\mathrm{Mw} / \mathrm{Mn}$; ${ }^{\mathrm{d}}$ specific optical rotation $\left(\mathrm{c}=1\right.$, in $\mathrm{H}_{2} \mathrm{O}$, at $\left.25^{\circ} \mathrm{C}\right)$; ${ }^{\mathrm{e}}$ made by microwave-assisted polymerization.

Table 2 indicates relatively low PDI values for the obtained proteinoids. This is unexpected since the polycondensation of the various amino acids is random and step-growth polymerization processes, as in the present case, result usually in very broad size distribution polymers [50]. The highest PDI (2.32) was observed for Prot1, composed of the single amino acid Lglutamic acid, while the PDIs of the other proteinoids composed of at least 2 amino acids were ranging between 1.01 and 1.27. All of the thermally-made proteinoids have relatively high molecular masses of 26-195 kDa. This indicates that the polymerization procedure by thermal heating used here provides relatively long polymer chains. This fact may serve as an advantage for different uses later, since polymers with such high molecular weights are usually mechanically stronger and resemble natural proteins. Table 2 indicates that the lowest molecular weight was observed for the proteinoid composed of the single amino acid L-glutamic acid (Prot1) and the highest one for the proteinoid composed of L-glutamic acid, L-aspartic acid and L-lysine (Prot 5). Prot2, which was synthesized by microwave-assisted polymerization, reached an abundantly higher molecular weight. In this procedure, a $500 \mathrm{kDa}$ proteinoid chain was prepared, about twice the size of the regular thermal proteinoid. This kind of procedure gives better yield over $60 \mathrm{~min}$, compared to the $3 \mathrm{~h}$ needed usually. It can be used further for higher molecular weights and more rigid proteinoids. However, unfortunately, this kind of proteinoid does not self-assemble into spherically-shaped particles.

As further indicated from Table 2, all of the proteinoids exhibit optical activity, although the amino acid monomers are known to racemize during the thermal process [51]. This fact can become a benefit later in the design of a stereospecific drug carrier, for example.

Fourier Transform Infra-Red (FTIR) measurements of the crude proteinoids were done by the Attenuated Total Reflectance (ATR) technique, using Bruker ALPHA-FTIR QuickSnapTM sampling module equipped with Platinum ATR diamond module. All proteinoids showed characteristic peaks of NH stretching at 3360 and $2990 \mathrm{~cm}-1$, amide CO stretching at $1565 \mathrm{~cm}-1$, 
an amide NH bending band at $1450 \mathrm{~cm}-1$ and $\mathrm{CO}$ bending at $500-700 \mathrm{~cm}-1$. A representative spectrum of Prot3 is shown in Figure 4.

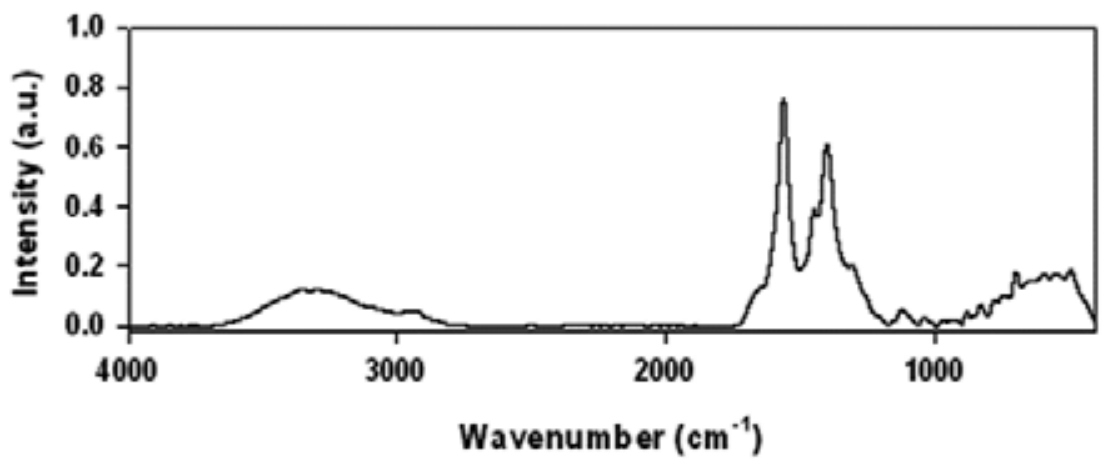

Figure 4. FTIR spectrum of Prot3.

The thermal behavior of the proteinoids was determined using Differential Scanning Calorimetry (DSC) and Thermo Gravimetric Analysis (TGA) with a TGA/DSC 1 STARe system (Mettler Toledo, Switzerland). The samples were heated between $25-400{ }^{\circ} \mathrm{C}$ at a rate of $10^{\circ} \mathrm{C} /$ min under nitrogen atmosphere. The results are shown in Table 3.

\begin{tabular}{lcclc}
\hline Polymer & $\mathrm{Tm}\left({ }^{\circ} \mathrm{C}\right) \mathrm{a}$ & $\Delta \mathrm{Hm}(\mathrm{J} / \mathrm{g}) \mathrm{a}$ & $\mathrm{Tdec}\left({ }^{\circ} \mathrm{C}\right) \mathrm{b}$ & $\begin{array}{c}\text { Weight loss }(\%) \\
\text { at } 400^{\circ} \mathrm{Cb}\end{array}$ \\
\hline Prot1 & 102 & -300.7 & 300 & 55 \\
\hline Prot2 & 89 & -428.7 & 297 & 57 \\
\hline Prot3 & 103 & -174.9 & 341 & 54 \\
\hline Prot4 & 217 & -183.4 & 330 & 57 \\
\hline Prot5 & 78 & -251.5 & 339 & 47 \\
\hline Prot6 & 241 & -90.1 & 373 & 64 \\
\hline Prot7 & 246 & -139.4 & 385 & 47 \\
\hline Prot8 & 117 & -420.5 & 268 & 90 \\
\hline PLLA & 150 & -57.2 & 349 & 25 \\
\hline
\end{tabular}

Table 3. Thermal properties of proteinoids produced by thermal polymerization. ${ }^{\mathrm{a}} \mathrm{Tm}$ and $\Delta \mathrm{Hm}$ were measured by

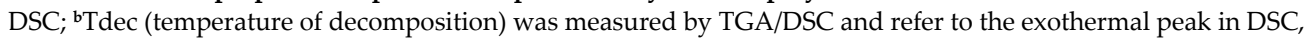
'commercial PLLA 2000 Da parameters were measured similar to the made proteinoids.

The melting temperatures of the different proteinoids range between $78-246^{\circ} \mathrm{C}$. The wide range of temperatures derives from the difference in the monomeric units used in each proteinoid. When using phenylalanine, as in Prot3, 4, 6 and 7, the resulted proteinoid gains significant rigidity in the overall structure, due to the aromatic rings which allow pi-stacking. Hence, these proteinoids melt at higher temperatures. When PLLA is incorporated into the proteinoid, as 
in Prot8 compared to Prot3, the Tm rises mildly $\left(103^{\circ} \mathrm{C}\right.$ and $117^{\circ} \mathrm{C}$, respectively), due to the presence of 2000 Da rigid polymer chains in the overall proteinoid structure.

The TGA/DSC measurements of the proteinoids show decomposition temperatures of $268-385^{\circ} \mathrm{C}$. Most proteinoids lose at this temperature range around $50 \%$ of their weight The decomposition measured at $400^{\circ} \mathrm{C}$ of most proteinoids is between $47-64 \%$, except Prot $4(25 \%)$. Pure PLLA decomposes at $349^{\circ} \mathrm{C}$ almost completely ( $90 \%$ weight loss). Prot 8 , composed of PLLA segments (10\% of the total monomer), has the lowest decomposition temperature of all proteinoids $\left(268^{\circ} \mathrm{C}\right)$. This can be explained by the non-uniformity of the structure of the whole proteinoid due to the inserted segments of 2000 Da PLLA within the random segments of polymerized amino acids.

The content of free carboxyl groups in the synthesized proteinoids is an essential factor in determining their solubility in different media, thus helping to understand their stability at different sites in the human body with different $\mathrm{pHs}$. In order to determine the free carboxyl groups in the synthesized proteinoids, a titrimetric method was carried out [40]. Briefly, to a known quantity of dry proteinoid, a known excess of $0.05 \mathrm{~N} \mathrm{NaOH}$ was added, followed by the addition of $37 \%$ formaldehyde solution. The unreacted $\mathrm{NaOH}$ was back-titrated with standard $0.05 \mathrm{~N} \mathrm{HCl}$. A blank titration was also performed. In addition, human serum albumin (HSA) was titrated for comparison. Table 6 indicates the carboxyl group contents of the synthesized proteinoids, showing higher values of $80-155 \mathrm{mmol} / \mathrm{g}$ compared with albumin. This is true also in Prot5-7, where lysine is also a part of the polymer. Moreover, aspartic and glutamic acid moieties in the proteinoids, along with lysine, impart the hydrophilic nature of the whole proteinoid. The biodegradability rate of various amino acid polymers increases with their hydrophilicity [40]. Therefore, it is more appropriate to choose these proteinoids as ideal biomaterials for drug delivery applications.

\begin{tabular}{lc}
\hline Polypeptide & [Carboxyl groups] (mmol/g) \\
\hline Albumin & 56 \\
\hline Prot1 & 150 \\
\hline Prot2 & 155 \\
\hline Prot3 & 90 \\
\hline Prot4 & 122 \\
\hline Prot5 & 88 \\
\hline Prot6 & 87 \\
\hline Prot7 & 80 \\
\hline Prot8 & 102 \\
\hline
\end{tabular}

Table 4. Carboxyl group content in the proteinoids and albumin.

\subsection{Incorporation of poly(L-lactic acid) into the proteinoids}

In order to effect the chemical and physical properties of the product, a thermal polymerization of L-glutamic acid and L-phenylalanine was carried out in the presence of low molecular 
weight poly(L-lactic acid) (PLLA, $2000 \mathrm{Da}$ ). The proteinoid-PLLA (Prot8) consists of $2.25 \mathrm{~g}$ of each amino acid and $0.5 \mathrm{~g}$ of PLLA. After polymerization, it was washed, dried and characterized as described earlier. The characterization of Prot8 is included in the tables above.

\section{Preparation and characterization of the proteinoid nano/micro-particles by a self-assembly process}

Proteinoid particles were prepared by a self-assembly mechanism. Briefly, $100 \mathrm{mg}$ of the dried proteinoid were added to $10 \mathrm{~mL} 10-5 \mathrm{~N} \mathrm{NaCl}$ solution. The mixture was then heated to $80^{\circ} \mathrm{C}$ until the crude proteinoid dissolves completely. Proteinoid particles were then formed by removal of the heating and leaving the mixture to cool to room temperature.

Hydrodynamic diameter and size distribution of the particles dispersed in double distilled (DD) water were measured at room temperature with a particle DLS analyzer model Nanophox (SympatecGmbH, Germany).

Dried particle size and size distribution were measured with a Scanning Electron Microscope (SEM). SEM pictures were obtained with a JEOL, JSM-840 Model, Japan. For this purpose, a drop of dilute particle dispersion in distilled water was spread on a glass surface, and then dried at room temperature. The dried sample was coated with carbon in vacuum before viewing under SEM. The average particle size and distribution were determined by the measurement of the diameter of more than 200 particles with image analysis software (Analysis Auto, Soft Imaging System $\mathrm{GmbH}$, Germany). Figure 5 exhibits the proteinoid particles made from self-assembly of Prot8. The procedure produced spherical proteinoid particles of $103 \pm 11 \mathrm{~nm}$ hydrodynamic diameter and $70 \pm 15 \mathrm{~nm}$ dry diameter. The dry diameter of the proteinoid particles is illustrated by the typical SEM photomicrograph shown in Figure 5A. The hydrodynamic diameter of these particles dispersed in water is illustrated by the typical light scattering measurement shown in Figure 5B. The difference in the particle size between the SEM and the light scattering measurements is probably due to the fact that SEM measurements determine the dry diameter, whereas light scattering measurements take into account the hydrated water layers adsorbed onto the particle's surface.

The density of the particles was determined by pycnometry [52]. Briefly, dry pre-weighed particles were put in a calibrated pycnometer, which was then filled with water. The density of the sample can then be calculated from the known density of the water, the weight of the pycnometer filled only with water, the weight of the pycnometer containing both the sample and water, and the weight of the sample, as described in the literature [52]. Density measurements indicated that all proteinoid particles possess a very low density, ranging from 0.001 to $0.014 \mathrm{~g} / \mathrm{mL}$ indicating that the particles formed are probably hollow, as already indicated for the proteinoids prepared by Fox et al [6,38]. The hollow nature of the particles is significantly important for applications such as ultrasound imaging agents, drugs and dyes encapsulation, controlled released, etc.

As suggested in our and in previous studies, the proteinoid forms particles of different sizes according to the nature of its surrounding. The hydrophobic portions of the crude proteinoid 
$\mathbf{A}$

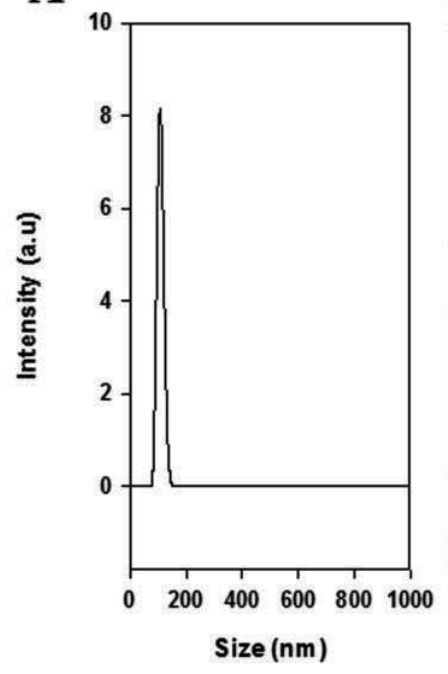

B

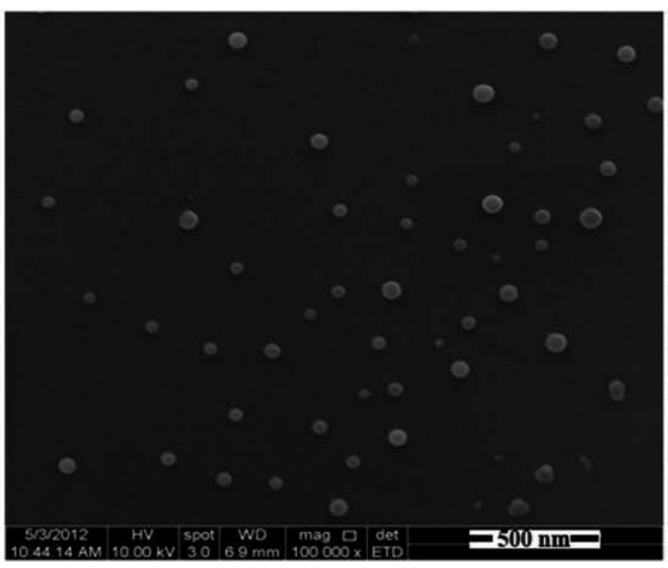

Figure 5. Hydrodynamic size histogram (A) and SEM image (B) of Prot8 particles.

are assembled within the particle matrix, while the polar hydrophilic groups (carboxyl and amines) are exposed to the aqueous environment, as illustrated before in Figure 2 [40].

\subsection{Particle stability in storage conditions}

Proteinoid particles aqueous dispersions $(1 \mathrm{mg} / \mathrm{mL})$ were put in a refrigerator at $4^{\circ} \mathrm{C}$ for 6 months. Samples were taken at different time periods, filtered through a centrifugation tube (Vivaspin 3000 Da MWCO) and the filtrate was checked by UV at 200-210 nm, to find aqueous soluble proteinoid. Also, the particle aqueous dispersions were checked by Nanophox for their size and size distribution. In order to check the particle stability after drying, the particles were lyophilized to dryness and then dispersed in an aqueous phase to their original concentration. The samples size and size distribution were then rechecked by Nanophox. Overall, the proteinoid particles remain in the same size after 6 months in storage at $4^{\circ} \mathrm{C}$. Also, the degradation and/or dissolution of the proteinoid particles in the aqueous continuous phase was tested by the filtration centrifugation method and resulted in negative results in the filtrate, meaning no degradation or dissolution occurs at this temperature over 6 months. When lyophilized to dryness, the proteinoid particles can be redispersed in water completely while the particle size and size distribution remain the same. This means that the particles can be stored as a freeze-dried powder as well, and redispersed when needed, without the need to add cryoprotectants as mentioned in the literature [53].

\subsection{Cytotoxicity of the proteinoid particles}

In vitro cytotoxicity of the proteinoid particles was tested by using human colon adenocarcinoma LS174T cancer cell line. The tests were done on Prot2, Prot4, Prot5, Prot7 and Prot8. The 
cell line is adherent to the used culture dishes. LS174T cells were grown in MEM that was supplemented with $10 \%$ heat-inactivated fetal bovine serum (FBS), $1 \%$ glutamine and $1 \%$ penicillin/streptomycin. Cells were screened to ensure they remained mycoplasma-free using Mycoplasma Detection Kit [54]. Cell cytotoxicity was assessed by measuring the release of cytoplasmic lactate dehydrogenase (LDH) into cell culture supernatants. LDH activity was assayed using the Cytotoxicity Detection Kit according to the manufacturer's instructions [55]. Cells ( $3 \times 105$ cells per well) were seeded and grown to $90-95 \%$ confluency in 24 well plates before treatment with the proteinoid particles. Cell cultures that were not exposed to the particles were included in all assays as negative controls. Cell cultures that were treated with $1 \%$ Triton-x-100 were used as positive controls. To test if the particles can interact with LDH kit compounds, cell cultures were exposed to a mixture containing maximal nano/microparticles concentration dispersed in PBS and 1\% Triton-x-100. The proteinoid particles were freshly dispersed in PBS (1.25 and $2.5 \mathrm{mg} / \mathrm{mL}$ ) and then added to the $95 \%$ confluent cell culture in culture medium. The cell cultures were further incubated at $37^{\circ} \mathrm{C}$ in a humidified $5 \% \mathrm{CO} 2$ incubator and then checked for cellular cytotoxicity at intervals of $24 \mathrm{~h}$. The percentage of cell cytotoxicity was calculated using the formula shown in the manufacturer's protocol [55]. All samples were tested in tetraplicates.

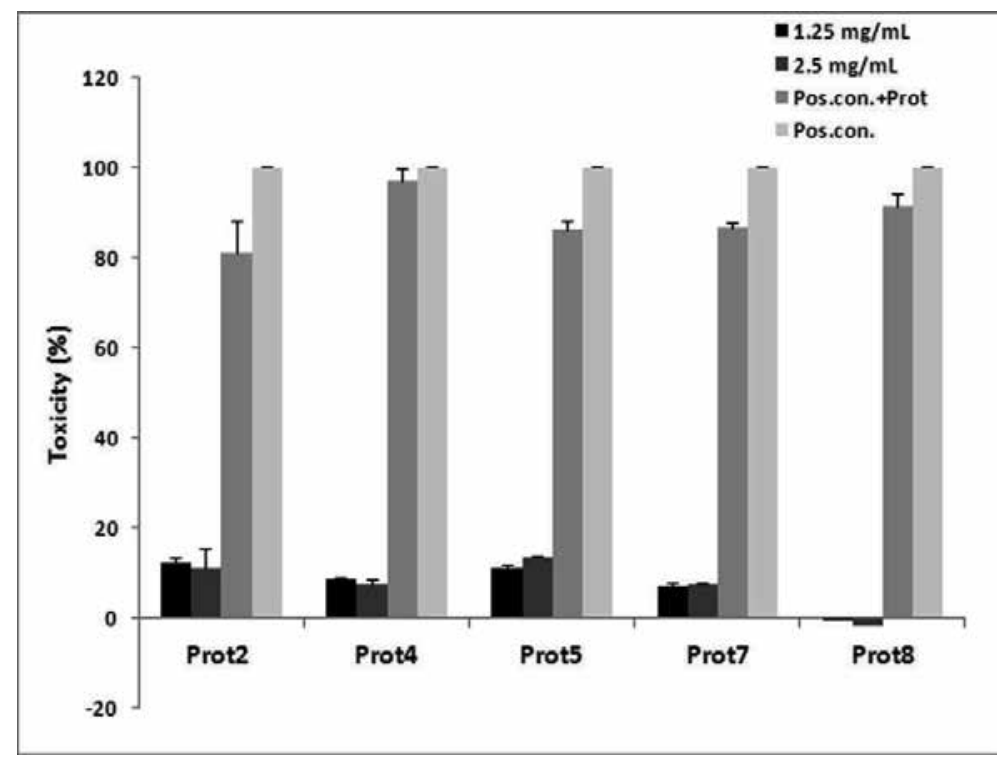

Figure 6. Cytotoxic effect of the proteinoid particles on colon adenocarcinoma LS174T cells measured by the LDH assay. Cells $\left(3 \times 10^{5}\right)$ were incubated for 24 and $48 \mathrm{~h}$ with the proteinoid particles dispersed in PBS $(1.25 \mathrm{mg} / \mathrm{mL}$ and 2.5 $\mathrm{mg} / \mathrm{mL}$ ) according to section 3.2.5.8. Cells were incubated with Triton-x-100 $1 \%$ as positive control (100\% toxicity). In addition, cells were incubated with Triton-x-100 1\% and each one of the proteinoids to revoke any interaction. Untreated cells (negative control) were similarly incubated. Each bar represents mean \pm standard deviations of 4 separate samples.

When tested by the LDH quantitative assay, Prot2, 4, 5, 7 and 8 particles dispersed in PBS at concentrations of 1.25 and $2.5 \mathrm{mg} / \mathrm{mL}$ had none, or minor cytotoxic effect on the human colon adenocarcinoma cell line LS174T (Figure 6). Treatment of the cells with Prot2 and Prot5 
particles at both concentrations produced the highest LDH levels (up to 13\% toxicity), when compared to untreated (blank) cells, indicating minor toxicity of these proteinoids to this cell line. Prot 8 had the lowest cytotoxic effect on the cells treated with both concentrations, almost zero toxicity. This proteinoid is therefore the most suitable for treating cells, considering its low toxicity.

\section{NIR flourescent proteinoid-PLLA particles}

\subsection{Synthesis of the NIR fluorescent Prot8 particles}

The optimal Prot8 particles were used to encapsulate ICG, a well-known NIR dye already in use in medical diagnostics. The NIR fluorescent particles were prepared by self-assembly of the crude Prot 8 , in the presence of ICG. Briefly, $100 \mathrm{mg}$ of the dried fabricated Prot 8 were resuspended in $10 \mathrm{~mL}$ of $10-5 \mathrm{~N} \mathrm{NaCl}$ solution. The mixture was then heated to $80^{\circ} \mathrm{C}$ while stirring for $15 \mathrm{~min}$. To this solution, $1 \mathrm{mg}$ ( $1 \%$ of the proteinoid polymer) of ICG was added. The mixture was then removed from the hot plate and was allowed to return to room temperature. During the cooling process hollow particles were formed and precipitated from solution. The obtained NIR fluorescent particles dispersed in water were then dialyzed versus $4 \mathrm{~L}$ of $10-5 \mathrm{NaCl}$ aqueous solution overnight at room temperature.

\subsection{Determination of the encapsulated ICG concentration in the NIR fluorescent Prot8 particles}

A calibration curve of free ICG was obtained by measuring the integrals of absorbance peaks of standard solutions $(0.5-10 \mu \mathrm{g} / \mathrm{mL})$ in PBS, at wavelengths $630-900 \mathrm{~nm}$. The concentration of the encapsulated ICG was determined by measuring the integral of the absorbance spectrum at $630-900 \mathrm{~nm}$ of a $1 \mathrm{mg} / \mathrm{mL}$ dispersion of the NIR fluorescent particles in PBS. An estimation of encapsulated ICG per mg of particles was determined according to the calibration curve.

\subsection{Characterization of the NIR fluorescent Prot8 particles}

Hydrodynamic and dry particle size and size distribution were determined by DLS and SEM, as mentioned above. For the SEM study, the diameter of more than 200 particles with image analysis software (AnalySIS Auto, Soft Imaging System GmbH, Germany). The self-assembly procedure produced spherical proteinoid particles of $145 \pm 20 \mathrm{~nm}$ hydrodynamic diameter and $70 \pm 15 \mathrm{~nm}$ dry diameter, as shown in Figure 7. The hydrodynamic diameter of these particles dispersed in water is illustrated by the typical light scattering measurement shown in Figure 7A. The dry diameter of the proteinoid particles is illustrated by the typical SEM photomicrograph shown in Figure 7B.

In addition, absorbance spectra were obtained using a Cary $100 \mathrm{UV}$-Visible spectrophotometer (Agilent Technologies Inc.). Excitation and emission spectra were recorded using a Cary Eclipse spectrofluorometer (Agilent Technologies Inc.). As indicated in Figure 8, no shift of absorbance of the ICG after encapsulation is observed compared to that of the free ICG. 

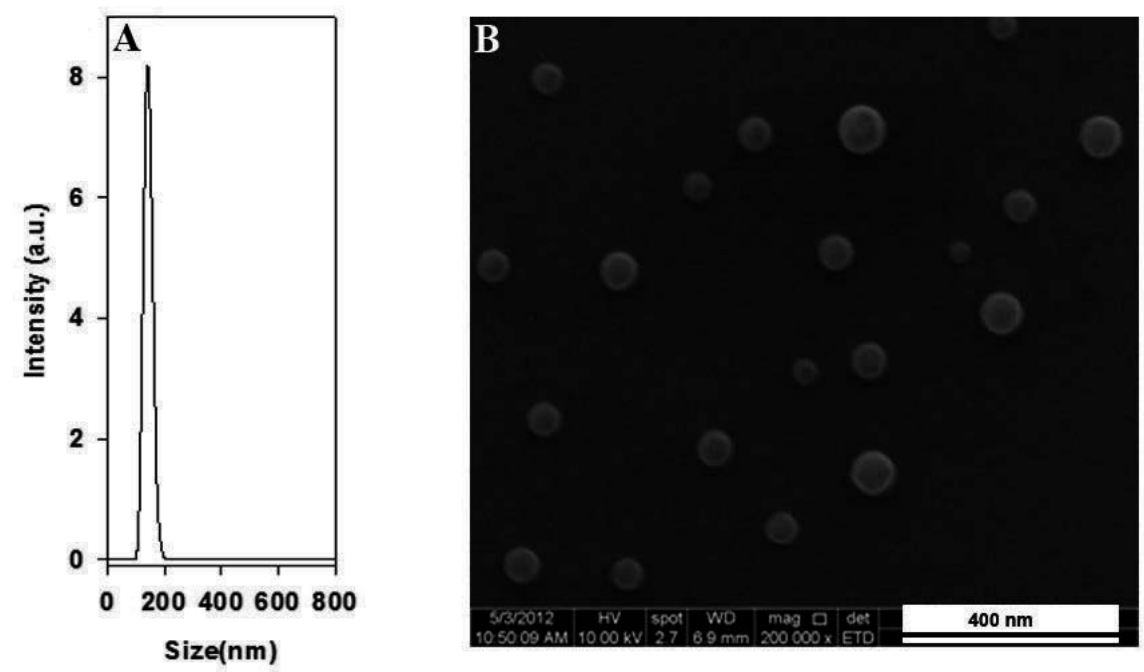

Figure 7. Hydrodynamic size histogram (A) and SEM image (B) of the P(EF-PLLA) NIR fluorescent nanoparticles.

However, due to the dye encapsulation process, the maximal absorbance peak of the free ICG changed from $779 \mathrm{~nm}$ to $718 \mathrm{~nm}$, probably since the ICG molecules get close to each other inside the nanoparticle interior and aggregation of the dye may occur causing this change in absorption peaks [56,57]. Furthermore, a $12 \mathrm{~nm}$ blue-shift of the emission spectrum of the NIR fluorescent particles compared to the free dye in solution is also observed.

The estimation of encapsulated ICG showed that the complete quantity of ICG used in the encapsulation procedure was encapsulated within the Prot8 nanoparticle interior. Following particle formation, leakage of the encapsulated ICG into PBS not-containing and containing $4 \%$ albumin at room temperature was not observed, indicating that the dye is strongly associated within the Prot8 particles, probably due to physical interactions between the dye and the polymer hydrophobic portions assembled in the core of the particles.

As suggested before, the proteinoid forms particles of different sizes according to the nature of its surrounding [40]. When discussing Prot8, the hydrophobic portions, in this case mainly the PLLA segments and the aromatic rings of the phenylalanine portion are assembled within the particle matrix, while the polar hydrophilic groups (mainly carboxylates) are exposed to the aqueous environment. This way, the self-assembly yields particles that encapsulate within them the ICG associated with the hydrophobic core via hydrophobic interaction, as illustrated in Figure 9.

\subsection{Optimization of the ICG concentration entrapped within the Prot8 particles}

In order to optimize the particles fluorescence intensity, different concentration $(0.5,1,2$ and $5 \% \mathrm{w} / \mathrm{w}$ relative to Prot8) of ICG were added to the Prot8 hot solution, prior to the formation of the particles through the self-assembly process, as described above. The NIR fluorescent 


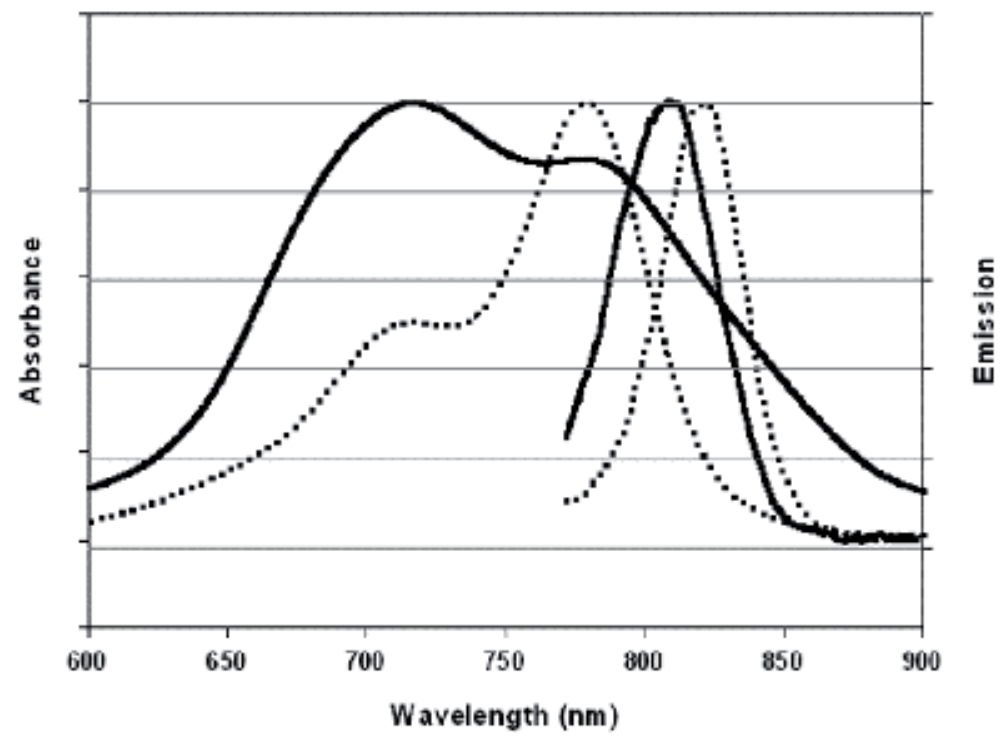

Figure 8. Absorbance and emission spectra of free ICG (dotted lines) and ICG-encapsulated Prot8 particles (solid lines) dispersed in water.

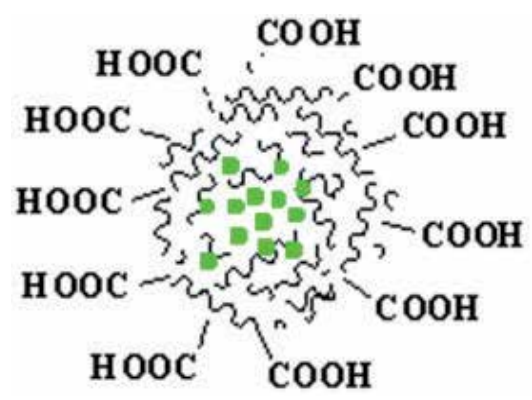

Figure 9. Schematic representation of the self-assembled NIR fluorescent particles. Hydrophobic moieties are represented by scribbled lines, ICG is represented by the interior green dots.

Prot8 particles dispersed in PBS were diluted to $1 \mathrm{mg} / \mathrm{mL}$ and their fluorescence intensities were measured at $809 \mathrm{~nm}$. The encapsulated ICG concentration that gave the maximum fluorescence intensity of the resultant NIR fluorescent particles was $1 \% \mathrm{w} / \mathrm{w}$ relative to Prot8. At higher dye concentrations, quenching of the fluorescence was observed, as the dye molecules encapsulated within the Prot8 nanoparticle are close to each other, resulting in nonemissive energy transfer between them.

\subsection{Photostability of the NIR fluorescent Prot8 particles}

In order to examine the photostability of the encapsulated ICG as opposed to free ICG, photobleaching experiments were performed for both the encapsulated and the free dye. For this purpose, an aqueous solution of ICG $(0.05 \mathrm{M})$ in PBS was prepared, and the 
fluorescence intensity with $\lambda$ ex set at $780 \mathrm{~nm}$ and $\lambda$ em set at $800 \mathrm{~nm}$ was measured. A dispersion of NIR fluorescent Prot8 particles in PBS was prepared, and diluted to give similar fluorescence intensity to the dye at the same wavelengths. The excitation and emission slits were opened to $20 \mathrm{~nm}$ and $5 \mathrm{~nm}$, respectively. Each of the samples was illuminated continuously with a xenon lamp, and the fluorescence intensity was measured over a period of $20 \mathrm{~min}$ by a Cary Eclipse fluorescence spectrophotometer (Agilent Technologies Inc.). Intensity values were normalized for comparison. Figure 10 illustrates that during illumination, the fluorescence intensity of the ICG-containing Prot8 particles remains intact while that of the free ICG decreased significantly. The photobleaching of ICG is significantly reduced by the encapsulation within the proteinoid-PLLA particles. The encapsulation probably protects the free dye from light-inducing factors such as oxygen, oxidizing or reducing agents, temperature, exposure time and illumination levels, which may reduce the fluorescence intensity irreversibly [58,59].

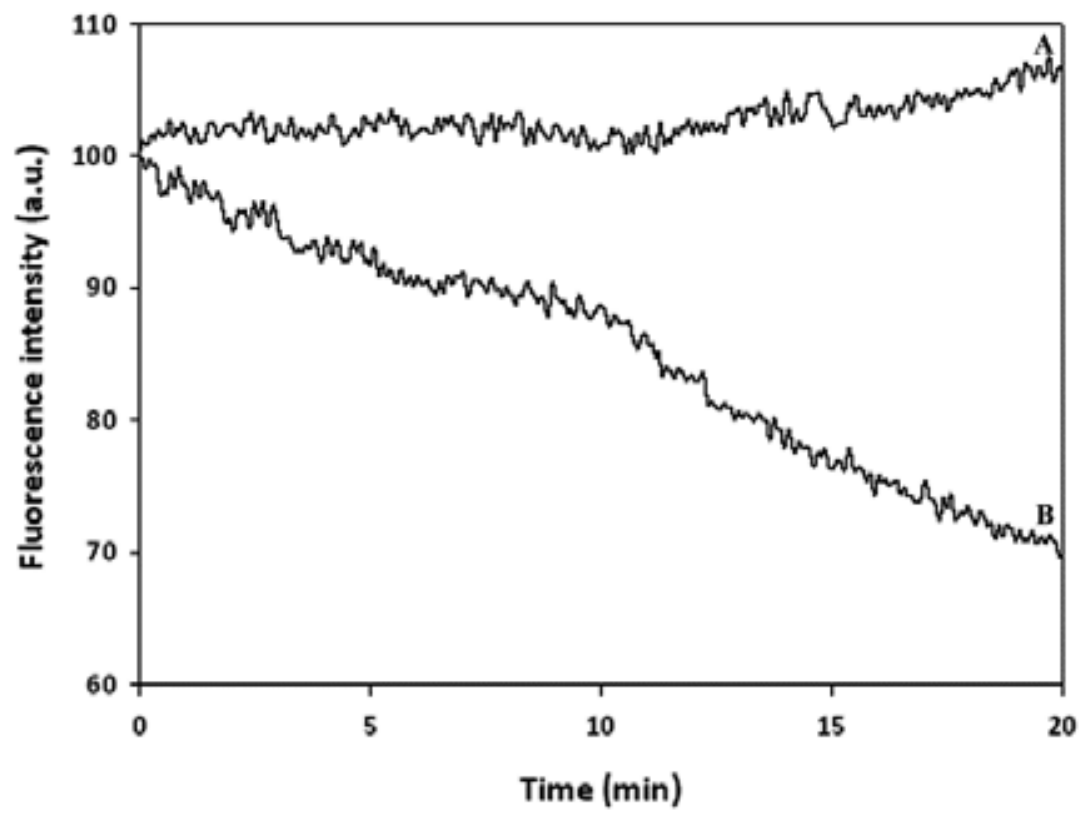

Figure 10. Photostability of the ICG-containing Prot8 particles (A) and free ICG (B) as function of time. Samples of ICG-containing Prot8 particles and free ICG were illuminated with a Xenon flash lamp for $20 \mathrm{~min}$, as described above.

\subsection{In vitro cytotoxicity of the Prot8 particles}

In order to revoke cell toxicity of the NIR Prot8 particles, in vitro cytotoxicity of the particles was tested by using human colorectal adenocarcinoma LS174t, SW480 and HT29 cell lines. The cell lines are adherent to the used culture dishes. LS174t cells were grown in Minimum 
Essential Medium (MEM) eagle supplemented with heat-inactivated fetal bovine serum (FBS, $10 \%)$, penicillin $(100 \mathrm{IU} / \mathrm{mL})$, streptomycin $(100 \mu \mathrm{g} / \mathrm{mL})$ and L-glutamine $(2 \mathrm{mM})$. SW480 cells were maintained in Dulbecco's MEM supplemented with heat-inactivated fetal bovine serum (FBS, 10\%), penicillin (100 IU/mL), streptomycin $(100 \mu \mathrm{g} / \mathrm{mL})$ and L-glutamine (2 mM). HT29 cells were maintained in McCoy's 5A medium supplemented with FBS (10\%), penicillin (100 $\mathrm{IU} / \mathrm{mL})$, streptomycin $(100 \mu \mathrm{g} / \mathrm{mL})$ and L-glutamine $(2 \mathrm{mM})$. Cells were screened to ensure they remained mycoplasma-free using Mycoplasma Detection Kit [54]. Cell cytotoxicity was assessed by measuring the release of cytoplasmic lactate dehydrogenase (LDH) as described above.

Figure 11 exhibits the cytotoxicity levels of the Prot8 particles at two different concentrations ( 1.25 and $2.5 \mathrm{mg} / \mathrm{mL}$ ). It can be seen that at both concentrations, the Prot8 particles have no significant cytotoxic effect on all three cell lines, compared to untreated (blank) cells, meaning that the particles may be used for biomedical applications as suggested, including drug delivery.

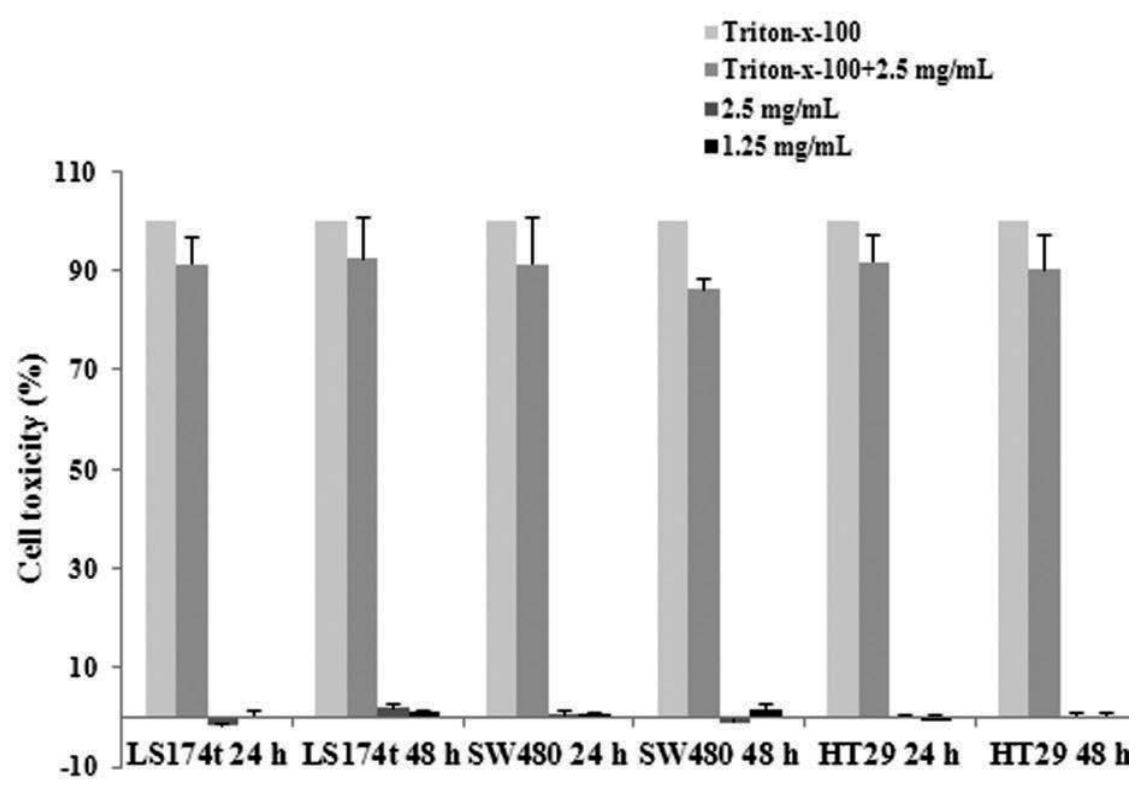

Figure 11. Cytotoxic effect of the NIR fluorescent Prot8 particles on human colorectal adenocarcinoma LS174t, SW480 and HT29 cell lines measured by the LDH assay. Cells $\left(3 \times 10^{5}\right)$ were incubated for 24 and $48 \mathrm{~h}$ with the Prot 8 particles (1.25 and $2.5 \mathrm{mg} / \mathrm{mL}$ in PBS). Cells were incubated with $1 \%$ Triton-x-100 as positive control (100\% toxicity). In addition, cells were incubated with Triton-x-100 1\% and the Prot8 particles $(2.5 \mathrm{mg} / \mathrm{mL})$ to revoke any interaction of the particles with the LDH kit components. Untreated cells (negative control) were similarly incubated. Each bar represents mean \pm standard deviations of 4 separate samples (originally published in [23]).

\subsection{In vivo biodistribution in a mouse model}

In order to examine the biodistribution in a living body, the NIR fluorescent Prot8 particles (2 $\mathrm{mg} / \mathrm{mL}, 0.01 \mathrm{mg} / \mathrm{kg}$ body weight per mouse) were injected i.v. into mice through the tail vein 
and checked at several time intervals over $24 \mathrm{~h}$. Male BALB/C mice (Harlan Laboratories, Israel) were utilized in this study under a protocol approved by the Institutional Animal Care and Use Committee at Bar-Ilan University. The biodistribution of the NIR fluorescent Prot8 particles was studied in normal 8-weeks-old mice, weighing 20-25 g at the time of experiment. Prior to the experiment, mice were anesthetized by intraperitoneal injection of Ketamine (40-80 $\mathrm{mg} / \mathrm{kg}$ body weight) and Xylazine (5-10 $\mathrm{mg} / \mathrm{kg}$ body weight), and the mice's skin was shaved with an electric animal clipper.

$100 \mu \mathrm{L}$ of either nanoparticle dispersion or free ICG solution $(0.01 \mathrm{mg} / \mathrm{kg}$ body weight, dissolved in PBS) were administered to the mice through tail vein injection at a concentration of $2 \mathrm{mg} / \mathrm{mL}$. During image acquisition, mice remained anesthetized by the intraperitoneal injection of Ketamine/Xylazine. Image cubes were obtained from the mice at several time points up to $24 \mathrm{~h}$ after injection. Each treatment group includes 3 mice for each time point $(5$ $\mathrm{min}, 20 \mathrm{~min}, 1 \mathrm{~h}$ and $24 \mathrm{~h}$ ); 2 uninjected mice served as negative control. The experiment was repeated twice, testing a total of 52 mice. At the end of the experiment, the mice were euthanized by cervical dislocation, and organs were taken for imaging (liver, spleen, kidney, duodenum, colon, brain, heart, tibia bone and blood).

Whole body fluorescence images were acquired using a Maestro II in vivo fluorescence imaging system (Cambridge Research \&Instrumentation, Inc., Woburn, MA). The system is equipped with a fiber-delivered $300 \mathrm{~W}$ xenon excitation lamp, and images can be acquired from $\lambda=500-950 \mathrm{~nm}$ by a 1.3 megapixel CCD camera (Sony ICX285 CCD chip). Each pixel within the image cube therefore has an associated fluorescence spectrum. The software for the Maestro system (Maestro 2.10.0) contains several algorithms to process the spectral data cubes to remove undesired auto-fluorescence signal and generate overlaid images for multiple fluorophores. A deep red excitation/emission filter set was used for our experiments ( $\lambda$ ex: $700-770 \mathrm{~nm}, \lambda \mathrm{em}>780 \mathrm{~nm}$ ). The liquid crystal tunable filter (LCTF) was programmed to acquire image cubes from $\lambda=780 \mathrm{~nm}-860 \mathrm{~nm}$ with an increment of $10 \mathrm{~nm}$ per image. The camera was set to $150 \mathrm{~ms}$ (whole body image), $15 \mathrm{~ms}$ (liver), 500ms (spleen), 7000ms (kidney), $10 \mathrm{~ms}$ (duodenum), $500 \mathrm{~ms}$ (colon), 1000ms (brain), 1000ms (tibia bones), $200 \mathrm{~ms}$ (heart) and $1000 \mathrm{~ms}$ (blood) exposure times. Fluorescence intensity measurements were performed using ImageJ $\mathrm{NIH}$ (National Institutes of Health) software.

Figure 12 shows whole body images of mice injected with the particles over time: at $5 \mathrm{~min}, 20$ min, $1 \mathrm{~h}$ and $24 \mathrm{~h}$ from injection. 5 min post injection, there is an initial burst of fluorescence which subsided quickly, while the majority of the fluorescent particles concentrated in the liver, at $20 \mathrm{~min} .24 \mathrm{~h}$ post injection, the fluorescence is almost non-existent, signifying the nanoparticle clearance from the body over $24 \mathrm{~h}$. Biodistribution was tested for free ICG as well, and no significant differences in distribution and kinetics were found between particles containing ICG and free ICG up to $24 \mathrm{~h}$ post injection. These findings were in complete agreement with previous reports of ICG and ICG-containing particles pharmacokinetics and biodistribution, as the free dye in solution, derivatives of the free dye and ICG-containing particles are all evacuated from the body after $1 \mathrm{~h}$ and completely vanished $24 \mathrm{~h}$ after i.v. injection $[60,61]$. 

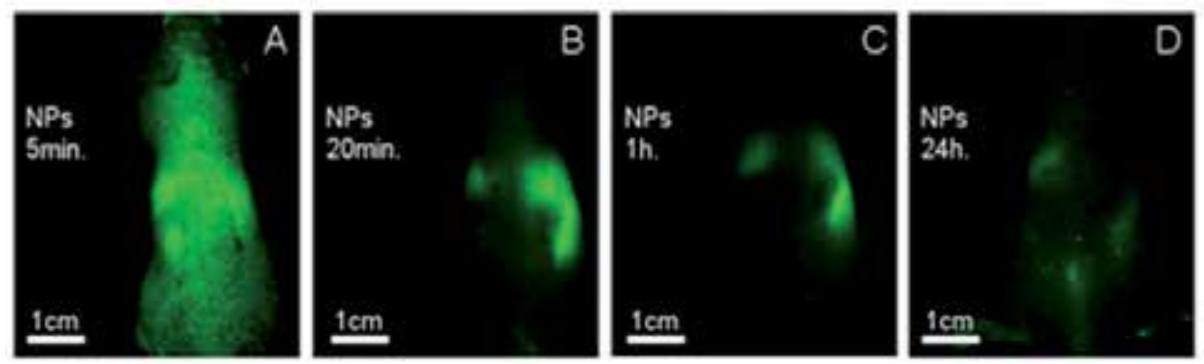

Figure 12. Typical whole body fluorescence images of the NIR fluorescent Prot 8 particles at $5 \mathrm{~min}, 20 \mathrm{~min}, 1 \mathrm{~h}$ and $24 \mathrm{~h}$ after i.v injection. 12 mice (each experiment group contained 3 mice) were anesthetized and treated with NIR fluorescent Prot8 particles $(2 \mathrm{mg} / \mathrm{mL}, 0.01 \mathrm{mg} / \mathrm{kg}$ body weight per mouse). Blood was drawn and organs were harvested at each time point. 2 uninjected mice served as negative control. 12 mice were injected correspondingly with free ICG solution, giving similar results (not shown). The experiment was repeated twice with similar results (originally published in [23]).

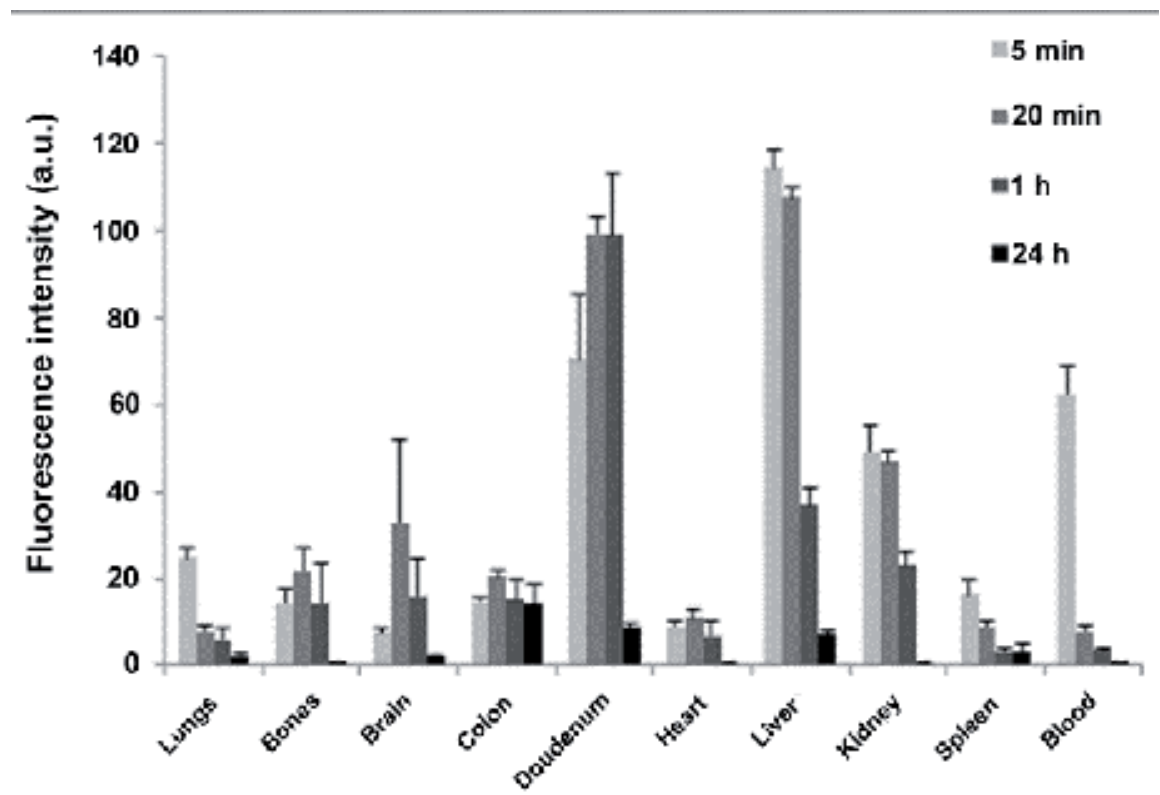

Figure 13. Fluorescence intensities of different organs taken at $5 \mathrm{~min}, 20 \mathrm{~min}, 1 \mathrm{~h}$ and $24 \mathrm{~h}$ post i.v. injection into mice tail veins. 12 mice (each experiment group contained 3 mice) were anesthetized and treated with NIR fluorescent Prot8 particles ( $2 \mathrm{mg} / \mathrm{mL}, 0.01 \mathrm{mg} / \mathrm{kg}$ body weight per mouse). Blood was drawn and organs were harvested at each time point. 2 uninjected mice served as negative control. The experiment was repeated twice with similar results (originally published in [23]).

Ex vivo fluorescence images of specific organs and blood were also obtained. Organs from mice were harvested and blood was drawn $5 \mathrm{~min}, 20 \mathrm{~min}, 1 \mathrm{~h}$ and $24 \mathrm{~h}$ post injection of the particles into the tail vein. Figure 13 shows the calculated fluorescence intensities of the lungs, bones, brain, colon, duodenum, heart, liver, kidney, spleen and blood screening. Evidently, this 
analysis shows that the particles penetrated and were found in all checked organs. It is shown clearly that by $20 \mathrm{~min}$ most of the inserted quantity of the fluorescent particles is cleared from the blood. The particles concentrate mostly at the liver and are probably evacuated from the body. Interestingly, it is also apparent that the particles pass the blood-brain barrier (BBB), since they are found in the brain at $20 \mathrm{~min}$ post injection. This may open up a scope of drug targeting to the brain for drug molecules which are usually blocked. Overall, it was demonstrated that following a single i.v. injection of the particles, fluorescence intensity at all organs decreased over time, and only traces of fluorescence could be seen after $24 \mathrm{~h}$.

\subsection{Conjugation of the tumor-targeting ligands to the particles}

PNA was covalently conjugated to the NIR fluorescent Prot8 particles by the cabodiimide activation method [62]. Briefly, EDC (1 mg) and Sulfo-NHS (1 mg) were each dissolved in 0.1 M MES (pH 6.0, $1 \mathrm{~mL}$ ) containing $0.5 \mathrm{M} \mathrm{NaCl}$. The EDC solution $(1 \mathrm{mg} / \mathrm{mL}, 10 \mu \mathrm{L})$ was added to an aqueous solution of PNA $(0.25 \mathrm{mg}, 62.5 \mu \mathrm{L})$, followed by the addition of the sulfo-NHS solution $(1 \mathrm{mg} / \mathrm{mL}, 25 \mu \mathrm{L})$. The mixture was then shaken for $15 \mathrm{~min}$, followed by the addition of the NIR fluorescent Prot8 particles $(2.5 \mathrm{mg}$ in $1 \mathrm{~mL}$ PBS). The mixture was then shaken for $90 \mathrm{~min}$. The obtained PNA-conjugated fluorescent particles were then washed from excess reagents by dilution and filtration through a 30-kDa filtration tube (VS2021 VIVA SPIN) at 1000 rpm (Centrifuge CN-2200 MRC) for 2 min, repeated three times. FITC-PNA, anti-CEA and anti-rabbit IgG were conjugated to the NIR fluorescent particles through a similar procedure. The concentration of bound PNA was determined with FITC-PNA by a calibration curve of FITC-PNA fluorescence using a multiplate reader (TECAN SpectraFluor Plus, Neotec Scientific Instruments). The concentrations of bound anti-CEA and anti-rabbit IgG were determined using a mouse IgG ELISA kit (Biotest, Israel). The calculated quantities of bound PNA and anti-CEA were 3.2 and $1.9 \mu \mathrm{g}$ per mg particles, respectively.

\subsection{Optical detection of human colon tumors in a chicken embryo model}

\subsubsection{The chicken embryo CAM model}

A chicken embryo CAM model was used to test the specific tumor detection by both the nonconjugated and the bioactive (PNA, anti-CEA or anti-rabbit IgG) conjugated NIR fluorescent Prot8 particles. Among most commonly used animal models, the chicken egg model allows the imaging of several tumors in a short time period and is less expensive [62]. Tumor cells were grafted on CAM according to the literature [62,64]. Briefly, fertile chicken eggs obtained from a commercial supplier were incubated at $37^{\circ} \mathrm{C}$ at $60-70 \%$ humidity in a forced-draft incubator. On day 3 of incubation, an artificial air sac was formed, allowing the CAM to drop. After 8 days of incubation, a window was opened in the shell and the CAM was exposed. Tumor cells were collected by trypsinization, washed with culture medium and pelleted by gentle centrifugation. Following removal of the medium, 5x106 cells were resuspended in 30 $\mu \mathrm{L}$ ice-cold Matrigel and inoculated on the CAM at the site of the blood vessels. Eggs were then sealed and returned to incubation. On day 6 post-grafting, day 14 of incubation, the tumor diameter ranged from 3 to $5 \mathrm{~mm}$ with visible neoangiogenesis. 
Figure 14 shows typical SW480 cell line derived tumors bordered by plastic rings on a chicken embryo CAM inside the egg.

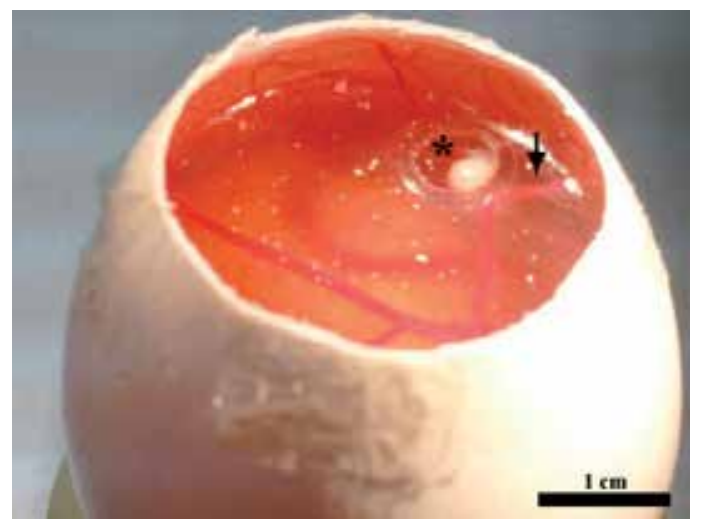

Figure 14. Light photograph of SW480 cell line derived tumors bordered by a plastic ring on chicken embryo CAM. Suspensions of $5 \times 10^{6} \mathrm{SW} 480$ cells suspended in Matrigel formed compact structures (asterisk) 8 days after transplantation with attraction of host blood vessels (arrow).

\subsubsection{CAM tumor detection}

Chicken embryos with 6-days-old human adenocarcinoma tumors (LS174t and SW480 cancer cell lines) implanted on the CAM were treated with the non-conjugated, PNA-conjugated and anti-CEA-conjugated NIR fluorescent Prot8 particles $(40 \mu \mathrm{L}, 2 \mathrm{mg} / \mathrm{mL})$. Additionally, nonpathological CAM treated with non-conjugated particles and untreated tumors served as control groups. After 40 minutes, the nanoparticle dispersions were removed and the tumors were washed with PBS. Then, the tumors and the non-pathological CAM were removed from the eggs, washed again with PBS and spread on a mat black background for observation using a Maestro II ${ }^{\mathrm{TM}}$ in vivo imaging system (Cambridge Research \& Instrumentation, Inc., Woburn, MA). A NIR excitation/emission filter set was used for the experiments ( $\lambda$ ex: 710-760 nm, $\lambda$ em $>750 \mathrm{~nm}$ ). The Liquid Crystal Tunable Filter (LCTF) was programmed to acquire image cubes from $\lambda=790 \mathrm{~nm}$ to $860 \mathrm{~nm}$ with an increment of $10 \mathrm{~nm}$ per image. Fluorescence intensity measurements were calculated as average intensity over the tumor surface area, using ImageJ software.

\subsubsection{In vivo optical detection of human colon tumors in a CAM model}

LS174t and SW480 colorectal cell lines were used to demonstrate the possible use of the NIR fluorescent Prot8 particles in tumor detection. As mentioned before, LS174t cells express certain receptors ( $\beta$-D-galactosyl-(1-3)-N-acetyl-D-galactosamine and CEA) at a much higher extent than SW480 cells $[62,65,66]$. This way, the chosen bioactive ligands PNA and anti-CEA, once conjugated to the Prot8 particles, can lead the particles specifically to the LS174t cancer cells. As shown in Figure 15, the LS174t tumors treated with bioactive-conjugated particles (B and C) gained higher fluorescence than SW480 tumors, compared to those treated with non- 
conjugated particles (A). This is accurate both for Prot8 particles conjugates with PNA (B) and anti-CEA (C), probably as a result of effective ligand-receptor interactions. In addition, the SW480 tumors treated with bioactive-conjugated Prot8 particles gained less fluorescence compared to those treated with the non-conjugated particles. The relative fluorescence intensities of the treated tumors by the conjugated and non-conjugated Prot8 particles are summarized in Figure 16. LS174t cells compared to SW480 cells gave fluorescence intensity ratios of 4:1 and 8:1 for PNA-conjugated particles and anti-CEA-conjugated particles, respectively. The non-conjugated Prot8 particles also labeled the tumors, however, the difference in the intensities between the types of tumors were not statistically significant. In this case, the overall fluorescence in both types of cells was higher than when treated with PNA-conjugated particles. This fact shows that even the bare non-conjugated particles penetrate the human cancer cell lines with a good extent. The possible reason is that the Prot8 particles can penetrate and label the cancerous cells specifically by either receptor-ligand interaction or utilization of these particles as nutrients for tumor growth, as they resemble biological proteins. The fluorescence intensity ratios between the types of cells show the significance of the nanoparticle surface. As seen in Figure 15D, no autofluorescence was observed in untreated tumors, signifying that all fluorescent signals are related to the fluorescent Prot8 particles labelling. Figure 15E shows that no non-specific labelling of non-pathological CAM tissue was observed, indicating the specificity of the Prot8 particles towards the tumor tissue.

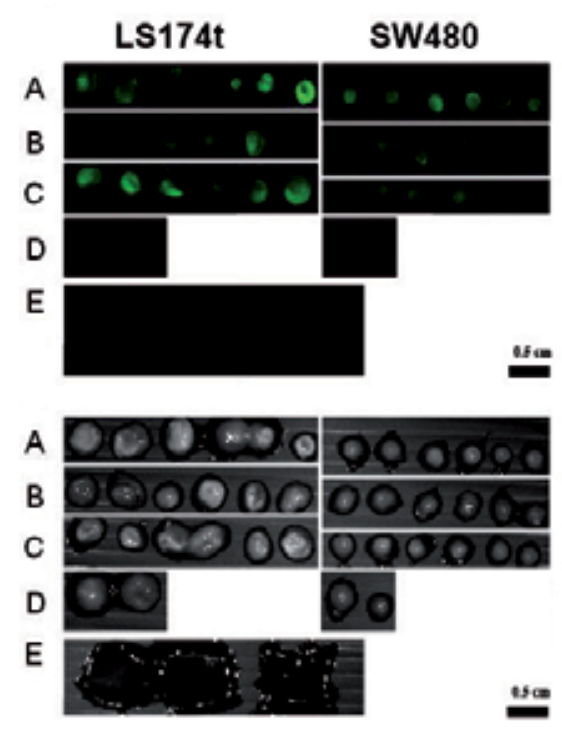

Figure 15. Fluorescent (upper) and greyscale (lower) images from a typical experiment of tumor cell lines LS174t and SW480 implants on chicken embryo CAM treated with non-conjugated (A), PNA-conjugated (B) and anti-CEA-conjugated (C) NIR fluorescent Prot8 particles. Images of untreated tumor cell lines are shown in (D). Images of non-pathological CAM treated with non-conjugated, PNA-conjugated and anti-CEA-conjugated particles are shown in (E). The experiment was repeated 5 times with similar results. 


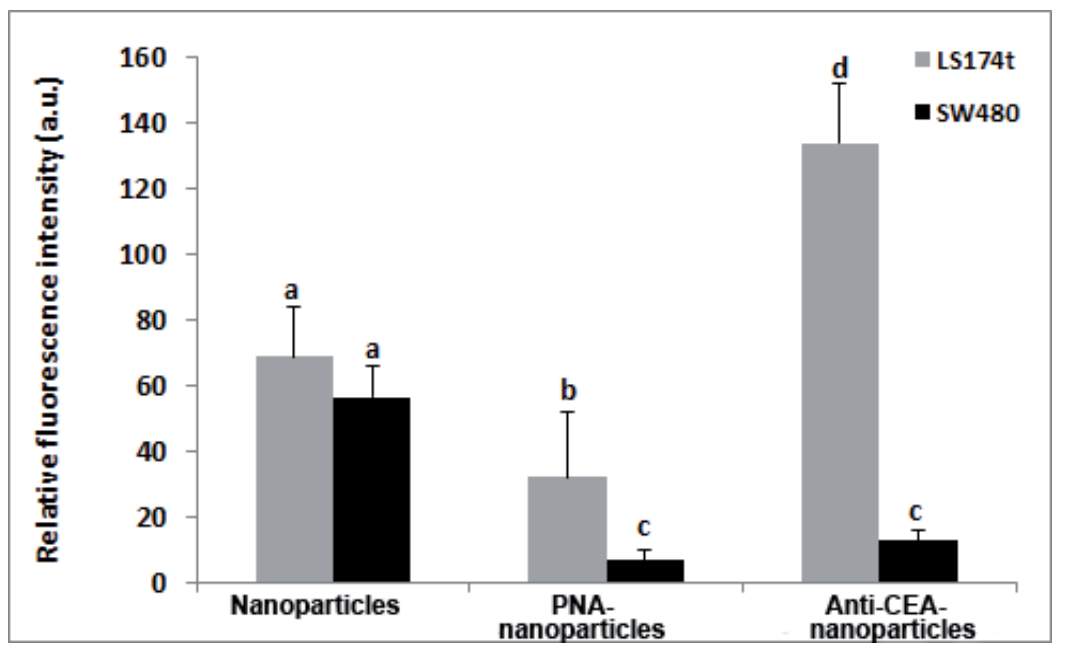

Figure 16. Relative fluorescence intensities of LS274t and SW480 tumors labeled with non-conjugated, PNA-conjugated and anti-CEA-conjugated particles. Data is presented as the mean value \pm SE. Values not sharing a common letter $(a, b$, $c$ or d) differ significantly from each other $(\mathrm{p}<0.05)$. The calculations are an average of 3 experiments.

In another set of in vivo experiments on LS174t and SW480 tumors implanted on the CAM model, the specific biomarker anti-CEA was tested against anti-rabbit IgG, serving as a nonspecific agent, as well as a control group of non-conjugated particles. As clearly illustrated in Figure 17, LS174t tumors treated with anti-CEA-conjugated particles (B) gained greater fluorescence compared to those treated with non-conjugated particles (A) or anti-rabbit IgGconjugated particles (C). This can be explained by the effective ligand-receptor interaction. Furthermore, the SW480 tumors treated with the anti-CEA-conjugated particles gained less fluorescence (about 3.5 times) compared to the LS174t tumors treated the same way. The fluorescent signal of LS174t tumors labeled by anti-CEA-conjugated particles was 4 times higher than that of the the tumors labeled by the anti-rabbit IgG-conjugated particles. Antirabbit IgG "blocks" the particle from interacting with the tumor receptors by the conjugation to the surface active moieties, thus serving as a negative control in colon tumor labelling.

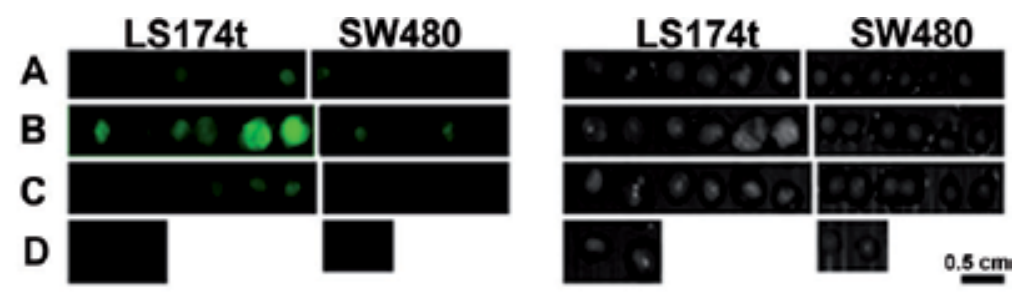

Figure 17. Fluorescent and grayscale images from a typical experiment of LS174t and SW480 human tumor cell lines implanted on chicken embryo CAM treated with the non-conjugated (A), anti-CEA-conjugated (B) and anti-rabbit IgGconjugated (C) NIR fluorescent Prot8 particles. Images of untreated tumors are shown in (D). The experiment was repeated 3 times with similar results. 


\subsection{Optical detection of human colon tumors in a mouse model}

Experiments were performed according to the protocols of the Israeli National Council for Animal Experiments by Harlan Biotech, Israel. Cancerous cells ( $30 \mu \mathrm{L}$ containing $2 \times 10^{6} \mathrm{LS} 174 \mathrm{t}$ cells) were injected into the mouse intestinal wall. 2 weeks later the nude mice were anaesthetized and treated with the bio-conjugated NIR fluorescent Prot8 particles $(0.1 \%, 200 \mu \mathrm{L})$, through the anus, using the guidance of a mini-colonoscope. $20 \mathrm{~min}$ later each colon was washed with PBS $(5 \times 1 \mathrm{~mL})$ and mice were allowed to recover for $4 \mathrm{~h}$. The mice were sacrificed and the colons were removed. Each colon was spread on a solid surface and imaging was performed using the Odysey Infra-red Imaging System (Li-Cor Biosciences, Lincoln, NE, USA) with excitation wavelength of $780 \mathrm{~nm}$ and emission wavelength of $800 \mathrm{~nm}$.

Figure 18 shows typical ( 8 out of 10 mice) fluorescent and grayscale images of the mice colons after treatment with anti- CEA (A) and anti-rabbit IgG (B) conjugated particles. As illustrated in Figure 18A, the anti-CEA-conjugated particles detected the tumors specifically and selectively with good signal to background ratio (SBR), the background refers to the surrounding non-pathological tissue. Moreover, as illustrated in Figure 18B, the "inactive" anti-rabbit IgGconjugated particles did not produce a significant signal of the tumors.

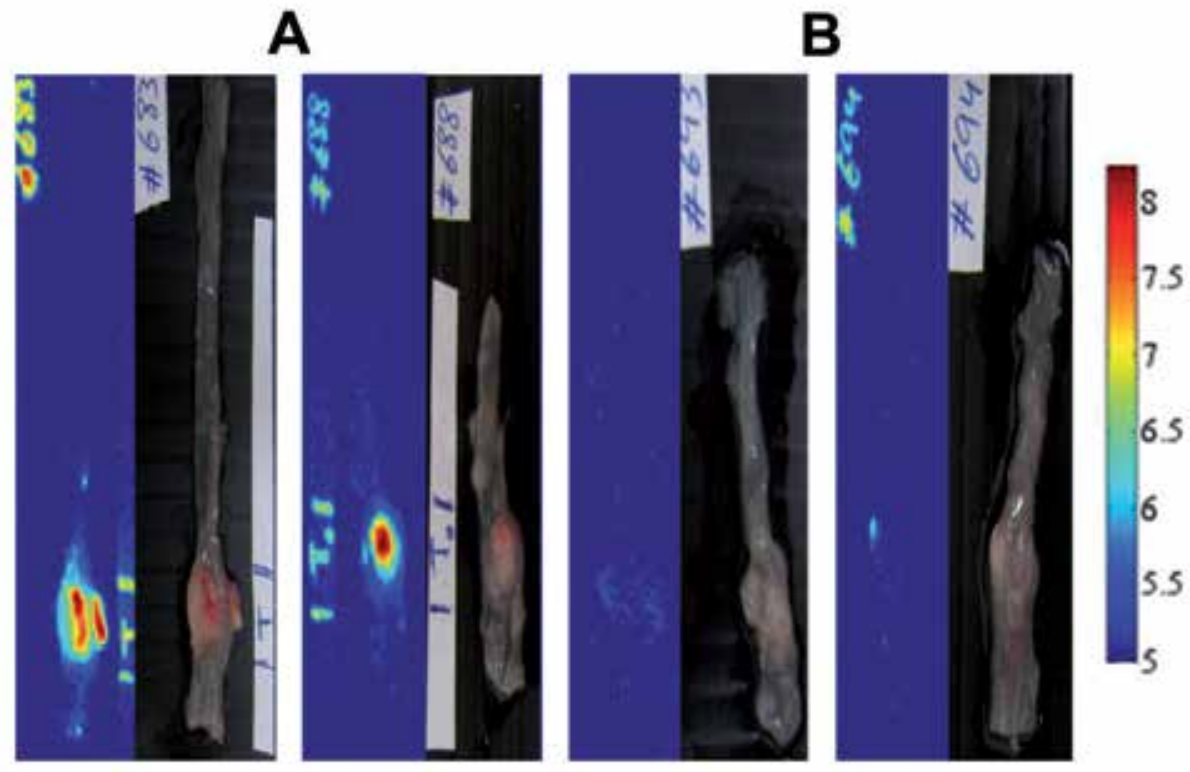

Figure 18. Fluorescent and grayscale images of typical LS174t colon tumors treated with anti-CEA (A) and anti-rabbit IgG (B)-conjugated NIR fluorescent Prot8 particles. 20 mice (10 in each experiment group) were anesthetized and treated with $0.1 \%$ particle dispersion in PBS, as described in section 3.2.5.14. 2 untreated mice served as a control group. 


\section{Summary and conclusions}

In the present study, new proteinoids were prepared using L-glutamic acid, L-aspartic acid, L-phenylalanine, L-lysine and low molecular weight poly(L-lactic acid). The polymerization was carried out by a simple straight-forward condensation polymerization in heat. The optimal conditions for the polymerization were tested, including changing the temperature and using microwave radiation. The proteinoids made are of high molecular weights with narrow size distributions and possess optical activity, which can be later used in specific drug delivery using chirality. The proteinoids are thermally stable and each proteinoid has enough carboxylic acid and/or amine functional groups, which can be later used to bind covalently desired molecules, such as drugs and dyes. The incorporation of 2000 Da PLLA into the proteinoid backbone presented a stable proteinoid as well. Proteinoids were manipulated in several ways to give proteinoid sphere-shaped nano/micro-particles, and to optimize this process. Specific conditions were found for the production of hollow particles of narrow size distribution. The incorporation of PLLA segments into the proteinoids increased the hydrophobic interior part and resulted in smaller size hollow particles. The particles were found to be non-toxic and stable over time. Also, encapsulation of different materials was carried out, giving organicfilled particles and fluorescent particles. In summary, proteinoid formation and cytotoxicity tests indicate that these particles are suitable for further in vivo testing.

The copolymer Prot8 containing 10\% PLLA, was chosen to be used for the biomedical study, since it provided the smallest particles with the biodegradability derived from the addition of PLLA to the proteinoid backbone. The proteinoid-PLLA copolymer was self-assembled in the presence of the NIR fluorescent dye ICG to yield NIR fluorescent Prot8 particles of $70 \pm 15 \mathrm{~nm}$ dry diameter. These new NIR fluorescent particles were found to be stable, avoiding leakage and photobleaching of the NIR dye over time. Furthermore, these particles are non-toxic, as shown by LDH and XTT assays for cytotoxicity and cell viability on human colorectal adenocarcinoma cell lines. The particles biodistribution in a mouse model was tested, following an i.v. injection, and the results showed that the particles penetrate a variety of organs, including the brain and bones. Nevertheless, $1 \mathrm{~h}$ post injection, the particles concentrate at specific sites of the body and are evacuated almost completely over $24 \mathrm{~h}$. It was established, using the chicken embryo CAM model and the tumor-implanted mouse model, that the NIR fluorescent particles may be very useful for tumor diagnosis in vivo due to their low autofluoresence of the background and the deep penetration into biomatrices. In the CAM model, when the NIR fluorescent proteinoid-PLLA particles are conjugated to a bioactive ligand (PNA or anti-CEA), they preserve their activity and specifically detect the cancer cells with upregulated receptors (LS174t vs. SW480). Non-conjugated and anti-rabbit IgG-conjugated NIR fluorescent particles (control particles) marked the cells without specific recognition at a much lower fluorescence intensity. In the mouse model, human tumor cells were implanted in mice colons, and the mice were treated with the NIR fluorescent particles. The results showed that the anti-CEA-conjugated particles specifically label the colon tumors, as opposed to those conjugated with anti-rabbit IgG antibodies. 
In summary, the chapter introduced a new series of newly-made proteinoids of a narrow selection of amino acid monomers, with high molecular weight and very narrow polydispersity index. Furthermore, this chapter has described proteinoid fluorescent particles as potential probes for precancerous colorectal adenomatous polyps or colon cancer tumors. Selective and specific fluorescent labelling of tumors is possible, with very low background signal, however improvements are still necessary for optimizing the targeting efficiency of the particles. Recent and ongoing developments in fluorescence imaging systems will open up the scope for effective detection of fluorescently labelled neoplasm. The proteinoid particles may further be developed to serve for both diagnostics and therapy combined.

\section{Author details}

Michal Kolitz-Domb and Shlomo Margel ${ }^{*}$

*Address all correspondence to: shlomo.margel@mail.biu.ac.il

The Institute of Nanotechnology and Advanced Materials, Department of Chemistry, USA

\section{References}

[1] Fox SW. How did life begin? Science. 1960;132:200-8.

[2] Fox SW. Proteinoid theory of origin of life and competing ideas. The American Biology Teacher. 1974;36(3):161-72.

[3] Fox SW. Thermal synthesis of amino-acids and the origin of life. Geochimica et Cosmochimica Acta. 1995;59(6):1213-4.

[4] Fox SW, Jungck JR, Nakashim T. From proteinoid microsphere to contemporary cell formation of internucleotide and peptide-bonds by proteinoid particles. Origins of Life and Evolution of Biospheres. 1974;5(1-2):227-37.

[5] Fox SW, Mccauley RJ, Fukushim T, Windsor CR, Montgome PO. Selective action in boundaries of particles of thermal proteinoid. Federation Proceedings. 1967;26(2):749.

[6] Fox SW, Nakashima T, Przybylski A, Syren RM. The updated experimental proteinoid model. International Journal of Quantum Chemistry. 1982:195-204.

[7] Fox SW, Waehneld TV. Thermal synthesis of neutral and basic proteinoids. Biochimica et Biophysica Acta. 1968;160(2):246-9.

[8] Fox SW, Harada K. Thermal copolymerization of amino acids to a product resembling protein. Science. 1958;128:1214. 
[9] Fox SW, Harada K. The thermal copolymerization of amino acids common to protein. Journal of the American Chemical Society. 1959;82(14):3745-51.

[10] Fox SW, Harada K. Thermal copolymerization of amino acids in the presence of Phosphoric Acid. Archives of Biochemistry and Biophysics. 1960;86:281-5.

[11] Fox SW, Harada K. Thermal polymerization of amino acid mixtures containing Aspartic Acid or a thermal precursor of Aspartic Acid. United States patent 3,052,655. 1962.

[12] Fox SW, Harada K. Method of making copolymers of amino acids containing Glutamic Acid. United States patent 3,076,790. 1963.

[13] Harada K, Fox SW. The thermal condensation of Glutamic Acid and Glycine to linear peptides. Journal of the American Chemical Society. 1957;80(11):2694-7.

[14] Harada K, Matsuyama M. Polycondensation of thermal precursors of amino-acids and characterization of constituent amino-acids. Biosystems. 1979;11(1):47-53.

[15] Slager J, Domb AJ. Biopolymer stereocomplexes. Advanced Drug Delivery Reviews. 2003;55(4):549-83.

[16] Kohn J, Langer R. Polymerization reactions involving the side-chains of Alpha-LAmino Acids. Journal of the American Chemical Society. 1987;109(3):817-20.

[17] Matsuno K. Electrical excitability of proteinoid microspheres composed of basic and acidic proteinoids. Biosystems. 1984;17(1):11-4.

[18] Przybylski AT. Excitable cell made of thermal proteinoids. Biosystems. 1985;17(4): 281-8.

[19] Przybylski AT, Fox SW. Excitable artificial cells of proteinoid. Applied Biochemistry and Biotechnology. 1984;10:301-7.

[20] Quirk S. Enhanced catalytic activity from proteinoid microspheres. Journal of Biomedical Materials Research Part A. 2013;101A(4):1133-43.

[21] Quirk S. Triggered release from proteinoid microspheres. United States patent 02314400 A1. 2007.

[22] Steiner S, Rosen R. Delivery systems for pharmacological agents encapsulated with proteinoids. Washington, USA patent 4,925,673. 1990.

[23] Kolitz-Domb M, Grinberg I, Corem-Salkmon E, Margel S: Engineering of near infrared fluorescent proteinoid-poly (L-lactic acid) particles for in vivo colon cancer detection. Journal of nanobiotechnology. 2014;12:1-13.

[24] Auras R. Poly (lactic acid). In: Encyclopedia Of Polymer Science and Technology.Wiley Interscience; 2010. 
[25] Kulkarni R, Moore E, Hegyeli A, Leonard F. Biodegradable poly (lactic acid) polymers. Journal of Biomedical Materials Research Part A. 1971;5(3):169-81.

[26] Bala I, Hariharan S, Kumar M. PLGA nanoparticles in drug delivery: The state of the art. Critical Reviews in Therapeutic Drug Carrier Systems. 2004;21(5):387-422.

[27] Danhier F, Ansorena E, Silva JM, Coco R, Le Breton A, Préat V. PLGA-based nanoparticles: an overview of biomedical applications. Journal of Controlled Release. 2012;161(2):505-22.

[28] Dibbern E, Toublan FJJ, Suslick KS. Poly(glutamic acid) nanospheres for biomedical applications. Abstracts of Papers of the American Chemical Society. 2004;228:U346U.

[29] Shikanov A, Kumar N, Domb AJ. Biodegradable polymers: An update. Israel Journal of Chemistry. 2005;45(4):393-9.

[30] Garlotta D. A literature review of poly (lactic acid). Journal of Polymers and the Environment. 2001;9(2):63-84.

[31] Tsuji H. Poly (lactic acid). In: Bio-Based Plastics: Materials and Applications. Kabasci S: John Wiley \& Sons; 2013. 171-239.

[32] Lim LT, Auras R, Rubino M. Processing technologies for poly (lactic acid). Progress in Polymer Science. 2008;33(8):820-52.

[33] Bahn PR, Pappelis A, Bozzola J. Protocell-like microspheres from thermal polyaspartic acid. Origins of Life and Evolution of Biospheres. 2006;36(5-6):617-9.

[34] Milstein SJ, Kantor ML. Proteinoid Microspheres and Methods for Preparation and Use Thereof. United States patent 5,601,846. 1997.

[35] Bamnolker H, Nitzan B, Gura S, Margel S. New solid and hollow, magnetic and nonmagnetic, organic-inorganic monodispersed hybrid microspheres: synthesis and characterization. Journal of Materials Science Letters. 1997;16(16):1412-5.

[36] Syren RM, Sanjur A, Fox SW. Proteinoid microspheres more stable in hot than in cold water. Biosystems. 1985;17(4):275-80.

[37] Bae SK, Kim JD. Aggregation behaviors and their $\mathrm{pH}$ sensitivity of cholesterol-conjugated proteinoids composed of glutamic acid and aspartic acid matrix. Journal of Biomedical Materials Research Part A. 2003;64A(2):282-90.

[38] Kokufuta E, Sakai H, Harada K. Factors controlling the size of proteinoid microspheres. Biosystems. 1983;16(3-4):175-81.

[39] Urry DW, Peng SQ. Nonlinear mechanical force induced pKa shifts: Implications for efficiency of conversion to chemical energy. Journal of the American Chemical Society. 1995;117(32):8478-9. 
[40] Kumar ABM, Rao KP. Preparation and characterization of $\mathrm{pH}$-sensitive proteinoid microspheres for the oral delivery of methotrexate. Biomaterials. 1998;19(7-9):725-32.

[41] Steiner S, Rosen R. Delivery systems for pharmacological agents encapsulated with proteinods. United States patent 4,925,673. 1990.

[42] Tallawi M. Proteinoid/hydroxyapatite hybrid microsphere composites. Journal of Biomedical Materials Research Part B: Applied Biomaterials. 2011;96(2):261-6.

[43] Mullerherold U, Nickel G. The stability of proteinoid microspheres. Biosystems. 1994;33(3):215-20.

[44] Nakashima T. Metabolism of proteinoid microspheres. Topics in Current Chemistry. 1987;139:57-81.

[45] Quirk S. Triggered release of small molecules from proteinoid microspheres. Journal of Biomedical Materials Research Part A. 2009;91A(2):391-9.

[46] Quirk S. Triggered release from peptide-proteinoid microspheres. Journal of Biomedical Materials Research Part A. 2010;92A(3):877-86.

[47] Toublan FJJ, Dibbern E, Argadine HM, Greenleaf JF, Simari RD, Suslick KS. Electrostatic adhesion of polyelectrolytes and colloids on protein microspheres. Abstracts of Papers of the American Chemical Society. 2004;228:U350-U.

[48] Quirk S. Diagnostic signal amplification with proteinoid microspheres. U.S. patent 20030138975A1. 2003.

[49] Janairo G, Sy ML, Yap L, Llanos-Lazaro N, Robles J. Determination of the Sensitivity Range of Biuret Test for Undergraduate Biochemistry Experiments. e-Journal of Science and Technology. 2011;5:77-83.

[50] Harris FW. State of the art: Polymer chemistry. Introduction. Journal of Chemical Education. 1981;58(11):836.

[51] Dose K. Chemical and catalytical properties of thermal polymers of amino acids (proteinoids). Origins of Life. 1974;5(1-2):239-52.

[52] Heiskanen J. Comparison of three methods for determining the particle density of soil with liquid pycnometers. Communications in Soil Science and Plant Analysis. 1992;23(7-8):841-6.

[53] Ma XH, Santiago N, Chen YS, Chaudhary K, Milstein SJ, Baughman RA. Stability study of drug-loaded proteinoid microsphere formulations during freeze-drying. Journal of Drug Targeting. 1994;2(1):9-21.

[54] Epsztejn S, Glickstein H, Picard V, Slotki IN, Breuer W, Beaumont C, et al. H-ferritin subunit overexpression in erythroid cells reduces the oxidative stress response and induces multidrug resistance properties. Blood. 1999;94(10):3593-603. 
[55] Decker T, Lohmannmatthes ML. A quick and simple method for the quantitation of Lactate-Dehydrogenase release in measurements of cellular cyto-toxicity and tumor necrosis factor (TNF) activity. Journal of Immunological Methods. 1988;115(1):61-9.

[56] Haritoglou C, Freyer W, Priglinger SG, Kampik A. Light absorbing properties of indocyanine green (ICG) in solution and after adsorption to the retinal surface-an exvivo approach. Graefe's Archive for Clinical and Experimental Ophthalmology. 2006;244(9):1196-202.

[57] Zweck J, Penzkofer A. Microstructure of indocyanine green J-aggregates in aqueous solution. Chemical Physics. 2001;269(1):399-409.

[58] Altınoğlu Eİ, Adair JH. Near infrared imaging with nanoparticles. Wiley Interdisciplinary Reviews Nanomedicine and Nanobiotechnology. 2010;2(5):461-77.

[59] Sharrna P, Brown S, Walter G, Santra S, Moudgil B. Nanoparticles for bioimaging. Advances in Colloid and Interface Science. 2006;123:471-85.

[60] Yaseen MA, Yu J, Jung B, Wong MS, Anvari B. Biodistribution of encapsulated indocyanine green in healthy mice. Molecular Pharmaceutics. 2009;6(5):1321-32.

[61] Mizrahi DM, Ziv-Polat O, Perlstein B, Gluz E, Margel S. Synthesis, fluorescence and biodistribution of a bone-targeted near-infrared conjugate. European Journal of Medicinal Chemistry. 2011;46(10):5175-83.

[62] Cohen S, Pellach M, Kam Y, Grinberg I, Corem-Salkmon E, Rubinstein A, et al. Synthesis and characterization of near IR fluorescent albumin nanoparticles for optical detection of colon cancer. Materials Science and Engineering: C. 2012;33:923-31.

[63] Durupt F, Koppers-Lalic D, Balme B, Budel L, Terrier O, Lina B, et al. The chicken chorioallantoic membrane tumor assay as model for qualitative testing of oncolytic adenoviruses. Cancer Gene Therapy. 2012;19(1):58-68.

[64] Noiman T, Buzhor E, Metsuyanim S, Harari-Steinberg O, Morgenshtern C, Dekel B, et al. A rapid in vivo assay system for analyzing the organogenetic capacity of human kidney cells. Organogenesis. 2011;7(2):140-4.

[65] Kaushal S, McElroy MK, Luiken GA, Talamini MA, Moossa A, Hoffman RM, et al. Fluorophore-conjugated anti-CEA antibody for the intraoperative imaging of pancreatic and colorectal cancer. Journal of Gastrointestinal Surgery. 2008;12(11):1938-50.

[66] Corem-Salkmon E, Perlstein B, Margel S. Design of near-infrared fluorescent bioactive conjugated functional iron oxide nanoparticles for optical detection of colon cancer. International Journal of Nanomedicine. 2012;7:5517-27. 

Chapter 4

\title{
Lipid-Based Nanoparticles and Microbubbles - Multifunctional Lipid-Based Biocompatible Particles for in vivo Imaging and Theranostics
}

\author{
Jaroslav Turánek, Andrew D. Miller, \\ Zuzana Kauerová, Róbert Lukáč, Josef Mašek, \\ Štěpán Koudelka and Milan Raška
}

Additional information is available at the end of the chapter

http://dx.doi.org/10.5772/59870

\section{Introduction}

\subsection{Summary}

Lipid-based nanoparticles (LNPs) are both biocompatible and biodegradable so very well suited to medicinal application in targeted drug delivery and in vivo imaging. Microbubbles (MB) are another emerging platform for functional delivery of drugs and in vivo imaging. This chapter is focused on the principles of LNP and MB construction, action and application, concluding with a section on the medical benefits that may result from bringing LNP and MB technologies together. Some applications are mentioned in brief to demonstrate the diagnostic and therapeutic potential of LNPs and MBs. More specific detail on applications is provided elsewhere in this book.

\section{LNP fundamentals}

Structural lipids such as cholesterol and glycerophospholipids are the major components of biological membranes. Without doubt, one of the great triumphs of nature is the manner in which lipid molecules such as these are able to form into cellular membranes sufficient to compartmentalize volumes within cells and between cells. In short, lipids are able to form the vast macromolecular assemblies that come to make up cellular membranes and other barriers 
in nature precisely because they have an unrivalled capability for self-association, driven by weak short range forces and the hydrophobic effect (Figure 1) [1]. This capacity for selfassociation can be exploited in the laboratory in order to create self-assembly LNPs (approx $100 \mathrm{~nm}$ in diameter) (Figure 2) that are proving to be a powerful platform to enable the functional delivery of active pharmaceutical ingredients (APIs) to target cells in vivo. Suitable APIs might include biopharmaceutical agents (e.g. DNA, RNA interference effectors), selected small molecule drugs (e.g. anticancer cytotoxic drugs), and/or imaging agents (e.g. magnetic resonance imaging contrast metals - gadolinium (III) $\left(\mathrm{Gd}^{3+}\right)$, radiometals, and/or fluorescent probes). The number of lipid variations is almost limitless, so too the number and variety of possible LNPs that may be produced for use in biological situations. Therefore, LNPs offer the potential opportunity for tailor-made preparation and production leading in the future even to the possibility of LNP-mediated personalized medicine, no less [2, 3].

a)

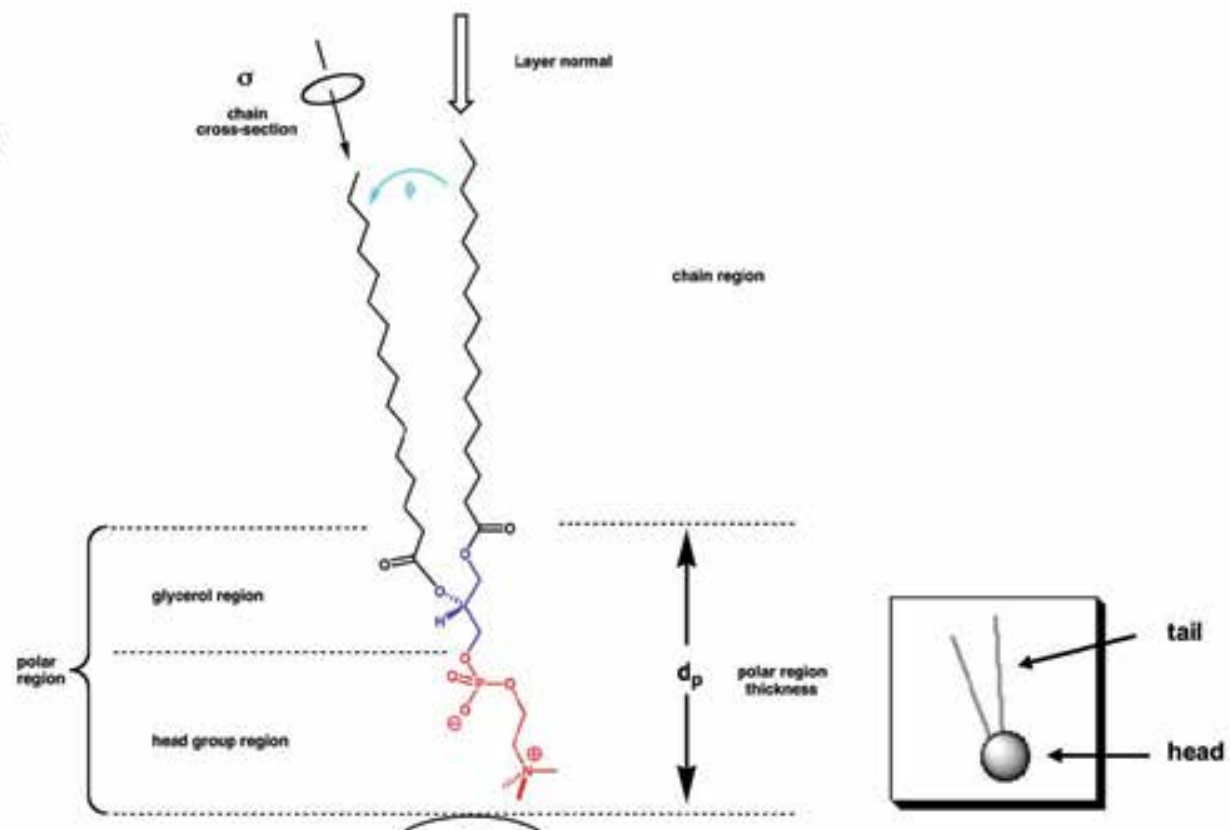

b)

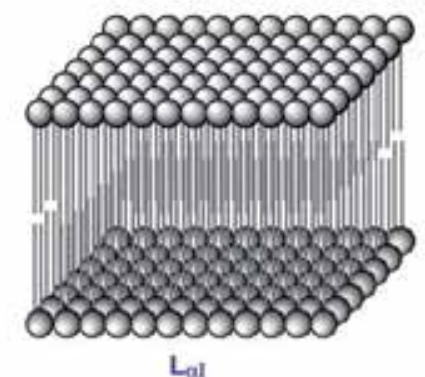

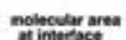

nolecular area

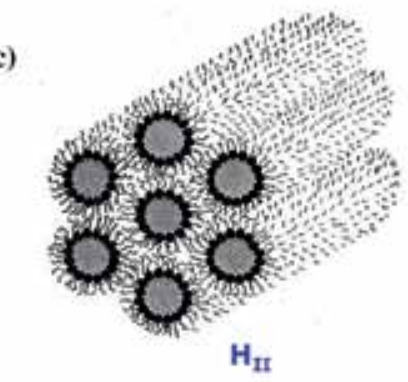

Figure 1. Principles of lipid structure and self-assembly. 
Structural lipids of all types consist broadly of a non-polar, hydrophobic "chain" or "tail" region attached to a "polar" or "head" region (a); biological membranes primarily adopt a normal topology lamellar L $\alpha$ I fluid mesophase (bilayer) structure (b); under certain circumstances biological membranes adopt other mesophases in particular the inverse topology hexagonal HII fluid mesophase (c), where hydrophobic chain regions face outwards and hydrophilic polar regions face inwards to form aqueous channels (the darker circles). (Diagrams reproduced from [1])

However, and there always is a however, LNPs that have seen service in vivo are turning out to have one fundamental design weakness which can be summarized by saying that those chemical modifications to LNP surfaces that are necessary for such nanoparticles to be stable to storage and in biological fluids, plus minimally visible to a host immune system, now turn out to limit the efficiency of functional delivery once LNPs reach their target cells. Accordingly, one of the primary ways to overcome this problem in recent years has been to introduce the concept of nanoparticle triggerability to LNP design [2, 4]. Nanoparticles possess triggerability (or are said to be triggerable) when designed for stability in biological fluids (from a desired point of administration to disease-target cells) then become triggered for the controlled release of associated APIs at target cells either through local changes in local endogenous (intrinsic) conditions, or through the application of an exogenous (extrinsic) stimulus trained onto target cell regions where nanoparticles are also located. What we are now learning is that MBs used in combination with ultrasound can be used in with LNPs, to provide a potent way to introduce LNP triggerability. Moreover, the introduction of ultrasound critically introduces an opportunity for real-time, diagnostic imaging of LNP mediated delivery of APIs to target cells in vivo. Nanoparticles that combine functional API delivery in vivo with real-time, diagnostic imaging are known as theranostic nanoparticles (TNPs) [5]. Most importantly, TNPs offer the opportunity for true image-guided therapy. As such this may well be a primary future of the MBs-ultrasound-LNP combination as the rest of this chapter will now aim to demonstrate.

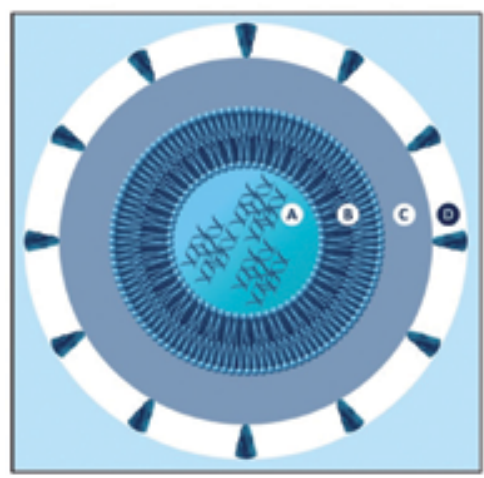

$A B$ systems; in vitro, ex vivo Local/regional use in vivo
A; active pharmaceutical ingredient

- e.g. nucleic acids, drugs, imaging agents

B; compaction/association agents

- lipids or lipid related in this instance

C; stealth/biocompatibility polymer layer

D; biological recognition ligand layer

ABCD nanoparticles constructed

from tool-kits of synthetic chemical components

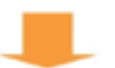

Tailor-made delivery solutions

$A B C / A B C D$ systems; in vivo

Figure 2. Functional lipid-based nanoparticle delivery systems. 
In functional lipid-based nanoparticles (LNPs), active pharmaceutical ingredients (APIs) (A) are condensed within functional concentric layers of chemical components designed for delivery into cells and intracellular trafficking (B components, primarily lipids and lipidrelated components), biological stability (C stealth/biocompatibility components-typically Polyethylene Glycol [PEG]) and biological targeting to target cells (D components, biological receptor-specific targeting ligands) $[2,3]$.

\section{Liposomes to LNPs}

Liposomes are the progenitors of all LNPs, and they are formulated directly from lipid constituents (Figure 3). Liposomes can be prepared by various techniques. The method based on hydration of lipid film represents the most exploited technique for preparation of liposomes. The lipids of known molarities are dissolved in organic solution which is subsequently slowly evaporated in vacuo to produce a thin film. The film is hydrated with a suitable aqueous buffer at temperature, which is generally above transition temperature of lipids used. Aqueous buffer contains also compound which are to be entrapped into liposomes. Size of liposomes can be reduced by freeze-thaw procedure followed by sonication, extrusion, microfluidisation or high pressure homogenisation. These procedures can tune size distribution of final liposomal preparation to desired values. The main parameters characterising liposomes are their size, spherical shape and degree of lamellarity. Liposomes may consist of one bilayer (unilamellar), several bilayers (oligolamellar) or multiple bilayers (multilamellar). Membrane rigidity can be adjusted using appropriate lipids and membrane fluidity may be controlled with the use of phospholipids with higher or lower transition temperature.
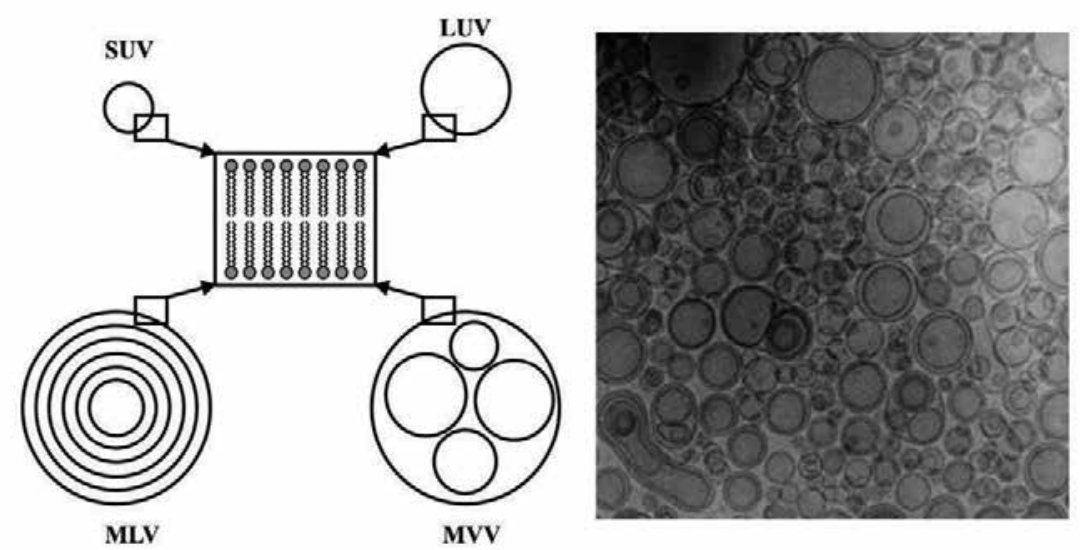

Figure 3. Various morphological types of liposomes and real appearance in cryoelectron microscopy.

Small unilamellar vesicles (SUV); Large unilamellar vesicles (LUV); Multilamellar vesicles concentric (MLV); Multivesicular vesicles (MVV) 
Liposomes are generally formulated on the nm scale and can be further size refined by passage through physical membrane pores of known size (extrusion). Liposomes are typically characterised by their size, spherical shape and degree of lamellarity. They may be composed of a single bilayer (unilamellar), a few bilayers (oligolamellar), or multiple bilayers (multilamellar). The rigidity of the membrane can also be modified with the use of suitable lipids; and the fluidity of the membrane may be controlled using phospholipids with higher or lower $\mathrm{L}_{\alpha \mathrm{I}}-$ $\mathrm{H}_{\mathrm{II}}$ mesophase transition temperatures. In general lipids of stearic acids (fully saturated $\mathrm{C} 18$ hydrocarbon chains) bestow rigidity to liposome structures (by favouring $\mathrm{L}_{\alpha \mathrm{I}}$ fluid mesophases), whilst lipids of oleic acid ( $\Delta 9$ unsaturated $C 18$ hydrophobic chains) can result in less stable structures (by favouring $\mathrm{H}_{\mathrm{II}}$ fluid mesophases). Liposomes have traditionally been used as some of the very earliest nanodrug delivery vehicles, encapsulating water-soluble drugs within their central aqueous cavities in order to improve drug pharmacokinetics [6-8]. Liposome spherical diameters can be investigated by using scattered light off the surface of the particles with photon correlation spectroscopy. Alternatively cryo-TEM can be used to provide visual images of liposome sizes and shape. The surface charge of liposomes can be obtained by measuring their zeta potential (electrokinetic potential). Liposome associated techniques and characteristics have all been employed in more recent time times to prepare more chemically diverse LNPs.

\section{Cellular imaging}

Cellular imaging is "the visualisation of specific cells in an intact animal". This term also collectively denotes the visualisation of any type of the entire cell under different conditions [9]. While cellular imaging applies to the imaging of whole cells, molecular imaging deals with the visualisation of molecules and sub-cellular components within individual cells [10]. The exploitation of molecular probes or imaging agents is essential for the use of both cellular and molecular imaging strategies. Despite the fact that the method of cellular imaging of extrinsic fluorophores is highly efficient, the depth of tissue from which the signal emitted represents often a limiting factor for this technique. The possible use of positron emission tomography (PET) or single-photon computed tomography (SPECT) methods is also limited due to shortlived radioisotopes and poor spatial resolution. However, three-dimensional images of tissues containing water can be obtained by magnetic resonance imaging (MRI). This technique is characterised by high levels of spatial resolution and imaging of depth tissues is achieved.

A primary limitation of MRI is an inherent lack of sensitivity that can be overcome through the use of contrast agents that enhance signal sensitivity and hence image quality. MRI contrast agents consist of molecules that incorporate a paramagnetic metal ion, most commonly gadolinium (III) $\left(\mathrm{Gd}^{3+}\right)$ or Iron $\left(\mathrm{Fe}^{3+} / \mathrm{Fe}^{2+}\right)$. The improvement in image quality derives from the modulating effects of the coordinated metal ions on longitudinal $\left(T_{1}\right)$ or transverse $\left(T_{2}\right)$ relaxation times associated with proton resonance signals emanating from bulk water molecules surrounding the coordinated metal ions. Even though contrast agents incorporating Gd3increase both 1//T1 and 1/T2 relaxivities, they are commonly used for T1-weighed (positive bright) contrast imaging, on condition that $1 / \mathrm{T} 1$ contribution is higher in tissue than the 
corresponding effect on 1/T2 enhancement. Due to the fact that more considerable increases in 1/T2 are induced by iron containing contrast agents, they are usually used in T2- weighed (negative dark) contrast imaging [11]. Despite the most frequently used MRI contrast agents are thermodynamically and kinetically stable low molecular weight $\mathrm{Gd}^{3+}$ complexes (that promote MRI contrast by non-specific enhancement of water proton relaxation rates within the blood pool), there has been a realization of the need for imaging LNPs suitable for the targeted delivery of imaging agents to sites of disease pathology such as cancerous lesions in order to obtain clear contrast images of their extent and location.

For cell populations to be imaged by MRI in vivo, these populations must be distinguishable from natural background noise signal. For this to happen, cells of interest should become labelled with appropriate agents, making use of controlling mechanisms such as enhanced permeability and retention (EPR) mechanism for imaging agents to reach target cells [12,13,14], followed by controlled target cell entry. According to Frangioni et al., the ideal cellular label should have the following features: a strong signalling effect, good biocompatibility, does not interfere with cellular genetics, remains retained only in the target cell population, and allows temporal imaging for long-lasting periods [15]. Controlled target cell entry is preferably by ligand-specific receptor-mediated cell entry processes, although alternative mechanisms of cell entry may also be acceptable (Figure 4) [16].

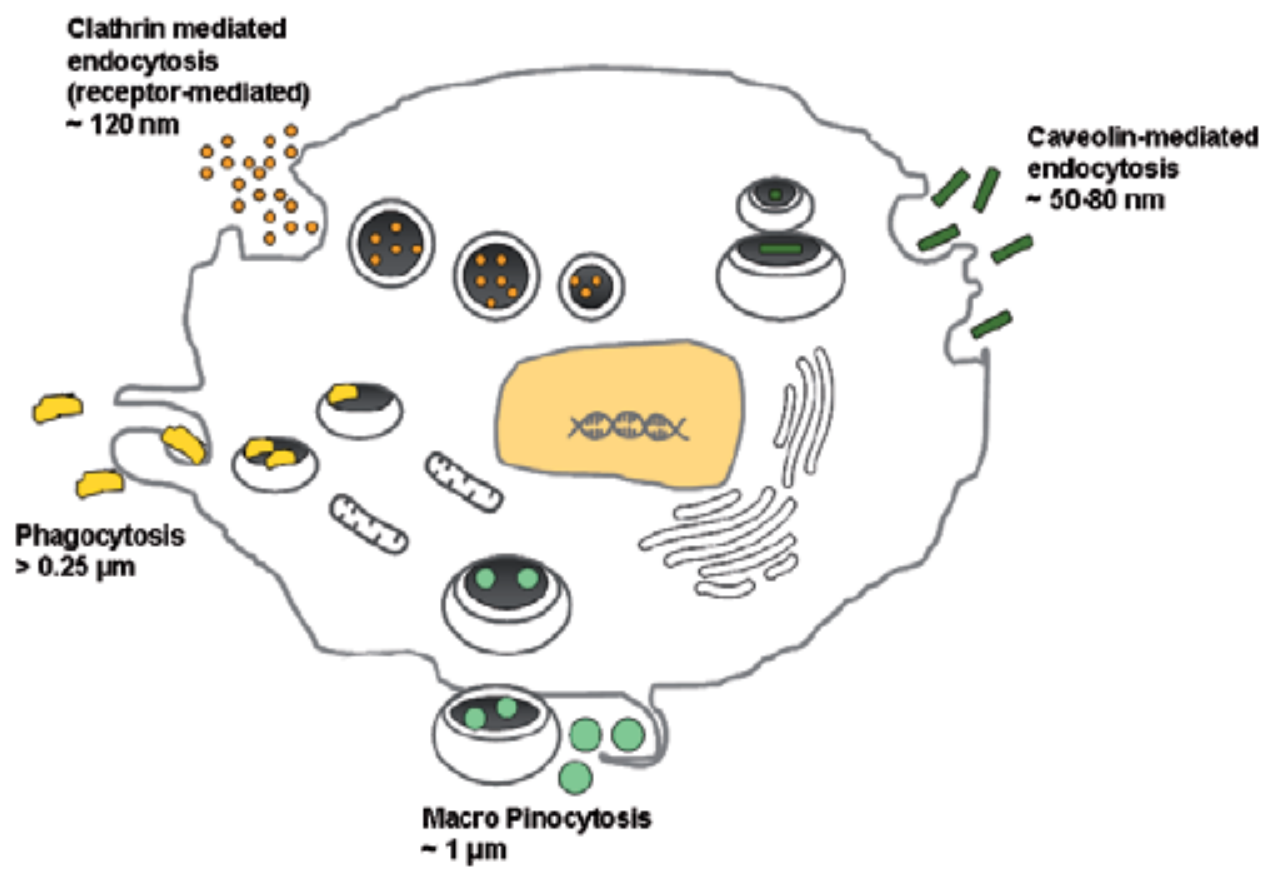

Figure 4. Cell entry mechanisms: the various uptake mechanisms of macromolecules and nanoparticles into cells are indicated according to their size (Adapted from [14]). 
LNPs are an ideal platform to combine multimodal imaging (e.g. MRI contrast agent and fluorescent probes) with multiple functionalities in order to effect multimodal cellular labelling. Targeting of LNPs to various tissues and cells (e.g. tumour) can be accomplished by conjugation of LNP surfaces with biological receptor-specific targeting ligands (e.g. monoclonal antibodies, folate, RGD peptides) [2,3]. One of the best examples of such an imaging LNP recently described in the literature is shown (Figure 5). This imaging LNP system (Gadonano$\mathrm{F}$ ) is a novel $\mathrm{Gd}^{3+}$-containing, imaging LNP system that was designed to act as a folate receptor (FR)-targeted, positive contrast agent to enable magnetic resonance imaging (MRI) of FRpresenting cancerous lesions (primary and metastatic) including breast, lung, intestinal, renal, and ovarian cancers.

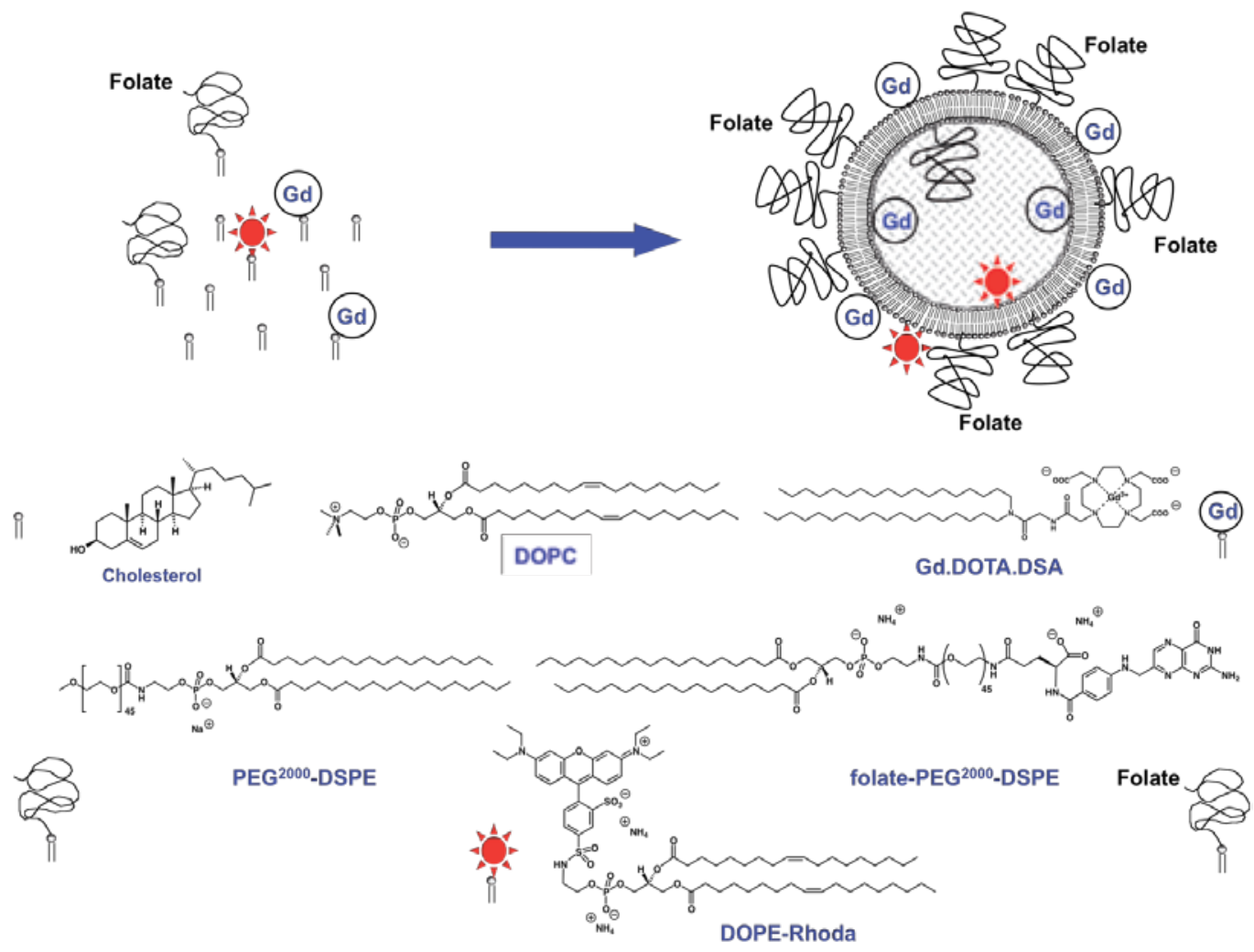

Figure 5. Multimodal imaging Gadonano-F LNPs for targeted delivery of MRI contrast agents to tumour cells.

Gadonano-F LNPs are prepared as above with the indicated lipid components. Double labelling is made possible using gadolinium metallochelating lipid (Gd.DOTA.DSA) for MRI positive contrast imaging and rhodamine fluorescent probe conjugated lipid (DOPE-Rhoda) for fluorescence imaging. Folate association with the Gadonano-F LNP surfaces is made possible using a polyethylene glycol (PEG) lipid (folate-PEG ${ }^{2000}-\mathrm{DSPE}$ ), ensuring that Gadonano-F LNPs are enabled for FR-specific targeting to tumour cells in vivo. The remaining PEG lipid (PEG ${ }^{2000}$-DSPE) ensures that the Gadonano-F LNPs possess stability in biological fluids (e.g. serum) (see [14]). 
There are a number of pathways by which LNPs could enter mammalian cells, namely by phagocytosis, pinocytosis, clathrin and calveolin independent endocytosis [9, 17]. Of these, phagocytosis is a process of engulfing and internalisation of large particles $(1 \mathrm{um})$ by cells. The majority of phagocytic cells of the immune system, including macrophages, neutrophils, monocytes, and microglia, are able to internalise foreign bodies via phagocytosis. Pinocytosis is a method of particle internalisation by non-phagocytic cells but this is a very non-specific mechanism of internalisation into cells [9]. On the other hand, clathrin-mediated endocytosis (receptor-mediated) and caveolin-mediated endocytosis are much the more specific means of cellular entry, of which the first is ideal for entry of LNPs to cells.

In original preclinical laboratory experiments, Gadonano-F LNPs were prepared and used to image xenograft tumours (IGROV-1) in mice. At just $2 \mathrm{~h}$ post-injection the Gadonano-F LNPs mediated a similar signal enhancement to that attained at $24 \mathrm{~h}$ post-injection by control (Gadonano LNPs, lacking folate ligand) (Figure 6). Specifically, Gadonano LNPs accumulated in tumour by the EPR mechanism ("passive" targeting) [12,13], and labelled cells for MRI over a period of $24 \mathrm{~h}$. These imaging LNPs were seen to access the entire hyperpermeable, living volume of the tumour while being excluded from the necrotic regions. By contrast, the FRtargeted Gadonano-F LNPs accumulated in the tumour and labelled cells for MRI from $2 \mathrm{~h}$ onwards, owing to EPR mediated tumour uptake from the blood pool followed by rapid FRmediated cell entry probably involving clathrin-mediated endocytosis. FR-targeted imaging LNPs accumulated in more perivascular and macrophage-rich locations [14,18].
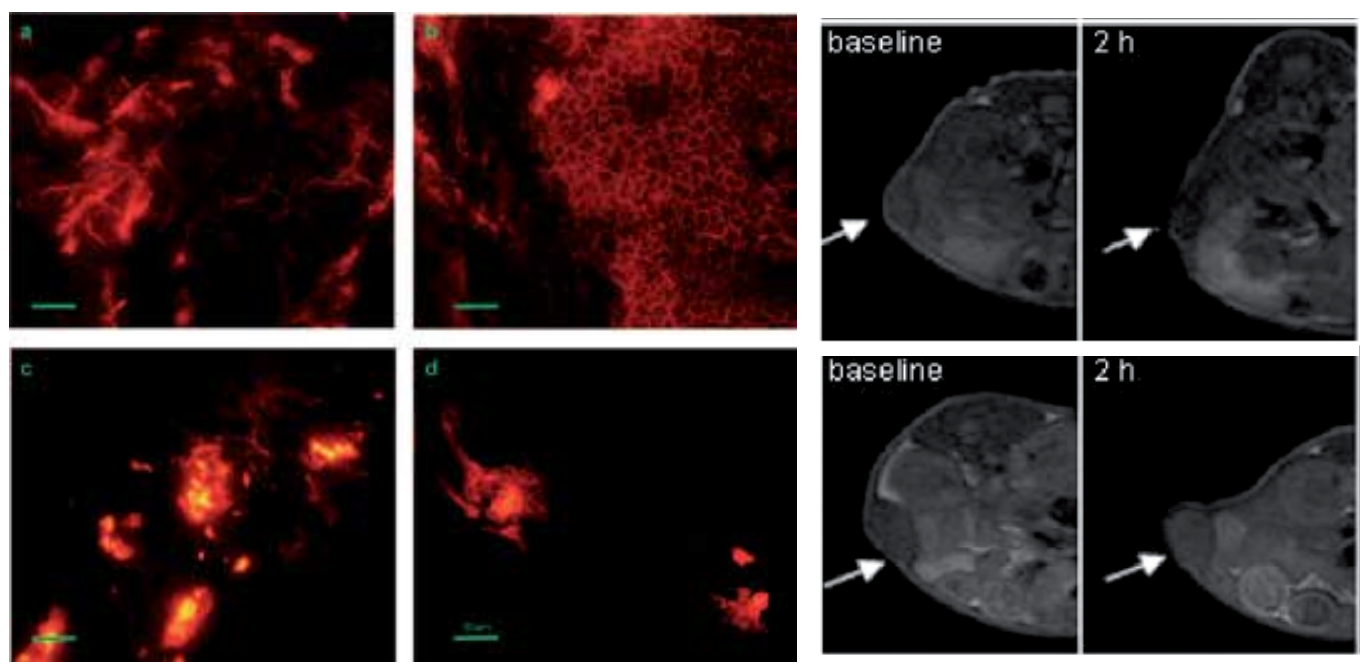

Figure 6. Fluorescence microscopy and MRI contrast imaging of $24 \mathrm{~h}$ post imaging LNP injection.

On the left $24 \mathrm{~h}$ post imaging LNP administration, (a) surface IGROV-1 tumor slices, Gadonano LNPs, (b) midtumor IGROV-1 slices, Gadonano LNPs, (c) surface IGROV-1 tumor slices, Gadonano-F LNPs, and (d) IGROV-1 midtumor slices, Gadonano-F LNPs (green bar represents $50 \mu \mathrm{m}$ ). On the right, MRI contrast images of IGROV-1 tumour bearing Balb/C nude mice: 
images are from control mice injected with Gadonano LNPs (top); images are of mice injected with Gadonano-F LNPs (bottom) (white arrow shows location of tumour) (Adapted from [18])

Rapid and effective imaging of FR-bearing tumours by Gadonano-F LNPs in preclinical experiments, has since led to these LNPs being prepared for clinical trial by GlobalAcorn Ltd under the name GA101 NANO-GdF (Gadonano LNPs are also being developed for clinical trial by GlobalAcorn Ltd under the name GA100 NANO-Gd). Rapid and specific imaging of diseased tissues and tumours will be of paramount importance for preclinical research and also future diagnostic and theranostic applications in clinic [2, 3].

\section{Microbubbles (MBs)}

Since the MBs were first proposed as suitable tools for ultrasound contrast agents more than three decades ago, there has been a remarkable progress in the field. In accordance with that, the research has been particularly concerned with the MB preparation techniques and their standardization, in order to ensure a steady composition and size distribution of MBs, both representing crucial features for the stability of MBs in vivo and thus providing a sufficient backscatter for ultrasound imaging. Although the very first MBs were generally considered to contribute to improvement of the imaging techniques, they were soon proved to serve as excellent drug and gene delivery systems both in diagnostics and therapy. Much has been discussed on ultrasound molecular imaging using MBs as contrast agents, including novel preparation techniques and their general design; however, the choice of a convenient system for drug and gene delivery and concept of MB functionalization is still an issue worth consideration. In this chapter, potential ways for application of MBs as contrast agents together with drug and gene delivery are shown, relating to the recently used functionalized MBs in vitro and in vivo.

MBs are small gas-filled microspheres whose size typically ranges between 1 and $10 \mu \mathrm{m}$ in diameter see (Figure 7). They comprise a gas-filled core, usually formed by inert, high molecular gases such as sulphur hexafluoride or perfluorocarbons, which due to their decreased solubility and low diffusion prolong the lifespan of MBs in circulation [19], by means of a stabilizing shell of lipid, polymer and/or protein [20,21]. The composition of the shell determines the stiffness and therefore the stability of MBs in blood flow, where instability is caused by exposure of MBs to the rigors of systemic circulation [19, 22]. Limits on stability can affect the scope of ultrasound imaging utilization in vivo since the half-life of MBs depends on their stability. Generally, lipid shelled MBs are considered thinner and more flexible, whilst polymer and protein-shelled bubbles have a thicker and more rigid shell [23]. A thick permeable shell is beneficial to enhance the MB stability (also reducing the gas diffusion) and thus might be used for ultrasound drug delivery purposes [19, 24, 25]. However, the shell ought to only slightly limit the vibration of MBs in the ultrasound field generated by echo imaging systems (otherwise they do not provide sufficient contrast).

Another important aspect for any $\mathrm{MB}$ formulation is their size distribution that dictates the acoustic properties of MBs [22]. As the MBs smaller than $1 \mu \mathrm{m}$ in diameter produce minimal 
acoustic contrast, the intent is to produce larger MBs [26-29]. On the other hand, the MB size distribution should not exceed $10 \mu \mathrm{m}$ of size in diameter; otherwise they get quickly filtered by the lungs and can pose a risk of embolus $[22,30]$. Furthermore, the size distribution is also believed to affect the drug delivery $[22,31,32]$.

\section{Ultrasound imaging}

In the field of molecular imaging, ultrasound represents a unique imaging technique as it provides real-time images in a non-invasive way using relatively cheap and easily portable equipment; thus, ultrasonography is considered to be currently most widely used diagnostic imaging modality [19]. However, since the liquid phase material with low compressibility, such as blood, scatters the sound waves poorly, contrast agents were proposed to improve the ultrasound images, offering the following general advantages: a very small intravenous injection is needed for imaging; and they are composed of non-toxic, biodegradable materials [30]. There are several types of ultrasound contrast agents, such as liquid emulsions, liposomes, and gas-filled MBs [33]. MBs will be described in detail in this chapter. Since the MB-based contrast agents are similar in size and shape to erythrocytes and also rheologically behave similarly to the blood, they appear to be of utmost convenience for ultrasound imaging systems, providing bright contrast [30]. Moreover, due to their larger size (compared to lipid and polymer based nanocarriers), systematically delivered MBs do not extravasate; instead they remain in circulation until they dissolve or are actively cleared by the mononuclear phagocyte system (MPS) [21, 34, 35].

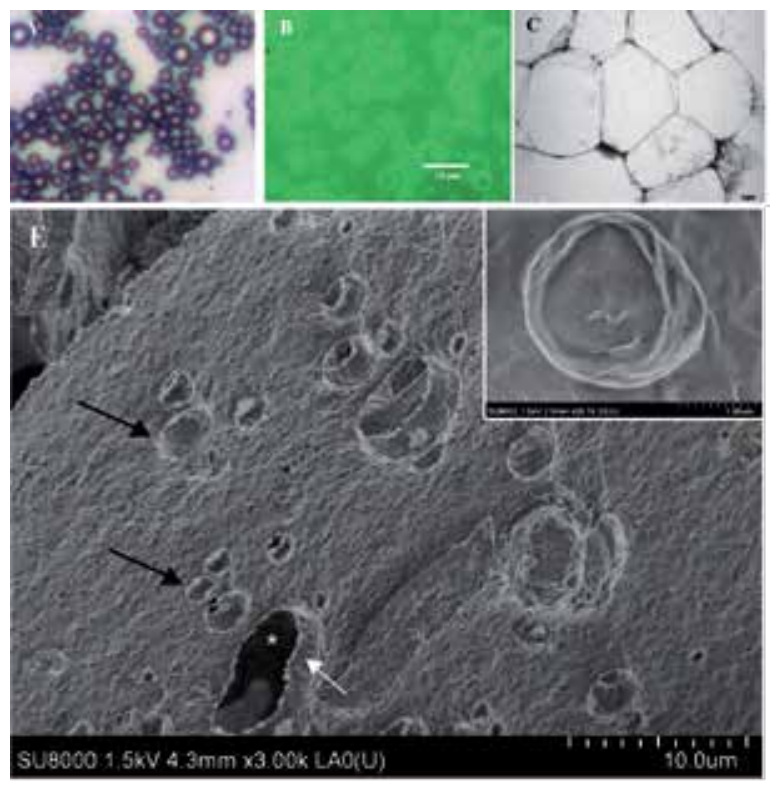

Figure 7. Pictures of MB obtained by various imaging techniques. 
a. Light microscopy of DPPC/ DPPC/1\% DOGS-NTA-Ni MB with carboxyfluorescein-PE $(1 \%)$

b. The same MBs as in (A) observed by epifluorescence microscopy.

c. TEM of MBs. During desiccation of the sample in the vacuum interior of electron microscope, the MB are pressed to each other, which results in the deformed polygonal shape

d. SEM picture of lyophilised MBs. Intact MB (black arrows), crushed MB shell (white arrow), hollow inside of MB (white asterisk). Insert: detail of the surface of lyophilised MB.

(Adapted from [48])

\section{MBs in the ultrasound field - Different aspects}

When exposed to low acoustic pressures, MBs tend to oscillate in a symmetrical, linear way [36], which means that their expansion and compression is inversely proportional to the local ultrasound pressure [37]; the phenomenon is known as a stable cavitation or non-inertial cavitation [38] and comprises stable, low amplitude oscillations of MBs (Figure 8) [23]. Such stable oscillations create a liquid flow around the MBs, the so called microstreams, which can apply a shear stress on cell membranes resulting in a transient opening of the cell membranes, as the shear stress related to micro streaming is relatively high compared to the shear stress associated with blood flow $[23,36,39]$. With the driving pressure turning into violent or inertial cavitation [38, 40], MBs undergo rapid expansion and contraction, which result in a violent bubble destruction [21]. The collapse of MBs is accompanied by shock waves generated in the fluid near the MB; furthermore, jet formation may occur, which can both contribute to a cell membrane perforation and increased permeabilization of blood vessels, thus improving extravasation of circulating drugs into target cells and tissues [20, 23, 41-44]. This phenomenon is called sonoporation [45] and has been proved visualisation of the pores by scanning electron microscopy, showing the pores in the plasma membrane (Figure 9) [46, 47]. Despite initial belief that inertial cavitation of MBs was required to enhance uptake of low molecular weight drugs, there is now mounting evidence that these stable oscillations may also stimulate the intracellular delivery of macromolecular drugs and nanoparticles [36]. Two mechanisms have been postulated to contribute to the uptake of cell impermeable molecules: the formation of small pores and endocytosis [46, 49]. Whilst the involvement of endocytosis was shown to be more extensive for larger molecules, the pore formation is considered the main mechanism for low-weight molecules, such as dextrans [49]. Moreover, when studied in a single-cell model, it was reported that a direct contact is needed to induce pore formation by stable cavitation, as larger distances would hamper direct mechanical cavitating $\mathrm{MB}$ and cell membrane and decrease the influence of microstreaming on the cell membrane (Figure 10) [36, 50-52]. Additionally, MBs facilitate cavitation related phenomena, such as temperature rise and free radical formation $[21,42,53]$; it is assumed that the free radicals increase the cell membrane permeability for $\mathrm{Ca}^{2+}$ in primary endothelial cells and cardiomyocytes [54-56]. 
Last but not least, acoustic radiation forces that may translate MBs in the direction of the propagating ultrasound wave should be taken into account $[21,36,57,58]$. Not only can the acoustic radiation forces be applied to adhere to a specific target, but they can also help to push the MBs towards the cell surface and thus stimulate interaction with the cell membrane and promote drug delivery to specific cells [59-62]. Through acoustic radiation forces, MBs can squeeze through endothelium and tunnel through soft tissue, possibly increasing the deposition of shell-loaded materials beyond the vasculature [21, 63, 64].

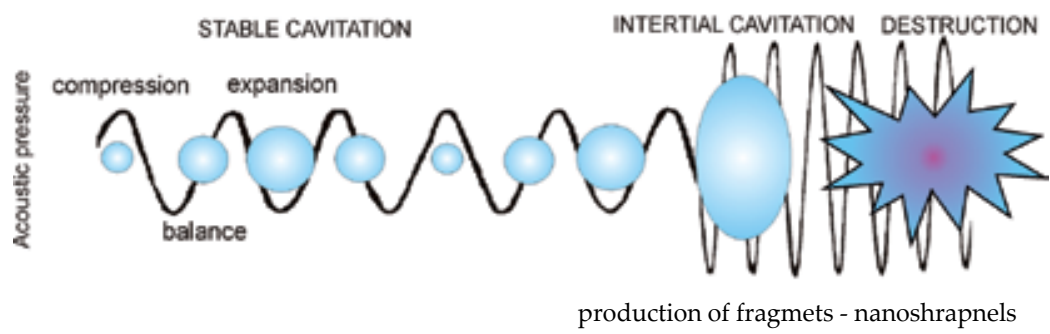

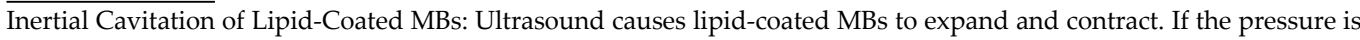
below a critical value, called the inertial cavitation threshold, then the bubble undergoes sustained oscillations - stable cavitation. If the pressure is above the inertial cavitation threshold, then inertial forces govern the collapse; this is typically associated with the bubble imploding and breaking into small fragments - nanoshrapnels. The inertial cavitation threshold pressure depends on the material properties of the lipid coating (e.g., area expansion modulus and surface tension), which are set by the monolayer composition (lipid chain length, PEG coating, nanoparticle bound to the MB surface)

Figure 8. Effect of acoustic pressure on $\mathrm{MB}$ oscillation and explosion.

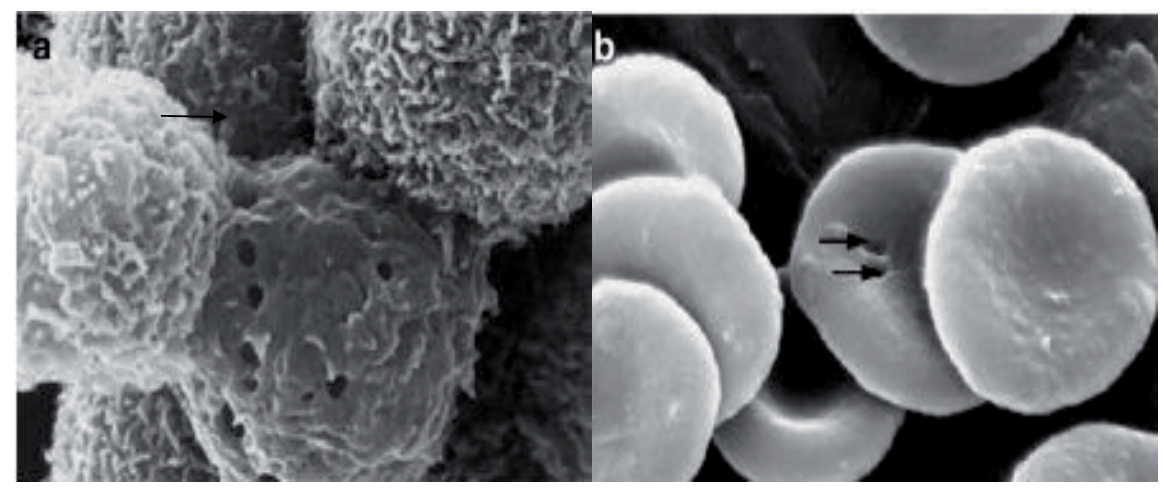

$\overline{\text { GSEM micrograph }}$ s of cells after sonoporation and fixation. The cells were insonated with $2.25 \mathrm{MHz}$ and $570 \mathrm{kPa}$ peak negative pressure pulses in the presence of MBs. (a) MAT B III cells; (b) red blood cells. Adapted from [45]. Arrows indicate pores in the cell membrane. (http://amazingbubbleworld.blogspot.cz/2012/05/classifications-of-cavitation.html)

Figure 9. MBs assisted sonoporation of cells. 


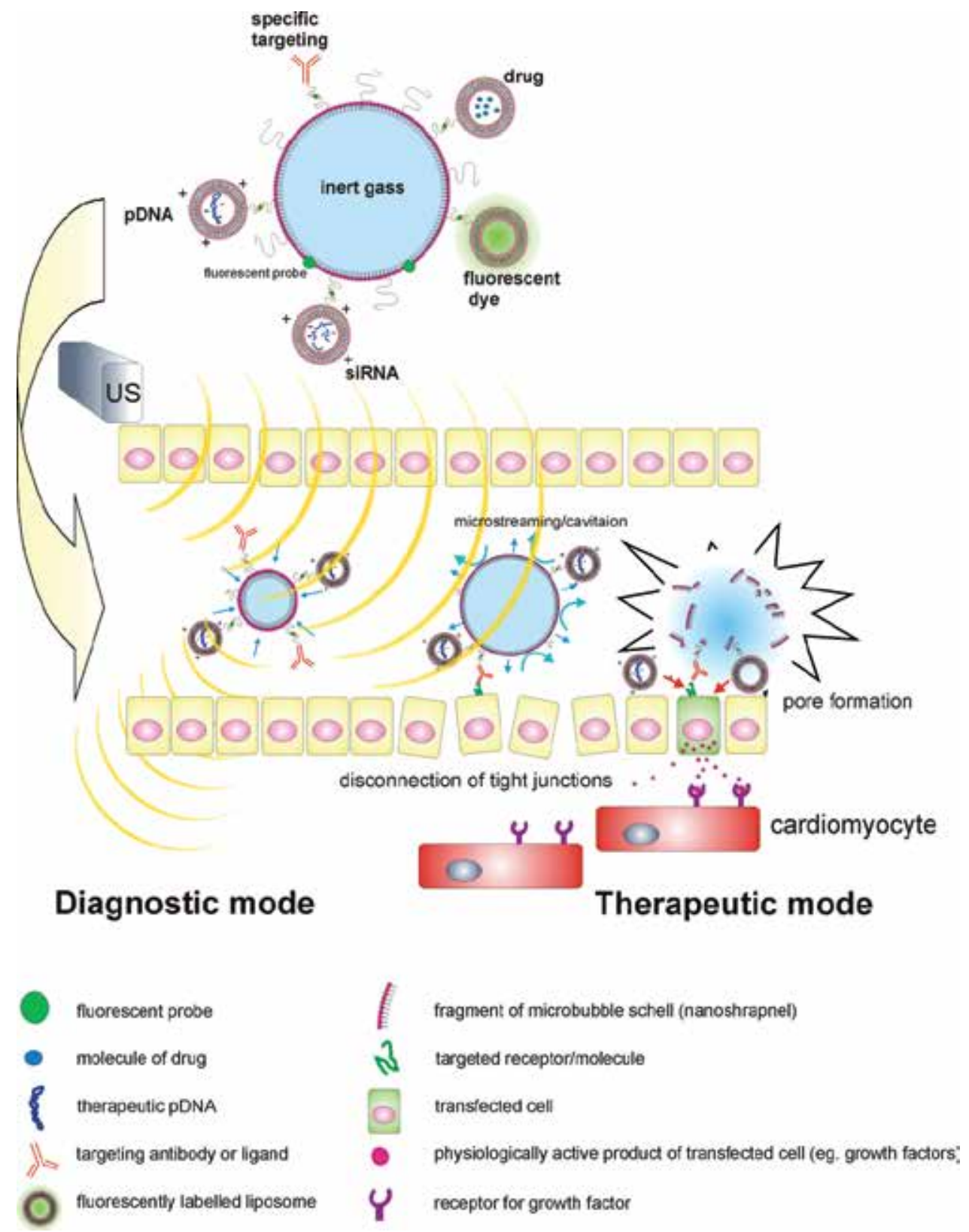

Figure 10. Various aspect of haw MBs can effect endothelial cell in capillary vessels. 


\section{Commercial preparations}

The use of MBs in echocardiography was first reported in 1968 [65]. Since that time great progress has been done in preparation of MBs for human and veterinary use. The following three commercial MB agents Optison TM (GE Healthcare, WI, USA), Definity ${ }^{\circledR}$ (Lantheus Medical Imaging, MA, USA) and SonoVue ${ }^{\circledR}$ (Bracco, Milano, Italy) currently hold the license for clinical diagnostic applications (Table 1). The size of the commercial MBs in these agents is usually larger than $1 \mathrm{um}$ with imaging durations being 5-10 min.

\begin{tabular}{ccccccc}
\hline MB type & Manufacturer & Shell material & Gas & $\begin{array}{c}\text { Mean size } \\
(\mu \mathrm{m})\end{array}$ & $\begin{array}{c}\text { Concentration } \\
(\mathbf{M B} / \mathbf{m L})\end{array}$ & $\begin{array}{c}\text { MB half life } \\
(\mathbf{m i n})\end{array}$ \\
\hline Optison $^{\mathrm{TM}}$ & GE Healthcare & Albumin & $\mathrm{C}_{3} \mathrm{~F}_{8}$ & $2.0-4.5$ & $5-8 \times 10^{8}$ & $2.5-4.5$ \\
Definity & $\begin{array}{c}\text { Lantheus Medical } \\
\text { Imaging }\end{array}$ & Phospholipid & $\mathrm{C}_{3} \mathrm{~F}_{8}$ & $1.1-3.3$ & $1.2 \times 10^{10}$ & $2-10$ \\
& Bracco & Phospholipid & $\mathrm{CF}_{6}$ & $2.0-8.0$ & $0.9-6 \times 10^{10}$ & $3-6$ \\
\hline
\end{tabular}

Table 1. Specifications of commercial and self-made MBs.

\section{Multifunctional MBs for ultrasound imaging and theranostics}

The concept of therapeutic agents being encapsulated in or conjugated with MBs has been developed over the past few years. In addition to the synergistic effects of ultrasound and MBs to enhance the permeability of biological barriers such as cell membranes, small blood vessels, and the BBB, as discussed above, MBs can serve as protective drug carriers. Drugs can be preincorporated into carriers such as liposomes, micelles, or microspheres, and these structures can then be easily attached to lipid MBs, usually via avidin-biotin interactions (Figure 11) [66]. Such advanced MB complexes have extremely high drug loading capacities and the advantage of being able to encapsulate both hydrophilic and hydrophobic drugs. Encapsulating unstable agents protects them from degradation in blood, thus prolonging their half-lives in vivo, improving treatment efficacy, and lowering the required dose [67]. Another advantage is that the encapsulated agents can be released during the ultrasound-triggered MB destruction process. Chemotherapeutic drugs can thus be directly and specifically delivered to target tissues via ultrasound-mediated perforations, whereas the uptake of the drugs by non-target tissues is reduced. The encapsulated agents are conjugated close to the shell of MBs, increasing the opportunity for microstreams, shock waves, and microjets to drive them toward the tissues and enhance their uptake in the ultrasound-treated region [68]. Since MBs act as ultrasound contrast agents, the drug delivery process can also be concurrently monitored by detecting the drug-loaded MBs [69]. 

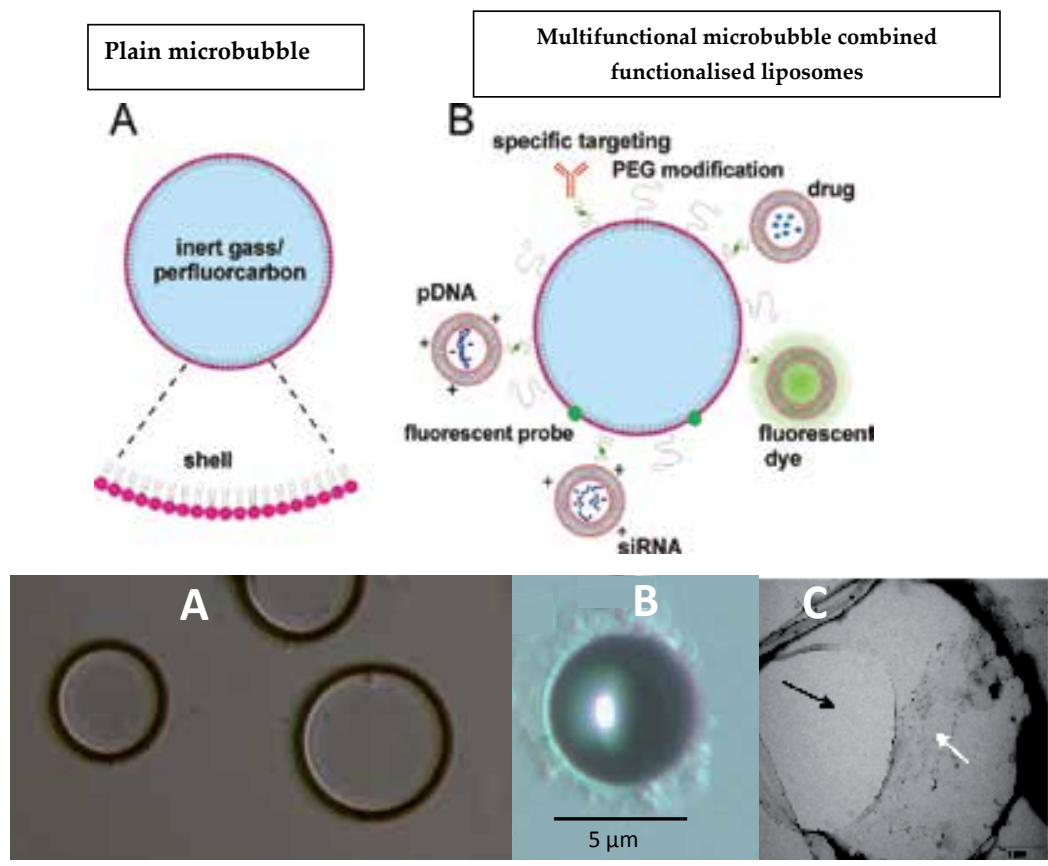

A) Structure of plain MB (upper - schematic picture; bottom - optical microscopy -Nomarski contrast)

B) Functionalised MB with bound liposomes (upper - schematic picture showing various possibility of functionalization and modification; bottom - MB with surface bound liposomes - optical microscopy -Nomarski contrast)

C) Transmission electron microscopy of the functionalised MB with bound liposomes. Black arrow - rupture of MB shell owing to vacuum in electron microscope; white arrows - liposomes bound onto the surface of MBs. (Adapted from [48])

Figure 11. Targeted and multifunctional MBs.

\section{Targeted MBs}

Despite the possibility of employing MBs for targeted ultrasound imaging and drug and gene delivery using the local ultrasound insonation, attaching targeting ligands on the surface of MBs, which leads to their accumulation in the target region (Figure 12), makes the MBs even more specific so that the range of clinical use of MBs is vastly expanded [23]. Owing to their size, MBs are not supposed to leave the vasculature; thus their use is limited to thrombus, endothelial cells, atherosclerotic plaques, blood clots, and organs such as spleen and liver [23, 47]. Recently there have also appeared papers referring to the use of MB-enhanced ultrasound for facilitated brain delivery [70,71]; although the exact mechanism is still unknown, the interaction between capillary walls and acoustically driven MBs was found to be one of the key factors which leads to the disruption of the blood-brain barrier - the main obstacle in the treatment of neurodegenerative diseases [70, 72]. Since the method is non-invasive, according to the authors, it could be the way for treatment of such diseases as Parkinson's disease or Alzheimer's disease. 


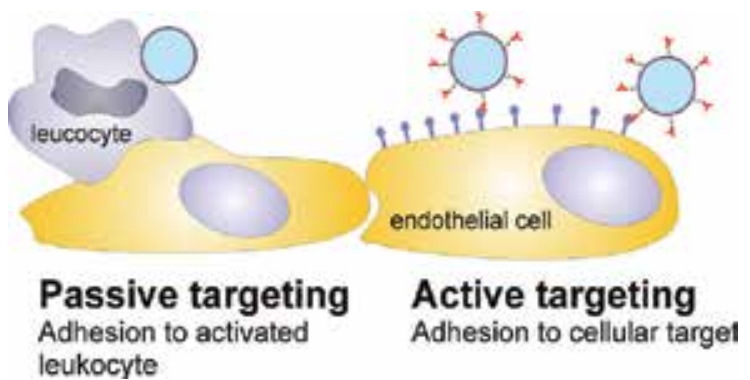

Figure 12. Passive and active targeting of MBs.

There are different ways how to attach the targeting ligands to the shell of MBs that are generally distinguished by the method of preparation. The targeting ligand can be either co-incorporated in the shell during the MB preparation, or attached to the surface of pre-formulated MBs by covalent or non-covalent methods [73, 74]. The simplest targeted MB design is based on the use of a phospholipid, phosphatidylserine, which is incorporated in the MB shell during preparation. Due to the fact that phosphatidylserine is a membrane marker of apoptosis, it promotes the phagocytic uptake of cells and liposomes by Kupffer cells and macrophages [74-76]. While circulating in the bloodstream, MBs containing phosphatidylserine are actively targeting and captured by the Kupffer cells in the liver, macrophages in the spleen and active neutrophils in the areas of inflammation, which is the reason why phosphatidylserine-shell agents are used to detect the foci that lack active phagocytic capacity, e.g. tumour nods in the liver [73, 77-79]. In these agents, the shell is the ligand (phosphatidylserine); since the molecules of phopshatidylserine are kept together by the hydrophobic, van der Waals, and other intermolecular interactions, the ligand is attached in a non-covalent manner [73].

Nevertheless, though prepared by straightforward shear-mixing in one simplestep, this method is convenient only for stable ligands, which are able to survive the extreme conditions during the MB preparations [23]. Particularly some proteins and enzymes might be denatured in the harsh conditions and might lose binding affinity [73]. Therefore a different approach has been applied, involving attachment of ligands after MB preparation: non-covalent (strept)avidinbiotin interaction and covalent binding [23, 73, 80, 81]. Considering the fact that the (strept)avidin-biotin interaction has the strongest effective dissociation constant, it has been the most widely used technique in biotechnology [82]. Biotin residues might be attached in one single step to antibodies, carbohydrates and many other targeting ligands, including the MB shell components $[73,83]$. Biotinylated antibodies are abundant and can be easily linked to premanufactured biotinylated bubbles via a streptavidin linker [84]. Nonetheless, this coupling scheme is useful only in preclinical research: streptavidin as a foreign protein is a possible immunogen which might lead to undesired immune response after multiple injections [85].

Another attractive non-covalent strategy is to use the interaction of a metallochelating lipid complex, such as nickel-nitrilotriacetyl lipid, with a multiplicity of histidine residues (His-tag) of a protein [48]. The method offers an easy way to attach a genetically engineered protein to the MB surface with a His-tag, a commonly applied insert in recombinant protein preparation. 
The advantage of metallochelating complex over protein-ligand complexes, e.g. (streptavidin - biotin, glutathion - glutathion transferase) consists in its specific binding and very low immunogenicity, if any [86]. However, except for studies using metallochelating liposomes $[86,87]$, very little research has been devoted to this issue in terms of MBs; therefore there still appears to be a high potential for the His-tag - MB applications in the foreseeable future.

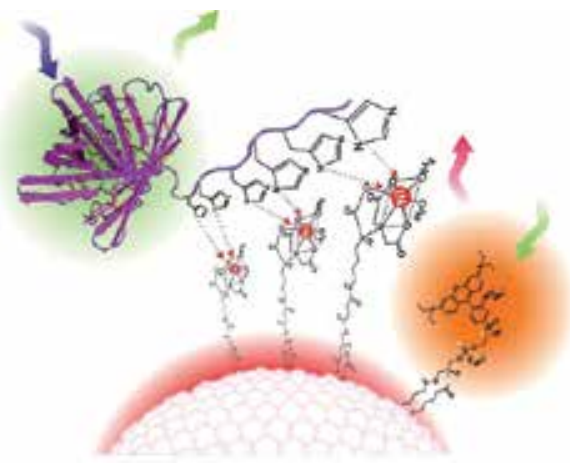

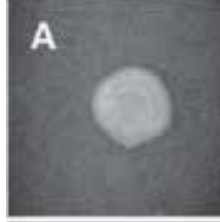
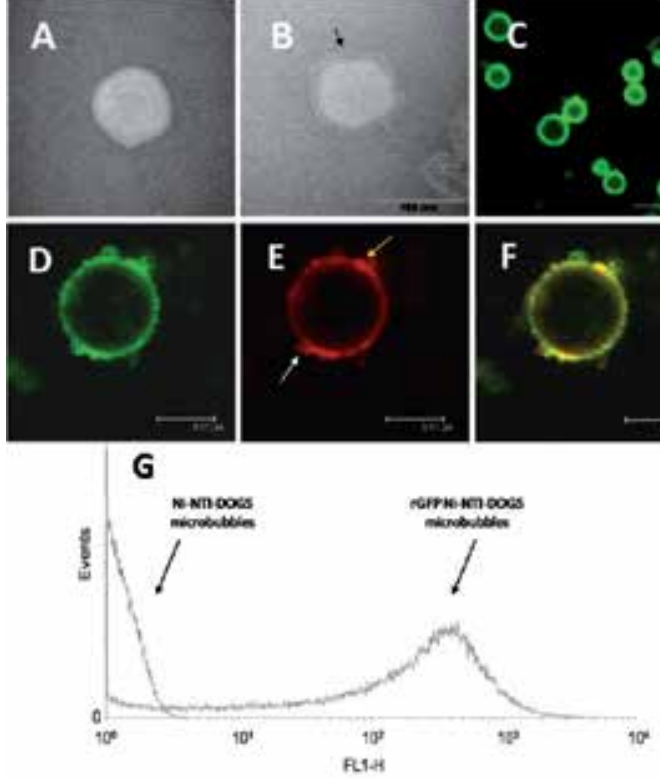

Figure 13. Schematic presentation of metallochelating MBs, confocal and electron microscopy of metallochelating MBs and coupling of proteins with HisTag anchor.

The rGFP-HisTag molecule bound onto the surface of metallochelating liposomes was directly detected by TEM $(\mathbf{A}, \mathbf{B})$. Fluorescence of rGFP was used to prove binding of this protein onto the MB (C). The binding did not affect the native conformation of rGFP as demonstrated by the preserved fluorescence characteristics of the protein. Homogenous population of highly fluorescent rGFP MB was detected by flow cytometry $(G)$. Nonspecific binding of rGFP on both DPPC liposomes as well as MB lacking DOGS-NTA-Ni was not observed by flow cytometry and confocal microscopy. Confocal microscopy confirmed co-localisation of bound rGFP-HisTag (green fluorescence) with the lipids forming MB monolayer. The monolayer was marked with LisR-PE (red fluorescence) (D, E, F). Confocal microscopy revealed that a small fraction of $\mathrm{MB}$ had also attached liposomes or residual lipid structures of collapsed $\mathrm{MB}$ on their surface (as seen in D, E, F). This is in a good agreement with the electron microscopy data (D) (Adapted from [48]).

Concerning the covalent coupling methods, the techniques are basically the same as the ones developed for the attachment of ligands to liposomes [88]. As the covalent coupling does not require foreign proteins or His-tags (Figure 13), the chances of undesired immune response to the ligand are low [84]. The simple covalent coupling with the formation of peptide bond between the carboxyl-carrying MBs and protein aminogroups is achieved by adding carbo- 
diimide chemistry [89]. First, a carboxyl group is activated with carbodiimide in the presence of $\mathrm{N}$-hydroxysulfosuccinimide, forming active ester [84]. The resulting ester then reacts with the protein aminogroup, forming an amide bond. This method is unfortunately unsuitable for clinical settings as well, as to achieve the desired number of targeting molecules per bubble, large quantities of expensive antibodies must be added to the reaction mixture, not to mention that only very few of them are actually coupled by the peptide bonding [73]. An alternative approach of covalent coupling scheme for antibody attachment to MB surface seems to be thiolmaleimide chemistry, developed for targeted liposome applications [90]. In this case, maleimide on the shell is coupled with a thiol group on the ligand, forming thiol-ether [74]. This technique is convenient with respect to a possible oriented coupling; if a ligand has a single thiol, then a single point attachment to the MB shell should retain affinity of the ligand to this target [84]. Overall, selective oriented coupling with of thiol-protein with a maleimide-carrying MB seems more attractive.

Despite the possibility of attaching the ligand directly to the MB surface, it might seem more suitable to connect the ligand to lipid via an extended spacer arm, such as poly(ethylene glycol) known as PEG. Up to $30 \mathrm{~nm}$ long [91], flexible extended PEG spacer arm separates the ligand and the anchor from each other [92], so that the spacer arm acts as a "fishing line" [93], improving the chances of reaching its target and $\mathrm{MB}$ adhesion and retention on the receptor-coated surfaces $[73,92,94]$. On the other hand, similar to the design of long-circulating liposomes, PEG chains, or PEG chain derivatives, can be incorporated into the shell of MBs in order to form a steric barrier against coalescence and adsorption of macromolecules, such as antibodies [47, 95]. In recent papers, the protective role by the PEG chains was shown when the targeting ligand was attached to shorter PEG chains that were shielded by a PEG overbrush layer (buried-ligand architecture), significantly increasing the $\mathrm{MB}$ in vivo stability, prolonging their circulation lifetime and reducing ligand-mediated immunogenicity; it was also reported that the extent of PEG protection to depended on the overbrush length [48, 96-99]. PEG overbrush architecture (PEG - polyethyleneimine with a high degree of substitution) was lately used for complexing and stabilizing the plasmid as well; as a result, the transfection was significantly enhanced [100]. One of the possible reasons for enhanced drug and gene delivery when PEG overbrush strategy is applied is the fact that targeting ligands are generally affixed to the free ends of the PEG chains to direct the MBs to a given target [97], so that the bubbles usually bear thousands of ligands on their surface. Thus cooperative binding of multiple ligands to a target might increase the affinity of particles to the target site, boosting the transfection efficacy. A combination of targeted $\mathrm{MB}$ agent BR55 and peptide ligand attached to the bubble shell via a PEG spacer are has even been applied in a prostate cancer clinical trial [101].

\section{Drug- and gene-loading strategies}

The main advantage of the use of MB-based particles as a delivery tool for drugs and genes lies in the dose reduction while maintaining its therapeutic efficacy; the method thus provides much less toxicity than conventional cytotoxic therapies and at the same time has more specificity, which has a great potential particularly in cancer treatment [84]. Generally, drugs 
and/or genes can be either (a) co-administered with MBs, (b) enclosed within the MB shell, (c) incorporated in the MB shell, (d) attached to the surface of the MBs (e) incorporated in liposomes or nanoparticles that are associated with the MB surface.

\subsection{Co-administration of drugs with MBs}

In this case, the MBs enhance the permeability of the endothelial wall in different manners; (1) by rupture of the endothelial wall, so that larger particles such as erythrocytes and polymer particles are able to extravasate [102]; (2) by MBs causing the temporary membrane opening when the low pressure is applied [103]; (3) by sonoporation, allowing for radionuclides or plasmid DNA to pass through [104, 105]. For more detailed description of the mechanisms concerned, please see the above part "Microbubbles in the Ultrasound Field".

\subsection{Enclosing drugs within the MB shell}

Instead of co-injecting drugs and MBs, the bubbles might be modified to contain drugs or DNA $[97,106-110]$. It is virtually possible to load the drugs within the interior of the MBs, which is very convenient since the drug is completely protected and is not released before applying the ultrasound [23]. However, since the MBs are relatively small, the space for drug loading is quite limited. This approach was applied for the first time in 1998 already by the team of Unger, who used lipid-shelled MB with an addition of paclitaxel containing oil phase. Nonetheless, Unger reported the methodology seems to be unsuitable for clinical setting for three reasons; firstly, as the monolayer of the lipid-shelled MB is very thin, only small amount of drug can be loaded; secondly, this strategy is limited only to hydrophobic drugs; last but not least, due to the very high mechanical indexes of ultrasound waves necessary for drug release, which resulted only in $30 \%$ of cell death, the in vivo use of such ultrasound setting appears unfeasible [110].

Recently, Kooiman et al. used oil-filled polymer microcapsule model for ultrasound-mediated delivery of lipophilic drugs; microcapsules with a shell of fluorinated end-capped poly(L-lactic acid) contained hexadecane oil as a drug-carrier reservoir. At higher diagnostic pressures, the microcapsules cracked and released the drug [111]. Despite offering a great potential in clinical use, there are limitations as this approach can be applied only for lipophilic drugs. In another paper, Tinkov et al. suggested the application of doxorubicin (DOX)-loaded phospholipid MBs for targeted tumour therapy. DOX as a potent chemotherapy was loaded into the DPPC/DPPG/ $\mathrm{PEG}^{2000}$-DPPE MBs and was tested in a pancreas carcinoma model of the rat. Although an agglomeration and capillary adhesion with embolisation/occlusion of the pulmonary arteries/ capillaries after intravenous infusion of MBs with any new formulation might appear, in this case no rat died or showed any signs of pulmonary vascular occlusion [112]. Therefore the strategy seems suitable for more extensive preclinical therapeutic studies.

\subsection{Nucleic acids and MBs}

Concerning gene delivery, [113] incorporated plasmid DNA inside the gas core of polymer MBs, so that the plasmid was even protected from the host nucleases; at the same time high plasmid load per bubble was achieved [113]. In a recent study, Sun et al. compared the 
transfection efficiency of commercial Definity MBs to modified cationic MBs with longer lipid chain; as a result, cationic bubbles were binding significantly more DNA, so that multi-fold enhancement was reached in gene delivery [114]. The results were consistent with the findings of Panje and Nomikou, et al. and the notion that gene delivery might be augmented with a higher local DNA concentration $[115,116]$. In another study, Florinas et al. designed a nonviral siRNA gene carrier using a combination of an arginine-grafted bioreducible polymer $(\mathrm{ABP}), \mathrm{MBs}$ and ultrasound, for targeting vascular endothelial growth factor (VEGF) in human ovarian cell line; as a result, MBs in combination with lipoplexes showed significantly higher loading capacity compared to naked siRNA; furthermore, only siRNA complexes with ABP and MBs showed significant knock down in human ovarian cell line compared to naked siRNA when incubated for a short time after sonication treatment [116]. The potential of MBs as gene carriers is still on its rise, offering a wide range of options by functionalization of the bubbles.

The drugs that have sufficient affinity for the lipid monolayer might be, in principle, incorporated directly in lipid-shelled MBs [106]. However, due to the fact that the lipid monolayer is rather thin, the amount of drug available for incorporation is very low. Furthermore, the thin shell might not be able to prevent the leakage of drugs from the bubble during their circulation. Therefore other strategies were developed, such as preparation of MBs with a thick lipid shell containing triglyceride oil phase and drug dissolved in it [117]. Nevertheless, this approach is restricted only to hydrophobic fat-soluble drugs, e.g. paclitaxel. A thick polymer shell represents another variety for drug incorporation suitable for water-soluble materials such as DNA and proteins.

Electrostatic interactions of the lipids and plasmid DNA (possessing an overall negative charge) are made possible by the positively charged groups of some synthetic lipids within the shell [109]. DNA can be loaded on the charged MB shell by simply mixing a plasmid with the lipid bubbles before use. After insonation, the surface-coupled DNA remains intact and at the same time is protected against enzymatic degradation [118, 119]. Moreover, the transfection efficiency is increased by the adherence of DNA to the MBs in comparison with co-administration of MBs and non-adsorbed DNA. At the same time, unbound plasmids should not be able to penetrate into non-targeted cells and tissues and should be degraded, resulting in high specificity with a low transfection in non-insonated regions [19]. Nevertheless, in comparison with the payload of DNA inside the gas core of the bubbles, the loading capacity is restricted to their surface area, which means that high numbers of MBs must be injected. The development of multi-layered assemblies by sequential adsorption of DNA on the positive MB template offers another alternative; because DNA can be directly incorporated as an anionic layer within these assemblies, layer-by-layer methods allow precise control over the loading (or dose) of DNA by controlling film thickness or the number of layers deposited during the fabrication. Besides that, these methods are entirely aqueous and, unlike conventional methods for the encapsulation of DNA in thin polymer films, do not require the use of organic solvents which residua could be presented in these materials post-assembly. [120].

In conclusion, an important advantage of ultrasound-mediated MB-based transfection is the ability to attain DNA delivery and expression only in the insonated areas and not in nontargeted regions. Even if the transfection efficacy is not very high but the encoded protein is 
produced in the target tissue in an amount sufficient to achieve functional biological response, therapeutic intervention should meet its success [121]. Considering the improvement of transfection efficacy via implementation of viral particles using positively charged assemblies, a significant transfection was observed [122]. However, one should be always careful since undesired immune responses after systemic administration might appear.

\subsection{Incorporation of drug loaded LNPs with MBs}

An intensive research has been devoted to the development of nanocarrier-MB hybrids, wherein drug-containing LNPs are physically attached to the surface of MB [21]. These hybrids therefore possess a high drug loading capacity of the nanocarriers and the systemic targeting capability and vascular permeabilizing effect of bubbles. Lum et al. were the first to attach loaded LNPs to MBs via avidin-biotin interaction; they reported on biotinylated lipid MBs to which avidinylated polystyrene beads were attached [117]. The biotinylated MBs were prepared by inclusion of DSPE-PEG-biotin in the lipid shell. Since up to $10^{5} \mathrm{LNPs}$ were bound to each of the bubbles, the drug loading capacity is reasonably enhanced [106]. LNPs seem highly advantageous as (1) they have been long proved as versatile drug delivery systems; (2) a plethora of knowledge is available on LNPs for drug delivery; (3) some LNPs are perfectly and already used in clinical practice. They might also enclose both amphiphilic and hydrophobic drugs in their lipid bilayer, as well as hydrophilic drugs in an aqueous core. Nevertheless, owing to the fact that biotin-avidin linkage might develop an undesired immune response, this approach is not suitable for clinical use. Therefore Geers et al. prepared doxorubicin (DOX)-LNPs loaded MBs containing pDNA or siRNA that in combination with ultrasound boosted both DOX cytotoxicity and pDNA and siRNA delivery to the cells in vitro $[108,119,123,124]$. In their last work, Geers et al. developed a self-assembly of functionalized phospholipids, DOX-loaded LNPs and perfluorobutan gas [125]. Compared to the other methods discussed above to load MBs with drugs, this strategy seems the most advantageous from the loading capacity point of view; though it was estimated that approximately 600 to 1300 LNPs might be bound per singe MB, the amount of loaded DOX is still rather limited. On the other hand, Klibanov et al. prepared liposome-MB pendants incorporating calcein, a hydrophilic self-quenching fluorescent dye, or thrombin, a crucial enzyme of the thrombosis cascade, and they achieved a significant payload volume - up to several cubic micrometres of internal liposome aqueous core space per bubble-LNP [126].

A different approach was introduced by Fan et al. who developed a novel DOX-loaded MB containing super-paramagnetic iron oxide (SPIO) nanoparticles for dual modality imaging and improved image-guided drug delivery [127]. The authors showed that the delivery of SPIO to brain tumours was improved using magnetic targeting in combination with focused ultrasound. Unfortunately, the improvement of DOX delivery using magnetic targeting was not the object of assessment. Hernot et al. prepared nanobody-targeted MBs using metabolic and site-specific biotinylation of nanobodies [128]. The researchers developed a system of carmelidderived single-domain antibody-fragments, i.e. molecular tracers, coupled to targeted MBs. Although they demonstrated the functionality of these modified MBs both in vitro and vivo, since the biotinylated nanobodies were linked to the surface of biotinylated MBs via streptavidin linker, these are not suitable for clinical application due to the undesired immune response. 


\subsection{Infusion of MBs}

Besides imaging applications, MBs showed to be a suitable tool for therapeutic use, e.g. targeted drug delivery, gene therapy, thrombolysis, and vascular permeability enhancement [129]. Certain US-contrast-imaging or therapeutic strategies, such as destruction-reperfusion or MB-enhanced drug delivery, require a continuous injection of the MBs over several minutes. Results of previous in vitro, animal, and human studies have shown that continuous infusion of a US contrast agent can markedly prolong vascular Doppler US enhancement. [130-132]. Most quantitative imaging strategies rely on the ability to administer a consistent dose of contrast agent. The spatial distribution of MBs inside a syringe varies over time owing to the buoyancy of the MBs. These changes are rapid and result in significant inhomogeneity in the concentration and size distribution of the MBs during continuous infusion. The population of MBs that is pumped from a horizontal syringe outlet differs from the initial population as the MBs float to the top of the syringe [133]. This effect is often overlooked and underestimated by experimenters. We believe that this is the main reason for contradictory results published by several authors that compare bolus versus continuous infusion methods of MB application [131]. A superior signal contrast of liver metastasis has been achieved by a continuous application of MBs to patients. US contrast agents for hepatic imaging are traditionally injected as a bolus.

For i.v. applications, precise concentration and size distribution of MBs is required. Continuous application of MBs might present a problem, owing to their strong tendency to float and accumulate directly below the surface. The concentration and size distribution of specific MBs injected into a patient's blood vessel can be quite different from the ones in the syringe.Recently, we have described the device called Infucon for continuous infusion of MBs which prevents flotation of MBs and their accumulation below the surface. The device has been tested on New Zealand White rabbits using both commercial Sono-Vue diagnostic MBs and PEGylated DPPC MBs prepared in our laboratory $[48,134]$.

The Infucon device consists of several parts: a programmable syringe pump (e.g. LAMBDA VIT-FIT Brno, Czech Republic) with a syringe filled with $\mathrm{SF}_{6}$, an Infucon stirred vessel positioned on magnetic stirrer (e.g. Heidolph MR 3000 D, Germany) and fixed in the position by a holder, and the cannula for application of MBs into the rabbit via ear vein. The schemes of the device together with its photographs are presented in Figure. 14. The role of the syringe pump is to force the gas (e.g. $\mathrm{SF}_{6}$ ) into the Infucon stirred vessel and to inject the MBs into bloodstream via cannula. The same gas which is used for filling MBs is used in the syringe to ensure stability of the MBs during the infusion. The main function of the turbine-like stirrer (which contains two teflon-coated ferrits) is to maintain a uniform dispersion of the MBs in the solution and to prevent their accumulation to the surface owing to their flotation. The stirrer is hung up on the collar of the carrying-off capillary outlet. The direction of rotation is selected to maintain down-stream movement of the fluid and to strip MBs from the surface. The device itself is designed to be hermetically insulated using an O-ring seal. The cell is made of inert materials (Teflon ${ }^{\circledR}$, polypropylene, glass, and Viton ${ }^{\circledR}$ ). All parts of the Infucon are compatible with chemical or heat sterilization. The filter installed in the Infucon head prevents possible air-born particles from entering the cell. The vial (conical bottom) is filled up with a solution 
of MBs (up to $1.5 \mathrm{~mL}$ ) via an inlet capillary. The flow rate (usually in the range of $100-200 \mu \mathrm{L} /$ $\min$ ) and the time of pump action are programmable parameters. The silicon piston of the syringe is properly lubricated to ensure very low friction and smooth movement preventing sudden jumps of the piston resulting in pulsation. A turbine-like stirrer fits the conical bottom of the vial, therefore very low dead volume of residual MB solution is obtained. The turbinelike stirrer freely hangs on the fringe of the Teflon outlet capillary. The capillary is tightly embedded into the Teflon body in order to be prevented from bending. There is also an inlet aperture for incoming gas in this Teflon body. The gas is delivered from the syringe through the inlet capillary linked tightly to the head part of the Infucon (Figure 15, 16). The stirred Infucon cell represents solution of the problem of the continuous infusion of MBs. This device is independent on mechanical stirring of the infusion syringe. Unlike the system based on the rotation of the entire syringe, Infucon cell provides a genuinely homogeneous MB dispersion. The MBs are prevented from flotation by stirring the suspension in a separate cell. The infusion is driven by inert gas filled in a syringe placed in the infusion pump. The inert gas used both for filling the MBs and driving the MB infusion is supposed to have stabilizing effect on the MBs during the process of infusion.
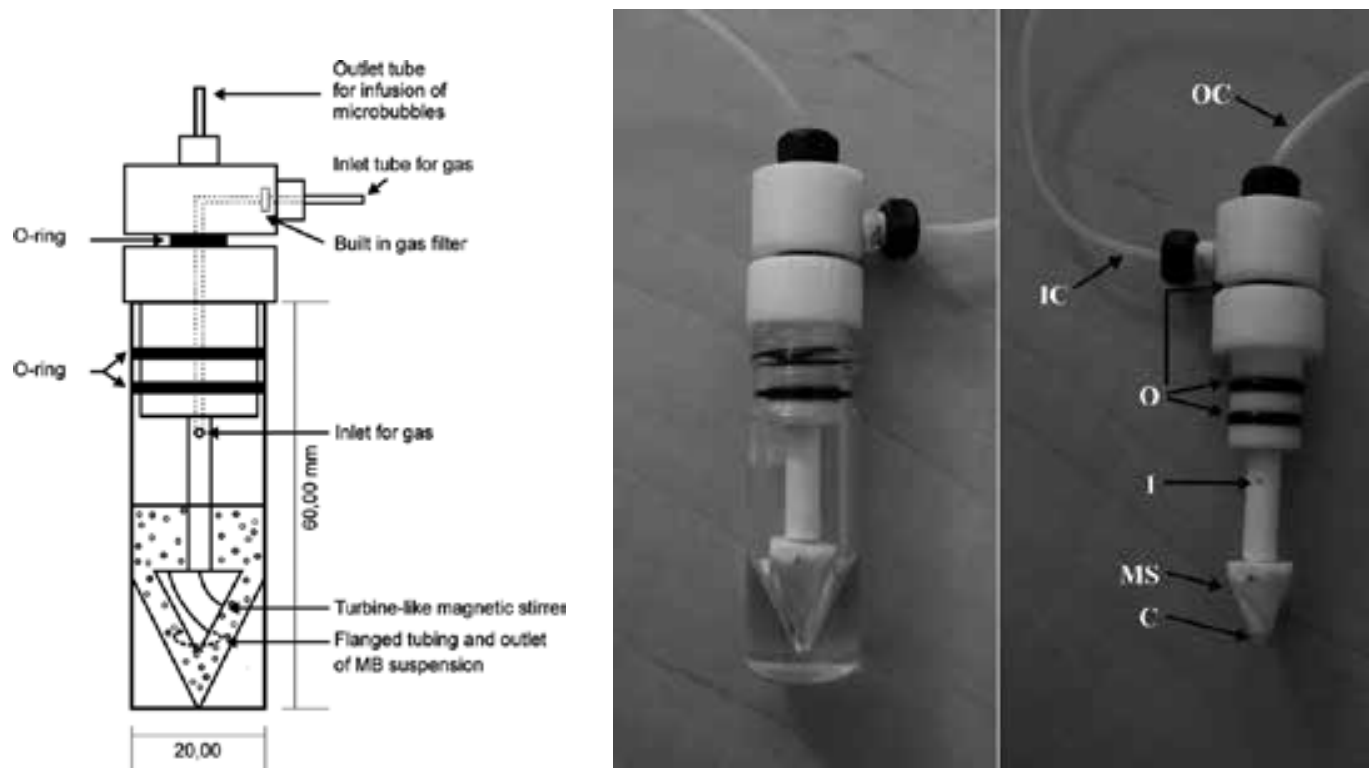

(A) Assembled device, (B) Head of the Infucon. (Adapted from [144])

OC - outlet capillary for the infusion of microbubles (connection to the cannula)

IC - inlet capillary for inert gas from a syringe inserted into the syringe pump

$\mathrm{O}-\mathrm{O}$-ring

I - inlet for gas

MS - turbine-like magnetic stirrer with blades

$\mathrm{C}$ - fringe of the outlet capillary (OC)

Figure 14. Schematic drawing of the stirred continuous injector Infucon and the photograph of the Infucon device. 

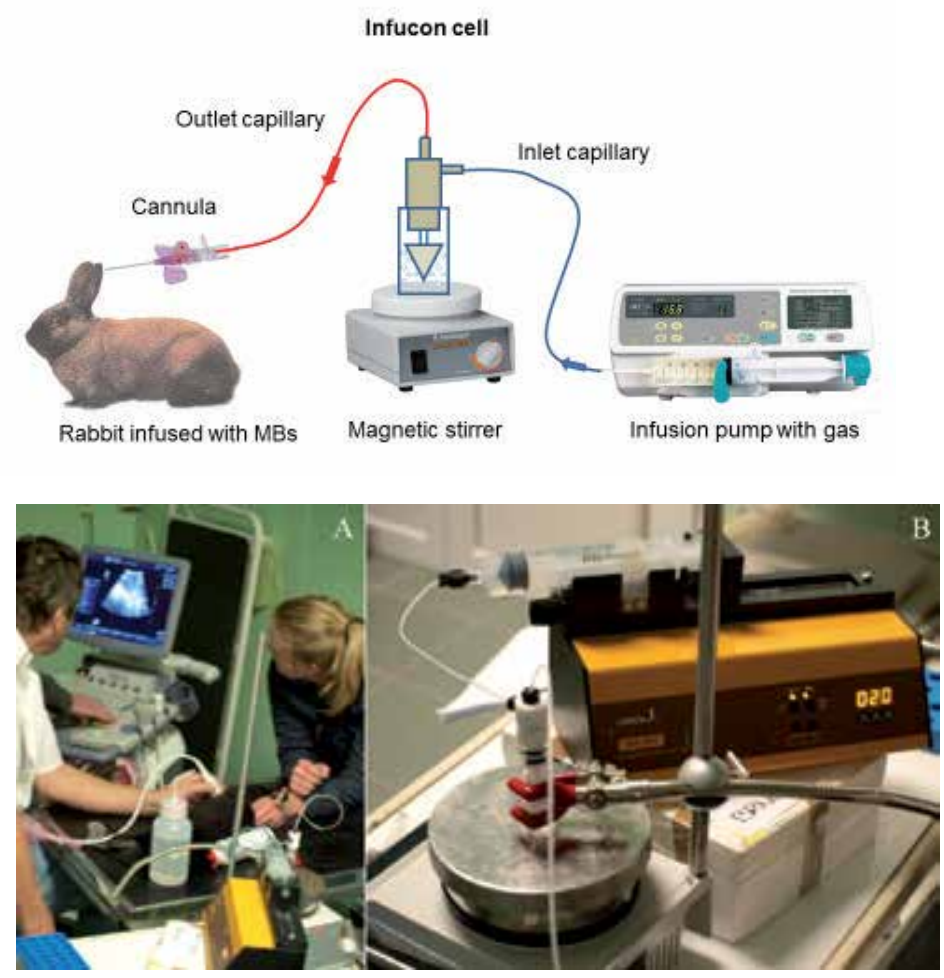

Blue line: gass pushing homogenaous dispersion of MBs

Red line: homogenaous dispesion of MBs driving from Infucon cell into rabbit ear vein via canula

Figure 15. Schematic and real view of the device used for i.v. infusion of MBs into the rabbit.

a. The use of Infucon device during the ultrasound scanning of the rabbit heart.

b. Integration of the Infucon device which is injecting the MB suspension via vena aricularis into the bloodstream of the rabbit. (Adapted from [144])

\section{Medicinal application}

Imaging the liver - This clinical application of MBs in radiology is most promising. Some portion of MBs is taken up by the liver and spleen. The precise mechanism is hitherto uncertain, but the involvement of the reticulo-endothelial system is probable. In clinical trials, this liver phase generally lasts about 30 minutes when the licensed agent Levovist is used and a few hours with some agents. The liver is very well seen with MB-specific imaging models, such as harmonic imaging, during this phase. The primary practical importance is that numerous focal liver lesions, especially metastases and hepatocellular carcinoma, appear as defects and the use of MBs considerably increases their visibility [135-137]. 
Imaging the heart - MBs can enhance Doppler flow signals in cardiac ultrasonography, and this can be useful in several situations, such as detecting valvular stenoses. The left ventricular cavity is highlighted by MB contrast agents and thus the blood-tissue boundary becomes much clearer. This is useful in detecting abnormalities in wall motion in a given region, assessment of ejection fraction, and finding left ventricular thrombus. The evaluation of left ventricular function is crucial for managing of a number of heart diseases. Both resting and inducible ischaemia can be accurately diagnosed by real time perfusion imaging in stress echocardiography which can be further enhanced by applying intermittent high power pulses destroying the majority of the MBs in a scan plane and subsequently watch refilling: the rate at which it occurs is a measure of microcirculatory flow speed [138].
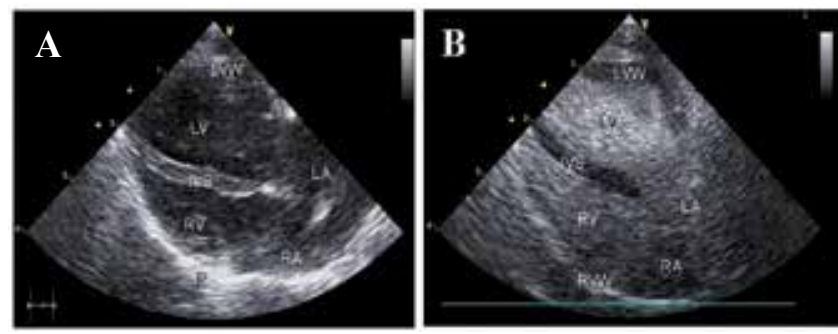

(A) control - an image of the heart without MBs.

(B) Heard filled by MBs during 10 minutes of continual infusion

Figure 16. The sonographs of the rabbit heart before and after the continual infusion of the pegylated micribubbles.Right parasternal long-axis window, 4 - chamber view:LA = left atrium, LV = left ventricle, RA = right atrium, RV $=$ right ventricle, IVS = intervetricular septum, $\mathrm{LVW}=$ left ventricular wall, $\mathrm{RVW}=$ right ventricular wall, $\mathrm{P}=$ pericardium - lung interface. (Adapted from [144])

\section{Therapeutic applications recent advances in molecular, multimodal and theranostic ultrasound imaging}

The importance of MBs usage in treatment is higher than their use as a diagnostic tool. At present, the different characteristic features of MBs (various sizes and the types of gases and shell materials) precondition them for use as targeting drug carriers. MBs can help in drug delivery in themselves (by acting as "cavitation nuclei") and as drug carriers for site-specific treatment. Due to these characteristics they can be employed as drug carriers for treatment of various diseases, such as inflammation, thrombus, lymph nodes, cancer, angiogenesis and atherosclerosis [136].

\subsection{Gene therapy}

The use of MBs in gene therapy is most challenging. The delivery of genetic material to a selected site represents the main problem in this emerging area. Drug delivery can be potentiated by ultrasound which creates transient non-lethal perforations in cell membranes facilitating penetration of large molecules and particles into the cells ("sonoporating"). During this process, high acoustic power is necessary, which is beyond that permitting for imaging. 
However, in the presence of MBs the amount of required power is substantially reduced because the MBs decrease the amount of energy needed for the process of cavitation, leading to MB collapse due to excessive oscillations induced by ultrasound pulses. Furthermore, cavitation of MBs in capillary beds leads to a capillary permeability increase, thus improving local access of the released therapeutic drug [139]. The clinical use of virus-based vectors for gene therapy is limited. This is due to the fact that immune response can be induced by viral proteins in the target tissue and elicit a strong inflammatory activation of endothelial cells. However, it has been shown that when e.g. plasmids and antisense oligonucleotides are used in non-viral delivery vehicles, transfection efficiency is decreased and the expression of the gene product is transient. Commonly used MB preparation Levovist enhanced the beta-Gal gene transfection efficiency more than 6-fold and Optison were used for gene transfer to cardiomyocytes. The transfection efficiencies are significantly higher than that of no $\mathrm{MB}$ and it is similar to Levovist $[97,100,114]$.

\subsection{Blood-brain barrier}

The blood-brain barrier (BBB) is a specialized system of capillary endothelial cells preventing harmful substances in the blood stream from entering the brain. They also supply the brain with nutrients necessary for proper brain functioning. The BBB regulates transport through both tight junctions and metabolic barriers and is often a rate-limiting factor in determining permeation of therapeutic drugs into the brain. It is a significant obstacle affecting delivery of both small molecules and macromolecular agents. Despite the fact that a variety of drugs could be potentially used to treat brain diseases, no method exists that would allow non-invasivetargeted delivery through the BBB.

Ultrasound can be used to locally deliver a drug or gene to a specific region of interest in the brain. The benefit of the MB technique is that it can be used on a very small area of the BBB. MBs are used in combination with ultrasound exposure. The effects of ultrasound can be focused upon the vasculature to reduce the acoustic intensity required for BBB opening production. Several pathways of transcapillary passage after ultrasound sonication have been recognized, including transcytosis, passage through endothelial cell cytoplasmic openings holeyed by "nanoschrapnels" produced by explosion of $\mathrm{MB}$, opening of tight junctions by oscillating MBs and free passage through injured endothelium [136, 140, 141].

\subsection{Thrombolysis and thrombus destruction}

The major cause of death in Western countries is thrombo-occlusive cardiovascular disease, with acute ST-elevation myocardial infarction (STEMI) being a serious medical condition leading to high morbidity and mortality. Two main treatment strategies have been developed during the past years. Their aim is to immediately restore coronary blood flow. In the first studies it was shown that fibrinolysis may be accelerated by ultrasound alone. Besides that, fibrinolysis can be even more increased by the addition of MBs which are destroyed by ultrasound induced high pressure. Three mechanisms leading to this increase have been proposed. The first is the occurrence of complete stress based on micro-jets formed after the destruction of the MBs by ultrasound. The second mechanism is the generation of reactive oxygen species in endothelial cells. The third proposed mechanism is a temperature increase, 
also occurring after the MBs collapse induced by ultrasound exposure. Whereas the full mechanism still remains to be elucidated, evidence indicates that the use of ultrasound alone and ultrasound combined with MBs can enhance clot dissolution by creating microholes in freshly formed thrombi $[142,143]$.

\section{Conclusions}

LNPs and MBs are examples of the successful transfer of basic and applied research into commercial medicinal products. Biocompatibility and biodegradability of LNPs together with versatility in modification and functionalization are features which put together favour their application in medicine. Combinations of LNPs and MBs make best use of both technologies to form new theranostic systems that could form the basis of future advanced therapeutics.

\section{Acknowledgements}

The chapter was prepared within collaborative projects: Centre of Excellence for International Research CZ.1.07/2.3.00/20.0164 to JT, MR and ADM. We gratefully acknowledge the support by Project Centre of Excellence for Nanotoxicology GACR GAP503/12/G147. Štěpán Koudelka was supported by European Regional Development Fund - Project FNUSA-ICRC (No. CZ. 1.05/1.1.00/02.0123), European Social Fund and the State Budget of the Czech Republic - Project FNUSA - ICRC - Support for neurological R\&D teams through new postdoc positions formation (No. CZ.1.07/2.3.00/30.0043). The author's team was supported by the project FIT.

The support by Global Acorn is kindly acknowledged and appreciated.

\section{Author details}

Jaroslav Turánek ${ }^{1 *}$, Andrew D. Miller ${ }^{2,3}$, Zuzana Kauerová ${ }^{1}$, Róbert Lukáčc ${ }^{1}$, Josef Mašek ${ }^{1}$, Štěpán Koudelka ${ }^{1,4}$ and Milan Raška ${ }^{5}$

*Address all correspondence to: turanek@seznam.cz

1 Veterinary Research Institute, Brno, Czech Republic

2 Institute of Pharmaceutical Science, King's College London, London, UK

3 GlobalAcorn Ltd, London, UK

4 International Clinical Research Center, St. Anne's University Hospital, Brno, Czech Republic

5 Department of Immunology, Faculty of Medicine and Dentistry, Palacky University, Olomouc, Czech Republic 


\section{References}

[1] Miller A, Tanner J. Essentials of chemical biology : structure and dynamics of biological macromolecules. Chichester: John Wiley \& Sons; 2008.

[2] Miller AD. Delivery of RNAi therapeutics: work in progress. Expert review of medical devices. 2013;10(6) 781-811.

[3] Miller AD. Delivering the promise of small ncRNA therapeutics. Therapeutic delivery. 2014;5(5) 569-89.

[4] Escriou V, Mignet N, Miller AD. Auto-associative lipid-based systems for nonviral nucleic acid delivery. In: Scherman D, editor. Advanced Textbook on Gene Transfer, Gene Therapy and Genetic Pharmacology. London: Imperial College Press; 2014. p. 221-54.

[5] Miller AD. Lipid-based nanoparticles in cancer diagnosis and therapy. Journal of drug delivery. 2013; 165981.

[6] Davis SS. Coming of age of lipid-based drug delivery systems - Commentary. Advanced drug delivery reviews. 2004;56(9) 1241-2.

[7] Lian T, Ho RJY. Trends and developments in liposome drug delivery systems. J Pharm Sci. 2001;90(6) 667-80.

[8] de Menezes DEL, Pilarski LM, Belch AR, Allen TM. Selective targeting of immunoliposomal doxorubicin against human multiple myeloma in vitro and ex vivo. Bba-Biomembranes. 2000;1466(1-2) 205-20.

[9] Modo MMJJ, Bulte JWM. Molecular and cellular MR imaging. Boca Raton: CRC Press; 2007. 421 p. p.

[10] Modo M, Hoehn M, Bulte JW. Cellular MR imaging. Molecular imaging. 2005;4(3) 143-64.

[11] Caravan P, Ellison JJ, McMurry TJ, Lauffer RB. Gadolinium(III) chelates as MRI contrast agents: Structure, dynamics, and applications. Chem Rev. 1999;99(9) 2293-352.

[12] Maeda H, Greish K, Fang J. The EPR effect and polymeric drugs: A paradigm shift for cancer chemotherapy in the 21st century. Adv Polym Sci. 2006;193 103-21.

[13] Iyer AK, Khaled G, Fang J, Maeda H. Exploiting the enhanced permeability and retention effect for tumor targeting. Drug discovery today. 2006;11(17-18) 812-8.

[14] Kamaly N, Miller AD. Paramagnetic liposome nanoparticles for cellular and tumour imaging. Int J Mol Sci, 2010; 11(4), 1759-1776.

[15] Frangioni JV, Hajjar RJ. In vivo tracking of stem cells for clinical trials in cardiovascular disease. Circulation. 2004;110(21) 3378-83. 
[16] Rogers WJ, Meyer CH, Kramer CM. Technology insight: in vivo cell tracking by use of MRI. Nat Clin Pract Card. 2006;3(10) 554-62.

[17] Mukherjee S, Ghosh RN, Maxfield FR. Endocytosis. Physiological reviews. 1997;77(3) 759-803.

[18] Kamaly N, Kalber T, Thanou M, Bell JD, Miller AD. Folate receptor targeted bimodal liposomes for tumor magnetic resonance imaging. Bioconjugate chemistry. 2009;20(4) 648-55.

[19] Hernot S, Klibanov AL. Microbubbles in ultrasound-triggered drug and gene delivery. Advanced drug delivery reviews. 2008;60(10) 1153-66.

[20] Sirsi S, Borden M. Microbubble Compositions, Properties and Biomedical Applications. Bubble science engineering and technology. 2009;1(1-2) 3-17.

[21] Sirsi SR, Borden MA. State-of-the-art materials for ultrasound-triggered drug delivery. Advanced drug delivery reviews. 2014;72 3-14.

[22] Chen JL, Dhanaliwala AH, Dixon AJ, Klibanov AL, Hossack JA. Synthesis and characterization of transiently stable albumin-coated microbubbles via a flow-focusing microfluidic device. Ultrasound in medicine \& biology. 2014;40(2) 400-9.

[23] Lentacker I, De Smedt SC, Sanders NN. Drug loaded microbubble design for ultrasound triggered delivery. Soft Matter. 2009;5(11) 2161-70.

[24] Perkins AC, Frier M, Hindle AJ, Blackshaw PE, Bailey SE, Hebden JM, et al. Human biodistribution of an ultrasound contrast agent (Quantison(TM)) by radiolabelling and gamma scintigraphy. Brit J Radiol. 1997;70(834) 603-11.

[25] Kauerova Z. Targeting nanoparticle drug carriers: Functionalized microbubbles and their application in ultrasonography: Thesis, Masaryk University, Brno; 2013.

[26] Kaya M, Feingold S, Hettiarachchi K, Lee AP, Dayton PA. Acoustic responses of monodisperse lipid-encapsulated microbubble contrast agents produced by flow focusing. Bubble science engineering and technology. 2010;2(2) 33-40.

[27] Streeter JE, Gessner R, Miles I, Dayton PA. Improving sensitivity in ultrasound molecular imaging by tailoring contrast agent size distribution: in vivo studies. Molecular imaging. 2010;9(2) 87-95.

[28] Gorce JM, Arditi M, Schneider M. Influence of bubble size distribution on the echogenicity of ultrasound contrast agents: a study of SonoVue. Investigative radiology. 2000;35(11) 661-71.

[29] Soetanto K, Chan M. Fundamental studies on contrast images from different-sized microbubbles: analytical and experimental studies. Ultrasound in medicine \& biology. 2000;26(1) 81-91.

[30] Klibanov AL. Ultrasound contrast agents: Development of the field and current status. Top Curr Chem. 2002;222 73-106. 
[31] Konofagou EE, Tung YS, Choi J, Deffieux T, Baseri B, Vlachos F. Ultrasound-induced blood-brain barrier opening. Current pharmaceutical biotechnology. 2012;13(7) 1332-45.

[32] Burke CW, Suk JS, Kim AJ, Hsiang YH, Klibanov AL, Hanes J, et al. Markedly enhanced skeletal muscle transfection achieved by the ultrasound-targeted delivery of non-viral gene nanocarriers with microbubbles. J Control Release. 2012;162(2) 414-21.

[33] Andre MP, Steinbach G, Mattrey RF. Enhancement of the echogenicity of flowing blood by the contrast agent perflubron. Investigative radiology. 1993;28(6) 502-6.

[34] Tartis MS, Kruse DE, Zheng H, Zhang H, Kheirolomoom A, Marik J, et al. Dynamic microPET imaging of ultrasound contrast agents and lipid delivery. J Control Release. 2008;131(3) 160-6.

[35] Kabalnov A, Klein D, Pelura T, Schutt E, Weers J. Dissolution of multicomponent microbubbles in the bloodstream: 1. Theory. Ultrasound in medicine \& biology. 1998;24(5) 739-49.

[36] Lentacker I, De Cock I, Deckers R, De Smedt SC, Moonen CT. Understanding ultrasound induced sonoporation: definitions and underlying mechanisms. Advanced drug delivery reviews. 2014;72 49-64.

[37] Lee JL, Lo CW, Ka SM, Chen A, Chen WS. Prolonging the expression duration of ultrasound-mediated gene transfection using PEI nanoparticles. J Control Release. 2012;160(1) 64-71.

[38] Miller DL, Nyborg WL. Theoretical investigation of the response of gas-filled micropores and cavitation nuclei to ultrasound J Acoust Soc Am 1983;73(5) 1537-44.

[39] Davies PF. Flow-mediated endothelial mechanotransduction. Physiological reviews. 1995;75(3) 519-60.

[40] Vokurka K. Comparison of Rayleigh's, Herring's, and Gilmore's models of gas bubbles. Acta Acustica united with Acustica. 1986;59(3) 214-9.

[41] Miller DL, Averkiou MA, Brayman AA, Everbach EC, Holland CK, Wible JH, Jr., et al. Bioeffects considerations for diagnostic ultrasound contrast agents. Journal of ultrasound in medicine : official journal of the American Institute of Ultrasound in Medicine. 2008;27(4) 611-32; quiz 33-6.

[42] Pitt WG, Husseini GA, Staples BJ. Ultrasonic drug delivery--a general review. Expert opinion on drug delivery. 2004;1(1) 37-56.

[43] Sirsi SR, Borden MA. Advances in Ultrasound Mediated Gene Therapy Using Microbubble Contrast Agents. Theranostics. 2012;2(12) 1208-22.

[44] Wu J, Nyborg WL. Ultrasound, cavitation bubbles and their interaction with cells. Advanced drug delivery reviews. 2008;60(10) 1103-16. 
[45] Mehier-Humbert S, Bettinger T, Yan F, Guy RH. Plasma membrane poration induced by ultrasound exposure: implication for drug delivery. J Control Release. 2005;104(1) 213-22.

[46] van Wamel A, Bouakaz A, Versluis M, de Jong N. Micromanipulation of endothelial cells: ultrasound-microbubble-cell interaction. Ultrasound in medicine \& biology. 2004;30(9) 1255-8.

[47] Klibanov AL. Targeted delivery of gas-filled microspheres, contrast agents for ultrasound imaging. Advanced drug delivery reviews. 1999;37(1-3) 139-57.

[48] Lukac R, Kauerova Z, Masek J, Bartheldyova E, Kulich P, Koudelka S, et al. Preparation of Metallochelating Microbubbles and Study on Their Site-Specific Interaction with rGFP-HisTag as a Model Protein. Langmuir : the ACS journal of surfaces and colloids. 2011;27(8) 4829-37.

[49] Meijering BDM, Juffermans LJM, van Wamel A, Henning RH, Zuhorn IS, Emmer M, et al. Ultrasound and Microbubble-Targeted Delivery of Macromolecules Is Regulated by Induction of Endocytosis and Pore Formation. Circ Res. 2009;104(5) 679-U226.

[50] van Wamel A, Kooiman K, Harteveld M, Emmer M, ten Cate FJ, Versluis M, et al. Vibrating microbubbles poking individual cells: Drug transfer into cells via sonoporation. J Control Release. 2006;112(2) 149-55.

[51] Zhou Y, Yang K, Cui J, Ye JY, Deng CX. Controlled permeation of cell membrane by single bubble acoustic cavitation. J Control Release. 2012;157(1) 103-11.

[52] Ward M, Wu J, Chiu JF. Experimental study of the effects of Optison concentration on sonoporation in vitro. Ultrasound in medicine \& biology. 2000;26(7) 1169-75.

[53] Miller DL. Overview of experimental studies of biological effects of medical ultrasound caused by gas body activation and inertial cavitation. Progress in biophysics and molecular biology. 2007;93(1-3) 314-30.

[54] Juffermans LJ, van Dijk A, Jongenelen CA, Drukarch B, Reijerkerk A, de Vries HE, et al. Ultrasound and microbubble-induced intra- and intercellular bioeffects in primary endothelial cells. Ultrasound in medicine \& biology. 2009;35(11) 1917-27.

[55] Juffermans LJ, Meijering DB, van Wamel A, Henning RH, Kooiman K, Emmer M, et al. Ultrasound and microbubble-targeted delivery of therapeutic compounds: ICIN Report Project 49: Drug and gene delivery through ultrasound and microbubbles. Netherlands heart journal : monthly journal of the Netherlands Society of Cardiology and the Netherlands Heart Foundation. 2009;17(2) 82-6.

[56] Juffermans LJM, Dijkmans PA, Musters RJP, Visser CA, Kamp O. Transient permeabilization of cell membranes by ultrasound-exposed microbubbles is related to formation of hydrogen peroxide. Am J Physiol-Heart C. 2006;291(4) H1595-H601.

[57] Postema M, Schmitz G. Bubble dynamics involved in ultrasonic imaging. Expert review of molecular diagnostics. 2006;6(3) 493-502. 
[58] Dayton PA, Allen JS, Ferrara KW. The magnitude of radiation force on ultrasound contrast agents. The Journal of the Acoustical Society of America. 2002;112(5 Pt 1) 2183-92.

[59] Rychak JJ, Klibanov AL, Hossack JA. Acoustic radiation force enhances targeted delivery of ultrasound contrast microbubbles: in vitro verification. IEEE transactions on ultrasonics, ferroelectrics, and frequency control. 2005;52(3) 421-33.

[60] Fan Z, Sun Y, Di C, Tay D, Chen W, Deng CX, et al. Acoustic tweezing cytometry for live-cell subcellular modulation of intracellular cytoskeleton contractility. Scientific reports. 2013;3 2176.

[61] Ferrara KW. Driving delivery vehicles with ultrasound. Advanced drug delivery reviews. 2008;60(10) 1097-102.

[62] Delalande A, Kotopoulis S, Rovers T, Pichon C, Postema M. Sonoporation at a low mechanical index. Bubble Sci Eng Technol. 2011;3 3-12.

[63] Caskey CF, Qin S, Dayton PA, Ferrara KW. Microbubble tunneling in gel phantoms. The Journal of the Acoustical Society of America. 2009;125(5) EL183-9.

[64] Arvanitis CD, Bazan-Peregrino M, Rifai B, Seymour LW, Coussios CC. Cavitation-enhanced extravasation for drug delivery. Ultrasound in medicine \& biology. 2011;37(11) 1838-52.

[65] Gramiak R, Shah PM. Echocardiography of the aortic root. Investigative radiology. 1968;3(5) 356-66.

[66] Panje CM, Wang DS, Pysz MA, Paulmurugan R, Ren Y, Tranquart F, et al. Ultrasound-mediated gene delivery with cationic versus neutral microbubbles: effect of DNA and microbubble dose on in vivo transfection efficiency. Theranostics. 2012;2(11) 1078-91.

[67] Tachibana K, Tachibana S. The use of ultrasound for drug delivery. Echocardiogr-J Card. 2001;18(4) 323-8.

[68] Frinking PJA, Bouakaz A, Kirkhorn J, Ten Cate FJ, de Jong N. Ultrasound contrast imaging: Current and new potential methods. Ultrasound in Medicine and Biology. 2000;26(6) 965-75.

[69] Ferrara K, Pollard R, Borden M. Ultrasound microbubble contrast agents: fundamentals and application to gene and drug delivery. Annual review of biomedical engineering. 2007;9 415-47.

[70] Wang S, Samiotaki G, Olumolade O, Feshitan JA, Konofagou EE. Microbubble type and distribution dependence of focused ultrasound-induced blood-brain barrier opening. Ultrasound in medicine \& biology. 2014;40(1) 130-7.

[71] Yao L, Song Q, Bai W, Zhang J, Miao D, Jiang M, et al. Facilitated brain delivery of poly (ethylene glycol)-poly (lactic acid) nanoparticles by microbubble-enhanced unfocused ultrasound. Biomaterials. 2014;35(10) 3384-95. 
[72] Tung YS, Vlachos F, Feshitan JA, Borden MA, Konofagou EE. The mechanism of interaction between focused ultrasound and microbubbles in blood-brain barrier opening in mice. Journal of the Acoustical Society of America. 2011;130(5) 3059-67.

[73] Klibanov AL. Preparation of targeted microbubbles: ultrasound contrast agents for molecular imaging. Medical \& biological engineering \& computing. 2009;47(8) 875-82.

[74] Klibanov AL. Molecular imaging with targeted ultrasound contrast microbubbles. Ernst Schering Research Foundation workshop. 2005(49) 171-91.

[75] Klibanov AL, Maruyama K, Beckerleg AM, Torchilin VP, Huang L. Activity of amphipathic poly(ethylene glycol) 5000 to prolong the circulation time of liposomes depends on the liposome size and is unfavorable for immunoliposome binding to target. Biochimica et biophysica acta. 1991;1062(2) 142-8.

[76] Allen TM, Williamson P, Schlegel RA. Phosphatidylserine as a Determinant of Reticuloendothelial Recognition of Liposome Models of the Erythrocyte Surface. P Natl Acad Sci USA. 1988;85(21) 8067-71.

[77] Lindner JR, Song J, Xu F, Klibanov AL, Singbartl K, Ley K, et al. Noninvasive ultrasound imaging of inflammation using microbubbles targeted to activated leukocytes. Circulation. 2000;102(22) 2745-50.

[78] Nakano H, Ishida Y, Hatakeyama T, Sakuraba K, Hayashi M, Sakurai O, et al. Contrast-enhanced intraoperative ultrasonography equipped with late Kupffer-phase image obtained by sonazoid in patients with colorectal liver metastases. World J Gastroentero. 2008;14(20) 3207-11.

[79] Yanagisawa K, Moriyasu F, Miyahara T, Yuki M, Iijima H. Phagocytosis of ultrasound contrast agent microbubbles by Kupffer cells. Ultrasound in Medicine and Biology. 2007;33(2) 318-25.

[80] Korpanty G, Grayburn PA, Shohet RV, Brekken RA. Targeting vascular endothelium with avidin microbubbles. Ultrasound in Medicine and Biology. 2005;31(9) 1279-83.

[81] Korpanty G, Carbon JG, Grayburn PA, Fleming JB, Brekken RA. Monitoring response to anticancer therapy by targeting microbubbles to tumor vasculature. Clinical cancer research : an official journal of the American Association for Cancer Research. 2007;13(1) 323-30.

[82] Bayer EA, Wilchek M, Skutelsky E. Affinity cytochemistry: the localization of lectin and antibody receptors on erythrocytes via the avidin-biotin complex. FEBS letters. $1976 ; 68(2)$ 240-4.

[83] Klibanov AL, Hughes MS, Marsh JN, Hall CS, Miller JG, Wible JH, et al. Targeting of ultrasound contrast material. An in vitro feasibility study. Acta radiologica Supplementum. 1997;412 113-20. 
[84] Unnikrishnan S, Klibanov AL. Microbubbles as ultrasound contrast agents for molecular imaging: preparation and application. AJR American journal of roentgenology. 2012;199(2) 292-9.

[85] Klibanov AL. Ligand-carrying gas-filled microbubbles: ultrasound contrast agents for targeted molecular imaging. Bioconjugate chemistry. 2005;16(1) 9-17.

[86] Krupka M, Masek J, Bartheldyova E, Turanek Knotigova P, Plockova J, Korvasova Z, et al. Enhancement of immune response towards non-lipidized Borrelia burgdorferi recombinant $\mathrm{OspC}$ antigen by binding onto the surface of metallochelating nanoliposomes with entrapped lipophilic derivatives of norAbuMDP. J Control Release. 2012;160(2) 374-81.

[87] Watson DS, Endsley AN, Huang L. Design considerations for liposomal vaccines: Influence of formulation parameters on antibody and cell-mediated immune responses to liposome associated antigens. Vaccine. 2012;30(13) 2256-72.

[88] Takalkar AM, Klibanov AL, Rychak JJ, Lindner JR, Ley K. Binding and detachment dynamics of microbubbles targeted to P-selectin under controlled shear flow. J Control Release. 2004;96(3) 473-82.

[89] Villanueva FS, Jankowski RJ, Klibanov S, Pina ML, Alber SM, Watkins SC, et al. Microbubbles targeted to intercellular adhesion molecule- 1 bind to activated coronary artery endothelial cells. Circulation. 1998;98(1) 1-5.

[90] Kirpotin D, Park JW, Hong K, Zalipsky S, Li WL, Carter P, et al. Sterically stabilized anti-HER2 immunoliposomes: design and targeting to human breast cancer cells in vitro. Biochemistry. 1997;36(1) 66-75.

[91] Jeppesen C, Wong JY, Kuhl TL, Israelachvili JN, Mullah N, Zalipsky S, et al. Impact of polymer tether length on multiple ligand-receptor bond formation. Science. 2001;293(5529) 465-8.

[92] Kim DH, Klibanov AL, Needham D. The influence of tiered layers of surface-grafted poly(ethylene glycol) on receptor-ligand-mediated adhesion between phospholipid monolayer-stabilized microbubbles and coated class beads. Langmuir : the ACS journal of surfaces and colloids. 2000;16(6) 2808-17.

[93] Lee RJ, Low PS. Folate-Mediated Tumor-Cell Targeting of Liposome-Entrapped Doxorubicin in-Vitro. Bba-Biomembranes. 1995;1233(2) 134-44.

[94] Ham AS, Klibanov AL, Lawrence MB. Action at a distance: lengthening adhesion bonds with poly(ethylene glycol) spacers enhances mechanically stressed affinity for improved vascular targeting of microparticles. Langmuir : the ACS journal of surfaces and colloids. 2009;25(17) 10038-44.

[95] Klibanov AL, Maruyama K, Torchilin VP, Huang L. Amphipathic polyethyleneglycols effectively prolong the circulation time of liposomes. FEBS letters. 1990;268(1) 235-7. 
[96] Chen CC, Borden MA. The role of poly(ethylene glycol) brush architecture in complement activation on targeted microbubble surfaces. Biomaterials. 2011;32(27) 6579-87.

[97] Unger E, Porter T, Lindner J, Grayburn P. Cardiovascular drug delivery with ultrasound and microbubbles. Advanced drug delivery reviews. 2014;72 110-26.

[98] Moghimi SM, Szebeni J. Stealth liposomes and long circulating nanoparticles: critical issues in pharmacokinetics, opsonization and protein-binding properties. Progress in lipid research. 2003;42(6) 463-78.

[99] Cavalli R, Bisazza A, Lembo D. Micro- and nanobubbles: a versatile non-viral platform for gene delivery. International journal of pharmaceutics. 2013;456(2) 437-45.

[100] Rychak JJ, Klibanov AL. Nucleic acid delivery with microbubbles and ultrasound. Advanced drug delivery reviews. 2014;72 82-93.

[101] Pillai R, Marinelli ER, Fan H, Nanjappan P, Song B, von Wronski MA, et al. A phospholipid-PEG2000 conjugate of a vascular endothelial growth factor receptor 2 (VEGFR2)-targeting heterodimer peptide for contrast-enhanced ultrasound imaging of angiogenesis. Bioconjugate chemistry. 2010;21(3) 556-62.

[102] Price RJ, Skyba DM, Kaul S, Skalak TC. Delivery of colloidal, particles and red blood cells to tissue through microvessel ruptures created by targeted microbubble destruction with ultrasound. Circulation. 1998;98(13) 1264-7.

[103] Marmottant P, Hilgenfeldt S. A bubble-driven microfluidic transport element for bioengineering. P Natl Acad Sci USA. 2004;101(26) 9523-7.

[104] Mayer CR, Bekeredjian R. Ultrasonic gene and drug delivery to the cardiovascular system. Advanced drug delivery reviews. 2008;60(10) 1177-92.

[105] van Wamel A, Bouakaz A, Bernard B, ten Cate F, de Jong N. Radionuclide tumour therapy with ultrasound contrast microbubbles. Ultrasonics. 2004;42(1-9) 903-6.

[106] Bohmer MR, Klibanov AL, Tiemann K, Hall CS, Gruell H, Steinbach OC. Ultrasound triggered image-guided drug delivery. European journal of radiology. 2009;70(2) 242-53.

[107] Shi WT, Bohmer M, van Wamel A, Celebi M, Klibanov AL, Chin CT, et al. Ultrasound therapy with drug loaded microcapsules. Ultrason. 2007 773-6.

[108] Vandenbroucke RE, Lentacker I, Demeester J, De Smedt SC, Sanders NN. Ultrasound assisted siRNA delivery using PEG-siPlex loaded microbubbles. J Control Release. 2008;126(3) 265-73.

[109] Lentacker I, De Smedt SC, Demeester J, Sanders NN. Microbubbles which bind and protect DNA against nucleases. J Control Release. 2006;116(2) E73-E5. 
[110] Unger EC, McCreery TP, Sweitzer RH, Caldwell VE, Wu Y. Acoustically active lipospheres containing paclitaxel: a new therapeutic ultrasound contrast agent. Investigative radiology. 1998;33(12) 886-92.

[111] Kooiman K, Bohmer MR, Emmer M, Vos HJ, Chlon C, Shi WT, et al. Oil-filled polymer microcapsules for ultrasound-mediated delivery of lipophilic drugs. J Control Release. 2009;133(2) 109-18.

[112] Tinkov S, Coester C, Serba S, Geis NA, Katus HA, Winter G, et al. New doxorubicinloaded phospholipid microbubbles or targeted tumor therapy: in-vivo characterization. J Control Release. 2010;148(3) 368-72.

[113] Hauff P, Seemann S, Reszka R, Schultze-Mosgau M, Reinhardt M, Buzasi T, et al. Evaluation of gas-filled microparticles and sonoporation as gene delivery system: Feasibility study in rodent tumor models. Radiology. 2005;236(2) 572-8.

[114] Sun RR, Noble ML, Sun SS, Song S, Miao CH. Development of therapeutic microbubbles for enhancing ultrasound-mediated gene delivery. J Control Release. 2014;182 111-20.

[115] Nomikou N, Tiwari P, Trehan T, Gulati K, McHale AP. Studies on neutral, cationic and biotinylated cationic microbubbles in enhancing ultrasound-mediated gene delivery in vitro and in vivo. Acta biomaterialia. 2012;8(3) 1273-80.

[116] Florinas S, Nam HY, Kim SW. Enhanced siRNA Delivery Using a Combination of an Arginine-Grafted Bioreducible Polymer, Ultrasound, and Microbubbles in Cancer Cells. Mol Pharmaceut. 2013;10(5) 2021-30.

[117] Lum AFH, Borden MA, Dayton PA, Kruse DE, Simon SI, Ferrara KW. Ultrasound radiation force enables targeted deposition of model drug carriers loaded on microbubbles. J Control Release. 2006;111(1-2) 128-34.

[118] Christiansen JP, French BA, Klibanov AL, Kaul S, Lindner JR. Targeted tissue transfection with ultrasound destruction of plasmid-bearing cationic microbubbles. Ultrasound in medicine \& biology. 2003;29(12) 1759-67.

[119] Lentacker I, De Geest BG, Vandenbroucke RE, Peeters L, Demeester J, De Smedt SC, et al. Ultrasound-responsive polymer-coated microbubbles that bind and protect DNA. Langmuir : the ACS journal of surfaces and colloids. 2006;22(17) 7273-8.

[120] Jewell CM, Lynn DM. Multilayered polyelectrolyte assemblies as platforms for the delivery of DNA and other nucleic acid-based therapeutics. Advanced drug delivery reviews. 2008;60(9) 979-99.

[121] Leong-Poi H, Kuliszewski MA, Lekas M, Sibbald M, Teichert-Kuliszewska K, Klibanov AL, et al. Therapeutic arteriogenesis by ultrasound-mediated VEGF165 plasmid gene delivery to chronically ischemic skeletal muscle. Circ Res. 2007;101(3) 295-303. 
[122] Taylor SL, Rahim AA, Bush NL, Barnber JC, Porter CD. Targeted retroviral gene delivery using ultrasound. J Gene Med. 2007;9(2) 77-87.

[123] Lentacker I, De Smedt SC, Demeester J, Van Marck V, Bracke M, Sanders NN. Lipoplex-loaded microbubbles for gene delivery: A Trojan horse controlled by ultrasound. Adv Funct Mater. 2007;17(12) 1910-6.

[124] Lentacker I, Vandenbroucke RE, Lucas B, Demeester J, De Smedt SC, Sanders NN. New strategies for nucleic acid delivery to conquer cellular and nuclear membranes. J Control Release. 2008;132(3) 279-88.

[125] Geers B, Lentacker I, Sanders NN, Demeester J, Meairs S, De Smedt SC. Self-assembled liposome-loaded microbubbles: The missing link for safe and efficient ultrasound triggered drug-delivery. J Control Release. 2011;152(2) 249-56.

[126] Klibanov AL, Shevchenko TI, Raju BI, Seip R, Chin CT. Ultrasound-triggered release of materials entrapped in microbubble-liposome constructs: a tool for targeted drug delivery. J Control Release. 2010;148(1) 13-7.

[127] Fan CH, Ting CY, Lin HJ, Wang CH, Liu HL, Yen TC, et al. SPIO-conjugated, doxorubicin-loaded microbubbles for concurrent MRI and focused-ultrasound enhanced brain-tumor drug delivery. Biomaterials. 2013;34(14) 3706-15.

[128] Hernot S, Unnikrishnan S, Du ZM, Shevchenko T, Cosyns B, Broisat A, et al. Nanobody-coupled microbubbles as novel molecular tracer. J Control Release. 2012;158(2) 346-53.

[129] Klibanov AL. Ultrasound molecular imaging with targeted microbubble contrast agents. Journal of nuclear cardiology : official publication of the American Society of Nuclear Cardiology. 2007;14(6) 876-84.

[130] Correas JM, Burns PN, Lai X, Qi X. Infusion versus bolus of an ultrasound contrast agent: in vivo dose-response measurements of BR1. Investigative radiology. 2000;35(1) 72-9.

[131] Albrecht T, Urbank A, Mahler M, Bauer A, Dore CJ, Blomley MJK, et al. Prolongation and optimization of Doppler enhancement with a microbubble US contrast agent by using continuous infusion: Preliminary experience. Radiology. 1998;207(2) 339-47.

[132] Kratzer W, Kachele V, Merkle E, Mason RA, Buchner M, von Tirpitz C, et al. Contrast enhanced power Doppler sonography: Comparison of different administration forms of the ultrasound contrast agent Levovist (R) (vol 172, pg 443, 2000). Rofo-Fortschr Rontg. 2000;172(11) 947-.

[133] Lohmaier S, Ghanem A, Veltmann C, Sommer T, Bruce M, Tiemann K. In vitro and in vivo studies on continuous echo-contrast application strategies using sonovue in a newly developed rotating pump setup. Ultrasound in Medicine and Biology. 2004;30(9) 1145-51. 
[134] Vlasin M, Lukac R, Kauerova Z, Kohout P, Masek J, Bartheldyova E, et al. Specific contrast ultrasound using sterically stabilized microbubbles for early diagnosis of thromboembolic disease in a rabbit model. Canadian journal of veterinary research $=$ Revue canadienne de recherche veterinaire. 2014;78(2) 133-9.

[135] Arita J, Ono Y, Takahashi M, Inoue Y, Takahashi Y, Saiura A. Usefulness of contrastenhanced intraoperative ultrasound in identifying disappearing liver metastases from colorectal carcinoma after chemotherapy. Annals of surgical oncology. 2014;21 Suppl 3 S390-7.

[136] Kiessling F, Fokong S, Bzyl J, Lederle W, Palmowski M, Lammers T. Recent advances in molecular, multimodal and theranostic ultrasound imaging. Advanced drug delivery reviews. 2014;72 15-27.

[137] Alzaraa A, Gravante G, Chung WY, Al-Leswas D, Morgan B, Dennison A, et al. Contrast-enhanced ultrasound in the preoperative, intraoperative and postoperative assessment of liver lesions. Hepatology research : the official journal of the Japan Society of Hepatology. 2013;43(8) 809-19.

[138] de Haas HJ, Narula J, Fuster V. From molecular imaging to pathogenesis and vice versa. Circulation Cardiovascular imaging. 2014;7(4) 581-5.

[139] Kooiman K, Vos HJ, Versluis M, de Jong N. Acoustic behavior of microbubbles and implications for drug delivery. Advanced drug delivery reviews. 2014;72 28-48.

[140] Aryal M, Arvanitis CD, Alexander PM, McDannold N. Ultrasound-mediated bloodbrain barrier disruption for targeted drug delivery in the central nervous system. Advanced drug delivery reviews. 2014;72 94-109.

[141] Burgess A, Hynynen K. Drug delivery across the blood-brain barrier using focused ultrasound. Expert opinion on drug delivery. 2014;11(5) 711-21.

[142] Gao SJ, Zhang Y, Wu JF, Shi WT, Lof J, Vignon F, et al. Improvements in Cerebral Blood Flow and Recanalization Rates With Transcranial Diagnostic Ultrasound and Intravenous Microbubbles After Acute Cerebral Emboli. Investigative radiology. 2014;49(9) 593-600.

[143] de Saint Victor M, Crake C, Coussios CC, Stride E. Properties, characteristics and applications of microbubbles for sonothrombolysis. Expert opinion on drug delivery. 2014;11(2) 187-209.

[144] Kauerova Z, Lukac R, Kohout P., Masek J, Koudelka S, Plockova J, et al. A prototype 'Infucon' device for continuous of microbubbles in vivo. International Journal of Pharmaceutics. 2013;441(1-2) 92-8. 
Chapter 5

\title{
Plasma Modified Textiles for Biomedical Applications
}

\author{
Pieter Cools, Rino Morent and Nathalie De Geyter \\ Additional information is available at the end of the chapter \\ http://dx.doi.org/10.5772/59770
}

\section{Introduction}

In the textile market industry, technical textiles are one of the fastest growing businesses. Part of that industry consists of textiles for medical and healthcare applications and are responsible for a continuous increase in its market potential [1]. Next to their need in hospital environments, there is a growing demand in other sectors such as the food and hotel industry, due to stricter hygiene regulations. In most cases biomedical textile meets a well-defined set of requirements such as minimizing non-specific protein adsorption, drug delivery coatings or the presence of active functional coatings and most importantly excellent biocompatibility (blood-, tissue-or cyto-compatibility) [2]. In general there are very few materials meeting all these characteristics, while at the same time offering the needed structural and mechanical properties. Furthermore, depending on the application, the production process has to be costeffective and approved by local legislation.

In order to meet all these requirements, numerous modification techniques have been developed in the past [3-5]. Most of these techniques lead to the incorporation of extra/new functionalities and might lead to a change in surface free energy. For most biomedical applications, the preservation of material bulk properties such as elasticity, strength, ductility, structural integrity etc. is critical. For biomedical end-products, the use of solvents and chemicals based surface treatment techniques are reduced to a strict list approved by local legislation. Chemical-free techniques such as $\gamma$-radiation, UV treatments, corona discharges etc. have led to some excellent results in the field of tissue engineering [6, 7]. One of those solvent-free techniques that have been around for over a century, has more recently found its way into the biomedical field: non-thermal plasma technology.

Over time, it has extensively been proven that non-thermal plasma technology can profoundly change the surface properties of polymer films (PP, PET, PU, etc.) as well as material characteristics (adhesion, printability, dyeing etc.) of more complex substrates such as industrially 
produced textile [8-14]. Alongside the growing interest in tissue engineering and the booming of the electrospinning industry at the end of last century, non-thermal plasma technology found its way into the biomedical field. Today non-thermal plasma treatment can be considered as a well-established technique for the surface treatment of (bio)materials.

Before the start of the $21^{\text {st }}$ century, the majority of contributions to scientific literature was focussing on oxygen plasma treatments at low pressures and the corresponding response on cell adhesion, growth and proliferation. Although today there is still a steady stream of publications on these low pressure oxygen plasmas, there is a growing interest in atmospheric pressure plasma treatments as they offer a number of practical advantages. In the next chapter part, a detailed overview will be given on plasma technology in general and the different treatments possible. After that, the chapter will continue on the use of plasma technology for (bio)medical textiles, according to the application. At the end there will be a critical conclusion and a look forward to the possible future of plasma technology for the biomedical textile industry.

\section{Non-thermal plasma technology}

\subsection{History and definitions}

A plasma is a gaseous mixture of ions, radicals, electrons and neutrals. Plasma is often referred to as the fourth state of matter, as its properties fundamentally differ from solids, liquids and gasses and the change of state can be obtained by adding energy to a gas, similar to the transition from solid to liquid to gas. In 1929, Langmuir was the first to actually define a plasma, but already in the $19^{\text {th }}$ century plasma was used on an industrial scale for the generation of ozone (Siemens) [15].

Plasma itself can be divided up into two categories: 1) thermal or equilibrium and 2) nonthermal or non-equilibrium plasma. Thermal or hot plasmas have temperatures of $4000 \mathrm{~K}$ or higher and are considered to be in a thermal equilibrium, meaning that both heavy ions and electrons have the same temperatures. Well known applications include plasma spraying, wide arc spraying, and thermal plasma chemical vapour deposition (TPCVD), thermal plasma synthesis of fine powders (nm), thermal plasma (toxic) waste destruction, thermal plasma densification of powders, thermal plasma metallurgy, thermal plasma extractive metallurgy etc. For non-thermal or cold plasma, only the electrons are accelerated via e.g. an applied electrical field, causing a thermal inequilibrium between the electrons and the heavy particles. This results in the formation of a plasma at lower temperatures. Due to this difference in operating temperature between thermal and non-thermal plasmas, they are often referred to as 'hot' and 'cold' plasmas respectively. Although referred to as cold plasma, temperatures of up to $1000 \mathrm{~K}$ can be reached. For biomedical applications, non-thermal plasma treatments are preferred with a degree of ionization of $1 \%$ or lower as this results in a discharge that can be sustained at room temperature (290-330 K), thus avoiding thermal degradation of thermosensitive materials. In the next paragraphs, the focus will be on the sources that are used to 
drive the discharge, as they are an excellent way to distinguish between the ways a plasma can be generated, independent of the set-ups possible.

\subsection{Plasma discharges}

The different non-thermal or cold discharges discussed in the following parts have all proven their usefulness as well as their limitations. Over time, applications have been found for each different type of discharge in all branches of the industry: automotive, packaging, textiles, aerospace, catalysis, waste treatment, (bio)medical etc. [9, 13, 16-18]. The number of plasma reactor designs is nearly limitless and complete reviews have been written on that topic alone, as design changes are made to optimize the plasma treatment for their specific application [19]. Most of the designs available today can be linked to one of the plasma sources discussed here.

\subsubsection{Corona and silent discharge}

A corona discharge reactor typically consists out of a cathode wire and an anode, which is normally the material that needs treatment. The first developed systems were powered by a DC source working in a pulsed mode and were operated at atmospheric pressure. When turned on, the system generates a lighting crown build out of many streamers, hence the name corona [9]. Pulses are used that are shorter than the time necessary to form an arc, thus avoiding the transition to the spark regime. In the middle of $20^{\text {th }}$ century, the first corona discharge systems were patented for the incorporation into industrial textile production systems [20]. Later on, the systems were adapted to work with high frequency sources (radio-frequency (RF), microwave (MW) and AC) and today a number of commercial systems are available from companies such as Tech Sales Company, Air Liquide, Acxys Technologies etc. These modern high-tech set-ups are able to quickly and efficiently treat delicate structures such as electrospun sheets. One of the main disadvantages of corona treatment is that the streamers always form at the same sports, resulting in an inhomogeneous treatment of the exposed surfaces. To solve this particular problem, there has been a shift to the usage of silent discharges [16].

A silent discharge, also known as a dielectric barrier discharge (DBD), is powered either via a high frequency $\mathrm{AC}$ or an RF source. What makes the DBD stand out against other systems, is its higher and broad pressure operating range $\left(5-10^{5} \mathrm{~Pa}\right)$ [21, 22]. In 1857, Siemens was the first to use a DBD in a successful attempt to generate ozone and to this day it remains one of the most important industrial applications of the DBD [15].

A DBD reactor typically consists of 2 electrodes, of which at least 1 is covered with a dielectric material such as glass, ceramic or quartz. The voltage used to drive the discharge can start as low as $0.5 \mathrm{kV}$ and can be increased up to a few $100 \mathrm{kV}$. The generated plasma is a collection of many small micro-discharges or streamers. The dielectric material is able to limit the discharge current, giving cause to very short-lived micro-discharges (1-10 ns) that are distributed homogeneously across the electrode. In some specific cases, the streamers can be avoided altogether and a true glow regime can be obtained, which is considered the best case for homogeneous treatments [9]. 
DBD set-ups have one major advantage compared to most other systems: the possibility to operate in a higher pressure range makes it possible to avoid extensive vacuum equipment. This results in a lower operating cost and faster treatment cycles, thus allowing them to be implemented in industrial surface modification processes. The low heat generation at elevated pressures allows for a wider range of applications, including plasma chemistry, grafting, polymerization, cleaning... These applications are not always as easily feasible in systems powered with a different source.

It should be noted that occasionally in literature also the term corona discharge or corona treatment is used in connection with DBDs, although most authors prefer to use this term only for discharges between bare metal electrodes without dielectric.

\subsubsection{DC discharge}

Non-thermal plasmas generated via a DC discharge are in most cases formed in a closed setup between two electrodes at very low pressures $\left(10^{-1}-10 \mathrm{pa}\right)$ [21, 22]. As the current is increased, different types of discharges can be obtained. The Townsend discharge is a selfsustaining discharge, typically characterized by a low current. A higher current results in a drop of voltage and a glow discharge is generated. The glow discharge regime is the desired regime for surface modifications, as it guarantees a homogeneous treatment al throughout the reactor. Increasing the discharge current still further results in a fast increase of voltage until an arc is formed, allowing for the charge to dissipate and the voltage drops almost completely. One of the biggest advantages today of DC discharges, is that it is a well understood process, allowing for a high control over the process and its different parameters.

The DC current can be driven through the system in a continuous manner, or it can be pulsed. For biomedical applications in general, there are two advantages in doing the latter: first of, higher discharge powers can be applied without the otherwise inevitable thermal damage caused by the heating of the electrodes and secondly, if used for the coating applications, it renders a more homogeneous coating. One of the main disadvantages of the DC driven systems is the direct exposure of the electrodes to the plasma environment, making them prone to corrosion if exposed to certain reactive monomers.

\subsubsection{Radiofrequency and microwave discharges}

Radiofrequency (RF) and microwave (MW) discharges are generated using high frequency electromagnetic fields [21-23]. RF discharges have a relatively wide frequency operating range between $1-100 \mathrm{MHz}$, but in most cases a fixed frequency of $13.56 \mathrm{MHz}$ is applied. Concerning the operating pressure, a wider range, compared to DC systems, $\left(1-10^{3} \mathrm{~Pa}\right)$ is possible, but with the exception of a few, high-vacuum equipment is needed, which is expensive, drastically increases treatment times and are hard to implement in continuous production processes. For the treatment of biomedical materials, it is most likely the most applied discharge, as it is the plasma treatment technique of choice for the popular oxygen plasma treatments and several systems are commercially available. 
Microwave discharges are operated at a higher frequency range, usually fixed at $2.45 \mathrm{GHz}$. The pressure range is more versatile compared to RF and DC discharges, with a range between $1 \mathrm{~Pa}$ and $10^{5} \mathrm{~Pa}$. Higher pressures lead in most cases to an increase of heat transfer from the electrodes to the substrate, making it a less than ideal situation for the treatment of textiles and nonwovens. This limitation results in the same treatment restrictions as the previously discussed discharges.

\subsubsection{Atmospheric pressure plasma jets}

To finalize this chapter part on plasma technology, some special attention will be given to atmospheric pressure plasma jets (APPJ's). Operating a plasma in a confined space has certain advantages when it comes to the control of the physics and chemistry taking place, but sometimes there are cases where it would be more desirable if the plasma could be free from any geometrical confinements. APPJ's, also referred to as plasma plumes are an ideal solution and are excellent tools for the treatment of geometrically larger and more complex surfaces such as textile fibers [24].

APPJ's can be powered with any of the sources discussed before, but all deal with the same problem: how to avoid the transition from glow to arc. For DC sources this can either be achieved via the use of hollow cathode discharges with sub $\mathrm{mm}$ dimension or the use of resistive barrier discharges. For the DBD systems driven by high frequency AC sources, the dielectric barrier itself is the solution, as it prevents the discharge current to increase to the point of arcing. Under some special circumstances the DBD's can generate an uniform diffuse plasma that is filament free. For the RF powered APPJ, either a set-up similar to the DBD setup can be used, or the metal electrodes are left bare. For the latter, cooling of the electrodes is required, as well as an excellent control of the flow rate in order to minimise the risk of arcing [24]. Finally it is also possible to generate a plasma plume, using a microwaves to drive the plasma, but it is limited to a strict set of geometrical parameters which has been described in more detail by Park et al. [25].

It would be possible to give an extended description on the different set-ups available today, but it would lead to far out of the scope of this chapter. Laroussi and Akan already wrote a complete review on the different set-ups available. Also Shütze el al. wrote a compact review on the physics behind several set-ups [22]. Since that time also a number of commercial systems have become available on the market (crf Plasmatreat ${ }^{\circledR}$, PlasmaSpot $^{\circledR}$, PlasmaStream $^{\circledR}$...). The applicability of the APPJ for the treatment of biomedical textile will be covered in the following chapter part 3: Plasma and textile: the biomedical applications.

\subsection{Plasma-material interactions}

In order to have an understanding of what is happening at the plasma-material interface, it is critical to have a basic knowledge about the possible effects the different active species have on a substrate exposed to them. 


\subsubsection{Plasma cleaning and etching}

During the production process and storage of (bio)materials, they can be exposed to a number of solvents, greases, volatiles components etc. These contaminants will adsorb and accumulate on the material surface over time, resulting in an altered, non-reproducible surface with a likely reduced product performance. A typical example in the biomedical field, is the adsorption of low molecular weight carbon species onto a pristine titanium sample, when exposed to ambient air. When used as an implant material, this surface pollution results in a reduced cell adhesion, proliferation and growth and in some cases even results in cell death [26,27].

Any volatile surface contamination that is exposed to a non-thermal plasma, will be removed in a few seconds [28]. Prolonged exposure to the plasma will not only result in the removal of the adsorbed contamination but will cause etching of the top layers of the material surface [29-32]. Depending on the density and hardness of the exposed material, more intense discharges and/or extended exposure are required to obtain a notable effect. As (biomedical) textiles are in most cases build out of relatively soft materials, the etching effect cannot be overseen and will introduce a certain nano-roughness on the fiber surface. For in-vitro and invivo applications this change in surface topography can have a benign effect, as it can amplify the other effects plasma has on cell adhesion and proliferation [33-35].

\subsubsection{Plasma activation}

Plasma activation or plasma treatment is the exposure of a surface to the reactive particles present in the plasma. This mixture of reactive particles will result in the incorporation of radical sites on the surface, up to the depth of a few $10 \mathrm{~nm}$. Depending on the gas used to maintain the plasma, these sites will react (in)directly with other radicals present, recombining into a broad variety of functional groups. These new functional groups have a high impact on surface properties such as wettability and surface free energy, which in turn might have a positive effect on material-material and material-cell interactions.

In most cases an increase in hydrophilicity is pursued to enhance the materials histological performance. For some applications such as the surface of heart valves, the insides of needles and tubes or artificial stents, any adhesion of cells and proteins is highly unwanted, as it can lead to blockages resulting in premature failure of the biomedical device. Instead of using typical gas feeds for plasma treatment (noble gasses, oxygen, dry air, nitrogen...), fluorinated gasses such as $\mathrm{CF}_{4}$ are used which result in the formation of super hydrophobic surfaces with water contact angles of $150^{\circ}$ and higher. These fluorinated surfaces prevent cells and proteins from effectively adhering on the surface and thus guaranteeing an optimal performance of the implant material [11, 36, 37].

Plasma activation is definitely not the only technique available for the introduction of new functional groups onto a surface, but as it is non-invasive and chemical-free, it guarantees the preservation of even the most delicate structures.

\subsubsection{Plasma grafting and polymerization}

Non-thermal plasmas are not only applied for plasma treatments, but can also be used as an initiation medium for radical polymerization, resulting in the deposition of a wide variety of 
thin films. In order to optimize the bonding between the thin film and the biomaterial, the deposition process is preceded by a plasma treatment, introducing radical sites that allow covalent bonding of the polymer to the substrate surface. The polymerization process itself can happen via two different reaction pathways: plasma polymer grafting simply uses the radical sites introduced via plasma treatment to initiate the chain reaction. In other words, during the polymerization process itself, no plasma is used and the monomer is not exposed to the plasma. This results in the incorporation of the monomer as such, thus preserving its functional groups.

For plasma polymerization this is not the case. The plasma is used as an initiation medium and remains active during the entire polymerization reaction. This has as a consequence that the monomer is exposed to the reactive plasma, forming initiation sites on both the substrate surface as well as on the monomer. In contrast to chemical initiation, plasma is not as specific as to where the radicals are formed, using any functional groups of the polymer precursor as well to initiate the chain reaction. This results in a highly cross-linked, pinhole free and completely amorphous thin film that significantly differs from its traditional counterpart and adheres to almost any surface. Varying the discharge power gives a high control over the amount of functionalities preserved in the film. From a biomedical viewpoint this is an interesting application, as functional group density plays a critical role in the growth and proliferation of cells and differs for the type of cells used.

\section{Plasma and textile: The biomedical applications}

\subsection{Wound dressing}

Optimal modern wound dressings should assure a moisture wound bed, help drainage, remove debris of the wound surface, provide optimal thermal stability, might be removed without trauma of the wound bed and wound edge, be antiallergenic and without immunogenicity [38]. Over the years wound dressings have experienced a continuous development stimulated by a better understanding of wound healing and bacterial growth mechanisms. In more recent years research has shifted to targeted therapy by including different pharmaceutical compounds (e.g., antiseptics, analgetics, or growth factors) in to wound dressings. The continuous presence, or controlled release of active substances, can tremendously stimulate the healing process.

Non-thermal plasma technology has been part of this development process in many different aspects of wound healing treatments. The review of the literature dealing with the use of plasma technology for the enhancement of wound dressings will be divided according to the purpose of the treatment: wound monitoring, enhancement antimicrobial properties, intermediate bonding, and adhesion.

\subsubsection{Antimicrobial properties}

Successful wound treatment cannot be achieved without keeping its two major aspects in mind: maintaining a decent hydrophilicity and a high antimicrobial efficiency. 
In two different papers Persin et al. compared a few different treatment methods on viscose fibers to address both of these aspects $[39,40]$. In a first reaction pathway, a two-step process consisting of an oxygen plasma treatment, followed by the immobilization of $\mathrm{AgCl}$ particles, is followed. The alternative pathway consists of a single-step treatment of the cellulose fibers with an ammonia plasma. The single-step treatment resulted in a 30-fold increase in water uptake while the immobilization of the silver particles only had a marginal effect. For the antimicrobial properties of the wound dressings, the reverse trend was found. The single-step treatment only had an effect on Gram-positive bacteria, while the steady release of silver ions resulted in a quasi-complete destruction of both Gram-positive and Gram-negative bacteria. The authors therefore suggest to use a different treatment for different wound-healing applications, depending on the risk of infection. In a third paper from the same authors, a closer look is taken on the ageing of the plasma treated surfaces and the effect on the water up-take [35]. The study showed that 4 days after the treatment, the water contact angle increased with $15^{\circ}$ and the oxygen concentration decreased with less than $1 \%$. These numbers suggest that there is a limited shelf-lifetime of the modified wound dressings. Hacker et al. followed an alternative strategy for the immobilization of Ag particles by plasma polymerizing PEG onto electrospun PU mats, followed by soaking in $\mathrm{AgNO}_{3}$ and $\mathrm{UV}$ treatment, resulting in the incorporation of metallic silver [41]. Water-uptake tests revealed a significant increase of hydrosorption after 24 hours. The steady release of silver particles had a detrimental effect on the viability of both Gram-positive and -negative bacteria while at the same time no cytotoxic effects were noted on the adhesion and proliferation of fibroblasts. Further on in the chapter, other strategies will be discussed to obtain antibacterial properties for other applications, but it is already clear that the incorporation of silver particles with the help of plasma technology is a successful pathway for the improvement of wound dressings.
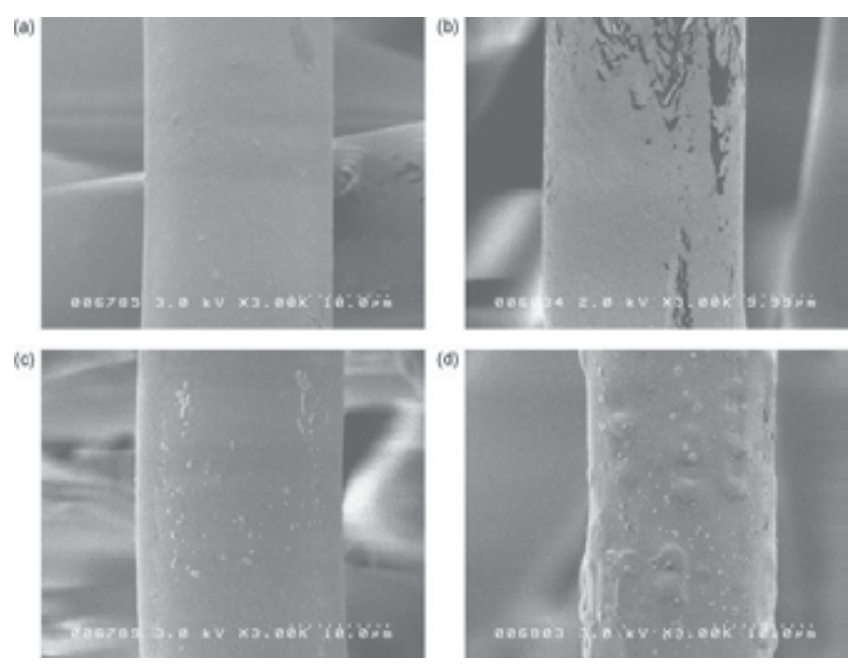

Figure 1. SEM micrographs (3000×) of the (a) plasma modified nonwoven, (b) AAc coated nonwoven, (c) PP-g-collagen nonwoven, and (d) PP-g-collagen-g-PNIPAAm nonwoven [126]. 


\subsubsection{Adhesion and intermediate bonding}

Next to increasing the hydrophilicity of surfaces, plasma are also known to promote the adhesion between layers. Gajanan et al. published two papers where plasma technology was used to improve the adhesion between an electrospun material and a woven support structure $[42,43]$. In the first article chitosan was used as a raw material for the electrospinning process. In the second article, the chitosan was mixed with silk fibroin. For both cases a $100 \%$ cotton gauze was used as support material. Out of the different DBD plasma treatments, using $\mathrm{He}$ $+1 \% \mathrm{O}$, the combination of a pre-and post-treatment gave the best results. Flex durability tests showed that there was a 4 -fold increase in adhesion between the electrospun material and the cotton gauze. On top of that SEM images showed that the plasma treatment resulted in a reduction of the fibers' delamination after repetitive flexing.

As explained earlier in section 2.3, plasma can be used to graft new side chains on polymer substrates. The goal of grafting can either be to introduce new functional groups and use their properties as such, or to introduce them as intermediates that can be used in consecutive reactions. In biomedical surface engineering the latter is quite popular as the list of products that can be used is limited, meaning that in most cases there is a sub-optimal affinity between coating and substrate. Chen et al. used a PP-non-woven as a substrate on which acrylic acid was grafted. The newly introduced carboxylic acid functionalities were used to either covalently bond collagen or chitosan, on which the thermo sensitive polymer PNiPAAm was immobilized of which each step is depicted in figure 1 . Above $32^{\circ} \mathrm{C}$, the wound dressing becomes hydrophobic and releases the stored moisture, which in turn resulted in an enhanced wound healing [44, 45]. Lin et al. used an oxygen low pressure plasma to activate a PE nonwoven substrate, followed by the grafting of $\mathrm{N}$-isopropyl acrylamide [46]. This intermediate was then used for the covalent bonding of bovine gelatin. In-vivo tests revealed that the covered wound healed completely (reached the maturation phase) and much faster compared to the PE control.

The results discussed above show that plasma technology can be a valid, solvent-free alternative for the permanent fixation of technically advanced layers onto standard substrates. Most of the studies on adhesion and intermediate bonding are relatively new and more research is needed to see if the obtained results can be extrapolated to other materials and applications.

\subsubsection{Wound monitoring}

In modern society the development of 'smart' materials is a hot topic (crf smartphones, smartwatches, smart-TV's etc.). In the wound care industry similar developments are taking place and several groups are doing research on so-called 'smart bandages'. The idea behind smart bandages is that they autonomously could monitor the wound and signal in case of infection or other irregularities. A few groups working on biosensors have successfully incorporated plasma technology in their biosensor production process.

Phair et al. used a corona discharge in ambient air to activate carbon fiber threads in order to improve their electro-analytical performance [47]. Via a redox probe set, the ion exchange rate was determined as a function of plasma treatment time. The results showed that the incorpo- 
ration of carboxyl groups resulted in better electron transfer kinetics. Exposing the prototype bandage to whole blood proved that they were capable of detecting urates.

Zhou et al. developed a biosensor based on self-quenching fluorocarbons [48]. These fluorocarbons were stored in lipid vessels which were immobilized on a pp-non-woven that was plasma coated with maleic anhydride using a commercial RF discharge system. Upon contact with both Gram positive and negative bacteria, the lipid vessels released the fluorocarbons, allowing for a visual infection detection mechanism. In combination with the simultaneous release of antibacterial products using the same release system, they believe it is possible to make a quasi-autonomous or 'smart' bandage.

The collection of reviewed papers shows that non-thermal plasma technology can play an important role in the development of smart bandages, allowing for low-cost personalized wound care treatment.

\subsection{Antimicrobial functionality}

Within the section on wound dressings already a part on antibacterial properties of plasma treated fibers has been included, but the applicability of these modified textiles goes much further than wound treatment alone and is highly wanted for other (bio)medical applications such as surgical gowns, sutures etc. as well as in the food industry and catering business [49]. The study on antimicrobial finishes of fibers and meshes has been quite extensive and between 25 and 30 papers will be reviewed here. A wide variety of substrates has been modified, ranging from natural-based products such as cellulose and wool to PP, polyesters, polysulfons, polyamides, carbon composites etc.

\subsubsection{Ag immobilization}

As for the modification itself, a distinction can be made between alterations involving the immobilization of metal nanoparticles $(\mathrm{Ag}, \mathrm{Cu})$ and all other treatments. To this day, the controlled release of silver particles is by far the most effective technique to inhibit the attachment and growth of both Gram positive and negative bacteria [50-65]. Most of the research groups use a non-thermal plasma to increase the wettability and nano-roughness of the textile substrate in order to enhance the uptake of silver nanoparticles (either via $\mathrm{AgNO}_{3}$ or $\mathrm{Ag}_{\mathrm{n}}$ ) or a plasma polymer coating with controlled release properties. Antibacterial tests involving micro-organisms such as E. Coli, S. Aureus and C. Albicans show in most cases a bactericidal efficiency of more than $99.9 \%$.

\subsubsection{Plasma activation, grafting and intermediate bonding}

Other research groups followed less known pathways with mixed success. Yao et al. did an Ar low pressure plasma pretreatment of PVDF-HFP/PU fibrous membranes, followed by the UV grafting of 4-vinylpyridine and a quaternization of the introduced pyridine group using hexyl bromide [66]. Anti-bacterial essays showed a killing efficiency of $99.9999 \%$ for both EColi and S. Aureus strains (See figure 2). Despite the excellent performance of the fibers, the 
commercial applicability remains limited due to relatively expensive production process in a competitive business environment.

Different research groups did a plasma pretreatment on cotton and polyproplyne (PP) nonwovens respectively, followed by the immobilization of natural products [67-69]. Vaideki et al compared the uptake of neem leaf extract before and after RF air plasma treatment and found that both the increase in wettability as well as the surface etching resulted in a superior adsorption of the extract [67]. Nitkyakalyani et al made a mix of herbal plant parts that were dried and grinded after which they were dissolved so the PP could be soaked in it [68]. A wide variety of both Gram positive and negative bacteria were tested and excellent antimicrobial activity was noted. Although the use of natural products is a pro in some cases, it is difficult to obtain a certain consistency in the production process and there is always a certain risk of product pollution. Strnad et al. treated cellulose fibers with an $\mathrm{RF}_{2}$ plasma to increase the adsorption of chitosan [69]. The antimicrobial tests revealed a modest effect against $\mathrm{S}$. Aureus and no effect against E-Coli. Antifungal tests using several fungi strings resulted in modest antifungal behavior.

Some groups simply applied a plasma treatment as such, using reactive gasses such as $\mathrm{CF}_{4}$ and hydrazine to obtain the desired antimicrobial effect. Uygun et al. did a pretreatment of chitosan powder using a RF hydrazine plasma [70]. Chemical analysis shows a significant increase in the number of primary amines present. Using the modified chitosan for the electrospinning of nano-fibers results in a nonwoven that has a better moisture uptake and an enhanced antibacterial effect for Gram-positive bacteria. Canal et al. and Virk et al. used an $\mathrm{Ar}-\mathrm{CF}_{4}$ post discharge plasma with mixed results. The treatment of wool, polyamide and Sontara ${ }^{\circledR}$ resulted in a heightened bacterial resistance, while the treatment of cotton had no effect at all [71, 72].

Just as described in the intermediate bonding paragraph of the wound treatment section, plasma is used for the grafting of intermediate layers that are used for the immobilization of antibacterial components. Degoutin et al. used a low pressure RF Ar plasma to graft acrylic acid onto a PP nonwoven [73]. The carboxylic acid functionalities were used for the immobilization of gentamicin, which is known to be $99 \%$ bactericidal as was confirmed by the testing with E. Coli. Gawish et al. used a He plasma for the grafting of glycylmethacrylate, which was used for the covalent bonding of cyclodextrines [74]. These macromolecules are known for the controlled release of active components, in this case biocides with antimicrobial and insect repellent properties. These experiments confirm that plasma grafting is a versatile technique that forms the basis for subsequent reactions.

In general it can be concluded that the incorporation of silver micro-and nano-particles is still the golden standard for the production of antibacterial textiles. Non-thermal plasma technology can be a useful tool for the immobilization of the metal particles and plasma deposited coatings are ideal for the controlled release. Plasma treatments as such can generate highly antimicrobial surfaces, as was proven, amongst others, by Yao et al. [66], but only after the right selection of precursors and discharge gasses. 

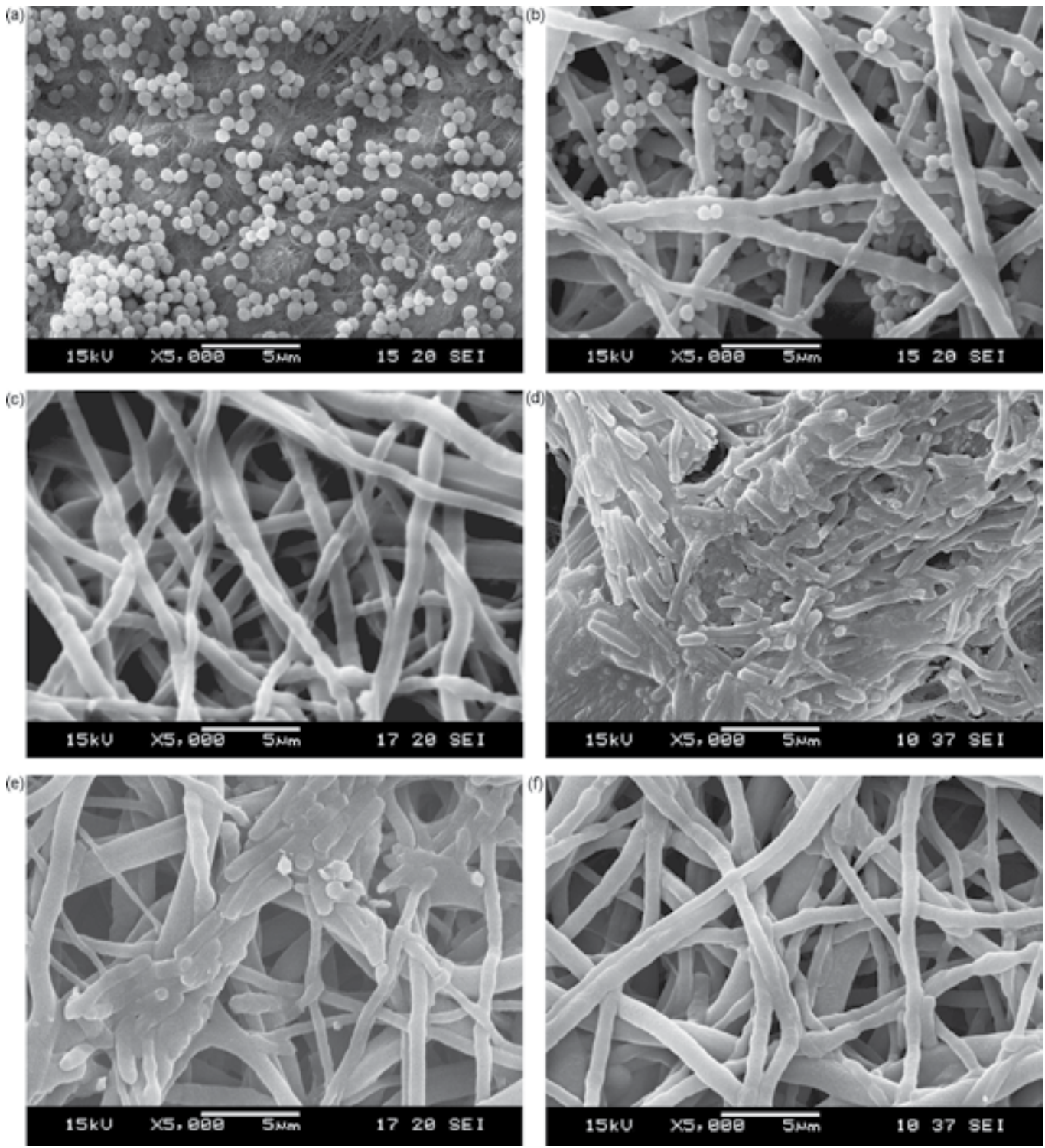

Figure 2. SEM images of (a) and (d) filter paper (control), (b) and (e) pristine, and (c) and (f) modified PU fibrous membranes after immersed in PBS suspension of (a)-(c) S. Aureus, or (d)-(f) E. Coli at 107 cells/mL for 4 h. PU fibrous membranes were electrospun from $10 \%(\mathrm{w} / \mathrm{v})$ solutions in THF and DMF $(1: 1, \mathrm{v} / \mathrm{v})$ [127].

\subsection{Cell culture and soft tissue engineering}

Technical textiles for tissue engineering applications are one of the youngest branches in the textile industry, but in a just a few decades they have become a major player on the biomedical market and the number of publications dealing on the theme of tissue engineering applications has exponentially grown. The idea behind tissue engineering is that (stem) cells are extracted 
from a patient with a malfunctioning organ. The retrieved healthy cells are seeded on a culture plate and grown to a full culture $[3,4,75]$. Once enough cells can be harvested, they are seeded onto a 3D nanofibrous scaffold. After a certain incubation period, allowing the cells to grow and differentiate into the scaffold, the nonwoven is implanted into the patient in order to restore the organ functionality or replace the organ as such. By treating patients with their own cells, immuno-response can be reduced to an absolute minimum and it is considered to be a constructive solution for the transplant waiting list issue.

In this specific chapter part, both the enhancement of cell culture applications as well as soft tissue engineering will be discussed together, as they are closely related and in some cases even overlap. This chapter part will therefore be subdivided into a first section dealing with plasma treatment as such and its effect on cell growth, adhesion, proliferation and differentiation and a second section handling plasma grafting and polymerization, talking in more detail about adhesion and homogeneity of the deposited coatings as well as the histological properties.

\subsubsection{Plasma treatment}

The most widely used polymer collection for the production of nonwoven scaffolds for tissue engineering is the biodegradable polymer family. Polylactic acid(PLLA), polylactic-glycolic acid (PLGA), Polycaprolacton (PCL)... are well established biomaterials due to the fact that after implantation they get broken down by the body in harmless end products (ideally $\mathrm{CO}_{2}$ and $\mathrm{H}_{2} \mathrm{O}$ ) that can be secreted by the body, making a second surgery no longer necessary [13, 76]. The mechanical and structural properties of these materials are sufficient for their field of applications. The biocompatibility and bioactivity on the other hand are mediocre at best and often inhibit the migration and differentiation of cells into the textile scaffold structure [77, 78]. The surface treatment of these scaffolds is rather complicated, as the pore size limits the infiltration efficiency of (wet)-chemical treatments and often degrade the structural stability of the delicate nano-fibers. Gas-based treatments such as non-thermal plasmas are promising to penetrate more easily into the electrospun scaffold structure and are at the same time known for the fact that they only alter the surface without affecting the bulk, guaranteeing the mechanical and structural integrity of the modified biomaterial [14, 79].

The contact angle of PCL electrospun scaffolds lies between $120^{\circ}$ and $140^{\circ}$, indicating a hydrophobic surface, which is not well liked by most cells. After treatment with either air, Ar, $\mathrm{NH}_{3}$ or $\mathrm{O}_{2}$ plasmas, all research groups were able to reduce the contact angle to less than $5^{\circ}$ [80-85]. The XPS results reveal an increase in the oxygen content with the incorporation of a mixture of $\mathrm{C}-\mathrm{O}, \mathrm{C}=\mathrm{O}$ and $\mathrm{O}-\mathrm{C}=\mathrm{O}$ functional groups. Prabhakaran et al. seeded neurolemmocytes (or Schwann) cells onto the nanofibrous scaffolds and found an increase in proliferation rate at all times, with a maximum of $17 \%$ compared to the untreated material after 10 days, compared to the untreated scaffolds [80]. They claim that the treatment is as effective as a collagen coating, making it a cost-effective alternative for nerve cell regeneration applications. Yan et al. found that after plasma treatment with $\mathrm{NH}_{3}+\mathrm{O}_{2}$ had no influence on the mechanical properties of the non-woven. After seeding with MC3T3 osteoblasts a 2-3 times increase in cell adhesion was found in the first 24 hours and after 7 days the proliferation was increased by a 
factor of 6, making the plasma treatment an excellent tool for the introduction of osteoinductive properties [81, 84]. Martins et al. came to the same conclusions after treating their PCL nano-textile scaffolds both with $\mathrm{Ar}$ and $\mathrm{O}_{2}$ plasma [82]. Seeding 3 different cell lines (L929, ATDC5 and Saos-2) covering a wide variety of cell-types, resulted in a significant increase in both adhesion and proliferation for all cells. Min et al. tested the $\mathrm{O}_{2}$ plasma treated PCL nanotextile with primary astrocytes and noted an increase in adherence and viability in the first 24 hours [83]. Jeon et al. used a nano-sized template to enhance the nano-roughness introduced by the plasma [85]. After seeding MG63 osteoblasts a dramatic increase in cell adhesion and proliferation were noted as well as an elongated morphology compared to both untreated PCL and PCL treated without the nano-sized template, showing that both the surface chemistry and topography have a significant influence on the histological performance of the PCL electrospun scaffolds. Finally Blackstone et al. used a $\mathrm{CF}_{4}$ plasma to further increase the contact angle of the non-woven in order to obtain a superhydrophobic scaffold [86]. After seeding a mixture of fibroblasts, keranocytes and MCF-7 cancer cells, they were able to sort out the cancer cells by applying a fixed amount of stress to the textile. The recovered cancer cells did not change in morphology, allowing for post-sorting analysis, making the development of a low cost cancer detection device possible.

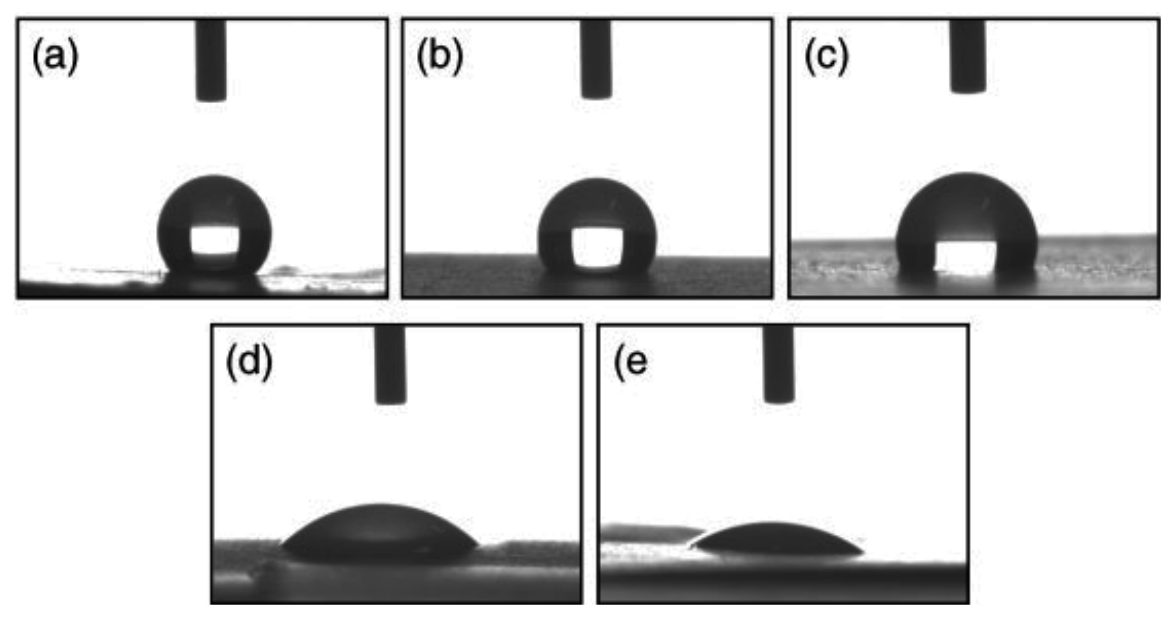

Figure 3. Photographs of water droplets taken immediately after contacting (a) non-treated, $(b, c)$ oxygen plasma-treated and (d,e) ammonia plasma-treated PLGA nanofibers. Treatment time was varied from (b,d) 30 to (c,e) $180 \mathrm{~s}$ [128].

PLLA and PLGA both exhibit the same hydrophobic properties as PCL non-woven, giving water contact angles situated between $130^{\circ}$ and $150^{\circ}$. After a plasma treatment with the typical discharge gasses $\left(\mathrm{O}_{2}, \mathrm{Ar}, \mathrm{NH}_{3 . .}\right)$ a decrease in contact angle was found to a minimum, ranging between $20^{\circ}$ and $45^{\circ}$ as depicted in figure 3 [87-89]. XPS reveals that PLLA is less robust compared to PCL as the initial increase in oxygen content is reversed when the substrate is over-treated, resulting in etching/degradation of the polymer structure [88]. Both Park et al. and Dolci et al. seeded fibroblasts (NIH3T3 and MEF) after $\mathrm{NH}_{3}$ and air treatments respectively and similar results for the increase in viability and elongated morphology were found [88, 
90]. Liu et al. did a study on the adhesion behavior before and after $\mathrm{O}_{2}$ plasma treatment of pMSC cells in the first hour after seeding [89]. Results revealed that both the adhesion and cell morphology were greatly improved as can be seen from figure 4 . These results show that plasma treatment is indeed a valid option for the culturing of stem cells.

As PLLA, PLGA and PCL all show similar histological effects after exposure to a wide variety of plasmas, it is no surprise that blends of PLLA and PCL exhibit similar behavior. Chandasekaran et al. exposed such a co-polymerized fiber mesh to an air plasma treatment and studied the effects on fibroblast growth and proliferation [91]. In both cases a significant increase was noted and stimulation of extra cellular matrix formation was found, opening up the possibility for skin tissue regeneration applications.

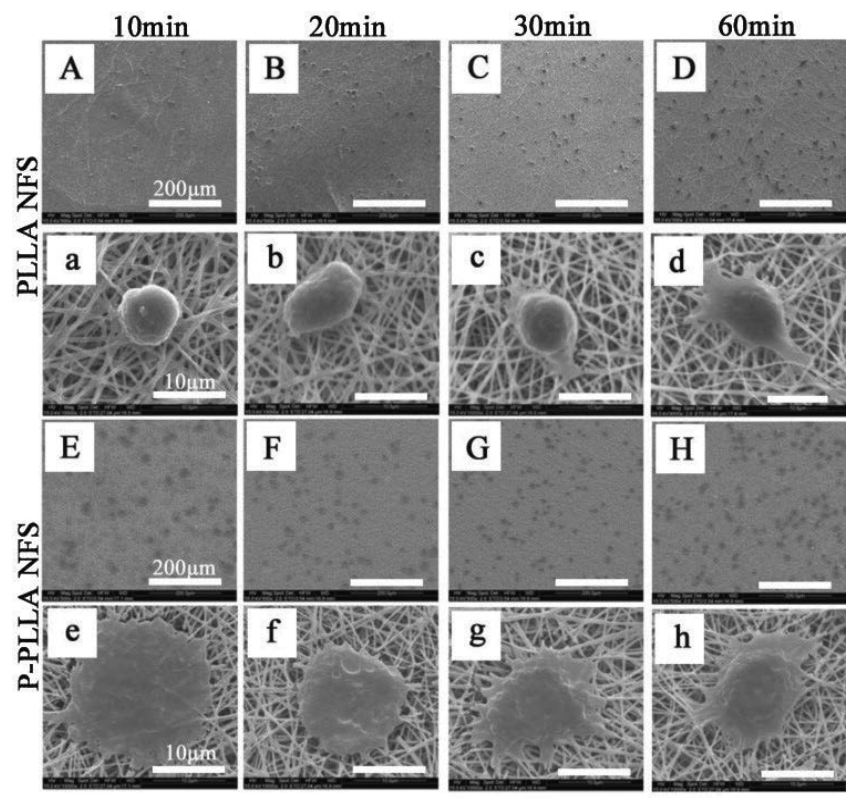

Figure 4. SEM images of pMSCs on Plasma treated PLLA nanofibers (NFS) and pristine PLLA NFS. (A-D) pMSCs on PLLA NFS after cultured for $10 \mathrm{~min}, 20 \mathrm{~min}, 30 \mathrm{~min}, 60 \mathrm{~min}$, respectively; (E-H) pMSCs on P-PLLA NFS after cultured for $10 \mathrm{~min}, 20 \mathrm{~min}, 30 \mathrm{~min}, 60 \mathrm{~min}$, respectively; $(\mathrm{a}-\mathrm{h})$ higher magnification for $(\mathrm{A}-\mathrm{H})$ [129].

The biodegradability of the textile scaffold material is not required in every case and sometimes has to be avoided all together (tissue culture 'plates', vascular grafts...) as the loss of the mechanical framework would result in the permanent failure of the implant. A polystyrene electrospun scaffold was treated by Baker et al. with a low pressure Ar plasma, followed by the seeding of smooth muscle cells [92]. The in-vitro tests revealed a significant increase in cells and an alignment with the electrospun material. The excellent results show that the polystyrene electrospun scaffold could be a valid alternative for 2D tissue culture plates. Zandén et al. treated a PU fiber mesh with an oxygen plasma in an attempt to improve the interaction with red blood cells [93]. SEM images showed that prolonged exposure to the plasma resulted in a reduction of the fiber diameter and finally the degradation of the structure. As was the case 
with PLLA and PCL, a significant increase in hydrophilicity was noted due to the incorporation of polar functional groups. The in-vitro tests revealed that there was no significant difference between the adhesion of red blood cells before and after treatment. This shows that not all plasma treatments have a positive effect on biocompatibility and that the treatment gas and operation parameters should be carefully selected.

The final paragraph of this chapter part on plasma treatment for tissue engineering will go over the possibilities to use non-thermal plasmas to stimulate the formation of apatite on flexible scaffold structures. Yang et al. immersed an Ar plasma treated PCL fiber structure in an SBF 10 solution for a period of 7 days [94]. In the first 24 hours, already a CaP coating had grown on the fibers, consisting of nano-apatite and dicalcium phosphate dehydrate. After 7 days a structure closely resembling bioapatite was found. Luo et al. performed the same analysis on air plasma treated PEEK (reinforced with carbon fiber) and whereas the untreated PEEK resulted in no apatite formation, a fully grown apatite layer could be found on the treated nonwoven scaffold [95]. Other research groups made a solution of PCL mixed with hydroxyapatite $/ \mathrm{CaCO}_{3}$ which was electrospun, resulting in a composite nano-textile which was followed by an oxygen/air plasma treatment [96, 97]. After seeding hFOB osteoblasts, a significant proliferation rate was noted, as well at the first signs of mineralization similar to human bone as depicted in figure 5 . These papers show that plasma activation of polymeric nonwoven scaffolds is an excellent tool for the promotion of apatite growth.
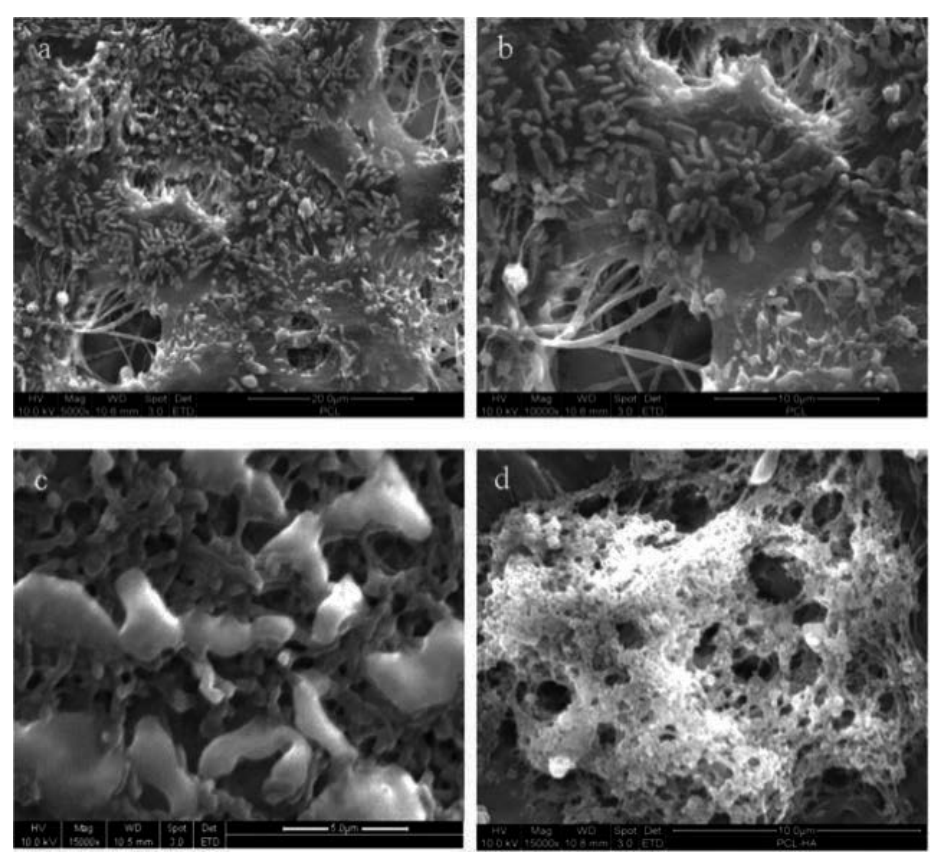

Figure 5. Mineralization of hFOB on PCL/HA-P nanofibrous scaffolds at different magnifications: (a) mineral deposition 5000× (6 days), (b) mineral deposition 10000× (6 days), (c) mineral deposition 15000× (6 days), (d) apatite-like morphology of natural bone $15000 \times$ (10 days) [130]. 
In general it can be concluded that non-thermal plasma treatments, both at lower and elevated pressures, are excellent tools for the stimulation of the histological properties of a wide variety of cells seeded onto flexible scaffolds for tissue culture and tissue engineering applications.

\subsection{Plasma grafting, polymerization and immobilization}

The tissue engineering nanofibrous scaffold materials subjected to plasma grafting and plasma polymerization are, in the majority of the papers reviewed, more or less the same ones used for plasma activation, PCL being the most popular one. A minority of papers investigated less obvious material choices that are inherently not (sufficiently) biocompatible, but once coated exhibit sufficient bioactive properties, as will be discussed in the last paragraph of this chapter part.

Guex et al. coated a PCL mesh using a combination of ethylene and $\mathrm{CO}_{2}$ in an Ar discharge in order to restore the functionality of damaged myocardium [98]. After seeding extracted mesenchymal stem cells onto the modified electrospun scaffold, it was implanted in a rodent model. Post-mortem analysis revealed a stabilized cardiac functionality as well as an attenuated dilation. Zander et al. covalently bonded lamilin proteins onto an air plasma treated PCL scaffold [99]. PC12 neuron-like cells were seeded onto the modified substrate and analysis showed a positive correlation between the neuron outgrowth and the concentration of the immobilized proteins. Xie et al followed a similar strategy, immobilizing dopamine [100]. The dopamine coating itself was then used to immobilize fibronectin, which significantly stimulated the attachment, spreading and cytoskeletal development of NIH 3T3 cells. Furthermore it was proven that the coatings could be used for the controlled release of active substances. Ma et al. used a combination of air plasma and wet carboiimide chemistry to graft gelatin onto both random and aligned PCL fiber meshes [101]. The spreading and proliferation of endothelial cells was greatly enhanced and the cells aligned themselves along the fibers, which was not the case for the untreated material. Finally Hegeman et al. performed a degradation study of PCL nanofibrous scaffolds coated with amine containing polymers and showed that the incorporation of oligomers in the deposited films can leach out, causing cell death [102]. Storage of the coated nano-textiles in liquid media removed the low molecular weight residue, solving the problem. Overall, the literature shows that PCL nonwoven scaffolds, either activated or coated, can be used for a wide variety of tissue engineering applications, exhibiting excellent bioactive properties. It is essential though to use the right set of parameters and avoid the inclusion of unreacted products as this can have a detrimental effect on the histological performance of the 3D electrospun scaffold.

Several research teams used non-thermal plasmas to immobilize bioactive macromolecules (collagen, cRGD peptides and heparin respectively) onto a PLLA nano-textile scaffold, after which they were seeded with either BOECs, hMSC or endothelial cells [78, 103-106]. In all cases a positive influence was found on the scaffold's histological properties. Park et al. also obtained an increase in adhesion and proliferation after seeding NIH 3 T3 fibroblasts onto PLLA nanotextile scaffolds that were grafted with an acrylic acid coating using a low pressure $\mathrm{O}_{2}$ plasma [107]. He et al and Chan et al. performed a similar procedure compared to Feng et al. to immobilize collagen onto PLLA-PCL electrospun scaffolds [108, 109]. The first group success- 
fully seeded hCAEC's, showing the possibilities for vascular grafts (see figure 6), while the Chan et al. studied the enhancement of the adsorption properties of MSC cells in the first hour after seeding, proving that coated nonwoven scaffolds are more efficient than plasma treated samples, which in turn are more effective then untreated samples.

A rather large number of publications can be found on a variety of other biomaterials that are not always biodegradable such as PDMS, PU, PET, silk fibroin, cellulose, PHBV... Most of these textile scaffolds get coated with well-known bioactive macromolecules such as collagen, galactose, lamilin, peptides, or polymer films containing functional groups that are well-liked by cells, such as primary amines or carboxylic acids [92, 110-118]. A whole spectrum of cells is seeded on the coated textile scaffolds, ranging from osteoblasts and fibroblasts to endothelial cells, nerve cells and even stem cells. Discussing all of them again would lead to far, especially as the effects are similar to the histological performance of the coated PCL and PLLA textiles. Therefore the results have been summarized in table 1 .

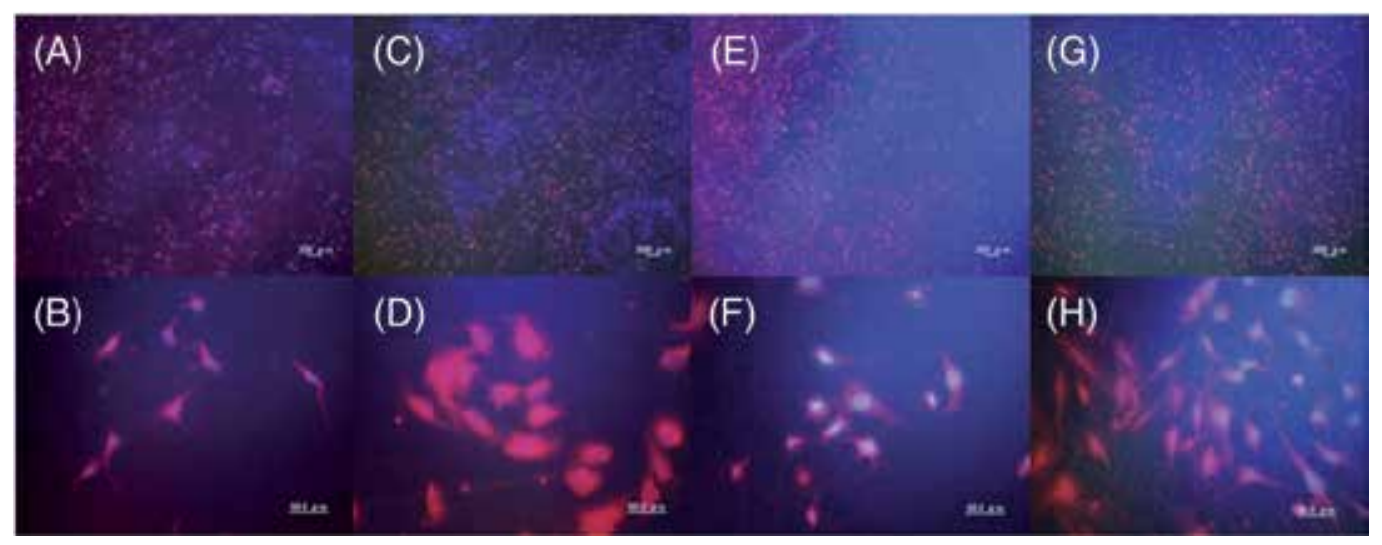

Figure 6. Fluorescent micrographs of HUVECs cultured on PU (A and B), plasma treated PU (P-PU) (C and D), P-PU/ PLGA (E and F), and plasma treated (P-PU/PLGA) films for $3 \mathrm{~s}(\mathrm{G}$ and $\mathrm{H})$. Cells were stained with Texas-Red Maleimide C2 for cell membrane and nuclei were stained with Hoechst33258. Images are 40× (A, C, E, and G) and 400× (B, D, $\mathrm{F}$, and $\mathrm{H}$ ) magnified [131].

\begin{tabular}{ccccccc}
\hline $\begin{array}{c}\text { Scaffold } \\
\text { material }\end{array}$ & $\begin{array}{c}\text { Plasma } \\
\text { treatment }\end{array}$ & $\begin{array}{c}\text { Deposited } \\
\text { coating }\end{array}$ & Cell line & $\begin{array}{c}\text { Histological } \\
\text { Effects }\end{array}$ & Authors & Reference \\
\hline \multirow{2}{*}{ Silk fibroin } & $\begin{array}{c}\text { RF Ar low } \\
\text { pressure }\end{array}$ & Heparin & $\begin{array}{c}\text { L929 } \\
\text { EVC }\end{array}$ & $\begin{array}{c}\text { Better } \\
\text { proliferation }\end{array}$ & Wang et al. & {$[110]$} \\
\hline \multirow{2}{*}{ PDMS } & RF Ar low & pNIPAm & aoSMC & $\begin{array}{c}\text { Smooth muscle } \\
\text { cell formation }\end{array}$ & $\begin{array}{c}\text { Rayatpishesh et } \\
\text { al. }\end{array}$ & {$[111]$} \\
\hline pressure & RF O low & Galactose & HepG2/C3A & $\begin{array}{c}\text { Improved } \\
\text { albumin secretion }\end{array}$ & Chien et al. & {$[112]$} \\
\hline
\end{tabular}




\begin{tabular}{|c|c|c|c|c|c|c|}
\hline $\begin{array}{l}\text { Scaffold } \\
\text { material }\end{array}$ & $\begin{array}{l}\text { Plasma } \\
\text { treatment }\end{array}$ & $\begin{array}{l}\text { Deposited } \\
\text { coating }\end{array}$ & Cell line & $\begin{array}{c}\text { Histological } \\
\text { Effects }\end{array}$ & Authors & Reference \\
\hline PCL + starch & $\begin{array}{l}\mathrm{RF} \mathrm{O}_{2} \text { low } \\
\text { pressure }\end{array}$ & $\begin{array}{l}\text { Vinyl sulphonic } \\
\text { \& phosphonic } \\
\text { acid }\end{array}$ & $\begin{array}{l}\text { Fibronectin } \\
\text { Vitronectin } \\
\text { Saos-2 }\end{array}$ & $\begin{array}{l}\text { Enhanced protein } \\
\text { adsorption \& } \\
\text { better cell growth } \\
\text { and proliferation }\end{array}$ & $\begin{array}{c}\text { López-Pérez et } \\
\text { al. }\end{array}$ & [113] \\
\hline PET & $\begin{array}{c}\mathrm{RF} \mathrm{C}_{2} \mathrm{H}_{4} \text { low } \\
\text { pressure }\end{array}$ & $\mathrm{NH}_{3}$ & HUVEC & $\begin{array}{c}\text { Better growth and } \\
\text { proliferation }\end{array}$ & Savoji et al. & [114] \\
\hline PES & $\begin{array}{l}\mathrm{MW}_{2} \text { low } \\
\text { pressure }\end{array}$ & Collagen & USSC & $\begin{array}{l}\text { Excellent } \\
\text { infiltration }\end{array}$ & Shabani et al. & [115] \\
\hline PS & $\mathrm{Ar}$ & Lamilin & $\begin{array}{l}\text { Smooth muscle } \\
\text { cells }\end{array}$ & $\begin{array}{c}\text { Enhanced } \\
\text { differentiated } \\
\text { phenotype }\end{array}$ & Baker et al. & [92] \\
\hline PSU & RF air cleaner & $\begin{array}{c}\text { Methacrylic acid } \\
\text { +F3GA }\end{array}$ & BSA & $\begin{array}{c}\text { Fast purification } \\
\text { small scale } \\
\text { proteins }\end{array}$ & Ma et al. & [116] \\
\hline Cellulose & APPJ Ar & f-Cyclodextrines & Fatty acids & $\begin{array}{c}\text { Excellent } \\
\text { inclusion \& no } \\
\text { cytotoxicity }\end{array}$ & Nada et al. & [117] \\
\hline PC-PU & $\begin{array}{l}\mathrm{RF} \mathrm{O}_{2} \text { low } \\
\text { pressure }\end{array}$ & PDMS & L929 & Cytocompatibility & Arjun et al. & [118] \\
\hline
\end{tabular}

Table 1. Overview of papers on the histological performance of plasma coated 3D electrospun scaffolds (no PCL and PLLA)

\subsection{Sutures}

Of all the textiles for biomedical applications, sutures are probably the most low-tech. The amount of research conducted to improve the performance of surgical sutures is therefore not as extensive. Traditionally sutures were either non-biodegradable, requiring removal afterwards or biodegradable, but lacking the necessary mechanical strength and flexibility [49]. Eventually glycolide and lactide polymers such as PLLA and PLGA found their way into the suture market, introducing the required mechanical properties combined with biodegradability. Yet, as has been discussed earlier in the chapter, these biodegradable polymers do not always exhibit the wanted bioactive surfaces. A small number of research groups have investigated if non-thermal plasma technology can help to further improve the performance of medical sutures, of which a brief overview will be given.

Loh et al. performed a study, using both activation and deposition, analyzing the hydrolytic degradation rate of commercially available synthetic absorbable sutures [119, 120]. Dexon (PGA), Vicryl (PGLA), PDS11 (PpDO) and Maxon were either coated with parylene or treated by a number of different plasma gasses. Using the right set of plasma treatment parameters significantly increased the degradation rate of Vicryl and PDS11, while for Dexon and Maxon 
only marginal differences were found. The plasma coating process, using parylene, resulted in an increase in tensile strength, most likely due to the hydrophobic character of the coating. Saxena et al. published 3 papers on plasma grafting of PP sutures [121-123]. In all the articles an $\mathrm{RF} \mathrm{O}_{2}$ plasma was used to activate the monofilament, followed by the immersion in an acrylic acid solution. The introduced carboxylic acid groups were then used to successfully immobilize chitosan. While the first 2 articles elaborates on the preservation of the mechanical properties and the surface chemical characterization, the second article focusses more on the antimicrobial, in-vitro and in-vivo properties. The viability of both E. Coli and S. Aureus were reduced with more than $90 \%$. The in-vitro studies revealed excellent adhesion and proliferation of MC3T3 cells and the in-vivo use in a rodent resulted in a better quality of tissue integration and a minimal inflammatory response. The grafting of acrylic acid onto a monofilament for improved antimicrobial functionality was inspired by Gupta at al. who grafted acrylic acid onto PET monofilaments, obtaining similar antimicrobial results [124, 125].
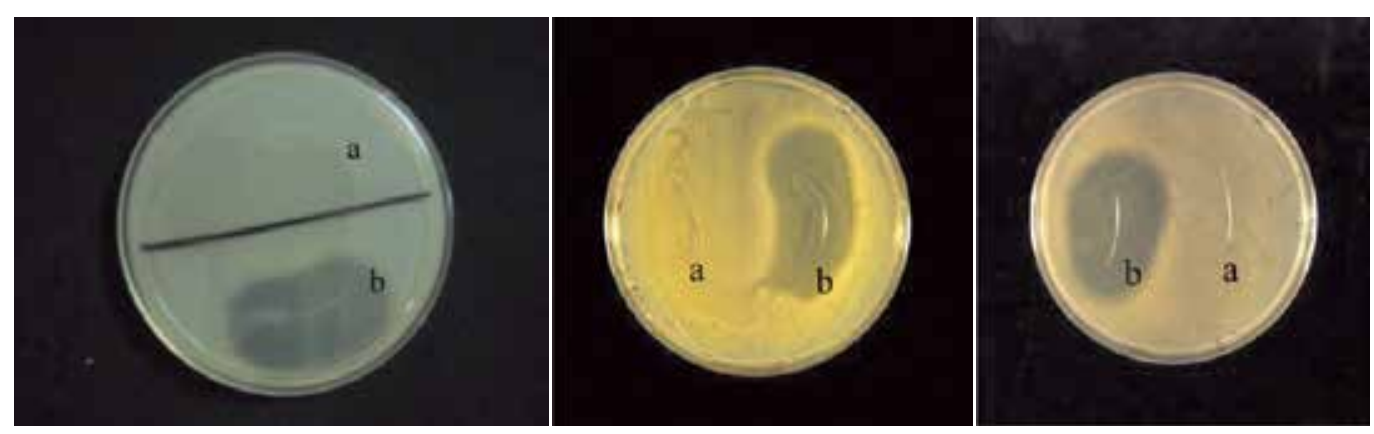

Figure 7. Left: Zone of inhibition against E. Coli (a) control PP suture and (b) drug-loaded PP suture (degree of grafting, 5\%). Middle: Zone of inhibition against K. Pneumonia (a) control PP suture and (b) drug-loaded PP suture (degree of grafting, 5\%). Right: Zone of inhibition against S. Aureus (a) control PP suture and (b) drug-loaded PP suture (degree of grafting, 5\%) [132].

Albeit being a rather low-tech application, plasma technology is still able to improve the performance of medical sutures. If the results found for tissue engineering applications would be applied for monofilament applications, it is beyond doubt that the biomedical properties could be further enhanced.

\section{Conclusion}

In this chapter a broad range of applications has been reviewed where non-thermal plasma technology could play a beneficial role in the biomedical performance of technical textiles. Albeit being more limited in the number of functional groups that can be incorporated and the limited stability over time, plasma activation still leads to improvements in cell viability, adhesion, proliferation and differentiation as well as better adsorption and chemical bonding of bioactive and bactericidal macromolecules. Plasma grafting and polymerization is equally able to do all of the above, while having access to a wider variety of functional groups and 
results in more stable surfaces. The incorporation of low molecular weight species into the coatings has to be avoided at all costs as they can have a detrimental effect on the cells viability. Up to now, the low pressure systems are by far the most used treatment systems, as the physics behind the process are well understood and multiple systems are commercially available. In the last decade there has been a growing interest in atmospheric pressure systems as they are more low-cost and can be more easily incorporated in textile production systems and it is our personal believe that atmospheric pressure systems such as the plasma jets will become the most prominently used set-ups. All in all it can be concluded that non-thermal plasma technology has earned its place in the (bio)medical textile market and will continue to do so in the future.

\section{Acknowledgements}

This chapter has received funding from the European Research Council under the European Union's Seventh Framework Program (FP/2007-2013) / ERC Grant Agreement n. 279022.

\section{Author details}

Pieter Cools, Rino Morent and Nathalie De Geyter*

*Address all correspondence to: nathalie.degeyter@ugent.be

Research Unit Plasma Technology, Department of Applied Physics, Ghent University, Ghent, Belgium

\section{References}

[1] R. Czajka, Development of medical textile market, Fibres \& Textiles in Eastern Europe $2005 ; 13(1), 49$.

[2] U. Vohrer, in: R. Shishoo (Ed.), Plasma technologies for textiles, Woodhead, Cambridge England, 2007.

[3] S. Agarwal, J.H. Wendorff, A. Greiner, Use of electrospinning technique for biomedical applications, Polymer 2008; 49 (26), 5603-5621.

[4] S. Agarwal, J.H. Wendorff, A. Greiner, Progress in the field of electrospinning for tissue engineering applications, Advanced Materials 2009; 21 (32-33), 3343-3351.

[5] Y. Ikada, Surface modification of polymers for medical applications, Biomaterials 1994; 15 (10), 725-736. 
[6] R.S. Benson, Use of radiation in biomaterials science, Nuclear Instruments and Methods in Physics Research Section B: Beam Interactions with Materials and Atoms 2002; 191 (1), 752-757.

[7] A.S. Hoffman, Macromolecular Symposia, Wiley Online Library, 1996, p. 443-454.

[8] T. Jacobs, N. De Geyter, R. Morent, S. Van Vlierberghe, P. Dubruel, C. Leys, Plasma modification of PET foils with different crystallinity, Surface and Coatings Technology 2011; 205 (Supplement 2), S511-S515.

[9] C. Tendero, C. Tixier, P. Tristant, J. Desmaison, P. Leprince, Atmospheric pressure plasmas: A review, Spectrochimica Acta Part B: Atomic Spectroscopy 2006; 61 (1), 2-30.

[10] N. De Geyter, Plasma modification of polymer surfaces in the subatmospheric pressure range, PhD, Applied Physics, Ghent University, Ghent, 2008.

[11] N. De Geyter, R. Morent, L. Gengembre, C. Leys, E. Payen, S. Van Vlierberghe, E. Schacht, Increasing the hydrophobicity of a PP film using a helium/CF4 DBD treatment at atmospheric pressure, Plasma Chemistry and Plasma Processing 2008; 28 (2), 289-298.

[12] C.-S. Ren, K. Wang, Q.-Y. Nie, D.-Z. Wang, S.-H. Guo, Surface modification of PE film by DBD plasma in air, Applied surface science 2008; 255 (5), 3421-3425.

[13] T. Desmet, R. Morent, N.D. Geyter, C. Leys, E. Schacht, P. Dubruel, Nonthermal plasma technology as a versatile strategy for polymeric biomaterials surface modification: a review, Biomacromolecules 2009; 10 (9), 2351-2378.

[14] R. Morent, N. De Geyter, T. Desmet, P. Dubruel, C. Leys, Plasma surface modification of biodegradable polymers: a review, Plasma Processes and Polymers 2011; 8 (3), 171-190.

[15] U. Kogelschatz, B. Eliasson, W. Egli, From ozone generators to flat television screens: history and future potential of dielectric-barrier discharges, Pure and Applied Chemistry 1999; 71 (10), 1819-1828.

[16] R. Morent, N. De Geyter, J. Verschuren, K. De Clerck, P. Kiekens, C. Leys, Non-thermal plasma treatment of textiles, Surface and Coatings Technology 2008; 202 (14), 3427-3449.

[17] T. Hammer, Non-thermal plasma application to the abatement of noxious emissions in automotive exhaust gases, Plasma Sources Science and Technology 2002; 11 (3A), A196.

[18] U. Kogelschatz, Atmospheric-pressure plasma technology, Plasma Physics and Controlled Fusion 2004; 46 (12B), B63. 
[19] D.B. Graves, M.J. Kushner, Influence of modeling and simulation on the maturation of plasma technology: Feature evolution and reactor design, Journal of Vacuum Science \& Technology A 2003; 21 (5), S152-S156.

[20] C.A. Castellan Patent 1949.

[21] H. Conrads, M. Schmidt, Plasma generation and plasma sources, Plasma Sources Science and Technology 2000; 9 (4), 441.

[22] A. Schutze, J.Y. Jeong, S.E. Babayan, J. Park, G.S. Selwyn, R.F. Hicks, The atmospheric-pressure plasma jet: a review and comparison to other plasma sources, Plasma Science, IEEE Transactions on 1998; 26 (6), 1685-1694.

[23] J. Hopwood, Review of inductively coupled plasmas for plasma processing, Plasma Sources Science and Technology 1992; 1 (2), 109.

[24] M. Laroussi, T. Akan, Arc-Free Atmospheric Pressure Cold Plasma Jets: A Review, Plasma Processes and Polymers 2007; 4 (9), 777-788.

[25] B.J. Park, D. Lee, J.-C. Park, I.-S. Lee, K.-Y. Lee, S. Hyun, M.-S. Chun, K.-H. Chung, Sterilization using a microwave-induced argon plasma system at atmospheric pressure, Physics of Plasmas (1994-present) 2003; 10 (11), 4539-4544.

[26] C. Larsson Wexell, P. Thomsen, B.-O. Aronsson, P. Tengvall, M. Rodahl, J. Lausmaa, B. Kasemo, L. Ericson, Bone Response to Surface-Modified Titanium Implants: Studies on the Early Tissue Response to Implants with Different Surface Characteristics, International Journal of Biomaterials 2013; 2013.

[27] M. Morra, C. Cassinelli, Evaluation of surface contamination of titanium dental implants by LV-SEM: comparison with XPS measurements, Surface and Interface Analysis 1997; 25 (13), 983-988.

[28] P. Cools, N. De Geyter, E. Vanderleyden, P. Dubruel, R. Morent, Surface Analysis of Titanium Cleaning and Activation Processes: Non-thermal Plasma Versus Other Techniques, Plasma Chemistry and Plasma Processing, 1-16.

[29] C. Constantine, D. Johnson, S. Pearton, U. Chakrabarti, A. Emerson, W. Hobson, A. Kinsella, Plasma etching of III-V semiconductors in $\mathrm{CH} 4 / \mathrm{H} 2 / \mathrm{Ar}$ electron cyclotron resonance discharges, Journal of Vacuum Science \& Technology B: Microelectronics and Nanometer Structures 1990; 8 (4), 596-606.

[30] F. Fracassi, R. d'Agostino, R. Lamendola, A. Filippo, C. Rapisarda, P. Vasquez, Plasma assisted dry etching of cobalt silicide for microelectronics applications, Journal of The Electrochemical Society 1996; 143 (2), 701-707.

[31] J. Lee, J. Hong, S. Pearton, Etching of InP at $\approx 1 \mu \mathrm{m} / \mathrm{min}$ in $\mathrm{Cl} 2 /$ Ar plasma chemistries, Applied physics letters 1996; 68 (6), 847-849.

[32] J.P. Chang, J.C. Arnold, G.C. Zau, H.-S. Shin, H.H. Sawin, Kinetic study of low energy argon ion-enhanced plasma etching of polysilicon with atomic/molecular chlor- 
ine, Journal of Vacuum Science \& Technology A: Vacuum, Surfaces, and Films 1997; 15 (4), 1853-1863.

[33] N.R. Washburn, K.M. Yamada, C.G. Simon Jr, S.B. Kennedy, E.J. Amis, Highthroughput investigation of osteoblast response to polymer crystallinity: influence of nanometer-scale roughness on proliferation, Biomaterials 2004; 25 (7), 1215-1224.

[34] M.T. Khorasani, H. Mirzadeh, S. Irani, Plasma surface modification of poly (1-lactic acid) and poly (lactic-co-glycolic acid) films for improvement of nerve cells adhesion, Radiation Physics and Chemistry 2008; 77 (3), 280-287.

[35] Z. Persin, M. Mozetic, A. Vesel, T. Maver, U. Maver, K.S. Kleinschek, Plasma Induced Hydrophilic Cellulose Wound Dressing, 2013.

[36] S. Mattioli, J. Kenny, I. Armentano, Plasma surface modification of porous PLLA films: analysis of surface properties and in vitro hydrolytic degradation, Journal of Applied Polymer Science 2012; 125 (S2), E239-E247.

[37] T. Jacobs, H. Declercq, N. De Geyter, R. Cornelissen, P. Dubruel, C. Leys, A. Beaurain, E. Payen, R. Morent, Plasma surface modification of polylactic acid to promote interaction with fibroblasts, Journal of Materials Science: Materials in Medicine 2013; 24 (2), 469-478.

[38] C. Erfurt-Berge, R. Renner, Recent Developments in Topical Wound Therapy: Impact of Antimicrobiological Changes and Rebalancing the Wound Milieu, BioMed research international 2014; 2014.

[39] Z. Peršin, U. Maver, T. Pivec, T. Maver, A. Vesel, M. Mozetič, K. Stana-Kleinschek, Novel cellulose based materials for safe and efficient wound treatment, Carbohydrate polymers $2014 ; 100,55-64$.

[40] Z. Persin, K.S. Kleinschek, Ammonia plasma treatment as a method promoting simultaneous hydrophilicity and antimicrobial activity of viscose wound dressings, Textile Research Journal 2013, 0040517513485631.

[41] C. Hacker, Z. Karahaliloglu, G. Seide, E.B. Denkbas, T. Gries, Functionally modified, melt-electrospun thermoplastic polyurethane mats for wound-dressing applications, Journal of Applied Polymer Science 2014; 131 (8).

[42] R. Nawalakhe, Q. Shi, N. Vitchuli, J. Noar, J.M. Caldwell, F. Breidt, M.A. Bourham, X. Zhang, M.G. McCord, Novel atmospheric plasma enhanced chitosan nanofiber/ gauze composite wound dressings, Journal of Applied Polymer Science 2013; 129 (2), 916-923.

[43] R.G. Nawalakhe, Novel Atmospheric Plasma Enhanced Nanofiber/Gauze Composite Wound Dressings, North Carolina State University, 2012.

[44] J.-P. Chen, W.-L. Lee, Collagen-grafted temperature-responsive nonwoven fabric for wound dressing, Applied Surface Science 2008; 255 (2), 412-415. 
[45] J.-P. Chen, C.-Y. Kuo, W.-L. Lee, Thermo-responsive wound dressings by grafting chitosan and poly $(<\mathrm{i}>\mathrm{N}</ \mathrm{i}>$-isopropylacrylamide) to plasma-induced graft polymerization modified non-woven fabrics, Applied Surface Science 2012; 262, 95-101.

[46] F.-H. Lin, T.-M. Chen, K.-S. Chen, T.-H. Wu, C.-C. Chen, An animal study of a novel tri-layer wound dressing material-non-woven fabric grafted with $<\mathrm{i}>\mathrm{N}</ \mathrm{i}>$-isopropyl acrylamide and gelatin, Materials chemistry and physics 2000; 64 (3), 189-195.

[47] J. Phair, C.P. Leach, M.F. Cardosi, J. Davis, Atmospheric pressure plasma treated carbon fibre weave: A flexible approach to wound monitoring, Electrochemistry Communications 2013; 33, 99-101.

[48] J. Zhou, T.N. Tun, S.-h. Hong, J.D. Mercer-Chalmers, M. Laabei, A.E. Young, A.T.A. Jenkins, Development of a prototype wound dressing technology which can detect and report colonization by pathogenic bacteria, Biosensors and Bioelectronics 2011; $30(1), 67-72$.

[49] S. Rajendran, S. Anand, Developments in medical textiles, Textile progress 2002; 32 (4), 1-42.

[50] M. Gorjanc, V. Bukošek, M. Gorenšek, M. Mozetič, CF4 plasma and silver functionalized cotton, Textile research journal 2010, 0040517510376268.

[51] Q. Shi, N. Vitchuli, J. Nowak, J.M. Caldwell, F. Breidt, M. Bourham, X. Zhang, M. McCord, Durable antibacterial Ag/polyacrylonitrile (Ag/PAN) hybrid nanofibers prepared by atmospheric plasma treatment and electrospinning, European polymer journal 2011; 47 (7), 1402-1409.

[52] L. Fras, T. Ristić, T. Tkavc, Adsorption and antibacterial activity of soluble and precipitated chitosan on cellulose viscose fibers, Journal of Engineered Fibers and Fabrics 2012; 7 (1).

[53] M.-R. Yang, K.-S. Chen, J.-C. Tsai, C.-C. Tseng, S.-F. Lin, The antibacterial activities of hydrophilic-modified nonwoven PET, Materials Science and Engineering: C 2002; 20 (1), 167-173.

[54] M. Kostić, N. Radić, B.M. Obradović, S. Dimitrijević, M.M. Kuraica, P. Škundrić, Antimicrobial textile prepared by silver deposition on dielectric barrier discharge treated cotton/polyester fabric, Chemical Industry and Chemical Engineering Quarterly 2008; 14 (4), 219-221.

[55] M. Kostić, N. Radić, B.M. Obradović, S. Dimitrijević, M.M. Kuraica, P. Škundrić, Silver-Loaded Cotton/Polyester Fabric Modified by Dielectric Barrier Discharge Treatment, Plasma Processes and Polymers 2009; 6 (1), 58-67.

[56] N. Radić, B.M. Obradović, M. Kostić, B. Dojčinović, M.M. Kuraica, M. Černák, Deposition of silver ions onto DBD and DCSBD plasma treated nonwoven polypropylene, Surface and Coatings Technology 2012; 206 (23), 5006-5011. 
[57] A. Kramar, V. Prysiazhnyi, B. Dojčinović, K. Mihajlovski, B. Obradović, M. Kuraica, M. Kostić, Antimicrobial viscose fabric prepared by treatment in DBD and subsequent deposition of silver and copper ions-Investigation of plasma aging effect, Surface and Coatings Technology 2013; 234, 92-99.

[58] K. Bazaka, M.V. Jacob, R.J. Crawford, E.P. Ivanova, Plasma-assisted surface modification of organic biopolymers to prevent bacterial attachment, Acta biomaterialia 2011; 7 (5), 2015-2028.

[59] E. Chadeau, N. Oulahal, L. Dubost, F. Favergeon, P. Degraeve, Anti- $<\mathrm{i}>$ Listeria innocua $</ \mathrm{i}>$ activity of silver functionalised textile prepared with plasma technology, Food control 2010; 21 (4), 505-512.

[60] V. Ilic, Z. Saponjić, V. Vodnik, S.a. Lazović, S. Dimitrijevic, P. Jovancic, J.M. Nedeljkovic, M. Radetic, Bactericidal efficiency of silver nanoparticles deposited onto radio frequency plasma pretreated polyester fabrics, Industrial \& Engineering Chemistry Research 2010; 49 (16), 7287-7293.

[61] M. Radetić, V. Ilić, V. Vodnik, S. Dimitrijević, P. Jovančić, Z. Šaponjić, J.M. Nedeljković, Antibacterial effect of silver nanoparticles deposited on corona-treated polyester and polyamide fabrics, Polymers for advanced technologies 2008; 19 (12), 1816-1821.

[62] M. Radetić, Functionalization of textile materials with silver nanoparticles, Journal of Materials Science 2013; 48 (1), 95-107.

[63] S. Lischer, E. Körner, D.J. Balazs, D. Shen, P. Wick, K. Grieder, D. Haas, M. Heuberger, D. Hegemann, Antibacterial burst-release from minimal Ag-containing plasma polymer coatings, Journal of The Royal Society Interface 2011; 8 (60), 1019-1030.

[64] S. Shahidi, A. Rashidi, M. Ghoranneviss, A. Anvari, M. Rahimi, M.B. Moghaddam, J. Wiener, Investigation of metal absorption and antibacterial activity on cotton fabric modified by low temperature plasma, Cellulose 2010; 17 (3), 627-634.

[65] J.D. Schiffman, Y. Wang, E.P. Giannelis, M. Elimelech, Biocidal activity of plasma modified electrospun polysulfone mats functionalized with polyethyleneimine-capped silver nanoparticles, Langmuir 2011; 27 (21), 13159-13164.

[66] C. Yao, X. Li, K. Neoh, Z. Shi, E. Kang, Surface modification and antibacterial activity of electrospun polyurethane fibrous membranes with quaternary ammonium moieties, Journal of Membrane Science 2008; 320 (1), 259-267.

[67] K. Vaideki, S. Jayakumar, R. Rajendran, G. Thilagavathi, Investigation on the effect of RF air plasma and neem leaf extract treatment on the surface modification and antimicrobial activity of cotton fabric, Applied surface science 2008; 254 (8), 2472-2478.

[68] D. Nithyakalyani, T. Ramachandran, R. Rajendran, M. Mahalakshmi, Assessment of antibacterial activity of herbal finished surface modified polypropylene nonwoven 
fabric against bacterial pathogens of wound, Journal of Applied Polymer Science 2013; 129 (2), 672-681.

[69] S. Strnad, O. Šauperl, L. Fras-Zemljič, Cellulose Fibres Functionalised by Chitosan: Characterization and Application, ISBN, 978-953.

[70] A. Uygun, M. Kiristi, L. Oksuz, S. Manolache, S. Ulusoy, RF hydrazine plasma modification of chitosan for antibacterial activity and nanofiber applications, Carbohydrate research 2011; 346 (2), 259-265.

[71] C. Canal, F. Gaboriau, S. Villeger, U. Cvelbar, A. Ricard, Studies on antibacterial dressings obtained by fluorinated post-discharge plasma, International journal of pharmaceutics 2009; 367 (1), 155-161.

[72] R.K. Virk, G.N. Ramaswamy, M. Bourham, B.L. Bures, Plasma and antimicrobial treatment of nonwoven fabrics for surgical gowns, Textile research journal 2004; 74 (12), 1073-1079.

[73] S. Degoutin, M. Jimenez, M. Casetta, S. Bellayer, F. Chai, N. Blanchemain, C. Neut, I. Kacem, M. Traisnel, B. Martel, Anticoagulant and antimicrobial finishing of non-woven polypropylene textiles, Biomedical Materials 2012; 7 (3), 035001.

[74] S. Gawish, S. Matthews, D. Wafa, F. Breidt, M. Bourham, Atmospheric plasma-aided biocidal finishes for nonwoven polypropylene fabrics. I. Synthesis and characterization, Journal of applied polymer science 2007; 103 (3), 1900-1910.

[75] S. Agarwal, A. Greiner, J.H. Wendorff, Functional Materials by Electrospinning of polymers, Progress in Polymer Science 2013 (0).

[76] B.D. Ulery, L.S. Nair, C.T. Laurencin, Biomedical applications of biodegradable polymers, Journal of Polymer Science Part B: Polymer Physics 2011; 49 (12), 832-864.

[77] C. Cheng, Z. Liye, R.-J. Zhan, Surface modification of polymer fibre by the new atmospheric pressure cold plasma jet, Surface and Coatings Technology 2006; 200 (24), 6659-6665.

[78] Q. Cheng, B.L.-P. Lee, K. Komvopoulos, Z. Yan, S. Li, Plasma surface chemical treatment of electrospun poly (L-lactide) microfibrous scaffolds for enhanced cell adhesion, growth, and infiltration, Tissue Engineering Part A 2013; 19 (9-10), 1188-1198.

[79] A. Sparavigna, Plasma treatment advantages for textiles, arXiv preprint arXiv: 0801.3727 2008.

[80] M.P. Prabhakaran, J. Venugopal, C.K. Chan, S. Ramakrishna, Surface modified electrospun nanofibrous scaffolds for nerve tissue engineering, Nanotechnology 2008; 19 (45), 455102.

[81] D. Yan, J. Jones, X. Yuan, X. Xu, J. Sheng, J.M. Lee, G. Ma, Q. Yu, Plasma treatment of electrospun PCL random nanofiber meshes (NFMs) for biological property improvement, Journal of Biomedical Materials Research Part A 2013; 101 (4), 963-972. 
[82] A. Martins, E.D. Pinho, S. Faria, I. Pashkuleva, A.P. Marques, R.L. Reis, N.M. Neves, Surface modification of electrospun polycaprolactone nanofiber meshes by plasma treatment to enhance biological performance, Small 2009; 5 (10), 1195-1206.

[83] S.K. Min, S.M. Jung, S.H. Kim, C.R. Kim, H.S. Shin, Implications of the oxygenated electrospun poly ( $\varepsilon$-caprolactone) nanofiber for the astrocytes activities, Journal of Biomedical Materials Research Part B: Applied Biomaterials 2013; 101 (7), 1267-1274.

[84] D. Yan, J. Jones, H. Li, J.C. Lee, Q. Yu, X. Yuan, J. Sheng, G. Ma, Plasma Surface Modification of Electrospun Poly ( $\varepsilon$-Caprolactone) Nanofibers and its Effect on Surface Bioactivity.

[85] H.J. Jeon, H. Lee, G.H. Kim, Nano-Sized Surface Patterns on Electrospun Microfibers Fabricated Using a Modified Plasma Process for Enhancing Initial Cellular Activities, Plasma Processes and Polymers 2014; 11 (2), 142-148.

[86] B. Blackstone, J. Willard, C. Lee, M. Nelson, R. Hart, J. Lannutti, H. Powell, Plasma surface modification of electrospun fibers for adhesion-based cancer cell sorting, Integrative Biology 2012; 4 (9), 1112-1121.

[87] K.E. Park, K.Y. Lee, S.J. Lee, W.H. Park, Macromolecular Symposia, Wiley Online Library, 2007, p. 103-108.

[88] H. Park, K.Y. Lee, S.J. Lee, K.E. Park, W.H. Park, Plasma-treated poly (lactic-co-glycolic acid) nanofibers for tissue engineering, Macromolecular Research 2007; 15 (3), 238-243.

[89] W. Liu, J. Zhan, Y. Su, T. Wu, C. Wu, S. Ramakrishna, X. Mo, S.S. Al-Deyab, M. ElNewehy, Effects of plasma treatment to nanofibers on initial cell adhesion and cell morphology, Colloids and Surfaces B: Biointerfaces 2014; 113, 101-106.

[90] L.S. Dolci, S.D. Quiroga, M. Gherardi, R. Laurita, A. Liguori, P. Sanibondi, A. Fiorani, L. Calzà, V. Colombo, M.L. Focarete, Carboxyl Surface Functionalization of Poly (Llactic acid) Electrospun Nanofibers through Atmospheric Non-Thermal Plasma Affects Fibroblast Morphology, Plasma Processes and Polymers 2014; 11 (3), 203-213.

[91] A.R. Chandrasekaran, J. Venugopal, S. Sundarrajan, S. Ramakrishna, Fabrication of a nanofibrous scaffold with improved bioactivity for culture of human dermal fibroblasts for skin regeneration, Biomedical Materials 2011; 6 (1), 015001.

[92] S.C. Baker, J. Southgate, Towards control of smooth muscle cell differentiation in synthetic 3D scaffolds, Biomaterials 2008; 29 (23), 3357-3366.

[93] C. Zandén, M. Voinova, J. Gold, D. Mörsdorf, I. Bernhardt, J. Liu, Surface characterisation of oxygen plasma treated electrospun polyurethane fibres and their interaction with red blood cells, European Polymer Journal 2012; 48 (3), 472-482. 
[94] F. Yang, J. Wolke, J. Jansen, Biomimetic calcium phosphate coating on electrospun poly ( $\varepsilon$-caprolactone) scaffolds for bone tissue engineering, Chemical Engineering Journal 2008; 137 (1), 154-161.

[95] H. Luo, G. Xiong, K. Ren, S.R. Raman, Z. Liu, Q. Li, C. Ma, D. Li, Y. Wan, Air DBD plasma treatment on three-dimensional braided carbon fiber-reinforced PEEK composites for enhancement of $<\mathrm{i}>$ in vitro $</ \mathrm{i}>$ bioactivity, Surface and Coatings Technology $2014 ; 242,1-7$.

[96] J. Venugopal, S. Low, A.T. Choon, A.B. Kumar, S. Ramakrishna, Electrospun-modified nanofibrous scaffolds for the mineralization of osteoblast cells, Journal of biomedical materials research Part A 2008; 85 (2), 408-417.

[97] K. Fujihara, M. Kotaki, S. Ramakrishna, Guided bone regeneration membrane made of polycaprolactone/calcium carbonate composite nano-fibers, Biomaterials 2005; 26 (19), 4139-4147.

[98] A. Guex, A. Frobert, J. Valentin, G. Fortunato, D. Hegemann, S. Cook, T. Carrel, H. Tevaearai, M. Giraud, Plasma-functionalized electrospun matrix for biograft development and cardiac function stabilization, Acta biomaterialia 2014; 10 (7), 2996-3006.

[99] N.E. Zander, J.A. Orlicki, A.M. Rawlett, T.P. Beebe Jr, Quantification of protein incorporated into electrospun polycaprolactone tissue engineering scaffolds, ACS applied materials \& interfaces 2012; 4 (4), 2074-2081.

[100] J. Xie, P.L. Michael, S. Zhong, B. Ma, M.R. MacEwan, C.T. Lim, Mussel inspired protein-mediated surface modification to electrospun fibers and their potential biomedical applications, Journal of Biomedical Materials Research Part A 2012; 100 (4), 929-938.

[101] Z. Ma, W. He, T. Yong, S. Ramakrishna, Grafting of gelatin on electrospun poly (caprolactone) nanofibers to improve endothelial cell spreading and proliferation and to control cell orientation, Tissue engineering 2005; 11 (7-8), 1149-1158.

[102] D. Hegemann, B. Hanselmann, S. Guimond, G. Fortunato, M.-N. Giraud, A.G. Guex, Considering the degradation effects of amino-functional plasma polymer coatings for biomedical application, Surface and Coatings Technology 2014.

[103] Z.-Q. Feng, H.-J. Lu, M.K. Leach, N.-P. Huang, Y.-C. Wang, C.-J. Liu, Z.-Z. Gu, The influence of type-I collagen-coated PLLA aligned nanofibers on growth of blood outgrowth endothelial cells, Biomedical Materials 2010; 5 (6), 065011.

[104] J.R.J. Paletta, S. Bockelmann, A. Walz, C. Theisen, J.H. Wendorff, A. Greiner, S. Fuchs-Winkelmann, M.D. Schofer, RGD-functionalisation of PLLA nanofibers by surface coupling using plasma treatment: influence on stem cell differentiation, Journal of Materials Science: Materials in Medicine 2010; 21 (4), 1363-1369. 
[105] Q. Cheng, K. Komvopoulos, S. Li, Plasma-assisted heparin conjugation on electrospun poly (l-lactide) fibrous scaffolds, Journal of Biomedical Materials Research Part A 2014; 102 (5), 1408-1414.

[106] B.J. Park, H.J. Seo, J. Kim, H.-L. Kim, J.K. Kim, J.B. Choi, I. Han, S.O. Hyun, K.-H. Chung, J.-C. Park, Cellular responses of vascular endothelial cells on surface modified polyurethane films grafted electrospun PLGA fiber with microwave-induced plasma at atmospheric pressure, Surface and Coatings Technology 2010; 205, Supplement 1 (0), S222-S226.

[107] K. Park, H.J. Jung, J.-J. Kim, K.-D. Ahn, D.K. Han, Y.M. Ju, Acrylic acid-grafted hydrophilic electrospun nanofibrous poly (L-lactic acid) scaffold, Macromolecular Research 2006; 14 (5), 552-558.

[108] W. He, Z. Ma, T. Yong, W.E. Teo, S. Ramakrishna, Fabrication of collagen-coated biodegradable polymer nanofiber mesh and its potential for endothelial cells growth, $\mathrm{Bi}$ omaterials 2005; 26 (36), 7606-7615.

[109] C.K. Chan, S. Liao, B. Li, R.R. Lareu, J.W. Larrick, S. Ramakrishna, M. Raghunath, Early adhesive behavior of bone-marrow-derived mesenchymal stem cells on collagen electrospun fibers, Biomedical materials 2009; 4 (3), 035006.

[110] S. Wang, Y. Zhang, H. Wang, Z. Dong, Preparation, characterization and biocompatibility of electrospinning heparin-modified silk fibroin nanofibers, International journal of biological macromolecules 2011; 48 (2), 345-353.

[111] S. Rayatpisheh, D.E. Heath, A. Shakouri, P.-O. Rujitanaroj, S.Y. Chew, M.B. ChanPark, Combining cell sheet technology and electrospun scaffolding for engineered tubular, aligned, and contractile blood vessels, Biomaterials 2014; 35 (9), 2713-2719.

[112] H.-W. Chien, J.-Y. Lai, W.-B. Tsai, Galactosylated electrospun membranes for hepatocyte sandwich culture, Colloids and Surfaces B: Biointerfaces 2014; 116, 576-581.

[113] P.M. López-Pérez, R.M. Da Silva, R.A. Sousa, I. Pashkuleva, R.L. Reis, Plasma-induced polymerization as a tool for surface functionalization of polymer scaffolds for bone tissue engineering: An in vitro study, Acta biomaterialia 2010; 6 (9), 3704-3712.

[114] H. Savoji, A. Hadjizadeh, M. Maire, A. Ajji, M.R. Wertheimer, S. Lerouge, Electrospun Nanofiber Scaffolds and Plasma Polymerization: A Promising Combination Towards Complete, Stable Endothelial Lining for Vascular Grafts, Macromolecular bioscience 2014 .

[115] I. Shabani, V. Haddadi-Asl, E. Seyedjafari, F. Babaeijandaghi, M. Soleimani, Improved infiltration of stem cells on electrospun nanofibers, Biochemical and biophysical research communications 2009; 382 (1), 129-133.

[116] Z. Ma, K. Masaya, S. Ramakrishna, Immobilization of Cibacron blue F3GA on electrospun polysulphone ultra-fine fiber surfaces towards developing an affinity membrane for albumin adsorption, Journal of membrane science 2006; 282 (1), 237-244. 
[117] A.A. Nada, P. Hauser, S.M. Hudson, The Grafting of Per-(2, 3, 6-O-allyl)- $\beta$ Cyclodextrin onto Derivatized Cotton Cellulose via Thermal and Atmospheric Plasma Techniques, Plasma Chemistry and Plasma Processing 2011; 31 (4), 605-621.

[118] G. Arjun, G. Menon, P. Ramesh, Plasma surface modification of fibroporous polycarbonate urethane membrane by polydimethyl siloxane: Structural characterization, mechanical properties, and in vitro cytocompatibility evaluation, Journal of Biomedical Materials Research Part A 2014; 102 (4), 947-957.

[119] I.H. Loh, H.L. Lin, C. Chu, Plasma surface modification of synthetic absorbable sutures, Journal of Applied Biomaterials 1992; 3 (2), 131-146.

[120] L. Zhang, C. Chu, I.H. Loh, Effect of a combined gamma irradiation and parylene plasma treatment on the hydrolytic degradation of synthetic biodegradable sutures, Journal of biomedical materials research 1993; 27 (11), 1425-1441.

[121] S. Saxena, A.R. Ray, A. Kapil, G. Pavon-Djavid, D. Letourneur, B. Gupta, A. Meddahi-Pellé, Development of a New Polypropylene-Based Suture: Plasma Grafting, Surface Treatment, Characterization, and Biocompatibility Studies, Macromolecular bioscience 2011; 11 (3), 373-382.

[122] S. Saxena, A.R. Ray, B. Gupta, Chitosan immobilization on polyacrylic acid grafted polypropylene monofilament, Carbohydrate Polymers 2010; 82 (4), 1315-1322.

[123] S. Saxena, A.R. Ray, B. Gupta, Graft polymerization of acrylic acid onto polypropylene monofilament by RF plasma, Journal of applied polymer science 2010; 116 (5), 2884-2892.

[124] B. Gupta, A. Srivastava, N. Grover, S. Saxena, Plasma induced graft polymerization of acrylic acid onto poly (ethylene terephthalate) monofilament, Indian journal of fibre \& textile research 2010; 35 (1), 9.

[125] B. Gupta, R. Jain, H. Singh, Preparation of antimicrobial sutures by preirradiation grafting onto polypropylene monofilament, Polymers for Advanced Technologies 2008; 19 (12), 1698-1703.

[126] Reprinted from Applied Surface Science, 2008. 255(2): p. 412-415. Chen, J.-P. and W.L. Lee., Collagen-grafted temperature-responsive nonwoven fabric for wound dressing., Copyright (c) 2008, with permission from Elsevier.

[127] Reprinted from Journal of Membrane Science, 2008. 320(1): p. 259-267 Yao, C., et al., Surface modification and antibacterial activity of electrospun polyurethane fibrous membranes with quaternary ammonium moieties., Copyright (c) 2008, with permission from Elsevier.

[128] Reprinted from Surface and Coatings Technology, 2007. 249-250: 103-108 Park, K.E., et al, Surface Characteristics of Plasma-Treated PLGA Nanofibers. in Macromolecular Symposia., Copyright (C) 2007 WILEY-VCH Verlag GmbH \& Co. KGaA, Weinheim 
[129] Reprinted from Colloids and Surfaces B: Biointerfaces, 2014. 113: p. 101-106 De Geyter, Liu, W., et al., Effects of plasma treatment to nanofibers on initial cell adhesion and cell morphology., Copyright (C) 2014, with permission from Elsevier.

[130] Reprinted from Journal of biomedical materials research Part A, 2008. 85(2): p. 408-417 De Venugopal, J., et al., Electrospun-modified nanofibrous scaffolds for the mineralization of osteoblast cells., Copyright (c) 2007 Wiley Periodicals, Inc..

[131] Reprinted from Surface and Coatings Technology, 2010. 205, Supplement 1(0): p. S222-S226 Park, B.J., et al., Cellular responses of vascular endothelial cells on surface modified polyurethane films grafted electrospun PLGA fiber with microwave-induced plasma at atmospheric pressure., Copyright (C) 2010, with permission from Elsevier.

[132] Reprinted from Polymers for Advanced Technologies, 19(12): p. 1698-1703. Gupta, B., R. Jain, and H. Singh, Preparation of antimicrobial sutures by preirradiation grafting onto polypropylene monofilament., Copyright (c) 2008 John Wiley \& Sons, Ltd. 
Biosensors, Electronics and Telemetry 



\title{
Chapter 6
}

\section{Electronic Sensor Interfaces With Wireless Telemetry}

\author{
Ifana Mahbub, Farhan Quaiyum, \\ Md Sakib Hasan and SyedKamrul Islam \\ Additional information is available at the end of the chapter \\ http://dx.doi.org/10.5772/59754
}

\section{Introduction}

Vital information monitoring has become an indispensable part of the next generation healthcare technologies. Remote monitoring of the vital health information facilitates personal in-home care, reduces the cost and time of frequently going to the hospitals and minimizes the difficulties of monitoring the health of the elderly persons. Recent research on contemporary implantable and wearable sensors for monitoring various physiological parameters as well as improvement of wireless technology have led to the development of all-inclusive patient monitoring systems such as Wireless Body Area Network (WBAN) and Body Sensor Network (BSN). One of the integral parts of these networks is implantable sensor. Applications of implantable sensors include (but not limited to) monitoring of blood glucose level for diabetic patients, continuous in vivo monitoring of lactose in the bloodstream or tissues, pressure monitoring of blood vessels and electronic interfaces to monitor the nervous system. Monitoring of physiological parameters such as $\mathrm{pH}$ level in tissues, glucose and lactose in bloodstreams, heart rate and respiration rate not only improves the quality of life of the patients but also increases their lifespan. Even though astounding advancements have been made in medical electronics and instrumentation, invasive medical devices such as small lancets are still used to collect samples from human body for testing and diagnostic purposes. These devices increase the risk of infection in human body. For a diabetic patient the discrete measurements provided by the lancets are not sufficient for monitoring of blood glucose level. In order to get an idea of the blood glucose trend line, continuous monitoring of glucose level is highly desirable and minimally invasive implantable sensors are ideal fit for this application. The most important concern related to the use of implantable sensor is the health safety of the patients. The true success of an implant depends on the proper functioning of the sensor without having any adverse effect on the tissues surrounding the implant. 


\section{Implantable sensors for biomedical applications}

Recent developments in biomedical sensors and state-of-the-art CMOS technologies have led to the realization of minimally invasive implantable biomedical sensors for continuous monitoring of the patients. A continuous monitoring system allows the doctors to investigate the medical data of the patient online and thus provides savings in both time and money. The data acquired by the sensor from frequent monitoring also helps the hospital to efficiently record the medical history of a patient for future references. Figure 1 represents a detailed implantable sensor system with the combination of different biosensors and integrated circuits (ICs) which are designed to be implanted underneath the skin. Sub-micron CMOS technologies offer various advantages such as small form factor and reliable operation which make them very suitable for implantable medical applications. Biosensors on this platform include glucose, lactose, oxygen and $\mathrm{pH}$ sensors, etc. The state of the art research on implantable biosensor system focuses on its small form factor and light-weight for easy integration and biological safety [1]. Even though the development of deep sub-micron CMOS processes has significantly brought down the overall chip area, still the power unit such as lithium ion battery takes up a substantial area of the overall system. Therefore elimination of the battery as the power source can potentially reduce the system area significantly. Batteries also impose a potential risk of leakage which might result in serious health hazards to the patient and require periodic replacement. An eco-friendly solution of this potential problem involves the development of more efficient wireless powering methods or the design of low-power ICs. In contrast to a battery operated system, wireless powered system eliminates the hassle of frequent replacement of the power source and can be considered as a minimally invasive option with no risk of infection [1] [2]. Previously reported works present the use of inductive coupling (i.e. inductive link) [1] and optical coupling (i.e. solar cells) [2] as potential wireless powering approaches. A noninvasive, reliable, and efficient power supply along with a reliable data communication interface is a potentially important feature that an implantable sensor must possess.

A biosensor usually consists of an electrochemical sensor which generates an electrical signal that corresponds to the concentration of a particular electrolyte. Readout electronics such as potentiostats (amperometric or voltametric electrochemical sensors) help maintain a constant potential difference between two electrodes to facilitate the chemical reaction to take place and provide output in the form of a current (amperometric) or a voltage (voltametric) signal. A typical potentiostat consists of three electrodes: a working electrode (WE), a counter electrode $(\mathrm{CE})$, and a reference electrode (RE). The potential difference between the working and the reference electrodes stimulates the chemical reaction and the counter electrode provides the corresponding output current signal, which is then delivered to the signal processing unit (SPU). The SPU modulates the data so that it can be transmitted outside of the body wirelessly to a receiver, which can be a smart phone or a similar electronic device. Peripheral ICs such as power supply units, sensor activation circuits etc. are also integrated together to realize this scheme on a system-on-a-chip (SoC) platform. 


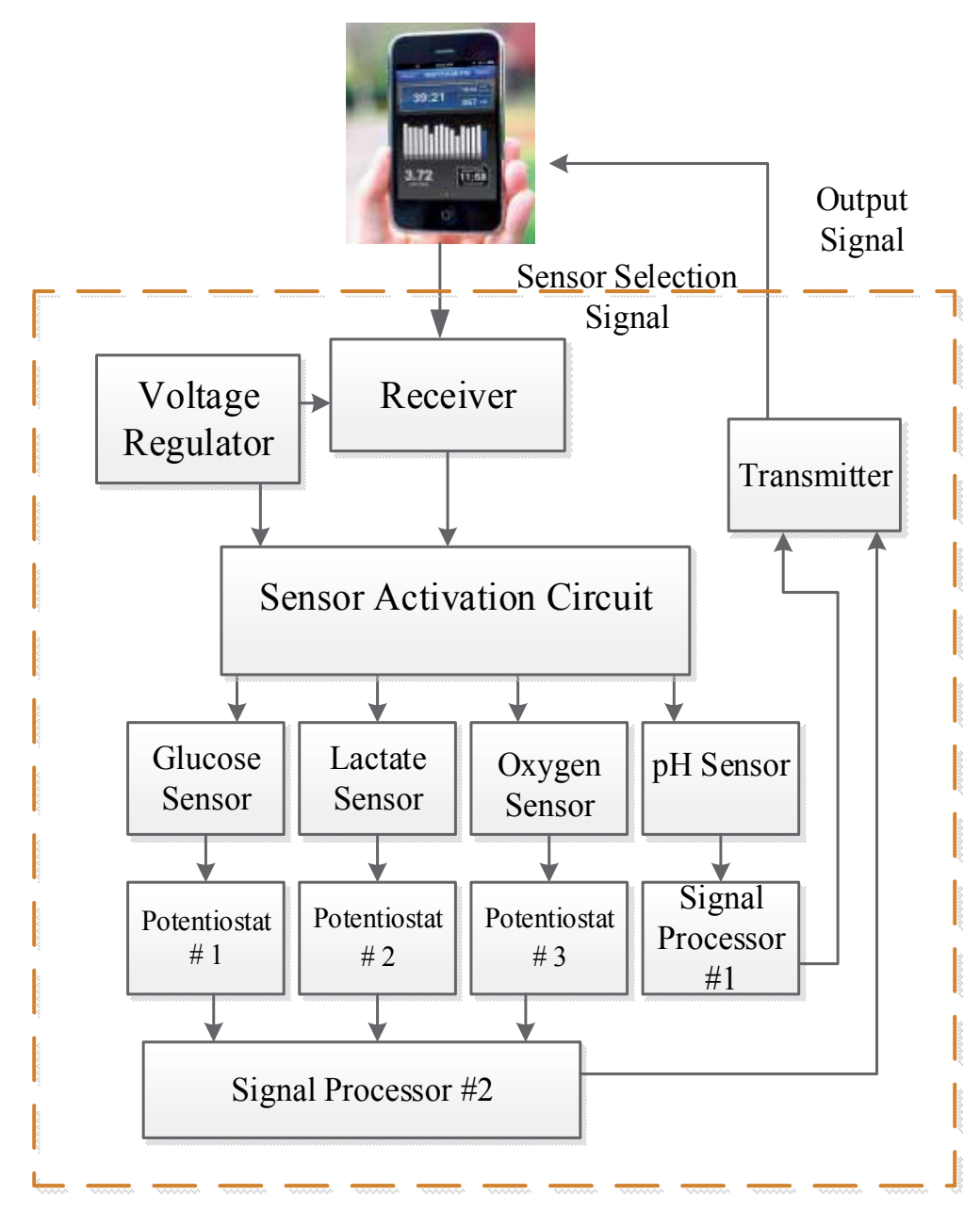

Figure 1. Implantable biosensor system (inside the dashed red rectangle) [3].

\section{Low-power circuit design techniques}

Even though the amplitude of the signal generated by the implantable biosensors depends on the concentration of various physiological parameters, nonetheless the signal needs to be amplified and converted to a digital signal for further processing. Low-voltage and low-power circuit design techniques are required to be employed in the design of the signal processing circuitry of the implantable devices for long-term reliable operations. Depending on the application the most appropriate circuit topology needs to be chosen to meet the design challenges. Various innovative low-power circuit design methodologies have appeared in literatures to meet the requirement of long term operations. Their working principles are discussed in the following sections. 
From the perspective of a circuit designer, employing of short-channel transistors is an appealing and efficient method to reduce the SoC chip area. The added advantage of these transistors is that the power supply voltage also downscales proportionally with the reduction of the transistor channel length. Thus hot electron effect and time-dependent dielectric breakdown (TDDB) [4] do not deteriorate the robustness and reliability of the devices. However, the threshold voltage, $\mathrm{V}_{\mathrm{T}}$ of the MOSFET does not scale down as aggressively as the channel length or the power supply voltage [5], which puts a constraint on the number of transistors that can be cascaded in a given process. Some of the techniques for low-power circuit design include bulk-or body-driven technique, floating gate technique, subthreshold biasing scheme of the transistors etc. The following sections summarize various low-power circuit design techniques reported in literature.

\subsection{Bulk-driven technique}

The bulk (or body-)-driven scheme is a technique that allows circuit designers to implement ultra-low voltage and ultra-low power system. In the bulk-driven circuit design scheme, the bulk or the body terminal of a MOSFET is enabled as an AC input. The gate of the device is kept at a certain potential so that the transistor is 'on' during the entire operation. This technique eliminates the threshold voltage limitation mentioned earlier and helps achieve ultra-low supply voltage requirement while cascading a good number of transistors. Circuit designers have used this technique to design a low-voltage, low-power amplifier with a supply voltage of as low as $1 \mathrm{~V}$ [3]. A major drawback of this approach is the lower body transconductance $\left(g_{\mathrm{mb}}\right)$. In addition, the bulk-driving voltage needs to be kept within a certain range so that the body diodes of the MOSFET are reverse-biased during the variation of the AC input signal of the body terminal. Therefore, this technique can only be applied to a limited number of applications [6].

\subsection{Floating gate technique}

Although the implementation of the floating gate technique is mostly seen in the integrated memory cell applications, it can also be used for designing low-power circuits for implantable sensors. Floating gate is the polysilicon gate of the MOSFET that is surrounded by silicon dioxide $\left(\mathrm{SiO}_{2}\right)$. Once the charge has been deposited on the floating gate, it can be stored permanently. Therefore this technique is suitable for flash memory cells. The amount of this charge can be adjusted by an ultraviolet (UV) light or a large gate voltage. The stored charge on the floating gate can be used to reduce the threshold voltage of the transistor. Thus this technique helps reduce the DC supply voltage requirement as well as the total power consumption [7].

\subsection{Subthreshold design technique}

Another method that is being used to implement the low-power circuit is the subthreshold design technique. In subthreshold region (or weak inversion region) design, the gate-to-source voltage of the MOSFET is biased below the threshold voltage $\left(V_{\mathrm{GS}} \leq V_{\mathrm{TH}}\right)$ of the transistors. This level of gate-to-source voltage can weakly invert the transistor channel underneath the gate. 
This process of inversion of the channel is also known as weak inversion. Previously it was assumed by the researchers that this condition put the MOSFET in to cut-off region and therefore no current flows through the device. Now, it is well known that there is actually a small current that flows through the channel of the MOSFET mostly due to the diffusion of electrons from the drain to the source. A common expression for this subthreshold current of a MOSFET is shown in equation 1 :

$$
I_{D}=I_{D 0} \frac{W}{L} e^{\left(\frac{v_{G S}-v_{T H}}{n_{T}}\right)}
$$

Where $I_{\mathrm{D} 0}$ is the current that flows when the gate-to-source voltage is equal to the threshold voltage, $n$ is a technology specific slope parameter, and $U_{\mathrm{T}}$ is thermal voltage $\left(U_{\mathrm{T}} \approx k T / q \approx 26\right.$ $m V$ at room temperature where $k$ is the Boltzmann constant, $T$ is the temperature in Kelvin and $q$ is the charge of an electron which is equal to $1.6 \times 10^{-19}$ coulomb). The exponential relationship between the drain current and the gate-to-source voltage causes subthreshold biased circuits to be extremely sensitive to noise and matching. In comparison with the strong inversion circuit design technique that requires the gate-to-source voltage of the transistor to be much higher than the threshold voltage, this technique can achieve higher transconductance efficiency for the same level of current. Combined with rational circuit design and layout approach, this method can be used for implementing low-power analog circuits for implantable sensors.

\section{Wireless interface for implantable sensor}

For tether-less operation and to avoid skin infections, the data signal from the signal processor needs to be wirelessly transmitted to the outside environment. Several research works have been reported in recent years to meet the design requirements of wireless operation in biomedical applications. These systems should be miniaturized, light-weight, low-power and reliable for long term operation. The radiated power needs to be less than the limit set by FCC (Federal Communications Commission) for wireless telemetry. Wireless communication is one of the most prevailing means of data transmission for biomedical sensors. Wireless transmitters and receivers can be found everywhere from short-distance medical endoscopic applications [8] to long-distance cell phone communication. Technological advances in silicon manufacturing have made it possible to design low-power, low-cost integrated circuit for biomedical sensing application. Examples include electroencephalography (EEG), electrocardiography (ECG) and biometric information sensing for early detection of diseases such as tumor, cancer, and Alzheimer. Most of these applications require low data rates of a few $\mathrm{Hz}$ to a few $\mathrm{kHz}$. ECG monitoring typically needs 12-bit resolution of ADC with $250 \mathrm{~Hz}$ data sampling rate for a transmission data rate of $3 \mathrm{kbps}$ [9]. However the minimum energy per bit requirements for implantable sensor put a constraint on the transmitter power consumption to extend the battery life time. Since it would not be feasible to change the battery of the sensor often for an implantable sensor, low-power circuit design is an essential requirement. Considering most of the power harvesting techniques as well as battery storage capacities, a power 
budget of only $100 \mu \mathrm{W}$ could be available for each sensor node [10]. Although one can relax the power constraints by larger battery/energy-harvester size, the importance of a low-power communication scheme cannot be overlooked for a compact design of the sensor node. Despite the urge for the design of a low-power low-data rate transceiver with traditional narrowband architecture, the best transceiver design in this domain for implantable sensors still consumes about $500 \mu \mathrm{W}$ of power [11].

The first demonstration of a fully customized mixed-signal silicon chip that had most of the attributes required for a wearable or implantable BSN was described in [12]. The system blocks include low-power analog sensor interface for temperature and $\mathrm{pH}$ sensing, a data multiplexing and conversion module, a digital platform based around an 8-b microcontroller, data encoding for spread-spectrum wireless transmission, and an RF section requiring very few offchip components as shown in Figure 2. A programmable direct-sequence spread-spectrum (DS-SS) transmitter is integrated into the SoC in order to improve the reliability of the wireless transmission [13]. The transmitter is comprised of a data encoder and an RF section. The minimum data rate from the encoder is approximately $3.67 \mathrm{kbps}$. The amplification stage of the RF section is designed to be a near-class-E RF power amplifier driven by the digital output of the encoder. The gain budget of the amplifier enables it to maintain high gain and linearity while limiting the total current. The on-chip RF section uses a relatively low frequency carrier for modulation. An $800 \mu \mathrm{m} \times 300 \mu \mathrm{m}$ on-chip spiral inductor transmits the signal that is detectable at a range of $0.5 \mathrm{~m}$ in air using a Winradio receiver with a conventional whip antenna at a data rate of up to $5 \mathrm{kbps}$. Even though the on-chip inductor is less efficient than an external antenna, it demonstrates the feasibility of integrated antennas on silicon [14], [15]. A dataacquisition device detects the signal from the SoC.

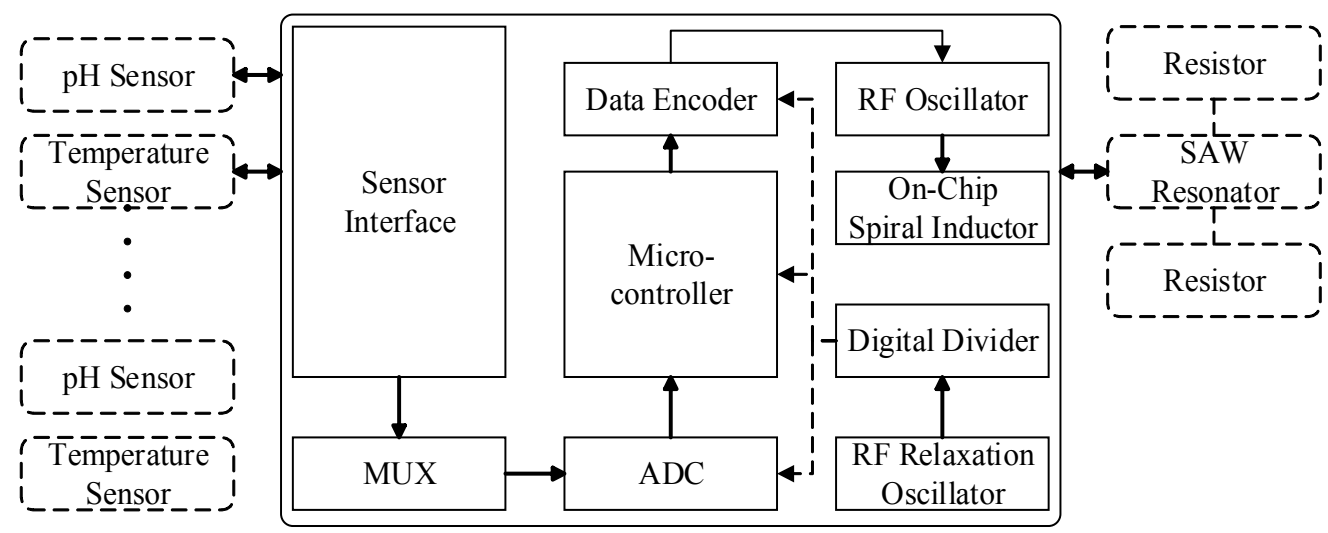

Figure 2. Schematic diagram of the system-on-chip architecture for body sensor networks [12].

An integrated CMOS ultra-wideband, high duty cycled, non-coherent wireless telemetry transceiver for wearable and implantable medical sensor applications was reported in [16]. A prototype wireless capsule for endoscopy was designed using the proposed transceiver and it demonstrated in vivo image transmission of $640 \times 480$ resolution at a frame rate of 
$2.5 \mathrm{fps}$ with $10 \mathrm{Mb} / \mathrm{s}$ data rate. This transceiver supports scalable data rate of up to $10 \mathrm{Mbps}$ with energy efficiency of $0.35 \mathrm{~nJ} / \mathrm{bit}$ and $6.2 \mathrm{~nJ} / \mathrm{bit}$ for transmitter and receiver, respectively. The block diagram of the transmitter is shown in Figure 3. The transceiver uses On/Off keying $(\mathrm{OOK})$ modulation scheme where a binary " 1 " is represented by a short pulse and binary " 0 " is represented by no pulse transmission. For improved performance of antennas with miniaturized size, UWB frequency band in 3-5 GHz is selected. The UWB pulse is generated by a fast on/off voltage-controlled oscillator (VCO) controlled by the TX data. A driving amplifier provides voltage amplification and isolation between the VCO and the antenna. Both the VCO and the driving amplifier consume power during pulse generation only. At the receiver end, the weak signal is first amplified by a variable gain lownoise amplifier (LNA) followed by a squarer performing the energy detection. A variable gain amplifier (VGA) amplifies the squarer output further and a slicer digitizes the final signal. The digital baseband provides synchronization, error correction coding, and interfaces with the external sensors. The transceiver chip consumes a die area of $3 \mathrm{~mm} \times 4$ $\mathrm{mm}$ when designed in a standard $0.18 \mu \mathrm{m}$ CMOS process. The transmitter draws an average power of $0.35 \mathrm{~mW}$ with the energy per bit of $0.35 \mathrm{~nJ} / \mathrm{bit}$ for up to $10 \mathrm{Mbps}$. The receiver average power consumption is as low as $6.1 \mathrm{~mW}$ with duty cycling under $1 \mathrm{Mbps}$ data rate and with the energy efficiency maintained at $6.2 \mathrm{~nJ} / \mathrm{bit}$.

\section{Transmitter}

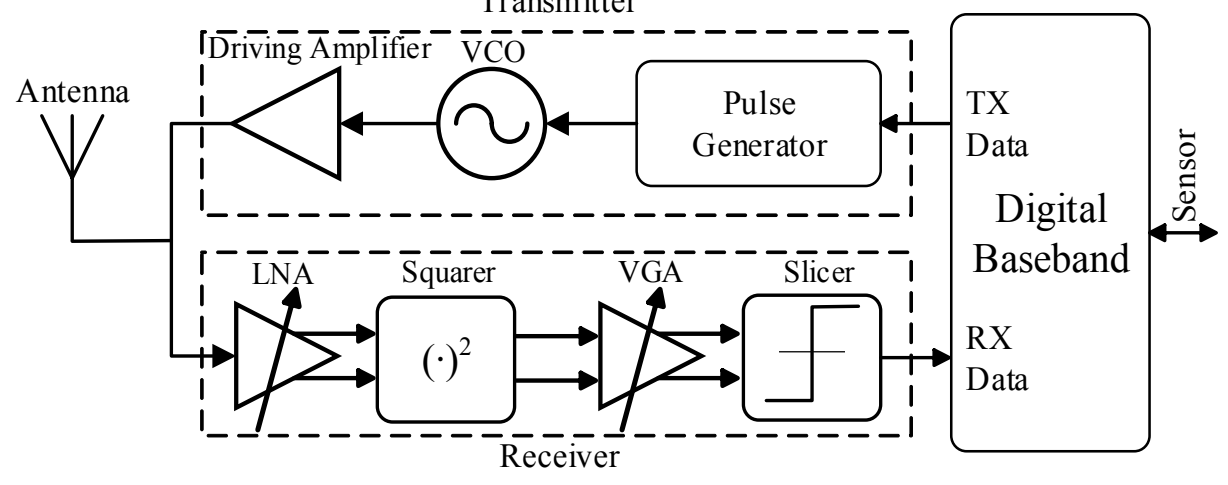

Figure 3. Block diagram of the ultra-wideband wireless telemetry transceiver [16].

Cleven et al. [17] presented a novel fully implantable wireless sensor system implanted into the femoral artery with computed tomography angiography intended for long-term monitoring of hypertension patients. The system was employed to measure intra-arterial pressure at a sampling rate of $30 \mathrm{~Hz}$ and an accuracy of $\pm 1.0 \mathrm{mmHg}$ over a range of $30-300 \mathrm{mmHg}$, and consumed up to $300 \mu \mathrm{W}$ power. The implant consists of two functional components: the pressure sensor tip and the transponder unit for communicating with the external readout station. Both the components are linked by a data cable. The full length of the sensor system is approximately $22 \mathrm{~cm}$. The telemetric unit has a diameter of approximately $2 \mathrm{~cm}$ and a thickness of approximately $4 \mathrm{~mm}$. The telemetry chip schematic including external components necessary for telemetric mode is presented in Figure 4. The analog output signal from 
the pressure sensor ASIC is digitized by the sensor readout block. At the same time, the bidirectional data pads provide the offset and the gain settings. The digital component of the chip, a state machine, provides the protocol for data transmission of the measured values. The HF front-end controls the telemetry components while generating the controlled supply voltage required for sensor readout. Using a transmission frequency of $133 \mathrm{kHz}$, the digitalized information is sent by telemetry to the receiver coil of the external readout electronics.

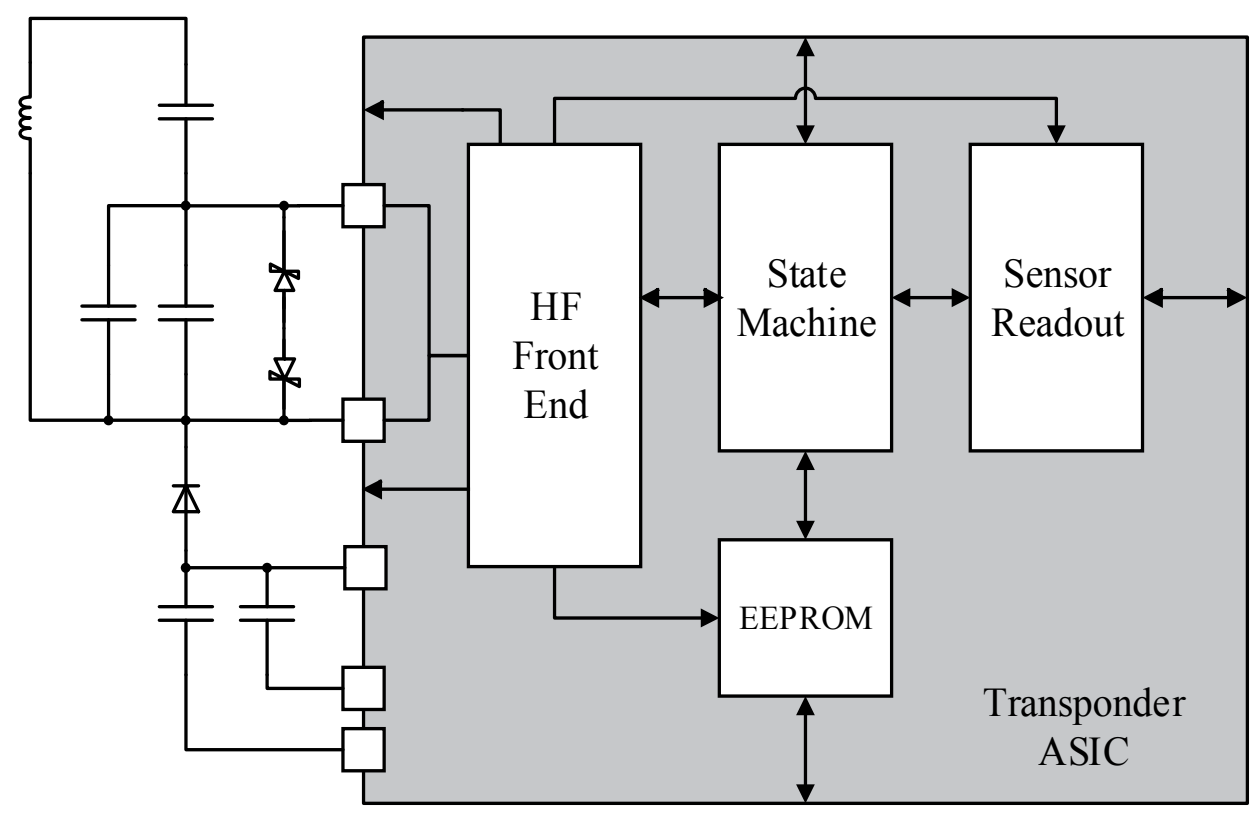

Figure 4. Schematics of transponder ASIC and external circuitry for long-term monitoring of hypertension patients [17].

A transcutaneous two-way communication and power system for wireless neural recording was reported in [18]. Figure 5(a) shows a schematic of the power and bidirectional data transfer system. Wireless powering and 1.25 Mbps forward data transmission (into the body) are achieved using a frequency-shift keying modulated class-E converter. The carrier frequency for reverse telemetry (out of the body) is generated using an integer-N phase-locked loop which provides the necessary wideband data link to support simultaneous reverse telemetry from multiple implanted devices on separate channels. The physical arrangement of the coils is illustrated in Figure 5(b). For the implanted device, coil 1 (L1) represents the external power coil, coil 2 (L2) is the implanted power coil, coil 3 (L3) is regarded as one of the external differential data coils, and coil 4 (L4) is the implanted data coil. A large AC current is generated in coil 1 using a class-E converter to transfer power to the implant. An AC current proportional to the coupling coefficient between the external and the implanted power coils is induced in coil 2. The resulting AC voltage is rectified and supplied to the application-specific integrated circuit (ASIC) to power it up. Frequency-Shift Keying (FSK) modulation of the $5 \mathrm{MHz}$ power carrier at a data rate of $1.25 \mathrm{Mbps}$ is performed to achieve forward data transfer and to send 
control data to the ASIC. The reference clock is multiplied up by an integer-N PLL in the ASIC circuitry to generate a reverse telemetry carrier between 50 and $100 \mathrm{MHz}$. The reverse telemetry uses either Amplitude-Shift Keying (ASK) or Binary-Phase-Shift Keying (BPSK) modulation scheme. To generate the reverse telemetry signal, the on-chip driver circuitry induces current in coil 4. Data is received by one of the two external differential data coils, coil 3. The purpose of a differential coil configuration is to cancel both the large power signal at its fundamental frequency and harmonics generated by the class-E converter that fall within the frequency range of the reverse telemetry.

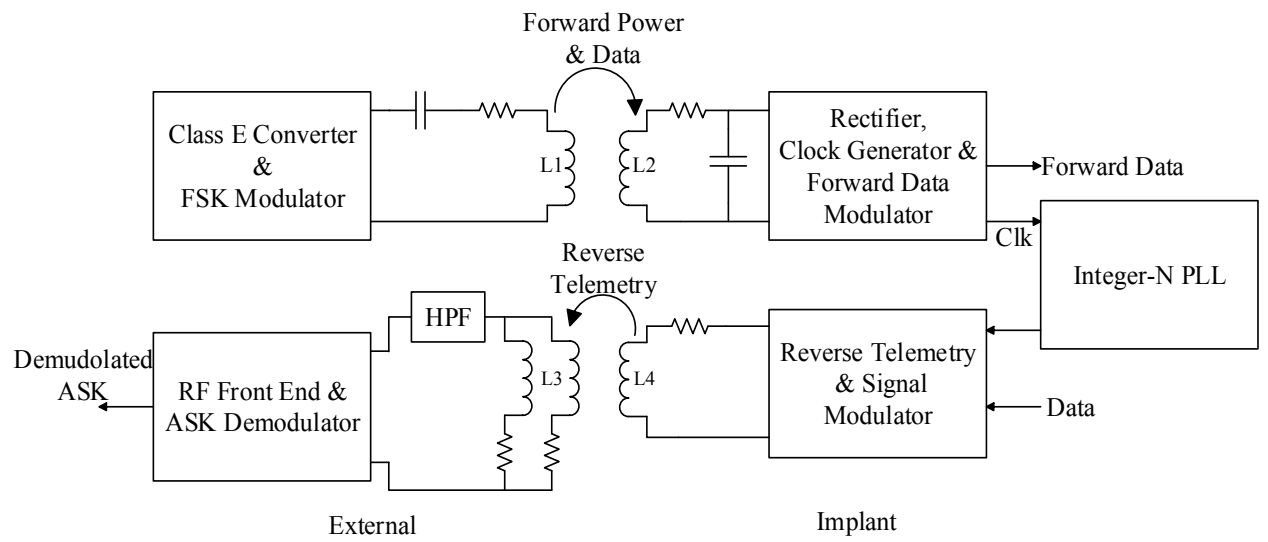

(a)

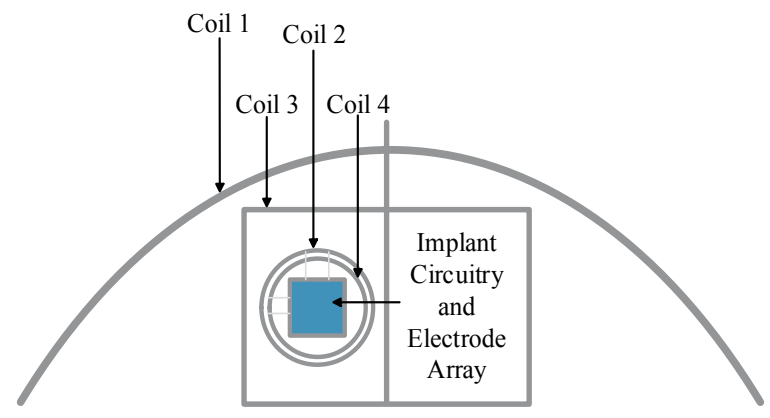

(b)

Figure 5. (a) Schematic of bidirectional data transfer system for wireless neural recording, (b) Physical diagram of dual inductive link coils [18].

Cao et al. prototyped a device for gastroesophageal reflux disease (GERD) monitoring in [19]. The system consists of an implantable, battery-less and wireless transponder with integrated impedance and $\mathrm{pH}$ sensors and a wearable, external reader that wirelessly powers up the transponder and interprets the transponded radio-frequency signals. The total size of the transponder implant is $0.4 \mathrm{~cm} \times 0.8 \mathrm{~cm} \times 3.8 \mathrm{~cm}$ and it harvests radio frequency energy to operate dual-sensor and load-modulation circuitry. The system is designed in a way that it can store data in a memory card and/or transmit data to a base station wirelessly. Figure 6 shows 
the block diagram of the sensor system. The coil antennas and the tuning capacitors form the resonant circuits. Relaxation oscillators are used as the frequency converters in the transponder. The system is designed to operate at $1.34 \mathrm{MHz}$ since the recommended maximum permissible exposure of magnetic fields is the highest in the frequency range of $1.34 \mathrm{MHz}$ to $30 \mathrm{MHz}$ [20]. A coil antenna is made using a 34-AWG magnet wire wound around the printed circuit board. The energy harvesting circuit consists of a series of diodes and capacitors $(100 \mathrm{pF})$ in a voltage multiplier circuitry that builds up the DC voltage from the received RF signals [21], [22], [23]. To maintain a constant DC level of $2.5 \mathrm{~V}$ for biasing the circuits, a voltage regulator is used.

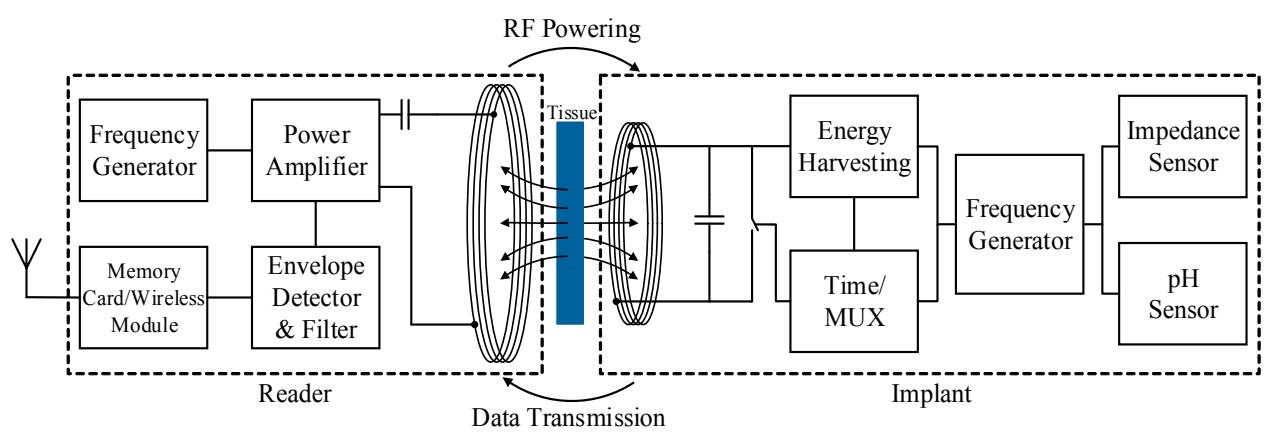

Figure 6. Block diagram of the gastroesophageal reflux disease (GERD) monitoring system [19].

Cheong et al. [24] presented an inductively powered implantable blood flow sensor microsystem with bidirectional telemetry. The microsystem is comprised of silicon nanowire (SiNW) sensors with tunable piezoresistivity, an ultra-low-power application-specific integrated circuit (ASIC), and two miniature coils that are coupled with a larger coil in an external monitoring unit to form a passive wireless link. The implantable microsystem operates at 13.56$\mathrm{MHz}$ carrier frequency. It receives power and command from the external unit and backscatters digitized sensor readout through the coupling coils. Cheong et al. fabricated the ASIC in a standard $0.18-\mu \mathrm{m}$ CMOS process and the chip occupied an active area of $1.5 \times 1.78 \mathrm{~mm}^{2}$ while consuming only $21.6 \mu \mathrm{W}$ of power. The overall system architecture consisting of an implantable wireless sensor microsystem and an external hand-held device is shown in Figure 7. The ASIC consists of a sensor interface circuit, an analog-to-digital converter (ADC), a digital baseband (DBB), a low-dropout (LDO) regulator, and front-end circuits for wireless powering and bidirectional telemetry. The external monitoring unit needs to be placed in close proximity to the implant microsystem to initiate the passive sensing operation. The RF power is transmitted by the external unit through the carrier at $13.56 \mathrm{MHz}$. The parallel resonant LC tanks and the rectifiers convert the received RF signal to a DC signal, and the LDO regulator powers the ASIC with regulated DC supply. Following the demodulation of the incoming modulated carrier, it is de-spread by the DBB to configure the system parameters such as integration time, amplifier gain, selection between two sensors, resonance tuning, and modulation index. At the same time, the clock is extracted from the incoming carrier and is provided to the DBB. Once the system parameters are set according to the received commands, the sensing operation 
ensues. A successive approximation register (SAR) ADC converts the analog voltage output from the sensor interface circuit into digital data. The digital data is spread and formatted by the DBB and is sent to the load modulator that backscatters the incoming RF carrier according to the sensor data bit stream from DBB.

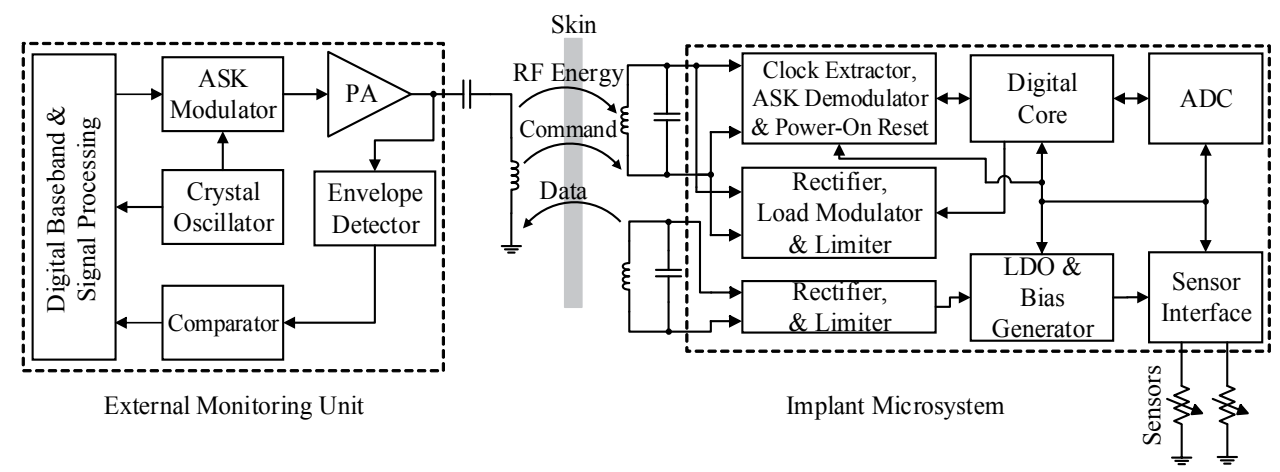

Figure 7. Architecture of the implantable blood flow monitoring system [24].

The above discussion presents an overall picture of the recent evolution in wireless technology for implantable sensors for monitoring of various physiological parameters. The background information and the current trends for the design of a wireless transmitters and receivers are also discussed. For a low-power, non-invasive and unobtrusive performance of an implantable sensor, wireless power transfer is mandatory. In the following sections, a brief discussion on various wireless power transfer and energy scavenging techniques are presented in terms of application requirements, available resources and radiation constraints.

\section{Wireless power transfer}

Previously, transcutaneous power cables were used in clinical implantable applications [25] at the expense of the introduction of a significant possibility for infection. Another alternative for power cables is an implanted battery. The use of batteries is usually intended to be avoided in implantable biomedical sensors as battery replacement is cumbersome and there is always a probability of leakage which can have serious health consequences. For this reason, various energy harvesting schemes and wireless powering techniques are employed in implantable sensors for battery-less operation. Energy harvested from body heat, breathing, arm motion, leg motion or from the motion of other body parts during walking or any other activity can be converted into a usable voltage to power up the sensor.

There are several possible sources of energy for sensorsincluding kinetic and thermal energy harvesters such as piezoelectric and pyroelectric transducers, photovoltaic cells etc. A summary is provided in the following table highlighting their sizes, produced energy or power and respective applications [26]. 


\begin{tabular}{cccc}
\hline Method & Density & Advantage & Disadvantage \\
\hline Piezoelectric & $200 \mu \mathrm{W} / \mathrm{cm}^{3}$ & No energy required from outside & Dependent on movement \\
\hline Thermoelectric & $60 \mu \mathrm{W} / \mathrm{cm}^{3}$ & No material to be replenished & Low efficiency less than $5 \%$ \\
\hline Kinetic & $4 \mu \mathrm{W} / \mathrm{cm}^{3}$ & No material to be replenished & Dependent on movement \\
\hline $\begin{array}{c}\text { Ambient RF energy } \\
\text { harvesting }\end{array}$ & ${ }^{*} 1 \mu \mathrm{W} / \mathrm{cm}^{2}$ & Harvesting energy from ambient EM & $\begin{array}{c}\text { Depends on EM wave } \\
\text { availability }\end{array}$ \\
\hline Visible light & $* 100 \mathrm{~mW} / \mathrm{cm}^{2}$ & Free & $\begin{array}{c}\text { Not available at night and in } \\
\text { cloudy days. }\end{array}$ \\
\hline Temperature variation & $10 \mu \mathrm{W} / \mathrm{cm}^{3}$ & No material to be replenished & $\begin{array}{c}\text { Low efficiency, Energy storage } \\
\text { required }\end{array}$ \\
\hline Airflow & $* 1 \mu \mathrm{W} / \mathrm{cm}^{2}$ & No material to be replenished & Implantation is difficult \\
\hline Heel strike & $* 7 \mathrm{~W} / \mathrm{cm}^{2}$ & Good source of energy & Dependent on movement. \\
\hline${ }^{\text {Energy Density per Unit Area }}$ & & & \\
\hline
\end{tabular}

Table 1. Comparison of Different Sources of Energy

All the above methods presented in Table 1 have their benefits as well as disadvantages. The method which is free of most of these disadvantages is the wireless power transfer (WPT). WPT is clean, controllable, independent of patient's movement, always available and more efficient than all the sources of energy that have been mentioned in Table 1. Although WPT has lower efficiency compared to the battery, it does not have the risk factors that are associated with a battery. Especially for sensors that come in direct contact with blood, any leakage can cause chemical burning, poisoning etc. and may eventually lead to death. A battery usually lasts for 5 to 7 years and then surgical procedure is required for its removal and replacement. On the other hand, WPT usually lasts for 15 to 20 years and consequently it is much cheaper than the batteries.

WPT is a technique for supplying energy from the source to the destination without any interconnecting wires. Nicola Tesla first demonstrated WPT using his resonant transformer called 'Tesla coil'. In this design, resonant inductive coupling was used to excite the secondary side of a transformer. With the passage of time, many researchers came up with different applications for the use of WPT and now WPT is used when a wire interconnection is inconvenient, risky or impossible. WPT is now used in induction heating coils, wireless chargers for consumer electronics, biomedical implants, radio frequency identification (RFID), contact-less smart cards etc. Several types of wireless power transfer techniques have been briefly discussed in the following section.

\subsection{Classifications of wireless power transfer}

There are two major methods for wireless power transfer - electromagnetic induction and electromagnetic radiation. Electromagnetic induction can be subdivided into three categories 
- electrodynamic, electrostatic and evanescent wave coupling. Electromagnetic radiation such as microwave power transfer and laser are also used for transferring power wirelessly. Figure 8 illustrates various types of wireless power transfer techniques.

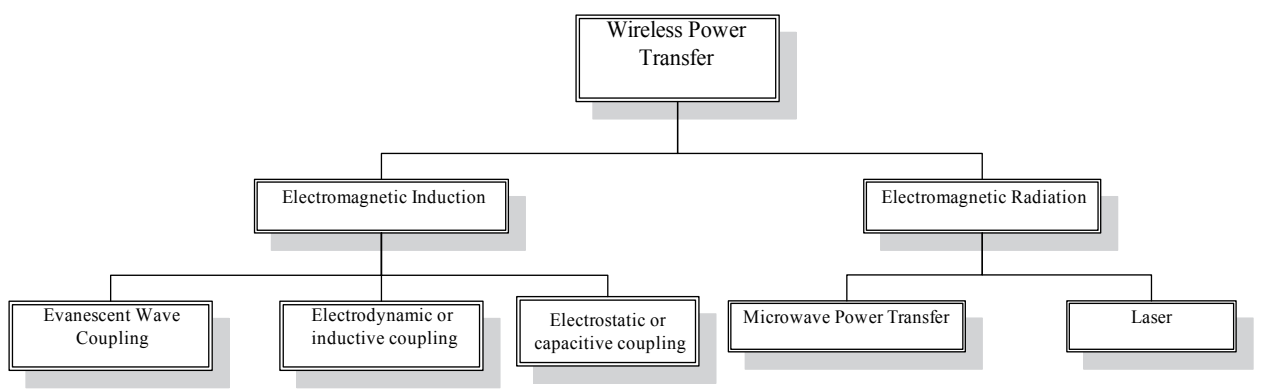

Figure 8. Types of wireless power.

Electromagnetic Induction

By varying the magnetic field an electromotive force can be produced across a conductor. This is called electromagnetic induction. The three possible ways to achieve that are summarized below:

a. Inductive coupling: This is achieved via near field radiation which causes coupling of energy between two inductors (coils) and is also known as the electrodynamic or magnetic coupling.

b. Electrostatic or capacitive coupling: This is the propagation of electrical energy through a dielectric medium. High voltage and high frequency alternating current gives rise to the electrical field for this form of WPT. Unintended parasitic capacitance (e.g. capacitance between two adjoining wires or PCB traces) can cause noise and has to be taken into account for high frequency circuit design.

c. Evanescent wave coupling: In this process, an exponentially decaying electromagnetic field is used to transmit the electromagnetic waves from one medium to another.

Electromagnetic Radiation

After an electromagnetic radiation is emitted, it can be absorbed by some charged particles. This type of radiation can propagate through vacuum at the speed of light. It has a time varying electric field component as well as a magnetic field component, which oscillates perpendicularly to each other and perpendicularly to the direction of energy and wave propagation. Two ways in which wireless power transfer using electromagnetic radiation can be accomplished are:

a. Microwave power transmission: It is the transmission of energy using electromagnetic waves with wavelengths ranging from $30 \mathrm{~cm}$ down to $1 \mathrm{~cm}$ or equivalently a frequency range of $1 \mathrm{GHz}$ to $30 \mathrm{GHz}$. It is used for directional power transmission to a remote destination. 
b. Laser: In this technique electricity is first converted into a laser beam which is then directed towards a photovoltaic cell. The receiver is an array of photovoltaic cells designed to convert the light back to a usable electrical energy. This method is also known as optical coupling.

Since inductive link is the most commonly used wireless power transferring technique for biomedical sensors, it is discussed in more detail in the following section.

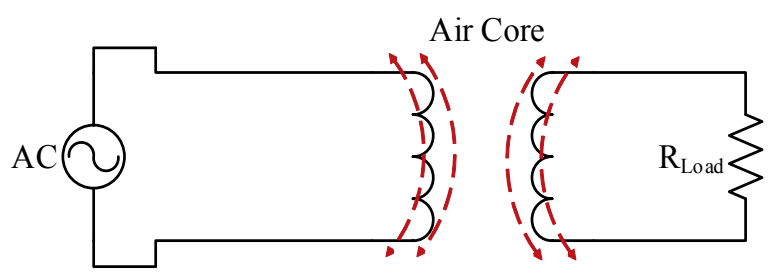

Figure 9. Basic inductive link caused by alternating electromagnetic field.

\subsection{Inductive link}

An inductive link comprises of a loosely coupled transformer consisting of a pair of coils as shown in Figure 9. An alternating source (AC) drives the primary coil and generates the desired electromagnetic field. A portion of the generated magnetic flux links the secondary coil and according to Faraday's Law of electromagnetic induction, the temporal change of magnetic flux induces a voltage across the secondary coil. The voltage induced in the secondary coil is proportional to the rate of change of magnetic flux in the secondary coil and the number of turns in that coil.

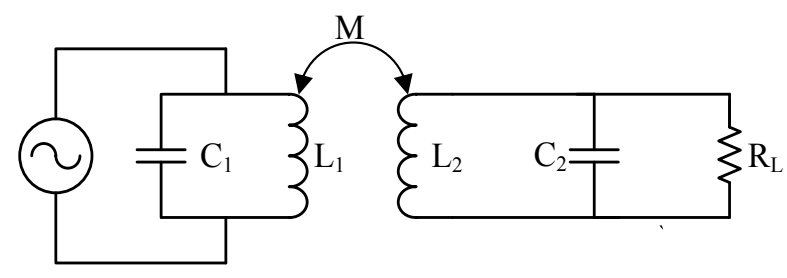

Figure 10. Schematic of basic inductive link based on series parallel resonance.

Figure 10 illustrates a basic inductive link based on series-parallel resonance. The maximum value of the mutual inductance, $M$ that can possibly be achieved between the two coils of inductance of $L_{1}$ and $L_{2}$ is $\left(L_{1} L_{2}\right)^{1 / 2}$ and this occurs when all the flux generated in the primary coil links with all the turns in the secondary coil. The ratio of mutual inductance to its maximum value is called the coupling coefficient $k$, which is a dimensionless quantity ranging from 0 to 1 and can be determined using the following equation:

$$
k={ }^{M} /\left(\begin{array}{lll}
L_{1} L_{2} & { }^{1}
\end{array}\right)^{1 / 2}
$$


The performance of the inductive link is dependent on the link efficiency, which is defined as the ratio of the power delivered to the load to the power supplied to the primary coil. For a parallel resonant circuit, the link efficiency of the secondary side can be written as [46],

$$
\eta=\frac{k^{2} Q_{1} Q_{2}}{\left(1+\frac{Q_{2}}{\alpha}+k^{2} Q_{1} Q_{2}\left(\alpha+\frac{1}{Q_{2}}\right)\right.}
$$

For a series resonant circuit, the link efficiency of the secondary side turns out to be,

$$
\eta=\frac{k^{2} Q_{1} \alpha}{\left(1+\frac{1}{Q_{2}}+k^{2} Q_{1}\right)\left(\alpha+\frac{1}{Q_{2}}\right)}
$$

In both Equation 3 and $4, Q_{1}$ is the quality factor of the primary coil, $Q_{2}$ is the quality factor of the secondary coil, $k$ is the coupling factor between the coils, $\alpha$ is a unit-less constant which is equal to $\omega C_{2} R_{\mathrm{L}}$, where $\omega$ is the angular frequency, $C_{2}$ is the capacitance of the secondary coil and $R_{\mathrm{L}}$ is the load resistance.

Inductive link is a common method for wireless powering of implantable biomedical electronics and data communication with the external world. WPT and data telemetry using inductive link have been demonstrated for various biomedical applications including visual prosthesis, cochlear implant, neuromuscular and nerve stimulator, cardiac pacemaker/ defibrillator, deep-brain stimulator, brain machine interface, gastrointestinal microsystem and capsule endoscopy[27-37]. A summary of various inductive link wireless power transfer applications and their respective carrier frequencies is presented in Table 2:

\begin{tabular}{cccc}
\hline Reference & Applications & Frequency & Inductor Type \\
\hline$[$ 28] & Neural prosthetic Implant & $2-20 \mathrm{MHz}$ & Ferrite core \\
\hline$[$ 29] & Cochlear Implant & -- & -- \\
\hline$[37]$ & Retinal prosthesis & $1 \mathrm{MHz}$ & Litz wire \\
\hline$[$ 36] & Biomedical implant & $5 / 10 \mathrm{MHz}$ & -- \\
\hline$[$ 35] & Neural implant & $4 \mathrm{MHz}$ & Copper magnet wire \\
\hline$[$ 32] & Endoscope & $1.055 \mathrm{MHz}$ & Litz wire \\
\hline$[$ 33] & Gastrointestinal microsystems & $58.418 \mathrm{KHz}$ & Copper wire \\
\hline$[$ 38] & Neural recording & $4 \mathrm{MHz}$ & Litz wire \\
\hline$[39]$ & Implantable system & $13.56 \mathrm{MHz}$ & On-chip \\
\hline$[40]$ & Neural prosthesis & $25 \mathrm{MHz}$ & Wire \\
\hline$[41]$ & Implantable prosthesis & $1 \mathrm{GHz}$ & Bond wire \\
\hline$[42]$ & Neuroprosthetic implantable device & $13.56 \mathrm{MHz}$ & PCB \\
\hline$[43]$ & Neural recording & $2.64 \mathrm{MHz}$ & Off-chip power, on-chip data \\
\hline$[44]$ & Neural recording & $<10 \mathrm{MHz}$ & Wire \\
\hline
\end{tabular}

Table 2. Wireless Power Transfer for Different Biomedical Implants 
The design of an inductive link is required to meet the power requirement of any of the above mentioned applications. There are several parameters which play key roles in determining the performance of an inductive link. A qualitative analysis of these key factors is presented in the following section.

\subsubsection{Performance dependence on different factors}

The factors that affect the performance of an inductive link wireless power transfer are as follows:

a. Diameter of coils: The diameters of the receiver and the transmitter coils are important parameters affecting the voltage gain of an inductive link [28]. Both the self inductance and the mutual inductance are proportional to the diameters of the coils. Therefore increasing the diameters boosts the link efficiency. In case of an implantable system, due to the constraint on the size of the implant there are more stringent limitations on the receiver coil size compared to those of the transmitter coil.

b. Number of turns: The number of turns is another important factor since the mutual inductance is proportional to the product of the number of turns in the transmitter and the receiver coils. Therefore increasing the number of turns will improve the performance.

c. Spacing and alignment: Spacing between the primary and the secondary coils and their alignment also significantly affect the coupling between them. Therefore the position of the implantable sensor in terms of the external wireless power transferring module is an important factor that needs to be taken in to account. Compared to an exact coaxial alignment, similar or even better performance can be achieved if the receiver coil is present within the circumference of the transmitter coil [28]. In case of implantable sensors, any movement of the patient can cause misalignment between the transmitter and the receiver which in turn can alter the mutual inductance and the link gain. Two types of misalignment that affect the link efficiency are lateral misalignment and angular misalignment [25] as illustrated in Figure 11. After a certain lateral or angular misalignment, the performance degradation of the wireless inductive link becomes proportional to the magnitude of the misalignment.
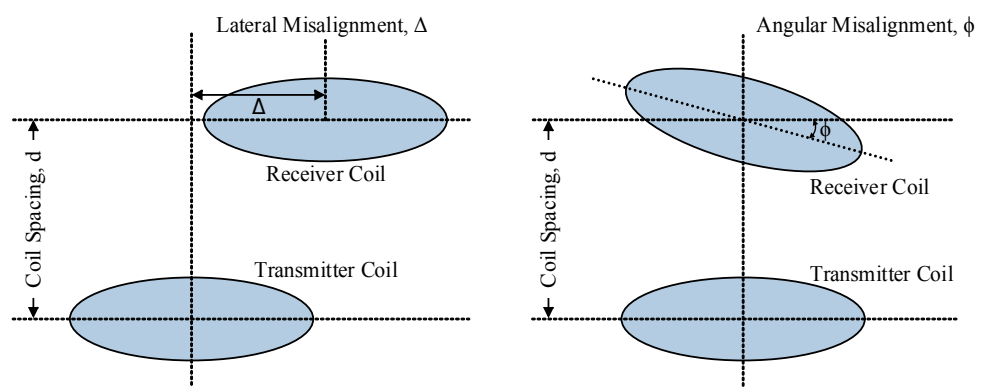

Figure 11. Indcutive coupling bewtween two coils with lateral misalignment and angular misalignment. 
d. Quality Factor: Higher Quality factors (Q) of the primary and the secondary coils can significantly improve the link efficiency of an inductive link [27], [47]. Therefore designing coils with high $\mathrm{Q}$ values at the desired frequency of operation is required to achieve a satisfactory power transfer. Another disadvantage of low $Q$ circuit is that it makes the output voltage susceptible to load changes [45].

e. Operating frequency: The maximum power allowed to penetrate through human tissue depends on the operating frequency. It also dictates the size of the coil, the mutual impedance and the voltage transfer ratio. Since the quality factor depends on the operating frequency, the efficiency and the bandwidth of the link are influenced as well. Thus, the frequency of operation plays an important role in the design of wireless power transfer.

f. Other relevant factors: The primary coil of an inductive link is usually driven by low-loss switching amplifiers. It is necessary to have a high driver efficiency to ensure satisfactory power transfer efficiency of an inductive link. With a tuned amplifier load, the drive transistor only draws current when there is no voltage across it [48], improving the overall system efficiency significantly in the process. Different topologies for the amplifier have been reported in literature: class-C [27], [48], class-D [45], [50], class-E [35], [37], [49] and class-CE [47]. Sokal et al. reported a class-E amplifier with a very high efficiency and insensitivity to small timing errors [51]. Variation in output impedance has a pronounced effect on class-E amplifiers. When the power demand of the implantable sensor or the coupling factor between the coils changes the equivalent load seen by the driver amplifier also varies. Lastly, as far as implantable biomedical applications are concerned, the overall system gain and the efficiency also depend on the physical configuration of the two coils and the surrounding materials.

There are some major challenges associated with the implementation of an efficient wireless power transfer system in implantable sensors. The next section summerizes the major limitations.

\subsection{Limitations of wireless energy transfer}

There are several factors that impose serious limitations on the widespread use of wireless energy transfer for implantable sensors. First of all, it is not often possible to scale the transmitter or the receiver down to a small enough size to make it suitable for its implementation in a miniaturized system. Secondly, the range of energy transfer has not yet been demonstrated to exceed a few meters, which poses a major challenge for its practical implementation. Ongoing research is focused on finding more compact solution for wireless energy transfer covering a greater range. Another problem with wireless energy transmission is that its typical efficiency varies between $45 \%$ and $80 \%$ falling short of a conventional battery or wire based technology. Future innovations resulting in the reduction in size as well as increase in efficiency and operating range will undoubtedly make wireless energy transfer suitable for plenty of new potential sensor applications. 


\section{Health issues related to wireless power transfer}

While designing wireless power transfer system for biomedical applications, the associated health risks have to be taken into consideration. Since RF energy can quickly heat up the biological tissues due to the thermal effect, the exposure to very high levels of RF radiation can be harmful. Since attenuation increases with frequency, most of the existing work in Wireless Body Area Networks (WBAN) considers only the Medical Implant Communication System (MICS) band (402-405 MHz) or sub-gigahertz bands. Federal Communications Commission (FCC) regulates the time and the amount of exposure of the electromagnetic radiation to human tissues at various frequencies [52]. American National Standard Institute (ANSI) standard C95.1-1982 sets the electromagnetic field strength limits for frequencies between $300 \mathrm{kHz}$ and $100 \mathrm{GHz}$ [53], [54]. For frequencies below $300 \mathrm{MHz}$, the electric and the magnetic fields have to be accounted for separately. The ANSI standard C95.1-1991 sets the electric and the magnetic field strength limits for the general public for the frequency range of 3 kHz-300 GHz [55]. Table 3 illustrates the IEEE standard C95.1-1991.

\begin{tabular}{|c|c|c|c|c|c|}
\hline \multirow{2}{*}{$\begin{array}{l}\text { Frequency Range } \\
(\mathrm{MHz})\end{array}$} & \multirow{2}{*}{$\begin{array}{c}\text { Electric Field } \\
\text { Strength, E (V/m) }\end{array}$} & \multirow{2}{*}{$\begin{array}{c}\text { Magnetic Field } \\
\text { Strength, H (A/m) }\end{array}$} & \multicolumn{2}{|c|}{ Power Density, S (mW/ $\left.\mathrm{cm}^{2}\right)$} & \multirow{2}{*}{$\begin{array}{c}\text { Averaging Time } \\
|E|^{2},|H|^{2} \text { (minutes) }\end{array}$} \\
\hline & & & E-field & H-field & \\
\hline $0.003-0.1$ & 614 & 163 & 100 & $1 \mathrm{E} 6$ & 6 \\
\hline $0.1-3.0$ & 614 & $16.3 / \mathrm{f}$ & 100 & $10000 / \mathrm{f}^{2}$ & 6 \\
\hline $3-30$ & $1842 / \mathrm{f}$ & $16.3 / \mathrm{f}$ & $900 / \mathrm{f}^{2}$ & $10000 / \mathrm{f}^{2}$ & 6 \\
\hline 30-100 & 61.4 & $16.3 / \mathrm{f}$ & 1 & $10000 / \mathrm{f}^{2}$ & 6 \\
\hline $100-300$ & 61.4 & 0.163 & \multicolumn{2}{|c|}{1} & 6 \\
\hline $300-3 K$ & -- & -- & \multicolumn{2}{|c|}{$f / 300$} & 6 \\
\hline $3 \mathrm{~K}-15 \mathrm{~K}$ & -- & - & \multicolumn{2}{|c|}{10} & 6 \\
\hline $15 \mathrm{~K}-300 \mathrm{~K}$ & -- & -- & \multicolumn{2}{|c|}{10} & $616000 / \mathrm{f}^{1.2}$ \\
\hline
\end{tabular}

Table 3. IEEE Standard C95.1-1991: Limit of Maximum Permissible Exposure at Controlled Environment on Human Body [55].

An important parameter that is used to measure the effect of radio frequency exposure on human is SAR (specific absorption rate). SAR is a quantity that is used to measure the amount of energy absorbed by a body which is exposed to radio frequency (RF) electromagnetic field. It is defined as the power absorbed per mass of the tissue with units of watts per kilogram (W/ $\mathrm{kg}$ ) or milliwatts per gram $(\mathrm{mW} / \mathrm{g})$ and can be expressed as,

$$
S A R=\int \frac{\sigma(r)|E(r)|^{2}}{\rho(r)} d r
$$

Here $\sigma$ is the electrical conductivity of the sample, $E$ is the RMS electric field and $\rho$ is the sample density. In case of whole-body exposure, a standing human adult can absorb a maximum RF 
radiation rate of approximately $80 \mathrm{MHz}$ to $100 \mathrm{MHz}$. As a result, RF safety standards are often most stringent for these frequencies. SAR has to be within an acceptable range for biological tissues [56], [57]. A whole-body average SAR of $0.4 \mathrm{~W} / \mathrm{kg}$ has been set as the restriction that provides adequate protection for occupational exposure [58]. An SAR level of $1.6 \mathrm{~W} / \mathrm{kg}$ has been set as the FCC limit for public exposure from cellular telephones [59]. Two areas of the body, the eyes and the testicles, are particularly vulnerable to RF heating due to the relative lack of available blood flow to dissipate the excessive heat. The detrimental effects of RF exposure which does not cause significant heating (referred to as 'non-thermal' effects) are still unproven.

\section{Biocompatibility issues related to implantable sensor}

Since there is direct contact between the implanted device and biological tissue, a compatibility assessment of the sensors needs to be performed prior to being deployed inside the human body. This biocompatibility assessment is defined in [60] as, "the ability of a material to perform with an appropriate host response in a specific application". Some of the major characteristics of the bulk and surface materials which can possibly influence host response and some of the important characteristics of host responses are listed in [61]. The biocompatibility of an implantable sensor depends on parameters such as the part of the human body where the implant is deployed and the surface material of the sensor itself. Also the shape and size of the sensor also have to be optimum to make the sensor compatible with human body. Surface chemistry and composition of the outer material also need to be kept in mind so that it does not react with the tissue and blood that come in contact with the implant. Finally, sterility issues, contact duration and degradation of the material that surrounds the sensor need to be taken into account while designing a biocompatible implantable sensor [62-63]. Several protocols related to the biocompatibility issues that are scrutinized thoroughly before an implantable device could be used in the human body have been developed by the U.S. Food and Drug Administration (FDA). Materials appropriate for long-term reliable implantable devices according to [61] include a) titanium alloys for dental implants, femoral stems, pacemaker cans, heart valves, fracture plates, spinal cages, b) cobalt-chromium alloys for bearing surfaces, heart valves, stents, pacemaker leads, c) platinum group alloys for electrodes, d) nitinol for shape memory applications, e) stainless steel for stents and orthopedic implants, f) alumina for bearing surfaces, g) calcium phosphates for bioactive surfaces, h) polyurethane for pacemaker lead insulation, i) PMMA for bone cement, intraocular lenses, j) silicone for soft tissue augmentation, insulating leads, ophthalmological devices. On May 28, 2014 FDA approved the first implantable wireless device with wireless monitoring feature to measure pulmonary artery pressure for heart patients [64]. With the ongoing research on finding biocompatible materials it can be easily inferred that there will be numerous implantable devices in the global market in near future. 


\section{Conclusion}

In summary, the chapter includes the discussion on various implantable sensors that are currently being used in biomedical applications. Various low-power low-voltage signal processing schemes to convert the signal from the sensors into usable data signal have been discussed as well. This is followed by an analysis of current state-of-the-art research on wireless telemetry for implantable sensors. Different power management schemes have been explored at the later part of the chapter. Finally, the chapter concludes with a brief discussion on biocompatible issues related to implantable sensors.

\section{Author details}

Ifana Mahbub*, Farhan Quaiyum, Md Sakib Hasan and SyedKamrul Islam

*Address all correspondence to: imahbub@vols.utk.edu

Department of Electrical Engineering and Computer Science, University of Tennessee, Knoxville, TN, USA

\section{References}

[1] Haider MR., Islam SK., Mostafa S., Zhang M., Oh T. Low-Power Low-Voltage Current Readout Circuit for Inductively Powered Implant System. IEEE Transactions on Biomedical Circuits and Systems 2010; 4(4) 205-213.

[2] Shamsuddin AKM., Tamura T., Nakajima K., Togawa T. Preliminary Study of Transcutaneous Optical Coupling for Implantable Devices Using GaAs Solar Cell. IEEE Engineering in Medicine and Biology Society 1995; 1/39-1/40.

[3] Liang Z. Low-Voltage Bulk-Driven Amplifier Design and Its Application in Implantable Biomedical Sensors. PhD diss., University of Tennessee 2012.

[4] Hu C., Future CMOS Scaling and Reliability. Proceedings of the IEEE 1993; 81(5) 682-689.

[5] Chatterjee S., Pun KP., Stanic N., Tsividis Y., Kinget P. Analog Circuit Design Techniques at 0.5V, Analog Circuits and Signal Processing, Springer 2007.

[6] Waltari M., Halonen K. 1-V 9-bit Pipelined Switched-Opamp ADC. IEEE Journal of Solid-State Circuits 2001; 36(1) 129-134.

[7] Hasler P., Lande TS. Overview of Floating Gate Devices, Circuits and Systems. IEEE Transactions on Circuits and Systems II, 2001; 48(1) 1-3. 
[8] Chi B., Yao J., Han S., Xiang X., Li G., Wang Z. Low-power Transceiver Analog Front-end Circuits for Bidirectional High Data Rate Wireless Telemetry in Medical Endoscopy Applications. IEEE Transactions on Biomedical Engineering 2007; 54(7) 1291-1299.

[9] Lande TS., Hjortland H. Impulse Radio Technology for Biomedical Applications. IEEE Biomedical Circuits and Systems Conference, (2007) 67-70.

[10] Dokania RK., Wang XY., Tallur SG., Apsel A., A Low Power Impulse Radio Design for Body-Area-Networks. IEEE Transactions on Circuits and Systems I: Regular Papers 2011; 58(7) 1458-1469.

[11] Bae J., Cho N., Yoo HJ. A 490uW Fully MICS Compatible FSK Transceiver for Implantable Devices. Symposium on VLSI Circuits 2009, 36-37, Kyoto, Japan.

[12] Lei W., Guang-Zhong Y., Jin H., Jinyong Z., Li Y., Zedong N., Cumming DRS. A Wireless Biomedical Signal Interface System-on-chip for Body Sensor Networks. IEEE Transactions on Biomedical Circuits and Systems 2010; 4(2) 112-117.

[13] Aydin N., Arslan T., Cumming DRS. A Direct-Sequence Spread-Spectrum Communication System for Integrated Sensor Microsystems. IEEE Transactions on Information Technology in Biomedicine 2005; 9(1) 4-12.

[14] Biebl EM. Integrated Active Antennas on Silicon. 1997 SBMO/IEEE MTT-S International Microwave and Optoelectronics Conference Proceedings, vol. 1, August 11-14, 1997, Natal, Brazil.

[15] Kim J., Rahmat-Samii Y. Implanted Antennas Inside a Human Body: Simulations, Designs and Characterizations. IEEE Transactions on Microwave Theory and Techniques 2004; 52(8) 1934-1943.

[16] Gao Y., Zheng Y., Diao S., Toh W., Ang C., Je M., Heng C. Low-Power Ultra-Wideband Wireless Telemetry Transceiver for Medical Sensor Application. IEEE Transactions on Biomedical Engineering 2011; 58(3) 1291-1299.

[17] Cleven NJ., Muntjes JA., Fassbender H., Urban U., Gortz M., Vogt H., Grafe M., Gottsche T., Penzkofer T., Schmitz-Rode T., Mokwa W. A Novel Fully Implantable Wireless Sensor System for Monitoring Hypertension Patients. IEEE Transactions on Biomedical Engineering 2012; 59(11) 3124-3130.

[18] Rush AD., Troyk PR. A Power and Data Link for a Wireless-Implanted Neural Recording System. IEEE Transactions on Biomedical Engineering 2012; 59(11) 3255-3262.

[19] Cao H., Landge V., Tata U., Seo YS., Rao S., Tang SJ., Tibbals HF., Spechler S., Chiao J. An Implantable, Batteryless, and Wireless Capsule with Integrated Impedance and $\mathrm{pH}$ Sensors for Gastroesophageal Reflux Monitoring. IEEE Transactions on Biomedical Engineering 2012; 59(11) 3131-3139. 
[20] 2005 IEEE Standard for Safety Levels with respect to Human Exposure to Radio Frequency Electromagnetic Fields, 3 kHz to $300 \mathrm{GHz}$, IEEE Std C95.1, 2006.

[21] Ativanichayaphong T., Wang J., Huang W., Rao S., Chiao JC. A Simple Wireless Batteryless Sensing Platform for Resistive and Capacitive Sensors. IEEE Sensors Conference Proceedings, October 26-28, 2007, Atlanta, GA.

[22] Ativanichayaphong T., Tang SJ., Hsu LC., Huang WD., Seo YS., Tibbals HF., Spechler S., Chiao JC. An Implantable Batteryless Wireless Impedance Sensor for Gastroesophageal Reflux Diagnosis. IEEE MTT 2010 International Microwave Symposium, May 23-28, 2010, Anaheim, CA.

[23] Cao H., Tharkar S., Fu T., Sheth M., Oseng M., Landge V., Seo YS., Chiao JC. A Wireless Strain Sensor System for Bladder Volume Monitoring. IEEE MTT 2011 International Microwave Symposium, June 5-10, 2011, Baltimore, MD.

[24] Cheong JH., Sheung S., Ng Y., Liu X., Xue RF., Lim HJ., Khannur PB., Chan KL., Lee AA., Kang K., Lim LS., He C., Singh P., Park WT., Je M. An Inductively Powered Implantable Blood Flow Sensor Microsystem for Vascular Grafts. IEEE Transactions on Biomedical Engineering 2012; 59(9) 2466-2475.

[25] Soma M., Galbraith DC., White RL. Radio-Frequency Coils in Implantable Devices: Misalignment Analysis and Design Procedure. IEEE Transactions on Biomedical Engineering, BME-34, 1987, 276-282.

[26] Islam AB. Design of Wireless Power Transfer and Data Telemetry System for Biomedical Applications. PhD Dissertation, UT Knoxville, 2011.

[27] Ko WH., Liang SP., Fung CDF., Design of Radio-frequency Powered Coils for Implant Instruments. Medical \& Biological Engineering \& Computing, 15, 1977, 634-640.

[28] Heetderks WJ. RF Powering of Millimeter-and Submillimeter-sized Neural Prosthetic Implants. IEEE Transactions on Biomedical Engineering 1988; 35(5) 323-327.

[29] Zierhofer CM., Hochmair-desoyer IJ., Hochmair ES. Electronic Design of a Cochlear Implant for Multichannel High-rate Pulsatile Stimulation Strategies. IEEE Transactions on Rehabilitation Engineering 1995; 3(1) 112-116.

[30] Schulman J.H., The Feasible FES System: Battery Powered BION Stimulator. Proceedings of the IEEE 2008; 96(7) 1226-1239.

[31] Liu WT.,Vichienchom K., Clements M., DeMarco SC., Hughes C., McGucken E., Humayun MS., DeJuan E., Weiland JD., Greenberg R. A Neuro-stimulus Chip with Telemetry Unit for Retinal Prosthetic Device. IEEE Journal of Solid-State Circuits 2000; 35(10) 1487-1497.

[32] Lenaerts B., Puers R. An Inductive Power Link for a Wireless Endoscope. Biosensors \& Bioelectronics 2007; 22(7) 1390-1395. 
[33] Ma GY., Yan G., He X. Power Transmission for Gastrointestinal Microsystems Using Inductive Coupling. Physiological Measurement 2007; 28(3) N9-N18.

[34] Mandal S., Zhak S., Sarpheskar R., Circuits for an RF Cochlea. Proceedings of 2006 IEEE International Symposium on Circuits and Systems 2006, 3610-3613, Island of Kos, Greece.

[35] Sauer C., Stanac'evic' M., Cauwenberghs G., Thakor N. Power Harvesting and Telemetry in CMOS for Implanted Devices. IEEE Transactions on Circuits and Systems I: Regular Papers 2005; 52(12) 2605-2613.

[36] Ghovanloo M., Najafi K. A Wideband Frequency-shift Keying Wireless Link for Inductively Powered Biomedical Implants. IEEE Transactions on Circuits and Systems I: Regular Papers 2004; 51(12) 2374-2383.

[37] Kendir GA., Liu W., Wang G., Sivaprakasam M., Bashirullah R., Humayun MS., Weiland JD. An Optimal Design Methodology for Inductive Power Link with Class-E Amplifier. IEEE Transactions on Circuits and Systems I: Regular Papers 2005; 52(5) 857-866.

[38] Li P., Principe JC., Bashirullah R. A Wireless Power Interface for Rechargeable Battery Operated Neural Recording Implants. Proceedings of the 28th IEEE EMBS Annual International Conference 2006, 6253-6256, New York, USA.

[39] Sawan M., Hashemi S., Sehil M., Awwad F., Hajj-Hassan M., Khouas A. Multicoilsbased Inductive Links Dedicated to Power up Implantable Medical Devices: Modeling, Design and Experimental Results. Biomedical Microdevices 2009; 11(5) 1059-1070.

[40] Mandal S., Sarpeshkar R. A Bidirectional Wireless Link for Neural Prostheses that Minimizes Implanted Power Consumption. IEEE Biomedical Circuits and Systems Conference 2007, 45-48, Montreal, Quebec, Canada.

[41] O'Driscoll S., Meng TH. Adaptive Signal Acquisition and Wireless Power Transfer for an Implantable Prosthesis Processor. Proceedings of 2010 IEEE International Symposium on Circuits and Systems, 2006, 3589-3592, Paris, France.

[42] Jow UM., Ghovanloo M. Optimization of Data Coils in a Multiband Wireless Link for Neuroprosthetic Implantable Devices. IEEE Transactions on Biomedical Circuits and Systems 2010; 4(5) 301-310.

[43] Harrison RR., Watkins PT., Kier RJ., Lovejoy RO., Black DJ., Greger B., Solzbacher F. A Low-power Integrated Circuit for a Wireless 100-electrode Neural Recording System. IEEE Journal of Solid-State Circuits 2007; 42(1) 123-133.

[44] Sodagar AM., Wise KD., Najafi K. A Wireless Implantable Microsystem for Multichannel Neural Recording. IEEE Transactions on Microwave Theory and Techniques 2009; 57(10) 2565-2573. 
[45] Galbraith DC., Soma M., White RL. A Wide-band Efficient Inductive Transdermal Power and Data Link with Coupling Insensitive Gain. IEEE Transactions on Biomedical Engineering 1987; 34(4) 265-275.

[46] Schuylenbergh KV. Optimization of Inductive Powering of Small Biotelemetry Implants. PhD Thesis, K.U. Leuven ESAT-MICAS, 1998.

[47] Vandevoorde G., Puers R. Wireless Energy Transfer for Stand-alone Systems: A Comparison Between Low and High Power Applicability, Sensors and Actuators APhysical 2001; 92(1-3) 305-311.

[48] Schuylenbergh KV., Puers R. Self-tuning Inductive Powering for Implantable Telemetric Monitoring Systems. Sensors and Actuators A-Physical 1996; 52(1-3) 1-7.

[49] Ziaie B., Rose SC., Nardin MD., Najafi K. A Self-oscillating Detuning-insensitive Class-E Transmitter for Implantable Microsystems. IEEE Transactions on Biomedical Engineering 2001; 48(1) 397-400.

[50] Donaldson N., Perkins T. Analysis of Resonant Coupled Coils in the Design of Radio Frequency Transcutaneous Links. Medical and Biological Engineering and Computing 1983; 21(5) 612-627.

[51] Sokal NO., Sokal AD. Class E-A New Class of High-efficiency Tuned Single-ended Switching Power Amplifiers. IEEE Journal of Solid-State Circuits 1975; 10(3) 168-176.

[52] Guidelines for Evaluating the Environmental Effects of Radio Frequency Radiation, F. C. Commission, 1996.

[53] American National Standard Safety Levels With Respect to Human Exposure to Radio Frequency Electromagnetic Fields, 300 kHz to 100 GHz, ANSI Std. C95.1-1982, 1982.

[54] Sawicki D. Police Traffic SPEED RADAR Handbook.

[55] IEEE Standard for Safety Levels With Respect to Human Exposure to Radio Frequency Electromagnetic Fields, 3 kHz to 300 GHz, IEEE Std. C95.1-1991, pp. 1,1992.

[56] Gabriel S., Lau RW., Gabriel C. The Dielectric Properties of Biological Tissues: 2. Measurements in the Frequency Range $10 \mathrm{~Hz}$ to $20 \mathrm{GHz}$. Physics in Medicine and Biology 1996; 41(11) 2251-2269.

[57] Tang QH., Tummala N., Gupta SKS., Schwiebert L. Communication Scheduling to Minimize Thermal Effects of Implanted Biosensor Networks in Homogeneous Tissue. IEEE Transactions on Biomedical Engineering 2005; 52(7) 1285-1294.

[58] ICNIRP. Guidelines for Limiting Exposure to Time-varying Electric, Magnetic and Electromagnetic Fields (up to $300 \mathrm{GHz}$ ), Health Physics 2009; 97(3) 257-259.

[59] FCC, Available online: http://www.fcc.gov/cgb/sar/. 
[60] Williams DF. Definitions in Biomaterials. In: Williams DF. (ed.) Progress in Biomedical Engineering. Amsterdam: Elsevier, 1987.

[61] Williams DF. On the Mechanisms of Biocompatibility, Biomaterials 2008; 29(20) 2941-2953.

[62] Sieminski AL., Gooch KJ. Biomaterial-microvasculature Interactions. Biomaterials 2000; 21(22) 2233-41.

[63] Rihova B. Immunocompatibility and Biocompatibility of Cell Delivery Systems. Advanced Drug Delivery Reviews 2000; 42(1-2) 65-80.

[64] Press Announcements - "FDA Approves First Implantable Wireless Device with Remote Monitoring to Measure Pulmonary Artery Pressure in Certain Heart Failure Patients." Available online: http://www.fda.gov/newsevents/newsroom/ pressannouncements/ucm399024.htm. 

Chapter 7

\title{
Biomedical Sensor, Device and Measurement Systems
}

\author{
Gaofeng Zhou, Yannian Wang and Lujun Cui \\ Additional information is available at the end of the chapter \\ http://dx.doi.org/10.5772/59941
}

\section{Introduction}

\subsection{Sensor definition and classification}

Sensors are very critical components in all devices and measurement systems. They have been widely used in a lot of fields such as science, medicine, automated manufacturing, environmental monitoring, and so on. Some cheap sensors are finding their ways applying into all sorts of consumer products, from children's toys, dishwashers to automobiles. To some extent, sensors are multidisciplinary and interdisciplinary field of endeavor. This chapter introduces sensor's basic definition and features, biomedical sensors, equivalent components in circuit, signal filters and amplifiers, biomeasurement systems and design.

There are a lot of terms which are often used for sensors including transducer, meter, detector, and gage. Defining the term sensor is a very difficult task. At present, there is not a uniform definition which is agreed by all of us. The most widely used definition is that which has been applied to electrical transducer by the Instrument Society of America(ANSI MC 1, 1975): "Transducer - A device which provides a usable output is in response to a specified measurand." Furthermore, national standard of China points out that sensors consist of sensing component, converting device and electronic circuit. A transducer is more generally defined as a device which converts energy from one form to another. Output of sensor can be an optical, electrical, chemical, or mechanical signal. In the field of electrical engineering, the measurand is physical, chemical, or biological property or condition measured; hence output of biological signal should be an electrical signal, too.

The words sensor and transducer are both commonly used in the context of measurement systems, and often in an interchangeable manner. Transducer is used more in the United States, and sensor has great popularity in Europe and China. The blurring of lines between the exact meaning of sensors and transducers leads to a degree of confusion. Most but not all sensors are transducers, employing one or more transduction mechanisms to produce an electrical 
output signal. According to the basic sensing principle, sensors are classified into mechanical sensors, electrochemical sensors, biosensors, optical sensors, semiconductor sensors, magnetic sensors, and thermal sensors. From different viewpoints, there are different classifying methods. According to the physical parameters measured by sensors, sensors are classified into resistance displacement sensor, inductive displacement sensor, capacitive displacement sensor, piezoelectric pressure sensor, laser interferometer displacement sensor, bore gagging displacement sensor, ultrasonic displacement sensor, optical encoder displacement sensor, optical fiber displacement sensor, optical beam deformation sensor, flow sensor, imaging sensor, temperature sensor, intelligent sensor and chemical ingredient sensor. Biomedical sensors are used to gain the information on body and pathology, which is a branch of biomedical engineering. Biomedical sensors are classified into physical sensor, chemical sensor and biosensor. Physical sensor could be employed to measure blood pressure, body temperature, blood flux, blood viscosity, biological magnetic field, etc. Chemical sensor is utilized to detect the ingredient and concentration of body liquid such as $\mathrm{PH}$ value, $\mathrm{Ca}^{+}$concentration, glucose concentration, etc. Biosensor is used to sense enzyme, antigen, antibody, hormone, DNA, RNA and microbe. In nature, biosensor is a kind of chemical sensor, which is mainly used to detect biological signals.

\subsection{Sensor package and specifications}

Packaging of certain biomedical sensor is an important consideration during the design, fabrication, and use of the device. Obviously, the biomedical sensor has to be safe, soft, and reliable for biomedical sensors often touch the body skin or inner organs of patients. In the development of implantable biosensor, an additional key issue is to consider the biocompatibility of sensor and operational lifetime in body. When a biomedical sensor is implanted into the body, it inevitably contacts with body fluids. Then body will affect the function of biomedical sensor, or sensor will affect the site that it is implanted. For example, protein absorption and cellular deposition can alter the permeability of sensor packaging that is designed to both protect sensor and allow free chemical diffusion of certain analytics between body fluids and the biosensor. Unsuitable packaging of implantable biomedical sensor could lead to drift and a gradual loss of sensor sensitivity and stability overtime. Furthermore, inflammation of tissue, infection, or clotting in a vascular site could produce some harmful or adverse effects on biomedical sensor. Hence, the material used in the construction of sensor's outer body must be biocompatible because they play a crucial pole in determining an overall performance and longevity of implantable biomedical sensor. One convenient method is to utilize various polymer covering material and barrier layers to prevent the toxic sensor components from coming into body. It's very important that packaging material of biomedical sensor must prevent the chemical diffusion of harmful ingredient between biomedical sensor and outer body.

Accurate medical diagnostic procedures require the stringent specifications on the design and use of biomedical sensor. Depending on the intended applications, the performance specifications of biomedical sensor may be evaluated to ensure that the measurement meets the design specifications. 
In order to understand sensor's performance characteristics, it is very important to learn some of the common terminology associated with sensor specifications. The following definitions are commonly used to describe sensor characteristics and select sensor for particular applications.

\section{(1) Measurement range}

The range of sensor corresponds to the minimum and maximum operation limits that sensor is expected to measure accurately. For example, a pressure sensor may have a nominal performance over the operating range from $0 \mathrm{~Pa}$ to $10 \mathrm{MPa}$.

\section{(2) Sensitivity}

Sensitivity refers to the ratio of output change for a given input change. Another way to define sensitivity is to find the slope of calibration line relating the input to the output, as illustrated in figure 1.A high sensitivity implies that a small change in input causes a large change in output.

For example, a pressure sensor may have a sensitivity of $0.4 \mathrm{~V} / \mathrm{Pa}$; that's to say, the output of this sensor will change $0.4 \mathrm{~V}$ for $1 \mathrm{~Pa}$ change in input pressure. If the calibration curve is linear seen in figure 1 (a), then sensitivity of sensor will be constant, whereas the sensitivity of sensor will vary with the input when the calibration is nonlinear, as in figure 1 (b). Alternatively, sensitivity can be defined as the smallest change in input that will result in a detectable change in sensor output.

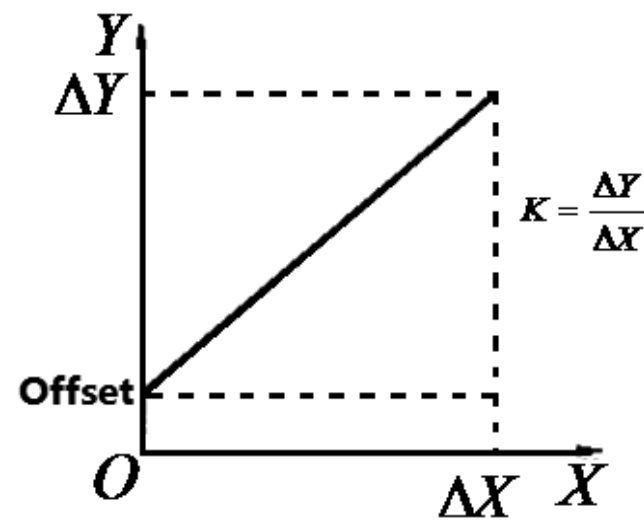

(a) Linear sensor

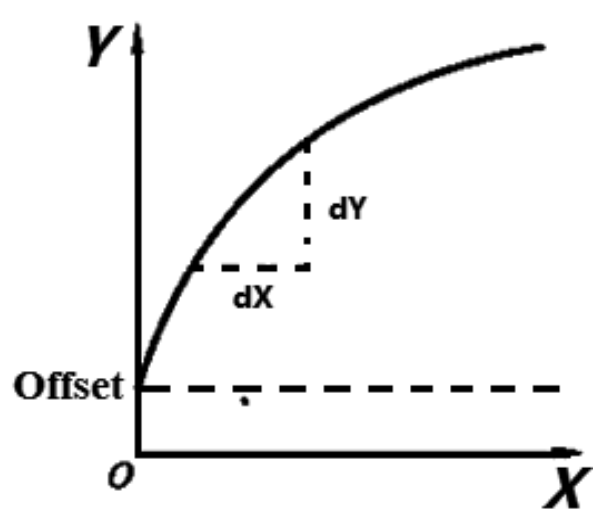

(b) Nonlinear sensor

Figure 1. Input versus output calibration curve of a typical sensor

\section{(3) Accuracy}

Accuracy refers to the difference between the true value and the actual value measured by sensor. Classically, accuracy is expressed as a ratio between the preceding difference and the true value; it is specified as a percent of full-scale readings. Here, note that the true value could be traceable to a primary reference standard. 


\section{(4) Precision}

Precision refers to the degree of measurement reproducibility under the same conditions. Very reproducible readings indicate a high precision. Precision should not be confused with accuracy. For an example, measurement may be very precise but not necessary accurate.

\section{(5) Resolution}

When the input is increased from some arbitrary nonzero value, the output of a sensor will not change until a certain input increment is exceeded. Accordingly, resolution is defined as the smallest distinguishable input change that can be detected with certainty.

\section{(6) Reproducibility}

Reproducibility describes how close measurements are when same input is repeatedly exerted into same sensor under same conditions. When the range of measurement is small, the reproducibility is very high. For example, a temperature sensor may have a reproducibility of $\pm 0.1 \mathrm{~V} /{ }^{\circ} \mathrm{C}$ for a measurement range from $20^{\circ} \mathrm{C}$ to $80^{\circ} \mathrm{C}$. Here, what need to be noticed is that reproducibility can vary depending on the measurement range. In other words, readings can be highly reproducible over one range and less reproducible over a different operating range.

\section{(7) Offset}

Offset refers to the output value when input value is zero, seen in figure 1 (a) and (b).

\section{(8) Linearity}

Linearity of sensor also called nonlinear error of sensor's characteristic curve; it is a measurement of the maximum deviation between calibration curve and fitting curve. Usually, linearity of sensor is expressed as a percent of full-scale readings or a percent of the actual readings. Linearity could be expressed as the following equation:

$$
\sigma_{\text {Linearity }}= \pm \frac{\Delta L_{\max }}{Y_{F . S}} \times 100 \%
$$

Here, $\sigma_{\text {Linearity }}-$-linearity of sensor; $\Delta L_{\max }=\max \left(V_{c a l}-V_{\text {fit }}\right), \Delta L_{\max }$ is the maximum error between calibration line and fitting line; $Y_{F . S}$ is the full-scale meaning value of sensor, $Y_{F . S}=Y_{\max }-Y_{o}, Y_{\max }$ is the maximum deviation of output, $Y_{0}$ is the deviation without any input value.

\section{(9) Response time}

The response time indicates that the time it takes a sensor to reach a percent of its final steadystate value when input of sensor is changed. For example, it takes 10 seconds for pressure sensor to reach 95 percent of its maximum value when a change in pressure of $1 \mathrm{~Pa}$ is measured. Ideally, a short response time indicates the ability of a sensor to respond quickly to change in input. 


\section{(10) Drift}

Drift refers to the change in sensor reading when the input keeps constant. Drift is divided into temperature drift and zero point drift. Zero point drift refers to the output without any input or with a constant input. Zero point drift could be expressed as the following equation:

$$
D_{\text {zero }}=\frac{\Delta Y_{0}}{Y_{F . S}} \times 100 \%
$$

Temperature drift refers to the change of output with the change of temperature. It means the deviation of sensor output, which could be expressed as the following equation:

$$
D_{\text {Temp }}=\frac{\Delta Y_{\max }}{Y_{F . S} \times \Delta T} \times 100 \%
$$

Here, $\Delta T$ is the change of temperature.

\section{(11) Hysteresis}

In some sensors, the input-output characteristic follows a different nonlinear trend, depending on whether input increase or decrease, as in figure 2. For example, a certain pressure sensor could produce a different output voltage when the input pressure varies from zero to full scale and then back to zero. When the measurement is not perfectly reversible, the sensor will show its hysteresis. If a sensor exhibits hysteresis, the input-output relationship is not unique, but depends on the direction change to the input value of sensor.

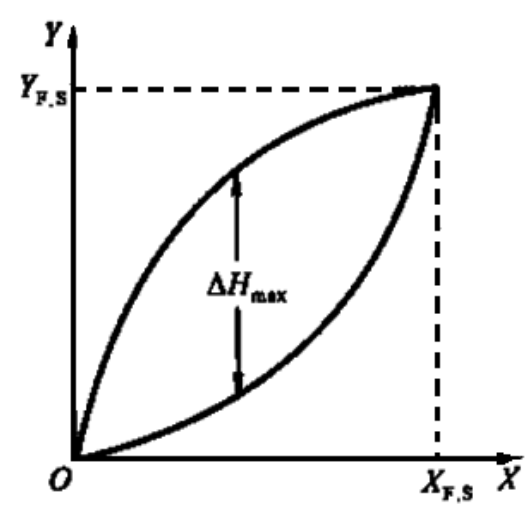

Figure 2. Input versus output response of a sensor with hysteresis

\subsection{Special features of biosensor}

Biosensor is a kind of device which senses biomaterial and its concentration, and which converts the biosignal into electrical signal. Biosensor has the function of acceptor and 
converter, which configuration is seen in figure 3. In biosensor, the physicochemical change of the biologically active material resulting from the interaction with the analyte must be converted into an electrical output signal by an appropriate converter. Biosensor's sensing components mainly have enzymes, cells, antibodies, DNA (Deoxyribonucleic acid), chemical electrode, microbe and other biologically active agents in analytical devices. In the course of detecting the parameters of analytes, biomaterial should be always immobile. In order to develop biosensor, some biotechnology has to be studied and applied, such as DNA biosensor, $\mathrm{PH}$ sensor, microelectrode, and so on.

The special features of biosensor are the following:

1. Biological active material immobilized is used as catalyst, and expensive reagents could be repeatedly used to detect same biological parameters.

2. Biosensor has intensive specificity. Biomaterial only senses definitive ingredient and it is not affected by color and concentration of measured material.

3. Biosensor could quickly analyze the result of the measurand.

4. Biosensor's accuracy is very high, which relative error could reach one percent.

5. Biosensor's analyzing system is very simple.

6. The cost of biosensor is very low.

According to biological sensing component, biosensor may be divided into five classes: enzyme sensor, microbe sensor, cell sensor, tissue sensor, and immune sensors. According to the signal converter of biosensor, biosensor may be also divided into five classes: bioelectrode sensor, semiconductor biosensor, optical biosensor, piezoelectric biosensor and thermal biosensor. According to the interaction between sensing component and measured material, biosensor can be divided into two classes: affinity biosensor and catalytic biosensor.
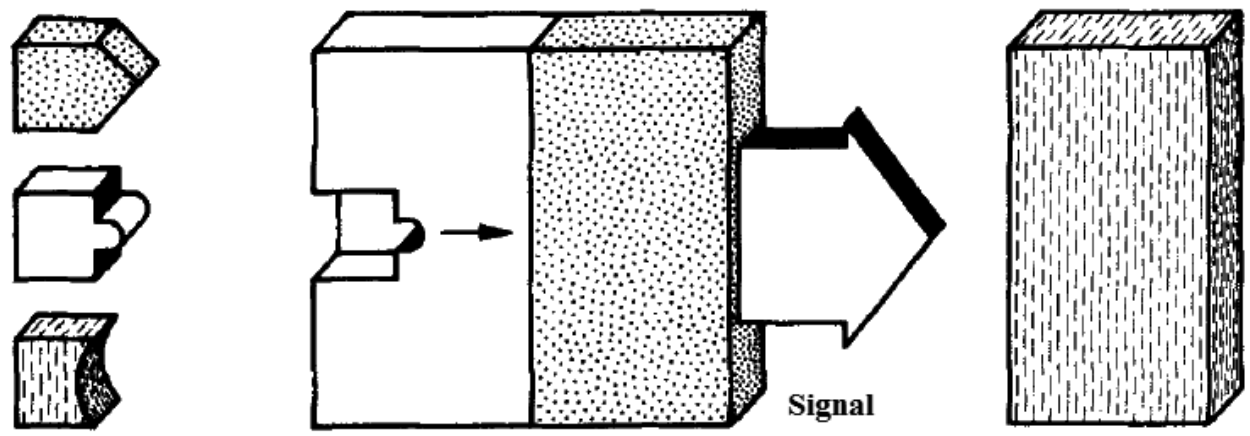

\section{Biological sensing component Receptor}

Converter

Electronics

Figure 3. Common configure of a biosensor 


\subsection{Biomedical sensor's application}

In biomedical field, main applications of biomedical sensor are as follows:

1. Detecting the information of clinical chemistry. In the field of medical clinic and basic research, the biology's information needs to be detected to ensure the present state of given biology. For example, before operating on a patient, a doctor needs to know the body temperature and blood pressure. Under this condition, clinic thermometer and blood sensor has to be employed to help doctor quickly detect body temperature and blood pressure of patient.

2. Continuously monitoring some parameters of biology outside and inside. In biomedical field, heart frequency has to be monitored continuously by heart sound sensor for a few days after operation. In military, some viruses need to be found by biosensor to hold back the attacking from enemy.

3. Control. In medicine, people usually utilize some parameter detected by biomedical sensor to control or adjust physiological course of body. In the food industry, biomedical sensor could be utilized to measure some enzyme and its concentration to control the process of fabricating food and to analyze the nutritional ingredient of food. In military, biomedical sensor could be employed to detect the situation of battle field to adjust the strategy of spying or attacking enemy.

Of course, biomedical sensor such as PH sensor could be also employed to detect our atmosphere and condition to improve our living situation.

\section{Biomedical sensors}

\subsection{Biomedical sensor classification}

Many different kinds of sensors can be used in biomedical application. According to the sensing principle in biomedical application, biomedical sensors can be classified into physical sensors and chemical sensors, seen in table 1 .

It's possible to categorize all sensors as being physical or chemical. In the case of physical sensors, quantities such as geometric, mechanical, thermal, and hydraulic variables are measured. In biomedical applications these variables can include things such as muscle displacement, blood pressure, core body temperature, blood flow, cerebrospinal fluid pressure, and bone growth velocity. Two types of physical sensors deserve special mention with regard to their biomedical application: sensors of electrical phenomena in the body, usually known as electrodes, play a special role as a result of their diagnostic therapeutic applications. The most familiar of these are sensors used to pick up the electrocardiogram, an electrical signal produced by the heart. The other type of physical sensor that finds many applications in biology and medicine is optical sensor. These sensors can utilize light to collect information, and, in the case of fiber optic sensors, light is the signal transmission medium as well. 
The second major classification of sensing device is chemical sensors. In this case sensors are concerned with the chemical quantities such as identifying the presence of chemical composite, detecting the concentration of various chemical species, and monitoring the chemical activities in the body for diagnostic and therapeutic application. A wide variety of chemical sensors are classified in many ways. Chemical sensors are used to detect chemical components being measured and chemical composition measured in the gas phase. Electrochemical sensors are utilized to measure chemical concentration, or more precisely, activities based on chemical reactions that interact with electrical systems. Photometric chemical sensors are optical devices that detect chemical concentrations based on changes in light transmission, reflection or color. Other types of physical chemical sensors such as the mass spectrometer utilize various physical methods to detect and quantify chemicals associated with biologic systems.

\begin{tabular}{ll}
\hline Class of sensor & Biomedical sensor \\
\hline Physical sensors & \\
\hline & Geometric \\
\hline Mechanical \\
\hline Thermal \\
\hline Hydraulic \\
\hline Ehemical sensors & Electric \\
\hline & Optical \\
\hline & Gas \\
\hline Electrochemical \\
\hline Photometric \\
\hline Other physical chemical methods \\
\hline Biopotential electrodes & Body surface biopotential electrode \\
\hline Metal plate \\
\hline Intracavitary and intratissue electrode \\
\hline Microelectrode \\
\hline \\
\hline Enzyme \\
\hline Protein \\
\hline Antigen \\
\hline Antibody \\
\hline Ligand \\
\hline Cell \\
\hline DNA \\
\hline
\end{tabular}

Table 1. Classifications of biomedical sensor 
Although bioanalytic sensors are essentially chemical sensors, they are often classified as a separate major sensor category. These devices incorporate biologic recognition reaction such as enzyme-substrate to identify complex biochemical molecules. The use of biologic reactions gives bioanalytic sensors high sensitivity and specificity in identifying and quantifying biochemical substances.

\subsection{Oxygen and carbon dioxide sensor for blood}

Measurements of arterial blood gas $\left(\mathrm{pO}_{2}\right.$ and $\left.\mathrm{pCO}_{2}\right)$ and $\mathrm{pH}$ are frequently performed by on critical patients in both the operating rooms and intensive care unit. They are selected and used by the physician to adjust mechanical ventilation or administer pharmacological agents. Such measurement could provide information about the respiratory and metabolic imbalance in the body and reflect the change of blood oxygen increment and carbon dioxide $\left(\mathrm{CO}_{2}\right)$ elimination.

Noninvasive sensors for measuring $\mathrm{O}_{2}$ and $\mathrm{CO}_{2}$ in arterial blood are based on the discovery that gases such as $\mathrm{O}_{2}$ and $\mathrm{CO}_{2}$ can easily diffuse from body skin. Diffusion occurs due to a partial pressure difference between the blood in the superficial layers of the skin and the outermost surface of the skin. Such idea has been used to develop two types of noninvasive electrochemical sensors $\mathrm{pO}_{2}$ and $\mathrm{pCO}_{2}$. The discovery that blood changes its color depending on the percent of oxygen has led to the development of several optical methods to measure the oxygen saturation in blood.

\subsubsection{Oxygen sensor for blood}

The method for measuring blood oxygenation is very great important in assessing the circulatory and respiratory condition of a patient. The blood from the lungs to the tissues in two distinct states transports oxygen. Under normal physiological conditions, approximately $2 \%$ of the total amount of oxygen carried by the blood is dissolved in the plasma. This amount is proportional to the blood $\mathrm{pO}_{2}$. The $98 \%$ remain is carried inside the erythrocytes in a loose reversible chemical combination with hemoglobin $(\mathrm{Hb})$ as oxyhemoglobin $\left(\mathrm{HbO}_{2}\right)$. Thus, there are two methods for measuring the blood oxygenation: either using polarographic $\mathrm{pO}_{2}$ sensor or measuring oxygen saturation (the relative amount of hemoglobin dioxide $\mathrm{HbO}_{2}$ in the blood) by means of an optical oximeter.

$\mathrm{A} \mathrm{PO}_{2}$ sensor, also widely known as a Clark electrode, is used to measure the partial pressure of $\mathrm{O}_{2}$ gas in a sample of air or blood. This sensor is categorized as an amperometric sensor and requires an external polarization bias source. The measurement is based on the principle of polarography as illustrated in figure 4 . The electrode utilizes the ability of oxygen $\mathrm{O}_{2}$ molecules to react chemically with $\mathrm{H}_{2} \mathrm{O}$ in the presence of electrons to produce hydroxyl $\left(\mathrm{OH}^{-}\right)$ions. This electrochemical reaction, called an oxidation/reduction or redox reaction, generates a small current and requires an externally applied constant polarizing voltage source of about $0.6 \mathrm{~V}$.

Oxygen is reduced (consumed) at the surface of a noble metal (such as platinum or gold) cathode (this electrode is connected to the negative side of voltage source) according to the following the chemical reaction: 


$$
\mathrm{O}_{2}+2 \mathrm{H}_{2} \mathrm{O}+4 e \leftrightarrow 4 \mathrm{OH}^{-}
$$

In this reduction reaction, one $\mathrm{O}_{2}$ molecule takes four electrons and reacts with two water molecules, generating four hydroxyl ions. The resulting $\mathrm{OH}^{-}$ions migrate and react with a reference $\mathrm{Ag} / \mathrm{AgCl}$ anode (this electrode is connected to the positive side of voltage source), causing a two-step oxidation reaction as follows:

$$
\begin{gathered}
\mathrm{Ag} \leftrightarrow \mathrm{Ag}^{+}+e \\
\mathrm{Ag}^{+}+\mathrm{Cl}^{-} \leftrightarrow \mathrm{AgCl} \downarrow
\end{gathered}
$$

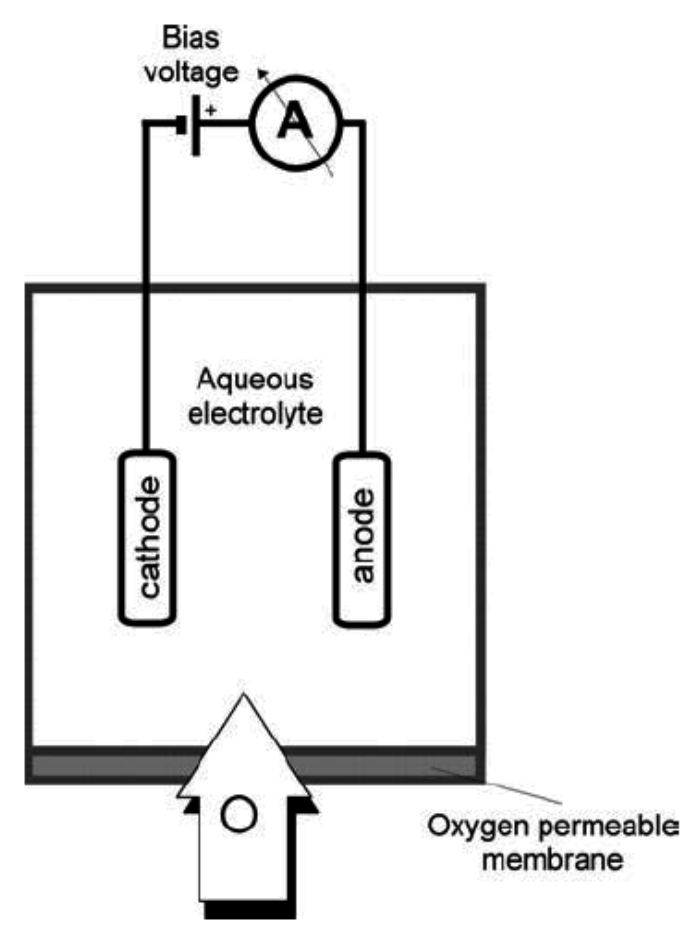

Figure 4. Sensing principle of Clark-type $\mathrm{pO}_{2}$ sensor

In this oxidation reaction, silver from the anode electrode is firstly oxidized to silver ions, and electrons are liberated to the anode. These silver ions are immediately combined with chloride ions to form a kind of compound precipitant silver chloride $\mathrm{AgCl}$ which precipitates on the surface of anode. The transient current between the anode and the cathode in the external circuit produced by this reaction is directly proportional to the number of $\mathrm{O}_{2}$ molecules constantly reduced on the surface of the cathode. The electrodes in the polarographic cell are immersed in an electrolyte solution of potassium chloride and surrounded by an $\mathrm{O}_{2}$-permeable or polypropylene membrane that permits gases to diffuse slowly into electrode. Thus, by 
measuring the change in current between the cathode and the anode, the amount of oxygen that is dissolved in the solution can be determined.

\subsubsection{Carbon dioxide sensor for blood}

Electrodes for measuring partial pressure of carbon dioxide $\mathrm{CO}_{2}$ in blood are based on measuring the $\mathrm{pH}$ as illustrated in figure 5 . The measurement is based on the observation that it forms a weakly dissociated carbonic acid $\left(\mathrm{H}_{2} \mathrm{CO}_{3}\right)$ that subsequently forms free hydrogen and bicarbonate ions when $\mathrm{CO}_{2}$ is dissolved in water according to the following reaction:

$$
\mathrm{CO}_{2}+\mathrm{H}_{2} \mathrm{O} \leftrightarrow \mathrm{H}_{2} \mathrm{CO}_{3} \leftrightarrow \mathrm{H}^{+}+\mathrm{HCO}_{3}^{-}
$$

As a result of this chemical reaction, the $\mathrm{pH}$ of the solution is changed. This change generates a potential between the glass $\mathrm{pH}$ and a reference electrode that is proportional to the negative logarithm of the concentration of the carbon dioxide $\mathrm{pCO}_{2}$ in the plasma.

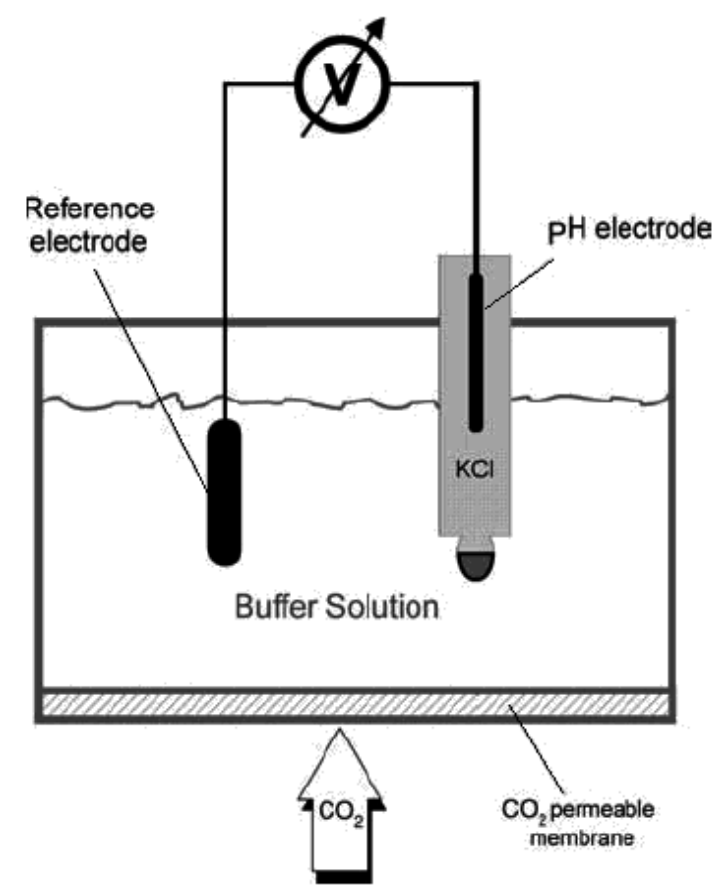

Figure 5. Sensing principle of a $\mathrm{pCO}_{2}$ electrode

\subsection{Heart sound sensor}

The expansion and shrinkage of heart necessarily lead to the vibration of artery that is formed by blood turbulence in vein. When the vibration of artery is transported to the surface of 
thoracic cavity, heart sound will take place. Heart sound is very valuable for doctor to diagnose many kinds of diseases in our body.

The range of heart sound is from $20 \mathrm{~Hz}$ to $200 \mathrm{~Hz}$. Low limit frequency of heart sound could reach about $4 \mathrm{~Hz}$ and high frequency limit is greater than $1000 \mathrm{~Hz}$. There are many kinds of medical heart sound sensors that are divided into two classifications: air conduction heart sound sensor and direct conduction heart sound sensor. Air conduction heart sound sensor consists of air chamber and common sensor. Such sensor has obvious defects: low sensitivity and easy disturb by surrounding circumstance. Hence, in clinic application, the most sensors applied are direct conduction heart sound sensor.

\subsubsection{Piezoelectric heart sound sensor}

The sensing structure of piezoelectric acceleration sensor is illustrated in figure 6. Such sensor is used to measure heart sound. Its structure is very simple, which consists of vibration mass block and piezoelectric crystal. A stress spring is utilized to exert a certain stress on vibration mass block between top shell and mass block. Such method could timely adjust the linear characteristic of sensing component. This sensor's gravity is less than $30 \mathrm{~g}$, and is used to detect heart sound and buffeting from body organisms.

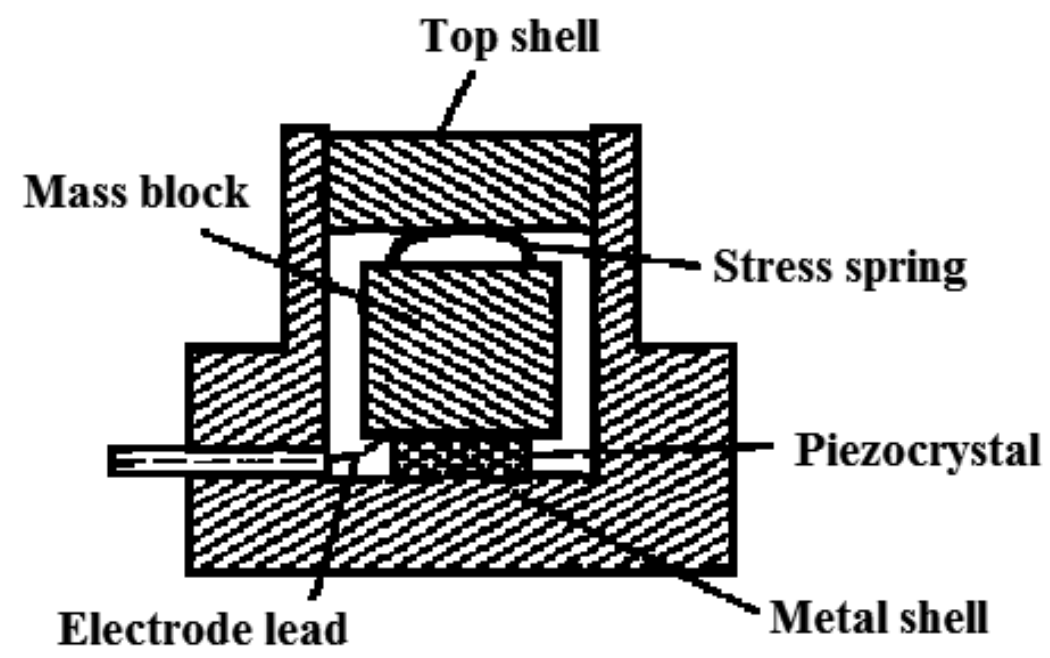

Figure 6. Sensing structure of piezoelectric acceleration sensor

\subsubsection{Fetus heart sound sensor}

Detecting fetus heart sound is very important in clinic application for doctor sometimes needs to grasp the present body status of fetus. PVDF piezoelectric thin film sensor is utilized to be fit for the measurement of fetus's heart sound as illustrated in figure 7. Its piezoelectric coefficient is the following: 


$$
\begin{aligned}
& d_{31}=(15 \sim 30) \times 10^{-12} \mathrm{C} / \mathrm{N} \\
& d_{33}=-(5 \sim 8) \times 10^{-12} \mathrm{C} / \mathrm{N}
\end{aligned}
$$

In this structure, silicon rubber converts the vertical motion of itself into the radial motion of PVDF piezoelectric thin film and then corresponding dynamic charge produced by PVDF thin film is proportional to the externally transient force. The voltage along thickness direction is output. Obviously, its work mode is $d_{31}$ work mode. For both thin film and silicon rubber are very soft, they could well touch the skin of body belly. Then fetus heart sound is gained to judge fetus heart. Design requirement of PVDF heart sound sensor is as follows:

1. In the same piezoelectric thin film, one part is used as driving electrode; another is utilized as receiving the sound wave from heart sound.

2. Dynamic response characteristics on the surface of belly should be considered to well mate belly skin and sensor.

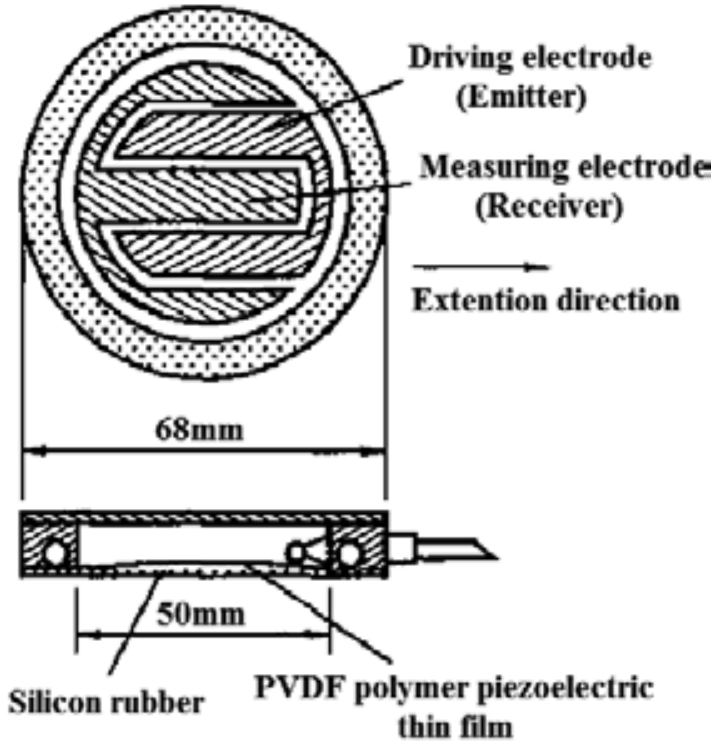

Figure 7. Sensing structure of PVDF piezoelectric acceleration sensor

With the development of sensing technology, more and more heart sound sensors are appearing in our life. Although they have different sensing principle, their functions are alike.

\subsection{Blood flow sensor}

If oxygen and nutrients are to reach tissues, the flow of blood must be maintained in body. Cardiac output flow is often measured as an index of cardiac performance, blood flow through arterial graft is used to ensure that a graft has been successfully inserted during surgery, or 
the blood flow in peripheral arteries and veins may be measured to assess vascular diseases. There are usually two kinds of measuring methods: one method is direct measurement, sensor is inserted into the blood pipe to sense transient blood flow; another one is indirect measurement, sensor is placed outside vein and senses blood flow by the parameter related to the blood flow. Here, an electromagnetic flow sensor as an example is introduced to demonstrate the measurement of blood flow.

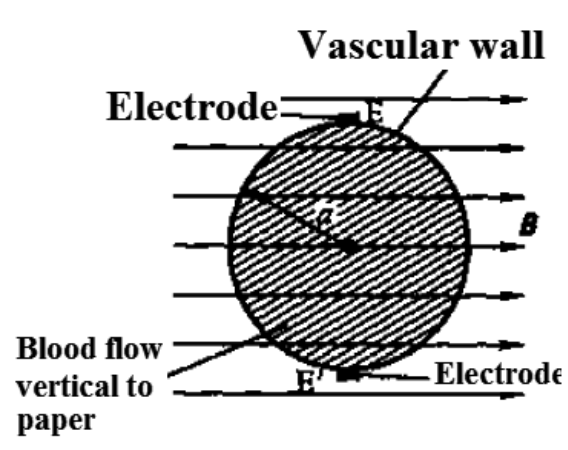

(a)

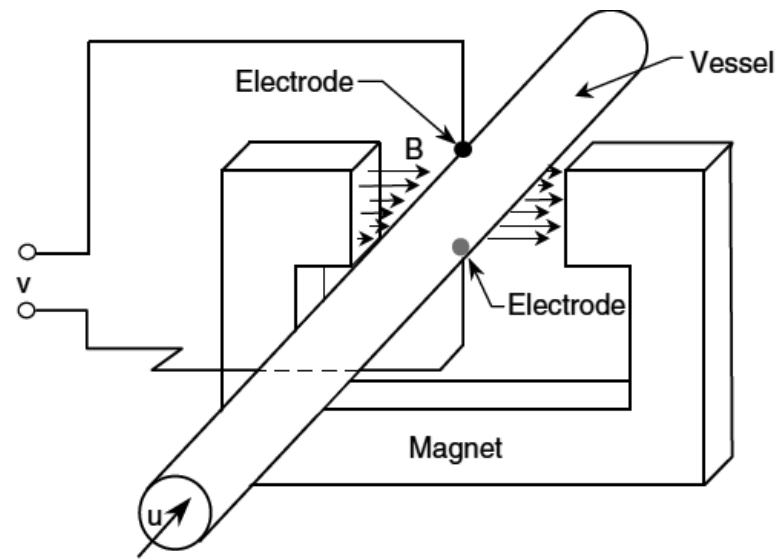

(b)

Figure 8. Sensing principle of electromagnetic flow sensor (a) Relation diagram between electrode and magnetic field intensity; (b) Three dimension diagram

Blood flow through an exposed vessel could be measured by means of electromagnetic flow sensor. Electromagnetic flow sensor can be used in biomedicine and science research studies to measure blood flow in major blood vessels near the heart. Such sensor requires that the tested vein must be peeled off and placed into the magnetic gap of sensor. According to the output voltage of sensor, the mean velocity of vein can be calculated and known. And then in terms of the section area tested of vein, the blood flow could be gained finally. According to above idea, the sensing principle of electromagnetic flow sensor is illustrated in figure 7.

Magnetic field intensity $B$ is exerted along the direction vertical to vein, two electrodes are installed at both sides of vein, and then potential between two electrodes could be tested:

$$
V=2 a B v_{a}
$$

Here, $B$ is the magnetic induction intensity at the magnetic gap; $a$ is the radius of tested vein; $v_{a}$ is the mean velocity of vein during given test time; $V$ is the output potential between two electrodes EE'.

Practically, this device consists of a clip-on probe that fits snugly around the blood vessel, as illustrated in figure 8 . The probe contains electrical coils to produce an electromagnetic field that is transverse to the direction of blood flow. This coil is usually excited by an AC current. 


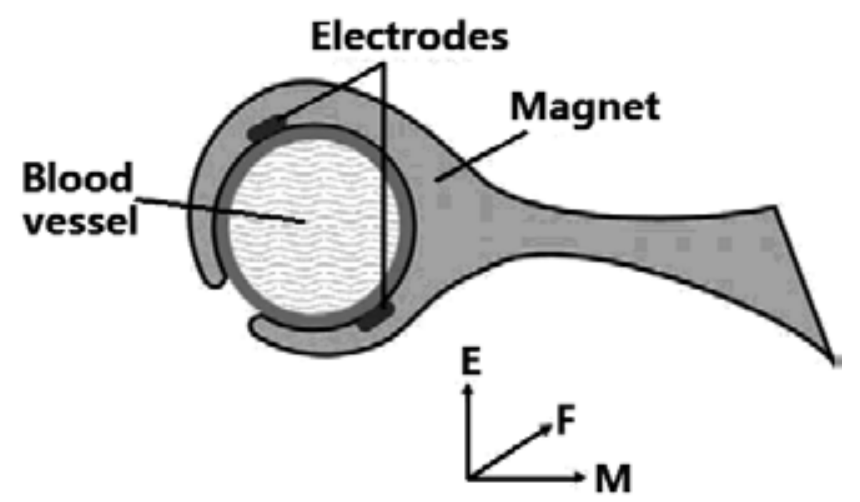

Figure 9. Electromagnetic blood flow sensor

A pair of very small biopotential electrodes is attached to the housing and rest against the wall of blood vessel to pick up the induced potential. The flow-induced voltage is an AC voltage at the same frequency as the excitation voltage. Utilizing AC method instead of DC excitation could help to remove any offset potential error due to the contact between the vessel wall and the biopotential electrodes.

Certainly, ultrasonic wave could be also used to detect blood flow of artery. In biomedical application, there are four kinds of ultrasonic wave blood flow sensors according to specific sensing principle and methods: (1) pulse time difference; (2) voice beam deflection; (3) phase shift; (4) Doppler frequency shift. Readers could research biomedical engineering handbook to learn more information.

\subsection{Respiration sensor}

Respiration measurement often includes two classes: physiological parameter measurement and gas ingredient from respiration system. What sensor the former utilizes is physical sensor, and what sensor the latter employs is chemical sensor and biological sensor. Here, respiration sensor which belongs to the first class is only introduced and explained. The measurement of respiration system is important basis of clinic diagnosis, and it is necessary for patients in the fields of surgery, baby and critically ill patient's monitoring, sports medicine, and medical research. The measurement of respiration system could be classified into three classes of parameters: respiration frequency, respiration flow and lung respiration volume.

In biomedical research or clinic monitoring, respiration frequency of patient needs to be sometimes detected to record the physiological status. Figure 9 illustrates a kind of sensor for respiration frequency based on thermistor sensing principle. Thermistor is mounted to the front-end of binder. When binder clamps the nares, airstreams from body flows through the surface of thermistor. According to the change of thermistor value, the respiration frequency would be measured. 


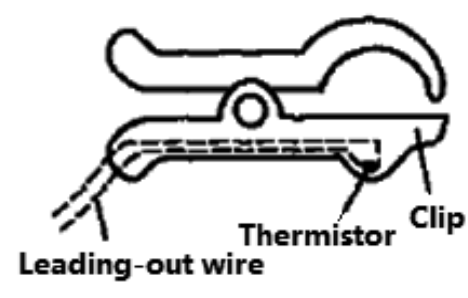

(a)

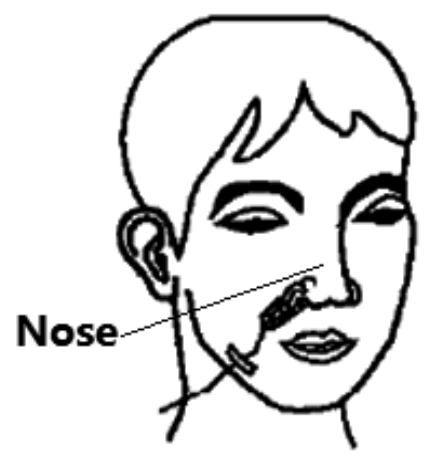

(b)

Figure 10. Thermistor sensor for respiration frequency (a)structure diagram, (b)measurement diagram

Of course, elastic strain instrument could be also utilized to detect the respiration frequency. Its sensing principle is such: resistance wire is fixed to the surface of elastic plastic pipe. And then mercury or other electrolyte is sealed into the elastic plastic pipe. After elastic plastic pipe is adhered to the front of breast, respiration would lead to the length change of elastic plastic pipe. Such length change causes the change of resistance wire which could show the change of respiration frequency. When resistance wire is introduced into a detecting circuit, the respiration frequency will be sensed and measured.

\subsection{Blood pressure sensor}

If blood circulation is to be maintained in the body, tissues are to be perfused with oxygen. Then correct pressure measurement has to be applied in the vascular system. The usual blood pressure methods have: liquid coupling direct measurement, pipe-end sensing measurement, indirect blood pressure sensing measurement. Liquid coupling direct measurement means that the pipe filled with liquid is inserted into the measured part and that the pressure is measured by liquid coupling of pipe end position in the body, which is the simplest method. Pipe-end sensing measurement employs pipe-end sensor to measure blood pressure. Pipe-end sensor which can convert the pressure signal into electronic signal is placed on the measured part. And then the electronic signal measured is transmitted to the external wire. Such method could avoid the distortion of signal of blood pressure. Pipe-end sensing measurement has a lot of advantages, but such method needs to activate the skin and relative sensors have to been placed into the body. Hence indirect blood pressure measurement is noted by people and continuously explored. Blood-pressure meter is a classic example of indirect blood pressure measurement, which is shown in figure 10.

In figure 10, the sensing principle is based on Coriolis sound. Gas is filled into cuff to hold back the arterial blood flow. And then gas in cuff is sent out slowly to monitor whether there appears arterial blood flow at the downstream of arterial blocking point. Here, the employed sensor is common mercury pressure meter. And such method is up to the actual experience of staff. 


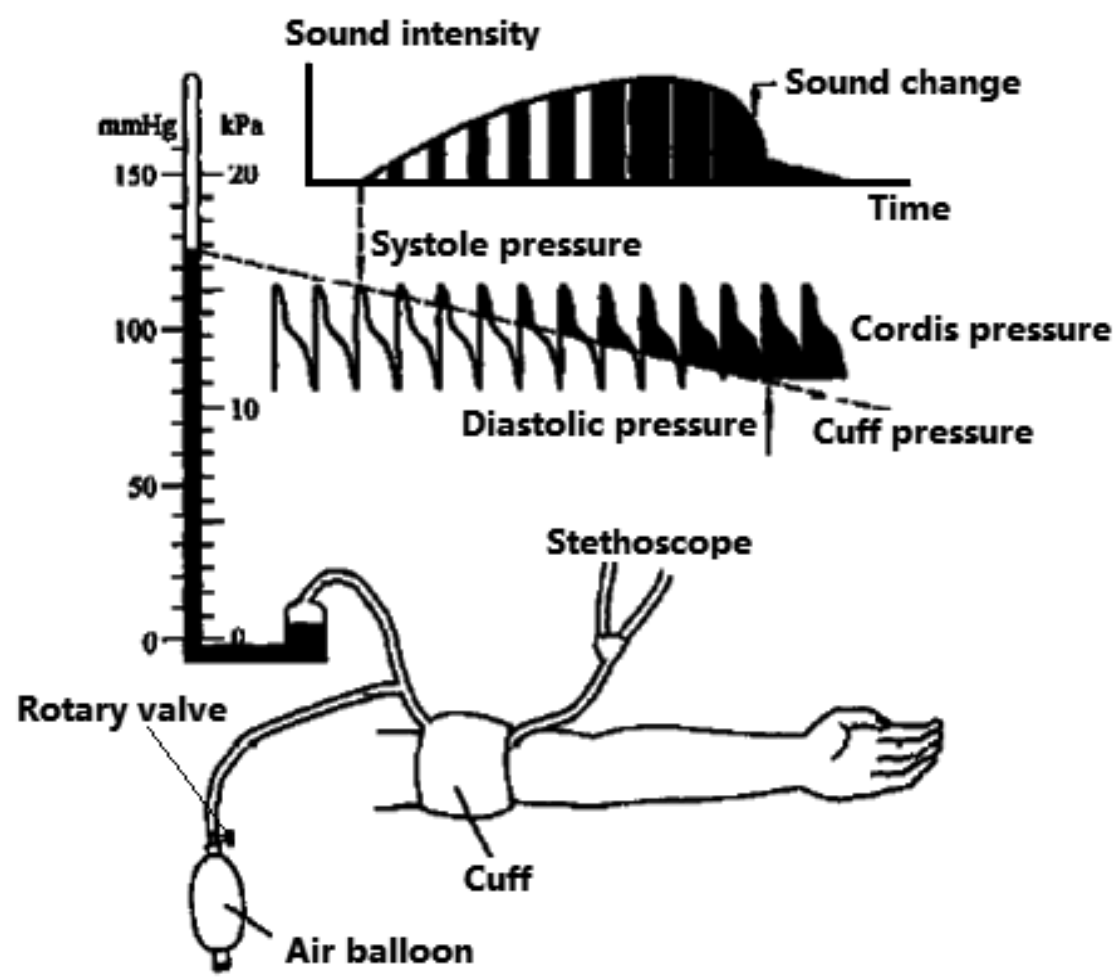

Figure 11. Sensing principle of indirect blood pressure measurement

When coriolis sound is heard, namely when blood flows through artery blood pipe, blood pressure in cuff is shrinking pressure in artery pipe. When blood flows recover normal level, blood pressure in cuff is diastolic pressure of artery. Systole pressure and diastolic pressure are recorded as blood pressure. Such method is not harmful to the skin or organ in the body.

\subsection{Electrochemical electrode}

Biopotential measurements are made using different kinds of specialized electrochemical electrodes. The function of electrodes is to couple the ionic potentials generated inside the body to an electronic instrument. Biopotential electrochemical electrode is classified either as noninvasive (e.g. skin surface) or invasive (e.g. microelectrode, wire electrode) electrodes. When a metal is placed in an electrolyte solution, a charge distribution is created next to the metal/electrolyte solution interface as illustrated in figure 11. The localized charge distribution causes an electronic potential by electrochemical electrode, called half-cell potential, to be developed across the interface between metal electrode and electrolyte solution.

The half-cell potentials of several important metals are listed in table 2. Here, a point needs to be pointed out that hydrogen electrode is considered to be a standard electrode against which the half-cell potentials of other metal electrodes are measured. 


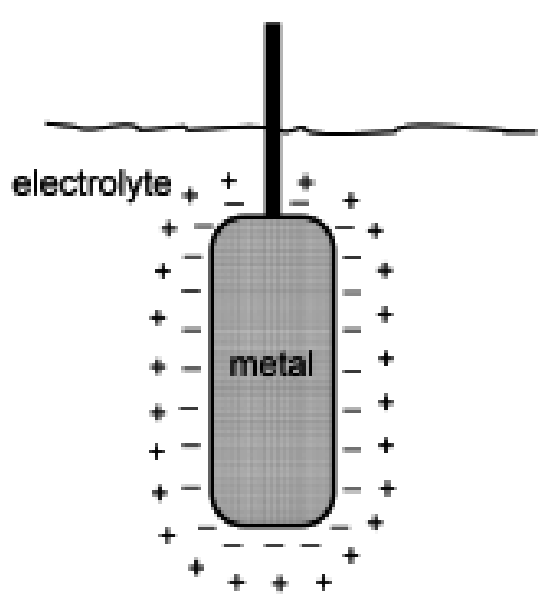

Figure 12. The charge distribution at a electrolyte/metal interface

Silver and zinc electrodes are immersed in an electrolyte solution. And then we may calculate the potential drop between two electrodes. From table 2, the half-cell potentials for silver and zinc electrodes are $0.799 \mathrm{~V}$ and $-0.763 \mathrm{~V}$ respectively. Hence, the half-cell potentials between two electrodes are equal to the following value:

$$
0.799-(-0.763)=1.562 \mathrm{~V}
$$

Typically, utilizing the electrochemical electrodes composed of the same metals could measure the half-cell potentials. Hence, the two half-cell potentials for these electrodes would be equal in magnitude. Some common electrodes are introduced here, which is utilized as a sensor.

\subsubsection{ECG electrodes}

A typical flexible biopotential electrode for ECG (electrocardiogram, ECG) recording is composed of certain polymers or elastomers which are made electrically conductive by the addition of a fine carbon or metal powder. These electrodes as illustrated in figure 13a are available with prepasted $\mathrm{AgCl}$ gel for quick easy application to the skin using a double-sided peel-off adhesive tape. The most common type of biopotential electrode is the silver/silver chloride electrode $(\mathrm{Ag} / \mathrm{AgCl})$, which is formed by electrochemically depositing a very thin layer silver chloride onto the surface of silver electrode as illustrated in figure 13b. These electrodes are recessed and imbedded in foam that has been soaked with an electrolyte paste to provide good electrical contact with the skin. The electrolyte saturated foam is also known to reduce motion artifacts which are produced during stress testing when the layer of the skin moves relative to the surface of the $\mathrm{Ag} / \mathrm{AgCl}$ electrode. This motion leads to the large interference in the recorded biopotential and, in the extreme cases, could severely degrade the measurement. 


\begin{tabular}{ll}
\hline Primary Metal and Chemical Reaction & Half-cell Potential \\
\hline $\mathrm{Al} \rightarrow \mathrm{Al}^{3+}+3 \mathrm{e}^{-}$ & -1.706 \\
\hline $\mathrm{Cr} \rightarrow \mathrm{Cr}^{3+}+3 \mathrm{e}^{-}$ & -0.744 \\
\hline $\mathrm{Cd} \rightarrow \mathrm{Cd}^{2+}+2 \mathrm{e}^{-}$ & -0.401 \\
\hline $\mathrm{Zn} \rightarrow \mathrm{Zn}^{2+}+2 \mathrm{e}^{-}$ & -0.763 \\
\hline $\mathrm{Fe} \rightarrow \mathrm{Fe}^{2+}+2 \mathrm{e}^{-}$ & -0.409 \\
\hline $\mathrm{Ni} \rightarrow \mathrm{Ni}^{2+}+2 \mathrm{e}^{-}$ & -0.230 \\
\hline $\mathrm{Pb} \rightarrow \mathrm{Pb}^{2+}+2 \mathrm{e}^{-}$ & -0.126 \\
\hline $\mathrm{H} \rightarrow 2 \mathrm{H}^{+}+2 \mathrm{e}^{-}$ & $0.000($ stand by definition $)$ \\
\hline $\mathrm{Ag} \rightarrow \mathrm{Ag}^{+}+\mathrm{e}^{-}$ & +0.799 \\
\hline $\mathrm{Au} \rightarrow \mathrm{Au}^{3+}+3 \mathrm{e}^{-}$ & +1.420 \\
\hline $\mathrm{Cu} \rightarrow \mathrm{Cu}^{2+}+2 \mathrm{e}^{-}$ & +0.340 \\
\hline $\mathrm{Ag}+\mathrm{Cl} \rightarrow \mathrm{AgCl}^{-} 2 \mathrm{e}^{-}$ & +0.223 \\
\hline
\end{tabular}

Table 2. Half-cell Potentials of Important Metals

\section{Mylar substrate with $\mathrm{AgCl}$ paste}

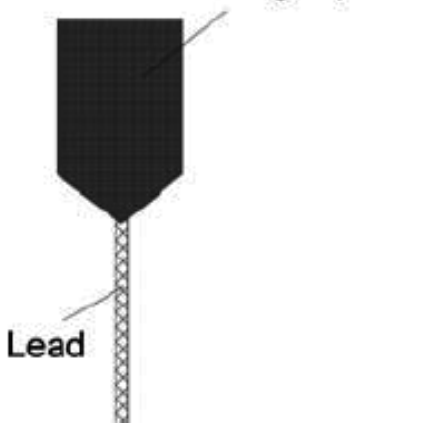

(a) Flexible Mylar electrode

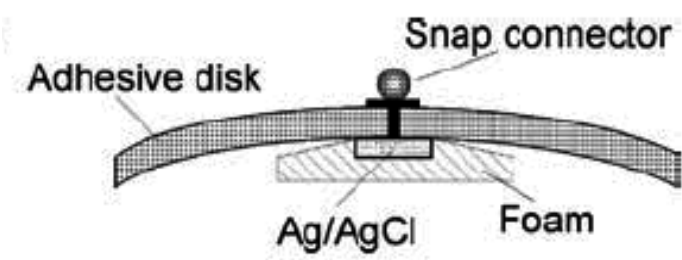

(b) Disposable snap-type $\mathrm{Ag} / \mathrm{AgCl}$ electrode

Figure 13. Biopotential skin surface ECG electrode

\subsubsection{EMG electrodes}

Electrochemical electrodes are also used to record electromyography (EMG) signals from different muscles in the body. The body and size of the recorded EMG signals depends on the electrical property of these electrodes and the recording location. For invasive recordings, proper skin preparation, which normally involves cleaning the skin with alcohol or the application of a small amount of an electrolyte paste, helps to minimize the impedance of the 
skin-electrode interface and to improve the quality of recording signal considerably. The most common electrodes used for the surface EMG recording and nerve conduction studies are circular discs, about $1 \mathrm{~cm}$ in diameter, that are made of silver or platinum. For direct recording of electrical signals from nerves and muscle fibers, a variety of percutaneous needle electrodes are available as illustrated in figure 14. The most common type of needle electrode is the concentric bipolar electrode as illustrated in figure 14a. This electrode is made from the thin metallic wires encased inside a larger canola or hypodermic needle. The two wires serve as the recording and reference electrodes.

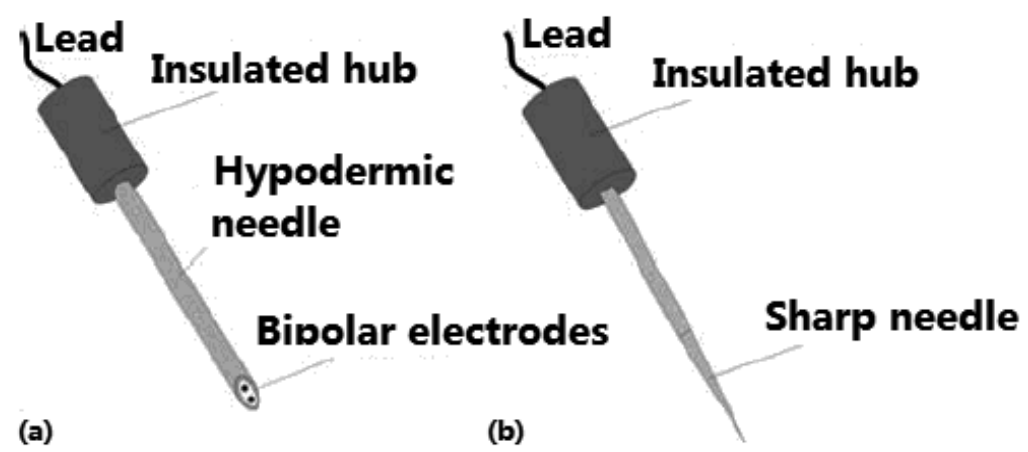

Figure 14. Intramuscular biopotential electrode:(a)bipolar electrode, (b)unipolar configuration

Another type of percutaneous EMG electrode is the unipolar needle electrode as illustrated in figure $14 \mathrm{~b}$. This electrode is made of a thin wire that is most insulated by a thin layer near the distal tip. Unlike bipolar electrode, this type of electrode requires a second unipolar reference electrode to form a closed electrical circuit. The second recording electrode is normally placed either adjacent to the recording electrode or attached to the surface of our skin.

\subsubsection{EEG electrodes}

The most commonly used electrode for recording electroencephalographic (EEG) signals from the brain are cup electrodes and subdermal needle electrodes. Cup electrodes are made of platinum or tin and are approximately $5-10 \mathrm{~mm}$ in diameter. The cup electrodes are filled with an electrolyte gel and can be attached to the scalp with an adhesive tape.

Recording the biopotentials from the scalp is very difficult because hair and oily skin hold back the good electrical contact. Hence, clinicians sometimes prefer to use subdermal needle electrodes (EEG electrodes) instead of the metal surface electrodes for EEG recording. These electrodes are both fine platinum or stainless-steel needle electrodes about $10 \mathrm{~mm}$ long by $0.5 \mathrm{~mm}$ wide, which are inserted under the skin to provide a better electrical contact.

\subsubsection{Microelectrodes}

Microelectrodes are biopotential electrodes with ultra-fine tapered tip that can be inserted into biological cells. These electrodes play a very important role in recording action potentials from 
single cells and are used in neurophysiologic studies to comprehend the course of biological information conversion and transmission in our body. The tip of these electrodes must be very small with respect to the dimensions of the biological cell to avoid cell damage and the same time sufficiently strong to penetrate the cell wall. The electrode which is applied to microbe studies is called microelectrodes. Generally, there are three types of microelectrodes: (1) glass microelectrodes, (2) metal electrodes, and (3) solid-state microprobes.

For glass microelectrodes, when the tip of such electrodes is inserted into an electrolyte solution, such as the intracellular cytoplasm of a biological cell, ionic current can flow through the fluid junction at the tip of the microelectrode. Such mode could establish a closed electrical circuit between two $\mathrm{Ag} / \mathrm{AgCl}$ wire electrodes inside the microelectrode and biological cell. For metal electrode, when the tip of such microelectrodes is usually sharpened down to a diameter of a few micrometers by an electrochemical etching process. The wires are then insulated up to its tip.

Solid-state microfabrication techniques commonly used in the production of the integrated circuits can be used to produce microprobes for multichannel recordings of biopotentials or for electrical stimulation of neurons in our brain or spinal cord. Most of solid-state microelectrodes are microsensor actually. Such probe consists of a precisely micromachined silicon substrate with four exposed recording sites. One of main advantages of microfabrication techniques is the ability to mass produce very small and highly sophisticated microsensors with highly reproducible electrical and physical properties.

\subsection{Enzyme sensor and microbial sensor}

Enzyme constitutes a group of more than 2000 proteins having so-called biocatalytic properties. These properties give the enzymes the unique and powerful ability to accelerate chemical reactions inside biological cells. Most enzymes react only with specific substrates even though they can be contained in a complicated mixture with other substances. It is important that soluble enzymes are very sensitive both to temperature and $\mathrm{pH}$ variations, and they can be inactivated by many chemical inhibitors. For practical biosensor applications, these enzymes are normally immobilized by insolubilizing the free enzymes via entrapment into an inert and stable matrix such as starch gel, silicon rubber, or polyacrylamide. This process is very important to ensure that the enzymes retains its catalytic properties and can be reusable.

The action of specific enzymes may be utilized to form a range of different biosensors. A typical example of enzyme-based sensor is a glucose sensor that uses the enzyme glucose oxidase. Glucose plays an important role in metabolic process. Currently, available glucose sensors are based on an immobilized enzyme, such as glucose oxidase, which acts as a catalyst. Glucose is detected by electromechanically measuring either the amount of gluconic acid or hydrogen peroxide $\left(\mathrm{H}_{2} \mathrm{O}_{2}\right)$ produced or by measuring the percent of oxygen consumed according to the following chemical reaction:

$$
\text { Glucose }+\mathrm{O}_{2}+\mathrm{H}_{2} \mathrm{O} \stackrel{\text { glucose oxidase }}{\leftrightarrow} \text { gluconic acide } \mathrm{H}_{2} \mathrm{O}_{2}
$$


A glucose sensor is similar to a $\mathrm{O}_{2}$ sensor and is shown in figure 15. Glucose and oxygen enter through outer membrane to interact with glucose oxidase enzyme. The remaining oxygen penetrates through the second oxygen-permeable membrane and is measured by the oxygen electrode.

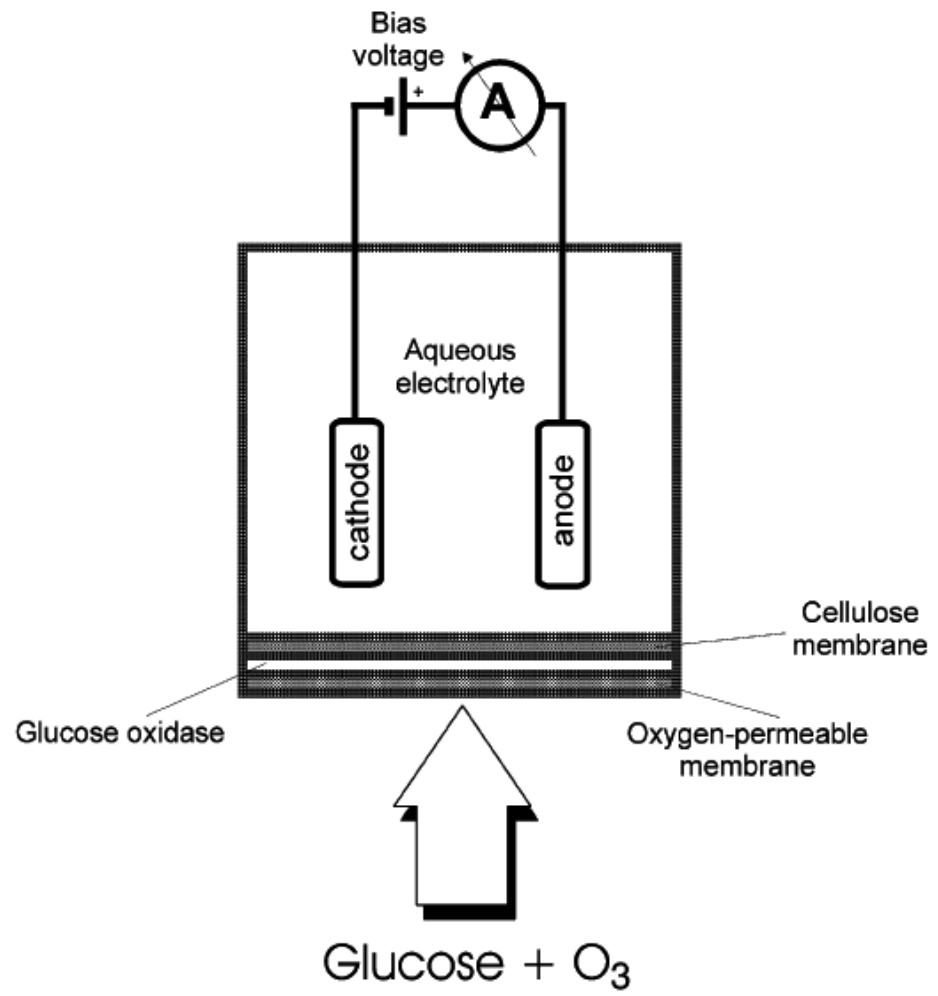

Figure 15. Sensing principle of glucose sensor

Biocatalytic enzyme-based sensors generally consist of an electrochemical gas-sensitive converter or an ion-selective electrode with an enzyme immobilized in or on a membrane that serve as the biological mediator. The analyte diffuses from the bulk sample solution into the biocatalytic layer where an enzymatic reaction takes place. The electroactive product that is formed (or consumed) is usually detected by an ion-selective electrode. A membrane separates the basic sensor from the enzyme if a new gas is produced (such as $\mathrm{CO}_{2}$ or $\mathrm{NH}_{3}$ ) or consumed (such as $\mathrm{O}_{2}$ ). Although the concentration of the bulk substrate drops continuously, the rate of consumption is usually negligible. The decrease is detected only when the test volume is very small or when the area of enzyme membrane is large enough. Thus this electrochemical analysis is nondestructive, and the sample is reused. Measurements are usually performed at a constant $\mathrm{pH}$ and temperature either in a stirred medium solution or in a flow through solution. In order to control biochemical process including some enzyme sensors, a number 
of microbial sensors have been continuously developed and applied to various environment, agriculture, food and pharmaceutical.

Microbial sensors typically involve the assimilation of organic compounds by microorganisms, followed by a change in respiration activity(metabolism) or the production of specific electrochemically active metabolites, such as $\mathrm{CO}_{2}, \mathrm{H}_{2}$, or $\mathrm{NH}_{3}$, that are secreted by the microorganism.

A microbial sensor is composed of immobilized microorganisms that serve as specific recognition elements and an electrochemical or optical sensing device that is used to convert the biochemical signal into electronic signal that can be processed. The operation of a microbial sensor can be described by the following five-step process:

1. The substrate is transported to the surface of the sensor;

2. The substrate diffuses the membrane to the immobilized microorganisms;

3. A reaction occurs at the organism;

4. The products formed in the reaction are transported through the membrane into the surface of detector;

5. The product is measured by the detector.

Here, an example of a microbial sensor is given to demonstrate the detecting course of microbial sensor including ammonia $\left(\mathrm{NH}_{3}\right)$ and nitrogen dioxide $\left(\mathrm{NO}_{2}\right)$ sensors that utilize the nitrifying bacteria as the biological sensing component. $\mathrm{A} \mathrm{NH}_{3}$ biosensor can be constructed on the base of nitrifying bacteria that uses ammonia $\left(\mathrm{NH}_{3}\right)$ as a source of energy and oxidizes ammonia as follows:

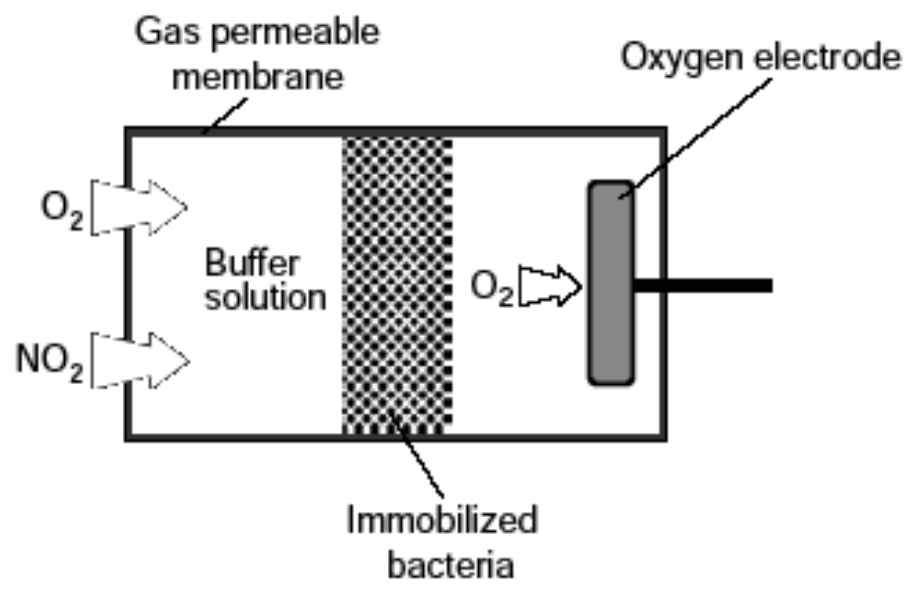

Figure 16. Sensing principle of a $\mathrm{NO}_{2}$ microbial biosensor

$$
\mathrm{NH}_{3}+1.5 \mathrm{O}_{2} \stackrel{\text { Nitrosomonas }}{=} \mathrm{NO}_{2}+\mathrm{H}_{2} \mathrm{O}+\mathrm{H}^{+}
$$


This oxidation process proceeds at high rate, and the amount of oxygen consumed by the immobilized bacteria can be measured directly by a polarographic oxygen electrode placed behind the bacteria.

Nitric oxide (NO) and $\mathrm{NO}_{2}$ are two principal pollution gases of nitrogen in the atmosphere. The principle of a $\mathrm{NO}_{2}$ biosensor is shown in figure 16. When a sample of $\mathrm{NO}_{2}$ gas diffuses through the gas-permeable membrane, it is oxidized by the nitrosomonas bacteria as follows:

$$
2 \mathrm{NO}_{2}+\mathrm{O}_{2} \stackrel{\text { Nitrosomonas }}{=} \mathrm{NO}_{3}
$$

Similar to an ammonia biosensor, the consumption of oxygen $\mathrm{O}_{2}$ around the membrane is determined by an electrochemical oxygen electrode.

\section{Charge, current, voltage, power and energy}

Many biomedical instruments utilize a sensor to convert a signal created by the body into an electrical signal. In medicine, the electrical circuits and electrical components are often utilized to detect the biomedical signal by sensor. After basic electrical components and biomedical sensors are connected together, a bioinstrumentation is then formed. Hence, describing a bioinstrumentation could begin with charge, current, voltage, power and energy. In this section, these basic variables will be introduced and explained.

\subsection{Charge and its conversion}

In our life, there are two kinds of charge, negative and positive, and they are carried by the protons and electrons, respectively. The negative charge, $q_{e}$, carried by the electron is the smallest amount of charge that exists and is measured in unit called coulombs $(\mathrm{C})$ :

$$
q_{e}=-1.6 \times 10^{-19} \mathrm{C}
$$

The symbol, $q(t)$, is used to represent the charge that change with time, and the symbol, $Q$, is used for constant charge. The charge carried by a proton is the opposite of a electron.

\subsection{Current and voltage}

\subsubsection{Current}

Electrical current, $i(t)$, is defined as the change in the amount of charge that passes through a given point or area in a given time period. Current is measured in amperes (A). By the definition, one ampere equals one coulomb/second $(\mathrm{C} / \mathrm{s})$ :

$$
i(t)=\frac{d q}{d t}
$$


and

$$
q(t)=\int_{t_{0}}^{t} i(\lambda) d \lambda+q\left(t_{0}\right)
$$

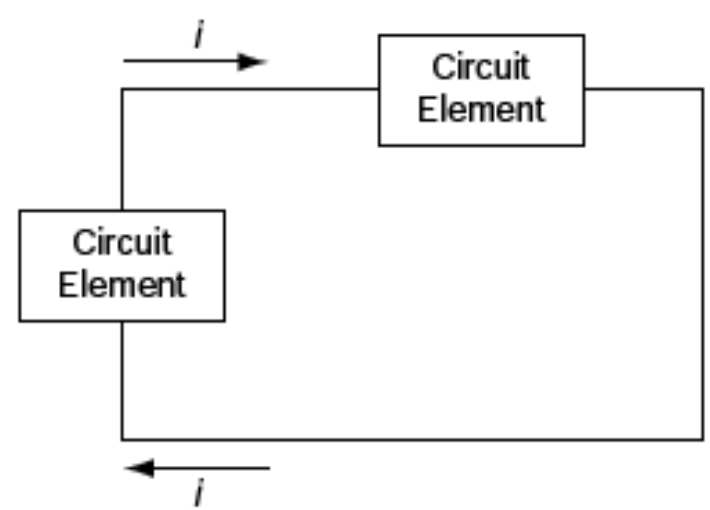

Figure 17. A simple circuit illustrating current flowing around a closed loop

In addition to the above definition, current also depends on the direction of flow, as illustrated in figure 17 Current is defined as positive if

a. A positive charge is moving in the direction of arrow;

b. A negative charge is moving in the opposite direction of arrow.

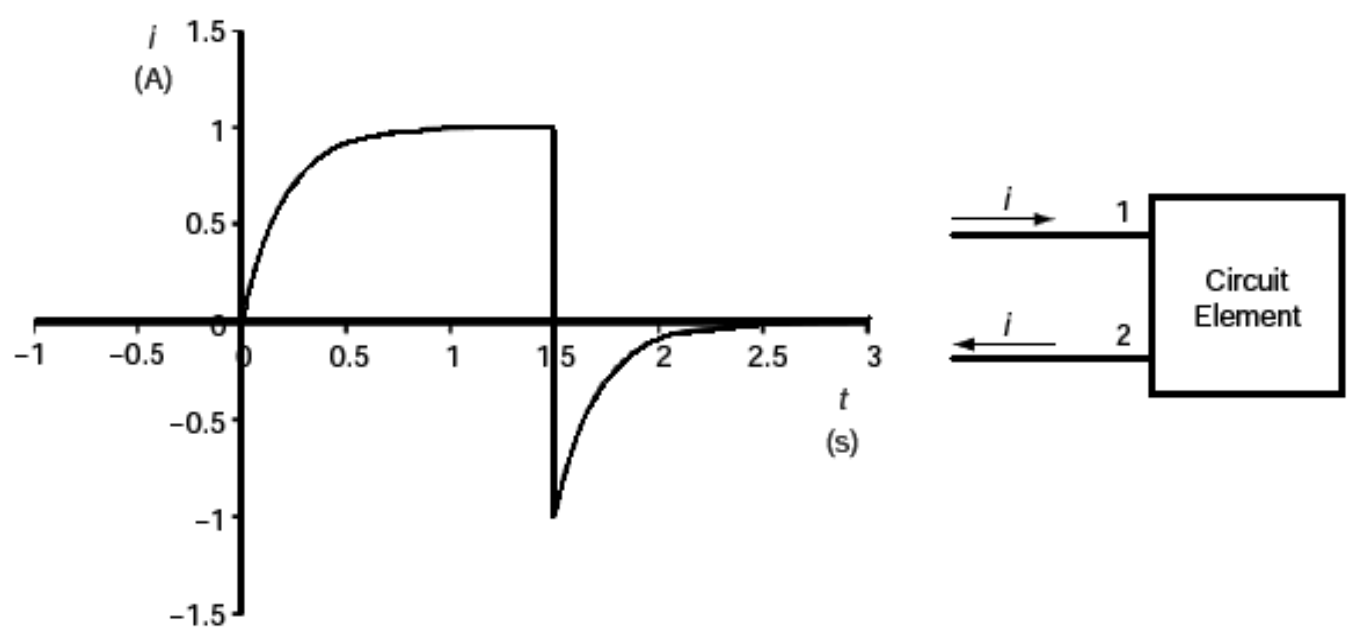

Figure 18. A sample current waveform and its electrical circuit 
Since these two cases cause the same outcome, there is no need to be concerned as to which is responsible for the current. In electrical circuits, current is carried by electrons in metallic inductors.

Consider the waveform in figure 18, with the current entering into terminal1 in the circuit on the right, the current in the time interval 0 to 1.5 second, is positive and enters terminal1. The current in the time interval 1.5 to 3 second, is negative and enters terminal 2 with positive value. If there are no current changes in the time interval 0 to $3 \mathrm{~s}$, the curve of current will be a line. Then the electrical circuit in figure 18 is constant which is called direct current (DC) indicating that it does not change with time. We denote a time-varying current with lowercase letter, such as $i$ or just $i(t)$.

\section{Kirchhoff's Current Law}

Current can only flow in a closed circuit. Kirchhoff's current law is used to ensure the relationship among every branch of circuit at same point. For current is continuous, any a point in circuit can not accumulate charge. Hence, at any time and any node, the sum of the currents which flow same node is equal to the sum of the currents which outflow from same node. This principle is known as Kirchhoff's current law (KCL).

In circuit as illustrated in figure 19, the current at the node, $a$, can be written as:

$$
I_{1}+I_{2}=I_{3}
$$

or, above formula is adjusted into the following equation:

$$
I_{1}+I_{2}-I_{3}=0
$$

Namely,

$$
\sum I=0
$$

At any time, the algebraic sum of the currents at a node is equal to zero. It should be clear for all currents whether they are all leaving or entering the node.

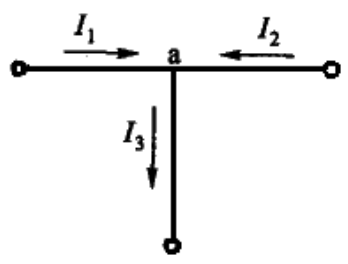

Figure 19. Node of circuit 


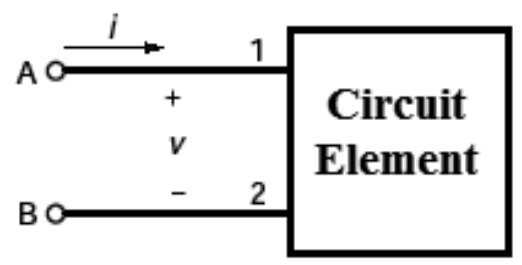

Figure 20. Voltage and current convention

In describing a circuit, we define its characteristics with terms node, branch, path, closed path, and mesh as follows:

- Node: A point at which two or more circuit elements have a common connection.

- Branch: A circuit element or connected group of circuit elements. A connected group of circuit elements usually connect nodes together.

- Path: A connected group of circuit elements in which none is repeated.

- Closed Path: A path that starts and ends at the sam node.

- Mesh: A closed path that does not contain any other closed paths within it.

- Essential Node: A point at which three or more circuit elements have a common connection.

- Essential Branch: A branch that connects two essential nodes.

Kirchhoff's current law could be also applied to any closed surface surrounding a part of the circuit. It's understood that the closed surface does not intersect any of the circuit elements.

\subsubsection{Voltage}

Voltage represents the work per unit charge associated with moving a charge between two points ( $\mathrm{A}$ and $\mathrm{B}$ in figure 20 ) and that is given as the following formula:

$$
V=\frac{d W}{d t}
$$

The unit of measurement for voltage is the volt $(\mathrm{V})$. A constant voltage source is denoted by the letter $\mathrm{V}$, while a time-varying voltage is denoted by the lowercase letter $v(t)$, or just $v$. In figure 20, the voltage, $v$ between two points (A and B) is the amount of energy required to move a charge from point $\mathrm{A}$ to point $\mathrm{B}$.

\section{Kirchhoff's Voltage Law}

Kirchhoff's voltage law is utilized to ensure the voltage relationship at any branch of circuit. Starting from any point of circuit, the sum of potential drop at the closed branch along the clockwise or counterclockwise direction is equal to the sum of potential rise. 


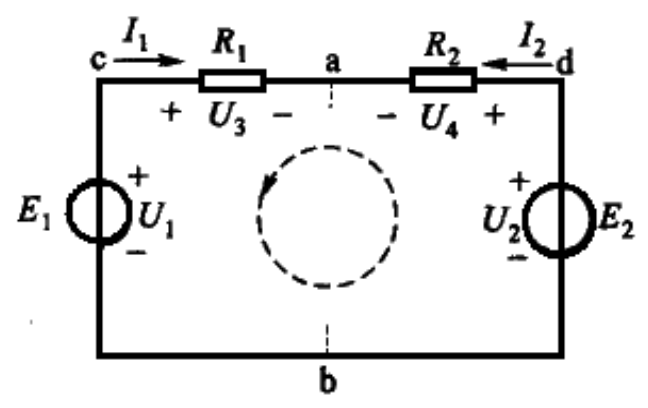

Figure 21. Circuit loop

In figure 21, the reference direction of electromotive force, current and branch voltage is marked. Cycling one circle along virtual line given in circuit, the following equation could be listed out:

$$
U_{1}+U_{4}=U_{2}+U_{3}
$$

Above equation could be also written into the following equation:

$$
U_{1}-U_{2}-U_{3}+U_{4}=0
$$

Namely, $\sum U=0$

According to above voltage equation, the algebraic sum of branch voltage is equal to zero along any a closed branch circuit. If it is stipulated that potential drop is negative, potential rise is positive.

Kirchhoff's laws are applied in electrical circuit analysis to determine unknown voltages and currents. Each unknown variable has its distinct equation. To solve for the unknowns using MATLAB, we create a matrix representation of the set of equations and solve them using the matrix calculation techniques.

\subsection{Power and energy}

Power is the rate of energy expenditure given as:

$$
p=\frac{d W}{d t}=\frac{d W}{d q} \frac{d q}{d t}=u i=i^{2} R
$$

Where, the letter, $p$, is power measured in watts $(\mathrm{W})$, and the letter, $w$, is energy measured in joules $(J)$. Power is usually determined by the product of voltage across a circuit element and the current through it. By convention, we assume that a positive value for power indicates that power is being delivered (or absorbed or consumed) by the circuit element. A negative value 
for power indicates that power is being extracted or generated by the circuit element which could be considered as a battery.

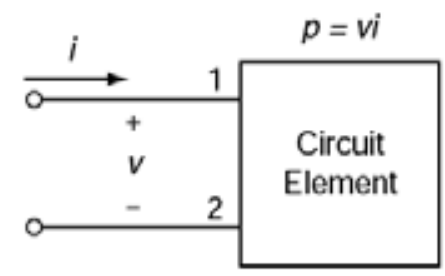

(a)

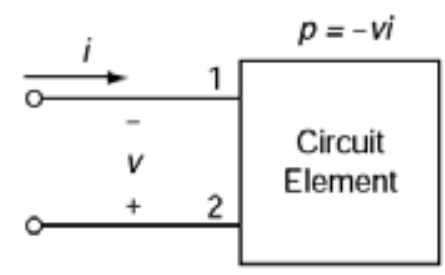

(c)

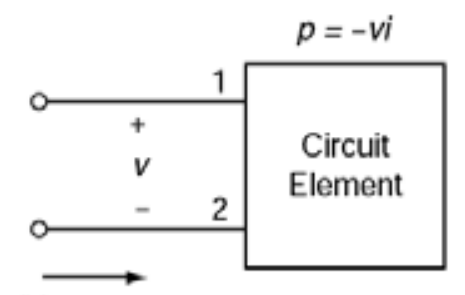

(b)

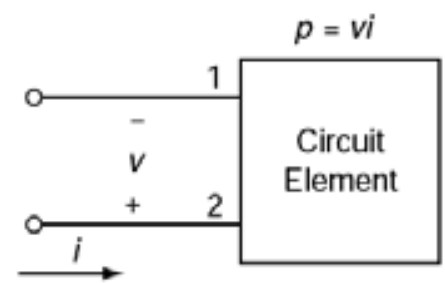

(d)

Figure 22. Polarity references for four cases of current and voltage. Cases (a) and (d) result in positive power being consumed by the circuit element. Cases (b) and(c) result in negative power being extracted from the circuit element.

Figure 22 illustrates the four possible cases for a circuit element's voltage and current configuration. According to the convention, if current and voltage are positive, with the arrow and polarity shown in figure 22, energy is absorbed. If either the current arrow or the voltage polarity is reserved, as in (b) and (c), energy is supplied to the circuit. If both the current direction and voltage polarity are reserved together as in figure 22(d), energy is absorbed.

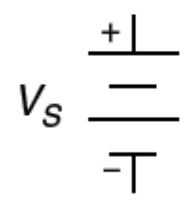

(a) Battery

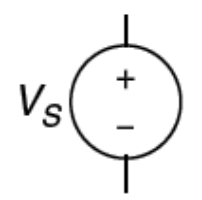

(b) Ideal voltage source

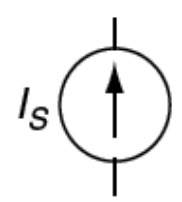

(c) Ideal current source

Figure 23. Basic symbol for independent source

A passive circuit element is defined as an element whose power is always positive or zero, which is dissipated as heat (resistance), stored in an electric field (capacitor), or stored in magnetic field (inductor). We define an active circuit element as one whose power is negative and capable of generating energy. Energy is given by the following equation: 


$$
W=\int_{-\infty}^{t} p d t
$$

In circuit, the basic source symbol is listed in figure 23.

\section{Resistance, inductors and capacitors}

\subsection{Resistance and its combination}

\subsubsection{Resistance}

In figure 24, the direction of current and voltage is the same. According to Ohm's law, the following formula could be given:

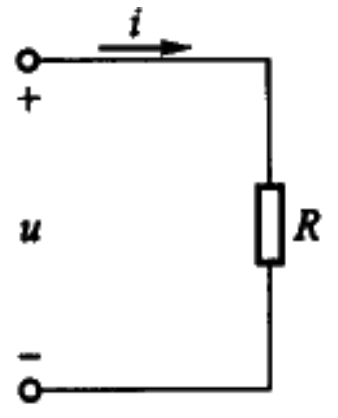

(a)resister component

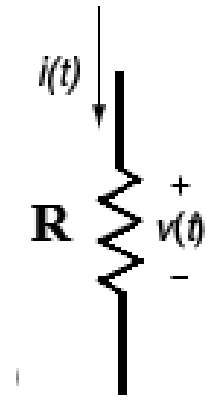

(b) Ideal resister with the Resistance

Figure 24. Resistance and its symbol

$$
u=i R
$$

The parameter of resistance is gained: $R=\frac{u}{i}$

This parameter is called resistance which has the property of holding back the current in circuit. And it is denoted with the symbol in figure $24 \mathrm{~b}$. Here, this relationship could be applied at very high voltage and current. Some electrictronic materials have a very small range of currents and voltages where they exhibit linear behavior. In reality, some material is linear only within a range of values. Outside this range, resistance is not linear. In circuit, we define:(1) having a $0 \mathrm{~V}$ voltage drop when $\mathrm{R}=0$;(2)having a 0current through resister when $\mathrm{R}=\infty$.

Each material has a property called resistivity(@) that indicates the resistance of the material. Conductivity is the inverse of resistivity, and conductance $(G)$ is the inverse of resistance. 
Conductance is measured in unit called siemens(S) and has the unit of $\mathrm{A} / \mathrm{V}$. In terms of conductance, ohm's law could be written as:

$$
i=G u
$$

For formula $u=i R$, if current is produced by both sides of this equation and they are integrated, the following equation could be given:

$$
\int_{0}^{t} u i d t=\int_{0}^{t} i^{2} R d t
$$

This formula demonstrates that electrical energy is all consumed by resister component. And the energy is converted into thermal energy, that's to say, resister is a consuming-energy component.

\subsubsection{Series and parallel combination of resistance}

If the same current flows from one resister to another, the two are said to be in series. If these two resisters are connected to the third and the same current flows through all of them, then the three resistors are in series. Consider figure 25 with three resisters in series, an equivalent circuit can be derived through Kirchhoff's Voltage Law as follows:

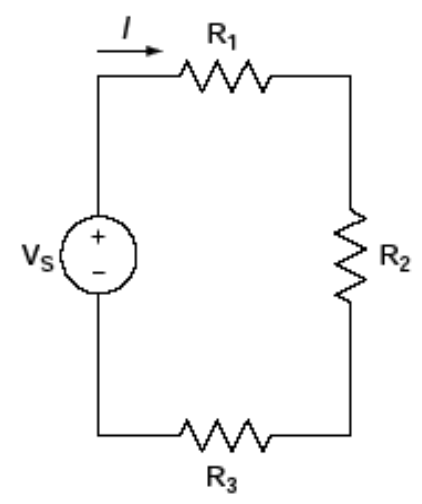

Figure 25. A series circuit

$$
-V_{s}+I R_{1}+I R_{2}+I R_{3}=0
$$

Above equation can be also rewritten as:

$$
R_{e q}=R_{1}+R_{2}+R_{3}=\frac{V_{s}}{I}
$$


Where, the equivalent resistance, $R_{e q}$, is the sum of three resister in figure 25 which is called equivalent resistance. In general, if there are $N$ resisters in series, their equivalent resistance is equal to the sum of all resistance, namely:

$$
R_{e q}=\sum_{i=1}^{N} R_{i}
$$

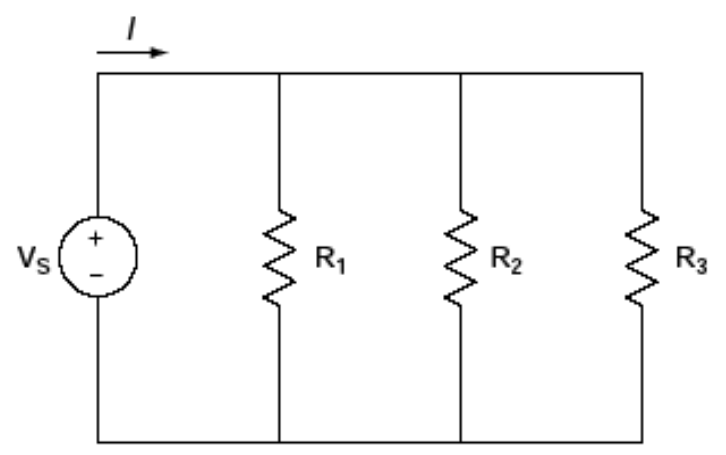

Figure 26. A parallel circuit

Two or more resisters are said to be parallel if the same voltage is across each of resisters. Consider the three parallel resisters as illustrated in figure 26, a equivalent circuit for figure 26 is derived through Kirchoff's Current Law as

$$
-I+\frac{V_{s}}{R_{1}}+\frac{V_{s}}{R_{2}}+\frac{V_{s}}{R_{3}}=0
$$

Above equivalent resistance can be also rewritten as:

$$
R_{e q}=\frac{V_{s}}{I}=\frac{1}{\frac{1}{R_{1}}+\frac{1}{R_{2}}+\frac{1}{R_{3}}}
$$

In general, if there are $N$ resisters in parallel,

$$
R_{e q}=\frac{V_{s}}{I}=\frac{1}{\frac{1}{R_{1}}+\frac{1}{R_{2}}+\cdots+\frac{1}{R_{N}}}
$$




\subsection{Capacitor}

A capacitor in figure 27 is a device that stores energy in the electrical field by charge separation when appropriately polarized by the voltage. Simple capacitors consist of parallel plates of conducting material that are separated by a gap filled with a dielectric material. Dielectric materials that are air or mica contain a large number of electric dipoles that become polarized in the presence of electric field. The charge separation caused by the polarization of the dielectric is proportional to the external voltage and given by the following equation:

$$
q(t)=C u(t)
$$

Where the symbol, $C$, represents the capacitance of element. The unit of measurement for capacitance is the farad or farads $(\mathrm{F})$.

$$
1 F=10^{6} \mu F=10^{12} p F
$$

When the charge or voltage of capacitor changes, the produced current in circuit is given as:

$$
i=\frac{d q}{d t}=C \frac{d u}{d t}
$$

This equation is given on the base of the same direction of current and voltage; otherwise there should be a negative symbol in this equation.

The capacitance of capacitor is determined by the permittivity of the dielectric, $\varepsilon$, that fills the gap between the parallel plate, the size of the gap between the plates, $d$, and the cross-section area of the plates, $A$, as

$$
C=\frac{\varepsilon A}{d}
$$

When a constant voltage is exerted on the both sides of capacitor and its current is zero, this capacitor is considered as an open circuit or DC circuit. In physical structure, capacitor consists of two conducting surfaces that store charge, separated by a thin insulating material that has a very large resistance.

For the equation $i d t=C d u$, if voltage is produced by both sides of this equation, the following equation could be given as:

$$
\int_{0}^{t} u i d t=\int_{0}^{u} C u d u=\frac{1}{2} C u^{2}
$$

Above equation demonstrates that the electric energy increases with the increase of voltage on the capacitor, and in the course, the capacitor component acquires electric energy from 
electric source. Formula $\frac{1}{2} C u^{2}$ is the electric energy in the capacitor. When voltage reduces on the capacitor, electric energy reduces. Namely, capacitor releases electric energy to electric source. Hence, capacitor is an energy storage element in circuit.

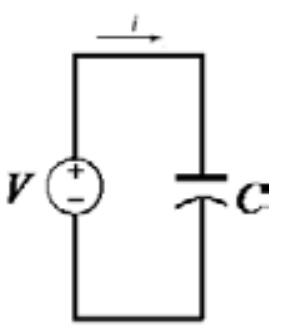

Figure 27. Circuit with a capacitor

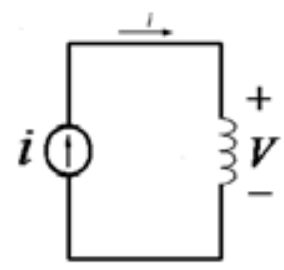

Figure 28. Circuit with inductor

For the equation $i d t=C d u$, integrating both sides yields the following formula:

$\int_{u_{0}}^{u} C d u=\int_{t_{0}}^{t} i d t$, or $u(t)=\frac{1}{C} \int_{t_{0}}^{t} i d t+u_{0}\left(t_{0}\right)$

If $t_{0}=0$, above equation can be simplified to

$$
u(t)=\frac{1}{C} \int_{t_{0}}^{t} i d t+u_{0}(0)
$$

and for $t_{0}=-\infty$, above equation reduces to

$$
u(t)=\frac{1}{C} \int_{-\infty}^{t} i d t
$$

The initial voltage in above equation, $u_{0}\left(t_{0}\right)$, is usually defined with the same polarity as $u$, which means $u_{0}\left(t_{0}\right)$ is a positive quantity. If the polarity of $u_{0}\left(t_{0}\right)$ is in the opposite direction, then $u_{0}\left(t_{0}\right)$ is negative. 


\subsection{Inductor}

An inductor in figure 28 is a passive element that is to store energy in magnetic field and is made by winding a coil of wire around a core that is a insulator or a ferromagnetic material. A magnetic field is established when current flows through the coil. The symbol is utilized to represent the inductor in a circuit. The unit of measurement for inductance is the Henry or Henries $(\mathrm{H})$. The relationship between voltage and current for inductor is given by

$$
u=L \frac{d i}{d t}
$$

The convention for writing the voltage drop across an inductor is similar to that of a resistor. Physically, current cannot change instantaneously through a inductor since an infinite voltage required. Mathematically, a step change in current through an inductor is possible by applying a voltage. For convenience, when a circuit has just DC currents (or voltages), the inductors can be replaced by short circuits, since voltage drops across the inductors are zero.

After producing current on the both sides of equation, the following expression can be acquired after integration:

$$
\int_{0}^{t} u i d t=\int_{0}^{i} L i d i=\frac{1}{2} L i^{2}
$$

Above expression demonstrates that magnetic energy increases with the increase of current through inductor component. In this course, electrical energy could be converted into magnetic energy, namely inductor acquires energy from the source. Formula $\frac{1}{2} L i^{2}$ is the magnetic energy of inductive element. When current decreases, magnetic energy decreases and then is converted into electric energy, namely inductor releases energy to the source. Hence, inductor is not a dissipative element, but a energy storage element, too.

For the equation $u d t=L d i$, integrating both sides yields the following formula:

$$
\int_{t_{0}}^{t} u(t) d t=\int_{i\left(t_{0}\right)}^{i(t)} L d i \text {, or, } i(t)=\frac{1}{L} \int_{t_{0}}^{t} u(t) d t+i\left(t_{0}\right)
$$

If $t_{0}=0$, above equation can be simplified to

$$
i(t)=\frac{1}{L} \int_{t_{0}}^{t} u(t) d t+i(0)
$$

and for $t_{0}=-\infty$, above equation reduces to

$$
i(t)=\frac{1}{L} \int_{-\infty}^{t} u(t) d t
$$


The initial current in above equation, $i\left(t_{0}\right)$, is usually defined with the same polarity as $i$, which means $i\left(t_{0}\right)$ is a positive quantity. If the polarity of the initial current $i\left(t_{0}\right)$ is in the opposite direction, then $i\left(t_{0}\right)$ is negative.

\section{Signal filters and operational amplifiers}

Biosignals are recorded as potentials, voltages, and electrical field strengths generated by nerves and muscles. The measurements involve voltages at very low levels, typically ranging from $1 \mu \mathrm{V}$ to $100 \mathrm{mV}$, with high source impedances and superimposed high level interference signals and noise. The signals need to be amplified to make them compatible with devices such displays, recorders, or A/D converters for computerized equipment. Amplifiers adequately to measure these signals have to satisfy very specific requirements. They have to provide amplification selective to the physiological signal, reject superimposed noise and interference signals, and guarantee protection from damages through voltage and current surges for both patient and electronic equipment. Amplifiers featuring these specifications are known as biopotential amplifiers.

\subsection{Basic signal amplifier}

The basic requirements that a biopotential amplifier has to satisfy are:

1. The physiological process to be monitored should not be influenced in any way by the amplifier.

2. The measurement signals should not be distorted.

3. The amplifier has to provide protection of patient from any hazard of electrical shock.

4. The amplifier itself has to be protected against damages that might result from high input voltages as they occur during the application of defibrillators or electrosurgical instrumentation.

A typical configuration for the measurement of biopotentials as illustrated in figure 29. Three electrodes, two of them are used to pick up the biological signal and the third providing the reference potential, connect the subject to amplifier. The input signal to the amplifier consists of five components:(1) the desired biopotential, (2)undesired biopotential, (3) a power line interference signal of $60 \mathrm{~Hz}(50 \mathrm{~Hz}$ in some countries) and its harmonics, (4)interference signal generated by the tissue/electrode interface, and (5) noise. Proper design of the amplifier provides rejection of a large portion of the signal interferences. The main task of designing deferential amplifier is to reject the line frequency interference that is electrostatically or magnetically coupled into subject. The desired biopotential appears as a voltage between two input terminals of differential amplifier and is referred to as the differential signal. The line frequency reference signal shows only small differences in amplitude and phase between the two measuring electrodes, causing approximately the same potential at both inputs, and thus 


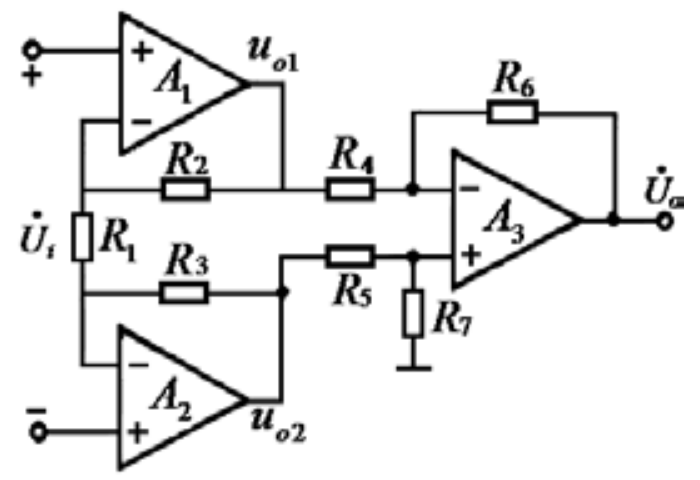

Figure 29. Typical configuration for the measurement of biopotentials

appears only between the inputs and ground and is called common mode signal. Strong rejection of the common mode signal is one of the most important characteristics of a good biopotential amplifier.

In order to provide optimum signal quality and adequate voltage level for further signal processing, amplifier has to provide a suitable gain range and needs to maintain a possible signal-to-noise ratio. The presence of the high level interference signals not only deteriorates the quality of the physiological signals, but also restricts the design of the biopotential amplifier. For example, electrode half-cell biopotentials limit the gain factor of the first amplifier stage since their amplitude can be several orders of magnitude larger than the amplitude of physiological signal. To prevent the amplifier from going to saturation, this component has to be eliminated before the required gain be provided for physiological signal.

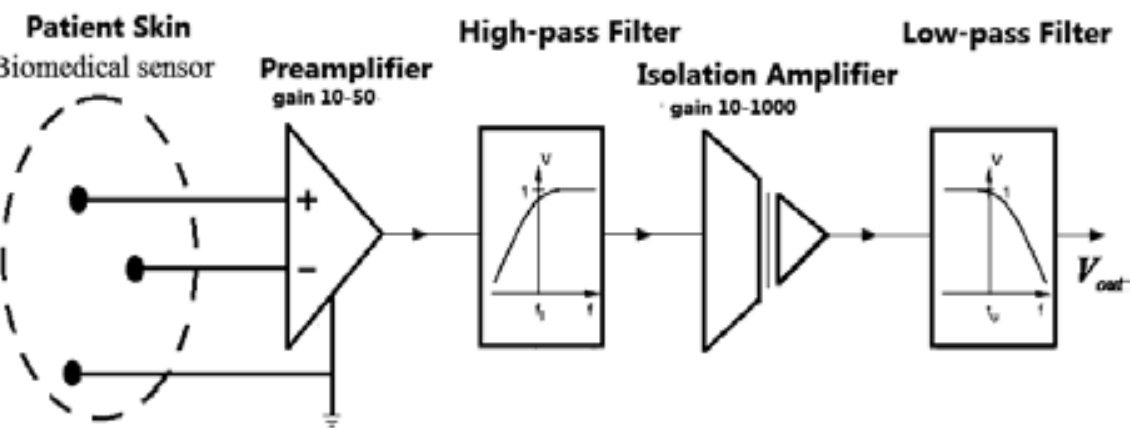

Figure 30. Schematic design of the main stages of a biopotential amplifier. Three electrodes connect the patient to a preamplifier stage. After removing DC and low frequency interference, biological signal is connected to an output lowpass filter through an isolation stage which provides electrical safety to the patient, prevents patient loops, and reduces the influence of interference signals.

A typical design of the various stage of a biopotential amplifier is shown in figure 30 . The three electrodes which provide the transition between the ionic flow of currents in biological tissue 
and electronic flow of currents in amplifier represent a complex electrochemical system. To a large extent, these electrodes determine the composition of the measured signal. The preamplifier represents the most critical part of a amplifier since it sets the stage for the quality of the biosignal. With proper design, the preamplifier can eliminate, or at least minimize, the most signal interfering with the measurement of biopotentials. In addition to electrode biopotentials and electromagnetic interference, noise which is generated by the amplifier and the connection between biological source and amplifier has to be taken into account when designing a preamplifier.

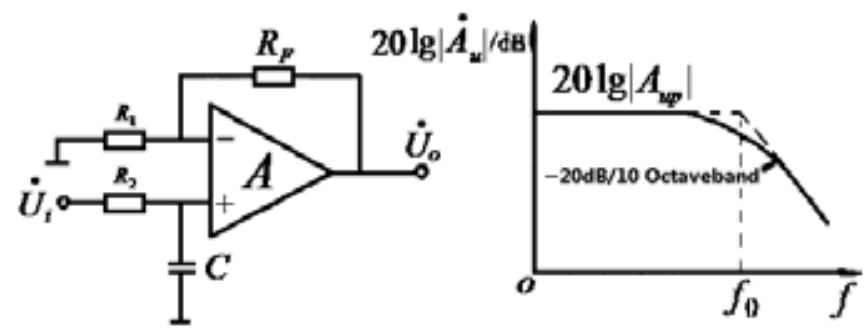

(a) One-order low-pass filter for its circuit and logarithmic amplitude-frequency characteristics

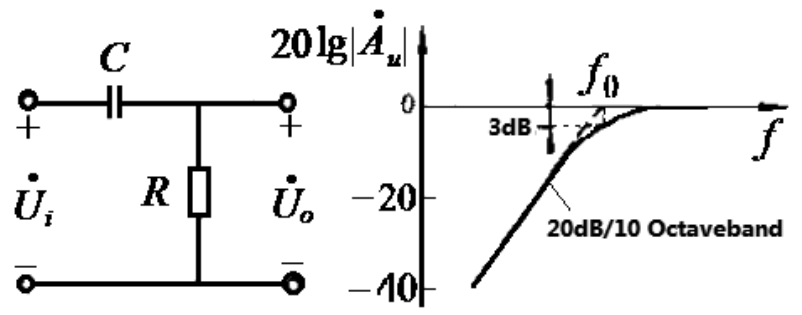

(b) One-order high-pass filter for its circuit and logarithmic amplitude-frequency characteristics

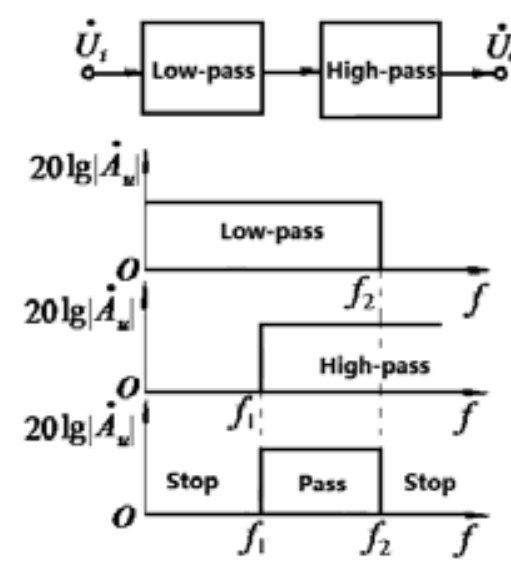

(c) Band-pass filter principle diagram

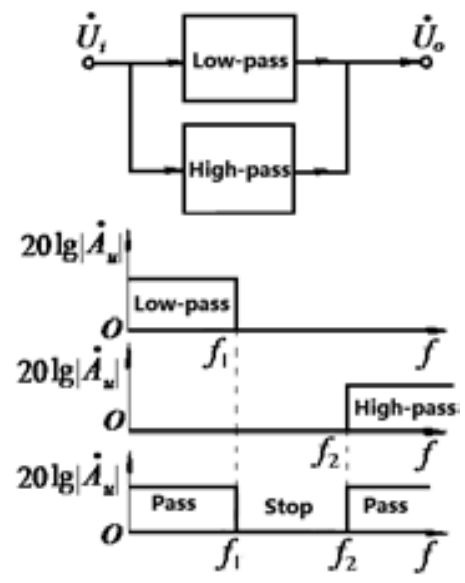

(d)Band elimination filter priciple diagram

Figure 31. Four types of filters and its amplitude-frequency characteristics 
After biosignals are preamplified, some unuseful signal have to be eliminated or filtered to highlight the useful biosignal. Such function can be realized by all kinds of filters. In circuit, according to the frequency range of signals there are four classes of filters: low-pass filter (LPF), high-pass filter (HPF), band-pass filter (BPF) and band elimination filter (BEF). These four types of filters are shown in figure 31.

\subsection{Operational amplifiers}

Operational amplifiers play an important role that they amplify a weak signal and adjust voltage or current in detecting circuit. An operation amplifier is an electronic device that consists of plenty of transistors, resistors, and capacitors. Fully understanding its operation requires that people have the knowledge of diodes and transistors. Circuit involving operational amplifier forms the cornerstone of any bioinstrumentation, from amplifiers to filters. Amplifiers used in biomedical applications have very high-input impedance to keep the current down from the system being measured. Most body signals have small magnitudes. For example, ECG has a magnitude in millivolts and the EEG has a magnitude in microvolt. Analog filters are often used to remove noise from a signal, typically through frequency domain analysis to design the filter.

\begin{tabular}{|c|c|c|c|}
\hline & Inverting input & Noninverting input & Differential input \\
\hline $\begin{array}{c}\text { Circuit } \\
\text { Constitution }\end{array}$ & $\begin{array}{c}\boldsymbol{u}_{\boldsymbol{i n}} \boldsymbol{R}_{1} \\
\text { Requi rement: } \\
\mathrm{R}_{2}=\mathrm{R}_{1} / \mathrm{R}_{\mathrm{F}}\end{array}$ & $\begin{array}{l}\mathrm{R}_{2}=\mathrm{R}_{1} / \mathrm{R}_{\mathrm{F}} \\
\text { Requirement: }\end{array}$ & $\mathrm{R}_{1}=\mathrm{R}_{1}^{\prime}, \mathrm{R}_{\mathrm{F}}=\mathrm{R}_{\mathrm{F}}^{\prime}$ \\
\hline $\begin{array}{l}\text { Voltage } \\
\text { amplification } \\
\text { factor }\end{array}$ & $\begin{array}{c}\qquad A_{u f}=\frac{u_{o}}{u_{i}}=-\frac{R_{F}}{R_{1}} \\
\text { Output and input voltages are inverse }\end{array}$ & $\begin{array}{c}\qquad A_{u f}=\frac{u_{o}}{u_{i}}=1-\frac{R_{F}}{R_{1}} \\
\text { Output and input voltages are } \\
\text { noninverse }\end{array}$ & $\begin{array}{c}A_{u f}=\frac{u_{o}}{u_{i}^{\prime}-u_{i}}=\frac{R_{F}}{R_{1}} \\
\text { When } \mathrm{R}_{1}=\mathrm{R}_{1}^{\prime}, \mathrm{R}_{\mathrm{F}}=\mathrm{R}_{\mathrm{F}}^{\prime}\end{array}$ \\
\hline$R_{i f}$ & $R_{i f}=R_{1}$ & $R_{i f}=\left(1+A_{o d} F\right) R_{i d}$ & $R_{i f}=2 R_{1}$ \\
\hline$R_{o}$ & Low & Low & Low \\
\hline $\begin{array}{l}\text { Performance } \\
\text { characteristics }\end{array}$ & $\begin{array}{l}\text { - Realize inverse proportional operation. } \\
\text { - Cite parallel voltage negative } \\
\text { feedback. } \\
\text { - Virtual ground, low common-mode } \\
\text { input voltage. } \\
\text { - Low input and output resistance. }\end{array}$ & $\begin{array}{l}\text { - Realize noninverse proportional } \\
\text { operation. } \\
\text { - Cite series voltage negative } \\
\text { feedback. } \\
\text { ם Virtual short, but not virtual } \\
\text { ground, high common-mode input } \\
\text { voltage. } \\
\text { - High input resistance and low } \\
\text { output resistance. }\end{array}$ & $\begin{array}{l}\text { - Realize subtract operation. } \\
\text { - Virtual short, but not virtual ground, } \\
\text { high common-mode input voltage. } \\
\text { - Low input and output resistance. } \\
\text { - High requirement of component } \\
\text { symmetry. }\end{array}$ \\
\hline
\end{tabular}

Table 3. Comparison of three types of scaling operation circuits 
The operational amplifier is an amplifier, but when it is combined with other circuit elements, it may integrate, differentiate, product, divide, sum, and subtract. In circuit, there are three basic types of proportional operation amplifiers: inverse scaling operation circuit, noninverse scaling operation circuit, and differential scaling operation circuit. These three types of operation circuit are compared as illustrated in table 3.

According to the information listed in table 3, we could know that operational amplifier is drawn with the symbol in figure 32. The input terminals are labeled the no inverting input $(+)$ and inverting input (-). The power supply terminals are labeled $V_{+}$and $V_{-}$, which are frequently omitted, since they do not affect the circuit behavior except in saturation conditions. Most people shorten the name of operational amplifier to the "op amp".

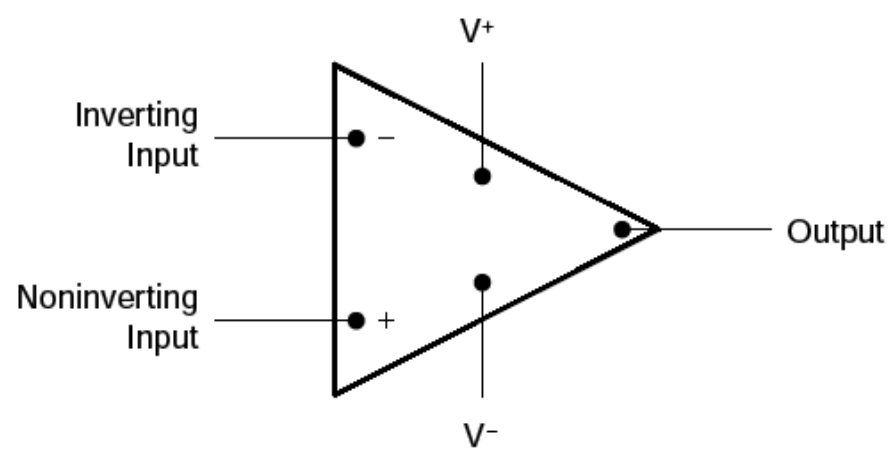

Figure 32. Circuit element symbol for the operational amplifier

Figure 33 shows an ideal mode of op amp, focusing on the internal behavior of input and output terminals. The input-output relationship is the following:

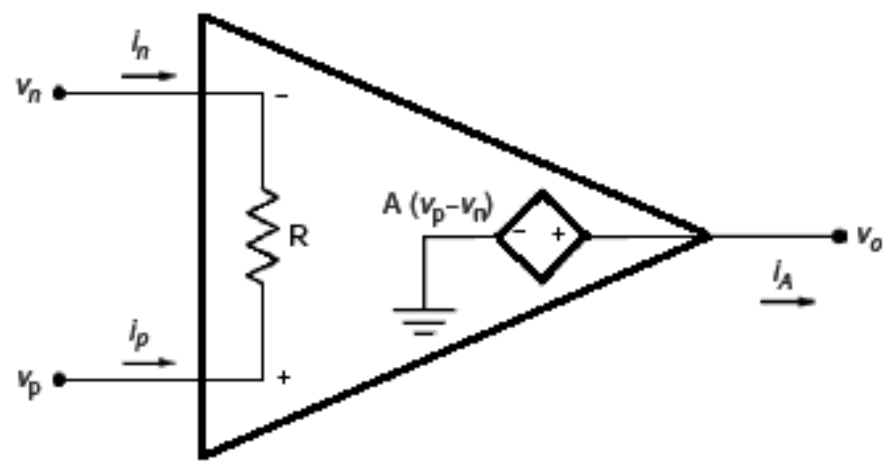

Figure 33. An internal mode of the op amp

$$
u_{o}=A\left(V_{p}-V_{n}\right)
$$




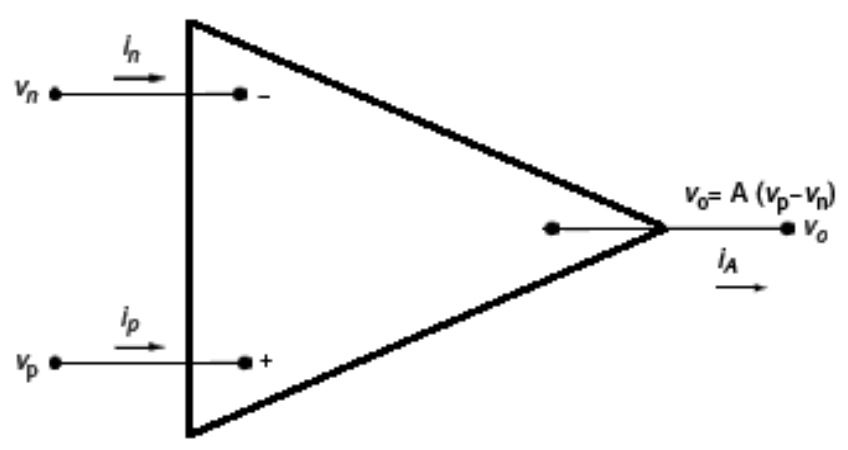

Figure 34. Idealized mode of the op amp

Since the terminal resistance is very large, we may replace it with an open circuit to simplify analysis, leaving us with the op amp model shown in figure 34.

With the replacement of the internal resistance in an open circuit, the input current is zero $\left(i_{p}=i_{n}=0 A\right)$. In addition, the output current $\left(i_{A}\right)$ of the op amp is not zero. Because the output current $\left(i_{A}\right)$ is not known, seldom is KCL applied at the output junction. In solving the op amp problems, KCL is always applied at input terminals.

\section{Example problem 1}

Find the output voltage $u_{0}$ for the following circuit.

\section{Solution}

Using the op amp model shown in figure 33, we may apply KCL at the inverting terminal and gain the following:

$$
i_{1}+i_{2}=0
$$

since currents do not flow into the op amp's input terminals. Replacing the current using ohm's law gives:

$$
\frac{V_{s}-V_{n}}{R_{1}}+\frac{V_{o}-V_{n}}{R_{2}}=0
$$

Multiplying by $R_{1} R_{2}$ and collecting like terms, we could gain:

$$
R_{2} V_{s}=\left(R_{1}+R_{2}\right) V_{n}-R_{1} V_{o}
$$

Now $V_{o}=A\left(V_{p}-V_{n}\right)$, and since the no inverting terminal is connected to the ground, $V_{p}=0$, 


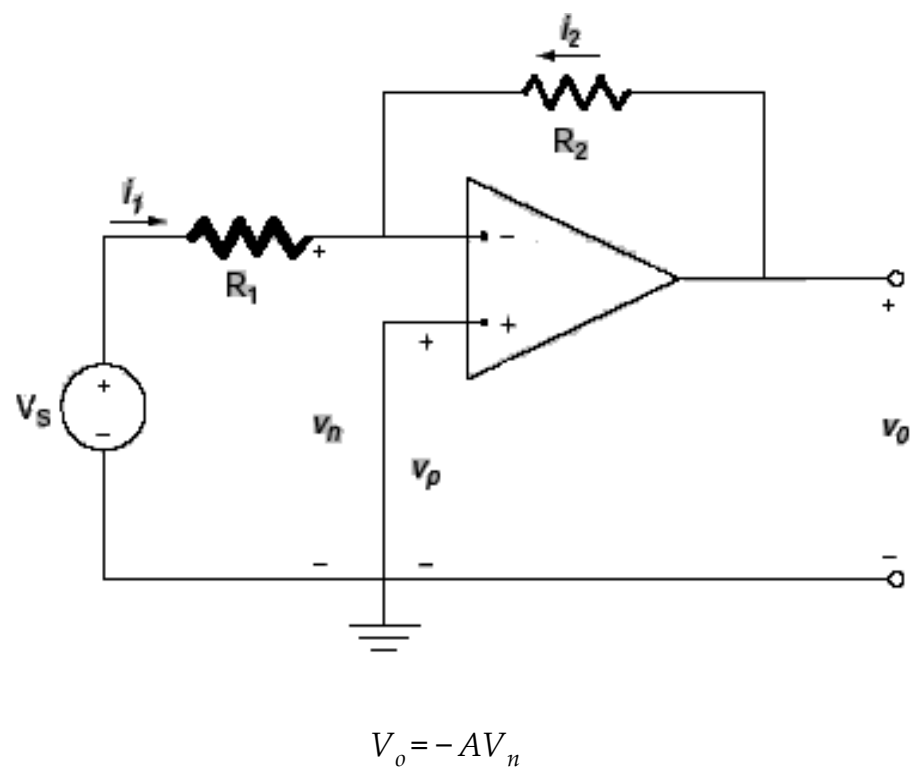

Substituting $V_{n}$ into the KCL inverting input equation gives

$$
R_{2} V_{s}=\left(R_{1}+R_{2}\right)\left(-\frac{V_{o}}{A}\right)-R_{1} V_{o}
$$

Namely, $V_{o}=-\frac{R_{2} V_{s}}{R_{1}+\frac{R_{1}+R_{2}}{A}}$

When a goes to infinity, the above equations could be simplified into:

$$
V_{o}=-\frac{R_{2}}{R_{1}} V_{s}
$$

Interestingly, with A going to infinity, output voltage $V_{o}$ remain finite due to the resistor $R_{2}$. This happens because a negative feedback path exists between the output and the inverting input terminal through resistor $R_{2}$. This circuit is called the inverting scaling operation circuit. Of course, similar circuits have differential circuit, integrating circuit, summing circuit, index circuit, logarithmic circuit and dividing circuit. These circuits could be found and read in the analog circuit textbook.

\subsection{Bioinformation acquisition}

Biological signals are often very small and typically contain some unwanted noise or interference. Such interference could determine the effect of obscuring relevant information that may 
be available in the measured signal. Noise can be extraneous in nature, arising from sources outside the body, such as thermal noise in sensors or noise in the electronic components of the acquisition system. Noise can be intrinsic to the biological media, meaning that it can arise from adjacent tissues or organs. ECG measurement from the heart can be affected by bioelectric activity from the adjacent muscles.

In order to extract the meaningful information from biological signals, sophisticated bioinformation acquisition techniques and equipment are commonly utilized and explored. Equipments with high-precision low-noise are very necessary to minimize the effect of unwanted noise. Basic components include sensors, amplifiers, analog signal conditioner, data acquisition, data storage and display, digital signal processing circuit. The bioinformation acquisition procedure is shown in figure 35.

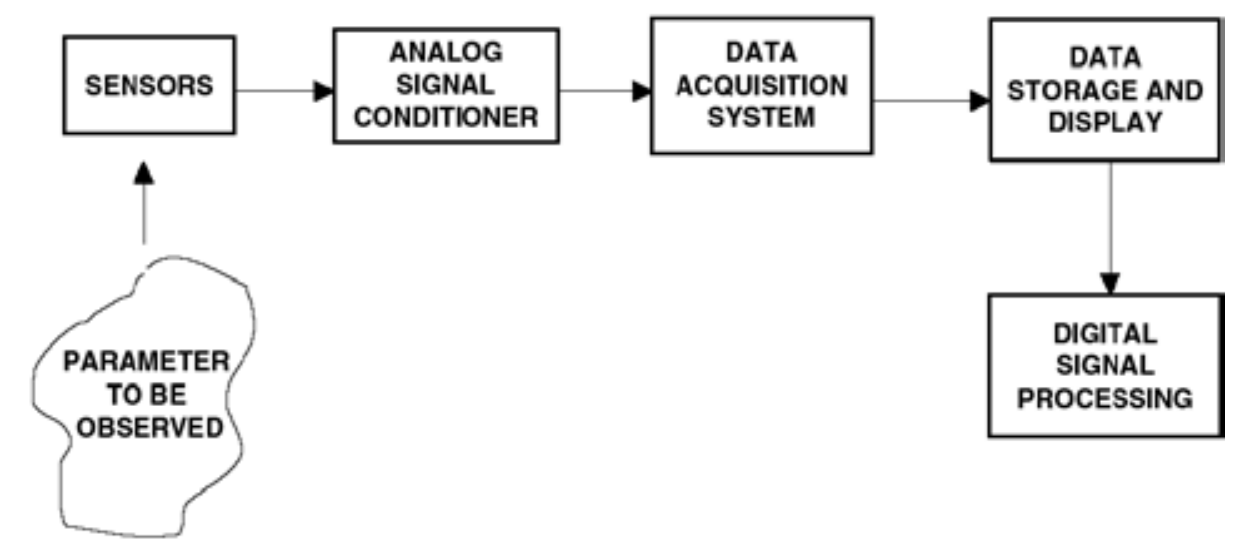

Figure 35. Procedure of obtaining biological information

In figure 35, sensors feel the biological signal that is being observed into an analog signal conditioner to adapt the requirement of data acquisition system. Here data acquisition system converts the analog signals into a calibrated digital signal that can be stored. Digital signal processing techniques are employed here to reduce the noise and extract additional information that can improve understanding of physiological meaning of original parameter. Throughout the data acquisition shown in figure 35, it's very critical that the information and structure of original biological signal of interests be faithfully preserved. Because these signals are often used to help people diagnose the pathological disorder. The procedure of analog signal conditioner, data acquisition system, analog amplifying and signal filtering, and A/D conversion should not generate misleading or untraceable distortion. Signal distortion would lead to an improper diagnosis on biological body.

In bioinstrumentation, after biological signal has been detected with an appropriate sensor, it is amplified and filtered. Operational amplifiers are electronic circuits that are used to adjust the amplitude or size of biological signal. Analog filter may be used to remove the noise hiding in biological signal or compensate for distortions caused by sensors. Amplification and 
filtering of biological signal may be necessary to meet the requirement of hardware specifications of signal conversion procedure. Continuous signal needs to be limited to a certain band of frequencies before signal can be digitized with an analog-to-digital converter, prior to storing in a digital computer.

\section{Biomeasurement system and instrumentation}

\subsection{Biomeasurement system constitution}

The biomeasurement system is shown in figure 36 to measure some biological signals such as quantity, property, or condition which are bioelectrical signal generated by muscles or the brain, or a chemical or mechanical signal that is converted into an electrical signal. Biomeasurement system is composed of sensor, analog processing circuit, A/D conversion, digital signal processing, output display, and data storage. A/D conversion is used in bioinstrumentation to acquire the enough system gain.

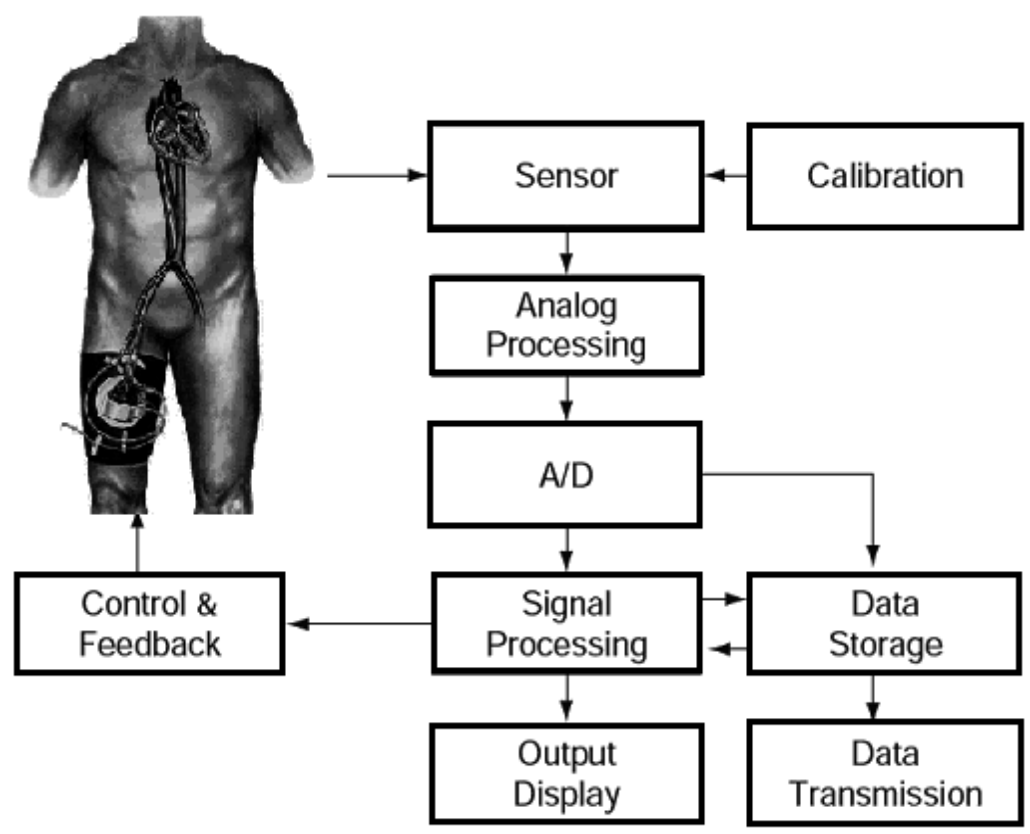

Figure 36. Basic biomeasurement system using sensors to measure a biological signal with data acquisition, storage and display capabilities, data transmission, along with control and feedback

With the invention of telephone and with appearance of internet, signal can be required with a device in one location, perhaps in patient's home, and transmitted into another device for transmission or storage. For example, if a biological signal from bioinstrumentation system in rural area could be transmitted into a diagnosis center in hospital, doctor would quickly judge some diseases to make patients gain an accurate treatment or diagnosis in time. 
Two other components play important roles in bioinstrumentation system. The first is the calibration signal. A signal with known frequency and amplitude is applied to the bioinstrumentation system at sensor's input. The calibration device permits the system components to be adjusted so that it's known that the output and input have a certain linear relationship. Without such information, it's impossible to convert the output of an instrument system into a meaningful representation of the measurand.

Another important component, a control or feedback element, is not a part of all instrument systems. These parts include pacemakers and ventilators that could stimulate the heart and lungs. Some feedback devices collect physiological data and stimulate a response - a beat or breath - when needed or are part of biofeedback systems in which patients are made aware of a physiological instrument, such as blood pressure, and uses conscious control to change the physiological response.

\subsection{Biomeasurement circuit}

Figure 36 shows the basic elements which constitute basic bioinstrumentation system. Circuits play a very important role in bioinstrumentation system. If a bioinstrumentation needs to be developed or improved to be fit for new condition, function circuits in different blocks from figure 36 have to be respectively designed to form bioinstrumentation system with relative indexes. Among all kinds of circuits, amplifiers and A/D converters are very important component for detecting the biological signal. Hence, amplifying circuits will be only introduced here in detail, and other function circuits could be read or utilized in relative books.

- Bioelectric amplifier

In order to record the bioelectric potential from the body, biological amplification is always required. The simplest form is shown in figure 37 which uses a single-input amplifier. Here amplifier only amplifies one input signal which is applied in the input and the reference "earth" or "ground".

In this amplifier, the resistor $R_{1}$ is required to allow the "bias current" to flow into the noninverting (+) input of the operational amplifier and the resistor $R_{2}$ is required to balance the resistor $R_{1}$ so that the bias currents do not produce a voltage difference between the two inputs of the amplifier. If there is no capacitor in the positive input, amplifier in figure 37 will become a voltage follower, that's to say, the bioelectric input signal could be transmitted completely to the output. Namely, the following equation is given:

$$
u_{\text {in+ }}=u_{\text {out }}
$$

Unfortunately, in circuit shown in figure 37, the resistor $R_{1}$ defines the maximum input impedance of the amplifier. The input impedance is an important consideration in bioelectric amplifiers because it can cause attenuation of a signal which is derived from electrodes with high impedances. For example, if the two electrode impedances were $10 \mathrm{k} \Omega$ and the input impedance of the amplifier was $1 \mathrm{M} \Omega$, then $1 \%$ of the signal would be lost by attenuation of 
two electrodes. The impedance presented by electrodes is termed the source impedance which has to be very much less than the input impedance of amplifier. Source impedance is very important when we consider differential amplifier shortly.

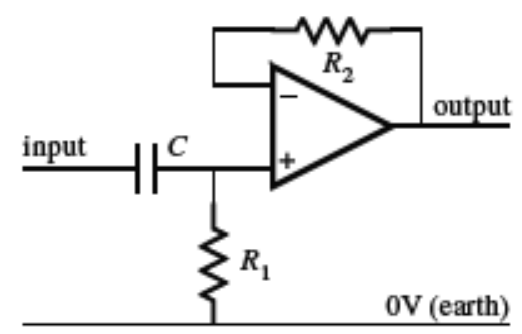

Figure 37. A simplest single-input amplifier

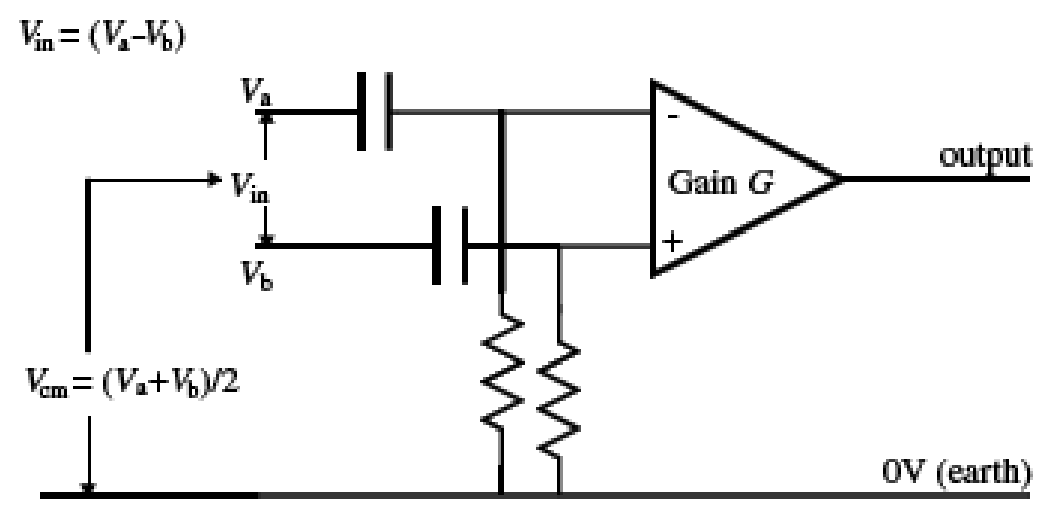

Figure 38. A differential amplifier

There is also capacitor introduced in figure 37 in series with the input signal. Capacitor $C$ blocks any DC (direct current) signal by acting as a high-pass filter with the resistor $R_{1}$. This function is usually referred as AC (alternative current) coupling. AC coupling will also cause some attenuation of the signal which may be important. We could determine the attenuation of signal by the following equations:

$$
\begin{gathered}
u_{\text {out }}=u_{-}=u_{+}, \frac{u_{\text {in }}}{R_{1}+\frac{1}{j \omega C}}=\frac{u_{+}}{R_{1}}, \text { then } \frac{u_{\text {out }}}{u_{\text {in }}}=\frac{R_{1}}{R_{1}+\frac{1}{j \omega C}}=\frac{R_{1}\left(R_{1}+j / \omega C\right)}{R_{1}^{2}+1 / \omega^{2} C^{2}} \\
\left|\frac{U_{\text {out }}}{U_{\text {in }}}\right|=\frac{1}{\sqrt{1+1 /\left(\omega^{2} R_{1}^{2} C^{2}\right)}}=\frac{1}{\sqrt{1+\omega_{0}^{2} / \omega^{2}}} \text {, where } \omega_{0}=1 / R_{1} C .
\end{gathered}
$$


If capacitor $C$ is $1 \mu \mathrm{F}$ and resistor $R_{1}$ is $1 \mathrm{M} \Omega$, then the attenuation of a $1 \mathrm{~Hz}$ signal will be $1.25 \%$. This is perhaps a significant attenuation for ECG which has considerable energy at $1 \mathrm{~Hz}$.

Unfortunately, even with capacitor $\mathrm{C}$ added, this type of amplifier is not suitable for recording small bioelectric signal because of interference from external electric fields. An electric electrode has to be connected to the amplifier via a wire and this wire is exposed to interfering signals. However, the interference will only appear on the input wire to the amplifier and not on the "ground" wire which is held at zero potential. An elegant solution to this problem is to utilize differential amplifier as shown in figure 38. The input of the type of amplifier has three connections marked '+', '-' and 'ground'.

\section{- Differential amplifier}

In figure 38, the signal which we wish to record is connected between the ' + ' and ' - ' points. Now both inputs are exposed to any external interfering electric fields so that the difference between the ' + ' and ' - ' input will be zero. This will not be quite true because the electric fields experienced by the two input wires may not be exactly the same, but if the wires are run close together then the difference will be small. Differential amplifier is not perfect in that even with the same signal applied to both inputs, with respect to ground; a small output signal can appear. This imperfection is specified by the common mode rejection ratio or CMMR. An ideal differential amplifier has zero output when identical signals are applied to the two ' + ' and ' inputs. CMMR could be defined as the following equation:

$$
C M M R=20 \log \left[\frac{\text { signal gain }}{\text { common }- \text { mode gain }}\right]
$$

Where, signal and common-mode gains are given by

$$
\begin{aligned}
& \text { signal gain }=\frac{U_{\text {out }}}{U_{\text {in }}}=\frac{U_{\text {out }}}{U_{+}-U_{-}} \\
& \text {common-mode gain }=\frac{U_{o u t}}{U_{c m}}=\frac{U_{o u t}}{\left(U_{a}+U_{b}\right) / 2}
\end{aligned}
$$

In practice, common-mode voltage $U_{c m}$ can be as large as $100 \mathrm{mV}$ or even more. In order to reject this signal and record a signal $\mathrm{V}_{\text {in }}$ as small as $100 \mu \mathrm{V}$, a high CMMR is required. If we wish the interfering signal to be reduced to only $1 \%$ of output voltage then

$$
\begin{aligned}
& \text { required-signal gain }=\frac{U_{\text {out }}}{U_{\text {in }}}=\frac{U_{\text {out }}}{U_{+}-U_{-}}=\frac{U_{\text {out }}}{100 \mu V} \\
& \text { required-CM gain }=\frac{U_{\text {out }}}{U_{c m}}=\frac{U_{\text {out }}}{1 \% U_{\text {in }}}=\frac{U_{\text {out }} / 100}{100 \mathrm{mV}}
\end{aligned}
$$

Hence, the required CMMR could be given as: 


$$
C M M R=20 \log \left[\frac{U_{\text {out }} / 0.1 m V}{U_{\text {out }} / 100^{2} m V}\right]=100 \mathrm{~dB}
$$

In fact, it's not always easy to achieve a CMMR of $100 \mathrm{~dB}$. As we have known, electrode source impedances have a very significant effect on CMMR and hence electrode impedance affects noise rejection.

Of course, in detecting biosignals, the AC coupling shown in figure 37 and figure 38 degrades the performance of the amplifiers. If the input impedance and bias current of amplifiers is sufficiently high, then they can be connected directly to the input electrodes, without producing electrode polarization. Furthermore, DC offset will occur from the electrode contact potentials, but if the amplifier gain is low $(<10)$ DC offset will be not a significant problem. The offset can be removed by AC coupling at later stage.

However, there are some safety arguments against the use of DC coupling. If a fault arises in the operational amplifier, then it's possible for the power supply to be directly connected to the patient and so give rise to a hazard. DC currents will cause electrolysis and result in tissue necrosis. AC coupling could avoid this problem and is often used. Nonetheless DC coupling is also often used in biomedical field.

\subsection{Bioinstrumentation design}

The purpose of using bioinstrumentation is to monitor the output of a sensor or sensors and to extract some useful information from signals that are produced by sensors.

Acquiring discrete-time signal and storing this signal in computer memory from a continuoustime signal is accomplished with analog-to-digital (A/D) converter. After analog signals have been processed which are based on analog filters such as low-pass or high-pass filters, $\mathrm{A} / \mathrm{D}$ converter uniformly samples the continuous-time waveform and transforms it into a sequence of numbers, one every $t_{k}$ seconds. The $\mathrm{A} / \mathrm{D}$ converter also transforms the continuous-time waveform into a digital signal, which is converted into computer words and stored in computer memory. To adequately capture the continuous-time signal, the sample frequency has to be carefully selected to ensure any signal information is not lost. The minimum sampling frequency is twice the highest frequency content of the signal based on the sampling theorem from communication theory. In reality, we often adopt the sampling frequency from five to ten times the highest frequency content of the signal so as to achieve better accuracy by reducing aliasing error.

- Biological signal categories in human body

The electrical, chemical and mechanical activity that occurs during this biological event often produces signals that could be detected and analyzed. Biological signals are the record of a biological event such as a beating heart or a contracting muscle. Hence, biological signals contain useful information which could reflect human's activities and physiology, that's to say, biological signal could be used for biomedical diagnosis. Biological signals are classified 
into bioelectric signals, biomagnetic signals, biochemical signals, biomechanical signals, bioacoustic signals and biooptical signals.

Nerve and muscle cells generate bioelectric signals that are the result of electrochemical changes within and between cells. When plenty of cells are stimulated, an electric field is then generated that propagates through biological tissues. These changes in extracellular potential may be measured on the surface of tissue or organism by using surface electrodes. The electrocardiogram (ECG) is an example of this phenomenon. Different organs in body, including the heart, brain, lungs, and liver, also generate weak magnetic fields that could be detected with magnetic sensors. The strength of magnetic field is much weaker than the corresponding physiological bioelectric signal. Magnetic sensors could be used to detect biomagnetic signals. Magnetocardiography (MCG) is a specific example of such phenomenon.

Biochemical signals contain information about changes in the concentration of various chemical agents in the body. The concentration of various ions such as calcium and potassium in cell can be measured and recorded. Oxygen sensor is used to detect oxygen concentration in body. Mechanical functions of biological systems, including motion, displacement, tension, force, pressure and flow, also produce measurable biological signals. Blood pressure sensor is a measurement of the force that blood exerts against the walls of blood vessels. Change in blood pressure can be recorded as a waveform by blood pressure sensor. Bioacoustics' signals are a special subset of biochemical signals which involve vibrations. Many biological events could produce acoustic noise. For example, the flow of blood through the valves in the heart can be used to determine whether motion is operating properly. Besides these, the respiratory system, joints and muscles could also produce bioacoustic signals that propagate through the biological medium and can be often measured at the skin surface by acoustic sensors. Biooptical signals are generated by the optical or light induced attributes of biological systems. Biooptical signals can occur or be introduced to measure a biological parameter with an external light medium such as the measurement of health of a fetus by red and infrared light.

\section{- Noise}

Measurement signals are always corrupted by noise in the bioinstrumentation system. Interference noise occurs when unwanted signals are introduced into systems by external sources such as telephone magnetic wave, power line and transmitted radio. Interference noise needs to be effectively reduced by careful attention to the circuit wiring configuration to minimize coupling effect.

Interference noise is introduced by power lines, fluorescent lights, AM/FM radio broadcasts, computer clock oscillator, laboratory equipment and cellphone. Electromagnetic energy radiating from noise source is injected into the amplifier circuit or into the patient by capacitive or inductive coupling. Even action potentials from nerve conduction in the patient generate noise at the sensor/amplifier surface. Filters are also used to reduce the noise and to maximize the signal-to-noise $(\mathrm{S} / \mathrm{N})$ rate at the input of the $\mathrm{A} / \mathrm{D}$ converter.

Low frequency noise could be eliminated by high-pass filter with the cutoff frequency set above the noise frequency. High frequency noise could be reduced by low-pass filter with the cutoff frequency set below the noise frequency and above the frequency of biological signal which 
is being monitored. Power line noise is a very difficult problem in biological monitoring because the $50-$ or- $60-\mathrm{Hz}$ frequency is usually at the range of biological signal which could be monitored. Band-stop filters are commonly used to reduce the power line noise. The notch frequency in the band-stop filters is set to the power line frequency of 50 or $60 \mathrm{~Hz}$ with the cutoff frequency located a few Hertz to either side.

The second type of noise is called inherent noise. Inherent noise arises from random processes that are fundamental to the operation of circuit's elements and thus is reduced by a good circuit design practice. While inherent noise is reduced, it can be never eliminated. Low-pass filters are used to reduce high-frequency components. However, noise signals within the frequency range of biological signals being amplified cannot be eliminated by this filtering approach.

\section{- Computer}

Computer is a main device which is used to display the biological signals being monitored. However some low or high level languages such as machine language, FORTRAN, visual C+ + , MATLAB or LabView, have to be used to realize the operation on the acquisition data from biological body. When computers are used to acquire physiological data, programming instruction tell computer when acquisition data should begin, how often samples should be taken from how many sensors, how long acquisition data should continue, and where the digitized data should be stored. The rate at which a system acquires sample depends on the speed of computer clock's frequency and the number of computer instruction that could be completed in order to realize a sample. Of course, some computers are utilized to control the gain on the input amplifiers so that biological signals could be adjusted during data acquisition. In other systems, the gain of data acquisition has to be adjusted.

\section{Chapter summary}

In this chapter, main biomedical sensors, devices and biological measurement systems are introduced to make readers understand present bioinstrumentation in market. The common biomedical sensors are narrated here to make readers grasp their basic sensing principle such as heart sound sensor, blood flow sensor and enzyme sensor. Furthermore, basic charge, current, voltage, power and energy used in biomedical engineering are explained to design some detecting circuits. Besides above, signal filters and operational amplifiers are also described and some advice or opinions are given out to give readers some available references. The basic detecting blocks of biomeasurement system are provided to quickly design relative bioinstrumentations. Readers need to carefully learn the content of biomedical sensors, signal filters, operational amplifiers for bioinstrumentation.

\section{Suggested reading}

[1] Wang Baohua. Biomedical measurement and instrument [M]. China Fudan press, shanghai, January, 2009. 
[2] Yang Yuxing. Biomedical sensor and measurement technology [M]. Beijing Chemical industry press, Beijing, 2005.

[3] John Enderle, Joseph Bronzino. Introduction to Biomedical Engineering [M]. Elsevier press, 2012.

\section{Author details}

Gaofeng Zhou ${ }^{1 *}$, Yannian Wang ${ }^{2}$ and Lujun Cui ${ }^{1}$

*Address all correspondence to: yaofabiaolunwen@163.com

1 School of Mechatronic Engineering, Zhongyuan University of Technology, Zhengzhou, China

2 School of Information Engineering, Zhengzhou University, Zhengzhou, China

\section{References}

[1] Chenlin Peng. Biomedical sensing principle and application [M]. Advanced Education Press, 2000.

[2] Richard C. Dorf. Sensors, Nanoscience, Biomedical Engineering, and Instruments [M]. CRC Press, 2006.

[3] Jiang Yuanhai, Huo Jiwen, Yi Lizhi. Medical Sensor [M]. Science Press, 1997.

[4] Waldemar Nawrocki. Measurements and sensors [M]. Artech House, 2005.

[5] Ping Wang, Qingjun Liu. Biomedical sensors and measurement [M]. Zhengjiang University Press, 2011.

[6] Deric P. Jones, Joe Watson. Biomedical sensors [M]. Momentum Press, 2009.

[7] Tatsuo Tagawa, Toshiyo Tamura, P. Ake Oberg. Biomedical sensors and instruments, second edition, CRC Press, 2011.

[8] Suxing Yang. Analog electronic circuit [M]. China Higher Eduction Press, 2006.

[9] Gabor Harsanyi. Sensors in Biomedical Applications Fundamentals Technology and Applications [M]. CRC Press, 2000.

[10] Claus Hélix-Nielsen. Biomimetic membranes for sensor and separation applications [M]. Springer, 2012. 



\section{Section 3}

Light Therapy 

Chapter 8

\title{
High Brightness LEDs Supplied by Electronics Converters Used in Tissue Healing and Cell Rejuvenation
}

\author{
Alexandre Campos, Yasmim Guterres, \\ Eduardo B. Bauer and Mauro C. Moreira \\ Additional information is available at the end of the chapter \\ http://dx.doi.org/10.5772/59817
}

\section{Introduction}

The rapid development of high brightness light emitting diodes (LEDs) makes feasible the use of LEDs, among other light sources (such as laser, intense pulse light and other incoherent light systems), for medical treatment and light therapy. This chapter provides an overview of LED application in human health and the results in healing and cell rejuvenation [1].

The technique of LEDs used in tissue treatments is called LED Therapy and from now on we will treat it this way. With the use of electronic converters that supply the high brightness LEDs it is possible to control the spectral composition, allowing to obtain the wavelengths desired to apply the human tissue to obtain efficacy in phototherapy. High brightness LEDs can be easily integrated in digital control systems for the purpose of providing a variation in spectral composition over a phototherapy treatment stage. The colors emitted by the high brightness LEDs operate differently, i.e. depending on which layer of the human tissue that will be applied to light and which type of which will combat disease. In the skin, the red light has restorative action, healing and analgesic, while blue has a bactericidal action [19]. The intensity of the beams of light emitted by LEDs on the skin is lower, since its cells maintain a good interaction with the light $[2,21]$. The high brightness LEDs respond effectively in the therapeutic application and can be used a variety of electronic converters that control the output current, output power, the duty cycle and other parameters that directly interfere in luminous efficiency and length of wave offering a positive response in phototherapy treatments.

LED Therapy is gaining ground every day in various areas, including in medicine, dentistry, physical therapy and aesthetics [20]. Biomedical engineering has made great strides in their research on the application of LEDs in the treatment of pre-malignant lesions and malignant, 
in the treatment of rejuvenation and acne, hair loss, skin lesions, wound healing in postoperative incisions in the patient's psychological recovery, where the lights are applied to the environment and on other fronts of medicine. In dentistry the development of modern photopolymerizable materials represents a great advance for restorative dentistry because they allow excellent aesthetics, ease in handling and control of the clinical time [3,23].

Advances in the medicine are encouraging and research in the area grows every day, the intent of this chapter is to present a study and results in healing and cell rejuvenation.

\section{Action of LED Therapy in human tissues}

The photobiostimulation caused by irradiation with LEDs has a number of outcomes such as increasing cell proliferation rate, the production rate of fibroblasts and collagen synthesis. LED Therapy is known for its anti-inflammatory and healing properties and it can be used as an independent procedure of the conventional treatments of clinical medicine, but it should always be accompanied by a health professional with knowledge of the tissue pathology, cell rejuvenation and photobiostimulation cell. Different applications of LED Therapy may be performed as a function of wavelength $(\mathrm{nm})$ of light penetration in tissue $(\mathrm{mm})$. The relationship between these parameters demonstrates that the penetration depth of light into tissue, as shown in Figure 5.

\subsection{Light penetration in human tissues generated by the action of high brightness LEDs arrangements}

The processes of refraction and reflection of light emitted by LEDs in human tissues are responsible for the dispersion of light, as shown in Figure 5. However, the action of light is peculiar because the response to these processes can vary from person to person undergoing therapy.

Despite the high dispersion, the degree of penetration is significant, approximately $50 \%$ of the incident radiation to reach the substrate directly below the skin of the patient [4]. The fabric layers to be submitted to receive a red light portion scattered radiation given where a small tissue area can absorb light in the dermis and epidermis. This is due to the presence of photoreceptor layers that can be constituted of amino acids, melanin, and other acids. Typically each type of photoreceptor is sensitive to a particular wavelength.

Thus, light can be absorbed by the tissue which depends on the color and wavelength. For example, red light, near infrared, easily penetrates the fabric because this light radiation is not blocked by blood and water as other wavelengths.

Wavelengths less than 630nm, such as yellow, blue and green are significantly blocked by hemoglobin in the blood, so that they do not penetrate deeply [5].

Wavelengths greater than 900nm are blocked by the liquid portion of the skin and connective tissues. Many wavelengths can emit a large amount of energy above the infrared range and 
cannot be seen by the human eye; this type of radiation may produce a certain amount of heat to interact with human tissue [5].

\subsection{Action of light emitted by high-brightness LEDs in human tissue}

The blue is in the range of 430 to $485 \mathrm{~nm}$. The green is in the range of 510 to $565 \mathrm{~nm}$. The yellow is between 570 to $590 \mathrm{~nm}$. The red is in the range of $620 \mathrm{~nm}$ to $700 \mathrm{~nm}$, to the point that it does not become visible anymore in the range of $740 \mathrm{~nm}$. Some companies that manufacture LEDs say that the yellow light helps remove wrinkles. There is also some interesting research, which emphasizes that the application of blue light helps in the elimination of a bacteria that causes some forms of acne [20].

The phototherapy with the narrow band blue light seems to be a safe treatment and one additional effective therapy for treatment of mild and moderate acnes. Some researchers suggest that the green LED light can help against cancer, but this color cannot penetrate more than the skin. Figures 1, 2, 3 and 4 show prototypes that emit light in the red, blue, green and amber colors.

\subsubsection{Action of red light emitted by high brightness LEDs}

Red light emitted by high brightness LEDs (in the range of 630nm wavelength) promotes increased cellular energy synthesis (ATP) restructuring fibroblast collagen and elastin fibers stimulating the production of new fibers. It also assists in enhancing the flow of blood and oxygen to the skin and capillaries, increasing cellular metabolism and strengthening the walls of the capillaries. The red light is also an excellent treatment for scarring, inflammation and hard-to-heal ulcers. The red light emitted by high brightness LEDs when in contact with tissue injury has regenerative and major impact on healing characteristics. Patients with physiological difficulties of tissue healing can get great results with this therapy.

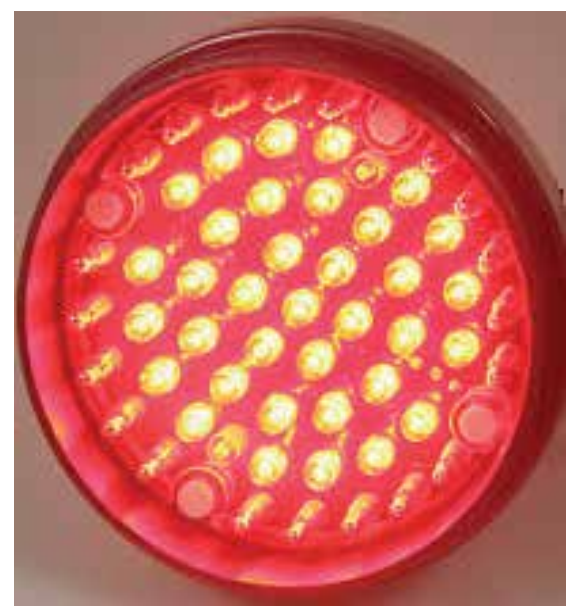

Figure 1. Arrangement of high brightness LEDs that emit red light. 


\subsubsection{Action of blue light emitted by high brightness LEDs}

Blue light emitted by high brightness LEDs (in the range of $470 \mathrm{~nm}$ wavelength) acts as a bactericide because its wavelength reaches the surface of the skin (epidermis) producing oxygen and generating oxidative stress destroying bacterias such as those that develop acne.

The action of blue light emitted by high-brightness LEDs is indicated for patients with inflammatory acne, as this therapy reduces bacterial growth and reduces inflammation. LED blue light therapy helps to kill the acne causing bacteria, reducing inflammation and providing a general rejuvenation effect on the skin. Blue light emitted by high brightness LEDs can be used to reduce cellulite and stretch marks and if combined with the red light the result will be more significant.

The action of light acts directly on the excessive production of fat by inhibiting excessive sebaceous secretion, oxygenation, aiding tissue regeneration, and stimulating the production of collagen and elastic fibers.

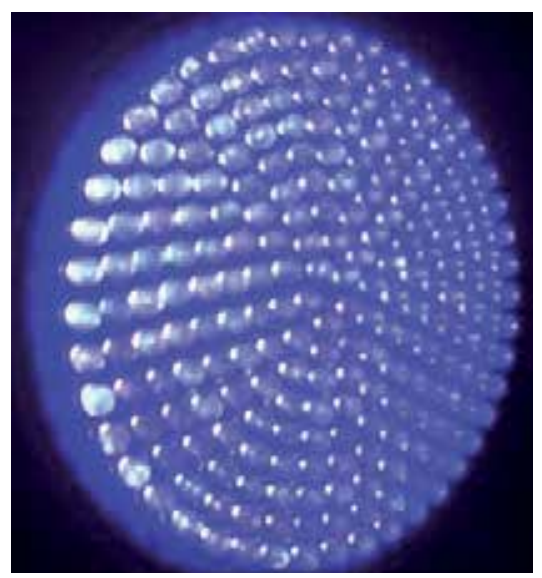

Figure 2. Arrangement of high brightness LEDs that emit blue light.

\subsubsection{Action of green light emitted by high brightness LEDs}

Green light emitted by high brightness LEDs (in the range of 530nm wavelength) can be used in the improvement of skin metabolism, pigmentation, and effects of aging. It provides a control of melanin absorption, aids in whitening pigmentation spots and provides an overall effect of hydration. It also prevents the formation of stains, freckles, and helps to reduce them. It keeps the skin smooth and moisturized.

\subsubsection{Action of amber light emitted by high brightness LEDs}

The amber LEDs have a wavelength of $617 \mathrm{~nm}$. The irradiation of amber has both draining and detoxifying properties, improving blood and lymph circulation, reducing edema, stimulating 


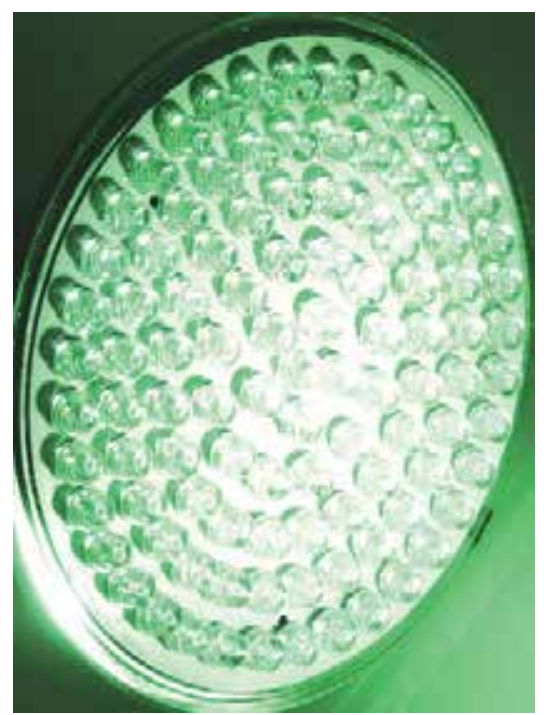

Figure 3. Arrangement of high brightness LEDs that emit green light.

the presence of water and causing a calming effect in case of redness caused by rosacea or sunburn.

For aging, the collagen fibers are presented densely packed together, to receive the amber light. The enzyme releases ions receivers which immediately adhere to the cytoplasmic membrane of the cells, creating a non-thermal thickening effect of collagen fibers. The reflection of light by the tissues in these conditions gives a healthy expression to patients. It has a draining action, stimulates blood and lymph circulation, and reduces swelling.

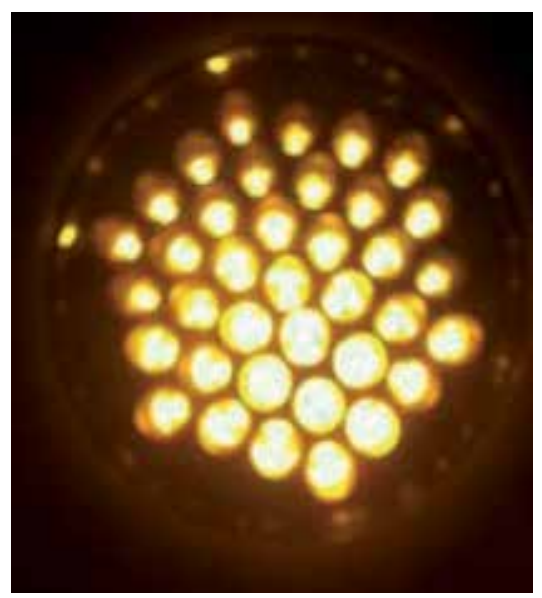

Figure 4. Arrangement of high brightness LEDs that emit amber light. 


\section{The importance of wavelength in the therapeutic application}

The response to the treatment with LED Therapy depends on several factors, but the most important is the wavelength and light penetration into the tissue beyond the physical, organic and genetic characteristics of the patients, which may provide a more efficient biological response one to another.

Some studies indicate that the wavelengths in the range of $620,680,760$ and $820 \mathrm{~nm}$ may be most suitable for health treatments [6]. Commercially available LEDs emit light at certain specific wavelengths, for example, 630, 660, 850 and $880 \mathrm{~nm}$. These values may not be accurate, it is possible to have a small variation during the operation in real time where this change can be due to temperature, input or output, electrical current and power dissipation. Currently the electronic converters and the quality of manufacture of high brightness LEDs can considerably reduce this variation. The wavelength of $630 \mathrm{~nm}$ generated by certain LEDs can affect peak $620 \mathrm{~nm}$ and $660 \mathrm{~nm}$ wavelength generated by the LEDs is approaching the peak of $680 \mathrm{~nm}$, for example [6]. When operating the LEDs with currents in milliampere range, you can improve the input waveform of [20], this fact is important to have control of the desired wavelength.

To perform the therapy a medical evaluation is required to get the correct diagnosis and indicate the most appropriate treatment and which wavelength should be applied.

\subsection{Relationship between the penetration depth and the wavelength in human tissues by applying light emitted by the high brightness LEDs}

The light penetration into human tissue is directly connected to the wavelength, that is, the larger the wavelength the longer is their penetration in the human tissue, as these wavelengths are specific and are within the visible light spectrum [6,22]. Therefore, the application of a given wavelength is directly related to the color. Each color has a certain wavelength respecting the chromaticity diagram. The color used depends on the type of treatment to be applied. The electronic converters can control the wavelength through duty cycle, which facilitates the relationship between the depth of penetration with the desired wavelength.

\subsection{Cell photobiostimulation}

There are areas of medicine and veterinary where the LED Therapy has an important role to play, as shown in Figure 6. These are: (i) wound healing, tissue repair, prevention of tissue death; (ii) the relief of inflammation, chronic diseases and injuries, with their pain and edema associated; (iii) relieving the pain and neurogenic some neurological disorders [7].

One of the photobiology principles is that visible light can have an effect in a living biological system. Photons must be absorbed by electronic absorption bands belonging to some molecular chromophore or photoreceptor. Figure 6 depicts the biological response as a function of wavelength, frequency, or energy of the photons and should resemble the absorption spectrum of the photoreceptor molecule [24].

The fact that a structured action spectrum can be built on the hypothesis of cell photoreceptors and absorption pathways stimulated by light. 


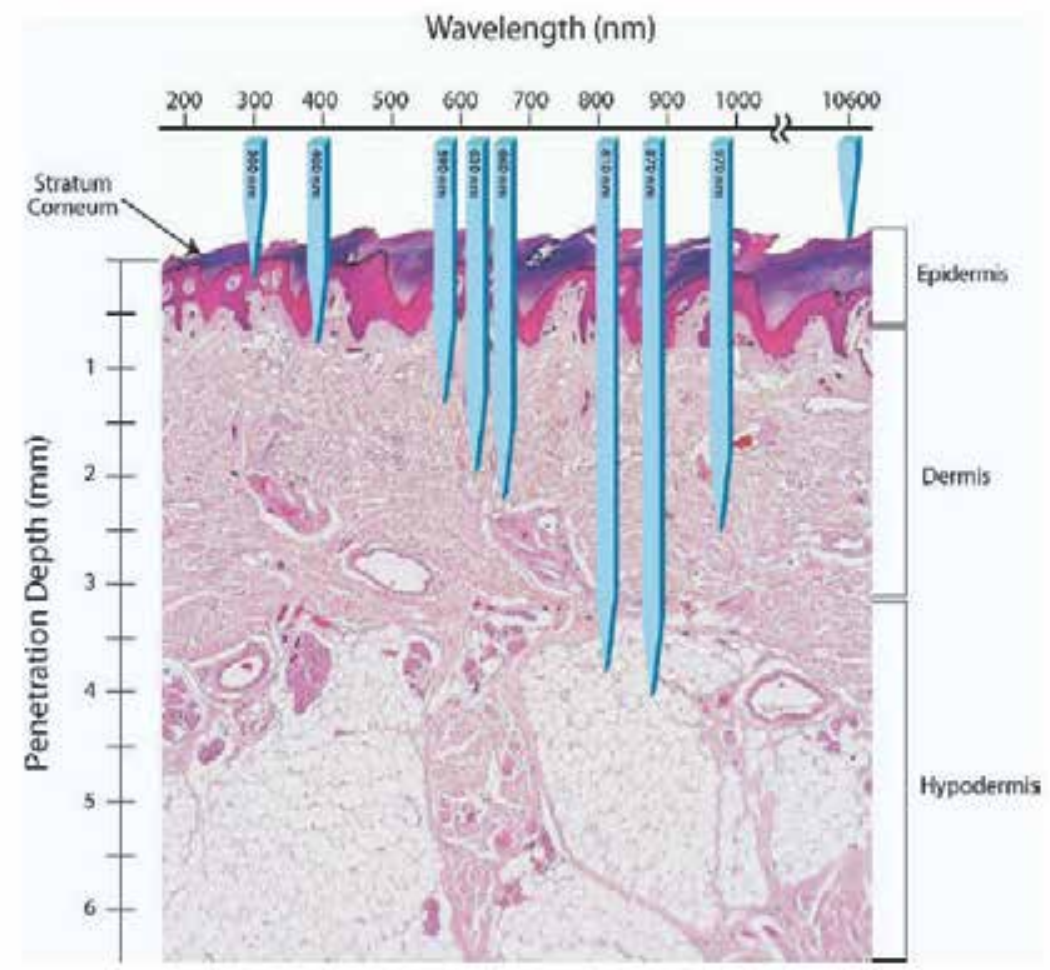

Figure 5. Action of color and depth of penetration in human tissue. Optical penetration depth.

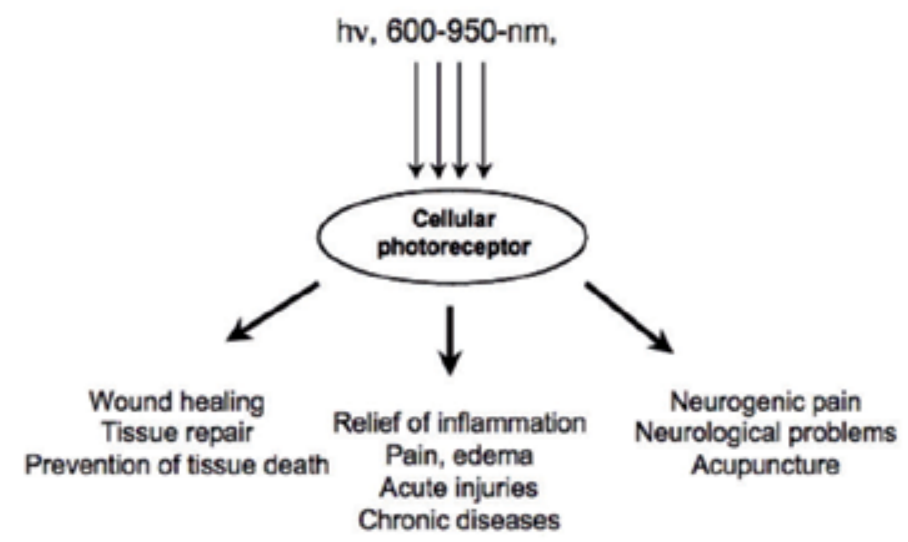

Figure 6. Schematic representation of the main areas of application of LED Therapy.

One should take into consideration some optical properties of tissues. Both the absorption and scattering of light in tissue are wavelength dependent (both much higher in the blue region of 
the spectrum than the red), chromophores (hemoglobin) has bands of high absorption and the wavelength less than 600nm [7]. For these reasons they are called "optical window".

The water begins to absorb wavelengths longer than $1150 \mathrm{~nm}$ and acts tissue at wavelengths in the range of red and NIR, wherein the tissue penetration becomes maximized (Figure 7). Furthermore, blue, green and yellow light may have significant effects on the cells because its therapeutic interaction is proven every day.

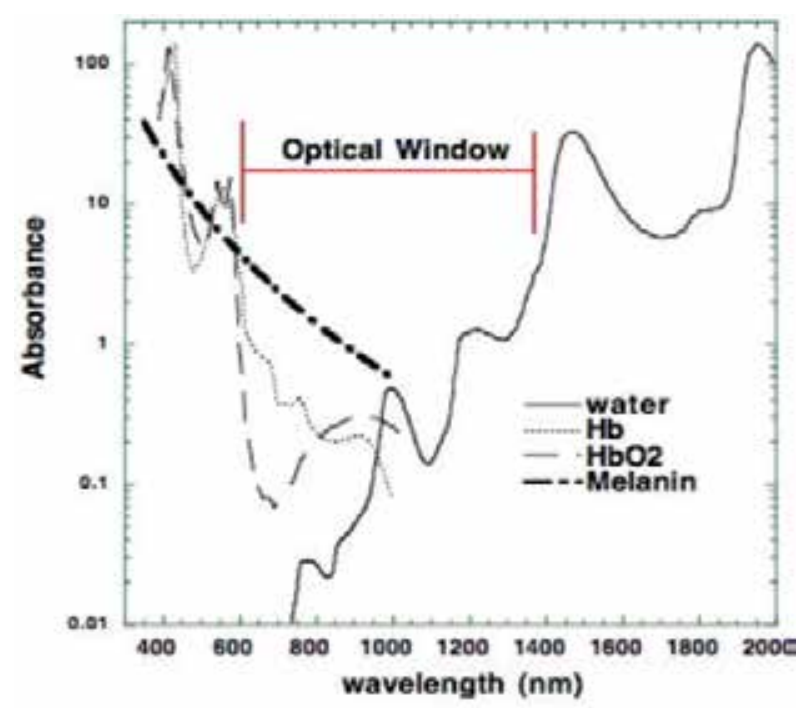

Figure 7. Optical window in tissue due to reduced absorption of red and NIR wavelengths (600-1200 nm) by tissue chromophores.

\section{Electronic converters used to supply high brightness LEDs}

The application of electronic converters switched to supply high brightness LEDs is critical because these converters have higher efficiency than linear converters. Thus, there are several possibilities of implementing converters DC/DC insulated and not insulated. The converters that are chosen must attend all the control and the power delivery to the high brightness LEDs [8]. Resonant converters help to reduce the peak power; they have low switching losses and low electromagnetic interference. Therefore, these topologies are useful for LED applications.

Depending on the characteristics of the electronic converters applied to LEDs more favorable characteristics should be observed to its applicability. Several converters can be used to supply the LEDs, preferably those that have a natural control over the output current.

DC/DC converter for high brightness LEDs should have a current source characteristic at its output, which already limits the inrush current, acting as an inherent protection circuit. To 
minimize the variations current it is preferable that its output current is not pulsed, which also minimizes the filter output current and allows the use of capacitors with longer life.

\subsection{Converters not isolated commonly used for supplying high brightness LEDs}

Buck converter, shown in Figure 8, is widely used in power high brightness LEDs. The current source attribute in the output makes this very interesting electronic converter, essentially because its output current can be continuous. Thus, the output capacitor $\mathrm{C}$ may have a small value and is unnecessary to use an electrolytic capacitor, which has the characteristic of a considerably short lifetime.

The output inductance $\mathrm{L}$ can be designed to provide a small ripple current, maintaining stable optical characteristics and the temperature of the LED junction.

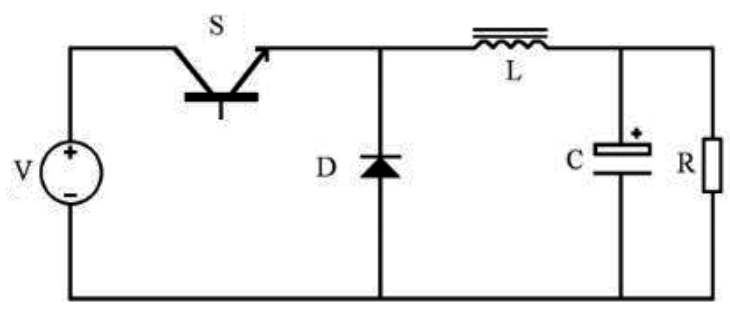

Figure 8. Buck converter.

If the output capacitor is removed from this type of DC/DC converter, the current on the LEDs is no longer purely DC because it contains a pulsating component. If the converter is a Boost or Buck-Boost converters, LED load is powered by an almost square with a sufficiently high reactance wave. The electronic converter CUK, in its basic structure consists of a fusion of two converters (Boost and Buck) in series using only a controlled switch. The union in series of these two converters permits the entry and exit can operate in continuous conduction mode and the static gain of the converter is the same Buck-Boost converter. Buck converter output enables obtaining a low current ripple in the LED, even for a small amount of C. ZETA converter comprises a Buck-Boost converter in series with an input Buck converter output. Similarly the CUK converter, the Buck converter on the output allows to obtain a low ripple current on the LED. SEPIC converter is composed of a Boost converter at the input, in series with a Buck-Boost converter output. All of these topologies may be used to supply the high brightness LEDs, some have more positive characteristics for the LEDs, such as the control of the output current.

\subsection{Converters isolated commonly used for supply high brightness LEDs}

Currently there is a considerable range of converters that can be used to supply LEDs, such as that with galvanic isolation. This sort of application employs the Flyback, Push Pull, Forward and Resonant converters [9]. The Figure 9 shows a system that supplies power to the LEDs using galvanic isolation. 


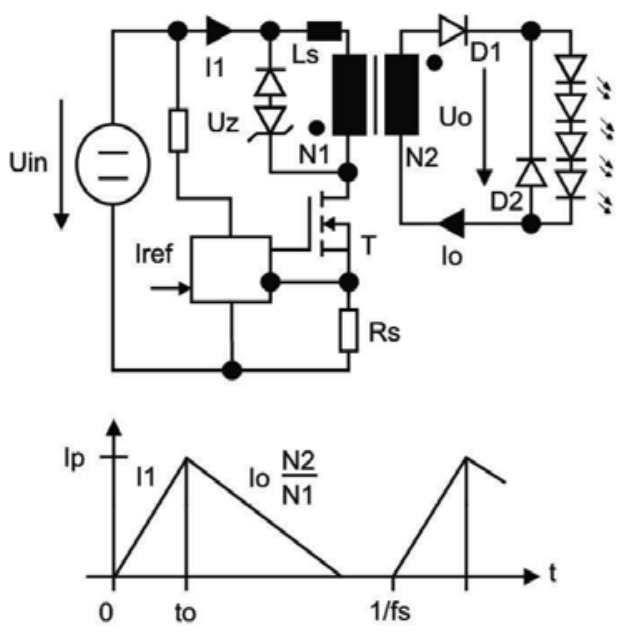

Figure 9. Representation of a LEDs supply the galvanic isolation system.

\subsection{Topologies used in research}

After reviewing several possible topologies for using as a power source for high-brightness LEDs two converters were chosen, one with galvanic isolation, the Flyback, and one without galvanic isolation, the Buck.

Flyback converter is more robust and has the advantage of being isolated, but it may show some noise in the output. Buck converter controls the output current better and offers a good response, but it has the disadvantage of not being isolated.

In summary, these two converters have been selected because their characteristics are more suited to supply the arrangements proposed by LEDs. The expected results should be quite satisfactory $[9,20]$.

\subsubsection{Flyback converter}

The Flyback converters of levels below 100W of power are widely used for the several applications and also for lighting with LED, normally, operating in discontinuous mode. This mode of operation is appropriate to control the current. The proposed topology is observed on Figure 10 and it was developed to supply the array of LEDs, which produce red light [9].

The red color has a greater wavelength (in the range of 647 to $780 \mathrm{~nm}$ ) and penetrates more deeply into the tissue. Thus, it is indicated for healing and recovering deep tissues [9].

The Flyback converter employed in the experiments owns a universal voltage input and its maximum output voltage is $5 \mathrm{~V}$.

The maximum output current is $2 \mathrm{~A}$. His frequency of switching is $100 \mathrm{kHz}$.

The proposed arrangement of red LED contains 90 high-intensity LEDs of $5 \mathrm{~mm}$, with wavelength in the range of 400 to $730 \mathrm{~nm}$. The current in each LED is around $20 \mathrm{~mA}$. 


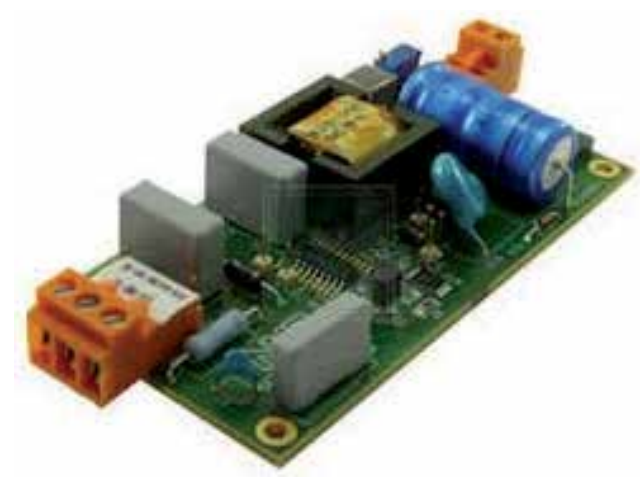

Figure 10. LEDs powered by a Flyback converter.

The source was designed to support up to 100 LEDs.

These tests are being conducted in patients with proper authorization and with the participation of five doctors, two surgeons, and three dermatologists, at Hospital Regional do Oeste in Chapecó, SC - Brazil.

The voltage produced on the LED was $4.1 \mathrm{~V}$ and current of the LEDs around 570mA. The values obtained were close to the simulation and design $[12,13]$.

\subsubsection{Buck converter}

The second converter developed has the Buck configuration as shown in Figure 11, with the following characteristics: Input Voltage DC-13V (after one stage rectified by with a Flyback converter) and the output voltage reaches $6 \mathrm{~V}$ and maximum output current reaches $1 \mathrm{~A}$ [20]. The frequency of switching is $52 \mathrm{kHz}$. The source has total isolation, even in short-circuit conditions in its terminals [10].

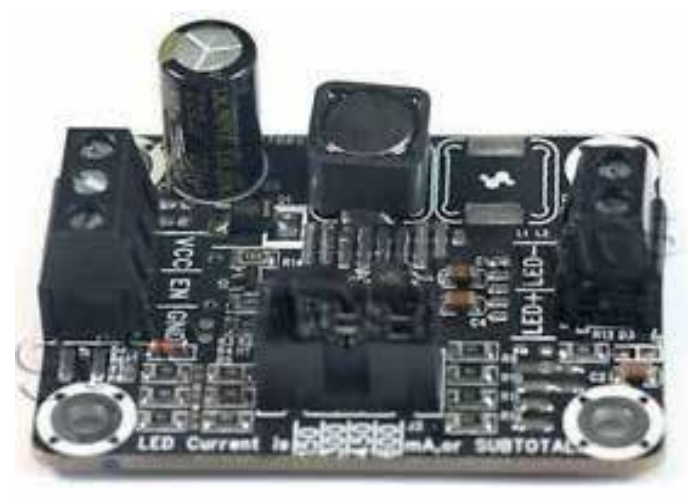

Figure 11. Buck converter.

This topology has the same versatility of a Flyback converter and supplies the array of LEDs. 
The voltage produced on the LED was 3.8V and current of the LEDs around 580mA. The values obtained were close to the simulation and design [11].

\section{LED Therapy automation using an embedded system integrated with a control software}

The main goal here was to provide a control interface of the therapy between the healthcare professional and the patient, giving them the possibility to monitor the phototherapy sessions progress and to treat the patient in a simple and effective way.

There are already lots of resources available in the market that use LEDs for skin disease treatment, like skin cancer or even aesthetic purposes, among others. When a new treatment is discovered, the creation of a new essay is necessary and therefore a new product is created. This project proposes a solution for this problem integrating hardware with control software. With the control software acting over the embedded system, it's possible to program new assays for each treatment; acting directly over the LED's, and dynamically minimizing the costs over new products every time a new treatment is discovered

\subsection{System overview}

The system is divided into two parts: the embedded system and the control. The embedded system contains a microcontroller, a LED board and an Ethernet Module for communication with the application [12]. The control consists of a database server and an application, as shown in Figure 12.

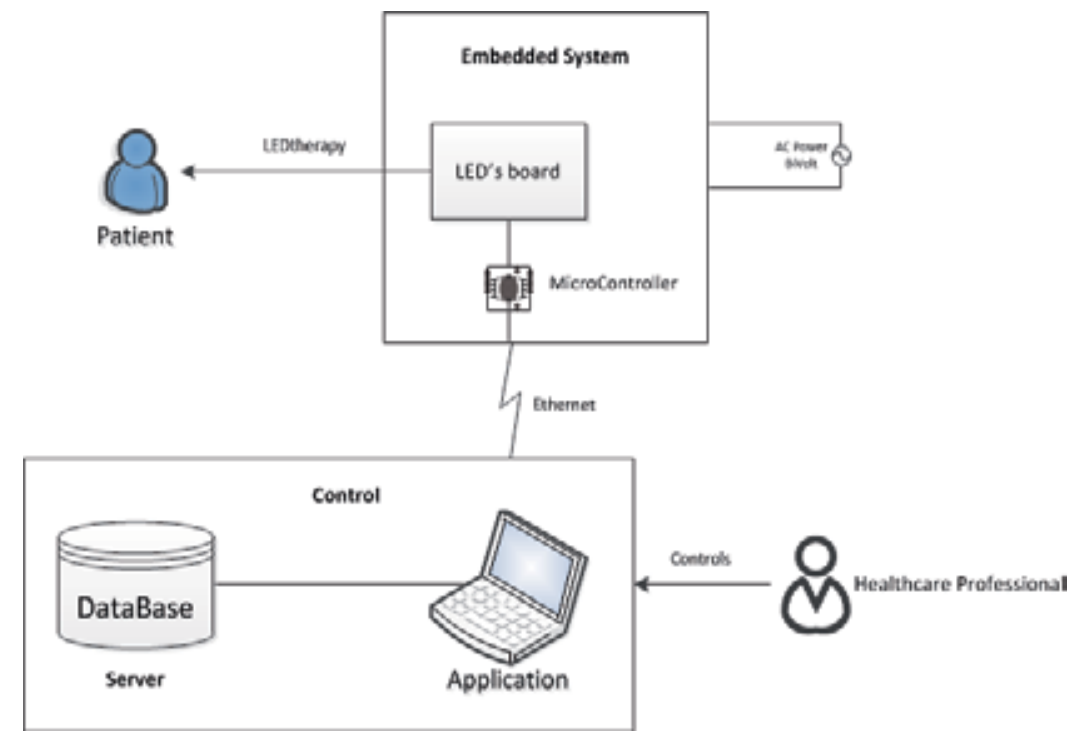

Figure 12. Automation of phototherapy with high brightness LEDs using an embedded system integrated with a software control. 
Through the control application, it is possible to register a patient or start a treatment session. The treatments are mapped to the database as essays that determine the variable values for each pathology. The variables are: the colors and the time of firing the LED arrays [25]. The communication between Embedded System and Application control is possible through the Ethernet Protocol [16].

\subsection{System model}

In this section the prototype details will be shown, such as: the application operation, embedded system operation and the database server details [16].

\subsubsection{Application}

The control application was written in JAVA language. The application aims to perform the user interface with the proposed system. Through the application the user can perform the following tasks:

- Register new patients, which are going to be stored in the database;

- Visualize the stored patients in the database;

- Connect the patient to a treatment; and

- Initiate a new session of the treatment.

The application stores the results of each treatment session.

\subsubsection{Embedded system}

The embedded system purpose is to communicate with the application and to execute the treatment over the patient. The embedded system is based on the Atmega328p AVR microcontroller present in the Arduino UNO board. It also has a LED board and an Ethernet Module ENC28J60 and resistors. The Figure 13 shows the prototype schematics. The database server aims to store all the patient data and the treatments as well. It's in the database that the LED treatment sequence is also stored. The chosen Data Base Management System (DBMS) was the PostgreSQL version 9, because it doesn't have license costs and it's capable of storing all the needed data $[13,14,16]$.

Tree arrays were assembled on a phenolite board with tree blue, yellow and red LED colors. Each array has thirty LEDs of the specified color which are powered by the corresponding Arduino pin and a common ground.

Where, (A) Ethernet ENC28J60 module, (B) Resistors, (C) Arduino UNO and (D) LEDs array. The LEDs board is composed by three arrays of high brightness LEDs with the following characteristics:

- Red array: The red light emitting LED array contains 30 high brightness LEDs of 5mm, with wavelength between $725 \mathrm{~nm}$ and $730 \mathrm{~nm}$. Operates at $3,3 \mathrm{~V}$ and $20 \mathrm{~mA}$. 
- Blue array: The blue light emitting LED array contains 30 high brightness LEDs of 5mm, with wavelength between $465 \mathrm{~nm}$ and $470 \mathrm{~nm}$. Operates at $4 \mathrm{~V}$ and $25 \mathrm{~mA}$.

- Yellow array: The blue light emitting LED array contains 30 high brightness LEDs of $5 \mathrm{~mm}$, with wavelength between $585 \mathrm{~nm}$ and 590nm. Operates at $3,6 \mathrm{~V}$ and $22 \mathrm{~mA}$.

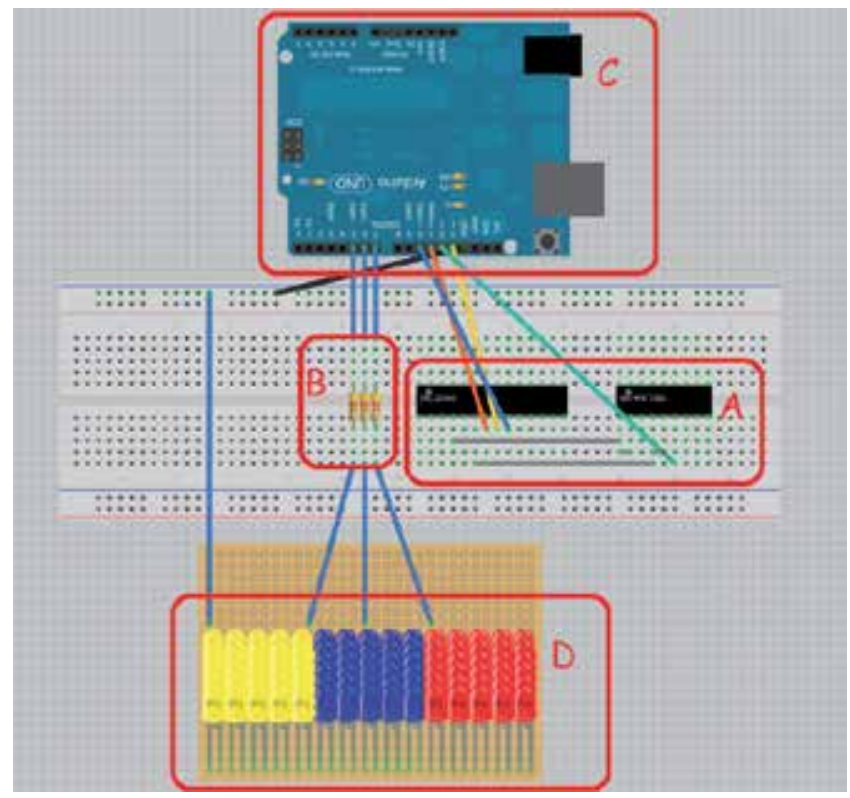

Figure 13. Prototype schematics.

Figure 14 shows the prototype used in the laboratory.

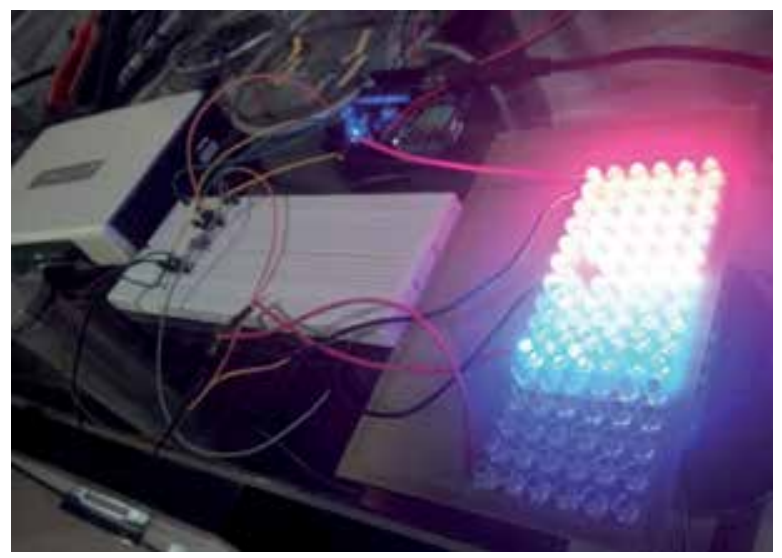

Figure 14. Numerous tests were performed with the prototype before manufacturing the device. 


\section{Types of treatment and response to therapy}

The concentration of light coming from the LED bulb can concentrate light to a certain point of light that can have a high ratio in millicandelas, but passing through the skin undergoes a dispersion of its light concentration. The rate control devices are important because the total light energy emitted by the LED or energy in Watts per square centimeter in units of $\mathrm{mW} / \mathrm{cm}^{2}$ is essential [15].

If the designer decides to use his knowledge to choose a less expensive way to manufacture power supply, then the power converter should be about 2 or 3 times more than the total of its light energy. The maximum light output of the output device is the source of half the power (W $=$ Volt $\mathrm{x}$ Amps) of the transformer. The $\mathrm{mW} / \mathrm{cm}^{2}$ is the total light energy in $\mathrm{mW}$ divided by the length and breadth of the array of LEDs in $\mathrm{cm}$.

\subsection{Criteria, control and response to treatment of the patients who were treated by red and blue light emitted by high-brightness LEDs}

Patients who are subject to the treatment will be properly classified with the criteria established by the doctors who assist in the implementation of therapy. Among them, age, sex, physical condition and mental health. The therapy was performed with LEDs Hospital Regional do Oeste in the city of Chapecó/SC, Brazil.

The first case is from a patient who had put breast prosthesis. The prothesis were large and a few days later, streaks appeared (Figures 15 and 16). Stretch marks are linear atrophies which arise due to the disruption of elastic fibers that support the middle layer of the skin. Generally, this breakup is from mechanical stress, such as excessive stretching of the skin, or physiological stress, stimulated by hormones. When the fibers are broken, they are filled with blood, becoming primarily redish, but over the time they acquire a white color. This patient underwent a medical evaluation and its treatment was approved. 15 sessions were held in 44 days. The application time for each session was 40 minutes. After the treatment the reduction was 95\% (Figures 17 and 18). The use of LED Therapy is completely painless and leaves no sequel. The Figures 19 and 20 show the results of treatment.

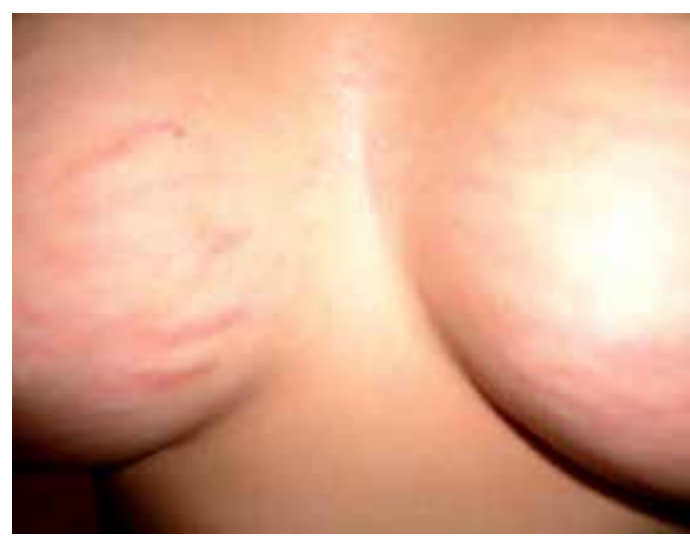

Figure 15. Patient with stretch marks on breasts before application of therapy. 


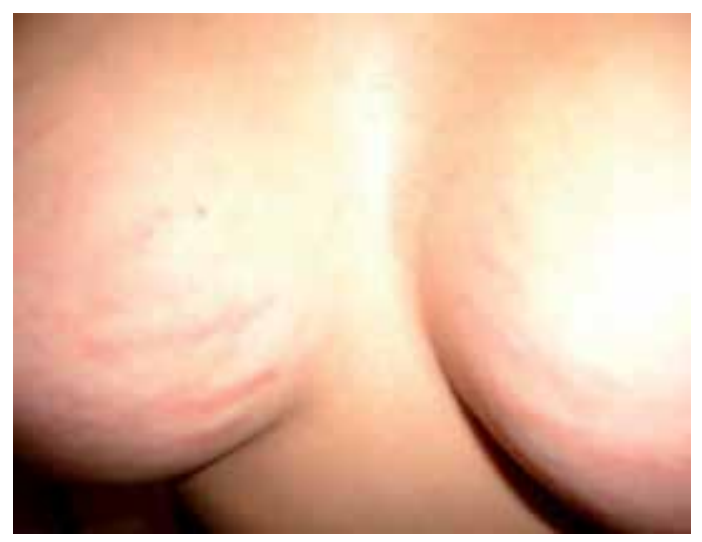

Figure 16. Patient developed stretch marks after placing silicone breast.

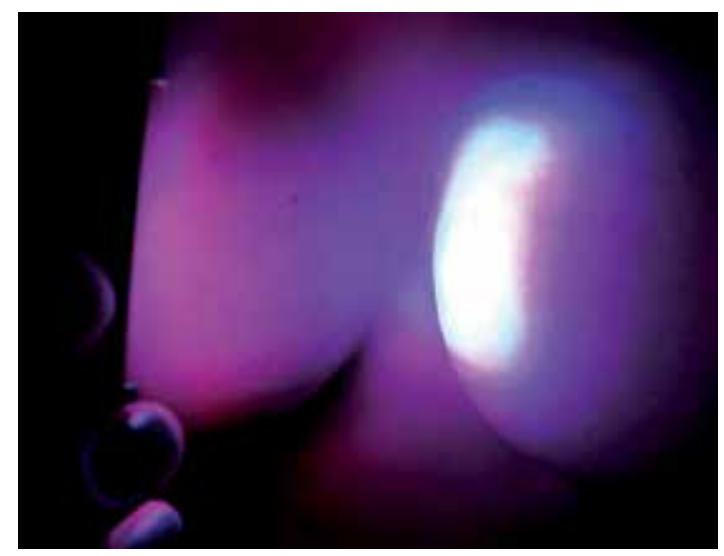

Figure 17. Application of therapy with LEDs that emit blue and red lights.

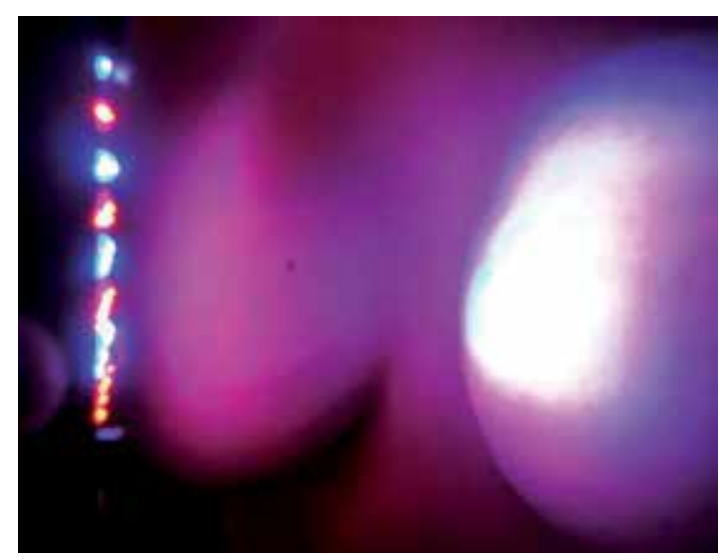

Figure 18. Application of the array of LEDs during treatment. 


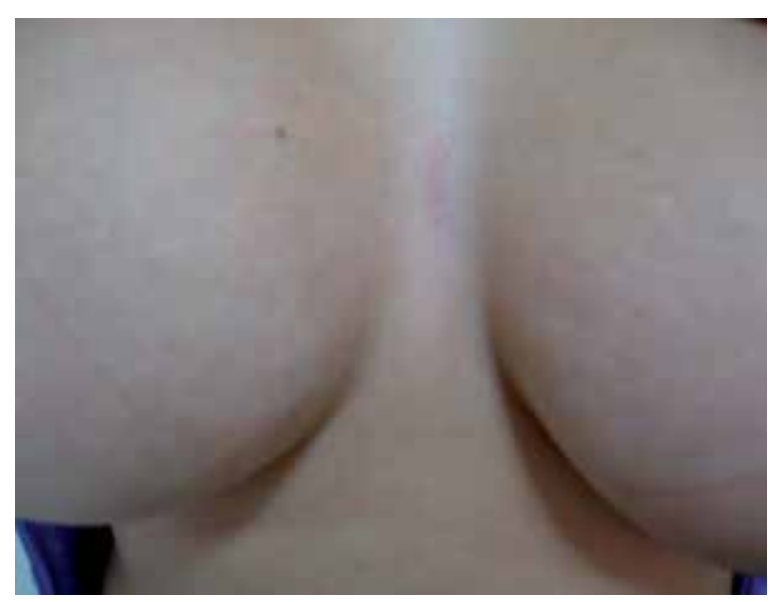

Figure 19. Result after treatment.

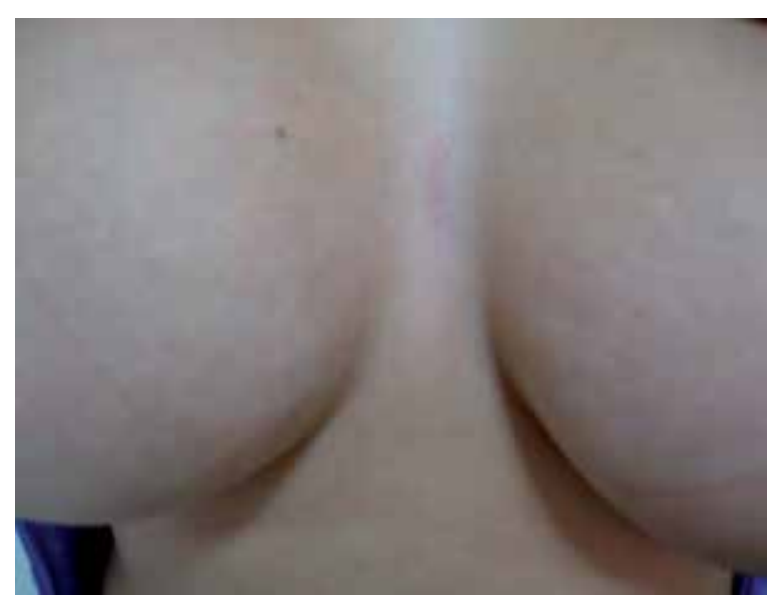

Figure 20. The striations were eliminated.

The second case is a patient who had stretch marks on her hip in the region of the buttocks. She underwent a medical evaluation and the treatment was initiated. 20 sessions were held for 49 days. Each session was 40 minutes long. A reduction of $87 \%$ of the hips as shown in Figure 21 has occurred.

In this procedure the Buck converter, which is supplied by the LEDs, was used that emit red and blue light.

All patients who underwent treatment fulfilled all requirements and recommendations of the medical staff. All International Laws and National research in human beings were observed. All protocols required by the Brazilian Ministry of Health and the Ethics Committees of Hospital Institutions. 

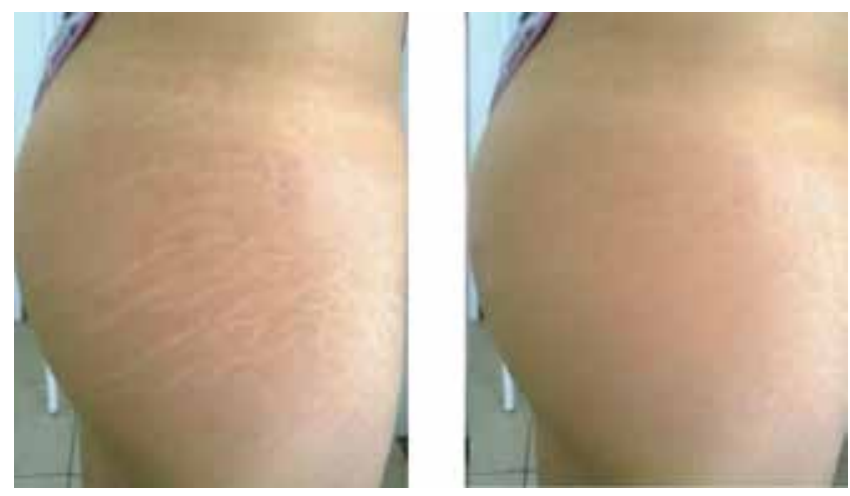

Figure 21. Outcome of treatment for stretch marks.

The Figure 22 shows some prototypes that were manufactured to perform the therapy.

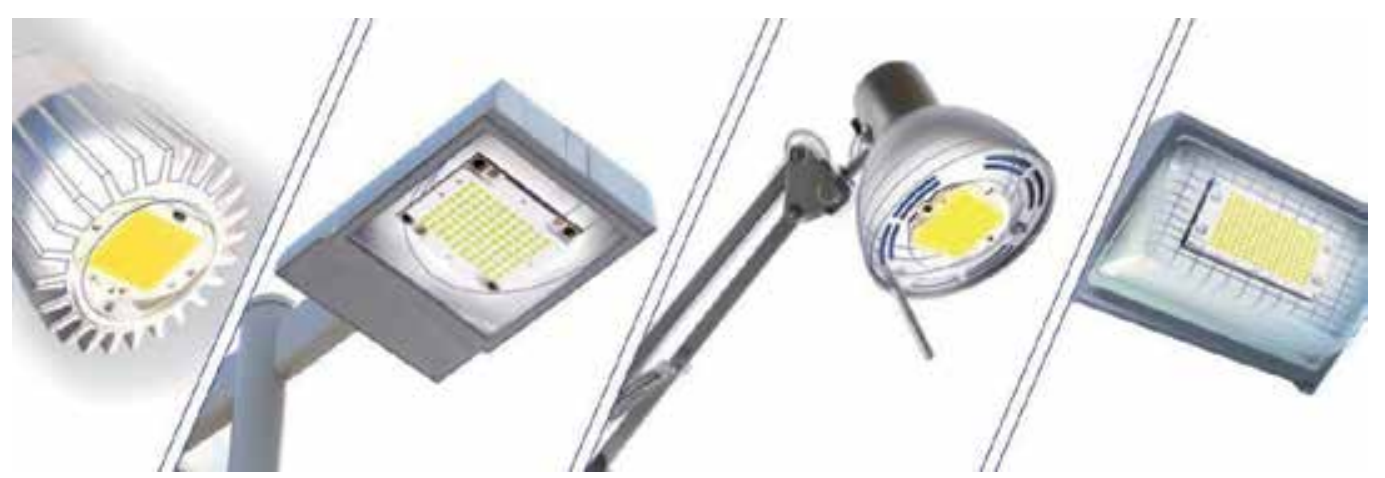

Figure 22. Prototypes that are being manufactured.

\section{The future of LED therapy}

The application of high brightness LEDs in human tissue has been growing every day. Several scientific institutions have explored this theme. Many researches are underway on the use of therapy with the LED in order to improve their application and to determine if there are other applications which may be used. The therapy is applied within the visible light spectrum and each color has its application and its therapeutic purpose.

The red color that is in a range of 600-700 nanometers, is effective in human tissues and shows excellent results in wounds and sores. The higher wavelengths, including infrared, are more sharp and can reach the bone. Studies also suggest that the spectrum between 400 to 500 nanometers, in the range of blue color, can be effective in the treatment of skin disorders, including acne, stretch marks, cellulite, skin blemishes and scarring. 
Probably in the next few years, LED Therapy will be the main treatment for wounds, such as post-surgical wounds and unhealed as diabetic ulcers. Scientists seek to test the technology of LED Therapy in other clinical situations, such as spinal cord injuries and treatment of Parkinson's disease, cerebrovascular accidents, brain tumors and regeneration of tissues and organs [17].

With the advancement and development of new applications of LEDs in health, manufacturers of these devices will increasingly improve these devices that every day demonstrate its usefulness and its application to human health.

\section{Conclusion}

The application of LEDs in interaction with the human tissues shows a great interest of manufacturers and researchers. The correct use of LEDs in this context directly depends on the tissue nature where it wants the light to interact [10].

Several parameters are important for satisfactory results, such as wavelength, the kind of color, temperature control of the LED, the characteristics of the used converter, control the brightness, output current, duty cycle and all the observations made in the previous sections of this work.

This is because a small spectral change can lead to a major shift in the lighting characteristics. LEDs are increasingly becoming a great option to help cure various diseases and to prevent others [18].

Thus, this work contributed to the development of LED application in human tissues showing that the effect of the emission of light through the high brightness LEDs offers a new treatment option for opening new ways of therapeutic technique LED Therapy applied to human tissues [20].

\section{Author details}

Alexandre Campos ${ }^{1}$, Yasmim Guterres$^{2}$, Eduardo B. Bauer ${ }^{3}$ and Mauro C. Moreira ${ }^{4^{*}}$

*Address all correspondence to: mcmoreira41@gmail.com

1 Federal University of Santa Maria/UFSM, Brazil

2 Federal University of Santa Catarina /UFSC, Brazil

3 University of Vale do Itajaí /UNIVALI, Brazil

4 Federal Institute of Santa Catarina /IFSC, Brazil 


\section{References}

[1] Moreira, M. C., Ricardo Prado and Alexandre Campos; "Application of High Brightness LEDs in the Human Tissue and Its Therapeutic Response " Book Chapter Applied Biomedical Engineering. Intech. Croatia (2011). p 1-20.

[2] Elder, D. et al. (2001). “Histopatologia da Pele de Lever”. Manual e Atlas. São Paulo: Manole.

[3] Bala, O.; Ölmez, A.; Kalayci, S.; Effect of LED and halogen light curing on polymerization of resin-based composites. Journal of Oral Rehabilitation 2005, 32: 134-140.

[4] Yoo, B. H.; Park C. M. ; Oh, T. J.; Han, S. H. ; Kang, H. H. (2002). "Investigation of jewelry powders radiating far infrared rays and the biological effects on human skin". Journal of Cosmetic Science, n.53. p. 175-184.

[5] HTM Indústria de Equipamentos Eletro-Eletrônicos Ltda. (2007). Manual do Equipamento Laser HTM, Amparo, São Paulo-SP.

[6] Heelspurs.com (2007), “Led Light Therapy”, LLC 3063 Pinehill Road Montgomery, AL 36109.

[7] Michael R. Hamblin, Mechanisms of Low Level Light Therapy, Department of Dermatology, Harvard Medical School, BAR 414 - Wellman Center for Photomedicine, Massachusetts General Hospital Boston (2008).

[8] SÁ JR., E. M. (2007) - “Projeto de Tese de Doutorado: Estudo de Novas Estruturas de Reatores Eletrônicos para LEDs de Iluminação". Programa de Pós-Graduação em Engenharia Elétrica, UFSC, Florianópolis-SC.

[9] Moreira, M. C.; Prado, R. N.; Campos, Alexandre; Marchezan, T. B.; Cervi, M. (2008) "Aplicação de LEDs de Potência nos Tecidos Humanos e sua Interação Terapêutica". In: XVII Congresso Brasileiro de Automática, Juiz de Fora-MG. Anais do XVII Congresso Brasileiro de Automática, p. 1-6.

[10] Moreira, M. C.; “Utilização de Conversores Eletrônicos que Alimentam LEDs de Alto Brilho na Aplicação em Tecido Humano e sua Interação Terapêutica" (2009). Tese de Doutorado, Universidade Federal de Santa Maria, p. 1-165.

[11] Moreira, M. C.; “Utilização de um Conversor Eletrônico que Alimenta LEDs de Alto Brilho na Cor Vermelha em Tecido Humano de Pessoas Idosas" (2010). Artigo publicado na 3를 Semana de Ciência e Tecnologia, IFSC, Chapecó-SC, Brazil, p. 1-6.

[12] Margolis, Michael. Arduino Cookbook. O’Reilly Media, Inc. (2011).

[13] ATMEGA328P, Datasheet. 8-bit Atmel Microcontroller with 4/8/16/32K Bytes In-System Programmable Flash. Rev. 8271DS-AVR-05/11, 2011.

[14] ENC28J60. Microchip Stand-Alone Ethernet Controller with SPI Interface. 2006. 
[15] YEH, N.G.; WU, C.; CHENG, T.C. Light-emitting diodes: their potential in biomedical applications. Renewable and Sustainable Energy Reviews, v.14, p.2161-2166, 2010.

[16] Eduardo B. Bauer, Alejandro Rafael Garcia Ramirez, Mauro C. Moreira; LED Therapy automation using an embedded system integrated with a control software. TCC, University of Vale do Itajaí /Univali, 2013.

[17] Daniela Litscher and Gerhard Litscher; Laser Therapy and Dementia: A Database Analysis and Future Aspects on LED-Based Systems. International Journal of Photoenergy Volume 2014, Article ID 268354, 5 pages, 2014.

[18] Lew Lim; The Potential of Treating Alzheimer's disease with Intranasal Light Therapy. MedicLights Research Inc., Toronto, Canada, August 2013.

[19] Sabino CP, Meneguzzo DT, Benetti E, Kato IT, Prates RA, Ribeiro MS ; Red laser attenuation in Biological tissues: study of the inflammatory process and pigmentation influence. Mechanism for Low-Light Therapy VII, edited by Hamblin MR et al. Proc. Of SPIE (8211, 821105), 2012.

[20] Campos, A., do Prado, R.N., Moreira, M.C.; Application of High-Brightness LEDs in Tissue Human and Their Therapeutic Interaction, Electron. Ballast Res. Group, Fed. Univ. of Santa Maria, Santa Maria, RS, Brazil. Industry Applications Society Annual Meeting. IAS 2009. IEEE, pages 1-6, Houston, TX - USA, DOI: 10.1109/IAS. 2009.5324855, 2009.

[21] Sontea, V., Chisinau, Moldova, Pocaznoi, I., Furtuna, D., Seryakov, A.; Effects of the Low Level Light therapy on skin wound using LED. E-Health and Bioengineering Conference (EHB), IEEE, pages 1- 4, Iasi, DOI: 0.1109/EHB.2013.6707243, 2013.

[22] Chotikasemsri, P., "Phototherapy and LED treatment optimization on gum fibroblast and osteoblast cell line, " Biomedical Engineering International Conference (BMEiCON), 2013 6th, vol., no., pp.1, 3, DOI: 10.1109/BMEiCon.2013.6687656, 2013.

[23] Ursarescu, I.-G., Solomon, S.; Pasarin, L., Scutariu, M., Martu, A., Boatca, R.-M., Martu, S. ; The effects of LED photo-activated disinfection on periodontal clinical parameters in patients with chronic periodontitis and osteoporosis. E-Health and Bioengineering Conference (EHB), vol., no., pp.1, 3, 21-23 Nov. 2013 DOI: 10.1109/ EHB.2013.6707311, 2013.

[24] Freisleben, J.; Hamacek, A.; Vik, R.; Cerny, J.; Kroupa, M.; Dzugan, T., "Design of a singlet oxygen generator based on LED emitters, " Electronics Technology (ISSE), 35th International Spring Seminar on, vol., no., pp.47, 49, DOI: 10.1109/ISSE. 2012.6273106, 2012.

[25] Hsi-Chao Chen; Cheng-Jyun Liou; Investigation of illumination efficiency on the LED therapy with different array types. Ninth International Conference on Solid State Lighting, Volume no 7422. San Diego, CA - USA, 2009. 

Chapter 9

\title{
Photodynamic Nanomedicine Strategies in Cancer Therapy and Drug Delivery
}

\author{
Rodica-Mariana Ion \\ Additional information is available at the end of the chapter \\ http://dx.doi.org/10.5772/59624
}

\section{Introduction}

Cancer is a group of diseases which cause an abnormal and uncontrolled cell division coupled with malignant behavior such as invasion and metastasis [1]. World Health Organization (WHO) estimates that in 2012, the total number of cancer deaths in the European Union (EU) was 1283101 [2]. For the treatment of cancer various methods have already been discovered and many others are in the process of discovery e.g. chemotherapy (alkylating agents, antimetabolites and natural products - as plant products and micro-organisms), hormonal therapy (with steroids, hormones, anti-estrogens, anti-androgens, gonadotropin releasing hormones analogues, non-steroidal aromatase inhibitors), immunotherapy (interferon, growth factor inhibitors, vaccines, interleukin-2) and different therapies: radiation therapy, photodynamic therapy, surgery, chemotherapy and some traditional therapies [3]. But the anticancer drugs can fail to kill cancer cells for various reasons, the transport of the anticancer drug being governed by physiological and physicochemical properties of the target cell and of the drug itself [4]. These properties include pressure, charge, size, configuration, electrochemical properties, hydrophilicity, etc. For the therapeutic agents delivery to the tumor cells, the following problems can be addressed, as follows:

a. Drug resistance at the tumor levels (non cellular based mechanisms);

b. Drug resistance at cellular level (cellular based mechanisms);

c. Pharmacokinetic properties of the anticancer agent in the body [5].

The concept of the nanoparticles which permits higher absorption of the drugs in a specific tissue, and this concept has been applied for hyperthermia, radiation therapy, photodynamic therapy, etc. [6]. Meanwhile, the nanoparticles opened new horizons for drug delivery and bringing the term nanomedicines. Nanomedicine is the medical application for diagnosis and 
treatment of different human diseases by means of small particles, known as nanoparticles with sizes of 2-100 $\mathrm{nm}$.

The nanoparticles are known by their large surface area, high reactivity, high solubility, reduced side effects and low toxicity [7-9]. The main nanoparticles applied in nanomedicine are: polymeric nanoparticles, liposomes and lipid nanoparticles, micelles, microcapsules, magnetic particles, and carbon nanoparticles (fullerenes, carbon nanotubes, carbon nanofibers, etc) and the nanoassemblies [10-12].

Photodynamic therapy (PDT) as a part of photochemotherapy, is a concerted method where, in addition to light and an administered drug, oxygen is required. PDT represents a concerted action of light, with a sensitizers and an oxygen active specie (singlet oxygen) which preferentially actions on tumor cells and not on healthy cells. The administered drug is generally a substance which can efficiently photosensitize the formation of singlet oxygen (or other reactive species derived from oxygen), and such species react with different biological targets, and cause cellular damage and finally, the cellular death. Activation of the photosensitizers by light is an essential condition for a successful PDT. Doses of light energy applied in PDT are commonly within $60-200 \mathrm{~J} / \mathrm{cm}^{2}$, though doses may vary from 25 to $500 \mathrm{~W} / \mathrm{cm}^{2}$ depending on indications, tissues and light sources [13].

Under such circumstances, this chapter offers the most up-to-date coverage of photodynamic therapy including information on how nanosensitizers, have evolved within the field of cancer therapy and more recently for drugs controlled release in this field, by using personal data correlated with literature reports.

\section{Short history}

Photodynamic therapy is dating from ancient time, the Indian civilizations reported from the first time the combined action of psoralens with sunlight to treat vitiligo [14].

Niels Fiensen used UV light to treat small pox, pustular infections eruptions, cutaneous tuberculosis, and for its results he obtained the Nobel Prize in Medicine in 1903. Similar results obtained Niels Raab in 1905, by using eosin as sensitizer and combining his results with Jesionek and J.Prime results for skin tumors and epilepsy generated by light induced dermatitis [17]. Meyer-Betz was the only experimentalist who tested this method on himself, by injecting haematoporphyrin, reporting the observed effects: oedema, erythema and light sensitivity [18]. Later, Campbell and Hill studied the PDT effects on microcirculation, reporting the thrombosis and vascular shutdown [19].

Lipson in 1966 went on to treat a patient with a large cancer of the breast following an injection of a derivative of haematoporphyrin $(\mathrm{HpD})$. The modern era of photodynamic therapy was established by Dr. T.J.Dougherty, at the Division of Radiation Biology at Roswell Park Memorial Institute, Buffalo, USA, who reported that a systematically injected porphyrin on activation with red light caused complete eradication of transplanted experimental tumors [20]. 


\section{Mechanism of photodynamic effect}

The photodynamic effect mainly results from energy and/or electron transfer of the lowest excited triplet state $T_{1}$ of the photosensitizer to an organic substrate or molecular oxygen. In the photodynamic therapy occur three types of mechanisms:

- type I mechanism - electron transfer $(e T)$ where the photosensitizer excited state generates a radical species, for example by electron transfer from (or to) a substrate, or by hydrogen atom abstraction from a substrate. The radical species then reacts with ground state oxygen so that the overall reaction is a photochemically initiated autoxidation:

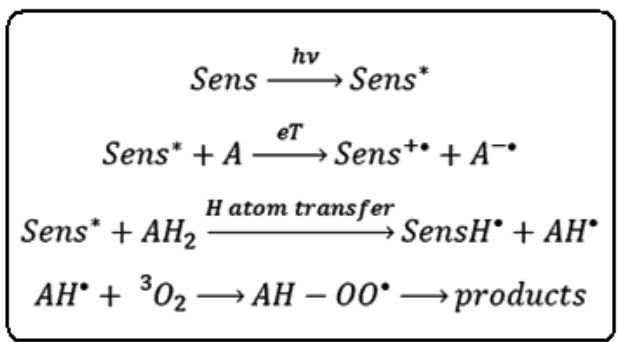

(Sens = sensitizer; $\mathrm{A}=$ biomolecule; ${ }^{3} \mathrm{O}_{2}=$ triplet excited state of oxygen)

Sheme 1. The type I mechanism of PDT

- In type II mechanism - energy transfer (ET) an energy transfer occurs from the excited photosensitizer to molecular oxygen, to give the sensitizer in its ground state and singlet oxygen. In this mechanism electronic excitation energy is transferred from the excited triplet $T_{1}$ of the sensitizer (generated by intersystem crossing isc from the ecited singlet $S_{1}$ ) to triplet molecular oxygen, to give the sensitizer in its ground state $S_{0}$ and singlet oxygen ${ }^{1} \mathrm{O}_{2}$.

$$
\begin{gathered}
\text { Sens }\left(\mathrm{S}_{0}\right) \stackrel{{ }^{h v}}{\longrightarrow} \operatorname{Sens}\left(\mathrm{S}_{1}\right) \stackrel{\text { isc }}{\longrightarrow} \operatorname{Sens}\left(\mathrm{T}_{1}\right) \\
\text { Sens }\left(\mathrm{T}_{1}\right)+{ }^{3} \mathrm{O}_{2} \longrightarrow \text { Sens }(\mathrm{S} 0)+{ }^{1} \mathrm{O}_{2} \\
A+{ }^{1} \mathrm{O}_{2} \longrightarrow \text { products }
\end{gathered}
$$

Sheme 2. The type II mechanism of PDT

Major biological targets are membranes that undergo rupture and the cells are destroyed through the membranes around the mitochondria and the lysosomes. These organelles induce subsequent cellular destruction by necrosis or apoptosis [21-24].

Except these two types of mechanisms, there is another one: type III mechanism, which take place when the oxygen is absent in the system. 


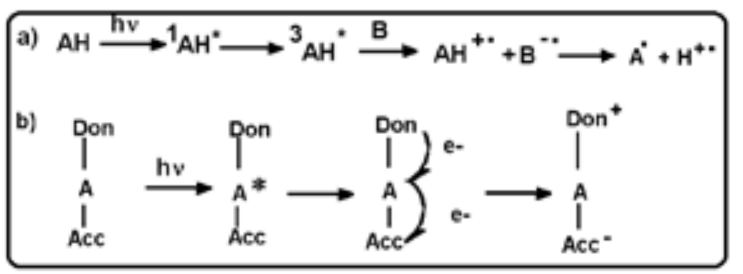

Sheme 3. The type III mechanism of PDT (a)AH = porphyrin; B = quinone (b)Don = donor (cysteine); $\mathrm{A}=$ porphyrin; Acc $=$ acceptor (methyl viologen)

\section{Photosensitizers}

\subsection{Conventional photosensitizers}

All the sensitizers could be natural or synthetic compounds, with proper absorption properties from a light source. They should be pure compounds, soluble in body fluids, with high capacity to be incorporated in malignant cells. Also, they should be fluorescent and able to generate singlet oxygen, which is the excited state of oxygen efficient on malignant cells [25]. Taking into account all these criteria and knowing the compatibility with human body, the porphyrins are known as ideal sensitizers for photodynamic therapy.

The general chemical structure for some porphyrins and phthalocyanines as PDT agents are represented in Figure 1.

First Generation Photosensitizers, includes Photofrin ${ }^{\circledR}$ and $\mathrm{HpD}$ and exist as complex mixtures of monomeric, dimeric, and oligomeric structures. At $630 \mathrm{~nm}$, their effective tissue penetration of light is small, $2-3 \mathrm{~mm}$, limiting treatment to surface tumors. Although Photofrin ${ }^{\circledR}$ has a low $\varepsilon_{\max }\left(\right.$ at $630 \mathrm{~nm} \sim 3000 \mathrm{M}^{-1} \mathrm{~cm}^{-1}$ ), generate singlet oxygen with high quantum yield, $\Phi_{\Delta}=0.89$. In spite of its safe applications in bladder, esophageal and lung cancers, Photofrin tends to be applied to head human part and thoracic part affected by cancer [26].

The Second Generation Photosensitizers, includes porphyrins and related compounds (porphycenes, chlorins, phthalocyanines, so on), many of them being under clinical tests. From the porphyrins family, meta-tetra(hydroxyphenyl)porphyrin ( $m$-THPP) and 5,10,15,20tetrakis(4-sulfanatophenyl)-21H,23H-porphyrin $\left(\mathrm{TPPS}_{4}\right.$ ) are the most used second generation PDT sensitizers (Figure 2). $m$-THPP, however, caused skin phototoxicity, and was 25 to 30 times more potent than $\mathrm{HpD}$ in tumor photonecrosis when irradiated at $648 \mathrm{~nm}$ [27]. $\mathrm{TPPS}_{4}$ exhibited lower photochemical efficiency than meso-substituted porphyrins containing fewer sulphonated groups [28].

Except the free-bases, the porphyrins can be chelated with a variety of metals, the diamagnetic ones enhancing the phototoxicity. Paramagnetic metals are shortening the lifetime of the triplet state and as result can make the dyes photoinactive [21]. The presence of axial ligands to the centrally coordinated metal ion is often advantageous, since it generates some degree of steric 


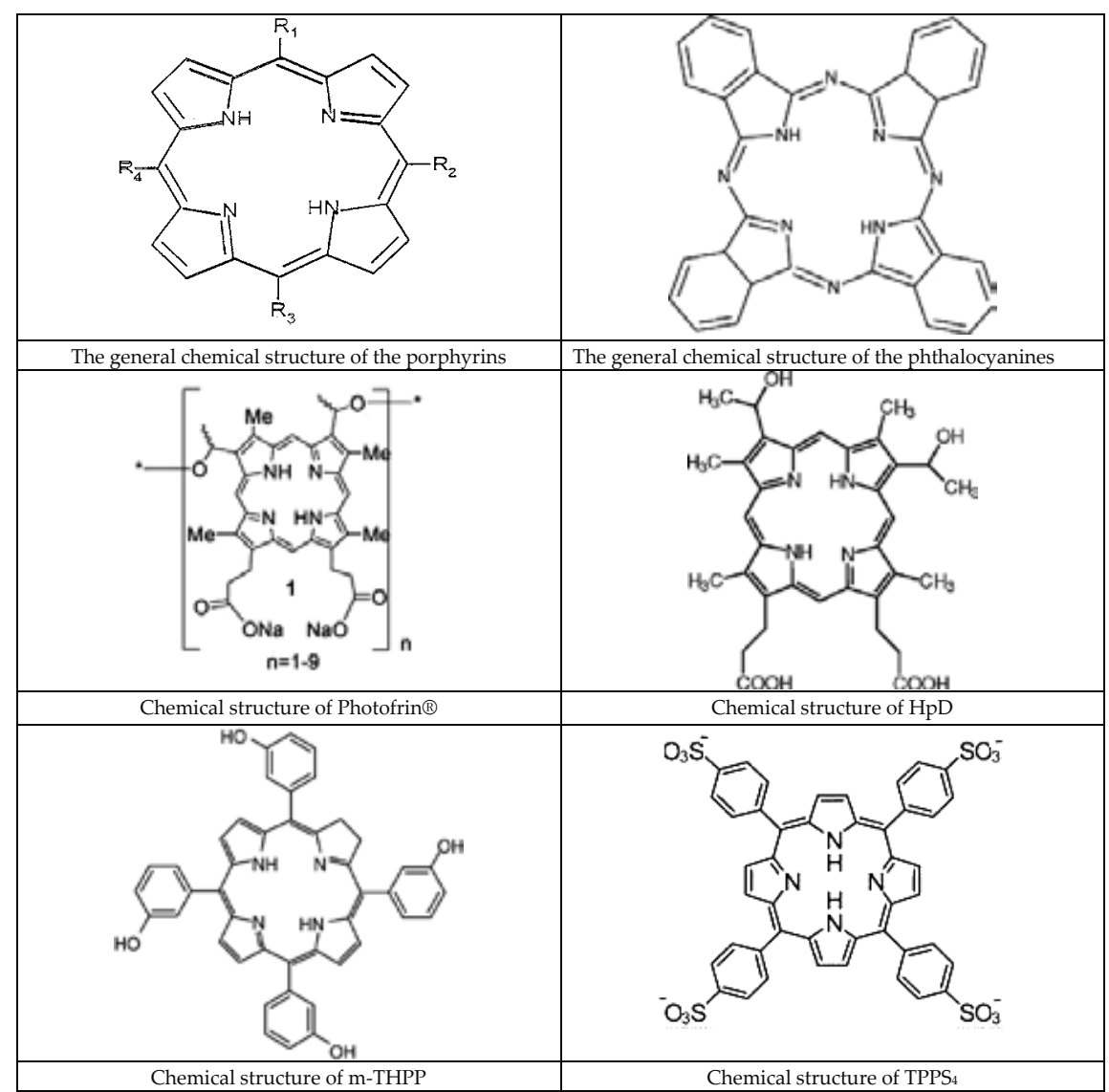

Figure 1. The chemical structure of some porphyrins and phthalocyanines

hindrance to intermolecular aggregation, without impairing the photophysical properties of the dye [21].

Phthalocyanines (Pc) are currently recognized as one of the best sensitizers used in PDT, have a long-wavelength band with a large extinction coefficient $\left(\sim 10^{5} \mathrm{M}^{-1} \cdot \mathrm{cm}^{-1}\right)$ and generally a low dark toxicity [29-32].

Their absorption maxima are in the region $670-700 \mathrm{~nm}$, with very high molar coefficients. A representative compound is aluminium phthalocyanine tetrasulphonated $\mathrm{AlPcS}_{4}$, $\mathrm{commer}^{-}$ cially known as Photosens, in spite of its skin sensitivity, proper absorption maxima at $676 \mathrm{~nm}$, it is well applied in Russian clinics for stomach, skin, oral and breast cancers [33].

Another clinical phthalocyanine is silicon phthalocyanine 4 (Pc4) which was successful tested in different skin cances (pre-malignant - actinic keratosis, Bowen disease) or even in malgnant forms of cutaneous cancers $[34,35,36]$.

The central metal ions play an important role in the photophysical properties of phthalocyanines. In metallophthalocyanines the central metal $(\mathrm{M})$ has one or two axial ligands or one or 
more ring substituents or both. When a diamagnetic ion is in the center of the ring (e.g., $\mathrm{Zn}$, $\mathrm{Al}, \mathrm{Ga})$, the phthalocyanine generally possesses a high triplet state yield $\left(\varphi_{\mathrm{T}}>0.4\right)$ with a long lifetime $\left(\tau_{\mathrm{T}}>200 \mu \mathrm{s}\right)$ and enough energy $\left(110-126 \mathrm{~kJ} / \mathrm{mol}^{-1}\right)$ to generate ${ }^{1} \mathrm{O}_{2}\left(94.5 \mathrm{~kJ} / \mathrm{mol}^{-1}\right.$ is required) [37-40]. Silicon phthalocyanine allows two appropriate axial ligands, which forbid the ring staking which decrease the clinical efficiency [41-44]. The triplet-state lifetimes of an axially substituted silicon phthalocyanine typically vary from 100 to $200 \mu$ s and the yields from 0.2 to 0.5 [43]. Some synthetic silicon phthalocyanine and naphthalocyanine (Figure 2) have been used in some laboratory experuiments on K562 culture cellk with excellent results [45, 46].

Third generation photosensitizers contains available drugs that are modified them with antibody conjugates, biologic conjugates, etc. [47,48]. These terms are still being used although not accepted unanimously and dividing photosensitizing drugs into such generations may be very confusing. The nanostructures are increasingly being used as carriers for the development of $3^{\text {rd }}$ generation PS, as the most important drug delivery systems used as carriers for PS in the field of anticancer PDT.

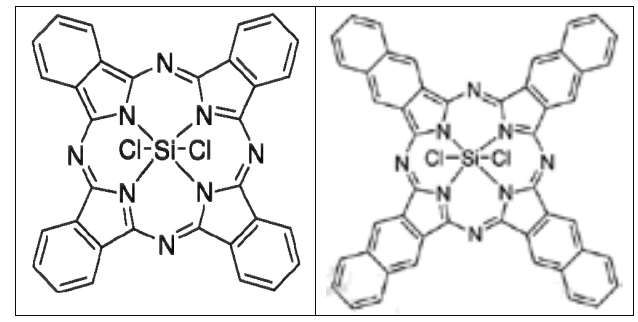

Figure 2. The chemical structures of $\mathrm{Cl}_{2} \mathrm{SiPc}$ (left) and $\mathrm{Cl}_{2} \mathrm{SiNc}$ (right)

\section{Nanoparticles in PDT}

The nanoparticles can be classified into:

- - 'Hard nanoparticles' - inorganic materials that keep unchanged their original shape and size,

- - 'Soft nanoparticles' - organic materials that could be functionalized capacity, with versatile size and shape under different conditions; $\mathrm{pH}, \mathrm{T}$, pressure. Nanoparticles have unusual properties that can improve the drug delivery.

\subsection{Hard nanoparticles}

Inorganic Nanoparticles is the generic term for several nanoparticles including for example metal oxide- and non-oxide ceramics, metals, gold and magnetic nanoparticles.

Ceramic nanoparticles: Ceramic-based nanoparticles have some advantages over organic carriers: particle size, shape, porosity, and mono-dispersibility. They are water-soluble, 
extremely stable, and known for their compatibility in biological systems without being subjected to microbial attack. For conventional drug delivery, the carrier vehicle should release the encapsulated drug at the target tissue. The works done by Prasad's group is one of the few examples for the application of ceramic nanoparticles to PDT [49]. Their silica-based nanoparticles (diameter ca. $30 \mathrm{~nm}$ ) have been entrapped with the hydrophobic photosensitizing anticancer drug 2-devinyl-2-(1-hexyl-oxyethyl) pyropheophorbide via a controlled hydrolysis of triethoxyvinylsilane in micellular media. The resulting silica- based nanoparticles were monodispersed with uniform particle size. By irradiation with suitable wavelengths: 532 or $650 \mathrm{~nm}$, silica nanoparticles with porphyrin embedded, could be efficiently taken up by tumor cells and lead to cells death.

Silica nanoparticles $\left(\mathrm{SiO}_{2}\right)$, with the following advantages:

1. chemically inert, avoiding interactions with other molecules in the body.

2. available for their synthesis, allowing precise control their particles size, shape, porosity and polydispersity during the preparation.

3. allow to incorporate small molecules encapsulated within the own particle or covalently attached to the surface.

4. These interesting properties have made silica nanoparticles the most studied nanoparticlebased PDT systems.

The delivery of photosensitisers embedded in porous silica nanoparticles has many advantages:

- almost any type of photosensitiser can be used. Second, the concentration of photosensitiser can be modulated as needed (increasing or decreasing it).

- the silica surface offers the possibility for further functionalisation.

- When the photosensitisers are incorporated on to silica nanoparticles trough covalent bonds, it is possible to avoid the eventual release of the compounds in the media, and the consequent lost of efficacy or the appearance of side effects.

Gold nanoparticles: Gold nanoparticles have been targeted to breast cancer cells by incorporating a primary antibody to the ir surface in addition to a zinc phthalocyanine photosensitiser and a bioavailability and solubility enhancer, with promising results [50,51]. Gold particles with various diameters and uniform size distribution have been demonstrated to have novel and fascinating properties. The goal of the synthesis methods is to produce size controllable gold nanoparticles. Many methods are based on the reduction of tetrachloric acid $\left(\mathrm{HAuCl}_{4}\right)$ to form gold nanoparticles. The formation and stabilization of nanosized colloidal metal particles demands careful attention to the preparation conditions and to the presence of stabilizers. Nanoparticles of silver, gold, platinum, and copper have been prepared by various methods, but most of their shapes have been limited to spheres [52,53].

Magnetic nanoparticles: The magnetic nanoparticles offer the possibility of being directed towards a specific target in the human body and remaining eventually localised, by means of 
an applied magnetic field. Iron coated nanoparticles are therefore appropriate to be used as magnetic carriers of medical drugs, magnetic resonance imaging contrasts, biological labels etc, adsorbed into the carbon surface. As one of the most important materials, magnetite $\left(\mathrm{Fe}_{3} \mathrm{O}_{4}\right)$ nanoparticles have attracted a lot of attentions for their interesting magnetic properties and potential applications in the fields of biology, pharmacy and diagnostics [54]. The magnetite $\mathrm{Fe}_{3} \mathrm{O}_{4}$ with oleic acid nanoparticles analyzed by TEM showed a spherical shape with a narrow size distribution. Figure 3 shows $\mathrm{Fe}_{3} \mathrm{O}_{4}$ nanoparticle surrounded by $\mathrm{TPPS}_{4}, \mathrm{AFM}$ for $\mathrm{TPPS}_{4}$ and $\mathrm{Fe}_{3} \mathrm{O}_{4}$.

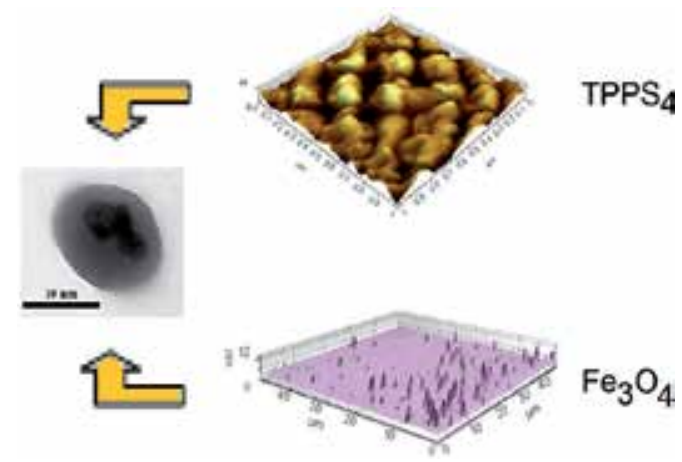

Figure 3. $\mathrm{Fe}_{3} \mathrm{O}_{4}$ nanoparticle surrounded by $\mathrm{TPPS}_{4}$ (left), AFM for TPPS 4 (right, up) and for $\mathrm{Fe}_{3} \mathrm{O}_{4}$ (right, down)

Organic Nanoparticles is the generic term for several nanoparticles including for example porphyrins, phthalocyanines and related sensitizer nanoparticles. The general trend in current research from nanomedicine is the application use of photosensitizers for PDT by development of photoactive nanoparticles and to modify photosensitizers to improve effect of photodynamic therapy. PS can be modified by encapsulated them in delivery agents such as liposomes [93], micelles [81], ceramic based nanoparticles [49], and polymer nanoparticles [57, 67]. Some examplification will be shown bellow.

\subsection{Soft nanoparticle}

\subsubsection{Polymeric carriers for drug delivery}

The polymeric carrier are divided into three groups:

1. Biodegradable polymers. These degrade under biological conditions to nontoxic products that are eliminated from the body.

2. Drug-conjugated polymers (Natural polymers). The used polymers are dextran, polyacrylamides and albumins, and offer a targeted drug controlled releasing by drug-polymer cleavage at the proper site.

3. Nondegradable polymers. These are stable in biological systems, and used as components of implantable devices for drug delivery. 
Macromolecular complexes of various polymers can be divided into the following categories according to the nature of molecular interactions: complexes formed by interaction of oppositely charged polyelectrolytes, charge transfer complexes, hydrogen-bonding complexes and stereocomplexes. Both synthetic and natural polymers could be used for the production of nanosystems. These polymers may be used alone or in combination to develop nanoparticles. Several fabrication techniques are developed and can generally be subdivided into two categories. The first category includes solvent evaporation or diffusion, ionotropic gelation, so on. The second one includes emulsion, interfacial polymerization and polycondensation [66].

\subsubsection{Biodegradable polymers}

Polymer nanoparticles involve natural or biocompatible synthetic polymers as: polysaccharides, poly lactic acid, poly lactides, poly acrylates, poly alkyl cyano acrylates, poly alkyl vinyl pyrrolidones or acryl polymers. The most important seems to be Poly(lactic-co-glycolic acid) (PLGA) which has shown several advantages over other biodegradable polymers that are routinely used for photosensitiser delivery [49] and has become the most popular polymer for PDT. The size of PLGA 50:50 nanoparticles with m-THPP as photosensitiser influences their photodynamic activity (bigger size, lower activity), but it also affects their interaction with the biological environment (protein absorption, cellular uptake or tissue distribution) [56]. Another important polymer - poly(vinyl alcohol) (PVA) - seems to have certain affinity for the p-THPP photosensitiser, inducing the adsorption of PVA on to the surface of the nanoparticle and leading to higher clearance of the complex [57, 76]. Many sensitizers from the second generation have been encapsulated into polymer nanoparticles, for example PLGA, the final size of the new system being $285 \mathrm{~nm}$, with a polidispersity index of 0.12 and a relatively reduced toxicity. A specific example is bacteriochlorophyll encapsulated into PLGA prepared by solvent evaporation method. This yielded to spherical particles of about $660 \mathrm{~nm}$ size with an encapsulation efficiency of $69 \%$ and higher singlet oxygen production $(\phi=0.26)$ [58]. Another porphyrin sensitizer, a synthetic one, 5,10,15,20-tetrakis(4-methoxyphenyl) porphyrin (TMPP) has been tested on chick embryo chloroallantoic membrane model, showing a longer retention time when is encapsulated into nanoparticles and an improvement of the vascular effects after light irradiation [59], due to the fact that the pathological tumoral vasculature is "leaky", most probably due to the pore size $100-780 \mathrm{~nm}$ and to the accumulation in the interstitial tumor tissue [60,61]. Also, pheophorbide a and chlorin e6 have been encapsulated in PLGA nanoparticles $[63,64]$. Similar results have been registered in choroidal neovascularization associated with AMD [62], where the lipophilic porphyrins show photothrombic effect and leakage from blood vessels.

\subsubsection{Natural polymers}

The naturally-occurring polymers of particular interest for delivery of some drugs could be the polysaccharides that include chitosan, hyaluronan, dextran, cellulose, pullulan, chondroitin sulphate and alginate, and polymers as casein and gelatin. They are nontoxic, biocompatible, biodegradable and hydrophilic.

Examples of the natural polymers used to prepare drugs-loaded nanoparticles are: 
Dextran sulphate is a polysaccharide that consist from linear 1,6-linked D-glucopyranose units with 2.3 sulphate groups per glucosyl residue, is non-toxic, water-soluble and biodegradable. Because it wears negatively charges, it is used for nanoparticle insulin delivery system based on complexation with oppositely charged polymers [65].

Alginate/chitosan nanoparticles may form complexes with cationic $\beta$-cyclodextrin polymers [66]. Some polyphenols have been entrapped in calcium alginate beads and to investigate their encapsulation efficiency and in vitro release [67]. Addition of $0.25-1 \%$ CS in coagulation fluid determined an improvement of encapsulation efficiency. This is probably due to increased ionic interactions between the carboxylate groups in the alginate and the protonated amine groups in the chitosan during gelation. In the presence of more chitosan, the process will go faster [68]. In vitro polyphenols released of prepared beads was carried out both in simulated gastric fluid (SGF) and simulated intestinal fluid (SIF). The total polyphenols release rate in SGF was between $40.7 \%-93.6 \%$ and in SIF was between 3.7 - 15.4\%, and the highest content of polyphenols was released in SGF. The release rate (RR) of polyphenols from microcapsules is influenced by the concentration of alginate, this phenomenon is in agreement with the previous study where it is reported that the release rate was quicker for beads prepared in low concentration of alginate but slower for beads prepared in high concentrations [69]. For microcapsules prepared by adding chitosan in coagulation fluid the best encapsulation efficiency $(85.2 \%)$ was obtained with $0.5 \%$ CS $(\mathrm{w} / \mathrm{v})$. Weak interactions between polyphenols and calcium alginate have allowed most of the polyphenols to be released in SGF. With the addition of CS in the coagulation fluid is observed a slight increase of polyphenols released in SIF.

Alginate is a linear copolymer composed of $\beta$-D-mannuronic acid and $\alpha$-L-guluronic acid joined by a 1-4 glycosidic bond. The composition is highly dependent on the used polysaccharide. The most common source of alginate is the cell wall of brown algae. Alginate is biocompatible, biodegradable and non-toxic polymer and has many biomedical applications due to the reactivity of its carboxylate side groups and its capacity to form spontaneous gelation when exposed to divalent cations such as calcium [70] and specific drug delivery [71]. Some nanoparticles were prepared by the ionotropic pre-gelation of alginate with calcium chloride followed by complexation between alginate and chitosan [69].

Chitosan (CS) is a copolymer consisting of $\beta(1 \rightarrow 4)$-linked 2-acetoamido-2-deoxy- $\beta$-Dglucopyranose (Glc-NAc; A-unit) and 2-amino-2-deoxy- $\beta$-D-glucopyranose (GlcN; D-unit) [70]. The primary amine groups make chitosan very useful in pharmaceutical applications [72]. CS nanoparticles proved cytotoxic properties on various tumor cell lines [73]. Moreover, this polysaccharide had been used as immunoadjuvant in laser immunotherapy with positive effects in the treatment of experimental tumors [74]. CS proved antioxidant properties.

\subsubsection{Nanovectors}

Polymeric micelles have many advantages such as small size (10 to $200 \mathrm{~nm}$ ) for passive accumulation in solid tumors by enhanced permeation and retention (EPR), improved stability, biodegradability and high flexibility for structural and chemical modifications $[75,76]$. Micelles polymers are usually formed into core shell structures by spontaneous 
assembly when its concentration is above critical micelle concentration (CMC). They have a number of unique features, including nano size, easy modification of the surface chemistry, core functionalities, and also, the and also, they serve as carriers and delivery systems [77]. They have been tested for solubilizing some anti-cancer drugs as: docetaxel (DOC), paclitaxel (PTX), camptothecin [78 -80]. Due to their hydrophilicity, the polymer micelles play an important part in RES recognition and in the blood circulation of drugs.

Porphyrins could be encapsulated into micelles as Triton X-100 at a critical concentration $2.7 \times 10^{-4} \mathrm{M}$. Above this concentration, porphyrins and micelles coexist in a dynamic equilibrium.

Also, from geometrical considerations, two possibilities can occur, i.e. the case of an oblate ellipsoid or that of a prolate ellipsoid. The model of an oblate ellipsoid is supported by energetically considerations, although the second model can't be neglected as beeing that of a host molecule for the porphyrin. The fluorescence lifetimes of porphyrins are in the range 13-17 ns in micelles and 9-12 ns in organic solvents, which means that the electron transfer should be available for about 10-9s before the excited molecule decays spontaneously back to its ground state. The lifetimes of all the porphyrins in Triton X-100 micelles could be attributed to the monomeric forms and are larger than the values obtained in pure solvents. The increasing of the porphyrins lifetime in micelles can be ascribed to the reduction in the diffusion-limited fluorescence quenching by oxygen in micellar samples (Figure 4).This could be an explanation for the low rate photodegradation of all these porphyrins [81].
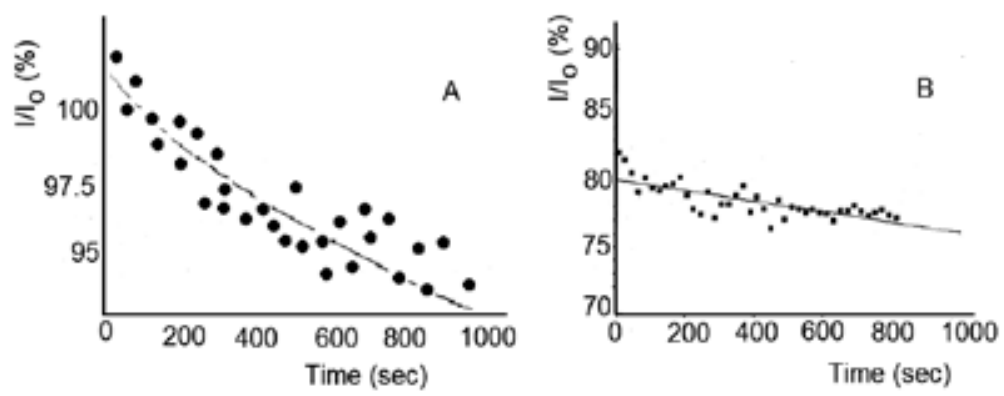

Figure 4. The fluorescence quenching of TPP-Mg in benzene (A) and Triton X-100 (B)

Liposomes are known as vesicles with clinical applications, formed by hydratation of phospholipids at higher temperature than their transition temperature. They have sizes of $100 \mathrm{~nm}$ and allow some drugs to be contained in the lipid space between bilayers. Liposomes are stable microscopic vesicles formed by phospholipids and similar amphipathic lipids. Liposome properties vary substantially with the composition, size, surface charge, and the preparation method. Liposomes are nanoconstructs (approximately $100 \mathrm{~nm}$ in diameter) with bilayered membrane structures composed of phospholipids with hydrophilic heads and hydrophobic anionic or cationic long chain tails [82]. Moreover, the hydrophobic membrane can encapsulate hydrophobic drug molecules and prevent leakage of hydrophilic agents from within the core. 
Based on their size and number of bilayers, the liposomes are divided into three classes.

1. Small unilamellar vesicles are surrounded by a single lipid layer and are $25-50 \mathrm{~nm}$ in diameter.

2. Large unilamellar vesicles as heterogeneous group of vesicles surrounded by a single lipid layer.

3. Multilamellar vesicles formed by several lipid layers separated from each other by a layer of aqueous solution.

Liposomes are used as pharmaceutical carriers due to their unique abilities to efficient encapsulate both hydrophilic and hydrophobic therapeutic agents, to offer protection to the encapsulated drugs from undesired effects of external conditions, because they can be functionalized with specific ligands that can target specific cells, tissues, and organs of interest, and because they could be coated with inert and biocompatible polymers such as polyethylene glycol (PEG), in turn prolonging the liposome circulation half-life in vivo. They can form desired formulations with needed composition, size, surface charge, and other properties [83]. Liposome vesicles are interesting and useful drug carriers because they can carry both hydrophilic molecules in their aqueous core and hydrophobic drugs among the fatty acid chains in the phospholipid bilayers [84]. Liposomes are vesicles which consist of one to several, chemically-active lipid bilayers. Some drug molecules can be encapsulated and/or solubilised within the bilayers according to their hydrophilic/lipophilic balance. Due to their hydrophobic properties, photosensitizers being poorly soluble in aqueous phases and due to their aggregation property have limited delivery in active form to the desired target $[85,86]$. Additionally, many photosensitizers have a low affinity to tumor sites inducing some damages of normal tissue following PDT in patients. Nanotechnology based formulations of photosensitizers are attractive systems for improved delivery of photosensitizers [87]. Liposomes are artificial vesicles composed of a lipid bilayer usually used for the formulation and delivery of all kind of drugs. The benzoporphyrin derivative monoacid ring A (BPD-MA) has been used for antiangiogenic PDT encapsulated in polycationic liposomes modified with cetyl-polyethyleneimine. The encapsulated photosensitiser was better internalised by human umbilical vein endothelial cells and was found inside the nucleus and associated with mitochondria [88]. The commercial liposomal preparation of the same photosensitiser (Visudyne; Novartis) is active against tumours in sarcoma -bearing mice [89]. Photofrin loaded into PEG modified liposomes presents enhanced phototoxicity compared to the free drug or when embedded in the same non-PEGylated liposomes [90]. Although the presence of the PEG inhibited the uptake of the nanoparticles by the tumour cells, it decreased the release of the photosensitiser from the liposome. Another porphyrin derivative (2,3-dihydro-5,15-di(3,5-dihydroxyphenyl)porphyrin (SIM01)) in dimyristoyl-phosphatidylcholine liposomes (DPPC) also yields better results in PDT than the photosensitizer alone, mainly due to a major accumulation in the tumour cells (human adenocarcinoma in nude mice) [91]. Liposomal TPP is effective in PDT of human a melanotic melanoma in nude mice; after being intravenously administered, authors demonstrated that their use can totally disintegrate tumours [92]. Also, TPP, TNP and ChL could be used in E.Coli destroying by PDT treatment (Figure 5), [93]. 


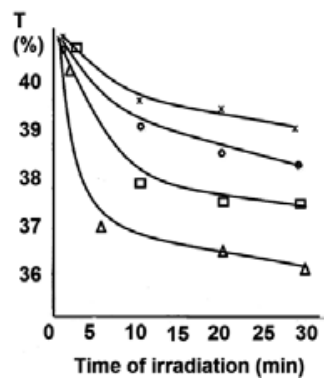

X=control; $\Delta=$ E.Coli+ TPP in DPPC; O= E.Coli + ChL in DPPC; $\square=$ E.Coli + TNP in DPPC

Figure 5. The destroying kinetics of E.Coli during PDT process with porphyrins

\subsection{Hydrogels and their applications in drug delivery}

Hydrogels are a desired material for biomedical and pharmaceutical applications due to their unique swelling properties and hydrated structure. Gels are macromolecular material with an intermediate material between a solid and liquid material. These gels are made up of a combination of local cross-linked polymer chains, noting that the junction zones are of size reduced. The structure of these gels is their property associated with swelling by incorporating a solvent. When the solvent comprises water in a proportion higher to $20 \%$, the gel will be called hydrogel. Hydrogels are elastic in nature due to the presence of the reference configurations, stored in the hydrogel, which will in turn even after the been distorted over a period of time. The natural polymers chitosan and alginate has been the most studied, for the manufacture of the hydrogel nanoparticles. Among synthetic polymers based nanoparticles, remember poly (vinyl alcohol) PVA, poly (ethylene oxide) PEO, poly (ethylene imide) (PEI), polyvinylpyrrolidone (PVP), poly (N-isopropyl acrylamide), which were used in order to release molecules incorporated.

Hydrogels are crosslinked polymeric networks that are insoluble in water but swell to an equilibrium size in the presence of excess water or biological fluids [94]. Research on hydrogels started in the 1960s with a landmark paper on poly(hydroxyethyl methacrylate) (PMMA) [95]. Due to the unique swelling properties and biocompatible structure, these materials have been extensively studied for biomedical and pharmaceutical applications, such as contact lenses, biosensors, artificial hearts, artificial skin and drug delivery devices [96]. Among them, hydrogels from poly (vinyl alcohol) are especially important due to their advantages of being water soluble, non-toxic, non-carcinogenic and biodegredable. Hydrogels based on poly (vinyl alcohol) (PVA) is characterized by transparency, in a three-dimensional polymeric structure and a water absorption capacity greater volume, without dissolving therein. Poly (vinyl alcohol) has a semi-crystalline nature with applications for encapsulation and controlled release of the porphyrins, especially in cancer therapy. Hydrogel loading procedure with 5,10,15,20-tetra-sulfonato-phenyl porphyrin $\left(\mathrm{TPPS}_{4}\right)$, sorption experiments, the retention efficiency of porphyrins on the PVA hydrogel, and controlled release of $\mathrm{TPPS}_{4}$ from the PVA hydrogel have been achieved and altready reported [97].

PVA becomes more porous and its pores become more larger after porphyrin retention. This observation is more stronger for $\mathrm{TPPS}_{4}$ than for the other porphyrins with smaller sizes [98, 
$99,100]$. SEM analysis showed a porous structure of the hydrogel, evidencing interconnected pores with a size distribution in the range of $80-950 \mathrm{~nm}$, Figure 6 . The retention efficiency (normalized to the swollen hydrogel mass) has been calculated according to the formula:

$$
R E(\%)+\frac{m_{\text {porphyrin_retained }}}{m_{\text {porphyrin_initial }} \cdot m_{\text {hydrogel }}} \cdot 100
$$

where: $m_{\text {Porphyrin_ initial }}$ is the initial amount of porphyrin to be found in the solution, and $\mathrm{m}_{\text {Porphyrin_retained }}$ is determined from the difference between the initial and the remaining amounts of porphyrin after retention. Release experiments were carried out by using a $\mathrm{TPPS}_{4}$-loaded PVA hydrogel, rinsed thoroughly after loading with distilled water, and then placed in the appropriate quantity of medium (distilled water). The sorption mechanism of the porphyrins onto the PVA hydrogel can be interpreted as having two components: physisorption and chemisorption. In physisorption, the porphyrin is encapsulated in the pores of the nanostructured hydrogels. This mechanism is mainly controlled by diffusion. The diffusion mechanism is not ideal (Fickian), but rather Stephan diffusion, because of the anisotropic porous structure of the gel [101]. The chemisorption mechanism consists of the hydrogen bonding between the $-\mathrm{OH}$ groups of the poly(vinyl alcohol) and the pyrrolic nitrogen of the porphyrin molecule. The nature and the intensity of the chemisorption depends largely on the conformation of the porphyrin molecule and the solvent used.
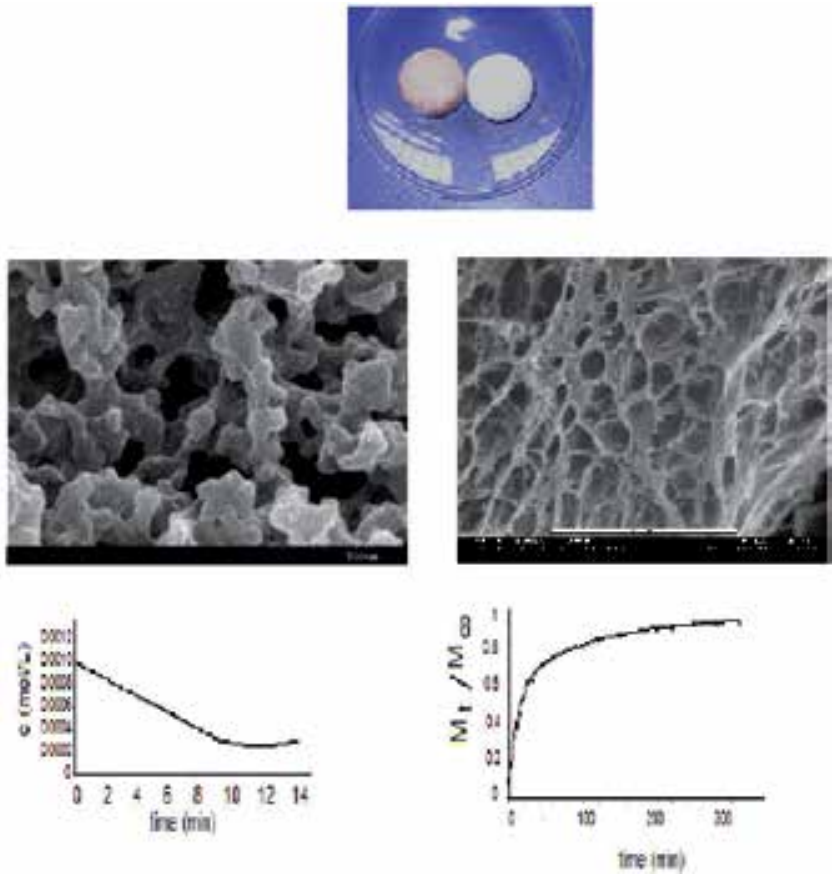

Figure 6. Aspect of PVA hydrogel with (left) and without TPPS 4 hydrogel (right)(up), SEM images of PVA 90 hydrogel $\left(\mathrm{TPPS}_{4}\right.$ ) before (left) and with $\mathrm{TPPS}_{4}$ (right) (middle) and kinetics of TPPS 4 loading in PVA hydrogel (left) and kinetic of $\mathrm{TPPS}_{4}$ release from the PVA hydrogel (normalized to encapsulated $\mathrm{TPPS}_{4}$ amount)(right) (down) 
Endothelial cell line, HUVEC, as adherent cells line and photodynamic therapy model, were grown on the surface of hydrogels mentioned, and monitorized by microscopic techniques, following the cellular membrane integrity. Also, the influence of $\mathrm{TPPS}_{4}$ forms on hydrogel properties are analyzed. For this purpose HUVEC cells pre-incubated with $\mathrm{TPPS}_{4}$ were illuminated with red light. PDT led to a dramatic change in the morphology of these endothelial cells. The photosensitizer accumulated in mitochondria and its fluorescence emission is detected in red region $(\sim 590 \mathrm{~nm})$, before (left) and after PDT protocol. A deformation of the cells, as a sign of the cellular death, is observed after PDT (right) (Figure 7).

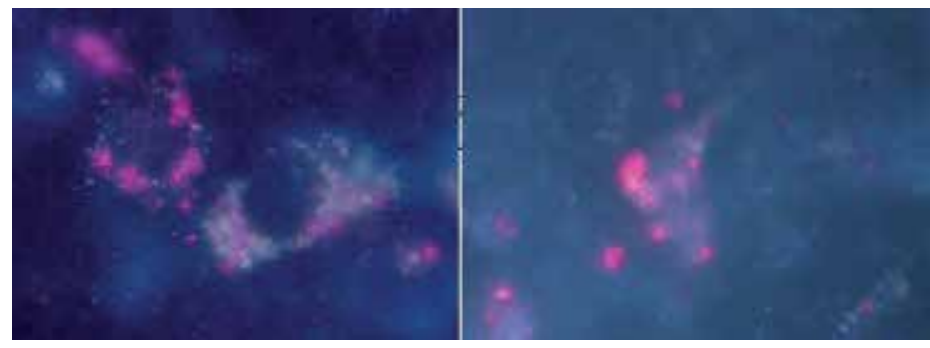

Figure 7. Laser scanning confocal microscopy of HUVEC before (left) and after (right) the PDT protocol with TPPS $_{4}$ hydrogels

\subsection{Non-biodegradable nanoparticles for photodynamic therapy}

Compared to biodegradable polymeric carrier systems, non-biodegradable nanoparticles have several advantages: they are very stable to fluctuations in temperature and $\mathrm{pH}$ [102], taking into account that the particle size, shape, porosity and mono-dispersibility can be controlled during their preparation [103]; they are not subject to microbial attack [104]; and the tiny pores in the ceramic particle, which are $0.50-1.00 \mathrm{~nm}$ in diameter, are too small to allow the drug to escape the matrix but are large enough to enable efficient oxygen diffusion to and from the particle [105]. Biodegradable polymer nanoparticles degrade readily to release the photosensitizers, whereas the shells in non-biodegradable particles are difficult to collapse. However, the efficiency of PDT is only attributable to the production of ${ }^{1} \mathrm{O}_{2}$; it is unnecessary to release the loaded photosensitizers, but it is essential that the oxygen can diffuse in and out of the nanoparticles. The lifetime of singlet oxygen is able to induce PDT-induced oxidative damage around few miliseconds in aqueous media [106]. The nanoparticles size is under $100 \mathrm{~nm}$ and have a negligible effect on the delivery of ${ }^{1} \mathrm{O}_{2}$. The use of non-biodegradable nanoparticles has some advantages with respect to their degradable counterparts. As the nanoparticle keeps its integrity, the photosensitizer has a permanent protection from the environment; besides, it is possible to use the nanoparticles as platforms to incorporate additional functionalities and they can be of smaller size. These nanoparticles kept their integrity over several months and were effective with just 5 minutes of irradiation. 5,10,15,20-tetrakis(1-methyl-4-pyridino)porphyrin tetra(p-toluenesulfonate) (TMPyP), has also been encapsulated in polyacrylamide -based nanoparticles. Its phototoxicity with two photon IR radiation was demonstrated in vitro by modulating the time of exposure to light [106]. The main difference between classic PS and nano-PS is their relative size (Figure 8). 


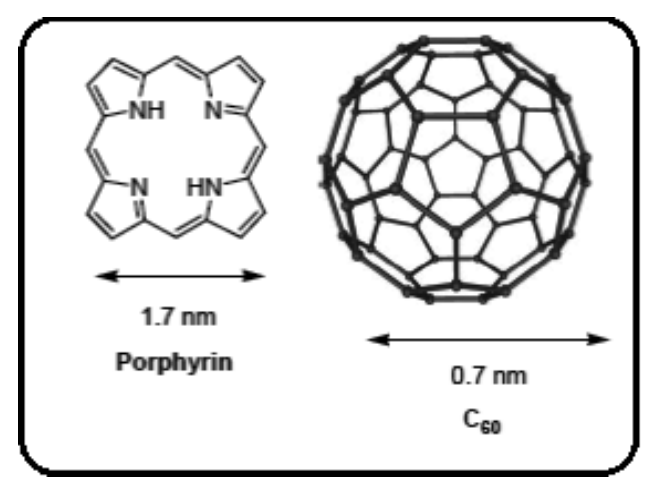

Figure 8. Comparison of the approximate sizes of a porphyrin and C60 nanoparticles

However, several strategies have now been developed to encapsulate photosensitizer into nanoparticles and also improve delivery to the required area and many formulations have been described whereby the nanomaterials have an additional active intermediary role in the photodynamic process [107]. Recent trends in the use of fullerene derivatives in medicine are related to development of nanoplatforms that contain drugs with different composition able to carry out selective delivery of them to some human organs. The main medicinal targets are cancer cells of different types. It is believed that in this aspect the fullerenes are of great interest because of their opportunity to participate in the composition of such nanoplatforms in several roles: cytotoxic agent as well as, conversely, an antioxidant (these roles may change depending on accumulation in different organs and tissues) ones; as transporter of drugs; as photo- or radiosensitizer (or protector). Recently, the conjugates of C60 with meso-aryl-porphyrins with long chain substituents were obtained for using in PDT, Figure 9, [108]. Apoptosis without participation of caspase-3 was observed when the human lymphoblast cell line (K562) was treated by TPP/PVP/C60 [109]. TPP generates singlet oxygen with high quantum yield (0.63) [110]. Three types of interactions were registered in this dyad: electrostatic, hydrogen bonds and the donor acceptor bonds between fullerene and other components [111]. Here the high ability of these compounds to the formation of photo-induced state with divided (isolated) charges was first noted. Cell survival was dependent on illumination rate and the phototoxic effect persisted even in an atmosphere of argon. Depending on the microenvironment of the sensitizer site localization, the tissue is damaged either through the mechanism of ${ }^{1} \mathrm{O}_{2}$ mediated photoreaction process or through ROS attack at a low concentration of oxygen. Apoptosis by caspase-3-dependent pathway (58\% of apoptotic cells) has been replaced by predominant necrotic phenomena in anaerobic conditions. Starting from the characteristics of fullerene compounds, we tried to study in vitro C60 fullerene and some functionalized derivatives (C60 complexes with PVP (poly-vinyl-pyrrolidone) and with the oxo-dimer (TPP$\mathrm{Fe})_{2} \mathrm{O}$ experimental models in vitro with normal and tumor cells and investigation of their toxicological profile, in order to identify novel anti-neoplasic therapeutic devices.

However, the complexity of the problem is that until now there is no predictive model of action of fullerene derivatives under concrete conditions for a specific cell type. Moreover, the set of 


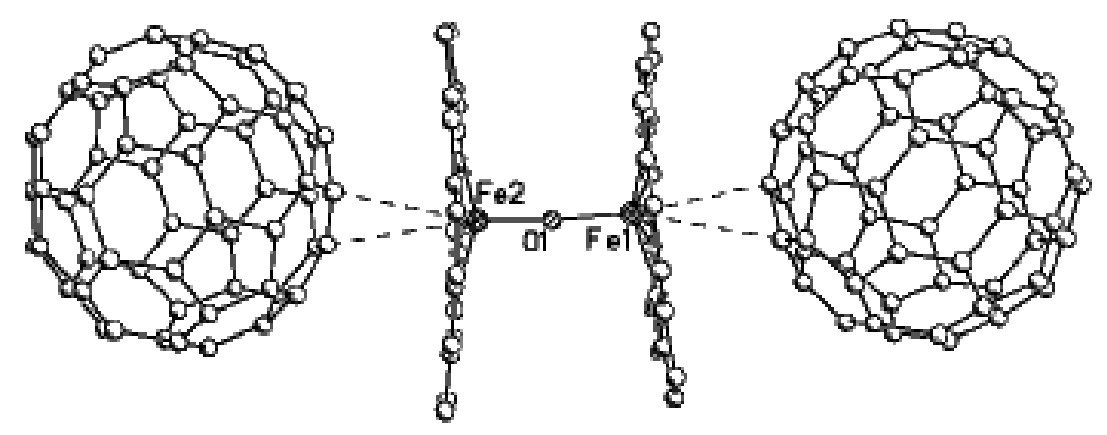

Figure 9. The proposed structure of $\mathrm{C} 60-(\mathrm{TPP}-\mathrm{Fe})_{2} \mathrm{O}$

possible mechanisms of the effect of fullerenes on the signaling pathways of apoptosis varied and depends on many factors that are difficult to administrate. However, the success of some fullerene derivatives against HIV, the selectivity to certain lines of cancer cells without damaging normal tissue, the possibility of using in theranostics suggest promising perspectives of fullerenes in the field of nanomedicine.

\section{Study of the photodynamic effects of selected photosensitizers on human biological samples}

\subsection{Incorporation of nanoparticles in human blood}

Significant nowadays research efforts are focused on finding new photosensitizers with antineoplastic activity and an acceptable toxicological profile. Although consistent information exists regarding PDT in solid tumors, relatively few data are available for PDT of blood cancers. Therefore, we carried out a comparative study on lymphoblastic K562 cells and human normal peripheral blood mononuclear cells (PBMC) treated at a density of $2 \times 10^{5}$ cells $/ \mathrm{mL}$ with 5,10,15,20-tetra-sulphophenyl-porphyrin $\left(\mathrm{TPPS}_{4}\right)$ and then irradiated with He-Ne laser light $(\lambda=632.8 \mathrm{~nm})$. The following cell functions were investigated: viability, multiplication, RNA synthesis, total RNA levels and apoptosis. Human normal PBMC subjected to $\mathrm{TPPS}_{4}$ loading and laser-irradiation develop a different cellular response, their viability and proliferative capacity not being altered by experimental PDT. Accordingly, it appears that $\mathrm{TPPS}_{4}$ is a non-aggressive compound for cellular physiology and becomes cytotoxic only by irradiation; moreover laser-activated $\mathrm{TPPS}_{4}$ affects only cells that have a tumoral pattern [112].

There are several differences between lymphocytes obtained from healthy donors undergoing artificial activation in vitro and genuinely leukemic cells. The cells membrane structure is different in healthy and malignant cells, resting and stimulated cells can be compared using fluorescence spectroscopy [27]. In the concentration range 10-100 $\mu \mathrm{g} / \mathrm{mL}, \mathrm{TPPS}_{4}$ loaded for $24 \mathrm{~h}$ in normal PBMC does not significantly alter cell viability (Figure 10). 


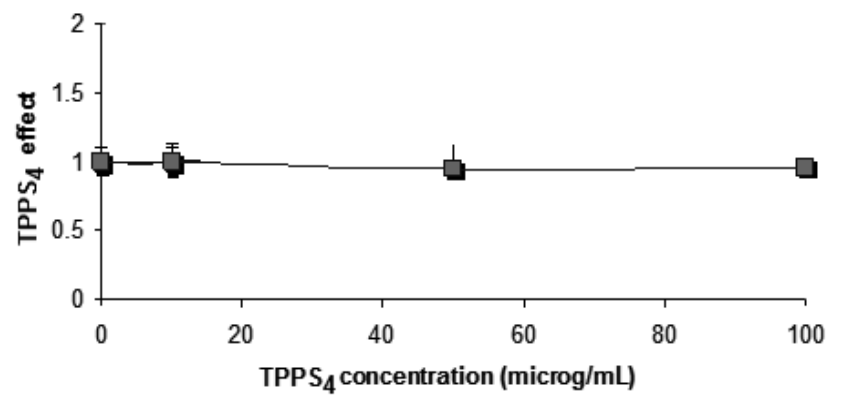

Figure 10. The viability of human normal PBMC loaded with various concentrations of $\mathrm{TPPS}_{4}$ for $24 \mathrm{~h}$

Irradiation of K562 cells, either loaded or not loaded with $\mathrm{TPPS}_{4}$, drops off cellular viability (assessed by the Trypan Blue exclusion test $4 \mathrm{hrs}$ after irradiation) for both tumor cells and normal PBMC (Figure 11). TPPS 4 -loaded K562 cells are almost similarly affected by irradiation as the corresponding control. $\mathrm{TPPS}_{4}$ alone has no significant effect on cell viability.

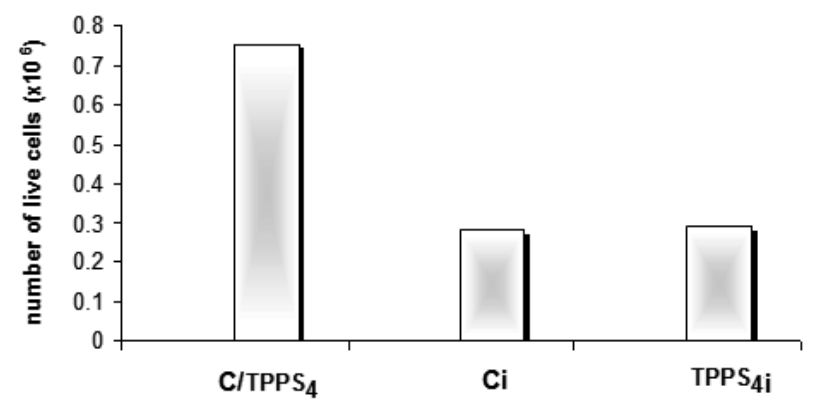

Figure 11. The viability of K562 cells and human normal PBMC at $4 \mathrm{~h}$ post-irradiation (assessed by the Trypan Blue exclusion test). $\mathrm{C} / \mathrm{TPPS}_{4}=$ non-irradiated cells; $\mathrm{Ci}=$ irradiated unloaded cells; $\mathrm{TPPS}_{4 \mathrm{i}}=$ irradiated loaded cells.

Human normal PBMC react differently at the PDT procedure than tumor cells. Their viability and capacity to incorporate uridine are not altered by laser-activating of $\mathrm{TPPS}_{4}$ loaded into cells (Figure 12).

4-5 $\mathrm{ml}$ of human peripheral blood were fast collected. Blood mononuclear cells (PBMC) were separated by gradient dental equipment screened using Histopaque 1077 (Sigma), washed twice with RPMI 1640 culture medium (Sigma) and then normalized to $10^{5}$ cells / $\mathrm{ml}$ RPMI 1640 culture medium. Samples containing cell lines loaded with various concentrations of $\mathrm{TPPS}_{4} 24 \mathrm{~h}$ were investigated in flow cytometry fluorescence recorded at wavelengths above $670 \mathrm{~nm}$. Increasing the concentration of $\mathrm{TPPS}_{4}$ fluorescence intensity increases cell suspension directly proportional both total fluorescence and fluorescence maximum. In flow cytometry, the regions of lymphocytes, granulocytes and monocytes are clearly distinguished. The fluorescence of sample can be measured by using properly chosen filter. Fluorescence is 


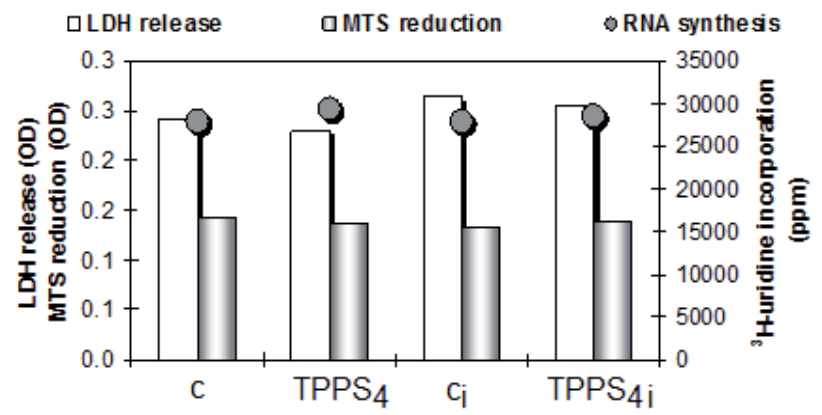

Figure 12. The viability and multiplication rate of normal PBMC at $24 \mathrm{~h}$ post-irradiation. $\mathrm{C}=$ non-irradiated unloaded cells; $\mathrm{TPPS}_{4}=$ non-irradiated loaded cells; $\mathrm{C}_{\mathrm{i}}=$ irradiated unloaded cells; $\mathrm{TPPS}_{4 \mathrm{i}}=$ irradiated loaded cells.

analyzed separately for each type of cells. A flow cytogram represents the graphical reprezentation of light scattering vs. right angle scattering and help to determine lymphocytes and granulocytes. The aspect of flow cytometry (mean fluorescence intensities) could offer data about dye aggregation or dye interaction with cellular membrane [41,113, 114]. The number of stained cells decrease from $91.02 \%$ (for control cells), to $89,27 \%$ for $10 \mu \mathrm{g} / \mathrm{ml} \mathrm{TPPS}_{4}, 87,80 \%$

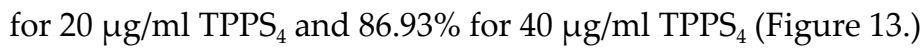
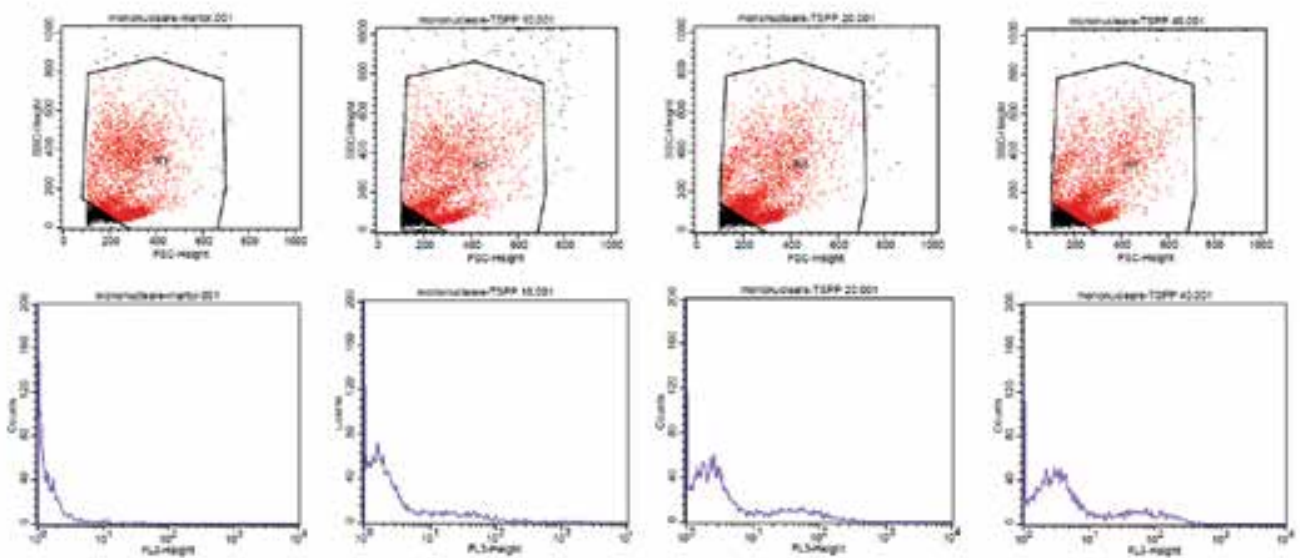

Figure 13. The flow cytometry results for the PMBC cells incubated with $\mathrm{TPPS}_{4}$ at different concentrations

Ion [115] and Frackowiak [27] evaluated the incorporation of sulphonated porphyrin TPPS $_{4}$ which is better incorporated in cells by comparison with the non-sulphonated ones, most probably due to the spatial forms of J-aggregated forms (helical forms) able to penetrate the membrane and recovering to the monomeric forms after penetration. It is shown that during irradiation cells are actively destroyed (Figures 14-17), [116]. 

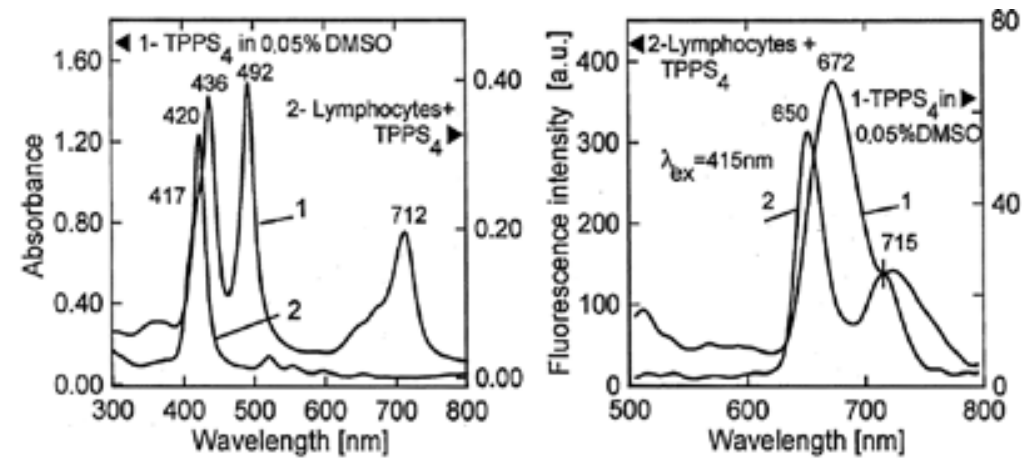

Figure 14. The absorption and emission properties of $\mathrm{TPPS}_{4}$

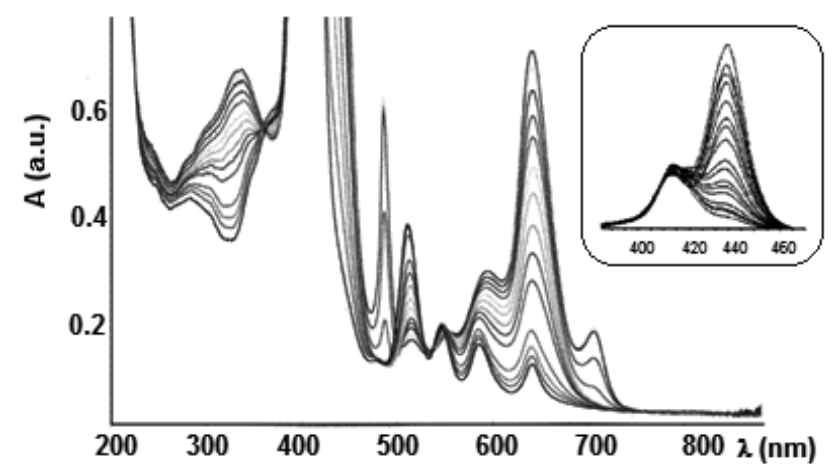

Figure 15. The aggregation equilibrium of $\mathrm{TPPS}_{4}$

For a longer photodynamic activity it is important to correlate the photophysical activity (the lifetimes of the excited states) with photochemical activity (singlet oxygen efficiency and the photodegradation rate. The aggregated forms (J-aggregates) of porphyrins favor the penetration of the membranes. The porphyrins incorporation in cells is well correlated with singlet oxygen generation capacity. In monomeric forms the non-sulfonated porphyrins are better incorporated than the sulfonated ones which are better incorporated in leukocytes than in granulocytes (Figures 18,19), [113].

The non-sulphonated porphyrins (TNP and TPP) in DMSO-water mixture (0.05\% DMSO) exist as monomeric and J-aggregated (dicationic - aggregated forms), the last ones with comparable fluorescence properties with the monomers [117]. 

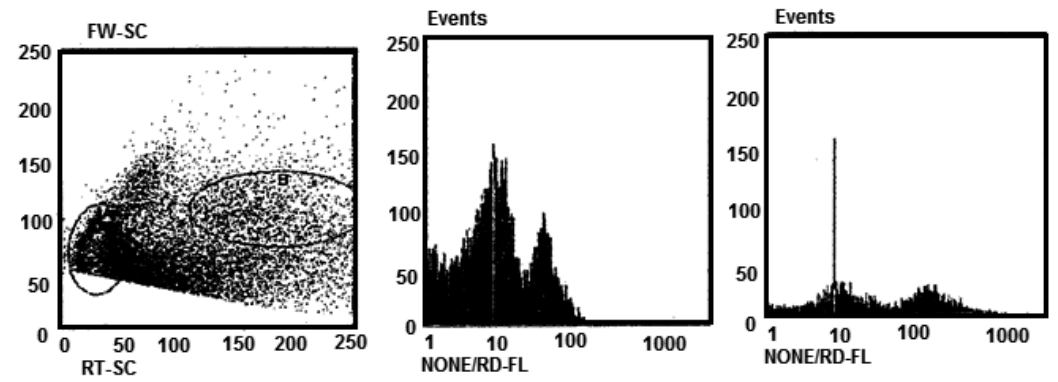

TPPS $_{4}$ monomer
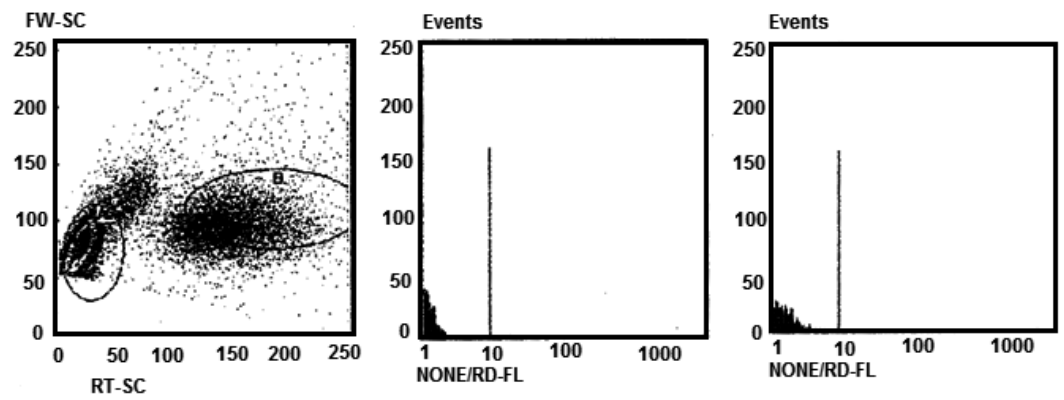

TPPS $_{4}$ aggregate

Figure 16. The flow cytometry of different forms of $\mathrm{TPPS}_{4}$
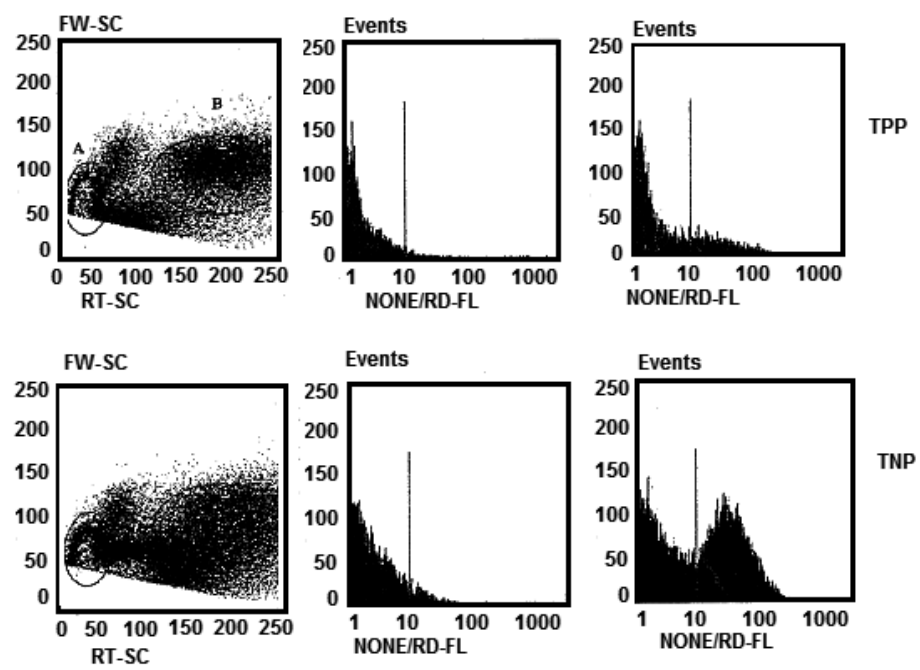

Figure 17. The flow cytometry of leukocytes and granulocytes incorporated with TPP and TNP 

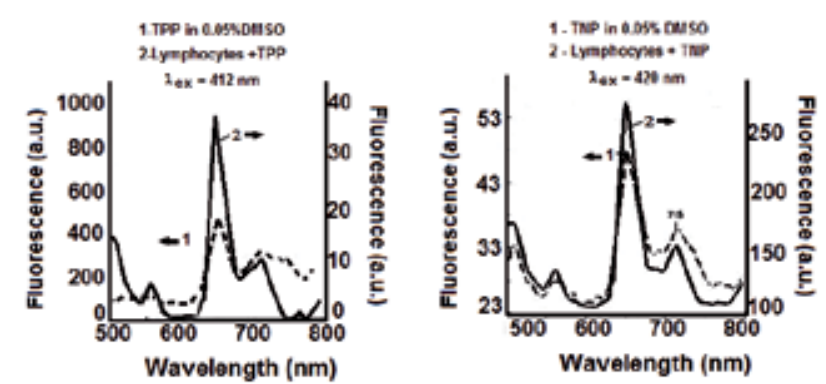

Figure 18. Emission properties of TPP and TNP in DMSO:water and in lymphocytes
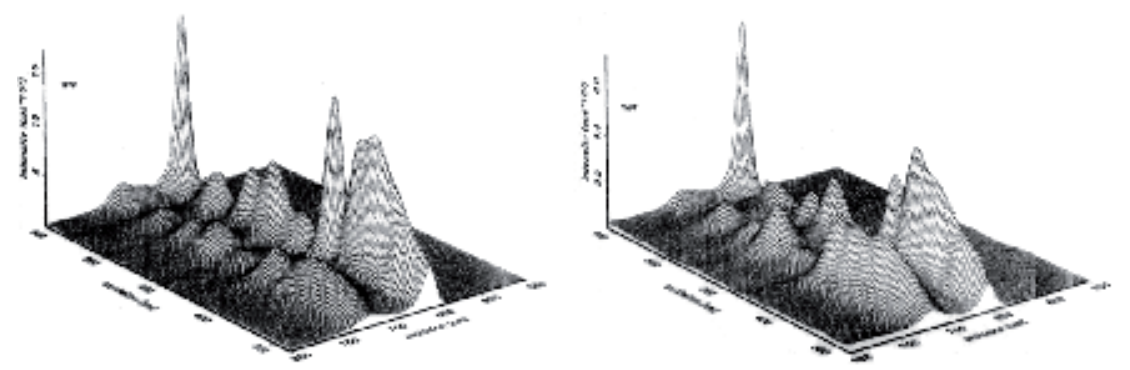

Figure 19. The correlation graphic of absorption and emission for TPP and TNP

\subsection{Application of B2 Vitamin in liposomes for ophthalmologic diseases}

After PDT treatment was possible to see by angrography a rapid and complete vasooclusion (Figure 20), because the vessels were filled with erythrocytes and due to platelet aggregates. Another observed effects are: vacuolization of mitochondria and endoplasmatic reticulum (ESR), clumping of nuclear chromatin and finally, a subconjunctival hemrrhage, chemosis and cyanotic color of the neovascular areas (Figures 21 and 22).

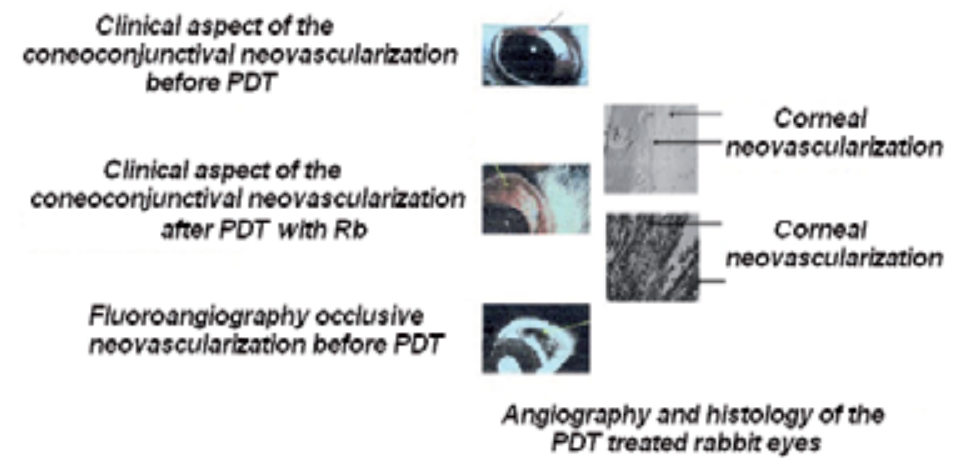

Figure 20. Fluorescein angiography after PDT treatment with Riboflavin on rabbit. Dark area delimitates the irradiated sites where hypofluorescence indicates vascular occlusion 

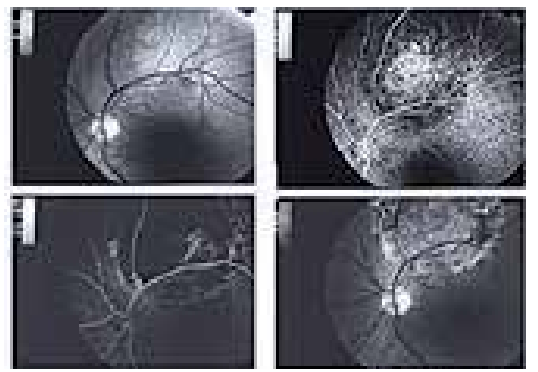

Figure 21. Angiofluorography for a patient with malignant melanoma before PDT treatment with Rb (left), and 6 months (right) after PDT treatment with Riboflavin.

From Figure 21 could be observed the dissapearance of peritumoral neovascularization and complete dissapearance of tumoral neovascularization and tumoral atrofia (6 luni). Angiography showed an immediate and complete vasooclusion (Figure 22).
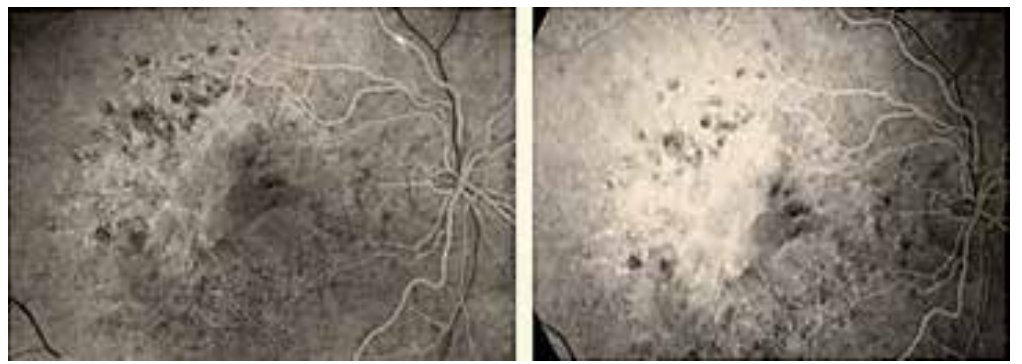

$\overline{\text { Before PDT: VPD }}=1 / 8$ fc, VOS =1/6 fc; 2 months after PDT: VOD=1/6 fc; VOS=1/12 fc; 4 months after PDT: VOD=1/10 fc; $\operatorname{VOS}=1 / 8 \mathrm{fc} ; 10$ months after PD: VOD=1/15 fc; VOS=1/20 fc

Figure 22. Macular degenerescence for a patient with neovascular membrane for right eye and atrofic pseudotumoral form at left eye

\subsection{Dermatological applications}

PDT produces cytotoxic effects through photodamage of cellular organelles and biomolecules. It is known that PDT mediates tumor destruction by three mechanisms: direct cell killing, tumor vasculature damage and immune response activation. The combination of the three mechanisms is required to obtain long-term tumor control [122].

Actinic keratosis (AK) is the most common skin lesion with malignant potential, with a prevalence ranging from $11 \%$ to $25 \%$ in the Northern Hemisphere and from $40 \%$ to $50 \%$ in Australia [123]. There are some factors responsible for skin lessions (squamous cell carconoma (SCC)) as follows: UV light, heat and pollutants resulted from carbon processing. The most sensitive persons are Fitzpatrick I and II phototype and men by comparison with women [124]. In time, these lesions could remain unchanged, could spontaneously regress or could progress to SCC and further developing on the support of pre-existing actinic keratosis. 
In our experimental approach, we have obtained from untreated skin biopsies a mean of $2.8 \times 10^{6}$ keratinocytes $/ \mathrm{cm}^{2}$ skin with a mean of $65 \%$ viability. After therapy from the same skin region and from the same surface isolated keratinocytes were less than half compared to the control skin, displaying as well a lower viability (Figure 23).

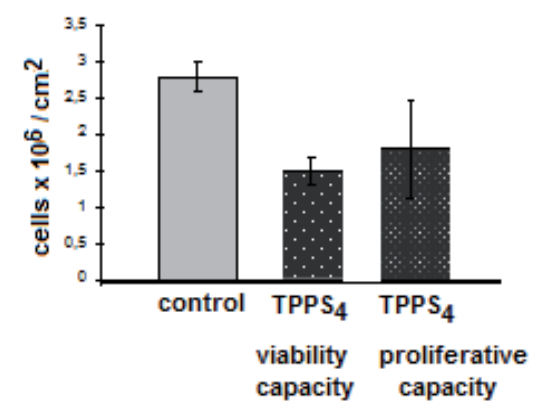

Figure 23. Primary keratinocytes isolated from normal human skin before (control) and after PDT with TPPS $_{4}$ (viability and proliferative capacity)

Primary keratinocytes were further cultivated until the culture could not be maintained. The proliferation capacity of primary keratinocytes extracted from PDT skin biopsies was significantly lower compared to control skin (Figure 23). Annexin- $V$ and propidium iodide labelling of isolated keratinocytes after in vivo PDT compared to control keratinocytes yielded to the following values: control = $100 \%$; An-PI- = $10 \%$; An+PI- = $18 \%$; An+PI+ = $65 \%$.

The tested $\mathrm{TPPS}_{4}$ showed an effective in vivo destructive effect on keratinocytes in the patient with actinic keratosis doubled by a good clinical response (Figure 24).
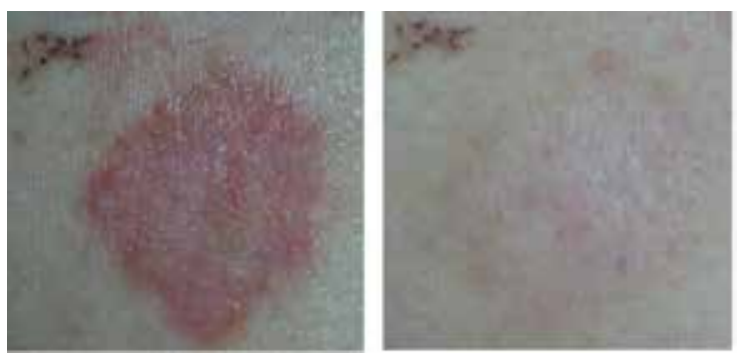

Figure 24. The aspect of the skin with AK before PDT (left) and after PDT (right) with TPPS $_{4}$

\section{Conclusions}

Photodynamic therapy is a recognized alternative used in successful cancer therapy, in a society when the clinicians are seeking new and efficient methods for saving people. There is 
no doubt that the long scientific efforts of photodynamic therapy will allow useful patient treatments in the future.

The main benefits of PDT are the following:

a. The patients can avoid surgery.

b. PDT patients usually don't even need to check into the hospital.

c. PDT can be repeated, unlike radiation and chemotherapy.

d. PDT can work in places where surgery would not be feasible, such as the trachea, the major airway leading from the voice box to the lungs.

e. The photosensitizing agent will selectively accumulate in cancer cells and not in surrounding normal tissues. Hence, cancer cells can be selectively destroyed while most normal cells are spared.

f. The treatment occurs only in the presence of light.

g. PDT might be applied in many cases where surgery, chemotherapy and $\mathrm{X}$ ray radiation are contra-indicated.

Side effects, limitations could be registered, especially for photosensitivity or sensitivity to light, the pain of PDT, which is usually mild to moderate, easily controlled with some drugs.

However, there are many problems for the clinical application of existing photosensitzers.

a. Most PS molecules are hydrophobic and can aggregate easily in aqueous media, decreasing its quantum yield. Moreover, the aggregated PS cannot be simply injected intravenously.

b. Selective accumulation of the PS molecules in deceased tissues is required to avoid collateral damage to healthy cells. Although third generation PS based on nanomaterials have been prepared for selective targeting, their selectivity is not high enough for clinical application.

c. Nanomaterials (organic or inorganic) are more promising because they are hydrophilic, possess enormous surface areas, and their surface can be modified with functional groups possessing a diverse chemical or biochemical properties;

d. Owing to their sub-cellular and sub-micron size, nanoparticles can penetrate deep into tissues through fine capillaries, and are generally taken up efficiently by cells.

\section{Acknowledgements}

This paper received the financial support of the projects: PN 09.09.01.13, PN 04.04.12.03, and PNII project 185/2014. 


\section{Author details}

Rodica-Mariana Ion ${ }^{1,2}$

Address all correspondence to: rodica-ion@icechim.ro; rodica_ion2000@yahoo.co.uk

1 ICECHIM, Nanomedicine Research Group, Bucharest, Romania

2 Valahia University, Materials Engineering Department, Targoviste, Romania

\section{References}

[1] M.W. Djojosubroto, Y.S. Choi, H.W. Lee, K.L. Rudolph, Telomeres and telomerase in aging, regeneration and cancer, Mol. Cell. 15 (2003), 164-175.

[2] M. Malvezzi, P. Bertuccio, F. Levi, C. La Vecchia, E. Negri, European cancer mortality predictions for the year 2012, Ann. Oncol. 23(4) (2012), 1044-1052.

[3] E. Espinosa, P. Zamora, J. Feliu, M. González Barón, Classification of anticancer drugs-a new system based on therapeutic targets, Cancer Treat. Rev. 29(6) (2003), 515-523.

[4] R.K. Jain, Transport of Molecules in the Tumor Interstitium: A Review, Cancer Res. 47(12) (1987), 3039-3051.

[5] I. Brigger, C. Dubernet, P. Couvreur, Nanoparticles in cancer therapy and diagnosis, Adv.Drug Deliv. Rev. 54(5) (2002), 631-651.

[6] R.M. Ion, Porphyrins for tumor destruction in photodynamic therapy, Current Topics on Biophysics, 24(1) (2000) 30-42.

[7] S. Svenson, Theranostics: Are we there yet?, Mol. Pharm. 10 (2013) 848-856.

[8] S.S. Kelkar, T.M. Reineke, Theranostics: Combining imaging and therapy, Bioconjug. Chem. 22 (2011) 1879-1903.

[9] R. Weissleder, V. Ntziachristos, Shedding light onto live molecular targets, Nat. Med. 9 (2003) 123-128.

[10] J. Klohs, A. Wunder, K. Licha, Near-infrared fluorescent probes for imaging vascular pathophysiology, Basic Res. Cardiol. 103 (2008) 144-151.

[11] R.F. Donnelly, P.A. McCarron, D.I. Morrow, S.A. Sibani, A.D. Woolfson, Photosensitiser delivery for photodynamic therapy. Part 1: Topical carrier platforms, Expert Opin. Drug Deliv. 5 (2008) 757-766. 
[12] C.P. Mccoy, C. Rooney, C.R. Edwards, D.S. Jones, S.P. Gorman, Light-triggered molecule-scale drug dosing devices, J. Am. Chem. Soc. 129 (2007) 9572-9573.

[13] R.M. Ion, M. Grigorescu, F. Scarlat, V.I.R. Niculescu, K. Gunaydin, Light, electron and photons beam effects on $\mathrm{TPPS}_{4}$ used in PDT, J.Balkan Union Oncology. 3(2) (2000) 129-135.

[14] M.L. Pascu, A. Popescu, L. Danaila, N. Carp, R.M. Ion, M.O. Pascu, A. Staicu, Photodynamic therapy studies on brain tumors using nitrogen pulsed lasers, SPIE Proceedings - the International Society for Optical Engineering. Florence 4166 (2000) 712-720.

[15] R.M. Ion, The photodynamic therapy of cancer -a photosensitization or a photocatalytic process?, Progr.Catal. 1 (1997) 55-68.

[16] O. Raab, Uber die Wirkung, fluorescirender Stoffe auf infusorien, Z. Biol. 39 (1900) 524-546.

[17] D. Dolmans, D. Fukumura, R.K. Jain, Photodynamic therapy for cancer, Nat. Rev. Cancer 3 (2003) 380-387.

[18] T.J. Dougherty, An update on photodynamic therapy applications, J. Clin. Laser Med. Surg. 20 (2002) 3-7.

[19] C. Hopper, Photodynamic therapy: A clinical reality in the treatment of cancer, Lancet Oncol. 1 (2000) 212-219.

[20] S.B. Brown, E.A. Brown, I. Walker, The present and future role of photodynamic therapy in cancer treatment, Lancet Oncol. 5 (2004) 497-508.

[21] R.M. Ion, Photodynamic therapy (PDT): A photochemical concept with medical applications, Revue Roumaine De Chimie 52(12) (2007) 1093-1102.

[22] M. Neagu, G. Manda, C. Constantin, E. Radu, R.M. Ion, Synthetic porphyrins in experimental photodynamic therapy induce different antitumoral effect, J. Porph. Phthal. 11(1) (2007) 58-65.

[23] S. Clichici, A. Filip, D. Daicoviciu, R.M. Ion, T. Mocan, C. Tatomir, The dynamics of reactive oxygen species in photodynamic therapy with tetra sulfophenyl-porphyrin, Acta Physiologica Hungarica 97(1) (2010) 41-51.

[24] S.K. Pushpan, S. Venkatraman, V.G. Anand, J. Sankar, D. Parmeswaran, S. Ganesan, T.K. Chandrashekar, Porphyrins in photodynamic therapy - A search for ideal photosensitizers, Curr. Med. Chem. Anti-Cancer Agents 2 (2002) 187-207.

[25] C. Matei, M. Tampa, C. Caruntu, R.M. Ion, S.R. Georgescu, G. Dumitrascu, C. Constantin, M. Neagu, Protein microarray for complex apoptosis monitoring of dysplastic oral keratinocytes in experimental photodynamic therapy, Biological Research 47 (2014) 1-9. 
[26] D. Frackowiak, R.M. Ion, A. Waszkowiak, Spectral properties of phthalocyanines oriented in stretched polymer films. Journal of Physical Chemistry B 106(51), (2002) 13154-13160.

[27] D. Frąckowiak, A. Planner, R.M. Ion, K. Wiktorowicz, Incorporation of dye in resting and stimulated leukocytes: in: Near-Infrared Dyes for High Technology Applications. NATO ASI SERIES, Ed. by S. Daehne, U. Resch-Genger, O. S. Wolfbeis., Kluwer Academic Publishers, Dordrecht/Boston/London 3/52 (1998) 87-114.

[28] S. Santoro, O. Bandieramonte, G. Melloni, E. Marchesini, R. Zunino, F. Lepera, P. de Palo, Photodynamic therapy by topical meso-tetraphenylporphinesulfonate tetrasodium salt administration in superficial basal cell carcinoma, Cancer Res. 50 (1990) 4501-4503.

[29] R.M. Ion, The Use of Phthalocyanines and Related Complexes in Photodynamic Therapy, in Photosensitizers in Medicine, Environment, and Security, T. Nyokong, V. Ahsen (Eds.), Springer (2012) 315-349.

[30] R.M. Ion, Synthesis, photophysical properties and photocatalytic activity of tungsten porphyrin (TPPWCl ${ }_{4}$ ), J. Porphyrins Phthalocyanines 17 (2013) 460-472.

[31] S. Agirtas, R.M. Ion, O. Bekaroglu, Spectral study of the supramolecular assemblies porphyrins-phthalocyanines for PDT, Mat.Sci. Eng.C:Biomimetic Materials Sensors Systems 7 (2000) 105-110.

[32] [32] R.M. Ion, M. Kocak, O. Bekaroglu, Catalytic decomposition of hydrogen peroxide with crowned ether phthalocyanines, Progr.Catal. 2 (1998) 34-45.

[33] M. Triesscheijn, P. Baas, J.H.M. Schellens, F.A. Stewart, Photodynamic therapy in oncology, The Oncologist 11 (2006) 1034-1044.

[34] T.J. Kinsella, E.D. Baron, V.C. Colussi, K.D. Cooper, C.L. Hoppel, S.T. Ingalls, M.E. Kenney, X. Li, N.L. Oleinick, S.R. Stevens, Preliminary clinical and pharmacologic investigation of photodynamic therapy with the silicon phthalocyanine photosensitizer Pc 4 for primary or metastatic cutaneous cancers, Front. Oncol. 1 (2011) 1-6.

[35] J.M. Fernandez, M.D. Bilgin, L.I. Grossweiner, Singlet oxygen generation by photodynamic agents, J. Photochem. Photobiol. B:Biology 37 (1997) 131-140.

[36] K. Plaetzer, B. Krammer, J. Berlanda, F. Berr, T. Kiesslich, Photophysics and photochemistry of photodynamic therapy: Fundamental aspects, Lasers Med. Sci. 24 (2009) 259-268.

[37] C. Matei, M. Tampa, R.M. Ion, M. Neagu, C. Constantin, Photodynamic properties of aluminium sulphonated phthalocyanines in human dysplazic oral keratinocytes experimental model, Digest Journal of Nanomaterials and Biostructures 7 (2012) 1535-1547. 
[38] R.D. Joyner, M.E. Kenney, Phthalocyaninosilicon compounds, Inorg. Chem. (1962) 236-240.

[39] C. Farren, S. FitzGerald, M.R. Bryce, A. Bee, A. Batsanov, Synthesis,structure and optical characterisation of silicon phthalocyanine bis-esters, Journal of the Chemical Society, Perkin Transactions 2 (2002) 59-65.

[40] K.A. Bello, I.A. Bello, Some observations on the visible absorption spectra and stability properties of the silicon phthalocyanine system, Dyes and Pigments 35 (1997) 261-266.

[41] D. Frackowiak, A. Waszkowiak, R.M. Ion, K. Wiktorowicz, I. Cofta, H. Manikowski, The interaction of Pc's with stimulated and resting human peripheral blood mononuclear cells, Acta Biochimica Polonica 48(1) (2001) 257-269.

[42] D. Frackowiak, A. Planner, A. Waszkowiak, A. Boguta, H. Manikowski, R.M. Ion, K. Wiktorowicz, Yield of ISC of Pc's evaluated on the basis of a time-resolved photothermal method, J.Photochem.Photobiol., A:Chem. 41 (2001)101-108.

[43] R.M. Ion, Nanomedicine between laboratory and clinical applications, in: Nanostructuring and Nanocharacterization, in Series in Micro and Nanoengineering (eds. M.Zaharescu, M.Ciurea, D.Dascalu), Ed. Academiei, Bucharest, Romania (2010) 241256.

[44] M. Ochsner, Photophysical and photobiological processes in the photodynamic therapy of tumours, Journal of Photochemistry and Photobiology B: Biology 39 (1997) $1-18$.

[45] R.M. Ion, A.A. Sorescu, A. Nuta, A silicon phthalocyanine and a silicon naphthalocyanine In: GV - Proceedings in GV - the 1st Global Virtual Conference. EDIS - Publishing Institution of the University of Zilina, Slovakia 2(2014) 390-395.

[46] A.A. Sorescu, A. Nuta, V. Raditoiu, R.M. Ion, Photophysical and photochemical properties of some silicon benzporphyrazine derivatives, in: Proceedings in Advanced Research in Scientific Areas, EDIS - Publishing Institution of the University of Zilina, Slovakia (2013) 294-299.

[47] J.G. Moser, $2^{\text {nd }}$ and $3^{\text {rd }}$ generation photosensitizers. Amsterdam: Harwood Academic Publishers (1998).

[48] L.A. Muehlmann, B.C. Ma, J.P.F. Longo, M.F.M. Almeida Santos, R.B. Azevedo, Aluminum-phthalocyanine chloride associated to poly(methyl vinyl ether-co-maleic anhydride) nanoparticles as a new third-generation photosensitizer for anticancer photodynamic therapy, International Journal of Nanomedicine 9(1) (2014) 1199-1213.

[49] I. Roy, T.Y. Ohulchanskyy, H.E. Pudavar, E.J. Bergey, A.R. Oseroff, J. Morgan, T.J. Dougherty, P.N. Prasad, Ceramic-based nanoparticles entrapping water-insoluble photosensitizing anticancer drugs: a novel drug-carrier system for photodynamic therapy, J. Am. Chem. Soc. 125(26) (2003) 7860-7865. 
[50] L. Dykman, N. Khlebtsov, Gold nanoparticles in biomedical applications: recent advances and perspectives, Chem Soc. Rev. 41 (2012) 2256-2282.

[51] M.E. Barbinta-Patrascu, I.R. Bunghez, S.M. Iordache, N. Badea, R.C. Fierascu, R.M. Ion, Antioxidant properties of biohybrids based on liposomes and sage silver nanoparticles, Journal of Nanoscience and Nanotechnology 13(3) (2013) 2051-2060.

[52] I.R. Bunghez, S.F. Pop, R.M. Ion, DNA as biotemplate for photochemically-induced generation of $\mathrm{Au}$ and/or Ag nanoparticles, Metalurgia International 18(2) (2013): 94-96.

[53] I.R. Bunghez, M.E. Barbinta-Patrascu, N. Badea, S.M. Doncea, A. Popescu, R.M. Ion, Antioxidant silver nanoparticles green synthesized using ornamental plants, Journal of Optoelectronics and Advanced Materials 14 (11-12) (2012) 1016-1022.

[54] R.M. Ion, D.V. Brezoi, A study on the photodynamic therapy of photosensitizer-coated magnetic nanoparticles, Journal of Optoelectronics and Advanced Materials 9(4) (2007) 936-939.

[55] N. Jux, B. Röder,Targeting Strategies for Tetrapyrrole-based Photodynamic Therapy, World Scientific Publishing, Co. Pte. Ltd, Singapore (2010) 325-401.

[56] M.J Bovis, J.H. Woodhams, M. Rojnik, P. Kocbek, J. Kos, S.G. Bown, M. Loizidou, MTHPC delivery via pegylated plga nanoparticles for in vivo photodynamic therapy (PDT), British Journal Of Surgery (2013) 43 - 44.

[57] A. Vargas, F. Delie, Potential Use Of Biodegradable Nanoparticles For The Photodynamic Therapy Of Eye Diseases, Arch. Soc. Esp. Oftalmol. 84 (2009) 169-176.

[58] E. Ricci-Júnior, J.M. Marchetti, Zinc(II) phthalocyanine loaded PLGA nanoparticles for photodynamic therapy use,Int J. Pharm. 310(1-2) (2006) 187-195.

[59] E. Paszko, C. Ehrhardt, M.O. Senge, D.P. Kelleher, J.V. Reynolds, Nanodrug Applications In Photodynamic Therapy. Photodiagn. Photodyn. Ther. 8 (2011) 14-29.

[60] V.P. Torchilin, Targeted pharmaceutical nanocarriers for cancer therapy and imaging, AAPS J. 9 (2007) 128-147.

[61] D. Kozlowska, P. Foran, P. MacMahon, M.J. Shelly, S. Eustace, R. O'Kennedy, Molecular and magnetic resonance imaging: The value of immunoliposomes, Adv. Drug. Deliv. Rev. 61 (2009) 1402-1411.

[62] B. Pegaz, E. Debefve, F. Borle, J.P. Ballini, H. van der Bergh, Y.N. Kouakou-Konon, Encapsulation of porphyrins and chlorins in biodegradable nanoparticles: the effect of dye lipophilicity on the extravasation and the photothrombic activity. A comparative study, J. Photochem. Photobiol. B:Biol. 80 (2005) 19-27.

[63] L.C. Bergstrom, I. Vucenik, I.K. Hagen, S.A. Chernomorsky, R.D. Poretz, In-vitro photocytotoxicity of lysosomotropic immunoliposomes contaning pheophorbide a with human bladder carcinoma cells, J. Photochem. Photobiol. B:Biol. 24 (1994) 17-23. 
[64] S. Hirohara, M. Obata, S. Ogata, K. Kajiwara, Ch. Ohtsuki, M. Tanihara, S. Yano, Sugar-dependent aggregation of glycoconjugated chlorins and its effect on photocytotoxicity in HeLa cells, J. Photochem. Photobiol. B: Biol. 84 (2006) 56-63.

[65] J. Balasubramanian, N. Narayanan, V. Mohan, A. Ranjit Mohan, B. Mandava Sree, Nanotechnology based Oral Delivery of Insulin - A Retrospective, Int. J. of Pharmacy and Analytical Research. 2(4) (2013) 144-150.

[66] C.P. Reis, R.J. Neufeld, A.J. Ribeiro, F. Veiga, Design of insulin-loaded alginate nanoparticles, CI\&CEQ. 12(1) (2006) 37-52.

[67] R. Stoica, S.F. Pop, R.M. Ion, Evaluation of natural polyphenols entrapped in calcium alginate beads prepared by the ionotropic gelation method, Journal Of Optoelectronics And Advanced Materials 15(7- 8) (2013) 893 - 898.

[68] O. Gaserod, A.Sannes, G. Skjak-Braek, Microcapsules of alginate-chitosan. II. A study of capsule stability and permeability, Biomaterials 20 (1999) 773-783.

[69] F.L. Mi, H.W. Sung, S.S. Shyu, Drug Release from Chitosan - Alginate Crosslinking Agent, Carbohydrate Polymers, 48(1) (2001) 61-72.

[70] G.F. Payne, S.R. Raghavan, Chitosan: a soft interconnect for hierarchical assembly of nano-scale components, Soft Matter. 3 (2007) 521-527.

[71] [71] S. Arora, S. Gupta, R.K. Narang, R.D. Budhiraja, Amoxicillin loaded chitosan-alginate polyelectrolyte complex nanoparticles as mucopenetrating delivery system for H. Pylori, Scientia Pharmaceutica 79(3) (2011) 673-694.

[72] M. George, T.E. Abraham, Polyionic hydrocolloids for the intestinal delivery of protein drugs: alginate and Chitosan. J. Control Release (2006) 1-14.

[73] I. Nenu, T. Popescu, M.D. Aldea, L. Craciun, D. Olteanu, C. Tatomir, P. Bolfa, R.M. Ion, A. Muresan, A. Filip, Metformin associated with photodynamic therapy - A novel oncological direction, Journal of Photochemistry and Photobiology B: Biology, 138 (2014) 80-91.

[74] W.R. Chen, M. Korbelik, K.E. Bartels, Enhancement of laser cancer treatment by a chitosan-derived immunoadjuvant, Photochem. Photobiol. 81 (2005) 190-195.

[75] V.P. Torchilin, Micellar Nanocarriers: Pharmaceutical Perspectives, Pharmaceutical Research 24(1) (2007) 1-16.

[76] D. Bechet, P. Couleaud, C. Frochot, V.L. Viriot, F. Guillemin, M. Barberi-Heyob, Nanoparticles as vehicles for delivery of photodynamic therapy agents, Trends in Biotechnology 26(11) (2008) 612-621.

[77] H.S. Yoo, T.G. Park, Biodegradable polymeric micelles composed of doxorubicin conjugated PLGA-PEG block copolymer, Journal of Controlled Release 70 (2001) 63-70. 
[78] S. Kim, J.Y. Kim, K.M. Huh, G. Acharya, K. Park, Hydrotropic polymer micelles containing acrylic acid moieties for oral delivery of paclitaxel, Journal of Controlled Release 132 (2008) 222-229.

[79] P. Satturwar, M.N. Eddine, F. Ravenelle, J.C. Leroux, pH-responsive polymeric micelles of poly(ethylene glycol)-b-poly(alkyl(meth)acrylate-co-methacrylic acid): Influence of the copolymer composition on self assembling properties and release of candesartan cilexetil, European Journal of Pharmaceutics and Biopharmaceutics 65 (2007) 379-387.

[80] J. Lee, S.C. Lee, G. Acharya, C.J. Chang, K. Park, Hydrotropic solubilization of paclitaxel: analysis of chemical structures for hydrotropic property, Pharmaceutical Research 20(7) (2003) 1022-1030.

[81] R.M. Ion, Nanosystems Based On Micelle Metallo-Porphyrins, The Scientific Bulletin of VALAHIA University - Materials and Mechanics 8(11) (2013) 33-38.

[82] A. Puri, K. Loomis, B. Smith, J.H. Lee, A. Yavlovich, E. Heldman, R. Blumenthal, Lipid-based nanoparticles as pharmaceutical drug carriers: From concepts to clinic, Crit. Rev.Ther. Drug Carrier Syst. 26 (2009) 523-580.

[83] T.M. Allen, P.R. Cullis, Liposomal drug delivery systems: From concept to clinical applications, Adv. Drug Deliv. Rev. 65 (2013) 36-48.

[84] G. Petrisor, R.M.Ion, C.H. Brachais, G. Boni, L. Plasseraud, J.P. Couvercelle, O. Chambin, In vitro release of local anaesthetic and anti-inflammatory drugs from crosslinked collagen based device, Journal of Macromolecular Science, Part A: Pure and Applied Chemistry 49 (9) (2012) 699-705.

[85] D.K. Chatterjee, L.S. Fong, Y. Zhang, Nanoparticles in photodynamic therapy: An emerging paradigm, Adv. Drug Deliv. Rev. 60 (2008) 1627-1637.

[86] R.K. Chowdhary, C.A. Green, C.G. Morgan, Dye-Sensitized destabilization of liposomes bearing photooxidizable lipid head groups, Photochem. Photobiol. 58 (1993) 362-366.

[87] R.M. Ion, M.A. Ionita, B.Carstocea, Photochemical and photodynamic properties of B2 Vitamin in liposomes, Oftalmologia XLIX(3) (2003) 29-35.

[88] M.O. Obochi, A.J. Canaan, A.K. Jain, A.M. Richter, J.G. Levy, Targeting activated lymphocytes with photodynamic therapy: susceptibility of mitogen-stimulated splenic lymphocytes to benzoporphyrin derivative (BPD) photosensitization, Photochem. Photobiol. 62 (1995) 169 - 175.

[89] T.A. Ciulla, R.P. Danis, M. Criswell, Changing therapeutic paradigms for exudative age-related macular degeneration: antiangiogenic agents and photodynamic therapy, Expert. Opin. Investig. Drugs. 8 (1999) $2173-2182$. 
[90] Y. Sadzuka, K. Tokutomi, F. Iwasaki, I. Sugiyama, T. Hirano, H. Konno, N. Oku, T. Sonobe, The phototoxicity of photofrin was enhanced by PEGylated liposome in vitro, Cancer Letters 241(1) (2006) 42-48.

[91] L. Bourré, S. Thibaut, M. Fimiani, Y. Ferrand, G. Simonneaux, T. Patrice, In vivo photosensitizing efficiency of a diphenylchlorin sensitizer: interest of a DMPC liposome formulation, Pharmacol. Res. 47(3) (2003) 253-261.

[92] P. Ježek, M. Nekvasil, E. Škobisová, E. Urbánková, M. Jirsa, M. Zadinová, P. Poučková, I. Klepáček, Experimental photodynamic therapy with MESO-tetrakisphenylporphyrin (TPP) in liposomes leads to disintegration of human amelanotic melanoma implanted to nude mice, International Journal of Cancer 103 (2002) 693-702.

[93] R.M. Ion, A. Stirbet, Photodynamic action of light radiation on E.Coli with pophyrin sensitizers, St.Cerc.Biotechnol., 29-30 (1997) 106-121.

[94] N.A. Peppas, A.G. Mikos, Preparation methods and structure of hydrogels, in: N.A. Peppas (Ed.), Hydrogels in Medicine and Pharmacy, CRC Press, Boca Raton, FL (1986) 1-27.

[95] O. Wichterle, D. Lim, Hydrophilic gels for biological use, Nature 185 (1960) 117-118.

[96] N.A. Peppas, Y. Huang, M. Torres-Lugo, J.H. Ward, J. Zhang, Physicochemical Foundations And Structural Design Of Hydrogels In Medicine And Biology, Ann. Rev. Biomed. Eng. 02 (2000) 9-29.

[97] R.M. Ion, S.C.F. Patachia, $\mathrm{TPPS}_{4}$ Controlled Release from PVA Hydrogels in Photodynamic Therapy of Cancer, in: International Conference on Advancements of Medicine and Health Care through Technology, S. Vlad and R.V. Ciupa (eds.), IFMBE Proceedings 44, Springer International Publishing Switzerland (2014) 307-310.

[98] S. Varga, S.C.F. Patachia, R.M. Ion, Development of controlled release devices for photodynamic therapy of cancer Pollack Periodica 2 (2007) 131-140.

[99] S. Varga, S.C.F. Patachia, R.M. Ion, Nanostructured PVA hydrogel materials as vehicles for the encapsulation and controlled release of porphyrin-based cancer therapeutics, Bulletin of the Transilvania University of Brasov: Supplement Bramat 4 (2007) 153-160.

[100] S.C.F. Patachia, S. Varga, R.M. Ion, Porphyrin encapsulation in nanostructured hydrogels. J.Optoel. Adv. Mat. 9(6) (2007) 1816-1820.

[101] R.M. Ion, P.D.V. Repossi, G. Arguello, Porphyrin-tyrosine conjugate as synergic photosensitizer in photodynamic therapy, Revista De Chimie, 58(11) (2007) 1030-1034.

[102] R. Bhuvaneswari, Y. Gan, K. Soo, M. Olivo, T. Alejandro Diaz-Moscoso, Soft versus hard nanoparticles in cancer PDT, Int. J. Med. Biomed. Res. 1(1) (2012) 2312-2321. 
[103] M. Wainwright, Photodynamic Therapy: The development of new photosensitisers. Anti-Cancer Agents in Medicinal Chemistry (Formerly Current Medicinal Chemistry - Anti-Cancer Agents) 8 (2008) 280-291.

[104] S. Perni, P. Prokopovich, J. Pratten, I.P. Parkin, M. Wilson, Nanoparticles: their potential use in antibacterial photodynamic therapy, Photochem. Photobiol. Sci. 10 (2011) 712 - 720 .

[105] P. Couleaud, V. Morosini, C. Frochot, S. Richeter, L. Raehm, J.O. Durand, Silicabased nanoparticles for photodynamic therapy applications, Nanoscale 2 (2010) 1083-1095.

[106] A. Vargas, M. Eid, M. Fanchaouy, R. Gurny, F. Delie, In vivo photodynamic activity of photosensitizer - loaded nanoparticles: Formulation properties, administration parameters and biological issues involved in PDT outcome, Eur. J. Pharm. Biopharm. 69 (2008) 43 - 53.

[107] W. Chen, Nanoparticle Based Photodynamic Therapy for Cancer Treatment, American Scientific Publishers (2007).

[108] R.M. Ion, D. Daicoviciu, A. Filip, S. Clichici, A. Muresan, Oxidative stress effects of fullerene-porphyrin derivatives in photodynamic therapy, J. Porphyrins Phthalocyanines 16 (2012) 870-877.

[109] R.M. Ion, Photodynamic therapy (PDT): a photochemical concept with medical applications, Rev. Roum. Chim. 52 (2007) 1093-1102.

[110] R.M. Ion, R.C. Fierascu, M. Neagu, C. Constantin, C. Stavaru, Porphyrin (TPP)-polyvinylpyrrolidone (PVP)-fullerene (C60) triad as novel sensitizer in photodynamic therapy, Sci. Adv. Mater. 2 (2010) 223-229.

[111] C. Constantin, M. Neagu, R.M. Ion, M. Gherghiceanu, C. Stavaru, Fullerene-porphyrin nanostructures in photodynamic therapy, Nanomedicine 5(2) 2010 307-317.

[112] C. Constantin, M. Neagu, G. Manda, R.M. Ion, D. Iordachescu, The effect of laser activation of 5,10,15,20-tetra-sulphophenyl-porphyrin loaded in K562 cells and human normal mononuclear cells, Roumanian archives of microbiology and immunology 63(3-4), (2004) 159-68.

[113] R.M. Ion, A. Planner, K. Wicktowicz, D. Frakowiak, The incorporation of various porphyrins into blood cells measured via flow cytometry, absorption and emission spectroscopy, Acta Biochimica Polonica 45(3) (1998) 833-845.

[114] J. Moan, H. Anholt, Phthalocyanine fluorescence in tumor during photodynamic therapy, Photochem. Photobiol. 51 (1990) 379-381.

[115] R.M. Ion, Spectral analysis of the porphyrins incorporation into human blood, J.Biomed Optics, 4(3) (1999) 319-325.

[116] A.G. Filip, S. Clichici, D. Daicoviciu, R.M. Ion, C. Tatomir, L. Rogojan, I. Opris, T. Mocan, D. Olteanu, A.Muresan, Possible in vivo mechanisms involved in photody- 
namic therapy using tetrapyrrolic macrocycles, Brazilian Journal of Medical and Biological Research 44(1) (2011) 53-61.

[117] S.F. Pop, R.M. Ion, M. Neagu, C. Constantin, Photodynamic Therapy on B16 Cells with Tetrasulphonated Porphyrin and Different Light Sources, Journal of Materials Science and Engineering 4(3) (Serial No.28) (2010) 10-16.

[118] M.A. Ionita, R.M. Ion, B. Carstocea, The use of riboflavin as drug in ocular anti-tumoral and anti-neovascularisation area - RO121892/2003.

[119] R.M. Ion, M.A. Ionita, B.Carstocea, M.L.Pascu, L.Danaila, A.Bucur, Clinical aspects of photodynamic therapy - Romanian Experience, Oftalmologia XLVII(2) (2004) 53-60.

[120] M.A. Ionita, B. Carstocea, R.M. Ion, V.I.R. Niculescu, Photodynamic occlusion of ocular neovascularisation with B2 vitamin, Oftalmologia XLXIV(3) (2002) 82-86.

[121] D. Boda, M. Neagu, C. Constantin, A. Diaconeasa, S. Ianosi, C. Amalinei, B. Stanoiu, E. Crauciuc, O. Toma, R.M. Ion, New Photosensitizers Versus Aminolevulinic Acid (ALA) In Experimental Photodynamic Therapy Of Actinic Keratosis - A Case Report, Analele Stiintifice ale Universitatatii „Alexandru Ioan Cuza, Sectiunea Genetica si Biologie Moleculara X (2009) 61-69.

[122] M. Susan, I. Baldea, S. Senila, V. Macovei, S. Dreve, R.M. Ion, R.Cosgarea, Photodamaging effects of porphyrins and chitosan on primary human keratinocytes and carcinoma cell cultures, International Journal of Dermatology, 50(3) (2011) 280-286.

[123] D. Brand, A.B. Ackerman, Squamous cell carcinoma, not basal cell carcinoma, is the most common cancer in humans, J. Am. Acad. Dermatol. 42 (2000) 523-526.

[124] M. Lebwohl, Actinic keratosis: epidemiology and progression to squamous cell carcinoma, Br. J. Dermatol. 149(suppl 66) (2003) 31-33. 



\section{Section 4}

Computing and Analysis Techniques 



\title{
Data Reduction Techniques in Neural Recording Microsystems
}

\author{
Mohsen Judy, Alireza Akhavian and Farzad Asgarian \\ Additional information is available at the end of the chapter \\ http://dx.doi.org/10.5772/59662
}

\section{Introduction}

Nowadays, implantable devices developed for electrically interfacing to the brain are of great interest. Such devices, also known as brain-machine interfaces (BMI), are expected to revolutionalize so many aspects of the human life, such as the way we interface with the external world, and how we cure deseases and disabilities such as the Parkinson's desease, paralysis, and blindness. General concept of intra-cortical neural recording using implantable microsystems along with an example of such systems is illustrated in Figure 1. In a wide variety of applications for such systems, there is a need for recording neural activities from a certain region of the brain with enough spatial resolution. To be able to come up with meaningful information from the region of interest in the brain, implantable neural recording devices are typically designed to record from tens to hundreds of recording sites [1-3].

\subsection{General building blocks}

A neural recording system, in general, comprises two parts: a neural recording implant, and an external setup. Implantable cortical neural recoding microsystems (the implant) typically consist of three main parts:

- neural recording front-end; This module is in charge of sensing extracellular neural activities, and consists of a recording microelectrode array followed by analog signal preconditioning circuitry. A 4-site silicon probe fabricated based on the Michigan approach [4] is shown in Figure 2 [5].

- neural signal processing module; This is where most of the signal handling and signal processing tasks take place, e.g., analog signal processing, analog/digital conversion, and digital signal processing. 

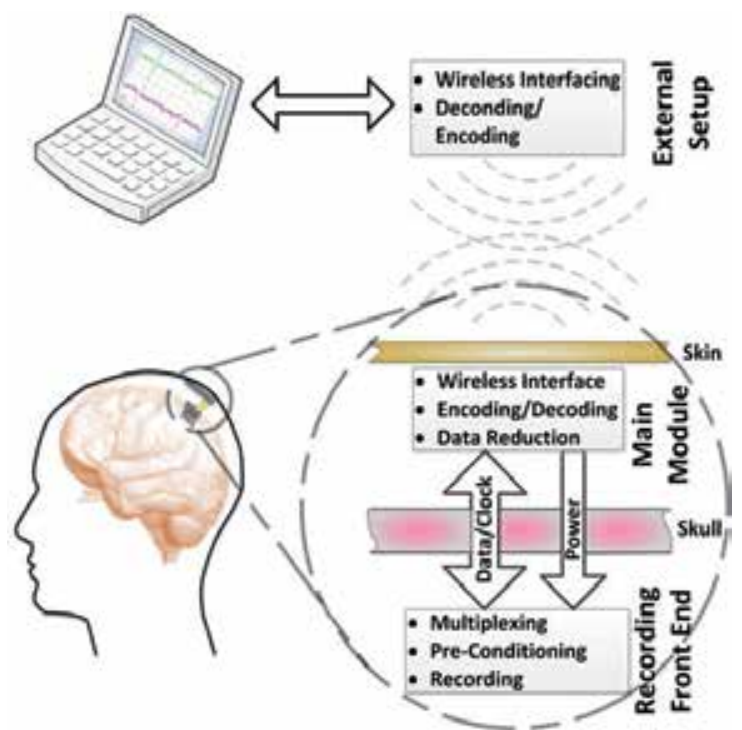

(a)

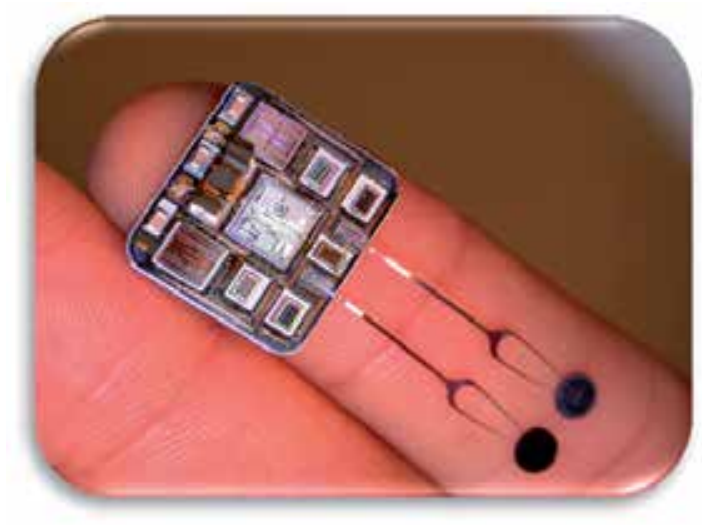

(b)

Figure 1. (a) Intra-cortical neural recording using an implantable microsystem [6] (b) a 64-channel neural recording microsystem developed at the University of Michigan [1]

- wireless interface module; This module is used for data exchange with the external setup and in some cases for supporting power telemetry from the outside to the implant.

\subsection{Challenges in the development of high-density neural recording microsystems}

As the number of recording channels for a wireless neural recording microsystem increases, many aspects of the design of the system will be challenging. From among the more important design challenges, one can point to the low power consumption and small physical dimensions of the system. For tens to hundreds of recording channels, transferring a huge amount of neural 


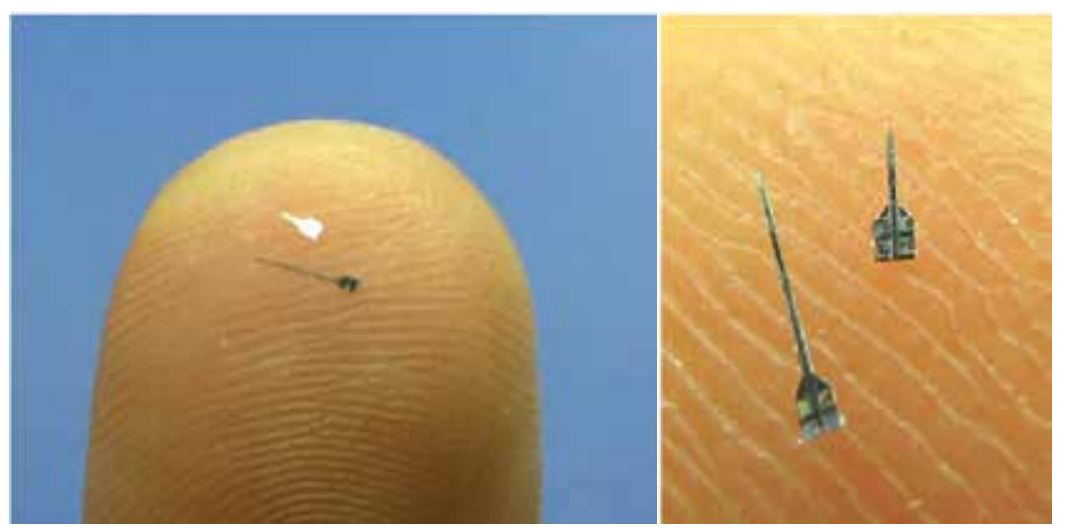

Figure 2. Passive silicon probes with four $20 \mu \mathrm{m} \times 20 \mu \mathrm{m}$ recording sites [5]

data through a wireless link is also a design bottleneck. This is simply due to the fact that an implantable neural recording device needs to transmit the recorded neural information to the external world through wireless connection, and the frequency band used for wireless communication is not unlimited. One of the efficient ways to overcome this problem is to either compress the data being telemetered or at least to extract and transmit only the useful information needed for the target application.

\section{Spike reporting}

An intra-cortically-recorded neural signal, in general, comprises three major components: action potentials (also known as neural spikes or simply spikes), local field potential (LFP), and background noise. It is believed that most of the important information in neural signals is reflected in the occurrence rate of neural spikes. As a result, in some applications (e.g., prosthetic applications) only the occurrence of spikes is detected and reported to the external world. In some other applications (e.g., neuroscientific research), however, researchers and scientists need more information on how or where the neural activities occur.

Recording the entire neural signal (action potentials superposed with background noise) is the maximum function expected from a general neural recording system, which allows for studying different components of a neural signal including the background noise. For multichannel wireless neural recording implants, because of the limited bandwidth available for transmitting the neural data, the number of recording channels will be limited if the entire signal is intended to be telemetered. In many applications, the rate of spike occurrence is the most important information that is expected from a neural recording system to provide. Hence, it will be much more bandwidth-efficient if the spikes are detected by the implanted recording system and only the occurrences of the spikes are reported to the external host rather than transmitting the entire neural signal. 


\subsection{Spike detection}

In addition to the small action potentials with the amplitude of around $100 \sim 500 \mu \mathrm{V}$, a neural signal contains background noise and probably low-frequency baseline variations. To prepare the neural signal for spike detection, it is amplified with a gain of around $40 \sim 60 \mathrm{~dB}$ and also its low-frequency (below 1 10Hz) and high-frequency (above 7 10kHz) contents are filtered out [7], [2]. Then, this preconditioned signal is delivered to a spike detector.

There are various spike detection approaches that can be classified into two major categories: feature-based spike detection methods, and spike detection by hard thresholding. In the former, a preprocessor searches the input neural signal for certain features of a spike to occur, while in the latter, a threshold level is defined and a spike is detected when the neural signal goes beyond the threshold.

Feature-Based Approaches. Only a few years after artificial neural networks (ANN's) were introduced as an efficient tool to implement artificial intelligence, due to the feature extraction capability that certain types of ANN's had, they showed to be attractive candidates for automatic spike detection either by themselves or in conjunction with preprocessors [8-9]. Kohonen and Grossberg networks with unsupervised learning, and Multi-Layered Perceptron network with "error back-propagation" as a supervised learning algorithm have been used to perform spike detection. Although there are cases where the raw neural signal is fed to the ANN for spike detection [9-10], it is mostly preferred to use a preprocessor for extracting certain spike features first, and then use an ANN for processing them and detecting the spikes, as illustrated in Figure 3. Because of their relatively large area- and power-consuming electronic implementations, this class of spike processors has never been used in implantable neural recording microsystems.

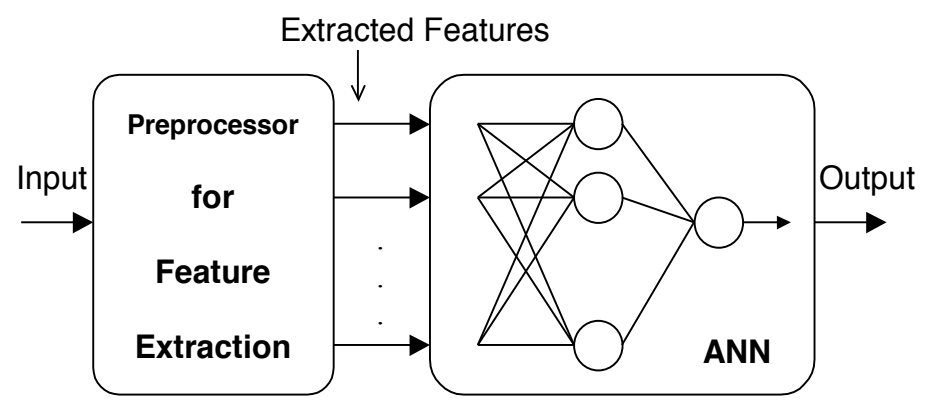

Figure 3. Employing neural networks for spike detection

Spike Detection Based on Nonlinear Energy Operator (NEO). Traditional spike detectors (explained above) usually need prior information about action potentials, which is usually not available before recording the neural signals in real applications. In contrast with these methods, which are mostly based on the amplitude or time-domain features of the neural signal, detection of action potentials, i.e., spikes, can also be performed based on the energy content of the signal. Direct square of the signal, absolute value operator, and variance 
estimator are the energy-based operators commonly used for the detection of bio-potentials. The nonlinear energy operator (NEO), also called the Teager energy operator (TEO) is one of the unsupervised action potential detectors exhibiting satisfactory performance in the case of low signal-to-noise ratio (SNR) for the neural signal and also convincing speed for the alignment of spikes in the real time. In its original form, continuous-time NEO is defined as:

$$
\psi(x(t))=\left(\frac{d x(t)}{d t}\right)^{2}-x(t)\left(\frac{d^{2} x(t)}{d t^{2}}\right) .
$$

which is sensitive to signal with short time interval and at a high-frequency band [11]. An efficient hardware implementation for an NEO neural signal processor employing custom $\mathrm{OTA}^{1}-\mathrm{C}$ analog circuits was reported in [12].

\subsection{Hard thresholding}

Four possible ways of spike detection by hard thresholding are illustrated in Figure 4. Spike detection is mostly performed to detect either positive (Figure 4 (a)) or negative (Figure 4 (b)) spikes [7], [2], [13]. Having such a fixed pre-assumption for the polarity of the spikes limits the operation of the system. Recognition of both positive and negative spikes, i.e., bi-phasic spike detection, can be realized in two major ways, illustrated in Figure 4 (c) and (d). In Figure 4 (c), the spike detector returns a logical "1" on the Spike Occurrence (S.O.) output upon the detection of a spike, no matter if it is positive or negative. This is a bandwidth-efficient way of bi-phasic spike detection, which requires almost the same bandwidth as the uni-phasic methods, but pays the price by losing the spike polarity. The simplest realization of this idea is to filter out the DC component of the input signal, find its absolute value, and then detect the spikes using one comparator and one threshold [14] as shown in Figure 5(a). Aside from the need for a precise full-wave rectifier in this realization, the fact that both the positive and the negative spikes are compared with the same threshold level might be considered as a drawback. Figure 5 (b) shows another realization of bi-phasic spike detection with no polarity, which uses two comparators and an OR gate and also allows for comparing positive and negative spikes with separate threshold levels. This approach is used in [7],[13] with positive and negative thresholds, $\mathrm{V}_{\mathrm{TH}, \mathrm{P}}$ and $\mathrm{V}_{\mathrm{TH}, \mathrm{N}}$, defined by a threshold value (THR) and a threshold offset (ThrOS), as shown in Figure 6.

The circuit shown in Figure 5 (b) can also be used with minor modifications to realize the complete bi-phasic spike detection method illustrated in Figure 4 (d), which returns two bits per detected spike. These two bits can be either Spike Occurrence (S.O.) and Spike Polarity (S.P.) as shown, or one bit assigned to detected positive spikes and the other to detected negative spikes.

1 Operational Transconductor Amplifier 


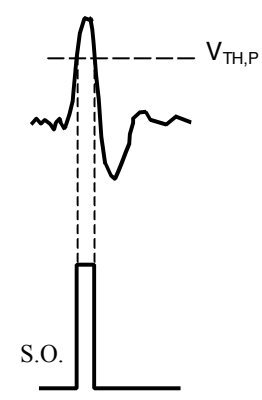

(a)

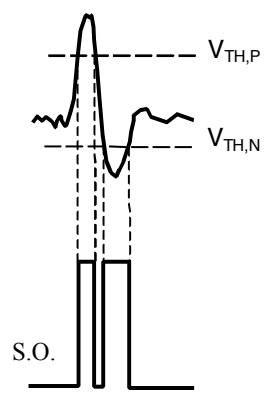

(c)

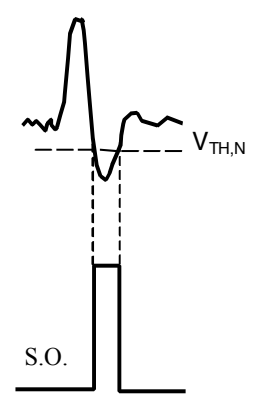

(b)

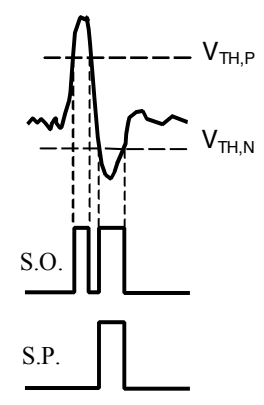

(d)

Figure 4. Spike detection approaches (a) Positive (b) Negative (c) Simple bi-phasic (d) Bi-phasic with spike polarity [13]

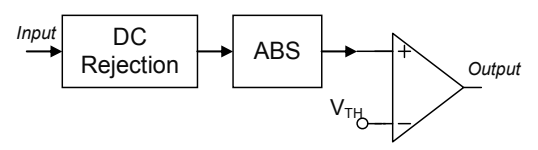

(a)

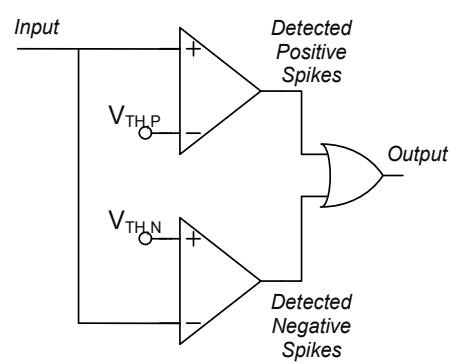

(b)

Figure 5. Implementations of biphasic spike detection with no polarity 
There is a variety of methods for generating the thresholds required for spike detection. The threshold can be either statically defined by the user [2], [13] or automatically set by the internal circuitry.

Automatic Threshold Setting. The 32-channel spike detector ASIC reported by [7] uses a straightforward approach for automatic threshold generation. In this method, the average (AVG) and the standard deviation (SD) of the neural signal is calculated, and then the two thresholds required for bi-phasic spike detection are set above and below the average value as:

$$
\mathrm{THR}=\mathrm{AVG} \pm \mathrm{k} . \mathrm{SD}
$$

where $\mathrm{k}$ is a constant. Typical value for $\mathrm{k}$ varies from 3 to 7 depending on the signal-to-noise ratio (SNR) of the recorded neural signal. Functional block diagram of this spike processor implementing the above method in digital domain is shown in Figure. Thirty two channels of preconditioned neural signals, which are already time-division multiplexed on four lines in analog domain by a recording front-end (not shown), are delivered to the spike detector ASIC. The four multiplexed inputs each carrying 8 channels of neural signals are first converted to digital by four A/D converters simultaneously. The Sample Distributor, which is synchronized with the time-division multiplexer on the recording front-end, demultiplexes the amplitude samples into 32 digital neural channels. Then, the digital spike detector computes the averages and standard deviations of the 32 channels separately and accordingly calculates their threshold values. After the Threshold Calculation program is executed, the Spike Detection program is run. The amplitude sample of each channel is compared to the associated threshold, and if it is beyond the threshold level, it is considered as a detected spike. As long as a channel is active, its amplitude samples are tagged with the associated channel address, and put in a buffer to be sent to a wireless interface.

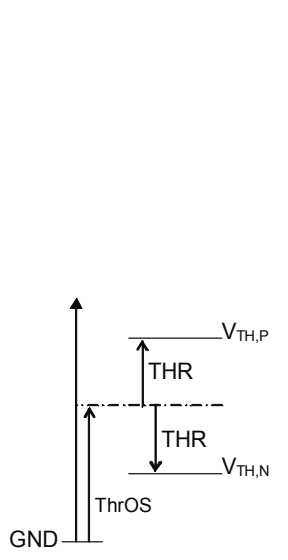

(a)

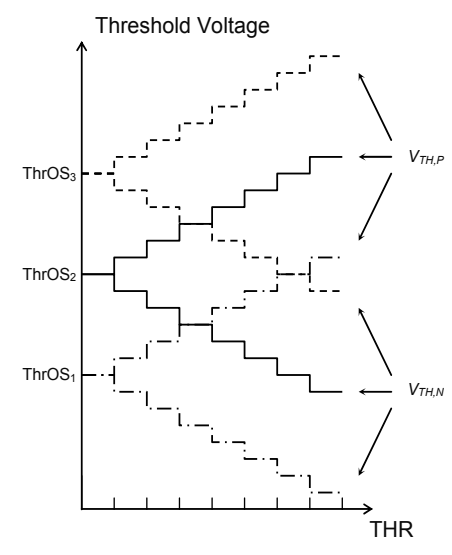

(b)

Figure 6. Defining positive and negative thresholds using a threshold value (THR) and an offset (ThrOS) [13] 


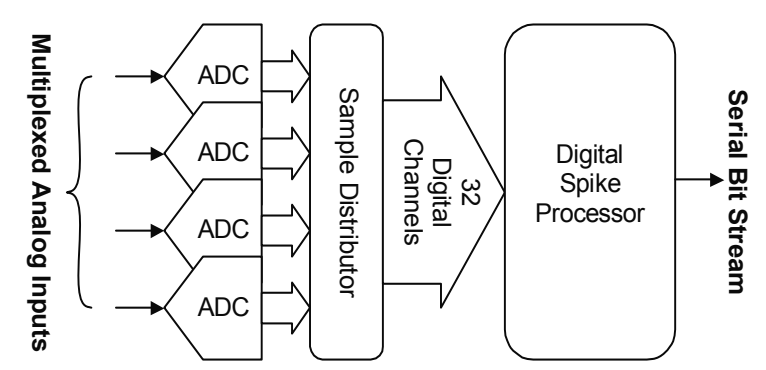

Figure 7. Functional block diagram of the 32-channel digital spike detector ASIC reported in [7]

In [15], a spike detector circuit is reported that computes the detection threshold in analog domain. Functional diagram of this circuit is shown in Figure 8. One of the advantages of this circuit is that unlike the spike detector in [7], the threshold is computed in real time. The circuit assumes that the input signal has already been amplified and band-pass filtered, and the background noise has a Gaussian distribution. Since the input signal is assumed to have no DC component, the noise can be described by its RMS value, $V_{1 \sigma}$, which is equivalent to its standard deviation, $\sigma$. In order to be well above the noise level, the threshold voltage is set to $V_{K \sigma}=K . V_{1 \sigma}$, with $\mathrm{K}$ set to 5 in [15]. Although it is assumed that the low-frequency baseline variations of the input signal have been already filtered out, further analysis of this method shows that the detection threshold can adaptively follow the baseline variations. There is, however, an upper bound on the frequency-amplitude product of the baseline variations that the adaptive threshold is capable of following [16]. Power dissipation of the implementation of this approach is very low, in the range of microwatt and occupies very small silicon area. The drawbacks of this method include the circuit's sensitivity to the absolute value of some of the circuit elements, which are usually subject to relatively large fabrication tolerances, and the difficulty in implementing the low-frequency low-pass filter required in the RMS block.

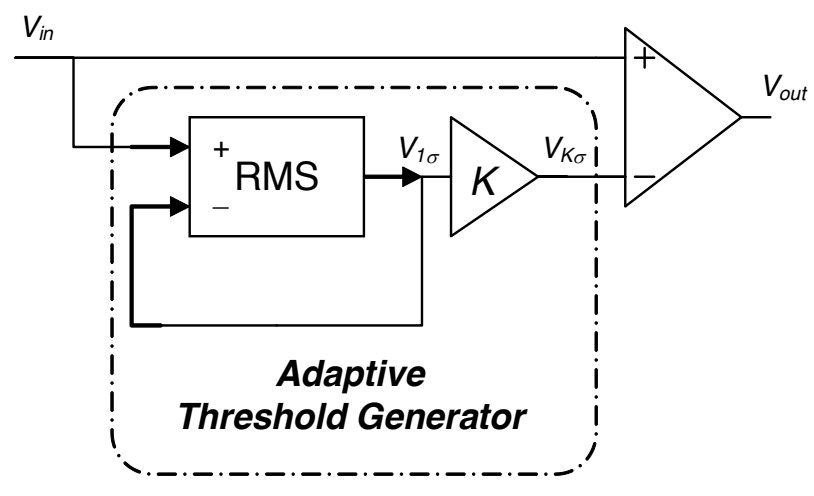

Figure 8. Functional diagram of the analog spike detector of [15]

Figure 9. shows another idea in analog spike detection, in which two OTA-based low-pass filters with different cut-off frequencies play the key role [17]. One filter has a higher cut-off 
frequency to remove high frequency noise, and the other has a lower cut-off frequency to make a local average. The difference between the high-pass filtered signal and its local average is provided by an OTA, and is recognized by another OTA as a detected spike when exceeds a certain reference value $\left(\mathrm{V}_{\text {ref }}\right)$. This method is robust against changes in both noise level and the input signal's DC offsets, both of which are likely to happen in long-term neural recording. The OTAs operate in subthreshold region to reduce power. The $\tau$ bias voltages are set off chip to enable adjustment of the cut-off frequencies after fabrication. In this circuit, the spike detection threshold level is set by $\mathrm{V}_{\text {bias }}$, which along with the other bias and reference voltages should be properly set, and probably fine-tuned for long-term recordings.

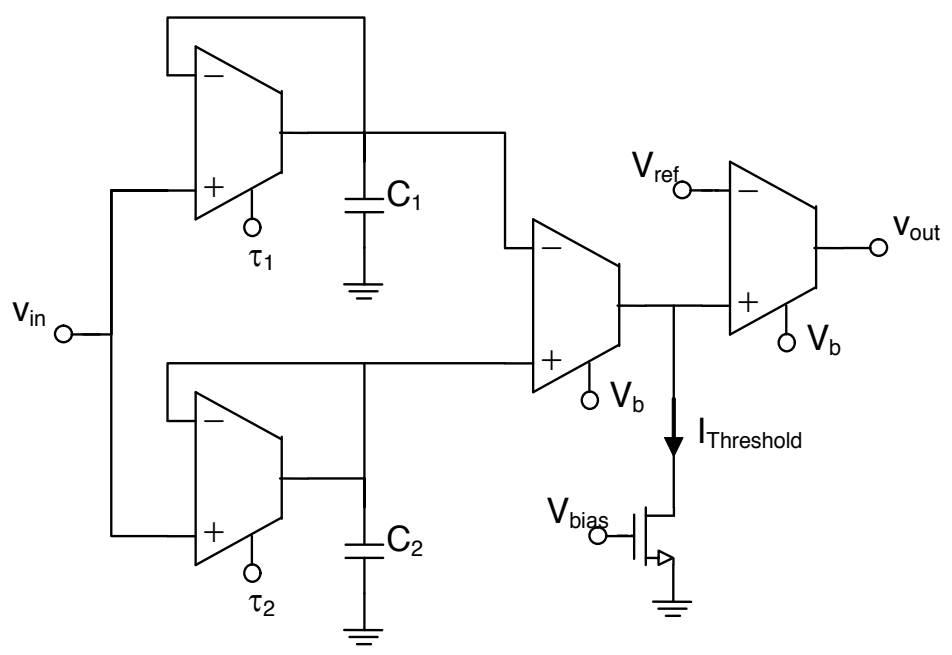

Figure 9. Schematic diagram of the analog spike detector of [16]

In both of the above analog approaches, there are device and circuit parameters that should be set by the user for proper operation, which make these circuits inappropriate for implantable applications.

\section{Mathematical approaches}

Mathematical transforms are among the most common methods of data compression. Recently, the Discrete Wavelet Transform (DWT) has been successfully employed in neural recording microsystems to compress the neural information, while preserving the wave shape of action potentials [18-26]. The DWT transforms discrete signals from the time domain into the time-frequency domain. One-level DWT for a given signal is achieved by convolving the signal samples through low-pass and high-pass decomposition filters [19,21]. The filtering is then followed by sub sampling to obtain the approximation and detail coefficients. For multilevel DWT, approximation coefficients should be fed to the same decomposition filters recursively [27]. Characteristics of the filters are determined by the wavelet basis. For neural 
signal compression, the optimal choice is a wavelet function that can approximate the action potentials waveform with minimum DWT coefficients and error. It has been shown that by proper selection of the wavelet basis, most of the spike energy is concentrated in a few large coefficients, while many small coefficients carry insignificant information and are mainly attributed to noise [19]. Therefore, in order to achieve higher data reduction rates, the DWT coefficients are passed through a thresholding stage. In this block, data coefficients smaller than desired certain threshold level are set to zero, while others are left unchanged. It is obvious that the threshold value plays an important role in the overall data compression rate, and also in the quality of the reconstructed signal. Hence, the threshold level should be set carefully based on the requirements of the target application.

Due to power and size constrains in biomedical implants, VLSI implementation of DWT is of great importance. In [20], it has been shown that from a signal compression standpoint, symmlet4 wavelet basis is advantageous over other wavelet functions for neural recording applications. It is believed that this is mainly because of the similarity of this function to the general wave shape of action potentials. For hardware implementation of symmlet4 function, lifting method is proposed in [21]. Furthermore, two different circuit designs, pipelined and sequential, are presented and compared for the lifting scheme. It is demonstrated that for single-level single-channel integer DWT, the pipelined approach consumes lower power, but occupies more silicon area compared with the sequential implementation. On the other hand, 4-level multi-channel implementation of the two designs indicates that the sequential approach requires significantly smaller chip area, while the power consumption of both is almost the same. As a result, the sequential execution architecture is employed in [23] to design a complete 32-channel compression system based on the 4-level symmlet4 DWT. The chip consumes $3 \mathrm{~mW}$ of power and occupies only $5.75 \mathrm{~mm}^{2}$ in a $0.5-\mu \mathrm{m}$ CMOS technology. Also, with a sampling rate of $25 \mathrm{KSample} / \mathrm{Sec}$ per channel and 10-bits data samples, the system provides data compression of more than 20 times, resulting in a total output bit rate of less than 370 kbps.

In [26], a neural signal compression method based on the Discrete Haar Wavelet Transforms (DHWT) is proposed. From the standpoint of data compression, Haar basis function may not perform as efficient as complex functions such as high-order Daubechies and symmlet, but due to its simple hardware implementation, it can be easily used for large number of neural channels with less concern about power and area. As discussed in [26] for two-point DHWT, data coefficients can be calculated by only a buffer, an adder, and a subtractor. Moreover, in order to compare Haar and symmlet4 basis functions, they have been both designed for processing a single channel with 8-bits data samples. Results indicate that before the thresholding stage, relative error (between the original signal and the corresponding reconstructed signal) for the DHWT is only $0.01 \%$ larger than the symmlet 4 case, which is obviously negligible. On the other hand, hardware implementation of the DHWT shows around $83 \%$ saving in number of transistors, and more than $90 \%$ in the occupied silicon area, when physically laid out in a $0.13-\mu \mathrm{m}$ CMOS technology. The complete 64-channel DHWT-based neural compressor achieves a compression rate of 112 with an error of $2.22 \%$. Additionally, the compressor circuit consumes as low as $0.12 \mathrm{~mW} @ 1.2 \mathrm{~V}$ supply voltage, and occupies less than $0.1 \mathrm{~mm}^{2}$ in a 0.13 - 
$\mu \mathrm{m}$ CMOS technology. Therefore, it can be said that with this architecture, the gain in circuit simplicity and the bit-rate improvement are much more significant than the penalty paid by the noise added to the signal. However, it is worth mentioning that, in general, the appropriate architecture should be selected based on the application.

\section{Hardware approaches}

To avoid adding extra power- and area-hungry signal processing blocks for data reduction, and at the same time preserving important information of the neural signals, there is a different category of data reduction techniques, known as hardware approaches. These are the approaches focused on modifying the hardware of the recording system in such a way that considerable bit-rate reduction can be achieved. Obvious advantages of these approaches are smaller silicon area and power consumption as compared with the mathematical approaches explained in the previous section.

To benefit from the advantages associated with digital signal processing and also digital data communication (as opposed to their analog counterparts), neural recording devices are commonly designed to convert neural signals into digital as the first step. As a result, analogto-digital converters (ADCs) are known as one of the key building blocks in such systems. Recently some efforts are put on designing application-specific ADCs to efficiently utilize the bandwidth allocated for wireless data telemetry. In this section, an efficient method for analogto-digital (A/D) conversion of neural signals is discussed. This method results in significant reduction of data-rate for multi-channel cortical neural recording microsystems.

\subsection{Anti-logarithmc quantization}

Although linear ADCs are typically used to digitize neural signals in neural recording microsystems, it is beneficial to design a nonlinear ADC for such specific signals. Choosing the best-suited nonlinearity function for a specific signal requires recognition of the concentration of information along the signal amplitude range. As illustrated in the left side of Figure 10, in general, signals can be categorized into three types according to how the information they carry is distributed along the amplitude range.

Type-I signals are named "Signals with Non-Concentrated Information (NCI)" due to their almost uniform distribution of information concentration. Important information for Type-II signals is concentrated at the lower side of the amplitude range. Audio signals are of this type, referred to as "signals with Information Concentration at Low Amplitudes (ICLA)". Conversely, for "signals with Information Concentration at High Amplitudes (ICHA)", i.e., Type-III signals, more information is present at higher side of the amplitude range, with neural signals as examples.

Figure 10 provides intuitive illustration of the choice of different quantization functions for the three signal types discussed. With a constant slope (i.e., linear) quantization function, an NCI signal is better digitized. Decreasing slope quantization functions such as logarithmic function are recommended for ICLA signals. These functions put more emphasis on lower 
amplitudes, where more information is concentrated. For example, compressing/expanding (companding) of audio signals in communications systems is based on logarithmic quantization, which increases the dynamic range and improves the SNR [28]. Whereas quantization functions with increasing slope along the input amplitude range, such as the exponential function, put more resolution in the quantization of the larger amplitudes and are preferred for ICHA signals.
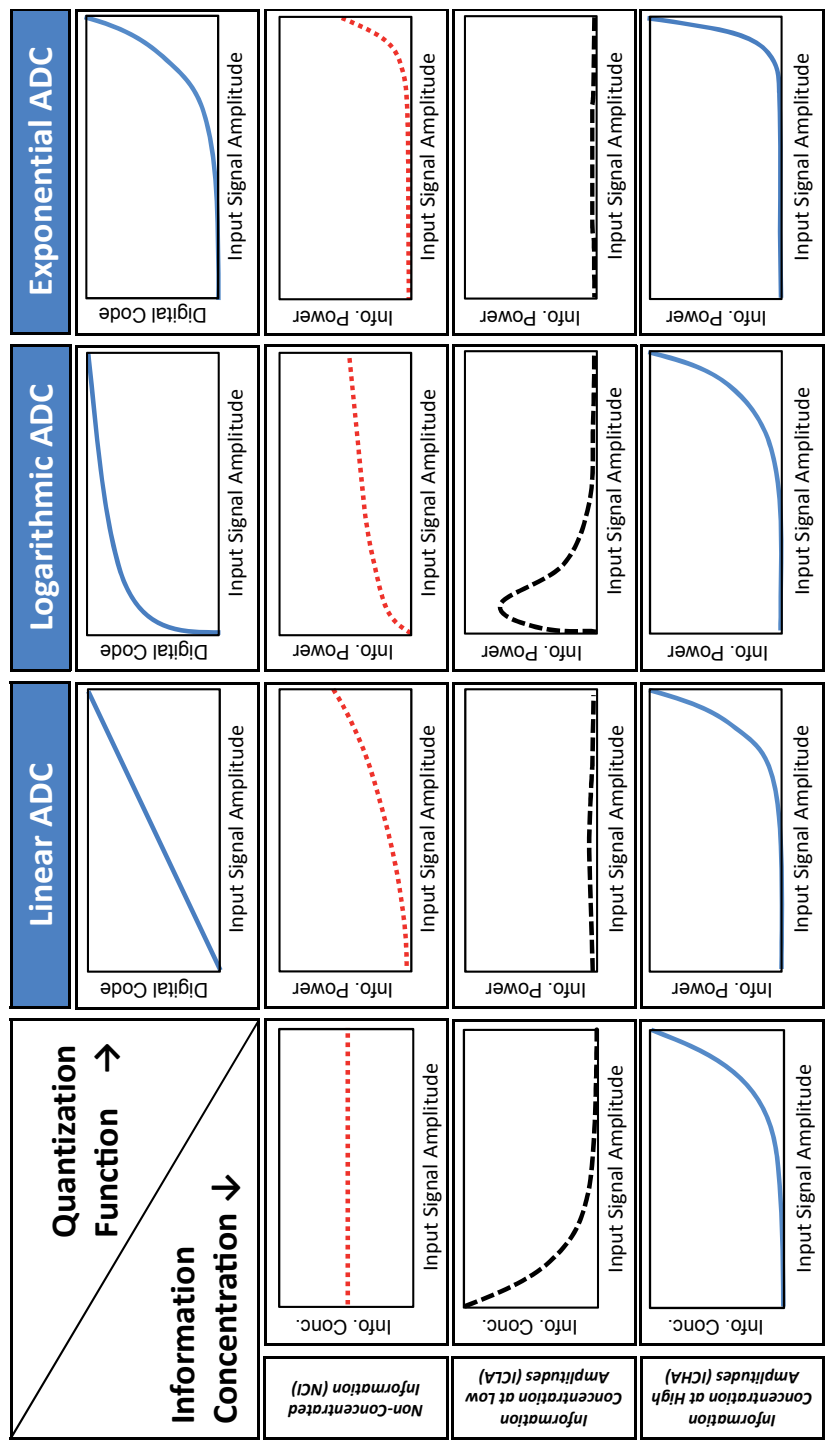

Figure 10. Selecting quantization function for NCI, ICLA, and ICHA signals 


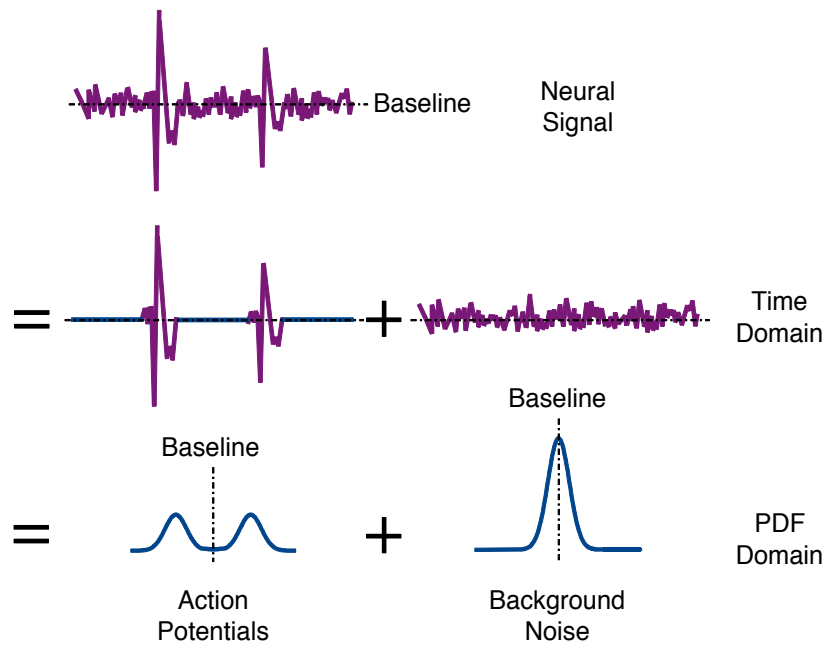

Figure 11. Neural signal composed of APs and B-Noise

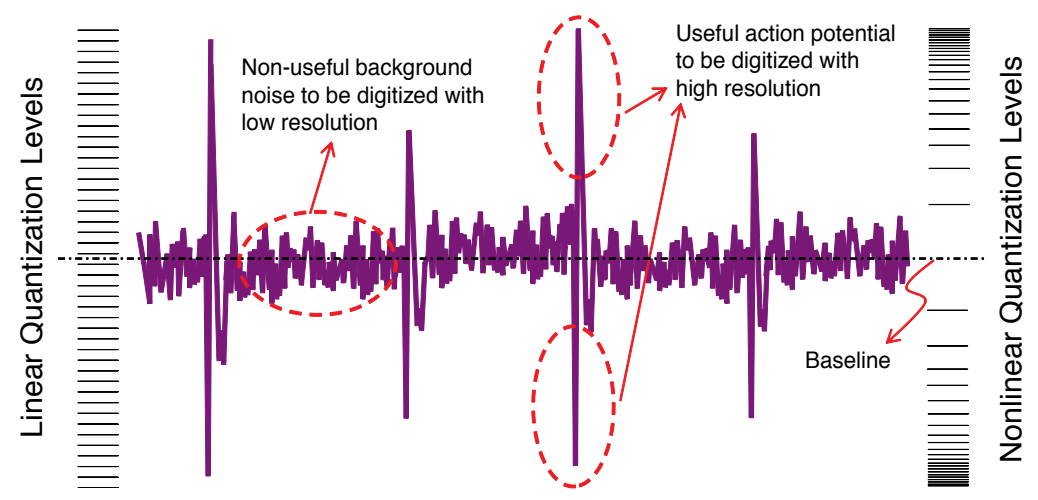

Figure 12. Quantization of a neural signal with linear and nonlinear quantization steps

Basic Idea. As shown in Figure 11, in time domain a typical intracortically-recorded extracellular neural signal can be divided into two parts: action potentials (APs) and background noise (B-Noise). In probability density function (PDF) domain, APs are concentrated at large amplitudes while B-Noise is concentrated at small amplitudes. In a wide variety of neuroscientific and neurophysiological studies, as well as in many neuroprosthetic applications, it is the APs that carry the useful information embedded in neural signals. As illustrated in Figure 12 , in implantable neural recording microdevices, neural signals are usually digitized using linear ADCs, i.e., ADCs with linear quantization characteristics. This means that the non-useful B-Noise is digitized with the same resolution as the useful APs are. In other words, when telemetering a digitized neural signal, part of the outgoing bit-rate is wasted to carry the noise content present in the neural signal. In [29] the idea of digitizing neural signals using an ADC 
with non-uniform quantization steps has been proposed. According to the classification presented in the previous section, neural signals are categorized under Type-III (i.e., ICHA). Hence, the best type of nonlinearity function for the quantization of neural signals is signals with increasing slopes such as parabolic and exponential functions.

Digitizing neural signals using exponential ADC (exp-ADC) helps saving the bandwidth in wireless data telemetry between the implanted device and the external host. Data reduction for an 8-bit exp-ADC is $24 \%$ as compared with its linear counterpart. Along with data reduction, anti-logarithmic quantization of neural signals significantly reduces the power consumption of the ADC, comparing with a standard linear ADC. This is due to less number of digital code transitions for the exp-ADC. Moreover, anti-logarithmic quantization increases the SNR of neural signal by reducing its noise content.

Converting Back to Analog. The transfer characteristics for conventional linear analog-todigital-to-analog (A/D/A) conversion process is a linear function, i.e., analog input signal is digitized by a linear ADC and then is converted back to the analog domain using a linear DAC. On the other hand, in a nonlinear A/D/A conversion process, analog input signal is digitized by a nonlinear quantization function. As shown in Figure 13 to convert back to the analog domain, digital signal should be passed through a nonlinear DAC with exact inverse characteristic. The resulted $\mathrm{A} / \mathrm{D} / \mathrm{A}$ conversion transfer characteristic is similar to that of a linear $\mathrm{A} / \mathrm{D} / \mathrm{A}$ conversion process, except that the quantization steps are non-uniform. In the case of anti-logarithmic $\mathrm{A} / \mathrm{D} / \mathrm{A}$ conversion, quantization steps are decreasing in length along the input amplitude range.
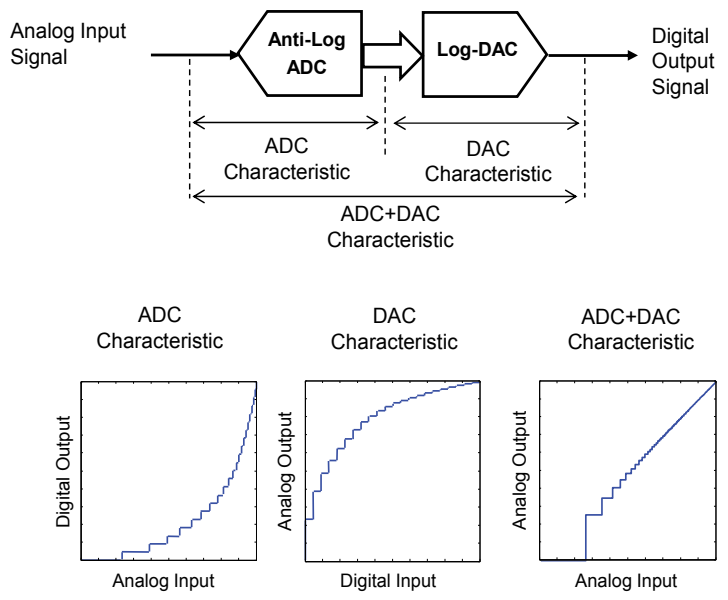

Figure 13. Transfer characteristics of an anti-logarithmic A/D/A conversion 

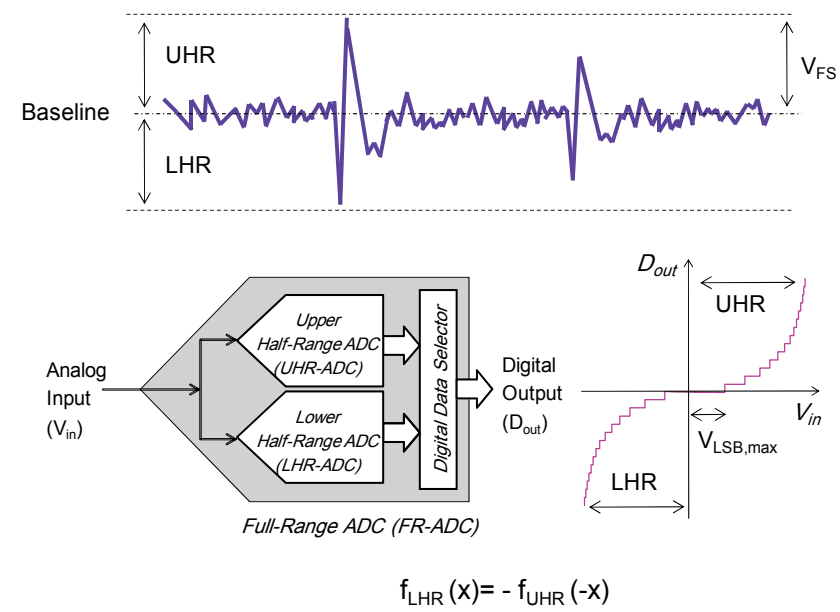

Figure 14. An FR-ADC designed to digitize neural signals using two HR-ADCs with exponential quantization function

Covering the Full Range. Assuming that the neural signal is preamplified and positioned around a certain baseline level, as illustrated in Figure 14, the nonlinear quantization function needs to be defined in an odd symmetric form around the baseline of the signal. Therefore, the nonlinear ADC needed to cover the entire input signal range, full-range ADC (FR-ADC), is realized using two complimentary half-range ADCs (HR-ADCs) each covering half of the input signal range. Hence, assuming that the basic nonlinear quantization function is used for the upper half-range $\mathrm{ADC}(\mathrm{UHR}-\mathrm{ADC}), \mathrm{f}_{\mathrm{UHR}}(\mathrm{x})$, the quantization function used for the lower halfrange ADC (LHR-ADC) will be:

$$
f_{\text {LHR }}(x)=-f_{U H R}(-x) \text {. }
$$

The nonlinear ADC discussed hereafter is assumed to be the ADC that covers the upper half of the input signal range, i.e., the UHR-ADC, unless otherwise stated.

Half-Range Characteristic Function. The input-output relationship for an N-bit HR-ADC with exponential quantization function is:

$$
\frac{b_{N-1} 2^{N-1}+\ldots+b_{0}}{2^{N}}=a \cdot\left[\exp \left(\frac{v_{i n}}{V_{F S}} \times k\right)-1\right],
$$

where $\left(b_{\mathrm{N}-1} \ldots b_{1} b_{0}\right)$ is digital representation of analog input, $v_{i n}$ and $V_{F S}$ is the full-scale input range for the UHR-ADC. To satisfy the boundary conditions for minimum and maximum values of $v_{\text {in }}$ it can be shown that: 


$$
k=\ln \left(\frac{1}{a}+1\right)
$$

Parameter $a$ sets the curvature of the characteristic function. The smaller this parameter is, the more rapid the exponential input-output relationship will be. Quantization steps along the input range are known, in general, as least significant bits (LSB), and are calculated as:

$$
V_{L S B, i}=\frac{V_{F S}}{k} \ln \left(\frac{1}{i+a 2^{N}}+1\right)
$$

for $i=0,1, \ldots, 2^{N}-1$. The largest and the smallest quantization steps, $L S B_{\max }$ and $L S B_{\min }$ are calculated using eq.(6) for $i=0$ and $2^{\mathrm{N}}-1$, respectively, as:

$$
V_{L S B, \max }=\frac{V_{F S}}{k} \ln \left(\frac{1}{a 2^{N}}+1\right)
$$

and

$$
V_{L S B, \min }=\frac{V_{F S}}{k} \ln \left(\frac{1}{(a+1) 2^{N}-1}+1\right) .
$$

In general, dynamic range (DR) of a nonlinear ADC is defined to be the ratio of the full-scale input voltage to the smallest resolvable signal, $\mathrm{V}_{\mathrm{LSB} \text {,min }}$ [32]. DR in the case of exp-ADC is achieved as:

$$
D R=\frac{V_{F S}}{V_{L S B, \min }}=\frac{k}{\ln \left(\frac{1}{(a+1) 2^{N}-1}+1\right)}
$$

The choice of the largest quantization step, $\mathrm{V}_{\mathrm{LSB} \text {, } \max }$ is perhaps the most critical decision in forming the quantization function for the exp-ADC. This is because of its key role in the reduction of the noise content of the neural signal. The largest LSB is responsible for the largest quantization error. It is along the $\mathrm{V}_{\mathrm{LSB} \text {, max }}$ that variations of the input signal are intentionally not seen and replaced with 0 (the baseline level in our design). By using this method, not only the quantization error is not a disturbing phenomenon for the signal, but it also plays a denoising role as it replaces the B-Noise around the baseline with 0 . To achieve significant reduction in the B-Noise power, $\mathrm{V}_{\mathrm{LSB} \text {, max }}$ is set to $3 \sigma$, where $\sigma$ is the standard deviation of the B-Noise PDF. This way, most of the B-Noise will be intentionally removed from the neural signal during the digitization process. 


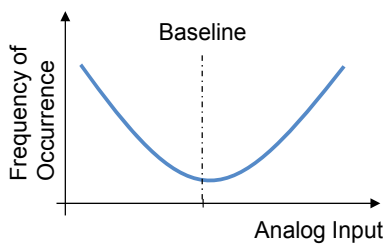

(a)

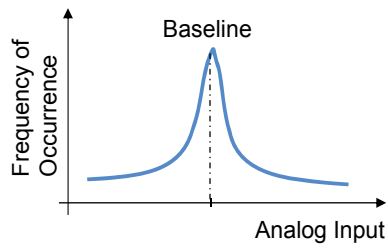

(b)
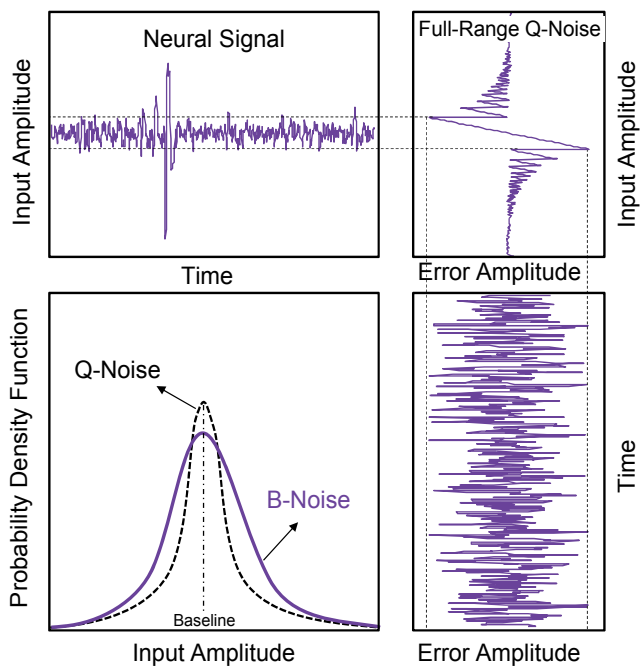

(c)

Figure 15. Q-Noise PDF for (a) logarithmic ADC and (b) exp-ADC; (c) Q-Noise for exp-ADC vs. B-Noise of a neural signal

Noise Analysis. PDF of quantization noise (Q-Noise) for linear ADC is uniform along the input amplitude range. This can be shown by eq. (10) which formulates PDF of the Q-Noise associated with code $\mathrm{n}$ in an N-bit linear HR-ADC [30]:

$$
P_{L i n, H R}(n)=\frac{1}{2}\left[\left(\frac{n}{2^{N}}\right)-\left(\frac{n-1}{2^{N}}\right)\right]=\frac{1}{2^{N+1}} .
$$

Uniform distribution of Q-Noise along the input amplitude makes linear ADCs suitable for digitizing NCI signals. For specific signals an NLADC might be useful in terms of SNR improvement. This advantage comes from the fact that NLADCs exhibit non-uniform Q-Noise distribution. In a Logarithmic ADC, Q-Noise energy is shifted to large amplitudes. As a result, logarithmic ADC has widely been used in digitizing of ICLA signals, such as audio. PDF of Q-noise associated with code $\mathrm{n}$ for an N-bit logarithmic HR-ADC is formulated in eq. (11) and is illustrated in Figure 15(a):

$$
P_{L o g, H R}(n)=\frac{a}{2}\left[\exp \left(\frac{k n}{2^{N}}\right)-\exp \left(\frac{k(n-1)}{2^{N}}\right)\right] .
$$


In the case of the anti-logarithmic N-bit HR-ADC, the Q-Noise PDF associated with code $\mathrm{n}$ is derived as:

$$
P_{E x p, H R}(n)=\frac{1}{2 k}\left[\ln \left(\frac{n}{2^{N} a}+1\right)-\ln \left(\frac{n-1}{2^{N} a}+1\right)\right] .
$$

As shown in Figure 15(b), exp-ADC shapes the Q-Noise in such a way that most of its energy concentrates at small amplitudes, making it suitable for digitizing ICHA signals. As an example, in neural signals, APs with large amplitudes are quantized with higher SQNR as opposed to the B-Noise with rather small amplitudes. Figure 15(c) illustrates that most of the noise content of the signal (B-Noise) lies within the very first LSBs. The interesting point here is that since it is some of the noise content of the neural signal that is lost during the quantization process, not only the associated quantization error is not undesirable, but it is also welcomed as it leads to noise-content reduction and consequently to significant improvement in the SNR of the neural signal being digitized.

Noise-Content-Reduction Ratio, NCRR, is a measure of capability of an ADC in reducing the noise content of the neural signal being digitized, and can be defined as [29]:

$$
\operatorname{NCRR~}(\mathrm{dB})=10 \log _{10}\left(\frac{\text { average noise power @ input }}{\text { average noise power @ output }}\right)
$$

where the average noise power at the input of the ADC is calculated as:

$$
\overline{n_{i}^{2}(t)}=\int_{-\infty}^{+\infty} n_{i}^{2}(t) P_{n_{i}}\left(n_{i}\right) d n_{i}
$$

In this equation, $P_{n i}\left(n_{i}\right)$ is the probability density function for noise content of the neural signal, $n_{i}(t)$. Similarly, the average noise power of the signal at the output of the ADC is derived to be:

$$
\overline{n_{o}^{2}(t)}=\int_{-\infty}^{+\infty} n_{o}^{2}(t) P_{n_{i}}\left(n_{i}\right) d n_{o}
$$

where $n_{0}(t)$ is the noise content of the neural signal after passing through the ADC:

$$
n_{o}(t)=n_{i}(t) \times[\text { ADC Characteristic Curve }]
$$


Figure 16 illustrates how the B-Noise is reduced as it passes through the exp-ADC, already formulated by equations (14)-(16).

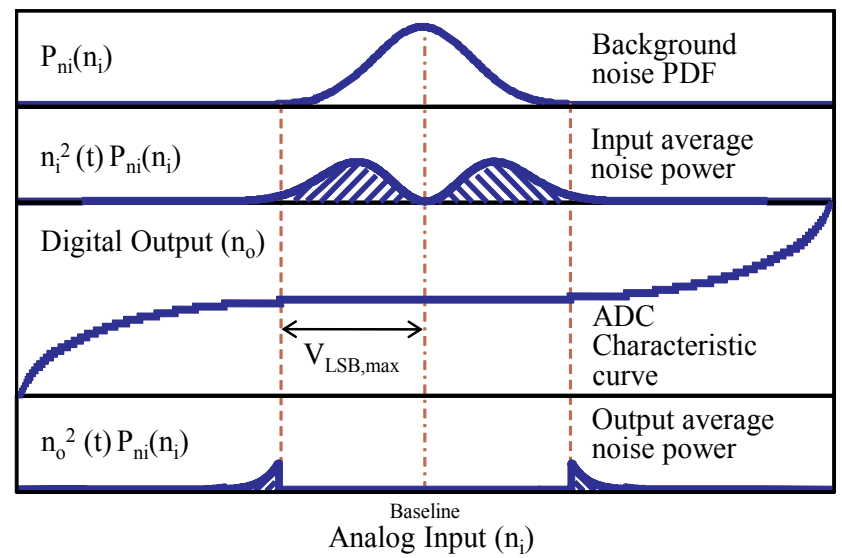

Figure 16. The process of B-Noise reduction as the signal passes through the NLADC, formulated by eqs. (14)-(16)

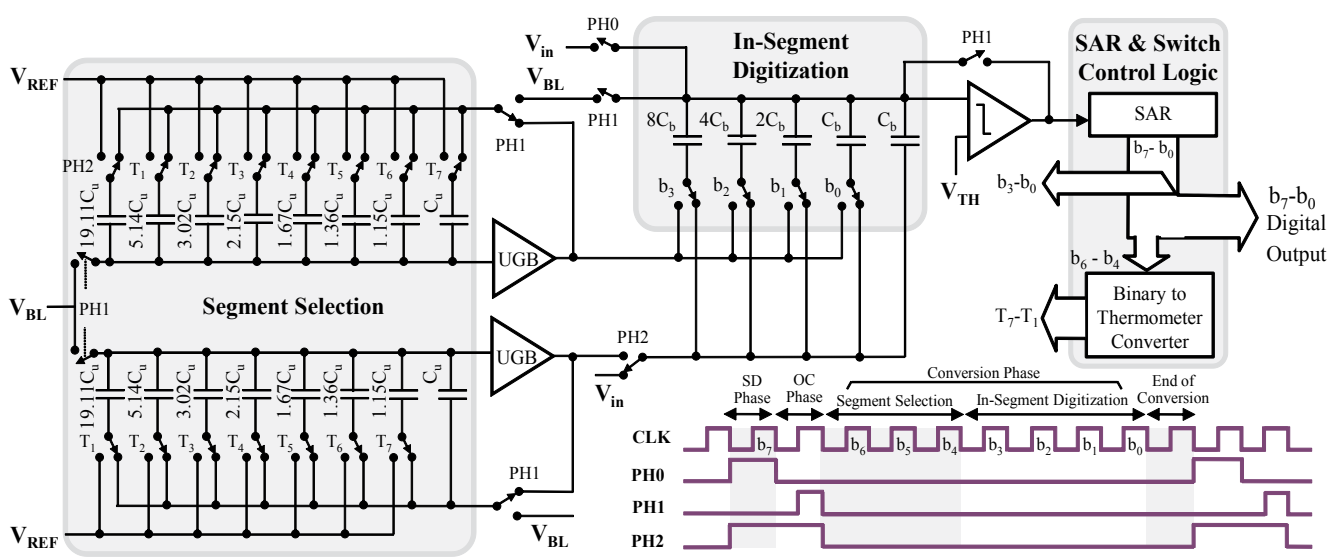

Figure 17. Circuit schematic for the two-step SAR ADC designed to implement exp-ADC and its simplified timing diagram

Circuit Design. To realize the proposed exp-ADC with reasonable power and silicon area, successive-approximation register (SAR) architecture was chosen [31]. In order to facilitate the realization of the exponential quantization function needed for the ADC, a piecewise-linear (PWL) approximation of the required function was implemented. As shown in the timing diagram of Figure 17, the proposed ADC operates in three phases: sign detection (SD), offset cancelation (OC), and conversion. In the SD phase, the analog input voltage, $\mathrm{v}_{\mathrm{in}}$, is compared with a certain threshold voltage, $\mathrm{V}_{\mathrm{TH}}$, which is temporarily set to the baseline voltage, $\mathrm{V}_{\mathrm{BL}}$. The result of this comparison determines the half range in which the input voltage is located. In 
the OC phase, an ordinary offset cancellation technique is applied on the comparator and buffers. In the conversion phase, successive approximation algorithm first finds the segment of interest, encoded by $b_{6} b_{5} b_{4}$, in 3 clock cycles. These 3 bits are converted to a 7-bit thermometer code, $\mathrm{T}_{7} \sim \mathrm{T}_{1}$, which will be used in the Segment Selection block to generate two analog voltages associated with the end points of the segment of interest. An in-segment linear A/D conversion process is then performed to determine the remained $4 \mathrm{LSBs}, \mathrm{b}_{3} \mathrm{~b}_{2} \mathrm{~b}_{1} \mathrm{~b}_{0}$, in 4 clock cycles. Finally, an end of conversion signal is generated to reset the ADC and prepare for the next conversion cycle. A low-voltage band-gap reference (BGR) was designed to generate the required baseline, reference and threshold voltages for the exp-ADC.

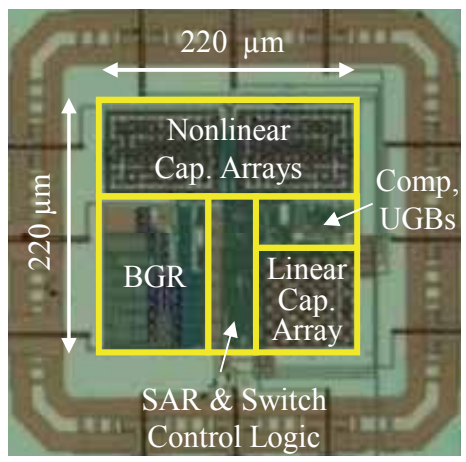

Figure 18. Chip photograph of the proposed NLADC

Experimental Results. The presented exp-ADC was fabricated in a standard 0.18- $\mu \mathrm{m}$ CMOS process. A chip photograph is shown in Figure 18, in which the chip occupies a total size of $220 \times 230 \mu \mathrm{m}^{2}$. The measured worst-case differential non-linearity (DNL) and integral nonlinearity (INL) are +0.8/-0.9 LSB and +4.3/-2.1 LSB, respectively. Table 1 summarizes the specifications of the NLADC and compares it with some of the nonlinear and linear ADCs reported in the literature.

A proof-of-concept prototype of a 4-channel neural recording system based on anti-logarithmic quantization was reported in [6]. As shown in the block diagram of Figure 19, a time-domain multiplexer (TDM) shares an anti-logarithmic ADC (AL-ADC) between 4 channels (each sampled at 20kSps). The output digital codes are then packed by the data packaging block to be transmitted to the outside world via a wireless link. At the external host, received signal is first recovered and then converted back to analog via a PC software. This inverse conversion is performed using a logarithmic DAC. Quantization characteristic function for the DAC is an exact inverse for that of the NLADC used in the recording system. To evaluate the operation of the system, neural signals recorded from the auditory cortex of a Guinea pig are used for in-vitro tests. Figure 20 shows the input signal to one of the neural channels before entering the nonlinear quantization process on the implant side along with the associated signal on the external setup after reconstruction. To verify the concept of the noise reduction caused by 
overlapping PDFs for B-Noise and Q-Noise, distributions of the measured quantization error along the input amplitude are depicted in Figure 21.

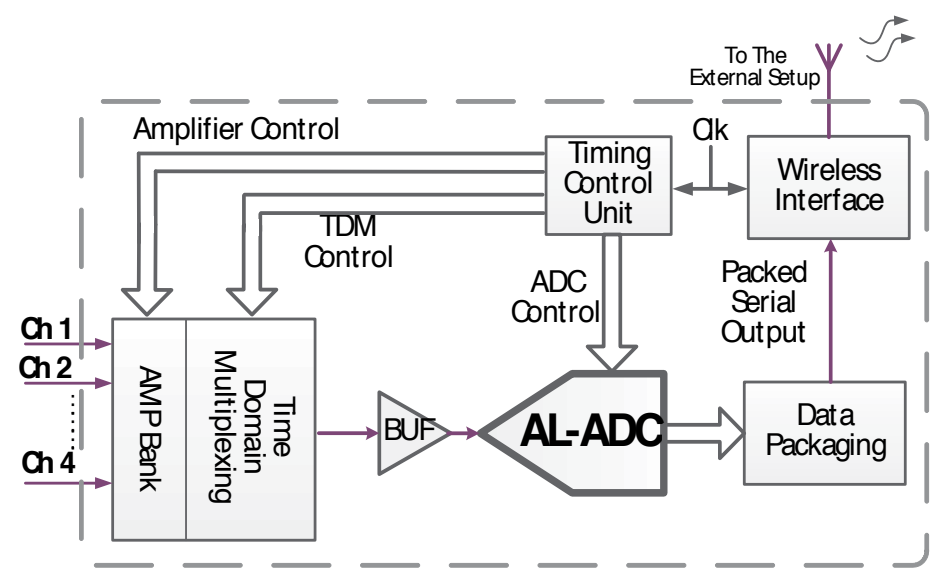

Figure 19. Block diagram of the prototyped neural recording system

\begin{tabular}{lccccc}
\hline \multicolumn{1}{c}{ Specification } & This Work & [34] & [35] & [36] & [37] \\
\hline Process $(\mu \mathrm{m})$ & 0.18 & 0.18 & 0.18 & 0.5 & 0.8 \\
\hline ADC Type & Exp. & Log. & Linear & Linear & Linear \\
\hline No. of Bits & 8 & 7 & 8 & 10 & 10 \\
\hline $\mathrm{P}_{\text {diss }}(\mu \mathrm{W})$ & 87.2 & 9.7 & 7.4 & 100 & 17.9 \\
\hline Area $\left(\mathrm{mm}^{2}\right)$ & 0.036 & 0.003 & 0.04 & 0.15 & 0.8 \\
\hline Supply $(\mathrm{V})$ & 1.8 & 1.8 & 1.8 & 3.3 & 2.8 \\
\hline Input Range $(\mathrm{V})$ & 1 & $\mathrm{~N} / \mathrm{A}$ & 0.6 & 2.5 & 0.8 \\
\hline DR $(\mathrm{dB})$ & 64.1 & 80 & 39.1 & N/A & 50.5 \\
\hline fs $(\mathrm{kS} / \mathrm{s})$ & 25 & 5 & 30 & 15 & 2.9 \\
\hline INL (LSB) & $+4.3 /-2.1$ & \pm 0.86 & \pm 1 & 0.6 & 0.98 \\
\hline DNL (LSB) & $+0.8 /-0.9$ & \pm 0.44 & N/A & 0.6 & 0.67 \\
\hline
\end{tabular}

Table 1. Performance Comparison

\section{Conclusion}

To overcome bandwidth limitation in the wireless telemetry of recorded neural data, a wide variety of data reduction techniques has been reported. These techniques range from spike reporting approaches such as spike detection and spike sorting techniques to mathematical approaches such as the discrete wavelet transform. Although it is proven that spike reporting 
approaches contain enough information to actuate prosthetic devices [33], in some other applications, such as neuroscientific studies, they are not satisfactory due to considerable loss of important information, e.g., spike wave shapes. Mathematical approaches, on the other hand, have been successful from the standpoint of data compression, while preserving wave shape of the spikes. Nonetheless, increasing the number of recording channels can result in the potential problems of these approaches: large silicon area and high power consumption. In contrast with all of the mentioned techniques, hardware approaches are capable of data reduction without adding any extra block to the microsystem. This is achieved by modifying the present hardware of the implant. An implementation of one of these approaches focusing on the ADC circuit of the system was presented and discussed in detail. The proposed method results in considerable reduction of bit-rate in multi-channel neural recording microsystems. Thus, efficient design of application-specific circuits for building blocks of neural implants should be taken into account as an appropriate method of data reduction.
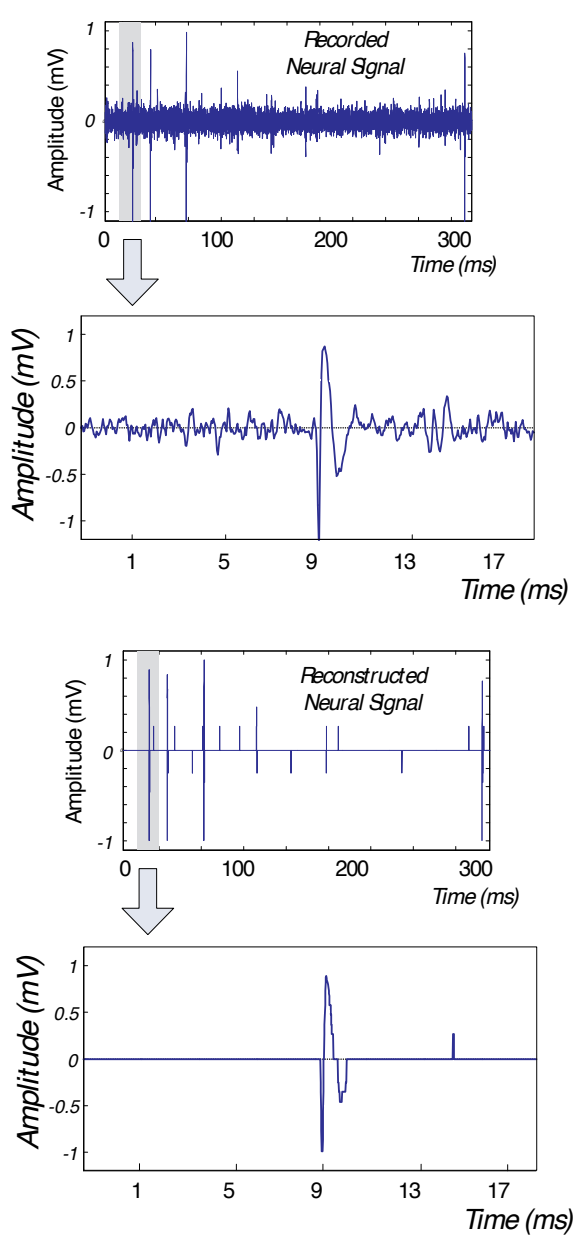

Figure 20. Recorded neural signal and reconstructed neural signal 


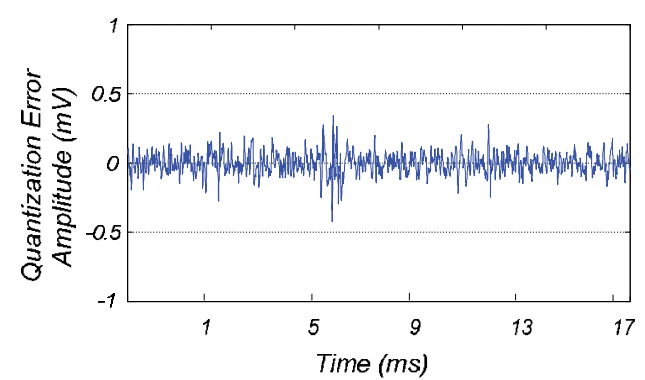

(a)

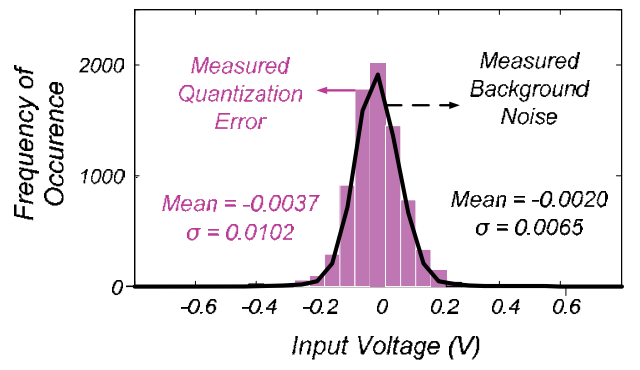

(b)

Figure 21. (a) Measured quantization error in the time domain (b) Distributions of the measured quantization error and the measured B-Noise

\section{Author details}

Mohsen Judy ${ }^{1}$, Alireza Akhavian² and Farzad Asgarian ${ }^{3}$

*Address all correspondence to: mjudy@ieee.org

1 EECS Dept., University of Tennessee, Knoxville, USA

2 ECE Dept., George Mason University, Fairfax,VA, USA

3 EECS Dept., University of Michigan, Ann Arbor, Michigan, USA

\section{References}

[1] Sodagar A.M., Perlin G.E., Yao Y., Najafi K., Wise K.D. An Implantable 64-Channel Wireless Microsystem for Single-Unit Neural Recording. IEEE Journal of Solid-State Circuits 2009; 44(9) 2591-2604. 
[2] Harrison R., Watkins P., Kier R., R. Lovejoy, Black D., Normann D., Solzbacher, F. A Low-Power Integrated Circuit for a Wireless 100-Electrode Neural Recording System. IEEE International Solid-State Circuits Conference 2006; 38. 958-965.

[3] Chae M.S., Yang Z., Yuce M.R. , Hoang L., Liu W. A 128-Channel 6 mW Wireless Neural Recording IC with Spike Feature Extraction and UWB Transmitter. IEEE Transactions on Neural Systems and Rehabilitation Engineering 2009; 17, 312-21.

[4] Wise K.D., Angell J.B., Starr A. An Integrated Circuit Approach to Extracellular Microelectrodes. IEEE Transactions on Biomedical Engineering 1970; 17, 238-247.

[5] Sodagar A.M. Integrated Circuit and System (ICAS) Lab Internal Report. K. N. Toosi University of Technology 2011; Tehran.

[6] Akhavian A., Judy M., Sodagar A.M. Anti-Logarithmic Quantization for Data Reduction in Multi-Channel Intra-Cortical Neural Recording Implants. Proceedings of the IEEE-EMBS International Conference on Biomedical and Health Informatics (BHI), Jan. 2012, Hong Kong, 416-419.

[7] Olsson III R., Wise K.D. A Three-Dimensional Neural Recording Microsystem with Implantable Data Compression Circuitry. IEEE Journal of Solid-State Circuits 2005; 40(12).

[8] Eberhart R.C, Dobbins R.W., Webber W.R.S. EEG Waveform Analysis Using CaseNet. Proceedings of the Annual International Conference of the IEEE Engineering in Medicine and Biology Society 1989; 2046-2047.

[9] Vaz F., Principe J.C. Neural Networks for EEG Signal Decomposition and Classification. Proceedings of the IEEE 17th Annual Conference on Engineering in Medicine and Biology Society1995; 1, 793-794.

[10] Eberhart, R.C., Dobbins, R.W. and Webber, W.R.S. Neural Network Design Considerations for EEG Spike Detection. Proceedings of the 1989 Fifteenth Annual Northeast Bioengineering Conference 1989; 97 - 98.

[11] Choi J.H., Jung H.K., Kim T. A New Action Potential Detector Using the MTEO and Its Effects on Spike Sorting Systems at Low Signal-to-Noise Ratios. IEEE Transactions on Biomedical Engineering 2006; 53, 738-746.

[12] B. Gosselin and M. Sawan. An Ultra Low-Power CMOS Automatic Action Potential Detector IEEE Transactions on Neural Systems and Rehabilitation Engineering 2009; 17, 346-353.

[13] Sodagar A.M, Wise K.D, Najafi K. A Fully Integrated Mixed-Signal Neural Processor for Implantable Multichannel Cortical Recording. IEEE Transactions on Biomedical Engineering 2007; 54(6), 1075-1088. 
[14] Iyad Obeid, Patrick D Wolf. Evaluation of spike-detection algorithms fora brain-machine interface application. IEEE Transactions on Biomedical Engineering 2004; 51, 905-911.

[15] Harrison R.R. A Low-Power Integrated Circuit for Adaptive Detection of Action Potentials in Noisy Signals. Proceedings of the 2003 International Conference of the IEEE Engineering in Medicine and Biology Society 2003; 3325-3329.

[16] Sodagar A.M. Analysis and Design of Analog Spike Detectors with Adaptive Threshold. Internal Report, Center for Wireless Integrated Microsystems (WIMS) 2005, University of Michigan; Ann Arbor, MI.

[17] Rogers C.L., Harris J.G. A Low-Power Analog Spike Detector for Extracellular Neural Recordings. Proceedings of the 2004 11th IEEE International Conference on Electronics Circuits and Systems 2004; 290-293.

[18] Roy J.F., Sawan M. A Fully Reconfigurable Controller Dedicated to Implantable Recording Devices. The 3rd International IEEE-NEWCAS Conference 2005; 303-306.

[19] Dumortier C., Gosselin B., Sawan M. Wavelet Transforms Dedicated to Compress Recorded ENGs from Multichannel Implants: Comparative Architectural Study. Proceedings of the 2006 IEEE International Symposium on Circuits and Systems 2006; 2129-2132.

[20] Oweiss K.G. A Systems Approach for Data Compression and Latency Reduction in Cortically Controlled Brain Machine Interfaces. IEEE Transactions on Biomedical Engineering 2006, 53(7), 1364-1377.

[21] Oweiss K.G., Mason A., Suhail Y., Kamboh A.M., Thomson K.E. A Scalable Wavelet Transform VLSI Architecture for Real-Time Signal Processing in High-Density IntraCortical Implants. IEEE Transactions on Circuits and Systems-Part I: Regular Papers 2007; 54(6), 1266-1278.

[22] Kamboh A.M., Raetz M., Oweiss K.G., Mason A. Area-Power Efficient VLSI Implementation of Multichannel DWT for Data Compression in Implantable Neuroprosthetics. IEEE Transactions on Biomedical Engineering 2007; 1 (2), 128-135.

[23] Kamboh A.M., Oweiss K.G., Mason A.J. Resource Constrained VLSI Architecture for Implantable Neural Data compression Systems. Proceedings of the 2009 IEEE International Symposium on Circuits and Systems 2009; 1481-1484.

[24] Kamboh A.M., Yang Y., Oweiss K.G., Mason A.J. Design of a Configurable Neural Data Compression System for Intra-Cortical Implants. Proceedings of the 2010 IEEE International Symposium on Circuits and Systems 2010; 3473-3476.

[25] Zhang, F., Aghagolzadeh, M. and Oweiss, K.G. An Implantable Neuroprocessor for Multichannel Compressive Neural Recording and On-the-fly Spike Sorting with Wireless Telemetry. Proceedings of the 2010 IEEE Biomedical Circuits and Systems Conference 2010; 3-6. 
[26] Shaeri M.A., Sodagar A.M., Abrishami-Moghaddam H. A 64-Channel Neural Signal Processor/ Compressor Based on Haar Wavelet Transform. Proceedings of the 2011 Annual IEEE Engineering in Medicine and Biology Society Conference 2011; 6409-6412.

[27] Mallat S. G. A Theory for Multiresolution Signal Decomposition: The Wavelet Representation. IEEE Transactions on Pattern Analysis and Machine Intelligence 1989; 2(7).

[28] Li B., Tenhunen H. A Second Order Sigma Delta Modulator Using Semi-uniform Quantizer with 81dB Dynamic Range at 32x OSR. Proc. of the 28th European SolidState Circuits Conference, Florence, Sept. 2002, 579-582

[29] Judy M., Sodagar A.M., lotfi R. A Nonlinear Signal-Specific ADC for Efficient Neural Recording. Proceedings of the 2010 IEEE Conference on Biomedical Circuits and Systems, Paphos, Cyprus Nov. 2010, 17-20.

[30] Burns M., Roberts GW. An Introduction to Mixed-Signal IC Test and Measurement, Oxford University Press, 2000.

[31] Judy M., Sodagar A.M., Lotfi R., Sawan M. Nonlinear Signal-Specific ADC for Efficient Neural Recording in Brain-Machine Interfaces, IEEE Transactions on Biomedical Circuits and Systems 2014; 8(3), 371-381.

[32] Lee J., Rhew H.G., Kipke D., Flynn M. A 64 Channel Programmable Closed-loop Deep Brain Stimulator with 8 Channel Neural Amplifier and Logarithmic ADC. Symposium on VLSI Circuits Digest of Technical Papers, Hawaii, June 2008. 76-77.

[33] Nurmikko A.V., Donoghue J.P., Hochberg L.R., Patterson W.R., Song Y., Bull C.W., Borton D.A., Laiwalla F., Park S., Ming Y., Aceros J. Listening to Brain Microcircuits for Interfacing with External World, Progress in Wireless Implantable Microelectronic Neuroengineering Devices. Proceedings of the IEEE 2010; 98 (3), 375-388.

[34] Guo J., Sonkusale S. An Area-Efficient and Low-Power Logarithmic A/D Converter for Current-Mode Sensor Array, IEEE Sensors Journal 2009; 9(12), 2042-2043.

[35] Robert P., Gosselin B., Ayoub A.E., Sawan M. An Ultra-Low-Power Successive-Approximation-Based ADC for Implantable Sensing Devices, in Proceedings of the 49th IEEE Int. Midwest Symposium on Circuits and Systems Aug. 2006, 7-11.

[36] R. R. Harrison, P. T. Watkins, R. J. Kier, R. O. Lovejoy, D. J. Black, B. Greger, and F. Sozbacher, "A Low-Power Integrated Circuit for a Wireless 100-Electrode Neural Recording System," IEEE J. Solid-State Circuits 2007; 42(1), 123-133.

[37] Bonfini G., Brogna A.S., Garbossa C., Colombini L., Bacci M., Chicca S., Bigongiari F., Guerrini N.C., Ferri G. An Ultralow-Power Switched Opamp-Based 10-b Integrated ADC for Implantable Biomedical Applications, IEEE Transactions on Circuits and Systems I: Regular Papers 2004; 51(1), 174-177. 


\title{
Multivariate Computing and Robust Estimating for Outlier and Novelty in Data and Imaging Sciences
}

\author{
Michelle Yongmei Wang and Chris E. Zwilling \\ Additional information is available at the end of the chapter \\ http://dx.doi.org/10.5772/59750
}

\section{Introduction}

Data science is an evolutionary step in interdisciplinary fields incorporating computer science, statistics, engineering, and mathematics. At its core, data science involves using automated and robust approaches to analyze massive amounts of data and to extract informative knowledge from them. Data science transforms traditional ways of analyzing problems and creates powerful new solutions. Diverse computational and analytical techniques contribute to data science. In this chapter, we review and also propose one type of data mining and pattern recognition strategy that has been under development in multiple disciplines (e.g. statistics and machine learning) with important applications ---- outlier or novelty detection [1-4].

In biomedical engineering, data science can make healthcare and medical imaging science not only more efficient but also more effective for better outcomes and earlier detection. Outlier and novelty detection in these domains plays an essential role, though it may be underappreciated and not well understood by many biomedical practitioners. From the healthcare point of view, an outlier probably reflects the need for heightened vigilance, if not full-fledged intervention. For example, an abnormally high glucose reading for a diabetic patient is an outlier which may require action. In high-dimensional medical imaging, developing automated and robust outlier detection methods is a critical preprocessing step for any subsequent statistical analysis or medical research.

An exact definition of an outlier or novelty typically depends on hidden assumptions regarding the data structure and the associated detection method, though some definitions are general enough to cope with varieties of data and methods. For example, outliers can be considered as patterns in data that do not conform to a well-defined notion of "normal" behavior, or as observations in a data set which appear to be inconsistent with the remainder of that set of data. Figure 1 shows outliers in a 2-dimensional dataset. Since most of the 
observations fall into clusters N1 and N2, they are two "normal" regions; while points in region $\mathrm{O} 1$ as well as points $\mathrm{o} 2$ and $\mathrm{o} 3$ are outliers (in red), due to their sufficiently far distance from the "normal" regions. Identifying observations inconsistent with the "normal" data, or detecting previously unobserved emergent or novel patterns is commonly referred to as outlier detection or novelty detection $[5,6]$. The distinction between novel patterns and outliers is that the novel patterns are often incorporated into the "normal" model after being detected whereas outliers are typically removed or corrected. This chapter aims to consider both detection schemes and sometimes treat them interchangeably for some general purposes.

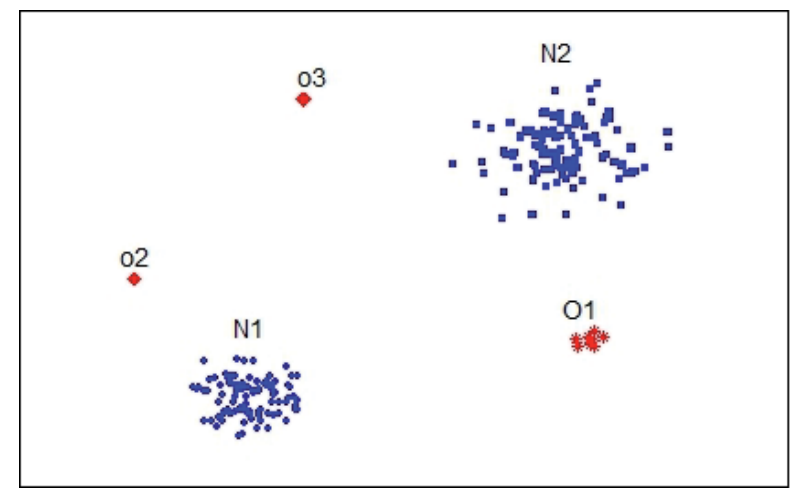

Figure 1. An example of outliers in a 2-dimensional dataset.

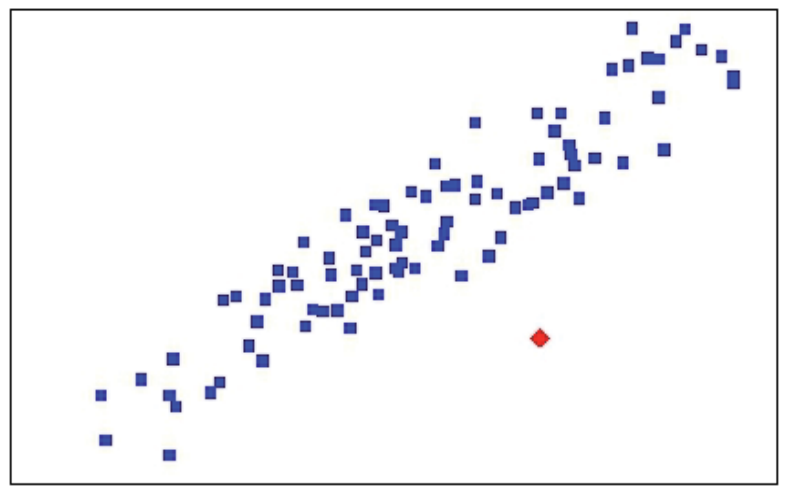

Figure 2. A 2-dimensional dataset with a multivariate outlier (lower right diamond in red).

Outlier and novelty detection methods can be divided into univariate and multivariate approaches [7-10]. The early univariate methods typically assume a known data distribution, for example, independently and identically distributed (i.i.d). In addition, many tests for detecting univariate outliers further assume the distribution parameters and the outlier types are known. However, these assumptions may be violated in real applications. Moreover, in many situations multivariate outliers can not be identified when each variable is examined independently. Multivariate analysis is usually required in these cases for precise outlier detection, which 
allows for interactions over different variables to be taken into account within the class of data. Figure 2 illustrates 2-dimensional data points, with the lower right observation (in red) a clear multivariate outlier but not a univariate one. When analyzing each measure separately with respect to the spread of values along the two dimensions, they are close to the center of the univariate distributions. Therefore, the relationships between the two variables shall be considered when testing for outliers, leading to multivariate methods that are the focus of this chapter.

Another related topic is robust statistics for estimation that can handle outliers or at least is less sensitive to the influence of outliers. Robust statistics perform well with data drawn from a wide range of probability distributions, especially for distributions that are not normally distributed. Robust statistical methods have been developed for many common problems, such as estimating data properties including location and scatter or estimating model parameters as in regression analysis [10,11]. One motivation is to produce statistical methods that are not unduly affected by outliers. Another motivation is to provide methods with good performance when there are small departures from a parametric distribution. A typical procedure or example of the former case is for multivariate estimation of location and covariance as well as for multivariate outlier detection. In this case, as a first step, the approaches often try to search for a minimum number of observations with a certain degree of confidence being outlier-free. Based on this starting subset, location and covariance can be estimated robustly. In a second step, outliers can be identified through computing the observations' distances with respect to these initial estimates.

In this chapter, we review and also propose statistical and machine learning approaches for outlier and novelty detection, as well as robust methods that can handle outliers in data and imaging sciences. In particular, robust statistical techniques based on the Minimum Covariance Determinant $(\mathrm{MCD})$ are introduced in Section 2, which include a classical and fast computation scheme of MCD and a few robust regression strategies. We present our newly developed multivariate Voronoi outlier detection (MVOD) method for time series data and some preliminary results in Section 3. This approach copes with outliers in a multivariate framework via designing and extracting effective attributes or features from the data; Voronoi diagrams allow for automatic configuration of the neighborhood relationship of the data points, facilitating the differentiation of outliers and non-outliers. Section 4 reviews varieties of machine learning methods for novelty detection, with a focus on probabilistic approaches. In Section 5, we present some existing and new technologies related to outliers and novelty in the area of imaging sciences. Section 6 provides concluding remarks of the chapter.

\section{Robust statistical methods using Minimum Covariance Determinant (MCD)}

The Minimum Covariance Determinant (MCD) estimator is a highly robust estimator of multivariate location and scatter. Since estimating the covariance matrix is the cornerstone of 
many multivariate statistical methods, the MCD has also been used to develop robust and computationally efficient multivariate techniques.

\subsection{MCD and its fast computing algorithm}

Given a dataset consisting of $p$ variables and $n$ observations, i.e. a $n \times p$ data matrix, we can represent this multivariate data as $X=\left(x_{1}, \ldots, x_{n}\right)^{\prime}$, where $x_{i}$, for $i=1, \ldots, n$, is the $i$ th observation and a $p$-dimensional vector. A classical distance measure, Mahalanobis distance (MD), is given in Equation (1); it only depends on the sample mean $\left(\hat{\mu}_{M D}\right)$ and sample covariance matrix $\left(\hat{\Sigma}_{M D}\right)$, both of which are computed from the entire set of data.

$$
M D(x)=\sqrt{\left(x-\hat{\mu}_{M D}\right)^{\hat{\Sigma}^{-1}}{ }_{M D}^{-1}\left(x-\hat{\mu}_{M D}\right)}
$$

A point with a larger Mahalanobis distance will lie further away from the center of the data cloud than a point with a smaller Mahalanobis distance. A robust distance (RD) measure is achieved if we substitute the MCD estimate of mean $\left(\hat{\mu}_{M C D}\right)$ and covariance $\left(\hat{\Sigma}_{M C D}\right)$ into Equation (1), which yields Equation (2).

$$
R D(x)=\sqrt{\left(x-\hat{\mu}_{M C D}\right)^{\prime} \Sigma_{M C D}^{-1}\left(x-\hat{\mu}_{M C D}\right)}
$$

The classical estimates can be sensitive to outliers, while the MCD estimate is robust $[8,12$, 13]. The MCD relies on a subset of the total observations. Choosing this subset makes the algorithm robust because it is less sensitive to the influence of outlying points. Figure 3 illustrates the difference between these two estimates; it is a scatterplot of the distances for an example dataset with 70 observations and 2 variables (i.e. $n=70, p=2$ ). The two ellipses are two outlier thresholds, determined by the 0.975 chi-square quantile with 2 degrees of freedom when the classical and robust estimates are used, respectively. The dashed blue ellipse marks off the $97.5 \%$ outlier threshold for the classical Mahalanobis distance, suggesting that two observations lying beyond the ellipse are outliers. The $97.5 \%$ outlier threshold for the robust distance measure is marked off by the solid red ellipse, suggesting ten points are outliers.

The MCD has a user-determined parameter, $h$, which specifies the size of the subset of data to base the estimate upon. It is constrained by $[(n+p+1) / 2] \leq h \leq n$. The $h$ observations are chosen such that the determinant of the sample covariance matrix is minimal (but not minimized in the formal sense, because it relies on a sampling algorithm instead of a loss function). The MCD is optimally designed for elliptically symmetric unimodal distributions, such as the commonly encountered multivariate normal distribution. The MCD is most robust when $h=[(n+p+1) / 2]$. But this causes low efficiency [14] (at least for normal probability distributions), which can be increased (while retaining high robustness) by applying reweighted estimators [15, 16]. Robust statistical estimators are commonly evaluated both on their breakdown value and influence functions. The MCD is a high breakdown estimator and its influence function appears bounded, which is desirable. An alternative strategy that employs 


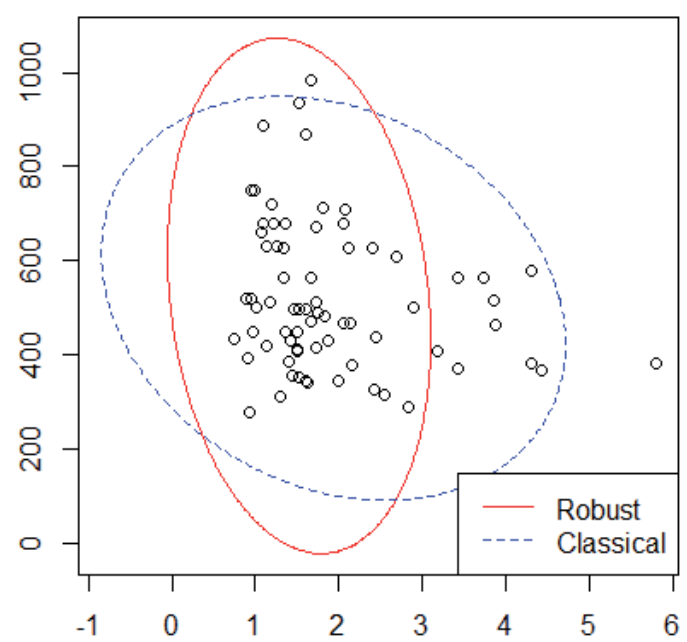

Figure 3. Outlier thresholds, as represented by ellipses, based on a classical and a robust scheme.

Delaunay triangulation to identify a robust outlier-free subsample in an adaptive way was presented in [17].

Computing the exact MCD is possible but computationally difficult, as it requires the evaluation of all $\left(\begin{array}{l}n \\ h\end{array}\right)$ subsets of size $h$. Even though the MCD is a powerful robust estimator, it has only become widely used since the development of the so-called Fast-MCD algorithm [18] which we summarize below. Assume we have a dataset $X=\left(x_{1}, \ldots, x_{n}\right)^{\prime}$ and let $H_{1} \subset\{1 \ldots n\}$ represent a $h$-subset of length constrained by $[(n+p+1) / 2] \leq h \leq n$. Denote this first $h$-subset as $H_{1}$ and it is randomly chosen from the entire dataset. Compute the mean $\hat{\mu}_{M C D, 1}$ and covariance matrix $\hat{\Sigma}_{M C D, 1}$ of $H_{1}$, as well as the determinant of $\hat{\Sigma}_{M C D, 1}$, denoted as $\operatorname{det}\left(\hat{\Sigma}_{M C D, 1}\right)$. Then compute the distance of all $n$ observations in the entire dataset (and not just the $h$ comprising the initial subset) using Equation (2). Next, these distances are ordered from smallest to largest. Retain an equivalent number of observations from this ordering as chosen in the initial $h$-subset; but instead of being chosen arbitrarily as in the initial subset, these are chosen such that they have the smallest distances as defined by the order statistics. Call this subset of observations $H_{2}$ and compute $\hat{\mu}_{M C D, 2}, \hat{\Sigma}_{M C D, 2}$ and $\operatorname{det}\left(\hat{\Sigma}_{M C D, 2}\right)$. Now Equation (3) must be true:

$$
\operatorname{det}\left(\hat{\Sigma}_{M C D, 2}\right) \leq \operatorname{det}\left(\hat{\Sigma}_{M C D, 1}\right)
$$

Going from $H_{1}$ to $H_{2}$ is called a C-step for "Concentration step", because the algorithm concentrates on the $h$ observations with the smallest distances and $\operatorname{det}\left(\hat{\Sigma}_{M C D, 2}\right)$ is more concentrated (or equivalently, has a smaller determinant). This C-step is repeated numerous or sufficient times, with each iteration using a different initial $h$-subset. The 10 subsets that yield the smallest determinants overall are retained and further concentrated until convergence is met. 


\subsection{Robust multivariate regression and Multivariate Least-Trimmed Squares (MLTS) estimator}

Section 2.1 introduced the robust MCD estimator and showed how the MCD can be computed efficiently. In this section, we review different frameworks for applying the MCD estimator to multivariate regression. These methods offer robust alternatives to standard multiple regression analysis.

We first look at robust multivariate regression in [19]. Suppose we have a full dataset of predictors and responses containing no outliers; computing the regression parameter estimates from the full dataset using a least squares procedure will yield accurate results. With outliers present in the dataset, the MCD is used to search for a subset of size $h$ whose covariance matrix has the smallest determinant with $h$ constrained by $[(n+p+q+1) / 2] \leq h \leq n$, where $p$ and $q$ are respectively the numbers of variables for the predictor and response matrices, and $n$ is the number of observations. Then, using this subset $h$, the sample mean and covariance estimates are calculated, which would allow for accurate estimation of the regression coefficients and covariance matrix of the errors in the presence of outliers.

Different from the above robust multivariate regression, the multivariate least trimmed squares (MLTS) estimator in [20] first fits a regression model to the subset of data and then calculates the covariance matrix of the residuals. The estimator is defined by minimizing a trimmed sum of squared Mahalanobis distances, and can be computed by a fast algorithm. Let us consider the classical multivariate regression framework. Assume we have a sample of data defined as $Z_{n}=\left\{\left(x_{i}, y_{i}\right) ; i=1, \ldots, n\right\}$ and let $X=\left(x_{1}, \ldots, x_{n}\right)^{\prime}$ denote the design (or predictor) matrix and $Y=\left(y_{1}, \ldots, y_{n}\right)^{\prime}$ denote the response matrix. The regression model is:

$$
Y=X \beta+\varepsilon
$$

The classical least squares estimator for the regression parameter is given by:

$$
\hat{\beta}_{L S}=\left(X^{\prime} X\right)^{-1} X^{\prime} Y
$$

and the classical estimator of the scatter matrix is:

$$
\hat{\Sigma}_{L S}=\frac{1}{n-p}\left(Y-X \hat{\beta}_{L S}\right)^{\prime}\left(Y-X \hat{\beta}_{L S}\right)
$$

These classical least squares estimators are sensitive to outliers. A robust alternative to these estimators based on the residuals is achieved as below. For any $\beta \in \mathbb{R}^{p+q}$, let $r_{i}(\beta)=y_{i}-\beta^{\prime} x_{i}$ denote the residuals from the fitted regression model. Furthermore let $\mathcal{H}=\{H \subset\{1, \ldots, n\} \mid \# H=h\}$ be the collection of all subsets of size $h$. For any $H \in \mathcal{H}$ denote $\hat{\beta}_{L S}(H)$ the least-squares fit solely on the observations $\left\{\left(x_{j}, y_{j}\right) ; j \in H\right\}$. In addition, for all $H \in \mathcal{H}$ and $\beta \in \mathbb{R}^{p+q}$ denote the covariance matrix of the residuals with respect to the fit $\beta$, belonging to subset $H$ as: 


$$
\operatorname{cov}(H, \beta)=\frac{1}{h} \sum_{j \in H}\left(r_{j}(\beta)-\bar{r}_{H}(\beta)\right)\left(r_{j}(\beta)-\bar{r}_{H}(\beta)\right)^{\prime}
$$

where $\bar{r}_{H}(\beta)=\frac{1}{h} \sum_{j \in H} r_{j}(\beta)$. If we let $\hat{\Sigma}_{L S}(H)=\operatorname{cov}\left(H, \hat{\beta}_{L S}(H)\right)$ for any $H \in \mathcal{H}$, the MLTS estimator is defined as:

$$
\hat{\beta}_{M L T S}\left(Z_{n}\right)=\hat{\beta}_{L S}(\hat{H})
$$

where $\hat{H} \in \underset{H \in \mathcal{H}}{\operatorname{argmin}} \operatorname{det} \hat{\Sigma}_{L S}(H)$. The covariance of the errors can be estimated by

$$
\hat{\Sigma}_{M L T S}\left(Z_{n}\right)=c_{\alpha} \hat{\Sigma}_{L S}(\hat{H})
$$

where $c_{\alpha}$ is a consistency factor. The observations corresponding to the residuals with the smallest determinant of the covariance matrix can then be used to give robust results for the regression parameters.

Using the MLTS as a means to estimate the parameters of the Vector Autoregressive (VAR) Model was presented in [21]. The VAR model is popular for modeling multiple time series. Estimation of its parameters based a typical least squares method is unreliable when outliers are present in the data. Development of robust procedures for multiple time series analysis is more crucial than for univariate time series analysis due to the data correlation structure. Experimental results in [21] show that applying the reweighted MLTS procedure to the VAR model leads to robust multivariate regression estimators with improved performance.

\section{Multivariate Voronoi Outlier Detection (MVOD) for time series}

In order to better analyze multivariate time series data, we have recently proposed a general outlier detection method based on the mathematical principles of Voronoi diagrams. It is general because different attributes or features can be extracted from the data for Voronoi diagram construction. These attributes or features can be designed based on the nature of the data and the outliers. This has the potential to increase the accuracy and precision of outlier detection for specific application problems.

\subsection{Background on Voronoi diagram}

Our new method requires a Voronoi diagram, which is composed of Voronoi cells [22]. A Voronoi diagram is a way of dividing space into regions. Assume we have a set $S$ of $n$ points, $p_{1}, \ldots, p_{n}$ in the Euclidean plane. Let $V\left(p_{i}\right)$ denote a Voronoi cell, which is a subdivision of the plane where the set of points $q$ are closer or as close to $p_{i}$ than to any other point in $S$. This is expressed formally in Equation (10): 


$$
V\left(p_{i}\right)=\left\{q \mid \operatorname{dist}\left(p_{i}, q\right) \leq \operatorname{dist}\left(p_{j}, q\right), \forall j \neq i\right\}
$$

where dist is the Euclidean distance function. The set of all Voronoi cells for all $n$ points comprises a Voronoi diagram.

Figure 4 shows part of a Voronoi diagram, assuming Euclidean distance between the points. If one used a different distance metric, the Voronoi diagram would be configured differently. The plane is decomposed into $n$ convex polygonal regions, one for each $p_{i}$. Vertices (or nodes) are called Voronoi vertices and are equidistant to three or more sites. Voronoi edges are the segments defined as the boundaries between two Voronoi cells and contain all the points in the plane equidistant to the two nearest sites. The boundaries of a Voronoi cell $V\left(p_{i}\right)$ cannot exceed $n-1$ edges. Three important theorems apply to Voronoi diagrams:

Theorem 1: Every nearest neighbor of $p_{i}$ defines an edge of the Voronoi polygonV $\left(p_{i}\right)$.

Theorem 2: Every edge of the Voronoi polygonV $\left(p_{i}\right)$ defines a nearest neighbor of $p_{i}$.

Theorem 3: Forn $\geq 3$, a Voronoi diagram onnpoints has at most $2 n-5$ vertices and $3 n-6$ edges.

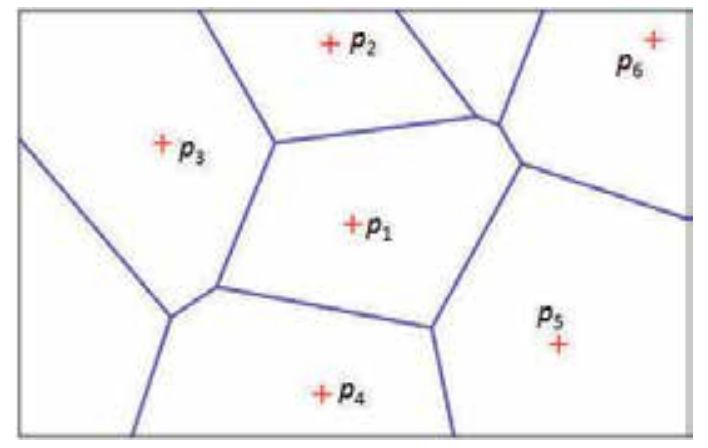

Figure 4. A subset of Voronoi cells from a Voronoi diagram.

\subsection{Our proposed MVOD method}

The Voronoi Outlier Index (VOInd) used in our Multivariate Voronoi Outlier Detection (MVOD) method is based upon the Voronoi notion of nearest neighbors. For a point $p_{i}$ of set $S$, the nearest neighbors of $p_{i}$ defined by the Voronoi polygon $V\left(p_{i}\right)$ are the Voronoi nearest neighbor of $p_{i}$, denoted as $V_{N N}\left(p_{i}\right)$. In Figure 4 the nearest Voronoi neighbors to point $p_{1}$ are $p_{2}, p_{3}, p_{4}, p_{5}$ and $p_{6}$. For each point in the data set, our method uses the nearest neighbors to compute an index (i.e. VOInd) of how likely that point is an outlier. It is multivariate because it aggregates information across all individual time series, thus retaining features which might be common to the entire interlocking set of variables.

Our method is based upon the geometric principles of Voronoi diagrams for defining the neighborhood relationship of the data points and this facilitates the assignment of group or 
data membership (i.e. outliers and non-outliers). Construction of a two dimensional Voronoi diagram requires two coordinates for each data point. Based on the nature of the data and the nature of the outliers to be identified, we can embed their attributes into the coordinates via extracting different valid features from the data. Here, we present one such case of the MVOD framework for feature extraction; but many others are also possible, including nonparametric forms. Figure 5 overviews the process and the rest of this subsection explains the steps in more detail.

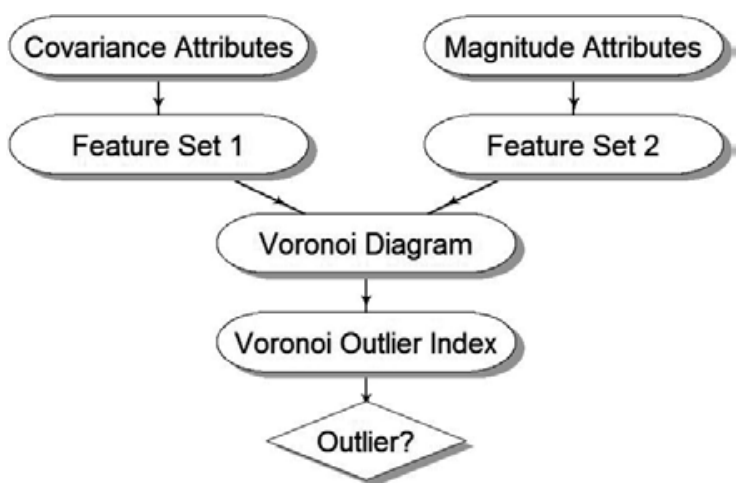

Figure 5. Flow chart of our MVOD procedure for outlier detection.

Feature Set 1 (feature value for the x-coordinate): In order to determine how a single observation (at the same time point, across all time series under consideration) affects the covariance matrix (which is a measure of inter-relationship among the individual time series), we remove a given point from the data set and then use Multivariate Least Trimmed Squares (MLTS) introduced in Section 2.2, which computes a determinant of the covariance matrix without that point, to yield a single feature value. Removing one observation at a time is not part of the original MLTS method; we introduced this modification into the procedure to show the effect of removing that observation from all the time series. This determinant is known as the generalized variance and can be interpreted as a volume. If we have outlying observations, the volume will be larger. But if we remove those outlying observations, the volume will be smaller.

Feature Set 2 (feature value for the y-coordinate): This is a two-step process. In Step 1, we take the absolute value of all time series points for all variables, which provides some information about the total magnitude each time series contributes. However, sometimes, magnitude alone is not sufficient for outlier detection as some data may have less extreme values than those data with the largest magnitudes but are actually outliers for the data [23]. One way to address this issue is by using the residual to calculate the feature value for the y-coordinate. So Step 2 consists of fitting an appropriate model to the multivariate time series data and then computing the residuals. Here, we estimate the parameters for a Multivariate Vector AutoRegressive (MVAR) model because our simulated data are generated from this type of model. Once these residuals are obtained for each time series, they are squared and then summed 
across all time series. Finally, the feature value for the y-coordinate is determined by multiplying together the results of Step 1 and Step 2. Specifically, let $y_{i}$ denote the $i$ th observation of the time series of length $n$, and $r_{i}$ denote the residual after fitting the MVAR model for each observation. Then, for all $i=1, \ldots, n$ we compute this feature value as in Equation (11):

$$
\sum_{1}^{n} a b s\left(y_{i}\right) \times \sum_{1}^{n} r_{i}^{2}
$$

Although a regression model is used here in Step 2 to extract the feature value, in fact, our method does not require this model. With either Step 1 or Step 2 alone, we will have a corresponding nonparametric or parametric basis, both of which could be suitable for different applications or datasets.

Voronoi Outlier Index (VOInd): Given the two feature sets (from the above procedures) that can be used as the $x$-coordinates and y-coordinates for the data, we construct the Voronoi diagram based on Section 3.1 and compute a Voronoi Outlier Index (VOInd) for each time series point. The VOInd for point $p_{i}$ has as its numerator the sum of the Euclidean distance (dist) between each point and all its nearest neighbors. This is divided by the denominator term which is the number of nearest neighbors, yielding an average density, as expressed in Equation (12):

$$
\operatorname{VOInd}\left(p_{i}\right)=\sum_{O \in V_{N N}\left(p_{i}\right)} \operatorname{dist}\left(p_{i}, o\right) /\left|V_{N N}\left(p_{i}\right)\right|
$$

Note that a Voronoi outlier factor is used in [24] as the index which, however, was completely univariate in nature, since the $\mathrm{x}$-and $\mathrm{y}$-coordinates were based on a univariate time series. One of our primary motivations for this study is to create a novel and general MVOD method, which can detect outliers in time series data in a multivariate framework with multiple, interlocking sets of variables.

\subsection{Experimental evaluation and results}

Simulation Setup and Data Generation: For each analysis, 5 multivariate autoregressive time series, each containing 100 observations, were simulated 25 times using published Matlab code [25]. The time series were generated using a Gaussian process with mean 0 and standard deviation 1 . The variance/covariance matrix contained all 1's on the diagonal and all 0 's on the off-diagonals. A total of 12 different unique multivariate time series were constructed, each with differing numbers of outliers and strength/magnitude of the outliers. 5, 10 or 15 outliers were introduced into a time series and the magnitude of those outliers was 1, 2, 3, 4 or 5 . All combinations of number of outliers and outlier magnitude were constructed; but they were never mixed. For instance, if we introduced 5 outliers of magnitude 3 into a simulated time series, only 5 outliers of magnitude 3 were used for all 25 simulated time series in that set. The observation to which the outliers were introduced into the time series was always determined randomly. Once the observations had been selected for outlier introduction, the same number 
of outliers for the given magnitude was added or subtracted (if the original observation was negative) to each of the five components of the multivariate time series.

Validation Criteria and Procedure: We validated and compared the performance of our new Voronoi Outlier Detection (MVOD) method with the MLTS, using True and False Positive Rates (TPR and FPR) as defined in Table 1.

\begin{tabular}{|c|c|c|c|c|}
\hline & & \multirow{2}{*}{\multicolumn{2}{|c|}{$\begin{array}{l}\text { Outlier in data? } \\
\text { (Gold Standard) }\end{array}$}} & \multirow{3}{*}{$\begin{array}{c}\text { Definition of TPR and FPR } \\
\text { True Positive Rate (TPR) } \\
=\mathrm{TP} /(\mathrm{TP}+\mathrm{FN})\end{array}$} \\
\hline & & & & \\
\hline & & Yes & No & \\
\hline \multirow{2}{*}{$\begin{array}{l}\text { Detected } \\
\text { Outlier? }\end{array}$} & Yes & $\mathrm{TP}$ & $\mathrm{FP}$ & \multirow{2}{*}{$\begin{array}{c}\text { False Positive Rate (FPR) } \\
=\mathrm{FP} /(\mathrm{FP}+\mathrm{TN})\end{array}$} \\
\hline & No & $\mathrm{FN}$ & $\mathrm{TN}$ & \\
\hline
\end{tabular}

Table 1. Definition of True (TPR) and False (FPR) Positive Rate.

The alpha parameter in the MLTS method determines both the size of the subset to use as well as a critical value in a chi-square distribution. If an observation is greater than this threshold in the chi-square distribution, then the MLTS method flags the observation as an outlier. However, it is critical to note that a one-to-one correspondence does not exist between the alpha value chosen, and the number of outliers flagged. For instance, one could set alpha at 0.10 but only have 2 out of 100 observations flagged as outliers. Partly for this reason we considered a range of alpha values and then averaged across this range to fairly compare with the MVOD method. For all simulated time series, we considered alpha between 0.01 and 0.20 .

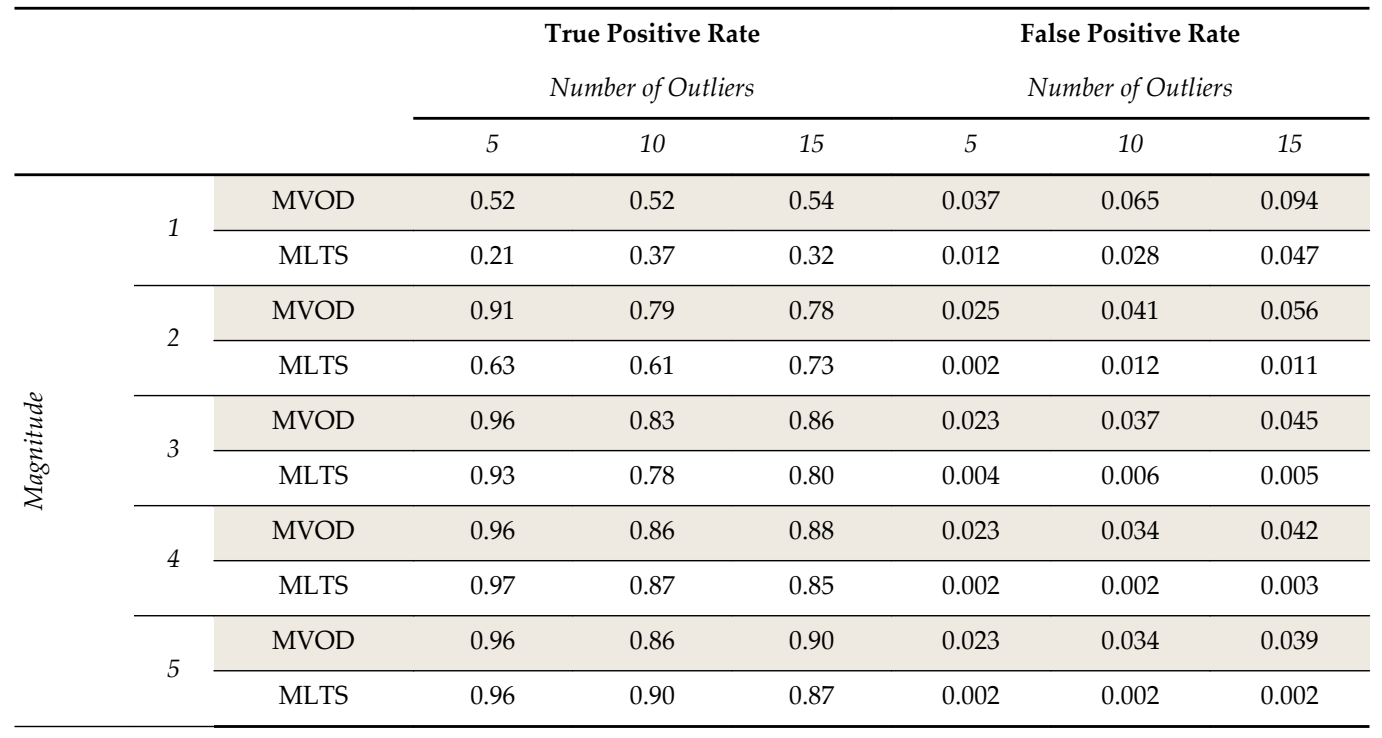

Table 2. True and False Positive Rates for MVOD and MLTS with 5, 10, or 15 outliers of magnitudes 1, 2, 3, 4, or 5. 
In the results presented next, we obtained the TPR and FPR for the two methods in the following way. For a given number of outliers with a specific outlier magnitude, we averaged a total of five cases. The five cases averaged always included the threshold (MVOD) or alpha value (MLTS) corresponding with the number of outliers, but also contained the preceding four cases as well. For instance, in the 10 outlier case, we took the results for threshold $=10$ (MVOD), as well as thresholds of 9, 8, 7 and 6. In the corresponding MLTS case, we would have taken alpha $=0.10,0.09,0.08,0.07$ and 0.06 . The TPR and FPR for each of these five cases for each method were averaged to obtain the values shown in Table 2.

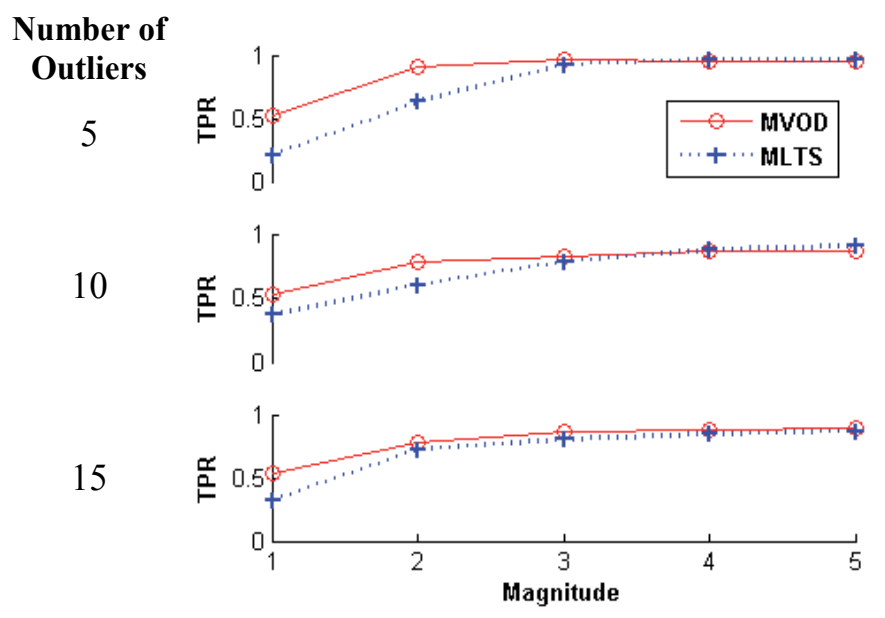

Figure 6. True Positive Rate (TPR, y-axis) for MVOD and MLTS for 5 outliers (top panel), 10 outliers (middle panel), and 15 outliers (bottom panel) with outlier magnitudes of $1,2,3,4$ or 5 (x-axis).

Results:Table 2 and Figure 6 show that in terms of the TPR, our method outperforms the MLTS when the outlier strength is low (i.e. magnitudes of 1 and 2 ) and has slightly better performance than the MLTS for medium outlier strength (i.e. magnitude of 3), while the two approaches are comparable when the outlier strength is high (i.e. magnitude of 4 and 5). As evident from Table 2, for the FPR, the two methods have similar behavior, with negligible difference on the order of $10^{-2}$. Additionally, the number of outliers $(5,10$ or 15$)$ does not have an obvious effect on either of the methods. In summary, the experiments demonstrate that our MVOD method can work effectively and accurately in detecting the outliers from the multivariate time series data. Compared to MLTS, the MVOD is more sensitive in detecting the small magnitude outliers, which are often difficult for an outlier detection algorithm. Furthermore, both our MVOD and the MLTS work reasonably well for a wide range of contamination levels. That is, both methods are quite robust to the number of outliers in the dataset.

\section{Machine learning methods for novelty detection}

Novelty detection can be considered as the task of classifying test data that differ in some respect from the data that are available during training. This may be approached within the 
framework of "one-class classification" [3], in which a model is built to describe "normal" training data. Novelty detection methods can be categorized into several areas such as probabilistic, distance-based, reconstruction-based, domain-based, and information-theoretic techniques. In this section, we mainly introduce the first category of probabilistic approaches, and briefly summarize the others.

\subsection{Probabilistic approaches}

Probabilistic approaches to novelty detection are based on estimation of the generative probability density function of the data, which may then be used to define thresholds for the boundaries of "normality" in the data space and test whether or not a test sample is from the same distribution. Statistical hypothesis tests are the simplest statistical techniques for novelty detection [5]. Among the different statistical tests for novelty detection, here we concentrate on more advanced statistical modeling methods involving complex, multivariate data distributions. Techniques for estimating the underlying data density from multivariate training data broadly fall into parametric and nonparametric methods. The former imposes a restrictive model on the data, leading to a large bias when the model does not fit the data; the later builds up a very flexible model with fewer assumptions but requires a large sample size for a reliable fit of all free parameters when the model size becomes large.

In parametric approaches, the widely used distribution form for continuous variables is Gaussian. The involved parameters are estimated from the training data via maximum likelihood estimates (MLE), for which a closed-form analytical solution is available for a Gaussian distribution. More complex data distribution forms can be modeled through mixture models (e.g. Gaussian Mixture Models, or GMMs for short), or other mixtures of different types of distributions (e.g. the gamma, the Poisson, the Student's t, and the Weibull distributions) [26, 27]. When the form of the data distribution is not available, Gaussian distribution is usually taken due to its convenient analytical properties. The parameters of the GMMs can be estimated with maximum likelihood methods (using optimization algorithms including conjugate gradients or expectation-maximization, EM) or with Bayesian methods (e.g. variational Bayes) [26]. Besides the requirement of large numbers of training examples in estimating model parameters, another limitation of parametric methods is that the chosen data distribution form and the model generating the data may not match well. Despite the limitations, GMMs have been a popular scheme for novelty detection. The other strategy for novelty detection is to utilize time-series approaches, for example, the stochastic process of Autoregressive Integrated Moving Average (ARIMA), which can be used to predict the next data point and determine whether or not it is artefactual [28]. State-space models are also typically used for novelty detection in time-series data, assuming there is some underlying hidden state that generates the observations and this hidden state evolving through time [29]. The Hidden Markov Model (HMM) and the Kalman filter are two common state-space models for novelty detection.

Non-parametric methods do not assume a fixed structure of a model; the model grows in size as necessary to fit the data and accommodate the data complexity. A common non-parametric approach for probabilistic density estimation is the kernel density estimator [26], which 
estimates the probability density function with a large number of kernels over the data space. The kernel density estimator places a kernel (e.g. Gaussian) on each data point and then sums the contributions from a localized neighborhood of the kernel. This is the so-called Parzen windows estimator [30], which has been used for novelty detection in a number of applications including mammographic image analysis [31]. One-class classification based on Gaussian Processes (GPs) has been developed and used recently [32]. This technique also takes a pointwise approach to novelty detection, which divides the data space into regions with high and low supports depending on whether those regions are close to those occupied by "normal" training data, or not. A related way of detecting novelty is based on the well-established area of changepoint detection [33], with the goal of determining whether the generative distribution of a sequence of observations has remained stable or has undergone some abrupt change. Here in addition to detecting whether a change has occurred or not, another aim is to estimate the time that the change has occurred. When applied in a batch or online setting, the idea of changepoint detection to the retrospective problem is identifying a test statistic suitable for testing the hypothesis that a change has occurred versus the one that no change has occurred. A likelihood ratio statistic, as well as others [33,34], would be appropriate.

\subsection{Other categories}

Distance-based approaches, such as clustering or nearest-neighbor methods [35-37], are another types of techniques that can be used for classification or for estimating the probability density function of data. The underlying assumption is that "normal" data are tightly clustered, while novel data occur far from their nearest neighbors. These methods use well-defined distance metrics to compute the distance (e.g. similarity measure) between two data points.

Reconstruction-based methods involve training a regression model with the training data [3, $38,39]$. The distance between the test vector and the output of the system (i.e. the reconstruction error) can be related to the novelty score, which would be high when "abnormal" data occurs. For instance, neural networks can be used in this way and show many of the same advantages for novelty detection as they do for typical classification applications. Another type of reconstruction-based novelty detection is subspace-based techniques. They assume that data can be projected or embedded into a lower dimensional subspace, which makes better discrimination of "normal" and "abnormal" data easier.

Domain-based methods often aim to describe a domain that contains "normal" data through a boundary around the "normal" class following the distribution of the data without explicitly providing a distribution [40,41]. These techniques are usually insensitive to the specific sampling and density of the interested class. The location to the boundary is the criterion for determining the class membership of unknown data. Novelty detection support vector machines (SVMs) are the "one-class SVMs", which set the location of the novelty boundary only based on the data lying closest to it in the transformed feature space. That is, the novelty boundary is determined without considering the data that are not support vectors.

Information-theoretic methods calculate the information content of a dataset with measures such as entropy, relative entropy, and Kolmogorov complexity, etc. [42, 43]. The key idea is that novel data alter the information content in a dataset significantly. A common procedure 
is: metrics are computed using the entire dataset and then the subset of points whose elimination from the dataset causes the largest difference in the metric are identified. The data contained in this subset is then assumed to be novel data.

\section{Robust estimator and outlier detection in high-dimensional medical imaging}

The statistical analysis of medical images is challenging, not only because of the high-dimensionality and low signal-to-noise ratio of the data, but also due to varieties of errors in the image acquisition processes, such as scanner instabilities, acquisition artifacts, and issues associated with the experimental protocol [44]. Furthermore, populations under study typically present high variability $[45,46]$, and therefore the corresponding imaging data may have uncommon though technically correct observations. Such outliers deviating from normality could be numerous. With emergence of large medical imaging databases, developing automated outlier detection methods turns out to be a critical preprocessing step for any subsequent statistical analysis or group study. In addition, medical imaging data are usually strongly correlated [47]; outlier detection approaches based on multivariate models are thus crucial and desirable. Procedures using the classical MCD estimator are not well-suited for such high-dimensional data.

In [48], several extensions to the classical outlier detection framework are proposed to handle high-dimensional imaging data. Specifically, the MCD robust estimator were modified so that it can be used for detecting outliers when the number of observations is small compared to the number of available features. This is achieved through introducing regularization in the definition and estimation of the MCD. Three regularization procedures were presented and compared: $l_{1}$ regularization $\left(R M C D-l_{1}\right) ; l_{2}$ regularization or ridge regularization $\left(R M C D-l_{2}\right)$; and random projections ( $R M C D-R P)$. The idea of $R M C D$ - $R P$ is to run the MCD estimator on datasets of reduced dimensionality, and this dimensionality reduction is done by projecting to a randomly selected subspace. In addition, the parametric approach of the regularized MCD estimators is compared to a non-parametric procedure, the One-Class SVM algorithm (see Section 4). Experimental results on both simulated and real data show that $l_{2}$ regularization performs generally well in simulations, but random projections outperform it in practice on non-Gaussian, and more importantly, on real neuroimaging data. One-Class SVM works well on unimodal datasets, and it has a strong potential if their parameters can be set correctly.

Outlier detection methods described above can serve as a statistical control on subject inclusion in neuroimaging. However, sometimes it is controversial regarding whether or not outliers should be discarded, and, if so, what tolerance to use. An alternative strategy is to utilize outlier-resistant techniques for statistical inference, which would also compensate for inexact hypotheses including data normality and homogeneous dataset. Robust techniques are especially useful when a large number of regressions are tested and assumptions cannot be evaluated for each individual regression, as with neuroimaging data.

Both individual subject and group analyses are required in neuroimaging. At a typical single subject level, a multiple regression model is used for the time series data at each voxel [49, 
50], and outliers (or other assumption violations) in the time series would impact the model fitness. Robust regression can minimize the influence of these outliers. At the group level, after spatial normalization, a common strategy is to first save the regression parameters for each subject at each voxel and then perform a test on the parameter values. Robust regression used at this level can minimize the influence of outlying subjects. Wager et al. [51] used simulations to evaluate several robust techniques against ordinary least squares regression, and apply robust regression to second-level group analyses in three real fMRI datasets. Experimental results demonstrate that robust Iteratively Reweighted Least Squares (IRLS) at the second level is computationally efficient; it increases statistical power and decreases false positive rates when outliers are present. Without the presence of outliers, IRLS controls false positive rates at an appropriate level. In summary, IRLS shows significant advantages in group data analysis and in the hemodynamic response shape estimation for fMRI time series data.

\section{Conclusions}

Outlier and novelty detection is a primary step in many data mining and analysis applications, including healthcare and medical research. In this chapter, statistical and machine learning methods for outlier and novelty detection, and robust approaches for handling outliers in data and imaging sciences were introduced and reviewed. Particularly, we also presented our new method for outlier detection in time series data based on the Voronoi diagram (i.e. MVOD). There are several key advantages of our method. First, it copes with outliers in a multivariate framework by accounting for multivariate structure in the data. Second, it is flexible in extracting valid features for differentiating outliers from non-outliers, in the sense that we have the option of using or not using a parametric model. Lastly, Voronoi diagrams capture the geometric relationship embedded in the data points. Initial experimental results show that our MVOD method can lead to accurate, sensitive, and robust identification of outliers in multivariate time series.

It is often difficult to reach a precise definition of outlier or novelty, and suggesting an optimal approach for outlier or novelty detection is even more challenging. The variety of practical and theoretical considerations arising in real-world datasets lead to the variety of techniques utilized [52]. Therefore, there is no single universally applicable detection method due to the large variety of considerations, which could include the application domain, the type of data such as dimension, and the availability of training data, etc. Based on the application and the nature of the associated data, developing suitable computational methods that can robustly and efficiently extract useful quantitative information from big data is still a current challenge and gaining increasing interest in data and imaging sciences.

\section{Acknowledgements}

This work is supported in part by a grant from the National Institute of Health, K25AG033725. 


\section{Author details}

Michelle Yongmei Wang ${ }^{1,2,3,4^{*}}$ and Chris E. Zwilling ${ }^{2}$

*Address all correspondence to: ymw@illinois.edu

1 Department of Statistics, University of Illinois at Urbana-Champaign, USA

2 Department of Psychology, University of Illinois at Urbana-Champaign, USA

3 Department of Bioengineering, University of Illinois at Urbana-Champaign, USA

4 Beckman Institute, University of Illinois at Urbana-Champaign, USA

\section{References}

[1] Aggarwal CC. Outlier Analysis. New York: Springer Science + Business Media; 2013.

[2] Markou M, Singh S. Novelty detection: a review---part 1: statistical approaches. Signal Processing 2003a; 83(12): 2481-2497.

[3] Markou M, Singh S. Novelty detection: a review---part 2: neural network based approaches. Signal Processing 2003b; 83(12): 2499-2521.

[4] Zwilling CE, Wang MY. Multivariate Voronoi outlier detection for time series. In: Proc. IEEE Healthcare Innovation Point-Of-Care Technologies Conference 2014; in press.

[5] Barnett V, Lewis T. Outliers in Statistical Data. John Wiley and Sons; 1994.

[6] Tarassenko L, Clifton DA, Bannister PR, King S, King D. Novelty Detection. In: Boller C, Chang F-K, Fujino Y (eds.) Encyclopedia of Structural Health Monitoring. 2009. Chapter 35.

[7] Davies L, Gather U. The identification of multiple outliers. Journal of American Statistical Association 1993; 88(423): 782-792.

[8] Rousseeuw P. Multivariate estimation with high breakdown point. In: W. Grossmann et al. (eds.) Mathematical Statistics and Applications. Budapest: AkademiaiKiado; 1985. Vol. B, p283-297.

[9] Ben-Gal I. Outlier detection. In: Maimon O, Rockach L (eds.) Data Mining and Knowledge Discovery Handbook: A Complete Guide for Practitioners and Researchers. Kluwer Academic Publishers; 2005. Chapter 1.

[10] Becker C, Fried R, Kuhnt S, editors. Robustness and Complex Data Structures. Berlin Heidelberg: Springer-Verlag; 2013. 
[11] Huber PJ, Ronchetti EM. Robust Statistics. John Wiley \& Sons, Inc.; 2009.

[12] Rousseeuw PJ. Least median of squares regression. Journal of the American Statistical Association 1984; 79: 871-880.

[13] Hubert M, Debruyne M. Minimum covariance determinant. Wiley interdisciplinary reviews: Computational statistics 2010; 2: 36-43.

[14] Croux C, Haesbroeck G. Influence function and efficiency of the Minimum Covariance Determinant. Journal of Multivariate Analysis 1999; 71: 161-190.

[15] Lopuhaa HP, Rousseeuw PJ. Breakdown points of affine equivariant estimators of multivariate location and covariance matrices. Annals of Statistics 1991; 19: 229-248.

[16] Lopuhaa HP. Asymptotics of reweighted estimators of multivariate location and scatter. Annals of Statistics 1999; 27: 1638-1665.

[17] Liebscher S, Kirschstein, Becker C. RDELA ---A Delaunay-triangulation-based, location and covariance estimator with high breakdown point. Statistics and Computing 2013; 23: 677-688.

[18] Rousseeuw PJ, Driessen KV. A fast algorithm for the minimum covariance determinant estimator. Technometrics 1999; 41(3): 212-223.

[19] Rousseeuw PT, Aelst SV, Driessen KV, Agullo J. Robust multivariate regression. Technometrics 2004; 46(3): 293-305.

[20] Agullo J, Croux C, Aelst SV. The multivariate least-trimmed squares estimator. Journal of Multivariate Analysis 2008; 99: 311-338.

[21] Croux C, Joossens K. Robust estimation of the vector autoregressive model by a least trimmed squares procedure. In: Proceedings in Computational Statistics 2008; p489-501.

[22] Preparata FP, Shamos MI. Computational Geometry-An Introduction. Springer; 1985.

[23] Pearson, RK. Exploring Data in Engineering, the Sciences and Medicine. Oxford University Press; 2011.

[24] Qu J. Outlier detection based on Voronoi diagram. In: Proceedings of the ADMA International Conference on Advanced Data Mining and Applications 2008; p516-523.

[25] Neumaier A, Schneider T. Algorithm 808: ARfit-A Matlab package for the estimation of parameters and eigenmodes of multivariate autoregressive models. ACM Transactions Mathematical Software 2001; 27: 58-65.

[26] Bishop CM. Pattern Recognition and Machine Learning. Springer, New York; 2006.

[27] Carvalho A, Tanner M. Modelling nonlinear count time series with local mixtures of poisson autoregressions. Comput. Stat. Data Anal. 2007; 51(11): 5266-5294. 
[28] Hoare S, Asbridge D, Beatty P. On-line novelty detection for artefact identification in automatic anaesthesia record keeping. Med. Eng. Phys. 2002; 24(10): 673-681.

[29] Quinn J, Williams C. Known unknowns: novelty detection in condition monitoring. In: Marti J et al. (eds.) Pattern Recognition and Image Analysis, LNCS 4477. 2007. p16.

[30] Parzen E. On estimation of a probability density function and mode. Ann. Math. Stat. 1962; 33(3): 1065-1076.

[31] Tarassenko L, Hayton P, Cerneaz N, Brady M. Novelty detection for the identification of masses in mammograms. In: Proceedings of the 4th International Conference on Artificial Neural Networks, IET. 1995. p442-447.

[32] Kemmler M, Rodner E, Denzler J, One-class classification with Gaussian processes. In: Asian Conference on Computer Vision (ACCV), vol. 6493. 2011. p489-500.

[33] Basseville M, Nikiforov IV. Detection of Abrupt Changes: Theory and Application. Prentice Hall, Englewood Cliffs; 1993.

[34] Reeves J, Chen J, Wang XL, Lund R, Lu QQ. A review and comparison of changepoint detection techniques for climate data. J. Appl. Meteorol. Climatol. 2007; 46(6): 900-915.

[35] Pires A, Santos-Pereira, C. Using clustering and robust estimators to detect outliers in multivariate data. In: Proceedings of the International Conference on Robust Statistics. 2005.

[36] Yong S, Deng J, Purvis M, Wildlife video key-frame extraction based on novelty detection in semantic context. Multimed. Tools Appl. 2013; 62(2): 359-376.

[37] Hautamaki V, Karkkainen I, Franti P. Outlier detection using k-nearest neighbor graph. In: Proceedings of the $17^{\text {th }}$ International Conference on Pattern Recognition, vol. 3. 2004. p430-433.

[38] Kit D, Sullivan B, Ballard D. Novelty detection using growing neural gas for visuospatial memory. In: Proceedings of the IEEE/RSJ International Conference on Intelligent Robots and Systems. 2011, p1194-1200.

[39] Xiao Y, Wang H, Xu W, Zhou J. L1 norm based KPCA for novelty detection. Pattern Recognit. 2013; 46(1): 389-396.

[40] Schölkopf B, Williamson R, Smola A, Shawe-Taylor J, Platt J. Support vector method for novelty detection. Adv. Neural Inf. Process. Syst. 2000; 12(3): 582-588.

[41] Le T, Tran D, Ma W, Sharma D. Multiple distribution data description learning algorithm for novelty detection. Adv. Knowl. Discov. Data Min. 6635. 2011. p246-257.

[42] He Z, Deng S, Xu X, Huang J. A fast greedy algorithm for outlier mining. Adv. Knowl. Discov. Data Min. 3918. 2006. p567-576. 
[43] Filippone M, Sanguinetti G. Information theoretic novelty detection. Pattern Recognition 2010; 43(3): 805-814.

[44] Wang MY, Zhou C, Xia J. Statistical analysis for recovery of structure and function from brain images. In: Komorowska MA, Olsztynska-Janus S (eds.) Biomedical Engineering, Trends, Researches and Technologies. 2011. p169-190.

[45] Chen G, Fedorenko E, Kanwisher NG, Golland P. Deformation-invariant sparse coding for modeling spatial variability of functional patterns in the brain. In: Proc. Neural Information Processing Systems Workshop on Machine Learning and Interpretation in Neuroimaging, LNAI 7263. 2012. p68-75.

[46] Staib LH, Wang YM. Methods for nonrigid image registration. In: Bayro-Corrochano E (ed.) Handbook of Goemetric Computing: Applications in Pattern Recognition, Computer Vision, Neuralcomputing, and Robotics. Springer-Verlag; 2005. p571-602.

[47] Wang MY, Xia J. Unified framework for robust estimation of brain networks from fMRI using temporal and spatial correlation analyses. IEEE Trans. on Medical Imaging 2009; 28(8): 1296-1307.

[48] Fritsch V, Varoquaux G, Thyreau B, Poline J-B, Thirion B. Detecting outliers in highdimensional neuroimaging datasets with robust covariance estimators. Medical Image Analysis 2012; 16(7): 1359-1370.

[49] Worsley KJ, Friston KJ. Analysis of fMRI time-series revisited - Again. NeuroImage 1995; 2(3): $173-181$.

[50] Worsley KJ, Poline JB, Friston KJ, Evans AC. Characterizing the response of PET and fMRI data using multivariate linear models. NeuroImage 1997; 6(4): 305-319.

[51] Wager TD, Keller MC, Lacey SC, Jonides J. Increased sensitivity in neuroimaging analyses using robust regression. NeuroImage 2005; 26: 99-113.

[52] Singh K, Upadhyaya S. Outlier detection: applications and techniques. International Journal of Computer Science Issues 2012; 9(1): 307-323. 
Chapter 12

\title{
Energy Transfer and Dissipation in non-Newtonian Flows in non-Circular Tubes
}

\author{
Mario F. Letelier \\ Additional information is available at the end of the chapter \\ http://dx.doi.org/10.5772/59907
}

\section{Introduction}

In general, physiological processes are basically isothermal and isobaric. Several mechanisms contribute to keep constant pressure in systems such as blood vessels, among them diameter distribution and peristaltic motion. In this, it is to be understood that pressure constancy refers to time-averaged or extreme values.

Many pathologies make the human body develop fever, which raises temporarily body temperature. It is common practice to use, in some cases, medicines that restore, albeit for a given period of time, normal temperature. Fever is not the only cause of body heat-transfer. This process also occurs with body adjustment to changes in ambient temperature, physical exertion, metabolism acceleration due to food ingestion, drug effects, and others. During these body changes, blood and other fluid vessels undergo heat-transfer processes that pose considerable difficulties to physical modeling. Some pertinent variables are fluid composition, vessel elasticity, peristaltic motion in some cases, unsteadiness, and complex vessel geometry.

Heat-transfer in tube-flow has been investigated for several decades. One main practical driving force for such studies is the need to understand and design efficient heat-transfer equipment, widely used in several industrial areas. Models of simple tube-flow heat-transfer problems are now textbook's standard contents.

These include laminar Newtonian steady flow in round tubes with simple boundary conditions. More recently, as it is reviewed in the next section, Newtonian and non-Newtonian flows in tubes of geometry other then circular have been modeled and analyzed. One important finding as to this chapter's objective is the effect of the interplay between tube geometry and non-linear viscoelasticity. 
Such coupling leads to the development of secondary flows, or helicoidal flows, that increase the transversal transport capacity of the flow, a phenomenon that can be applied, or related, to heat-transfer enhancement, or to other transversal transport processes, such as crosssectional transport of particles immersed in the fluid.

Especially in blood flow, cells and other components introduce viscoelasticity [1,2] that becomes relevant in smaller blood vessels. The understanding of its effect on the flow characteristics may become very relevant in cases such as heart arteries, and when artificial implants affect the blood flow. Also blood plastic effects appear is smaller vessels due to the aggregation of red blood cells at low shear rates, which develop a yield stress to be overcome for the flow to ensue.

Drawing on the above results, in this chapter it is presented a summary of relatively new analytical findings that may be useful for the better understanding of heat-transfer and complex flow phenomena in vessels that share some characteristics with biological vessels, particularly when these work under abnormal conditions. Also it is analyzed the effect of geometry in energy dissipation. This chapter is closely related to [3].

\section{Mathematical models}

Non-linear viscoelasticity and fluid plasticity are related in the following to heat- transfer and energy dissipation processes. Next are presented the mathematical models to be considered together with some remarks as to the corresponding state-of-the art, and relevant developments with related references.

\subsection{Viscoelastic flow}

The physical model considered is a straight tube of arbitrary cross-sectional shape, in which a non-Newtonian fluid moves along the axial coordinate $z$ impelled by a pressure gradient, which can be a function of $t=$ time. Secondary flows are induced when the necessary conditions operate. The flow is assumed laminar, incompressible and with constant properties. Considering Cartesian coordinates, the velocity field can be expressed as $(u, v, w)$ in which the velocity components align with the $(x, y, z)$ axes respectively. The temperature and velocity fields are, in general, dependent on $x, y, z$ and $t$.

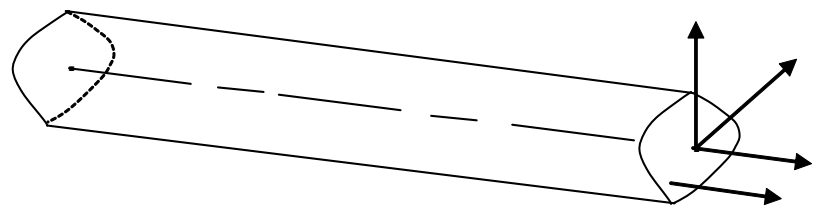

Figure 1. Definition diagram 
Two well-known problems are here relevant, i.e.,

1. Flow with constant wall heat-flux, in which the wall temperature is assumed constant at any cross-section, with prescribed axial variation.

2. The "Graetz problem", in which the flow is assumed isothermal up to a point $z=0$, from which on a different constant temperature is applied at the wall, so that the fluid is progressively heated or cooled.

Experimental findings concerning heat-transfer characteristics of aqueous polymer solutions flowing in straight tubes point at considerable enhancement as compared to its Newtonian counterpart driven by the same conditions and in the same geometry. Specifically it is reported that heat-transfer results for viscoelastic aqueous polymer solutions are considerably higher in flows fully developed both hydrodynamically and thermally, as much as by an order of magnitude depending primarily on the constitutive elasticity of the fluid and to some extent on the boundary conditions, than those found for water in laminar flow in rectangular ducts $[4,5]$. Heat-transfer phenomena in laminar flow of non-linear fluids has not been the subject of many investigations with the exception of round pipes, and the case of inelastic shearthinning fluids in tubes of rectangular cross-section, in spite of the widespread use of some specific contours in industry such as flattened elliptical tubes.

This statement is true for all cross-sectional shapes for both steady and unsteady phenomena including quasi-periodic flows. Heat-transfer with viscoelastic fluids has been declared to be a new challenge in heat-transfer research in the early nineties [6], but progress has been limited since that time. The physics of the phenomenon has not been entirely clarified.

Highly enhanced heat-transfer to aqueous solutions of polyacrylamide and polyethylene of the order of $40-45 \%$ as compared to the case of pure water in flattened copper tubes was observed by Oliver [7] and later by Oliver and co-workers as early as 1969. Recent numerical investigations in rectangular cross-sections of Gao and Hartnett $[8,9]$, Naccache and Souza Mendes [10], Payvar [11] and Syrjala [12] establish the connection between the enhanced heattransfer observed and the secondary flows induced by viscoelastic effects. The former researchers as well as Naccache and Souza Mendes predict for instance viscoelastic Nusselt numbers as high as three times their Newtonian counterparts. Gao and Hartnett $[8,9]$.report numerical results in rectangular contours which provide evidence that the stronger the secondary flow (as represented by the dimensionless second normal stress coefficient $\Psi_{2}$ ) the higher the value of the heat-transfer (as represented by the Nusselt number $\mathrm{Nu}$ ) regardless the combination of thermal boundary conditions on the four walls. Constant heat flux is imposed everywhere on the heated walls in their numerical experiments with the remaining walls being adiabatic. The combination of boundary conditions plays some role in the enhancement reported with the largest enhancement occurring when two opposing walls are heated. Despite these efforts heat-transfer characteristics of viscoelastic fluids in steady laminar flow in rectangular tubes remains very much an open question (quoted from Siginer and Letelier [13]). Coelho et al [14] presented an analytical solution for the Graetz problem for the MPTT fluid for a circular tube, steady flow, including several computations for negative heat flux (flow cooling). Valko [15] published a solution of the Graetz problem using a power-law fluid model; 
he determined the influence of the Brinkman number for several flow conditions in circular tubes. Kin and Özisik [17] published work on transient laminar forced convection of a powerlaw fluid in ducts with sudden change in wall temperature. In these and related references it is reflected the actual state-of-the-art in this subject.

Concerning numerical analysis, there is only one available commercial package for viscoelastic fluid flow computations, POLYFLOW. However POLYFLOW cannot handle even relatively high Weissenberg number flows and although convergent, gives erroneous results of the order of $400 \%$ as compared to analytical test cases, Filali et al. (2012). In addition it cannot handle heat-transfer in steady viscoelastic fluid flow in tubes. Thus to study problems of this type a numerical algorithm has to be built from scratch and tested for stability (Hadamard-type). Numerical analysis is not considered in this chapter.

Two well-known models of non-linear viscoelastic fluids are next described:

Modified Phan-Thien-Tanner (MPTT)

$$
2 \eta_{m} D=\left(1+\frac{{ }^{0} \lambda}{\eta_{m 0}} \operatorname{tr} \tau\right) \tau+\lambda\left(V \bullet \nabla \tau-\varphi \tau-\tau \varphi^{T}\right)
$$

Giesekus

$$
\begin{gathered}
\boldsymbol{\tau}+\lambda \check{\tau}+\frac{\alpha \lambda}{\eta_{0}} \boldsymbol{\tau} \bullet \boldsymbol{\tau}=\eta_{0} \boldsymbol{D} \\
\check{\boldsymbol{\tau}}=-\left((\nabla V)^{T} \bullet \boldsymbol{\tau}+\boldsymbol{\tau} \bullet \nabla \boldsymbol{V}\right)
\end{gathered}
$$

In the following, the MPTT model is applied to the flow field analysis. For the purposes of this presentation, there is some evidence [17] that both models lead to qualitatively similar results.

The applicable equation of motion for 3-D, steady and incompressible flow are, in cylindrical coordinates:

Continuity

$$
\nabla \bullet V=0
$$

Momentum

$$
V \bullet \nabla V=\nabla \bullet \sigma
$$

or, in expanded form

$$
\left.u \frac{\partial u}{\partial r}+\frac{v}{r} \frac{\partial u}{\partial \theta}+w \frac{\partial u}{\partial z}-\frac{v^{2}}{r}=\frac{1}{r}\left[\frac{\partial}{\partial r}\left(r \sigma_{r r}\right)+\frac{\partial}{\partial \theta}\left(\sigma_{\theta r}\right)+\frac{\partial}{\partial z}\left(r \sigma_{z r}\right)\right)\right]-\frac{\sigma_{\theta \theta}}{r}
$$




$$
\begin{gathered}
u \frac{\partial v}{\partial r}+\frac{v}{r} \frac{\partial v}{\partial \theta}+w \frac{\partial v}{\partial z}-\frac{u v}{r}=\frac{1}{r}\left[\frac{\partial}{\partial r}\left(r \sigma_{r \theta}\right)+\frac{\partial}{\partial \theta}\left(\sigma_{\theta \theta}\right)+\frac{\partial}{\partial z}\left(r \sigma_{z \theta}\right)\right]-\frac{\sigma_{r \theta}}{r} \\
u \frac{\partial w}{\partial r}+\frac{v}{r} \frac{\partial w}{\partial \theta}+w \frac{\partial w}{\partial z}=\frac{1}{r}\left[\frac{\partial}{\partial r}\left(r \sigma_{r z}\right)+\frac{\partial}{\partial \theta}\left(\sigma_{\theta z}\right)+\frac{\partial}{\partial z}\left(r \sigma_{z z}\right)\right]
\end{gathered}
$$

In the above, $u, v, w$ are the radial, tangential and axial velocity components, $\boldsymbol{\sigma}$ is the stress matrix and $P$ is the piezometric pressure. Scale factors applied are $a$ (base radius) for $r, w_{0}$ for the velocity components, and $\eta_{N} w_{0} / a$ for the stress components, in which $\eta_{N}$ is the Newtonian viscosity.

The MPTT model of viscoelastic fluid is next expressed, in dimensional variables, through the following equations, i.e. [18].

$$
\begin{gathered}
\sigma=-P \boldsymbol{I}+2 \eta_{N} \boldsymbol{D}+\boldsymbol{\tau} \\
2 \eta_{m} \boldsymbol{D}=f\left(\varepsilon_{0}, \operatorname{tr} \boldsymbol{\tau}\right) \boldsymbol{\tau}+\lambda \boldsymbol{\tau}^{\nabla} \\
\boldsymbol{\tau}^{\nabla}=\frac{\partial \tau}{\partial t}+\boldsymbol{V} \bullet \nabla \boldsymbol{\tau}-\left(\nabla \boldsymbol{V}^{T}-\xi \boldsymbol{D}\right) \tau-\tau\left(\nabla \boldsymbol{V}^{T}-\xi \boldsymbol{D}\right)^{T}
\end{gathered}
$$

where $\mathbf{D}$ is the rate of deformation tensor and $\tau$ is the non-Newtonian component of the shear stress.

Defining

$$
\boldsymbol{\varphi}=\nabla \bullet \nabla \boldsymbol{V}^{T}-\xi \boldsymbol{D}
$$

then, a more compact form of (10), is

$$
\tau^{\nabla}=V \cdot \nabla \tau-\varphi \tau-\tau \varphi^{T}
$$

In this $\eta_{m}$ is a viscosity, $\varepsilon_{0}, \xi$ are material parameters, and $\lambda$ is the relaxation time.

The function $f$, as defined in the MPTT model can be simplified for small values of $\varepsilon_{0}$, yielding

$$
f\left(\varepsilon_{0}, \operatorname{tr} \tau\right)=\exp \left(\frac{\varepsilon_{0} \lambda}{\eta_{m o}} \operatorname{tr} \tau\right)=\sum_{n=0}^{\infty} \frac{1}{n !}\left(\frac{\varepsilon_{0} \lambda}{\eta_{m o}} \operatorname{tr} \tau\right)^{n}=1+\frac{\varepsilon_{0} \lambda}{\eta_{m o}} \operatorname{tr} \tau+O\left(\varepsilon_{0}^{2}\right)
$$

where from

$$
2 \eta_{m} D=\left(1+\frac{\varepsilon_{0} \lambda}{\eta_{m 0}} \operatorname{tr} \tau\right) \tau+\lambda\left(V \bullet \nabla \tau-\varphi \tau-\tau \varphi^{T}\right)
$$


For this constitutive equation the viscosity is defined as

$$
\eta_{m}=\eta_{m 0} \frac{1+\xi(2-\xi) \lambda^{2} \kappa^{2}}{\left(1+\lambda^{2} \kappa^{2}\right)^{(1-m) / 2}}
$$

in which

$$
\kappa=\sqrt{2 \operatorname{tr} D^{2}}
$$

and where $\eta_{m 0}$ and $m$ are additional material parameters. If $m=1$, then (15) reduces to

$$
\eta_{m}=\eta_{m 0}\left[1+\lambda^{2} \xi(2-\xi) \mathcal{K}^{2}\right]
$$

Coming back to dimensionless variables and parameters (14) becomes

$$
2\left(1+2 \xi(2-\xi) W i^{2} \operatorname{tr} \boldsymbol{D}^{2}\right) \boldsymbol{D}=\left(1+\varepsilon_{0} W i \operatorname{tr} \tau\right) \boldsymbol{\tau}+W i \boldsymbol{\tau}^{\nabla}
$$

in which $W i$ is the Weissenberg number, or dimensionless relaxation time, defined as

$$
W i=\frac{w_{0} \lambda}{a}
$$

By combining equations (18), (8) and (5-7), it is obtained the set of working equations in full, i.e.

$$
\begin{gathered}
u \frac{\partial u}{\partial r}+\frac{v}{r} \frac{\partial u}{\partial \theta}+w \frac{\partial u}{\partial z}-\frac{v^{2}}{r}=F_{r}-\frac{\partial P}{\partial r}+\nabla^{2} u-\frac{u}{r^{2}}-\frac{2}{r^{2}} \frac{\partial v}{\partial \theta} \\
u \frac{\partial v}{\partial r}+\frac{v}{r} \frac{\partial v}{\partial \theta}+w \frac{\partial v}{\partial z}-\frac{u v}{r}=F_{\theta}-\frac{1}{r} \frac{\partial P}{\partial \theta}+\nabla^{2} v-\frac{v}{r^{2}}-\frac{2}{r^{2}} \frac{\partial u}{\partial \theta} \\
u \frac{\partial w}{\partial r}+\frac{v}{r} \frac{\partial w}{\partial \theta}+w \frac{\partial w}{\partial z}=F_{z}-\frac{\partial P}{\partial z}+\nabla^{2} w
\end{gathered}
$$

in which the functions $F_{r}, F_{\theta}$ and $F_{z}$ are the viscoelastic forcing functions that determine the transversal flow. For developed flow, all derivatives with respect to $z$, excepted for the pressure, must be put equal to zero.

The explicit expressions for the extra forcing terms are:

$$
F_{r}=\left(\nabla \bullet \tau^{\nabla}\right)_{r}=\frac{1}{r} \frac{\partial}{\partial r}\left(r \tau_{r r}^{\nabla}\right)+\frac{1}{r} \frac{\partial \tau^{\nabla}{ }_{\theta r}}{\partial \theta}-\frac{\tau^{\nabla}{ }_{\theta \theta}}{r}
$$




$$
\begin{gathered}
F_{\theta}=\left(\nabla \bullet \tau^{\nabla}\right)_{\theta}=\frac{\partial \tau^{\nabla}{ }_{r \theta}}{\partial r}+\frac{1}{r} \frac{\partial \tau^{\nabla} \theta_{\theta \theta}}{\partial \theta}+\frac{2}{r} \tau^{\nabla}{ }_{r \theta} r \\
F_{z}=\left(\nabla \bullet \tau^{\nabla}\right)_{z}=\frac{1}{r} \frac{\partial}{\partial r}\left(r \tau_{r z}^{\nabla}\right)+\frac{1}{r} \frac{\partial \tau^{\nabla}}{\partial \theta}
\end{gathered}
$$

\section{Energy}

For steady flow of an incompressible fluid with constant properties, the energy equation, in terms of the temperature $\mathrm{T}$ can be written as

$$
\rho_{0} C_{p} \frac{D T}{D t}=\Phi+\nabla \bullet(k \nabla T)
$$

Where $\varrho_{0}$ is the density, $C_{p}$ is the specific heat at constant pressure, $\phi$ is the dissipation function, and $\mathrm{k}$ is the thermal conductivity coefficient. Further, it is assumed negligible dissipation and constant $k$. Under these assumptions (26) becomes

$$
\rho C_{p}\left(u \frac{\partial T}{\partial r}+\frac{v}{r} \frac{\partial T}{\partial r}+w \frac{\partial T}{\partial z}\right)=k\left(\frac{\partial^{2} T}{\partial r^{2}}+\frac{1}{r} \frac{\partial T}{\partial r}+\frac{1}{r^{2}} \frac{\partial^{2} T}{\partial \theta^{2}}+\frac{\partial^{2} T}{\partial z^{2}}\right)
$$

\subsection{Viscoplastic flow}

Viscoplastic fluids are fluids that exhibit yield stress, which must be overcome before the material develops deformation. As already mentioned plasticity appears in small blood vessels due to red cell aggregation.

Also, many industrial fluids exhibit yield stress, and are found in areas such as mining (slurries), food (pastes), construction (concrete and mud), cosmetics, etc. Flow and heattransfer description in tubes and other configurations is compounded by their geometry, which brings in non-linear constitutive equations, except for very simple shapes. Some recent research in this field include flow around a cylinder by Tokpavi et al. [19] particle sedimentation, $\mathrm{Yu}$ and Wachs [20], flow in an eccentric annular tube, Wachs [21], and Walton and Bittleston [22], a general analysis for flow in non-circular ducts, Letelier and Siginer [23] and a preliminary analysis of the velocity field in non-circular pipes, Letelier, et al. [24]. Other pertinent references can be found in the previous ones.

The constitutive characteristics of viscoplastic flow determine complex structures of velocity and shear fields in tube flow when the tube cross-sectional contour differs from circular. This is true even for the case of the Bingham model of viscoplastic fluid, which is one of the simplest mathematical expressions for this kind of fluids.

Plasticity implies existence of fluid yield stress, which may induce both plug zones and stagnant zones within the tube cross-section, when in there the tube geometry determines zones of shear stress below the value of the yield stress. Such solid regions of the flow make it necessary to apply greater pressure gradients in order to keep a given rate of flow which, in turn, leads to greater energy dissipation and heating inside the fluid. 
In the following Bingham's model of fluid is used. Simple plastic flows in straight non-circular tubes do not develop secondary flows. The flow is, therefore, parallel when the motion is laminar, and only the axial component of the velocity exists. Under these conditions, the momentum equation, in terms of shear stress components, is

$$
\frac{\partial \tau_{r z}}{\partial r}+\frac{\tau_{r z}}{r}+\frac{1}{r} \frac{\partial \tau_{\theta z}}{\partial \theta}=-\frac{\partial P}{\partial z}
$$

The constitutive relations are

$$
\begin{gathered}
\tau_{r z}=-\left(1+\frac{N}{I}\right) \frac{\partial w}{\partial r} \\
\tau_{\theta z}=-\left(1+\frac{N}{I}\right) \frac{1}{r} \frac{\partial w}{\partial \theta}
\end{gathered}
$$

in which

$$
I=\left[\left(\frac{\partial w}{\partial r}\right)^{2}+\left(\frac{1}{r} \frac{\partial w}{\partial \theta}\right)^{2}\right]^{\frac{1}{2}}
$$

is an invariant related to the rate of deformation matrix. Also

$$
N=\frac{a}{\eta w_{0}} \tau_{y}
$$

where $\tau_{y}$ is the dimensional yield stress. The parameter $N$ is a dimensionless yield stress that greatly influence the flow characteristics.

The momentum and constitutive equations can be written in more compact form by using natural coordinates, i.e.

$$
\begin{gathered}
\frac{d \tau_{n z}}{d n}+\frac{\tau_{n z}}{\rho}=-\frac{\partial P}{\partial z} \\
\tau_{n z}=N-\frac{d w}{d n}
\end{gathered}
$$

In the above, $n$ is a coordinate normal to isovels and $\rho=$ radius of curvature of isovels

The dissipation function $\phi$ can be used as an index of energy dissipation. For parallel flow $\phi$ is given by

$$
\phi=\tau_{n} \frac{d w}{d n}=\tau_{n}\left(\tau_{n}-N\right)
$$




\section{Analysis of secondary flows and their effect on heat-transfer}

The equations of motion and energy are next solved by using a double perturbation method, as follows. First, and for all boundary conditions, the tube cross-section boundary is defined by

$$
G=1-r^{2}+\varepsilon r^{n} \sin n \theta=0
$$

In which $G$ is here labeled as a "shape factor", that can describe a wide array of shapes according to the given values of the parameters $n$ and $\varepsilon$ [1]. The shape perturbation parameter is $\varepsilon$, which can take values in between 0 and a limiting values for the curve (36) staying closed. The parameter $n$ must be given integer values in order to get regular shapes.

Next all velocity components are expanded in series in terms of the Weissenberg number, i.e.

$$
\begin{gathered}
u=W i u_{1}+W i^{2} u_{2}+\ldots \\
v=W i v_{1}+W i^{2} v_{2}+\ldots \\
w=w_{0}+W i w_{1}+W i^{2} w_{2}+\ldots
\end{gathered}
$$

Complementarily, all velocity component, at any order, are defined as

$$
V=G\left(f_{0}+\varepsilon f_{1}+\varepsilon^{2} f_{2}+\ldots\right)
$$

where $V$ is a generic velocity component, at any order in $W i$, and $f_{0}, f_{1}, \ldots$ are functions specific for every $\mathrm{V}$, to be determined by solving the momentum equations. Similarly, all other dependent variables are expressed in series, i.e.

$$
\begin{aligned}
& \tau=\tau_{0}+W i \tau_{1}+W i^{2} \tau_{2}+\ldots \\
& P=P_{0}+W i P_{1}+W i^{2} P_{2}+\ldots
\end{aligned}
$$

More details can be found in references $[13,25]$. The velocity field is first found by substituting (37-39) in (20-25) and related equations. For the axial velocity, the following expressions can be determined, i.e.

$$
\begin{gathered}
w_{0}=p\left(1-r^{2}+\varepsilon r^{n} \sin n \theta\right)=p G \\
p=-\frac{1}{4} \frac{\partial P}{\partial z}
\end{gathered}
$$




$$
\begin{gathered}
w_{1}=0 \\
w_{2}(r, \theta)=w_{0} p^{3}\left(\left[4(1-\xi)_{0}-\xi(2-\xi)\right]\left(1+r^{2}\right)+\left[\frac{\xi(2-\xi)(3 n-1)-4(1-\xi)\left(n^{2}+2 n-1\right)_{0}}{(n+1)}\right] r^{n} \sin (n \theta)\right)
\end{gathered}
$$

Further, albeit more involved, exact solutions can be found for $w_{3}$ and higher order terms in $W_{i}$. In these results, since the parameter $\varepsilon$ has a maximums value of 0,3849 for $n=3$, which decreases as $n$ increases, the series in $\varepsilon$ shown in brackets in (38) were developed up to $(\varepsilon)$ yielding accurate results.

Computations show that up to $0\left(W i^{2}\right)$ the transversal velocity field is zero. Viscoelastic forcing terms in (20-22) are non-zero from $0\left(W i^{3}\right)$ upwards, which implies $u_{1}=u_{2}=v_{1}=v_{2}=0$.

At third order inWi, the viscoelastic forcing terms become non-zero. These are

$$
\begin{aligned}
& F_{r 3}=\left(\nabla \bullet \tau{ }_{2}\right)_{r} \\
& F_{\theta 3}=\left(\nabla \bullet \tau{ }_{2}\right)_{\theta} \\
& F_{z 3}=\left(\nabla \bullet \tau \nabla_{2}\right)_{z}
\end{aligned}
$$

If the velocity components at third order inWi are expressed in terms of a stream-function $\Psi_{3}$, i.e.

$$
u_{3}=\frac{1}{r} \frac{\partial \Psi_{3}}{\partial \theta} v_{3}=-\frac{\partial \Psi_{3}}{\partial r}
$$

then it is found

$$
r \nabla^{4} \Psi_{3}=\frac{\partial\left(r F_{\theta 3}\right)}{\partial r}-\frac{\partial F_{r 3}}{\partial \theta}
$$

and

$$
r \nabla^{4} \Psi_{3}=8 \in(\xi-2) \xi^{2}(n-1) n(n+4) p^{4} r^{n+1} \cos (n \theta)
$$

The solution of (46) is

$$
\Psi_{3}(r, \theta)=\frac{1}{4} \xi^{2}(2-\xi) p^{4}\left[1-r^{2}+r^{n} \sin (n \theta)\right]^{2} \frac{n(n-1)(n+4)}{(n+1)(n+2)} r^{n} \cos (n \theta)
$$

Where from

$$
\begin{gathered}
u_{3}(r, \theta)=-\frac{\xi^{2}(2-\xi) p^{4} n^{2}(n+4)(n-1) w_{0}{ }^{2} r^{n-1} \sin (n \theta)}{4(n+1)(n+2)} \\
v_{3}(r, 0)=\frac{\xi^{2}(2-\xi) n(n+4)(n-1) p^{4} w_{0}\left[n-(n+4) r^{2}\right] r^{n-1} \cos (n \theta)}{4(n+1)(n+2)}
\end{gathered}
$$


Plots of (47) are shown in figures 2, 3, 4 and 5

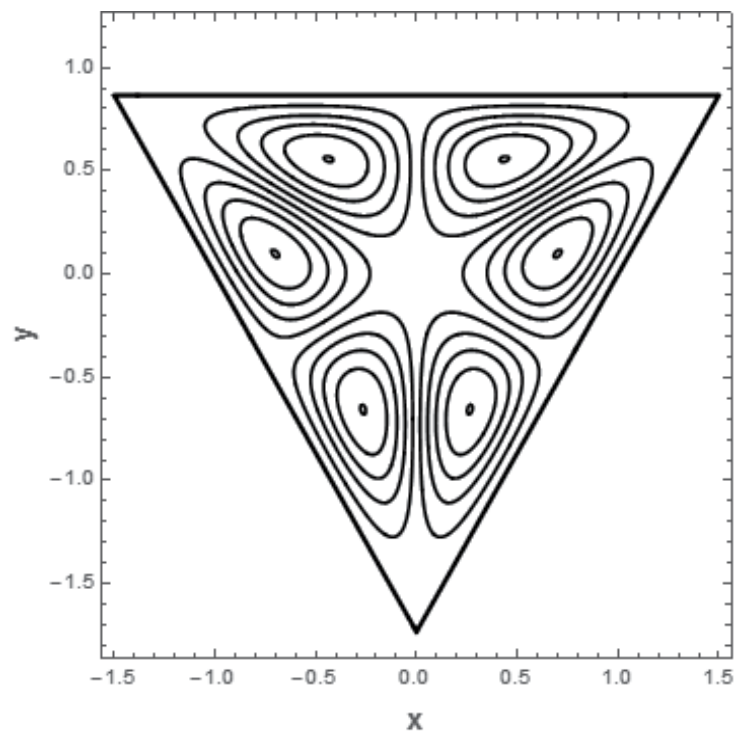

Figure 2. Characteristics plots of transversal streamlines for $n=3$ and $\varepsilon=0.3849$

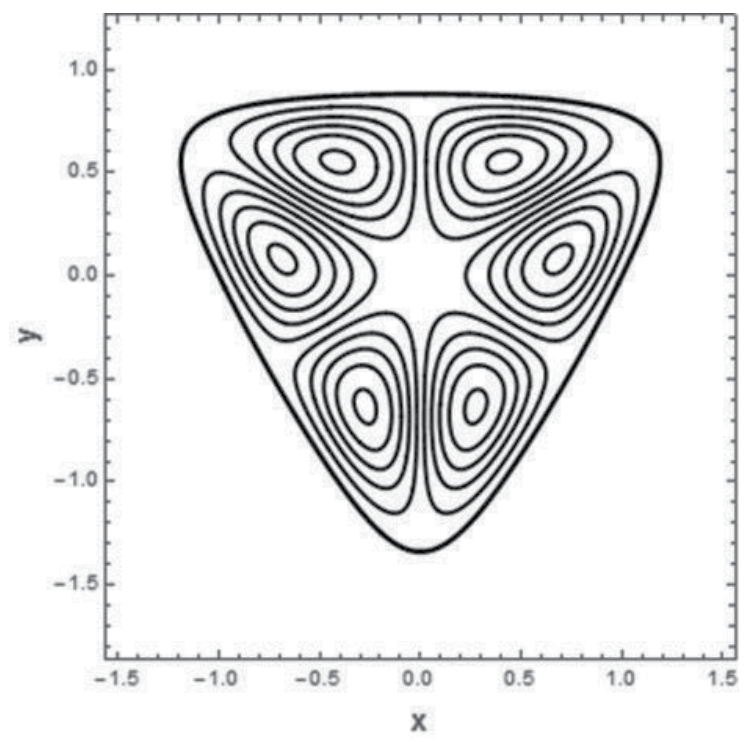

Figure 3. Characteristics plots of transversal streamlines for $n=3$ and $\varepsilon=0.33$ 


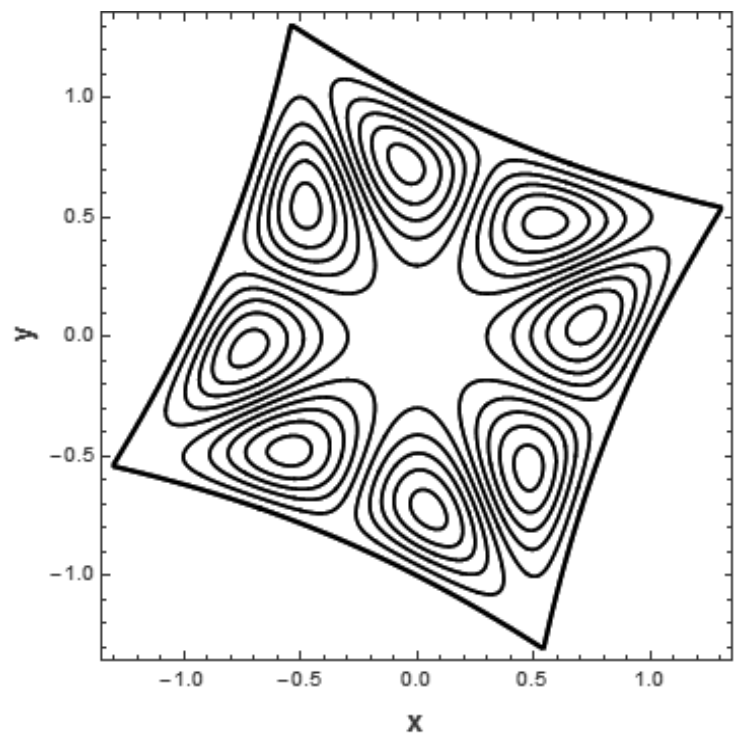

Figure 4. Characteristics plots of transversal streamlines for $n=4$ and $\varepsilon=0.25$

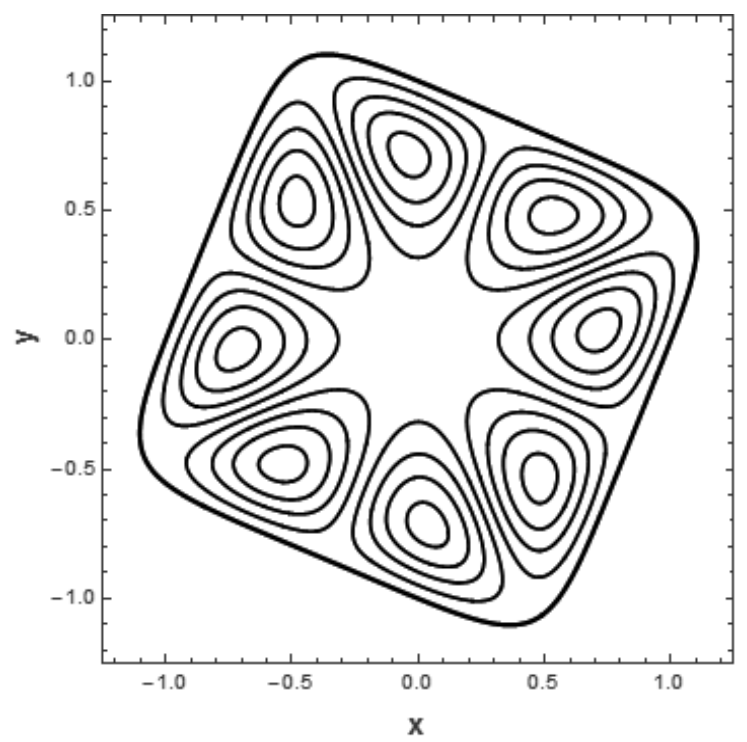

Figure 5. Characteristics plots of transversal streamlines for $n=4$ and $\varepsilon=0.2$

It is to be noted that the vortical shape does not change with the slip parameter $\xi$, the pressure coefficient $p$ or the Weissenberg number. As these parameters change, the strength of the 
vortices change. Also, other parameters being equal, the strength of the vortices increases significantly with $\varepsilon$, and thus transversal transport capacity. In all these cases the Reynolds number is $R_{e}=180$. Similar results are found for $n=4,5 \ldots$ in which the number of vortices is $2 n$.

In Figure 6 is shown the internal distribution of the normal axial shear stress for the case of $\varepsilon=0.3849$

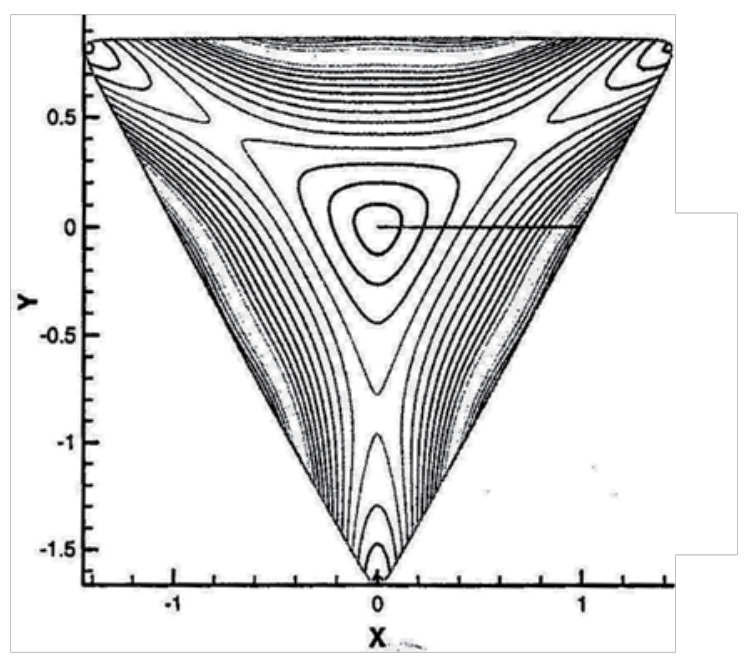

Figure 6. Characteristics plots of normal axial shear stress for $\mathrm{n}=3, \varepsilon=0.3849, \mathrm{Wi}=0.3$ and $\xi=0.2$

Curves are similar for all values of parameters, but the values of the normal axial shear stress vary with $W i$, being of the order of $70 \%$ greater than the Newtonian counterpart for $R e=180$ and $W i=0,3$.

The temperature field can be computed once the velocity field is known, through the energy equation (27). To this end, the temperature $\mathrm{T}$ is expressed as

$$
T=T_{0}+W i T_{1}+W i^{2} T_{2}+\ldots
$$

In the following the temperature field and heat-transfer are computed for the case in which there is a constant heat flux through de tube wall, so that the temperature difference between de wall and the average temperature, i.e., $T_{w}-T_{a}$ remains constant and also $\partial T_{a} / \partial z$ is constant. This is problem 1 described in the section Mathematical Models.

The above leads to

$$
\nabla^{2} T_{0}=a_{0} \operatorname{Pr} w_{0}
$$

in which 


$$
a_{0}=\frac{1}{T_{w}-T_{a}} \frac{\partial T_{a}}{\partial z}
$$

and $\mathrm{Pr}$ is the Prandtl number, i.e.

$$
\operatorname{Pr}=\frac{C_{p} \mu}{k}
$$

Applying the condition that the temperature, at all orders in Wi be zero at the tube contour, i.e., that the fluid temperature is equal to $T_{w}$ at the wall, then it is found

$$
T_{0}=\frac{P_{r} w_{0} p a_{0}}{16}\left[r^{2}-3+\varepsilon \frac{(n-3)}{(n-1)} r^{n} \sin n \theta\right]
$$

Higher order terms of $T$ can be found in similar way, but the expressions become very involved and are omitted here.

Heat exchange is computed through the Nusselt number $\mathrm{Nu}, \mathrm{i}$, e.

$$
N u=\frac{D_{n} h}{k}
$$

where

$$
\begin{gathered}
h=\frac{k \int \frac{d T}{d n} d \bar{p}}{\bar{p}\left(T_{w}-T_{a}\right)} \\
D_{n}=\frac{4 S}{\bar{p}}=\text { hydraulic diameter } \\
S=\iint r d r d \theta
\end{gathered}
$$

In the above $\bar{p}$ is de contour perimeter and $S$ is de cross-sectional area. A plot of $N u$ in terms of $W i$ is shown in Figure 7 for different values of the Reynolds number Re, defined as

$$
\operatorname{Re}=\frac{\rho_{0} w_{a} D h}{\eta_{m 0}}
$$

in which $w_{a}$ is the axial average velocity.

Numerical values related to Figure 7 are given in the following table, where they can be compared with the Newtonian case

Typical plots of temperature distribution appears in Figures 8 and 9. 


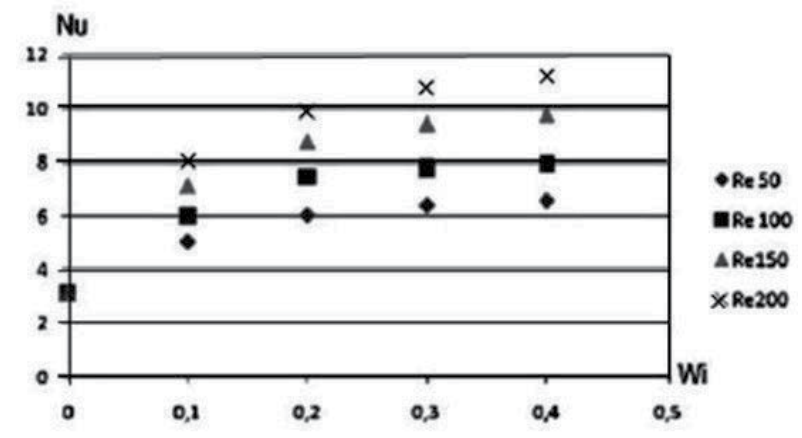

Figure 7. Please add caption

\begin{tabular}{|c|c|c|c|c}
\hline $\mathbf{N u}$ & $\mathbf{R e}$ & $\mathbf{R e}$ & $\mathbf{R e}$ & $\mathbf{R e}$ \\
\hline $\mathbf{W i}$ & 50 & 100 & 150 & 200 \\
\hline 0 & 3,1623 & 3,1623 & 3,1623 & 3,1623 \\
\hline 0.1 & 5,0498 & 6,0012 & 7,1403 & 8,046 \\
\hline 0.2 & 6,0340 & 7,4544 & 8,7734 & 9,895 \\
\hline 0.3 & 6,3993 & 7,7702 & 9,4245 & 10,7913 \\
\hline 0.4 & 6,5746 & 7,9199 & 9,7712 & 11,2114 \\
\hline
\end{tabular}

Table 1. Nusselt number values

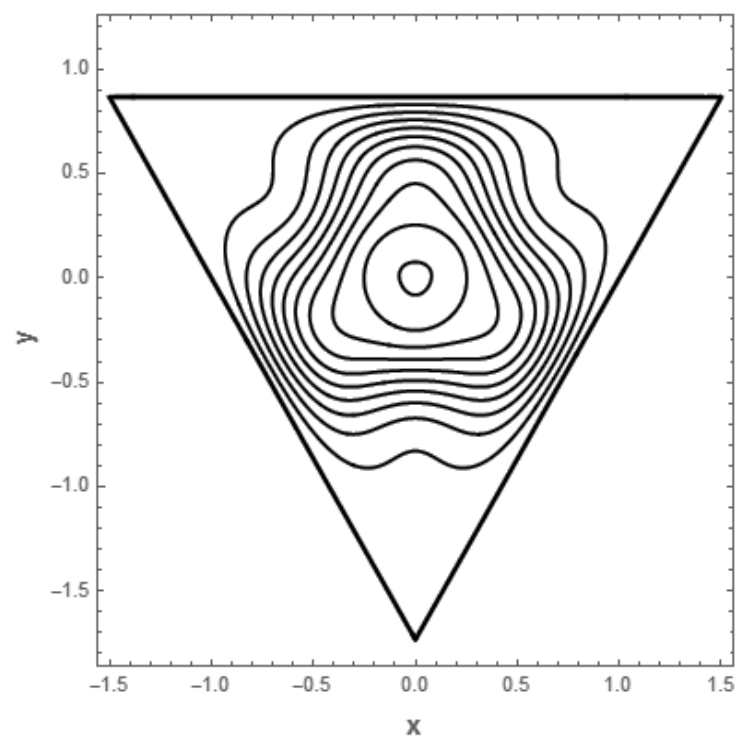

Figure 8. Characteristics plots of isothermal curves for $n=3$ and $\varepsilon=0.3849$ 


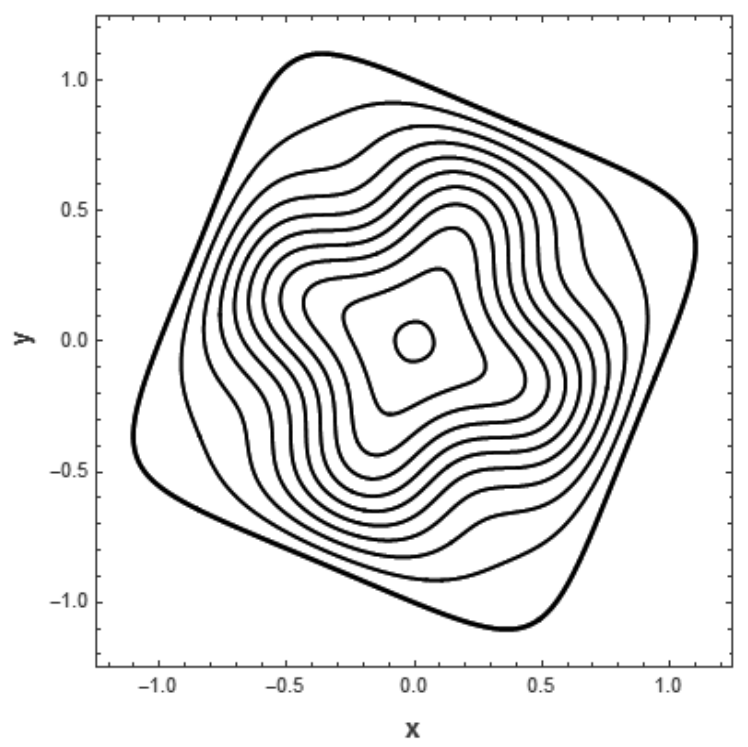

Figure 9. Characteristics plots of isothermal curves for $n=3$ and $\varepsilon=0.2$

These results, as initially presented and discussed in $[13,25,26]$ show that the Nusselt number, i.e., the heat-transfer between fluid and wall, increases as the viscoelastic parameter Wi increases, with an asymptotic trend, for a given value of the Reynolds number. As $R_{e}$ increases, also $N_{u}$ increases for a given value of Wi.

The relevance of these findings for biological flows may be related to vessel deformation due to wall elasticity and peristaltic motion.

The preceding analysis shows that a very small deviation of the cross-section contour from the circular geometry, as represented by the value of the parameter $\varepsilon$, may induce secondary flows, and so increase the transversal transport capacity of biological flows. It seems to be an open research area the study of physiological and morphological changes that, in this context, may induce pathologies that accelerate heat transfer inside the human body. Such changes may create conditions that improve or worsen transport processes that should lead to restore normal physiological states.

Previous results associated to the flow of viscoelastic fluid in channels of axially-varying crosssection [27] show also that axial change of geometry, as found in biological vessels, also augment transport capacity.

\section{Plastic flow}

The velocity field and related variables for the flow of a Bingham fluid in non-circular tubes is next found using a similar technique. The axial velocity is defined as 


$$
w=w_{0}+\varepsilon w_{1}+\varepsilon^{2} w_{2}+
$$

The constitutive equations $(29,30)$ can be expanded in series around the parameter $\varepsilon$ by using (58), where from [24]

$$
\begin{gathered}
\tau_{r z}=N-\frac{\partial w_{0}}{\partial r}-\varepsilon \frac{\partial w_{1}}{\partial r}+0\left(\varepsilon^{2}\right) \\
\tau_{\theta z}=-\frac{\varepsilon}{r}\left(\frac{\partial w_{0}}{\partial r}-N\right) \frac{\frac{\partial w_{1}}{\partial \theta}}{\frac{\partial w_{0}}{\partial r}}+0\left(\varepsilon^{2}\right)
\end{gathered}
$$

From the above the governing equation for $w_{1}$ are found, i.e.

$$
r\left(r-\frac{N}{2}\right)\left(\frac{\partial^{2} w_{1}}{\partial r^{2}}+\frac{1}{r} \frac{\partial w_{1}}{\partial r}\right)+\frac{\partial^{2} w_{1}}{\partial \theta^{2}}=0
$$

which can be solved by separation of variables.

The dissipation function, in terms of the normal axial shear stress, is given in equation (35) and can be computed, as an indicator of energy dissipation due to friction.

Typical results for the velocity field and dissipation function are shown in the following figures.

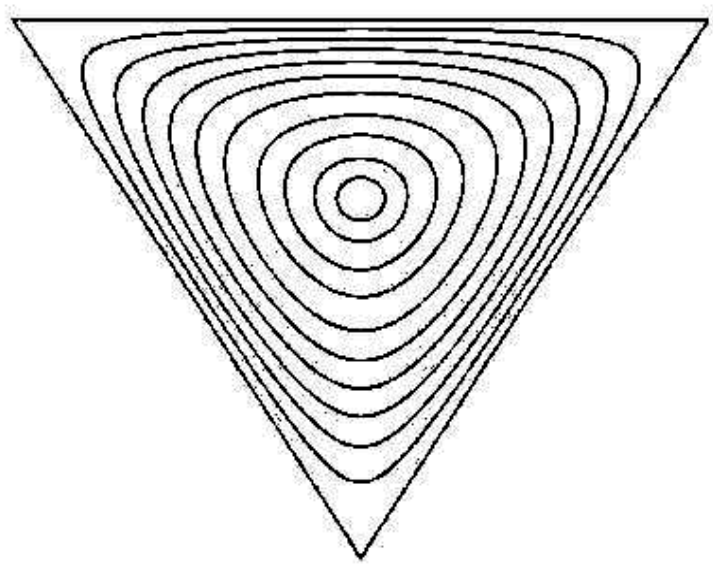

Figure 10. Plots of isovels for $\mathrm{n}=3, \quad \varepsilon=0.3849$ and $\mathrm{N}=0.2$ 


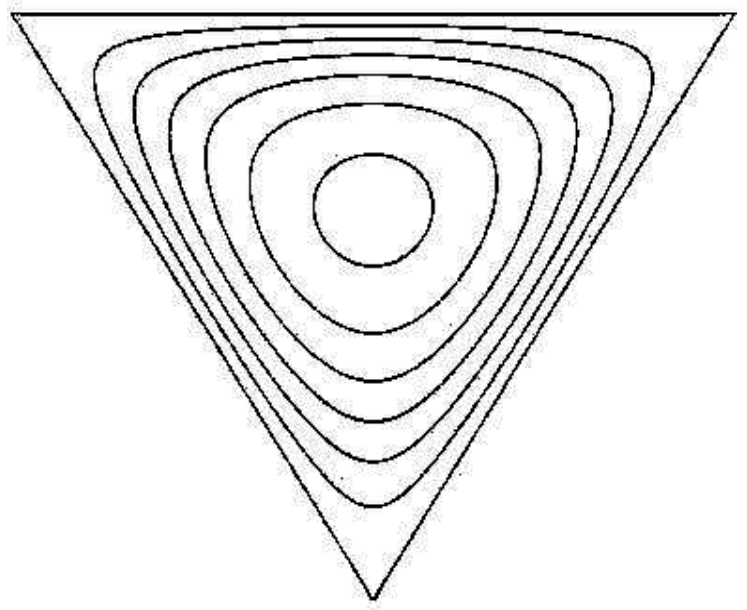

Figure 11. Plots of isovels for $n=3, \varepsilon=0.3849$ and $N=0.5$

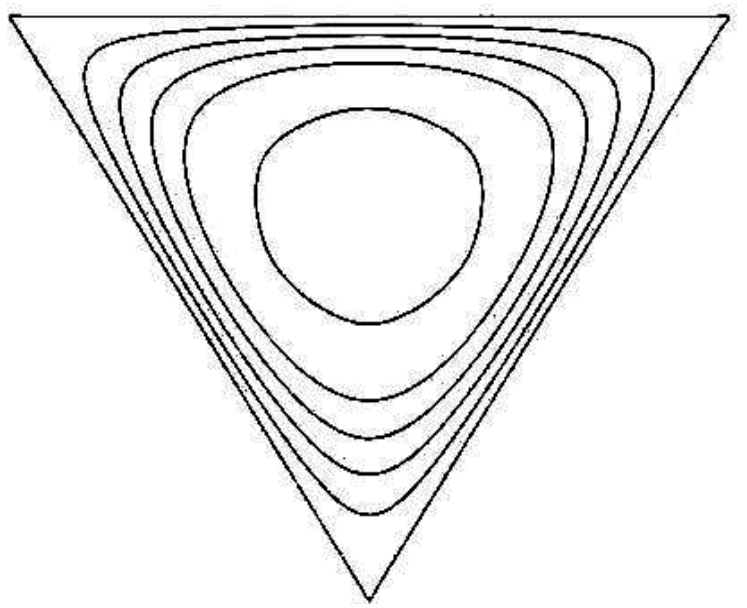

Figure 12. Plots of isovels for $n=3, \varepsilon=0.3849$ and $N=1.0$

One significant feature of plastic flows are plug (or solid) and stagnant zones, which, as a general rule, tend to decrease the rate of flow for a given pressure gradient, or require that more energy by supplied in order to keep constant the rate of flow.

Figure 13 shows that energy dissipation is greatly affected by the tube geometry. The maximum energy dissipation in the case of an equilateral triangle appears in the middle of the sides, where the axial shear stress is maximum. Also the shear stress is zero at the corners, which is a factor that determines stagnant areas close to such corners.

The plug zones are defined through the following joint conditions 


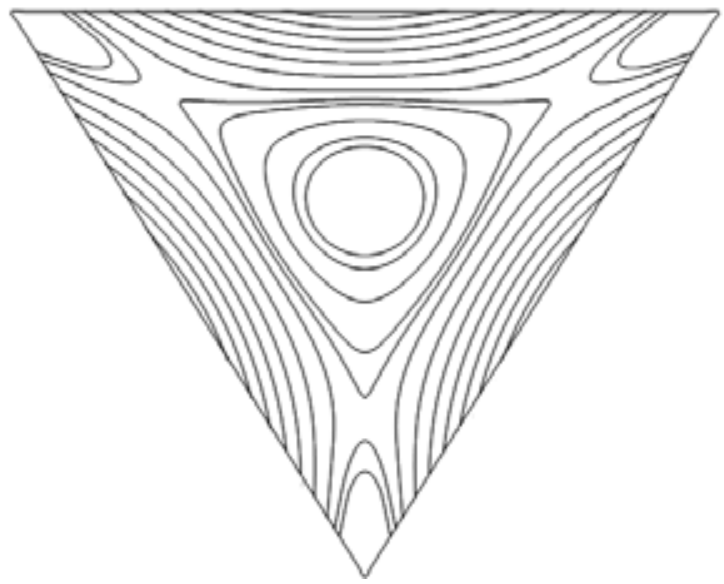

Figure 13. Plots of the dissipation function (35) for $n=3, \varepsilon=0.3849$ and $N=0.5$

$$
\frac{d w}{d n}=0 ; w=\text { const }
$$

which can be met up to certain value of $N$ according to the cross-section shape.

Abnormal vessel geometries may occur in biological flow arising from many sources. Especially in the case of stenosis, or geometry change due to solid deposition in artery walls, plastic effects may lead to blood clotting in corners, when the arteries are small.

\section{Concluding remarks}

Some analytical results concerning the effects of fluid elasticity and plasticity, coupled with tube cross-sectional geometry variation have been presented in this chapter.

The analysis is unified through some concepts that make it possible to explore, in a rather general fashion, the mechanisms of transversal transport that arise with the coupling of viscoelasticity and non-circular shape.

The circular shape is the most energy-efficient shape when only longitudinal mass transfer is considered. However, this not the case when transversal motion becomes important, as in heatexchange processes, or in cases when also particle distribution is relevant.

General results indicate that viscoelasticity tends to increase transversal transport, which can be demonstrated by analytical means. These in particular, show that starting at third order in $W_{i}$, secondary flows appear when the fluid exhibits non-linear viscoelasticity, as prescribed by the fluid models of Giesekus and Phan-Tien-Tanner. Such secondary, or transversal motions, when coupled with the temperature, through the energy equation, determine a temperature field that improve heat-transfer between the fluid and the tube wall. 
Plastic effects, on another perspective, do not induce transversal motion if the cross-section is not circular. Rather plasticity increases energy consumption when maintaining a given rate of flow is a priority. Shapes that include sharp corners lead to stagnant zones of fluids in the vicinity of those corners, thus decreasing the flow. The analytical method herein applied can be used for determining plug-zones of limiting values of the yield parameter $N$ for which such zones exist properly.

The application of these findings to biological flows have been commented in previous sections of the chapter.

\section{Acknowledgements}

The author acknowledges the financial support of the National Fund for Scientific and Technological Development-FONDECYT, and of the Department of Scientific and Technological Research-DICYT at the University of Santiago, Chile.

\section{Author details}

Mario F. Letelier

Address all correspondence to: mario.letelier@usach.cl

Department of Mechanical Engineering, Center for Research in Creativity and Higher Education, University of Santiago, Chile

\section{References}

[1] M. Marinkovic, K. Turner, J. Butler, J. Fredberg, S. Suresh, Viscoelasticity of the Human Red Blood Cell. American Journal of Physiology - Cell Physiology 293 (2007), 597-605.

[2] A. Ündar, W. Vaughn, J. Calhoon, The effects of cardiopulmonary bypass and deep hypothermic circulatory arrest on blood viscoelasticity and cerebral blood flow in a neonatal piglet model. Perfusion 15 (2000) 121-128.

[3] M. Letelier, J. Stockle, A Shape-Factor method for Modeling Parallel and AxiallyVarying Flow in Tubes and Channels of Complex Cross- Section Shapes. Biomedical Science. Engineering and Technology: InTech 19 (2011) 469-487. 
[4] J.P. Hartnett, M. Kostic, Heat transfer to a Viscoelastic Fluid in Laminar Flow Through a Rectangular Channel. International Journal Heat Mass Transfer 28 (19859 1147-1155.

[5] J.P Hartnett, M. Kostic, Heat Transfer to Newtonian and Non-Newtonian Fluids in Rectangular Ducts, Adv. Heat Transfer 19 (1989) 247-355.

[6] J.P. Hartnett, Viscoelastic Fluids: a New Challenge in Heat Transfer (1990 Max Jacob Memorial Award Lecture). Journal Heat Transfer 114 (1992) 296-303.

[7] D.R. Oliver, Non-Newtonian Heat Transfer: an Interesting Effect Observed in nonCircular Tubes, Trans. Inst. Chem. Eng. (1969) 47 (T18).

[8] S.X Gao, J.P. Hartnett, Steady Flow of non-Newtonian Fluids Through Rectangular Ducts, International Commun. Heat Mass Transfer 20 (1993) 197-210.

[9] S.X. Gao, J.P. Hartnett, Heat Transfer Behavior of Reiner-Rivlin Fluids in Rectangular Ducts, International Journal Heat Mass Transfer 3 (1996) 1317-1324.

[10] M.F Naccache, P.R. Souza Mendes, Heat Transfer to non-Newtonian Fluids in Laminar Flow Through Rectangular Ducts, International Journal Heat Fluid Flow 17(6) (1996) 613-620.

[11] P. Payvar, Heat Transfer Enhancement in Laminar Flow of Viscoelastic Fluids Through Rectangular Ducts, International Journal Heat Mass Transfer 40(3) (1997) 745-756.

[12] S. Syrjala, Laminar Flow of Viscoelastic Fluids in Rectangular Ducts with Heat Transfer: a Finite Element Analysis, International. Commun. Heat Mass Transfer 25(2) (1998) 191-204.

[13] D.A Siginer, M.F. Letelier, Laminar Flow of Non-Linear Viscoelastic Fluids in Straight Tubes of Arbitrary Contour, International Journal Heat Mass Transfer 54(9-10) (2011) 2188-2202.

[14] P.M. Coelho, F.T. Pinho, P.J. Oliveira, Thermal entry flow for a Viscoelastic fluid: the Graetz Problem for the PTT Model, International Journal of Heat and Mass Transfer 46 (2003) 3865-3880.

[15] P.P. Valko, Solution of the Graetz-Brinkman problem with the Laplace transform Galerkin method, International Journal of Heat and Mass Transfer 48 (2005) 1874-1882.

[16] W.S. Kiln, M.N. Özisik, Transient laminar forced convection to power-law fluids inside ducts resulting from a sudden change in wall temperature, Wärme- und Stofffibertragung 2 (1998) 159-165.

[17] M. Letelier, D.A. Siginer, J.S. Stockle, Secondary Flows in Straight Non-Circular Tubes-Giesekus Fluid. Asme International Mechanical Engineering Congress and Exposition, November 2013, San Diego, California, USA. 2013. 
[18] S.C. Xue, N. Phan-Thien, R.I. Tanner. Numerical Study of Secondary Flows of Viscoelastic Fluid in Straight Pipes by an Implicit Finite Volume Method. Journal of NonNewtonian Fluid Mech. 59 (1995) 191-213.

[19] D.L. Tokpavi, A. Magnin, P. Jay, Very Slow Flow of Bingham Viscoplastic Fluid Around a Circular Cylinder. Journal of Non-Newtonian Fluid Mechanics 154 (2008) 65-76.

[20] Z. Yu, A. Wachs, A Fictitious Domain Method for Dynamic Simulation of Particle Sedimentation in Bingham Fluids. Journal of Non-Newtonian Fluid Mechanics 145 (2007) 78-91.

[21] A. Wachs, Numerical Simulation of Steady Bingham Flow Through an Eccentric Annular Cross- Section by Distributed Lagrange Multiplier Fictitious Domain and Augmented Lagrangian Methods. Journal of Non-Newtonian Fluid Mechanics 142 (2007) 183-198.

[22] I.C. Walton, S.H. Bittleston, The Axial Flow a Bingham Plastic in a Narrow Eccentric Annulus. Journal Fluid Mechanics 222 (1991) 39-60.

[23] M. Letelier, D. Siginer, On the Flow of a Class of Viscoinelastic-Viscoplastic Fluids in Tubes of Non-Circular Contour. International Journal of Engineering Science 45 (2007) 873-881.

[24] M. Letelier, F. Godoy, C. Rosas, On the Existence Conditions for Plug Zones in Plastic Flow in Tubes of Non-Circular Cross-Section. 14 ${ }^{\text {th }}$ Pan-American Congress of Applied Mechanics PACAM XIV 2014, Santiago, Chile.

[25] D.A. Siginer, M.F. Letelier, Heat Transfer Asymptote in Laminar Flow of Non-Linear Viscoelastic Fluids in Straight Non-Circular Tubes, Int. J. Engineering Science 48(11) (2010) 1544 -1562.

[26] M. Letelier, F.N. Zapata, D.A. Siginer, J.S. Stockle, Analysis of Secondary Flows and Heat Transfer in Viscoelastic Flow with Viscous Dissipation in Non-Circular Tubes. ASME International Mechanical Engineering Congress and Exposition, November 2012, Houston, Texas, USA 2012.

[27] M.F. Letelier, R. Gaete, D.A. Siginer, Steady Viscoelastic Flow Channels of Arbitrarily Varying Cross-Section Shape. Asme International Mechanical Engineering Congress and Exposition, DC., ISBN Nº-7918-4663-6, Washington 2003. 
Chapter 13

\title{
Quantifying Stress in Crabs and Humans using Modified DFA
}

\author{
Toru Yazawa
}

Additional information is available at the end of the chapter

http://dx.doi.org/10.5772/59718

\section{Introduction}

Stress, which can be acute or chronic, is a term that has not been strictly defined, especially in the field of neuroscience. Scientists have not yet fully characterized the complete set of neuronal mechanisms that can explain stress. In this article, however, I use the term "stress" because it is the most appropriate one.

Stress is a physiological reaction of an organism to an uncomfortable or unfamiliar physical or psychological stimulus. Stress-inducing stimuli trigger reflex behavior, which results from alteration in the activity of the autonomic nervous system (ANS) and hormones. Reflex behavior includes a heightened state of alertness and increased heart rate. Acute stress is a short-term response that lasts for seconds, minutes, or days. In this article, "stress" refers to acute stress.

Scientists have not yet determined a method that can efficiently quantify stress. In fact, scientists have difficulty objectively defining whether an organism is expressing stress in response to stimuli. Through studies of crustacean hearts using electrophysiological methods, I have discovered that stressful and non-stressful states can be distinguished by heartbeat recordings: i.e., via electrocardiograms (EKGs). The methods used to measure stress in model animals can be applied to humans because model animals and humans are fundamentally similar in terms of autonomic nerve physiology. The autonomic cardio-regulatory function can simply described as the combined action of acceleration and inhibition. In this article, I present empirical results of stress observations in both model animals and humans. The presented data provide convincing evidence that stress can be quantified. 


\section{Brief history of crustacean heart physiology}

Cardiac nerves - comprising accelerator nerves (CA) and inhibitory nerves (CI) - govern the activity of the heart in an involuntary manner in both model animals and humans. CA and CI are cardiovascular components of the ANS that transmit psychological information to the heart. Alterations in the activity of these neurons can modulate momentary heart rate and contractile force in a reflex manner in crustaceans as shown in Figures 1-3. Because the degree of stress can be measured by monitoring heart rate variability, the heart can be called a reflection of the neural activity of an animal.

However, the cardiac regulatory function of the human ANS is not clearly understood because of the difficulty associated with recording ANS activity. Nevertheless, I have successfully recorded ANS activity in crustaceans. Both CA and CI are active when the heart is beating. This is evident in in situ recordings of nerve activity in hermit crabs in the 1970s (Figure 1, unpublished data). In decapod crustaceans, only one pair of nerves, termed the cardiac or regulator nerves, innervate the heart bilaterally. The crustacean system therefore appears simpler than the human cardiac nervous system. However, appearances can be deceiving: the fundamental mechanisms of the acceleration/inhibition functions are similar in humans and crustaceans, and the ANS controls the cardiac region in both animals. It is evident in Figure 1 that three different impulses varying in size indicate discharging: small (not marked), medium (shown by triangles), and large (not marked) impulses. A significant feature of Figure 1 is that the crab's heartbeat stopped completely during the period when the large-sized impulses were active, which is shown by a train discharge at a high frequency of approximately 50 or $60 \mathrm{~Hz}$. Based on these observations, I was able to identify the neurons (in this case, the large-sized impulses) that transmit inhibitory commands. CI and CA always function in balance with each other. Figure 1 shows that the ANS dynamically controls the heart.

Healthy human hearts do not stop beating; only the rate of the heartbeat changes. However, crustacean hearts can cease beating for a period of time (Figure 2). A key finding in my crustacean study was that specimens showed a pattern of intermittent heartbeat cessation under healthy, normal, non-stressed conditions. The cessation was induced by an intermittent burst discharge of CI as mentioned above (Figure 1). An important observation was that the presence of a human caused interruptions in this intermittency. In other words, stress from an approaching human caused an alteration in ANS function: i.e., the intermittent pattern changed to a continuous pattern [2] (Figure 3).

This intermittency was actually documented in the 1970s by Canadian crustacean heart researchers, J. L. Wilkens and B. McMahon, though they did not mention the role played by stress. (They found a strong relationship between cardiac and respiratory control.) The physiology of the crustacean ANS has been studied throughout the $20^{\text {th }}$ century by Carlson (1900s), Alexandrowicz (1930s), Maynard (1950s, 1960s), Young [3], Field and Larimer [4], and Yazawa and Kuwasawa [5]. However, these authors did not provide information on the relationship between heartbeat and behavior of crustaceans. 


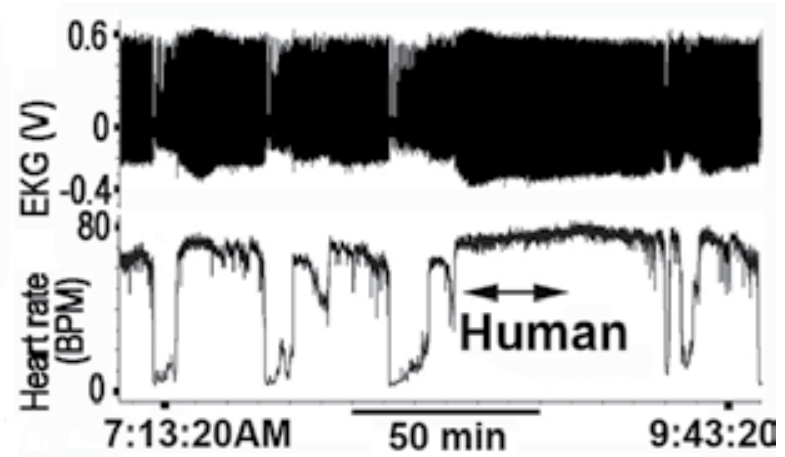

Figure 3. Intermittent heartbeat cessation of a Japanese spiny lobster (Panulirus japonicus) was interrupted by an approaching human shown in an EKG recording from a live specimen.

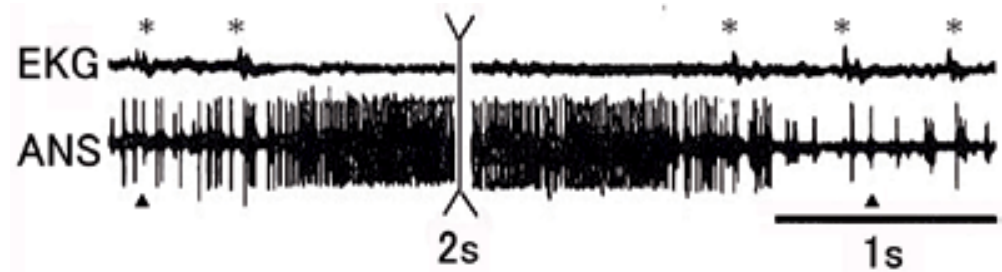

Figure 1. Simultaneous recording of heartbeat (EKG) and cardiac nerve activity (ANS) of the hermit crab (Dardanus crassimanus). Asterisks $\left({ }^{*}\right)$ indicate heartbeats.

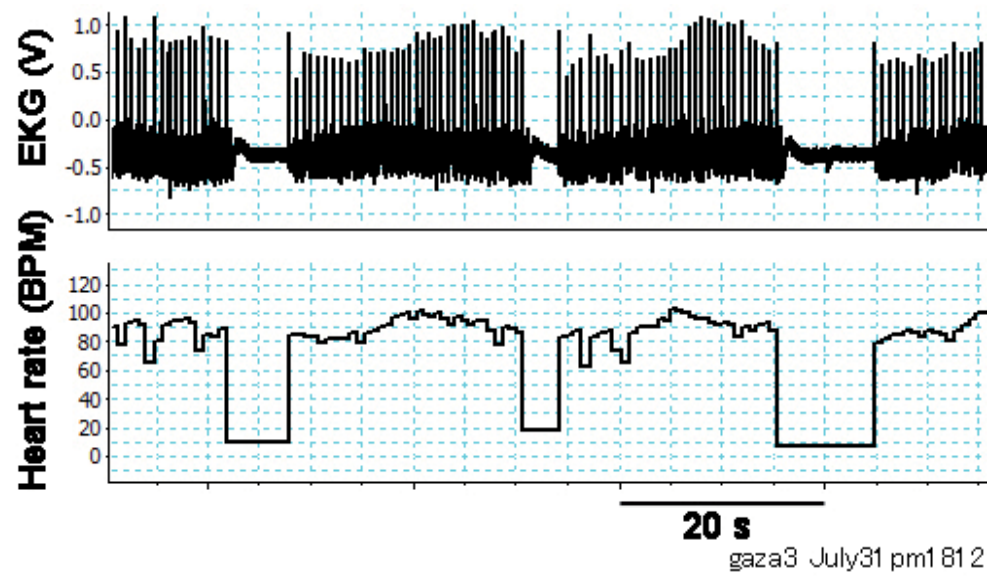

Figure 2. Intermittent cessation of heartbeat shown in an EKG recording from a live saw tooth Gazami crab (Scyll serrata) specimen.

In summary, I recorded impulses of the cardiac nerves of crustacean hearts. I observed that CI discharged a burst of impulses at a high-rate, $\sim 60 \mathrm{~Hz}$, and concomitantly, CA momentarily 
ceased their impulse discharge [1]. During the CI burst period, the heart rate disappeared or decreased significantly, although a rapid restitution of heartbeats occurred within one second. These brief cessations of heartbeats occurred intermittently and somewhat regularly during "stress-free" periods, for example, when the animal was hiding in a shelter. In contrast, the stress-free behavior was not exhibited if the lobsters or crabs were approached by humans. This response can be likened to the way cicadas stop singing when humans are in close proximity. These findings suggest that crustaceans are incredible specimens because their stress can be detected by electrocardiograms.

Crustacean and human hearts strongly resemble each other in structure and function. It is known that homologous genes (e.g., Nkx2-5, the NK2 homeobox gene) function to form the developing heart of all animals: [6]. In crustaceans and humans, both CA and CI connect with the cardiac pacemaker cells, but CA further proceed to the ventricular muscles beyond the pacemaker cells. Why do CA control the entire heart? The answer is that CA-muscle connections can implement direct modulation of contractile force, whereas CI merely suppress rhythm [5]. The resemblance between crustacean and human indicates that some knowledge obtained from crustacean should be applicable to human. See Recent report by Fossat et al.

(1) P. Fossat et al. "Anxiety-like behavior in crayfish is controlled by serotonin." Science 344, 1293-1297 (2014).

(2) P. FOSSAT et al. "Anxiety-like behavior in crayfish is controlled by serotonin." Society for Neuroscience, Poster 655.18/UU43 - Motivation and Emotions: Rodent Anxiety Models Tue, Nov 18, 1:00 - 5:00 PM 2014 Washington DC.

(3) OUTSIDE JEB. The Journal of Experimental Biology (2014) 217, 3389-3391

I studied electrocardiograms (EKG) of both animal models and humans and used modified Detrended Fluctuation Analysis (mDFA) to calculate the scaling exponent (SI) (originally, Peng et al., [7]). In this article, I show that the SI numerically is capable of distinguishing between healthy and unhealthy hearts, and between "stressed" and "relaxed" hearts. Further, I propose the mDFA to be a viable potential method for health/stress checking if incorporated into a device that can quantify stress through EKGs.

\section{Electrophysiology method}

Human heartbeats were recorded using a PowerLab system (AD Instruments, Australia). A set of three, ready-made $\mathrm{Ag}-\mathrm{AgCl}$ electrodes (Vitrode $\mathrm{V}$, Nihonkoden Co. Ltd. Tokyo) were used for EKG monitoring. Permanently mounted metal electrodes were glued on the crustacean carapace for EKG recordings. These EKG signals were transferred to the PowerLab system and PC. All subjects were treated according to the ethical regulations of Tokyo Metropolitan University. 


\section{Stress hormone measurement}

The stress response of the lobster to approach by a human (Figure 3) may involve the contribution of stress hormones, but combined hormone and EKG measurements have not been conducted. Therefore, I conducted micro-dialysis blood hormone measurements to determine whether hormones are involved in crustacean stress responses. I prepared four different HPLC-ECD (high performance liquid chromatography with electrochemical detection) machines. These machines were respectively set up to separate biogenic monoamines (dopamine, adrenaline, serotonin and their breakdown products), acetylcholine and related substances, amino acid neurotransmitters (glutamate, GABA, etc.), and peptide hormones. Blood samples were collected using a micro-dialysis probe for all HPLC analyses (Figure 4).

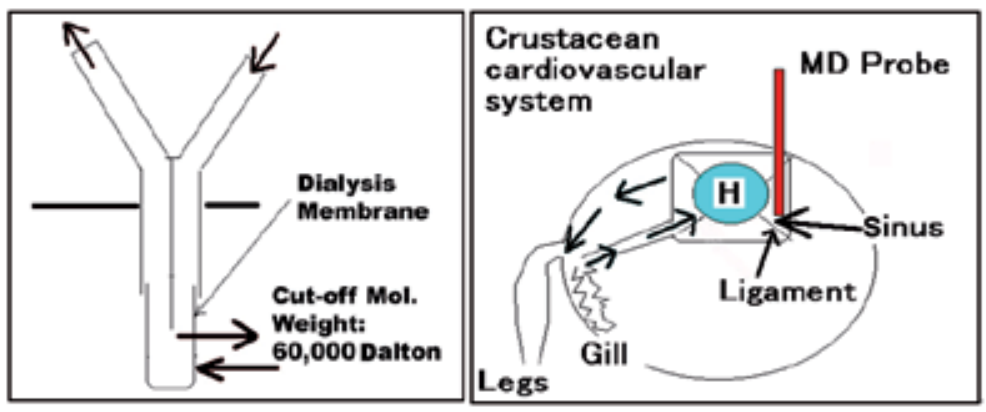

Figure 4. The micro-dialysis (MD) probe placed on the dorsal carapace of lobsters (left panel). The tip of the MD probe placed in the blood stream (right panel). H represents the heart chamber and arrows indicate the direction of flow.

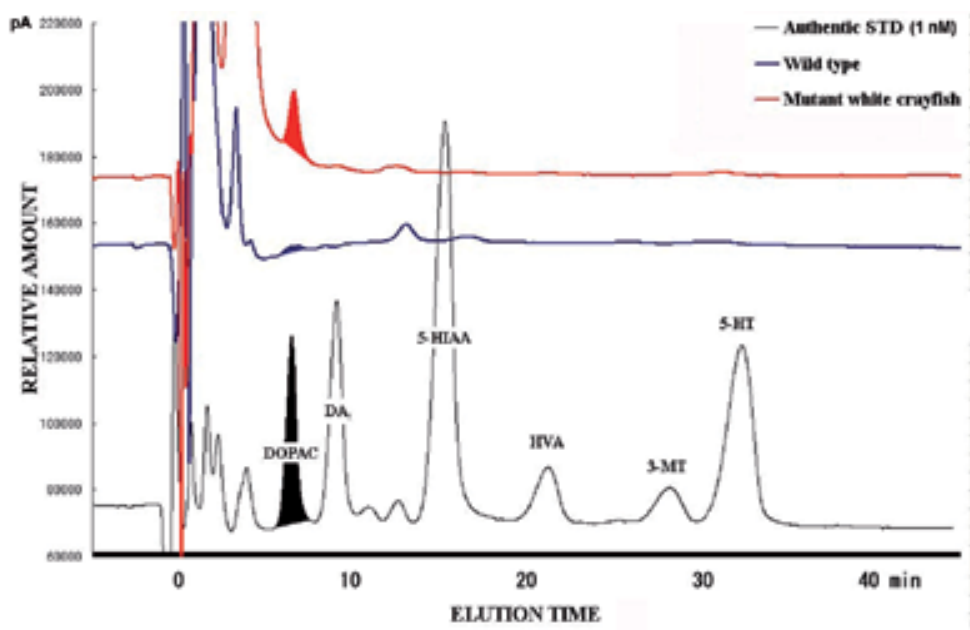

Figure 5. HPLC chart indicating the detection of DOPAC (a breakdown product of dopamine) using the HPLC system in a blood sample collected from a crayfish (Procambarus clarkii) specimen. 


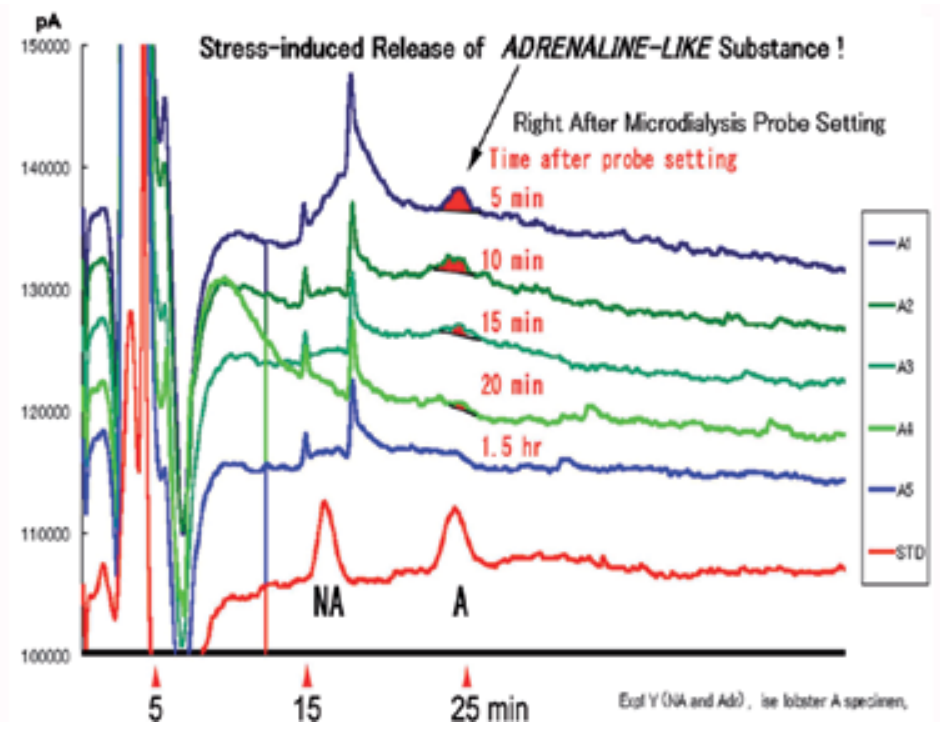

Figure 6. HPLC chart indicating the detection of adrenaline (A) in a Japanese lobster (Panulirus japonicus) specimen. Adrenaline release was induced by stress from human handling. Very little adrenaline was detected 20 min and 1.5 hour after the stress stimulus. Therefore, the adrenaline content measured at these times indicates the basal adrenaline content in the blood. Note: noradrenaline (NA) was not detected.

A

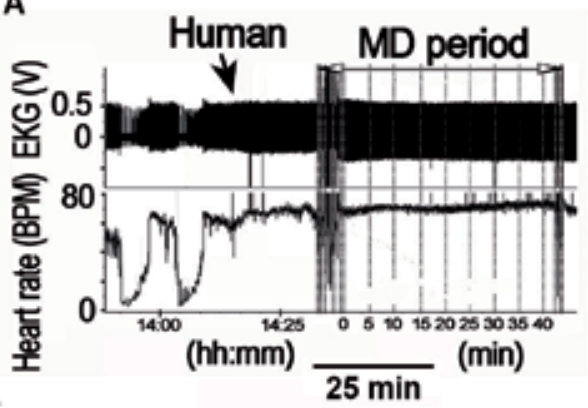

B

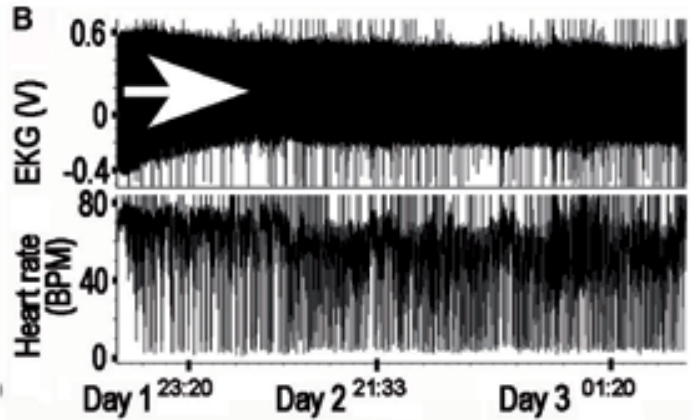

Figure 7. EKG recording and MD experiment in a Japanese spiny lobster (Panulirus japonicus). A, a human approached the lobster tank and conducted an MD experiment. B, continuous recording from A (note: the time scale is different).

Micro-dialysis HPLC (MD-HPLC) analysis detected a few substances in the blood samples before, during, and after stress stimulation. EKGs were continuously recorded to check stress responses. Figure 7 shows a lobster's response to a human, observed through EKG recordings. Significant features are evident in Figure 7. First, approach by a human interrupted the slow, repetitive heartbeat pattern (Figure 7A). Second, the micro-dialysis experiment resulted in continuous stress to the animal (MD period in Figure 7A). Third, the stressful reaction lasted for approximately one day, the period shown by a white arrow (Figure 7B). 


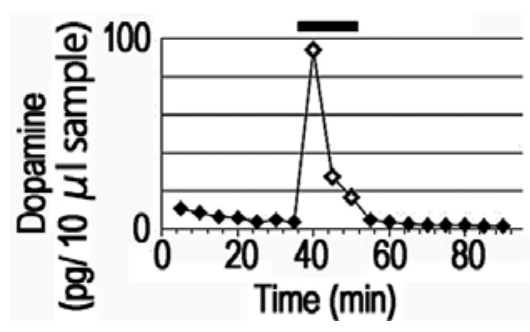

Figure 8. Micro-dialysis HPLC detection of dopamine in a Japanese spiny lobster (Panulirus japonicus) specimen. The bar indicates a stimulation period of $15 \mathrm{~min}$.

Figure 8 shows the stress-induced release of dopamine. Blood samples were collected for 5 min and collection vials were exchanged every $5 \mathrm{~min}$ by an automatic solution-sampling machine. A human entered the room containing the lobster for only a short time period (15 min); this period is indicated by the black bar. The human presence evidently irritated the lobster, triggering a sharp increase in dopamine concentration, but this was followed by a rapid decrease even though the stimulus was maintained for $15 \mathrm{~min}$. The same stimulus also induced adrenaline release, shown in Figure 6. The detection of stress-related hormones was confined to dopamine and adrenaline and the breakdown product DOPAC. No other hormones that directly correlated with stress behavior were detected-e.g., serotonin, noradrenaline, amino acid neurotransmitters, and peptide hormones. Further details about these experiments can be found in [8].

A characteristic feature revealed by the MD-HPLC experiments was that increased levels of stress hormones are not maintained at a consistently high level even though the stress stimulation was steadily maintained for $15 \mathrm{~min}$ (Figure 8). In conclusion, acute stress is governed mainly by the functioning of the nervous system, whereas chronic stress more likely relates to hormonal systems. Hormone release seems to be able to trigger a chain reaction in biochemical pathways that results in a state of chronic stress.

Based on the results of the MD-HPLC examinations, I suggest that stress can be quantified by measuring heartbeat rather than hormones. Stress can be determined using heartbeat fluctuation analysis; the method used was modified DFA (mDFA).

\section{Modified DFA and DFA}

In this article, I compare the methodologies of original and modified DFA. While most of the computation sequences in DFA and mDFA are similar, there is one difference in the computation process.

In DFA and mDFA, the scaling exponent (Peng used the Greek letter alpha) or scaling index (SI) is calculated from time series data that obey the scaling law. Here, I use SI to refer to both.

In the mid-1980s, Goldberger pioneered the application of nonlinear dynamics to clinical cardiology [9]. Thereafter, a voluminous literature appeared on chaos and nonlinear analysis 
in the life sciences [10]. The following literature provides information on nonlinear physics with respect to heart physiology and DFA: Peng [11], Glass [12], Stadnistski [13], Stanley [14], Goldberger [9], Katsuyama [15], Pérez [16], Liebovitch [17], Huikuri [18], Bigger [19], Scafetta [20], and other work cited in these references. In addition, the scaling exponent, DFA, and topics related to fractality research, for example, fractal, scaling, the Hurst exponent, and power spectral density, are well explained by T. Stadnistski [13]. DFA is based on the concepts of scaling and self-similarity [13]. Peng's DFA [7] deals with critical phenomena. (Details of the mathematics of DFA can be referenced elsewhere [9-19]). One DFA program, PhisoNet, provided by Goldberger, Peng and others is available on the internet. However, there is no web-based mDFA program available. Regardless, an mDFA program can be written with an understanding of DFA and programming skills (I am a biologist and received programming support from a graduate student, Katsunori Tanaka, who constructed an mDFA program under my supervision using $\mathrm{C}++$ programming language).

While the genesis of DFA was long ago, no one has since constructed a useful device/instrument to quantify stress using it. In this article, I argue that implementing power law concepts in DFA is a superior method for its practical use in biomedicine. In addition, I present the results of our mDFA applied to real-world data. I anticipate that this literature will initiate a public debate on whether to construct such a device/instrument, and hope that a functional DFA device will be constructed as a result. This work is being performed in collaboration with Symphodia Phil Confidential, Japan (President O. Takiguchi). Empirical data and the device concept were previously presented at a conference of the Society for Chaos Theory in Psychology and Life Sciences in Milwaukee, Wisconsin, USA (August 2, 2014).

Although DFA is not a recent development, the technique is somewhat difficult to understand. An important concept of DFA is that if data exhibit scaling characteristics and self-similar fluctuations [11,14], recorded signals and their magnified/contracted copies are statistically similar. In general, statistical parameters such as the average and variance of fluctuating signals can be calculated by taking the average and corresponding variance of the signals across a certain section. In DFA and mDFA, however, the average is the squared average of the data. The calculation of the statistical parameter thus depends on the size of the section.

To use DFA as a practical tool for instantaneous determination of heart condition, the appropriate section size, i.e. box size or number of heartbeats, needs to be determined. A practical DFA tool should NOT be subject-specific. Rather, it should be constructed for general use across the population. The section size, which is a restricted period of time, was determined to range from 30 to 270 beats after conducting hundreds of tests.

\section{EKG recording before mDFA}

mDFA requires accurate heartbeat interval measurements without missing a single heartbeat. Stable EKG recordings are needed for accurate detection of peaks in the data. If subjects move (e.g., talk, touch, walk, squat, exercise, etc.) commercially available medical-EKG machines are 


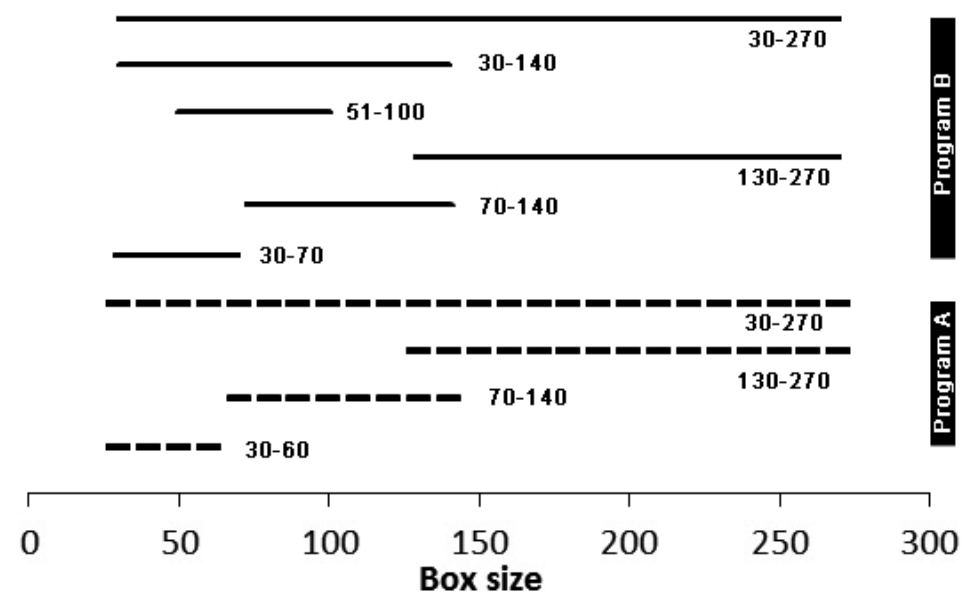

Figure 9. Range in box sizes used for mDFA (i.e., the slope from one particular box size to another).

not reliable because the EKG trace is frequently lost from the recorder chart or screen under these conditions. Accurate identification of peak heartbeat times were a necessary condition in this study. Therefore, I constructed a baseline-stable EKG recording amplifier with an input time constant of 0.1 or $0.22 \mathrm{~s}(\mathrm{C}=0.1 \mu \mathrm{F}, \mathrm{R}=1 \mathrm{M} \Omega$, or $\mathrm{C}=0.22 \mu \mathrm{F}, \mathrm{R}=1 \mathrm{M} \Omega$, respectively) and amplification-gain of 2000 times. This made it possible to record baseline-stable EKGs. I used a 1-kHz sampling rate. Based on my own electro-physiological observations, I knew that the peak time of an action-potential always fluctuates in the order of milliseconds in response to a stimulus. Therefore, I used a 1-kHz sampling rate (1000 dots per 1000 milliseconds). After detecting peak heartbeat intervals, I constructed an inter-heartbeat interval time series (such as the R-R peak interval of conventional medical EKGs). This time series was analyzed using the mDFA program.

To conduct mDFA and obtain reliable scaling exponent (SI) values, a recording of $\sim 2000$ consecutive heartbeats was required; this number was used throughout my research. If a dataset contained 4000 or 5000 heartbeats, these were divided into two data sets. The ideal (minimum essential) number of heartbeats for use in an mDFA instrument would be $\sim 2000$. In fact, various data lengths of EKGs, ranging from 700 to 5000 heartbeats are used. In this article, analysis based on 2000 beats as well as shorter and/or longer data lengths are presented in some figures. To obtain stable results in a practical health-determining device, a 30-min EKG recording is ideal (here, 1900-2100 beats were used).

There are several ways to detect heartbeat timing; however, I prefer stable baseline EKGs. In terms of a stable baseline, short and constant EKG recordings are superior to finger pulse and light-sensor (infrared) blood flow recordings. However, consecutive R-R intervals of $\sim 2000$ beats are required irrespective of the type of recording used.

Several mDFA computations were performed simultaneously: scaling exponents in various box sizes were computed (Figure 9). Initially (from 2004 to 2006), program A was used, which 


$\begin{array}{cllllllll}\begin{array}{c}\text { Program A } \\ 10\end{array} & 53 & 12 & 13 & 14 & 15 & 16 & 17 & 18 \\ 19 & 20 & 21 & 22 & 23 & 24 & 25 & 26 \\ 27 & 28 & 29 & 30 & 40 & 60 & 70 & 80 \\ 90 & 110 & 120 & 130 & 140 & 150 & 160 & 170 \\ 180 & 190 & 200 & 210 & 220 & 230 & 240 & 250 \\ 260 & 270 & 280 & 290 & 300 & 400 & 500 & 600 \\ 700 & 800 & 900 & 1000 & & & & \end{array}$

\begin{tabular}{cllllllll}
\multicolumn{2}{c}{ Program B } & 136 \\
10 & 11 & 12 & 13 & 14 & 15 & 16 & 17 & 18 \\
19 & 20 & 21 & 22 & 23 & 24 & 25 & 26 \\
27 & 28 & 29 & 30 & 31 & 32 & 33 & 34 \\
35 & 36 & 37 & 38 & 39 & 40 & 41 & 42 \\
43 & 44 & 45 & 46 & 47 & 48 & 49 & 50 \\
51 & 52 & 53 & 54 & 55 & 56 & 57 & 58 \\
59 & 60 & 61 & 62 & 63 & 64 & 65 & 66 \\
67 & 68 & 69 & 70 & 71 & 72 & 73 & 74 \\
75 & 76 & 77 & 78 & 79 & 80 & 81 & 82 \\
83 & 84 & 85 & 86 & 87 & 88 & 89 & 90 \\
91 & 92 & 93 & 94 & 95 & 96 & 97 & 98 \\
99 & 100 & 110 & 120 & 130 & 140 & 150 & 160 \\
170 & 180 & 190 & 200 & 210 & 220 & 230 & 240 \\
250 & 260 & 270 & 280 & 290 & 300 & 310 & 320 \\
330 & 340 & 350 & 360 & 370 & 380 & 390 & 400 \\
410 & 420 & 430 & 440 & 450 & 460 & 470 & 480 \\
490 & 500 & 600 & 700 & 800 & 900 & 1000 &
\end{tabular}

Figure 10. Box size (number of heartbeats) for the mDFA programs. Program A and Program B contained 53 and 136 boxes, respectively. Programming language $\mathrm{C}++$.

included box-ranges [30: 60], [70: 140], [130: 270], and [30: 270]. These four ranges were automatically and simultaneously computed. However, any box size range could be manually computed if necessary. Then, program B was added in 2006, which included box-ranges [30: 70], [70; 140], [130: 270], [51; 100], [30; 140], [30; 270] (Figure 9). These automatic ranges were arbitrarily determined. Until now, programs A and B have been used simultaneously. All existing EKG-mDFA results were computed using both programs.

Program A computed 53 box points and program B computed 136 box points (Figure 10). This increase in box number occurred because of an increase in the PC calculation speed due to a Windows software improvement. A device requires relatively rapid computation of the scaling exponent. An average scaling exponent was computed in each computation, calculated as the average of the scaling exponents across the range of box sizes (see Figure 22 and 23).

mDFA calculations use recordings of approximately 2000 beats, digital recordings at a $1-\mathrm{KHz}$ sampling ratio, and a box size range of [30: 270] in the following sections of this article, unless otherwise mentioned.

\section{Box size}

The mDFA program generated by K. Tanaka was based on an article by Scafetta and Grigolini published in 2002 [20]. These authors pointed out that DFA does not reliably detect the distribution of the Lévy time series [20]. The mDFA program is intended to observe the scaling behavior over a wide range of SI values. DFA focuses on critical phenomena and an SI value near 1.0, which is a Gauss distribution time series. In the current study, there were significant differences in the computation results between the mDFA and Peng's DFA, especially when diseased hearts were examined. If mDFA was used instead of DFA, diseased hearts exhibited a high SI (SI= 1.4, Table 1). 
The idea of using a box in mDFA (and DFA) computations is presented in Figure 11 for 2000heartbeat interval time series obtained from a human subject. Seven clear irregular beats are evident, i.e., so-called premature ventral contractions (PVCs) or ectopic heartbeat. The four black bars indicate that the 2000-beat data set was divided into four boxes. Here, there are 500 beats in each box; therefore, the box size is 500. Every box always has an identical number of beats in any mDFA/DFA computation. In mDFA (not DFA) computations using a PC, the box size is changed cyclically from 10 to 1000 as shown in Figure 10.

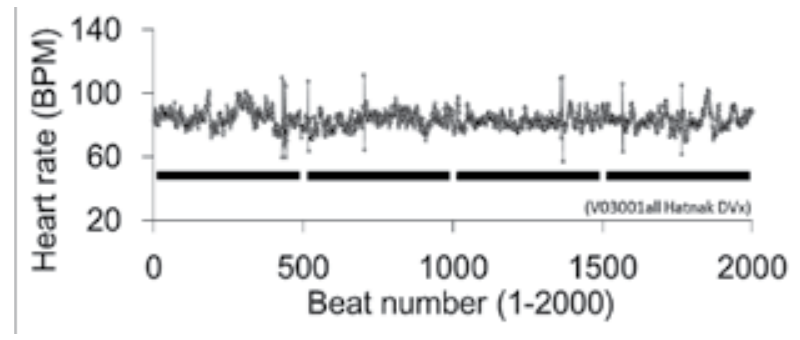

Figure 11. Interval time series and boxes.

\section{Arithmetic calculation and detrended data}

Here, the mDFA procedures are briefly shown. First, one can obtain heartbeat interval time series $\{X i\}$ and construct a "box" (Figure 12). Second, average heart rate $\langle X\rangle$ is computed (Figure 13). Third, the values $X i-<X>$ are computed (Figure 14). All data fluctuate around the zero line (see Figure 14).

Next, an integrated series (sigma) of all data fluctuating around the zero line $q_{i}=\sum_{k=1}^{i}\left(x_{k}-\langle x\rangle\right)$ can be obtained (Figure 15). Note the random walk-like steps. A regression line is computed for each box using a fourth order (biquadratic) polynomial (data not shown) (Figure 16). Then, the procedure is detrended by computing $s_{\mathrm{i}}=q_{\mathrm{i}}-q_{\mathrm{j}}$ (Figure 17).

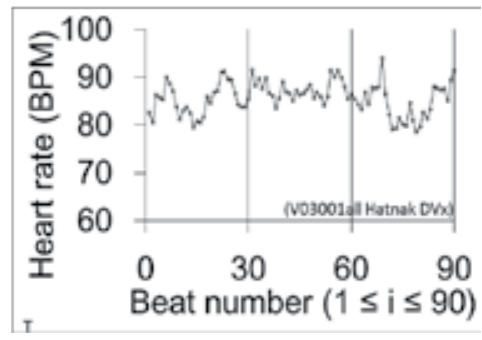

Figure 12. Heartbeat interval time series $\{X \mathrm{i}\}$ and boxes. Ninety beats from 2000 beats are shown. 


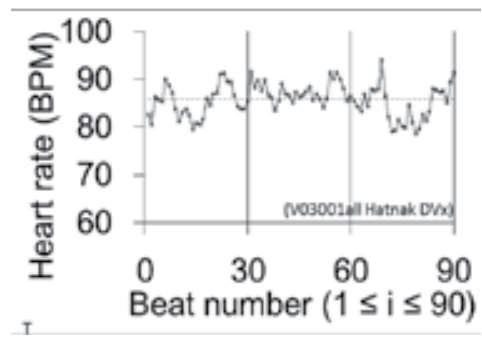

Figure 13. Average heart rate, i.e., $<X>$; note the dotted horizontal line.

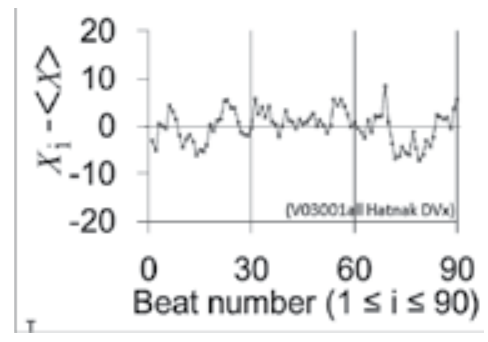

Figure 14. Average heart rate $\langle X>$ is deduced from $\{X i\}$, resulting in a time series fluctuation around zero.

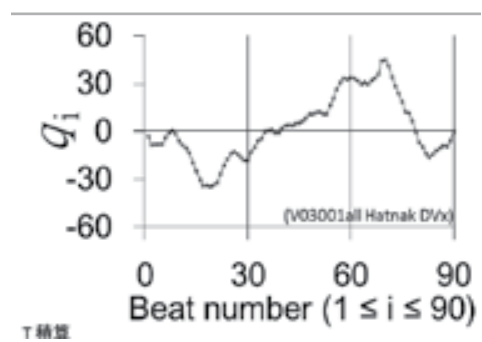

Figure 15. An integrated series, $\mathrm{q}_{\mathrm{i}}$.

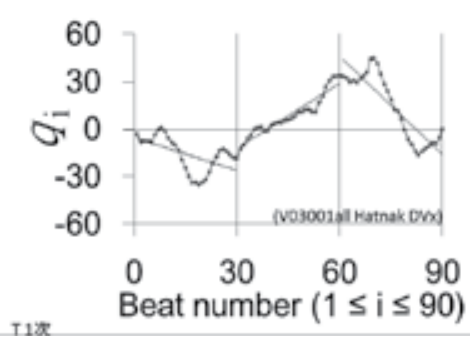

Figure 16. Regression lines, $\mathrm{q}_{\mathrm{j}}$. 


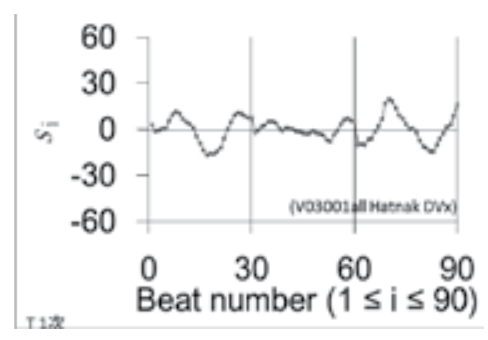

Figure 17. Explanation of detrended computations, $\mathrm{s}_{\mathrm{i}}=\mathrm{q}_{\mathrm{i}}-\mathrm{q}_{\mathrm{j}}$.

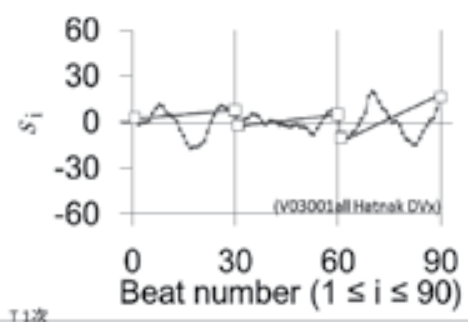

Figure 18. Computation of mDFA.

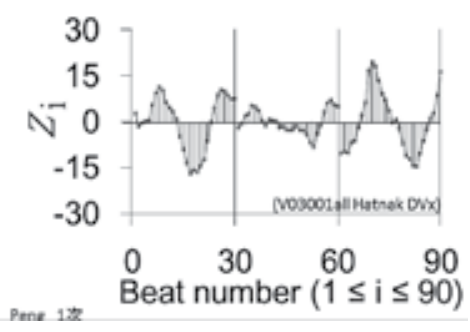

Figure 19. Peng's DFA showing the distance between each data point and the regression line.

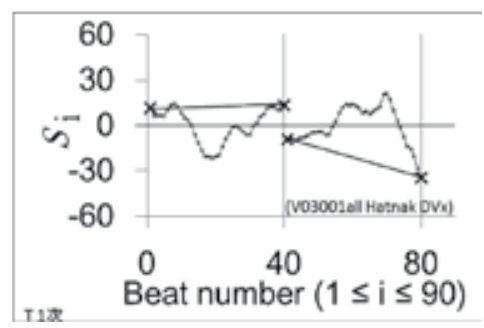

Figure 20. An example of mDFA with a box size of 40 .

Then, in mDFA (not DFA), the program calculates how many steps proceeded after traveling within a box (Figure 18). This is an mDFA-exclusive computation, which is not used in Peng's DFA. Peng's DFA program calculates the distance $\left(z_{\mathrm{i}}\right)$ between random walk-like lines $\left(y_{\mathrm{j}}\right)$ and 
regression lines $\left(y_{\mathrm{v}}\right)$ (note the numerous vertical lines in Figure 19) as $y_{\mathrm{j}}-y_{\mathrm{v}}$. The data presented in Figure 20 and 21 represent differences between mDFA and DFA with a box size of 40.

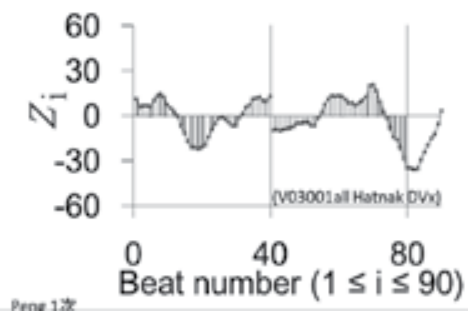

Figure 21. An example of Peng's DFA with a box size of 40 .

\section{Scaling}

Scaling graphs are constructed using the following procedures. The scaling exponent can be determined using an integrated series (Figure 15). The important variables are the number of boxes $(\mathrm{N} / \mathrm{n})$, where box size $(\mathrm{n})$ and interval data number $(\mathrm{N})$ are given by the integrated series. The box size (n) can be changed from 10 to 1000 in mDFA computations (see Figure 10). In addition, the variance, $\mathrm{F}(\mathrm{n})$ can be calculated for each box size (n). Then, the following graphs can be drawn: Logarithm (n) vs. Logarithm F(n), and linear regression lines across various box sizes (see Figure 22). These procedures was used to compute the scaling exponent SI (or Greek letter alpha, $\alpha$ ).

The equation used to determine the scaling exponent in Peng's DFA program (source code obtained from PhysioNet) is:

$$
F(n)=\sqrt{\frac{\sum_{k=1}^{N}\left(y_{k}-y_{k}^{\prime}\right)^{2}}{n}},
$$

where $y_{k}$ is the integrated series and $y_{k}^{\prime}$ is the regression line.

In the mDFA program, however, the following equation is used to determine the scaling exponent:

$$
S(n)=\sqrt{\frac{\sum_{j=0}^{\frac{N}{n-1}}\left\{\left(q_{j n+n}-q^{\prime}{ }_{j n+n}\right)-\left(q_{j n+1}-q^{\prime}{ }_{j n+1}\right)\right\}^{2}}{\frac{N}{n}}}
$$


where $\mathrm{F}(\mathrm{n}), \mathrm{S}(\mathrm{n}), q_{\mathrm{i}}$ and $q_{\mathrm{i}}^{\prime}$ are the same as Peng, but different letters are used for comparison between mDFA and DFA. Based on this equation, mDFA computations can be performed to determine the scaling exponents for practical use (see Figure 18 and Figure 20).

First, a graph of variance vs. box size (Figure 22) can be constructed, followed by determination of the scaling exponent (Figure 22 and Figure 23). For practical use of mDFA as a device, one can use the slope of [30:270] because the value is nearly always close to the average value. This condition was applied to most of the empirical results presented below.

In summary, in Peng's DFA, the SI is calculated from the variance, $\left\langle\left(\mathrm{x}_{\mathrm{i}}\right)^{2}\right\rangle$ and the random walk-like steps are considered. However, in the mDFA presented here, SI is calculated as $<\left(\mathrm{x}_{\mathrm{i}}\right.$ $\left.+j-x_{i}\right)^{2}>$. According to my physicist colleagues, the mDFA idea originates from a structure and function distribution and deals with scaling.

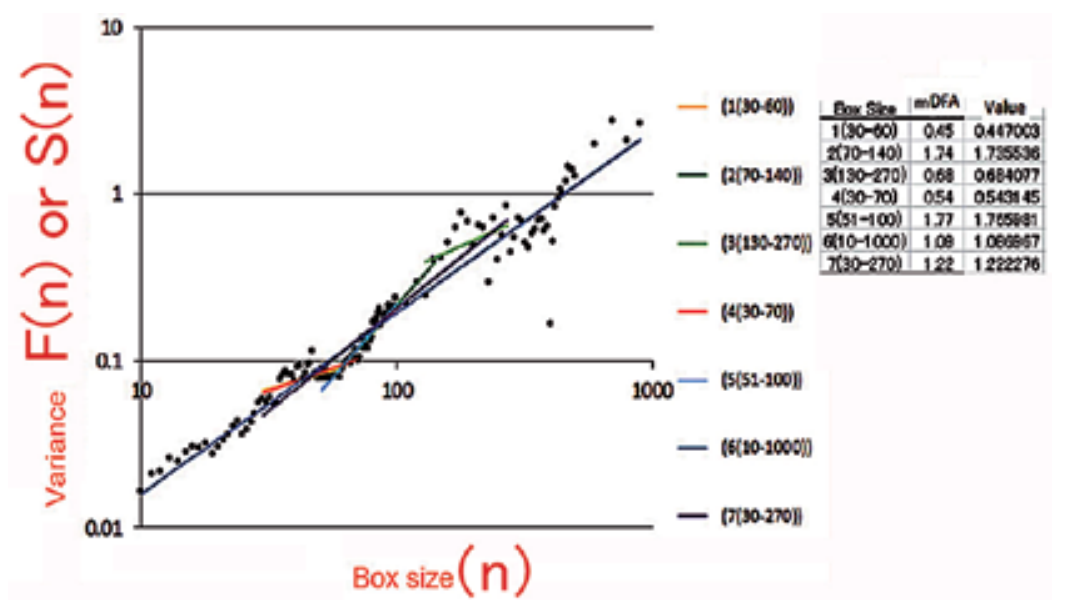

Figure 22. Determining SI in mDFA. Various SI values were computed simultaneously.

\begin{tabular}{lcc} 
Box size & \multicolumn{2}{c}{ Scaling exponent } \\
\hline $30-70$ & $\square$ & 1 \\
$70-140$ & 0.71 \\
$130-270$ & $\square$ & 1.21 \\
$51-100$ & 0.75 \\
$30-140$ & 0.91 \\
$30-270$ & $\square$ & 0.98 \\
\hline AVE & 0.92
\end{tabular}

Figure 23. Examples of SIs. 


\section{Stress quantification using mDFA}

Lobster EKGs are presented in Figure 24. The data presented in Figure 24A were recorded when specimens were in a non-stressful state when no human was present in the room. The data presented in Figure 24B were recorded during micro-dialysis blood sampling. The heartbeat interval time series obtained from the highlighted section is shown in Figure 25 for 578 beats. Under the no-stress condition, the lobster exhibited a dynamically changing pattern (Figure 25, red line) indicated by numerous spikes in the data. This is evidence that the heart received an inhibitory command from $\mathrm{CI}$. In contrast, the lobster under the MD stress condition did not exhibit such a dynamic pattern (Figure 25, black line). Inhibitory neural control was not evident, suggesting the stressed state is a state dominated by acceleratory neurons.

The scaling nature is expressed as a slope in the mDFA graph. The scaling nature of the no stress and MD stress states are presented in Figure 26. mDFA computation revealed a significant difference between the two states. Stress (caused by an approaching human) clearly decreased the slope (Figure 26 and 27). SI values of approximately 0.55 and 1.0 were obtained when a human did or did not approach the lobsters, respectively (Figure 27). These animal model experiments suggested that it may be possible to quantify human stress using the mDFA technique.
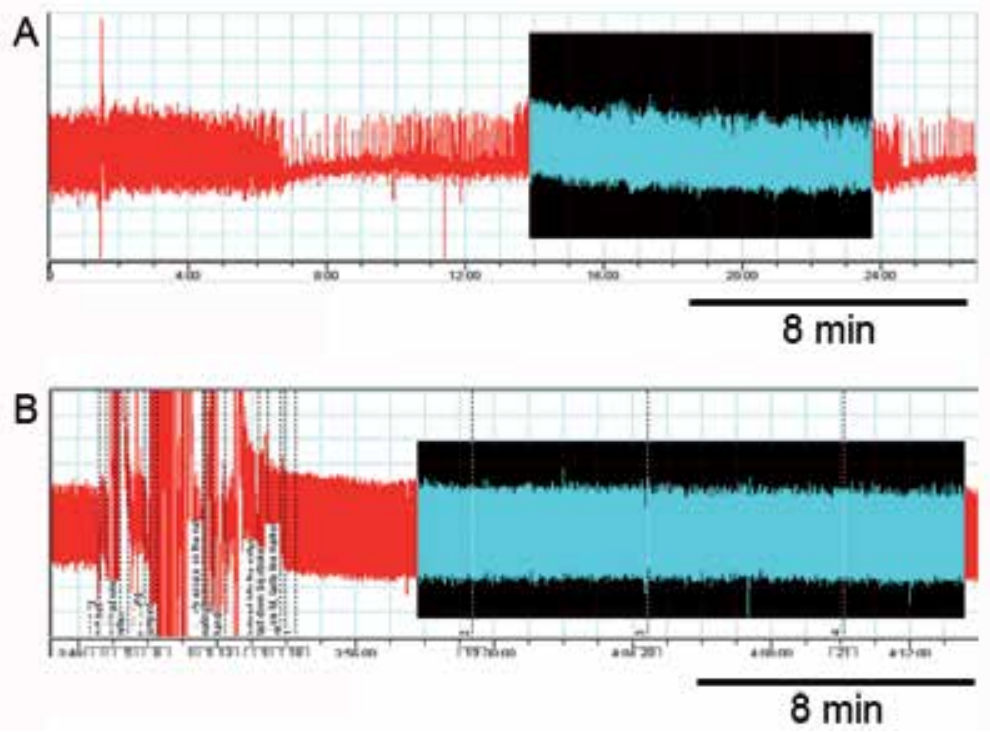

Figure 24. EKGs measured under no-stress (A) and MD-stress (B) conditions (these data were collected from the same lobster used in Figures 3 and 7). 


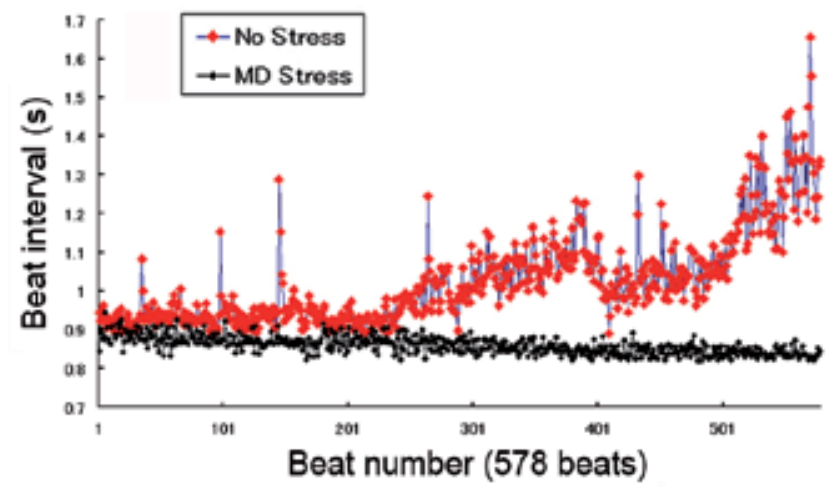

Figure 25. Interval time series obtained from data presented in Figure 24.
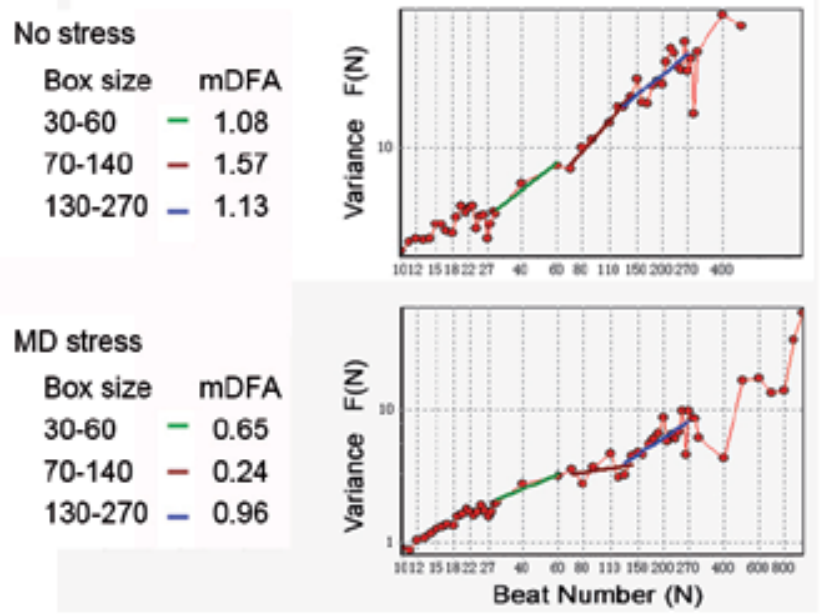

Figure 26. Results of mDFA (program A). Slope and SI for no-stress and MD stress conditions.

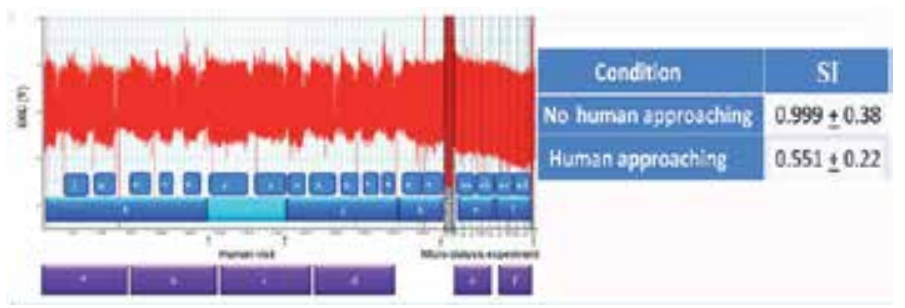

Figure 27. Scaling exponent (SI) computed from EKGs under no stress and MD stress conditions. SI computations from various time periods are shown by bars $(\mathrm{a}, \mathrm{b}, \mathrm{c},---\mathrm{ac}, \mathrm{ad})$. 


\section{Humans}

Human EKGs were analyzed using mDFA. Table 1 indicates considerable differences in SI values between stressed and non-stressed individuals. Stress significantly lowers SI. mDFA revealed that the President and Dean of the University had low SIs, whereas teaching-only professors had healthy SIs close to 1.0.

The Appendix in Table 1 shows that heart muscle injury (such as myocardial cell damage caused by ischemia or heart operations) can be identified using mDFA. People with a poor medical history have high SIs, considerably higher than 1.0. It is possible that the hearts of patients who suffered from cardiac disease and recovered through successful medical treatment may have scar-like non-muscle tissue; i.e., a fibrous matrix or debris following necrosis. This tissue might cause abnormal fluctuation of heartbeats, which can be detected by mDFA. Although there is no mathematical explanation as to why the SI values are so high, it is notable that mDFA was able to distinguish ischemic hearts from healthy ones. It is remarkable that injured hearts have exceptionally high SIs. In fact, they echo previous findings of heart damage in crabs and lobsters that died suddenly during experimentation (Presented at international conferences: Yazawa et al. Neurodynamical Control of the Heart of Healthy and Dying Crustacean Animals. CCCT2005 Proceedings Vol. 1, pp. 367-372; Best Papers of IIIS Conference WMSCI 2006, DFA on Cardiac Rhythm: Fluctuation of the Heartbeat Interval Contains Useful Information for the Risk of Mortality in Both Animal Models and Humans)

\begin{tabular}{|l|c|c|c|}
\hline \multicolumn{1}{|c|}{ Categories } & Age & Stress level (Interview) & SI \\
\hline Business owner (a company) No. 1 & 50 s, Male & Fairly low & 1.03 \\
\hline Business owner (a company) No. 2 & 50 s, Male & High & 0.72 \\
\hline Top management, President of a Univ. & 60s, Male & High & 0.84 \\
\hline Top management, Vice President of a Univ. & 40s, Female & High & 0.84 \\
\hline Middle management, Dean & 40s, Male & High & 0.72 \\
\hline Middle management, Secretary of president & 40s, Female & High & 0.76 \\
\hline Ordinary employee, Teaching only professor, No. 1 & 50 s, Male & Fairly low & 1 \\
\hline Ordinary employee, Teaching only professor, No. 2 & 50s, Female & Fairly low & 0.98 \\
\hline Table 1. Appendix: Cardiac disease & Age & Daily life & Sl \\
\hline Patient with stent-placement & 60, Male & OK & 1.26 \\
\hline Patient with bypass-surgery & 45, Male & OK & 1.38 \\
\hline Patient with inplantable cardioverter & 53, Male & OK & 1.22 \\
\hline Ventricular septal defect (20 y ago operation) & 48, Female & OK & 1.41 \\
\hline Healthy representative, housewife & 46, Female & OK & 1.03 \\
\hline
\end{tabular}

Table 1. Comparizon: Stress level and exponent value (Indonesia, 2012, working with Prof. A. Hutapea

The SI data presented in Table 1 (and explained in Figure 28) show that mDFA is able to detect job-related stress. Some of the subjects presented in Figure 28 are the same as those shown in Table 1. Interestingly, the people who had administrative and teaching-professor obligations were somewhat serious during the EKG measurements and exhibited low scaling exponents. However, teaching-only professors who appeared happy (they laughed and spoke during the 
EKG measurements) had healthy exponents with values near 1.0. It is likely that mDFA can quantify stress; however, additional empirical results on a greater number of samples are needed before a definite conclusion can be reached.

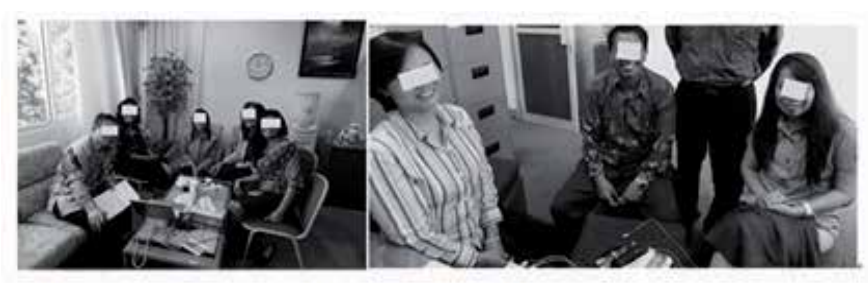

Job related stress (Indonesian University)
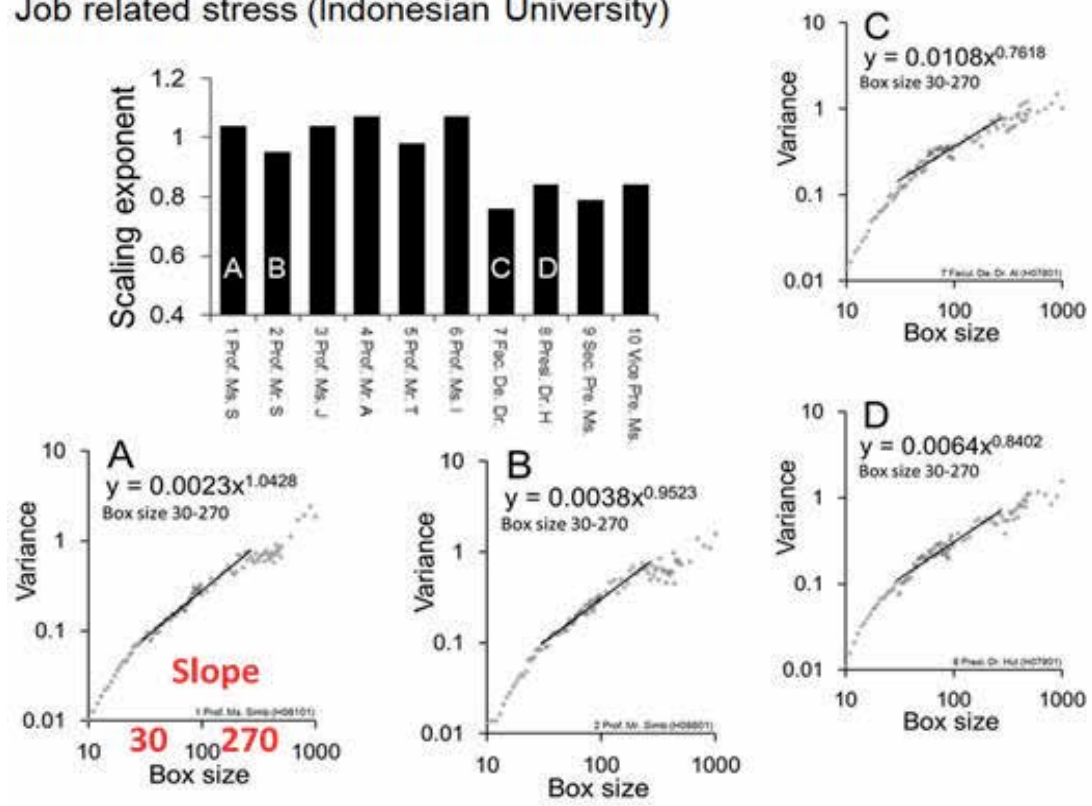

Figure 28. Job-related stress determined using mDFA (some are identical data shown in Table 1)

\section{The heart reflects neural activity: Individual stories}

A subject who volunteered for a long-term follow-up study had her EKG measured yearly under sitting and talking conditions, always on a September afternoon at approximately 3 PM. In 2006, she had a good SI and after that appeared normal in terms of mDFA. However, as can be seen in Figure 29, her SI changed dramatically over the years.

This subject did not disclose any personal issue until 2009 when she related that she experienced difficult conditions in her work place in 2007 and 2008 and wanted to relocate. In addition, she lost a loved one to illness in 2008 and left her office job at the end of 2009. Her 
environment improved greatly by 2010; her EKG was not measured in 2011, and she had a good SI in 2012 (data not shown).

Another subject was caring for her aging mother at home (Figure 30). The caregiver did not sleep from 28-29 September because her mother did not sleep. Consequently, the subject's SI was very low (3 PM on 29 September). However, she slept well the following two nights because her mother slept well. This subject visited my exhibition booth at Innovation Japan 2011.

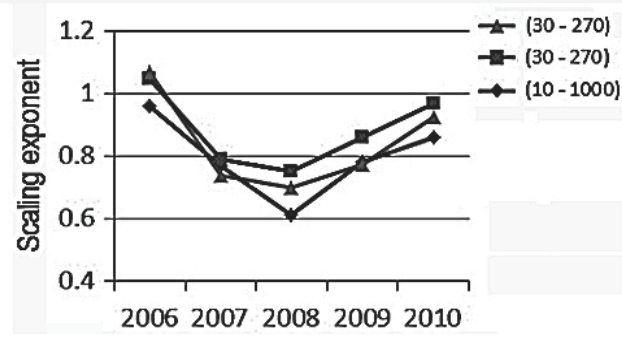

MDFA

A: 20061.1077

B: 20080.7119

C: 20090.7716
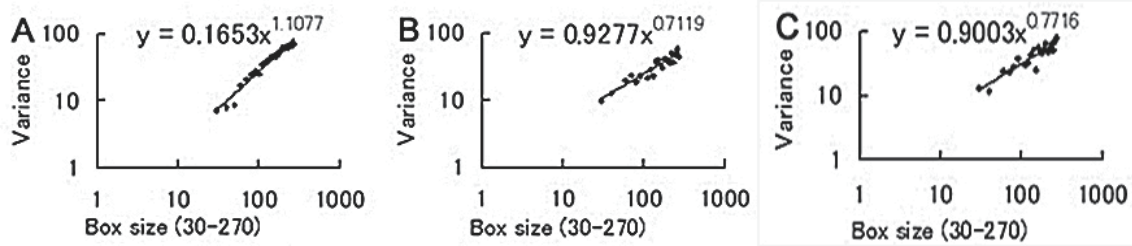

Figure 29. EKG data from a woman aged 27 years collected in September 2006 at 3 PM and five-year follow-up results.

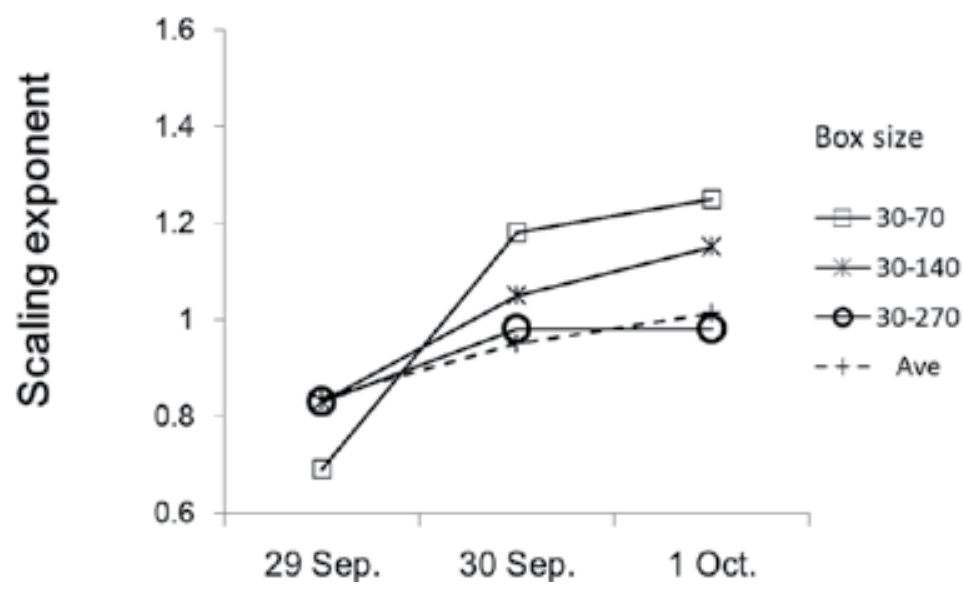

Figure 30. Sleep deprivation decreases SI. These data were collected from a woman in her 50s who was taking care of her aging mother. 


\section{A device}

I am currently collaborating with a company to construct a device equipped with a stablebaseline (meaning, the EKG trace is NOT lost from the recorder chart or screen) EKG amplifier and data logger. In the future, an mDFA program will be incorporated into the device. The device will instantaneously compute SI, and EKG data, mDFA results, and SI values will be stored on an SD memory card. All data will be transmitted to a PC through a web-based system (Figure 31).

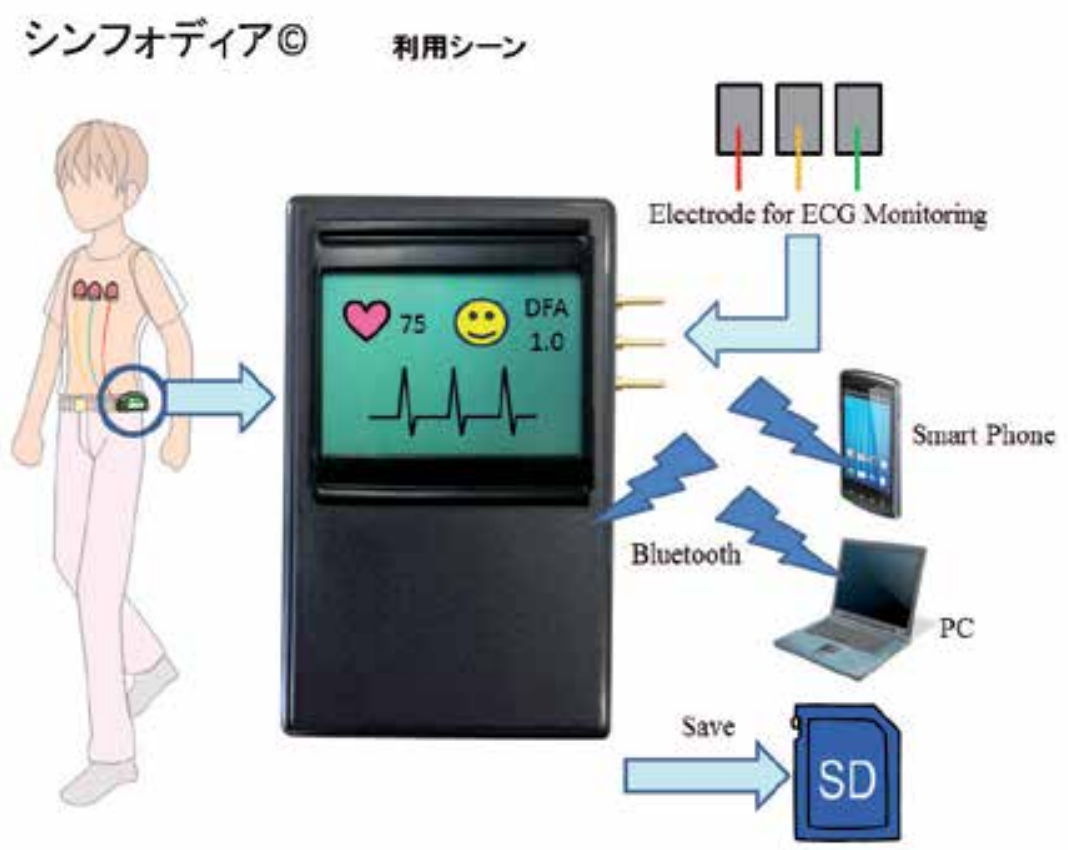

Figure 31. A device that records stable-baseline EKG (Symphodia Phil Confidential, Japan).

\section{Discussion}

Unique characteristics of mDFA are shown in Figure 18 and 20. In addition, the unique mDFA computation is specified in equation (2); DFA does not use this procedure.

mDFA calculates SI using a method that includes preparation of time series data, removing trends from the data, determining statistical variations, constructing a graph of $F(n)$ vs. (n), where $\mathrm{F}(\mathrm{n})$ is variance and $(\mathrm{n})$ is box size, and determining the slope, i.e., SI. In order to 
construct a gadget that rapidly calculates SI, I determined an appropriate scaling zone where a straight line and a regression line were drawn using the least-squares method; this zone is [30: 270] in beat per min (BPM).

Until now, a DFA-user must determine the box range before drawing a regression line to obtain the SI. I determined the best range to compute SI automatically is [30: 270]. Biological systems do not operate optimally because ranges that are too small or too large are avoided. As a biomedical tool, however, mDFA uses a special box-range [30: 270]. Currently, people can construct their own device to analyze their own cyclic phenomena. The device would automatically return SI values when people include time series data, and SI could be computed for any time series interval.

The time length of 270 heartbeats in the [30: 270] box range corresponds to $270 \mathrm{sec}$ if the heart beats at a steady ratio of $60 \mathrm{BPM}$, which is equivalent to $3-4 \mathrm{~min}$. This time length has a particular significance with respect to neuroscience, because internal biochemical reactions can retain a steady state for this length of time. I can wait for three minutes before instant noodles are cooked. A boxer fights with full power for three minutes. The neuron network functions under the three-minute law. Short-term memory and acute stress, for example, can last for three minutes but they can also decline after three min. The length [30: 270] (three min) could be a biologically important number.

\title{
15. Conclusion
}

mDFA and SI provide useful information. mDFA can be incorporated in the future into a device for checking health and stress levels. It is possible that anyone could construct their own mDFA program for developing such a device.

\section{Acknowledgements}

This work was supported by JSPS Grants No. 23500524 and No. 2635050. I thank DVX Inc. Tokyo, Japan, for financial support for research, No. 4DQ404, for 2012, 2013, and 2014.

\section{Author details}

\author{
Toru Yazawa*
}

Address all correspondence to: yazawa-tohru@tmu.ac.jp

Tokyo Metropolitan University, Japan 


\section{References}

[1] T. Yazawa, K. Kuwasawa, Intrinsic and extrinsic neural and neurohumoral control of the decapod heart, Experientia 48 (1992) 834-840.

[2] T. Yazawa, T. Katsuyama, Spontaneous and repetitive cardiac slowdown in the freely moving spiny lobster, Panulirus japnicus, Journal of Comparative Physiology Part A 187 (2001) 817-824.

[3] R. E. Young, Correlated activities in the cardioregulator nerves and ventilatory system in the norwegian lobster, Nephrops norvegicus (L.).Comparative Biochemistry and Physiology Part A: Physiology 61(1978) 387-394.

[4] L. H. Field, J. L. Larimer, The cardioregulatory system of crayfish: neuroanatomy and physiology. Journal of Experimental Biology 62 (1975) 519-530.

[5] T. Yazawa, K. Kuwasawa, The cardio-regulator nerves of the hermit crabs: anatomical and electrophysiological identification of their distribution inside the heart. Journal of Comparative Physiology Part A 154 (1984) 871-881.

[6] A service of the U.S. National Library of Medicine. http://ghr.nlm.nih.gov/gene/ NKX2-5 (accessed Sep 20 2014\%).

[7] C.-K. Peng, S. Havlin, H. E. Stanley, A. L. Goldberger, Quantification of scaling exponents and crossover phenomena in nonstationary heartbeat time series, Chaos 5 (1995) 82-87.

[8] T. Yazawa, Crustacean stress behavior. In: T. Hariyama, T. Shimozawa (eds.) Insect Mimetics. Advanced Biomimetics Series 3, NTS, Tokyo, Japan (2008) 542-548 (in Japanese).

[9] A. L. Goldberger, B. J. West, Application of nonlinear dynamics to clinical cardiology. Annals of the New York Academy of Science 504 (1987) 195-231.

[10] A. A. Cecen, C. Erkal, Nonlinear dynamical modeling in ECG analysis: A heuristic guideline. Nonlinear Dynamics, Psychology, and Life Sciences 12 (2008) 359-369.

[11] C.-K. Peng, S. Havlin, J. M. Hausdorff, J.E. Mietus, H.E. Stanley, A. L. Goldberger, Fractal mechanisms and heart rate control: Long-range correlations and their breakdown with disease. In: Rienzo M Di, et al. (eds.) Frontiers of blood pressure and heart rate analysis. IOS Press, Amsterdam, Netherlands (1997) 3-14.

[12] L. Glass, Dynamical disease-The impact of nonlinear dynamics and chaos on cardiology and medicine. In: C. Grebogi et al. (eds.) The impact of chaos on science and society. United Nations University Press, Tokyo (1997) 219-231.

[13] T. Stadnitski, Some critical aspects of fractality research. Nonlinear Dynamics, Psychology, and Life Sciences 16 (2012) 137-158. 
[14] H. E. Stanley, Phase transitions. Power laws and universality, Nature 378 (1995) 554.

[15] T. Katsuyama, Scaling analysis of unstable fluctuation of heartbeat in spiny lobster. Numazu College of Technology Report 38 (2004) 189-194 (in Japanese).

[16] O. B.-Perez, J. M. de Sa, J. L. R.-Alvarez, R. G.-Esteban, Changes in detrended fluctuation indices with aging in healthy and congestive heart failure subjects. Computers in Cardiology, 35 (2008) 45-48.

[17] L. S. Liebovitch, T. Penzel, J. W. Kantelhardt, Physiological relevance of scaling of heart phenomena, In: A. Bunde et al. (eds) The science of disasters: climate disruptions, heart attacks, and market crashes, Springer (2002) p331-352.

[18] H. V. Huikuri, T. H. Mäkikallio, C.-K. Peng, A.L. Goldberger, U. Hintze, M. Møller, Fractal correlation properties of R-R interval dynamics and mortality in patients with depressed left ventricular function after an acute myocardial infarction, Circulation 101 (2000) 47-53.

[19] J. T. Bigger Jr, R. C. Steinman, L. M. Rolnitzky, J. L. Fleiss, P. Albrecht, R.J. Cohen, Power law behavior of R-R interval variability in healthy middle-aged persons, patients with recent acute myocardial infarction, and patients with heart transplants. Circulation 93 (1996) 2142-2151.

[20] N. Scafetta, P. Grigolini, Scaling detection in time series: Diffusion entropy analysis. Physical Review E 66 (2002) 036130-1-10. 



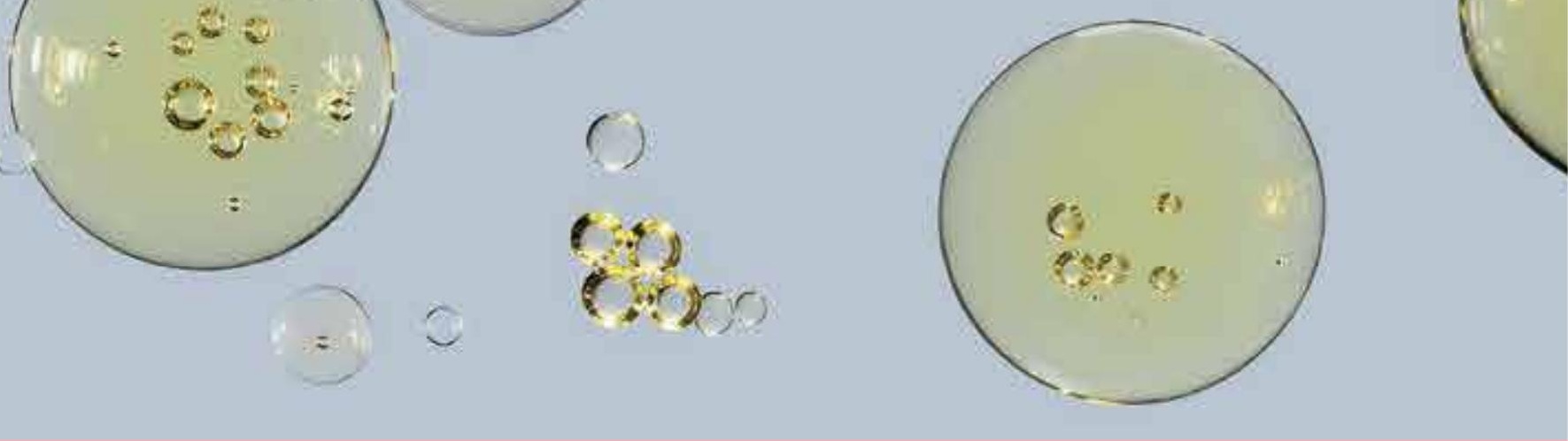

\section{Edited by Pier Andrea Serra}

The technological approach and the high level of innovation make bioengineering extremely dynamic and this forces researchers to continuous updating. It involves the publication of the results of the latest scientific research. This book covers a wide range of aspects and issues related to advances in bioengineering research with a particular focus on innovative technologies and applications. The book consists of 13 scientific contributions divided in four sections: Materials Science; Biosensors. Electronics and Telemetry; Light Therapy; Computing and Analysis Techniques.

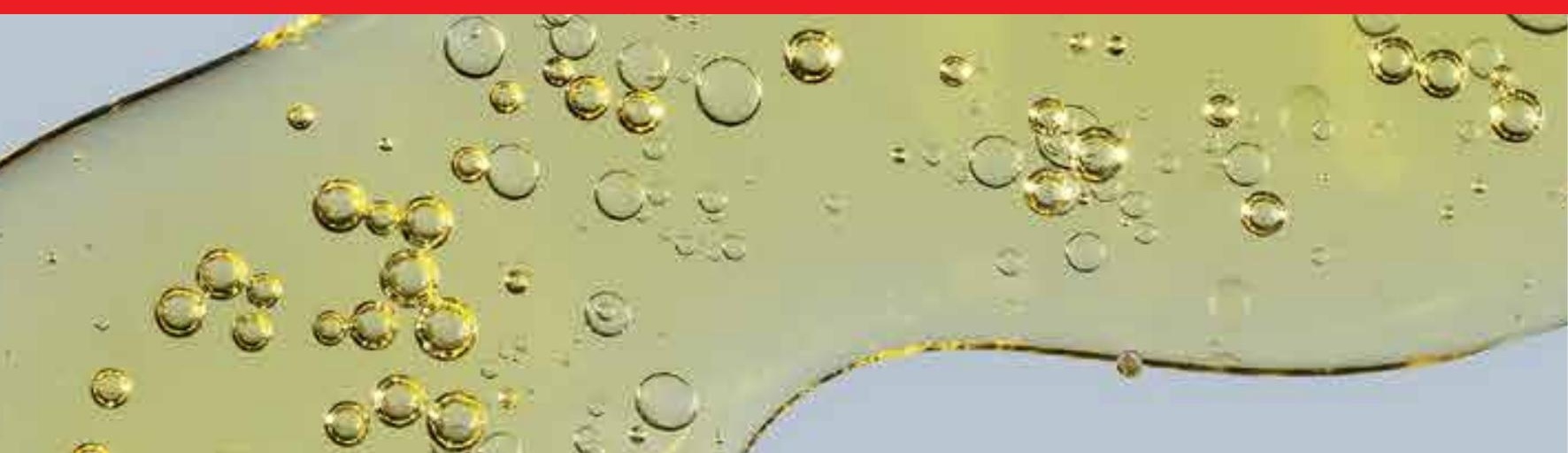

